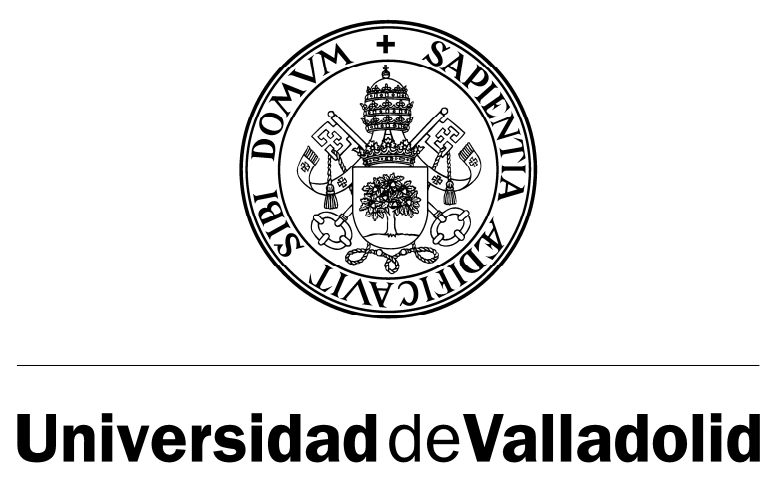

ESCUELA TÉCNICA SUPERIOR DE INGENIEROS INDUSTRIALES

DEPARTAMENTO DE CONSTRUCCIONES ARQUITECTÓNICAS, INGENIERÍA DEL TERRENO Y MECÁNICA DE LOS MEDIOS CONTINUOS

Y TEORÍA DE ESTRUCTURAS. AULA UVA-CIMNE

TESIS DOCTORAL

\title{
ANÁLISIS DE VIBRACIONES AEROELÁSTICAS EN ESTRUCTURAS ESBELTAS SOMETIDAS A CARGAS DE VIENTO
}

Presentada por Ali Vasallo Belver para optar al grado de doctor por la Universidad de Valladolid

Dirigida por:

Dr. Antonio Foces Mediavilla

Dr. Antolín Lorenzana Ibán 

A Patricia, mi prometida 

Agradecimientos

Deseo expresar mi más profunda y sincera gratitud al Profesor José Antonio Garrido García por haberme dado la posibilidad de realizar esta Tesis Doctoral e iniciado en los caminos de la investigación científica.

A mis directores de Tesis, el Dr. Antonio Foces Mediavilla y el Dr. Antolín Lorenzana Ibán, por su orientación científica, colaboración y apoyo durante la realización de la misma.

Al Dr. Eugenio Oñate Ibáñez por haberme acogido en el Centro Internacional de Métodos Numéricos en Ingeniería, lugar donde se inició este trabajo y al Dr. Riccardo Rossi por su inestimable ayuda.

A José Perada y a Mariano Cacho, compañeros del Departamento, por su disposición y en los que siempre he encontrado compañerismo y ayuda.

A mis padres y a mi hermano, en general, a toda mi familia y a mis amigos por su apoyo y paciencia durante todos estos años.

A Patricia, mi prometida, a quien dedico la Tesis, por su comprensión, confianza en mi y haber estado a mi lado en todo momento. Muchas gracias. 



\section{Resumen}

En este trabajo se estudian las vibraciones inducidas por el desprendimiento alternado de torbellinos en estructuras cilíndricas tales como chimeneas industriales y mástiles usando un algoritmo de acoplamiento en el contexto de los elementos finitos. Para ello, se resuelven las ecuaciones de Navier-Stokes en 2D para flujo incompresible alrededor de la sección transversal junto con las ecuaciones del movimiento de la estructura. Para resolver el campo fluido y poder determinar las cargas (presiones) sobre el contorno se utiliza un método de paso fraccionado de segundo orden con segregación de la presión. El movimiento del dominio del fluido es tenido en cuenta mediante una formulación ALE (arbitrariamente Lagrangiana-Euleriana). La estabilización de los términos de convección y difusión se consigue mediante subescalas ortogonales y se asume que la variación temporal de las subescalas es despreciable. En la simulación no se incluye ningún modelo de turbulencia. Para la resolución de la estructura se considera un modelo monodimensional de barras de sección de pared delgada, de forma que la estructura es modelada a través de elementos tipo barra. En cada instante, las presiones sobre el contorno en cada plano analizado se integran y se interpolan para determinar las cargas por unidad de longitud que excitan a la estructura. El problema dinámico se resuelve mediante el método estándar de Newmark de segundo orden. Para cada instante de tiempo se resuelve el fluido y se realiza el análisis dinámico de la estructura, a la vez que se actualiza la geometría de la malla de cada plano de fluido de acuerdo con el movimiento de la estructura. La técnica propuesta se valida mediante ejemplos en los que se consiguen obtener los resultados numéricos esperados tanto para estructuras rígidas como para otras más flexibles. En estas últimas, los movimientos resultantes son apreciables y afectan al modo en el que el viento excita a la estructura, produciéndose cierta interacción que da lugar a fenómenos aeroelásticos denominados de lock-in o sincronización y de amplificación del coeficiente de arrastre. Estos fenómenos experimentales son reproducidos con el método numérico propuesto. 


\begin{abstract}
Flow-induced vibrations of line slender structures such as chimneys, cables or masts have been studied using a simplified fluid-structure interaction algorithm. Navier-Stokes equations for incompressible flow have been solved in $2 \mathrm{D}$ in several transverse planes of the line-like structure. Afterwards, the resultant pressure field was introduced using standard FEM interpolation techniques, together with the dynamical behaviour of the structure and its boundary conditions. A fractional step scheme, which is based on the second order pressure splitting scheme, has been used to solve the fluid field. In each fluid plane, the displacements have been taken into account considering an Arbitrary Lagrangian Eulerian approach (ALE). The stabilization of convection and diffusion terms has been achieved by means of orthogonal subscales (OSS) assuming quasi-static ones, that is, the temporal variation of subscales is negligible. In this simulation, no turbulence model has been included. In order to solve the structural problem, a monodimensional element for thin wall section beam has been considered, employing several elements for modelling the whole structure. The standard second order Newmark method has been used to include the dynamical behaviour equations. The wind has been considered to be an uncompressible fluid acting on the structure in a series of planes (cells of vortex) that are independent among them, and perpendicular to the structure direction. For each period of time, the fluid problem was solved, and the aeroelastic analysis was carried out, and at the same time the geometry of the mesh of each fluid plane was updated according to the structure displacements. To illustrate the applicability of the technique described above, an example of the response of a cantilever beam subjected to the wind action is shown in a range where vibration behaviour is critical, resulting in lock-in and drag amplification phenomena.
\end{abstract}




\section{Índice}

\section{Capítulo 1. Introducción}

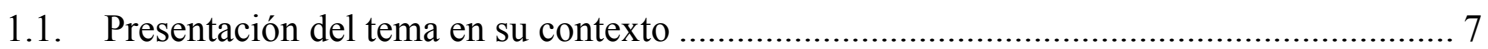

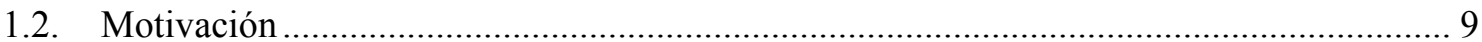

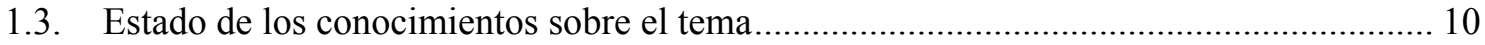

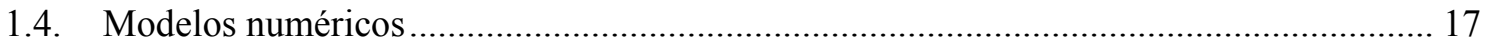

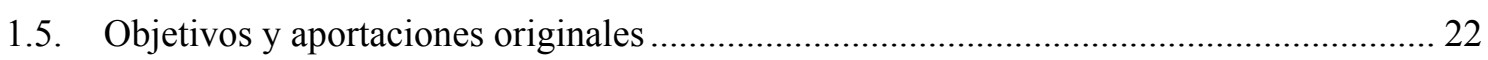

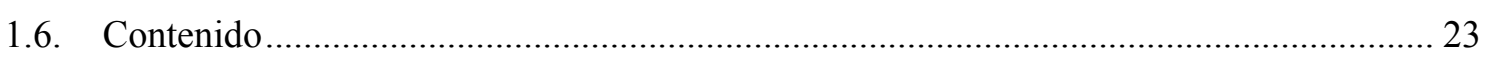

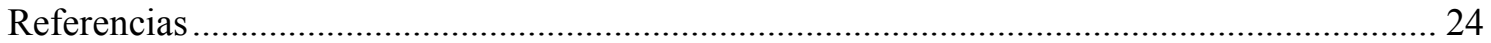

\section{Capítulo 2. Fenómenos Aeroelásticos}

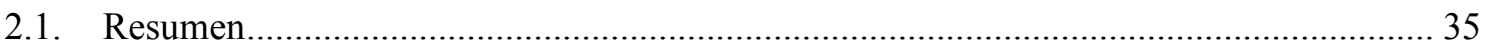

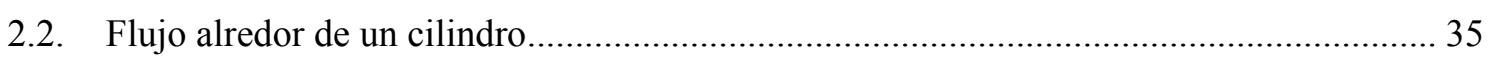

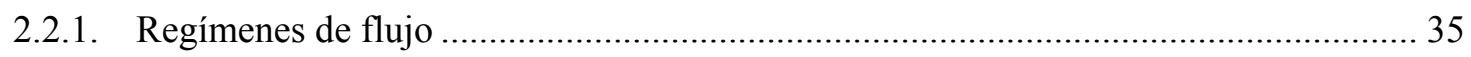

2.2.2. Variación de los coeficientes de fuerza en función del número de Reynolds............... 39

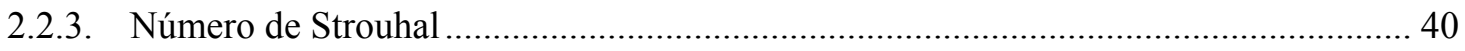

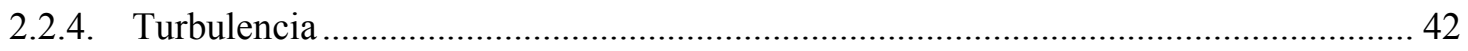

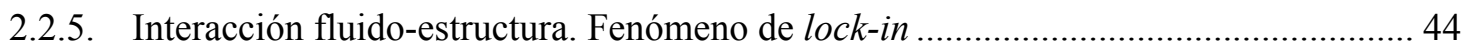

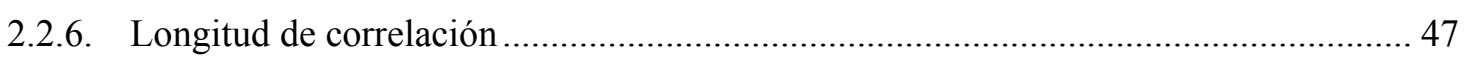

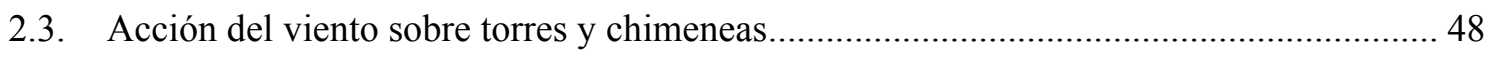

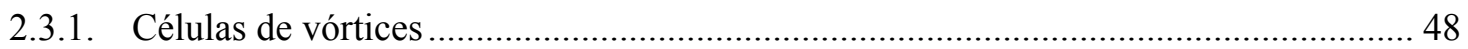

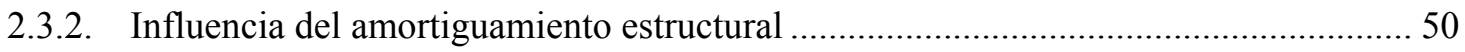

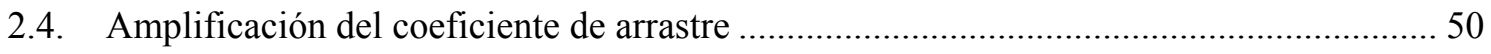

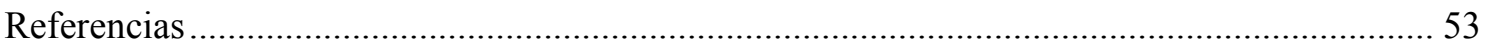

\section{Capítulo 3. Formulación Teórica del Modelo de Barras}

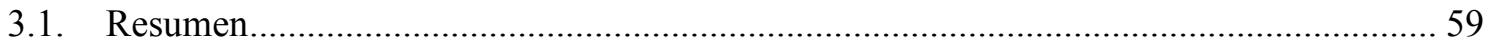

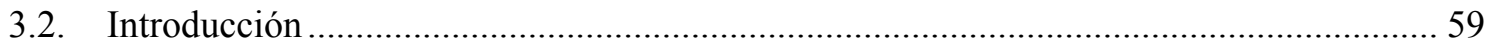

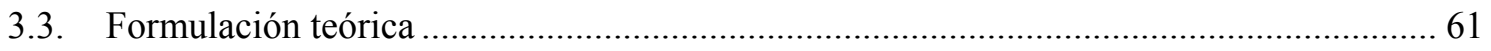

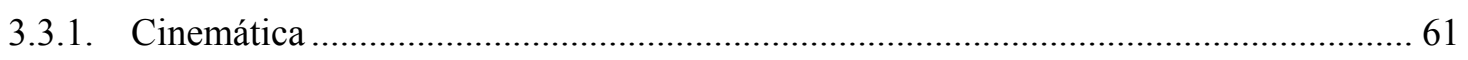

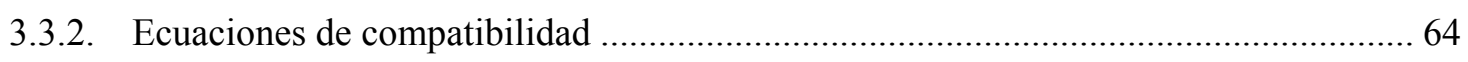

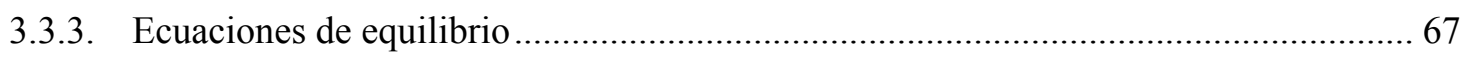

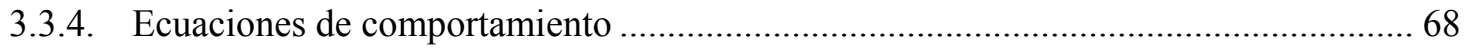

3.4. Análisis mediante el método de los elementos finitos ....................................................... 71

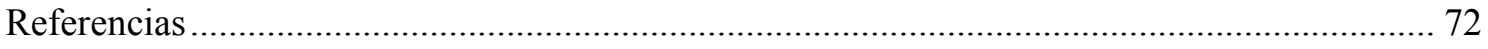




\section{Capítulo 4. Problema Dinámico}

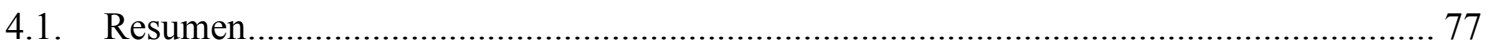

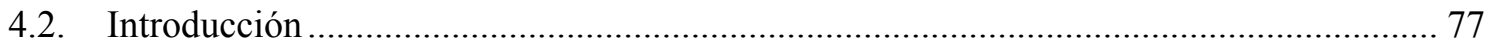

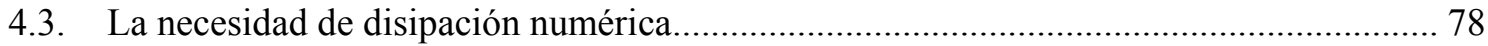

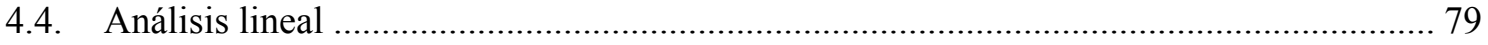

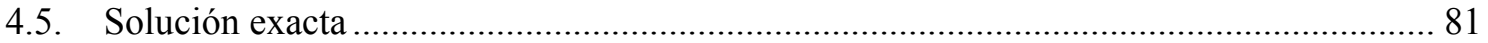

4.6. Uso de métodos numéricos para la resolución de EDO de orden alto .............................. 81

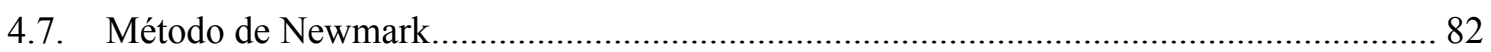

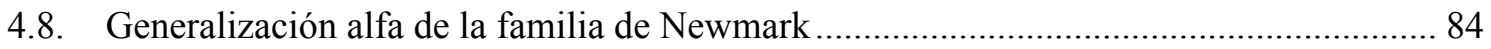

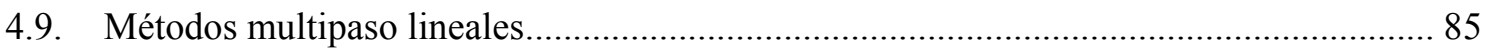

4.10. Otras Técnicas para abordar el problema de la precisión. Método de Ecuaciones

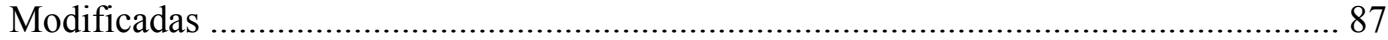

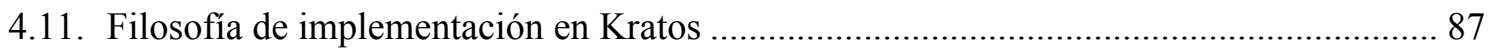

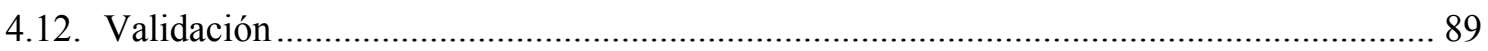

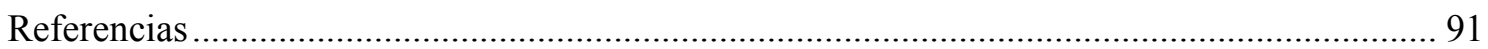

\section{Capítulo 5. Mecánica de Fluidos Computacional}

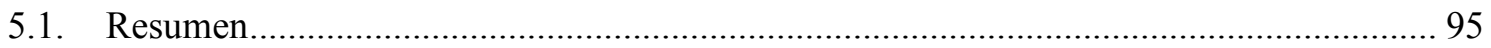

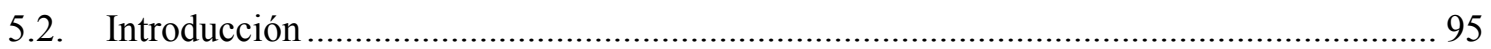

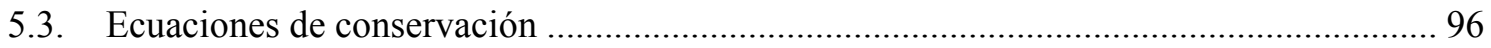

5.3.1. Ecuación de conservación de la masa ............................................................................. 97

5.3.2. Ecuación de conservación de la cantidad de movimiento............................................. 97

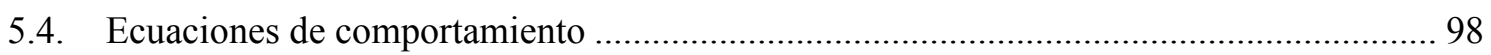

5.5. Ecuaciones de Navier-Stokes. Flujo incompresible _.......................................................... 100

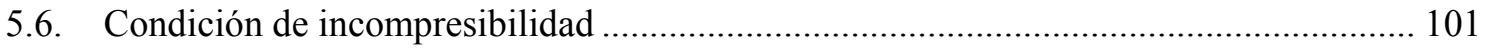

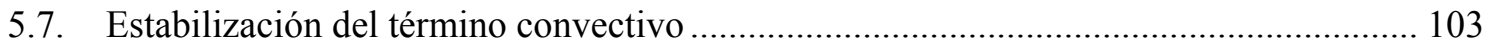

5.7.1. Técnicas de estabilización Streamline Upwind Petrov-Galerkin y Galerkin Least

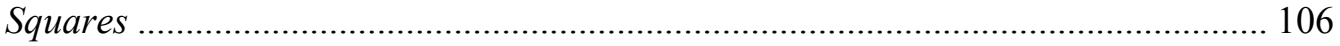

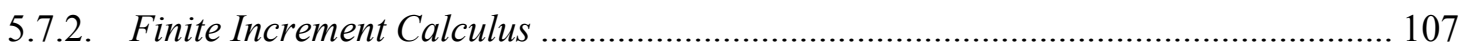

5.7.3. Variational Multiscale Methods ............................................................................... 108

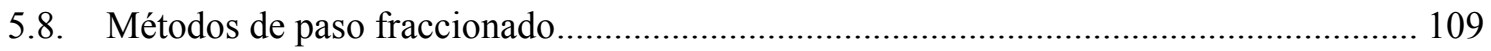

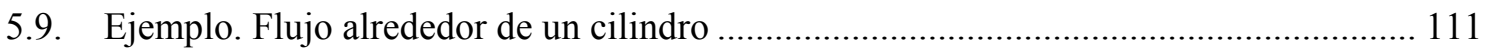

5.10. Formulación arbitrariamente Lagrangiana-Euleriana .................................................... 115

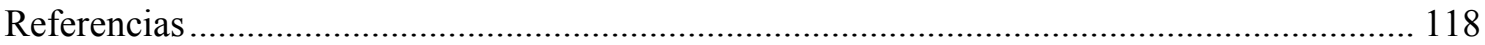




\section{Capítulo 6. Acoplamiento Fluido-Estructura}

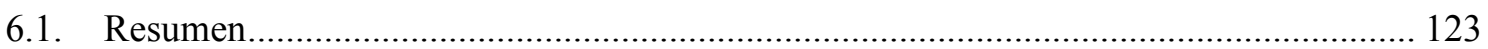

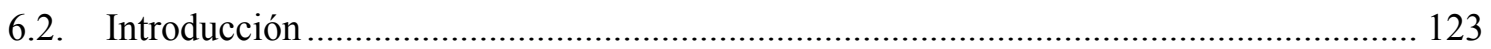

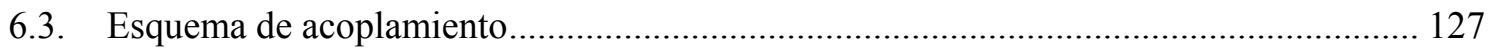

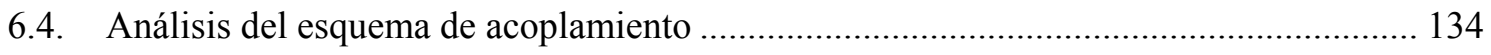

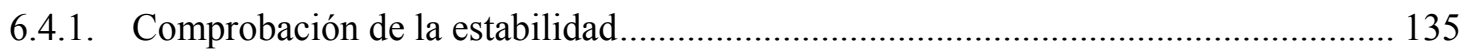

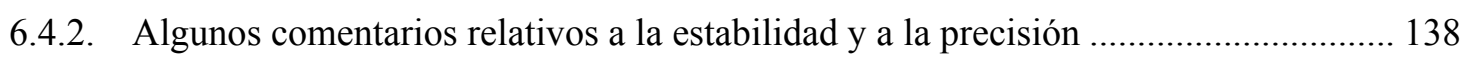

6.4.3. Análisis del algoritmo de acoplamiento de paso fraccionado ...................................... 140

6.4.4. Solución discreta del problema acoplado exacto ....................................................... 141

6.4.5. Solución aproximada del problema acoplado ........................................................... 142

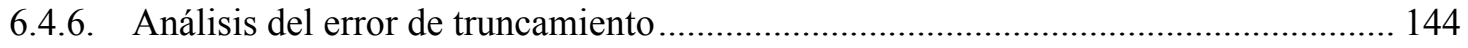

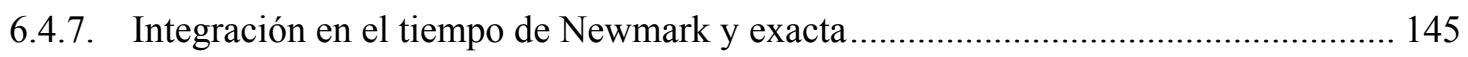

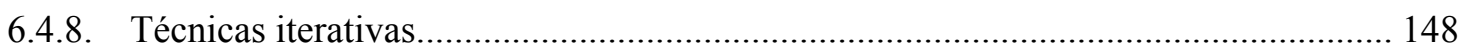

6.4.9. Uso de algoritmos disipativos para la integración estructural .................................. 153

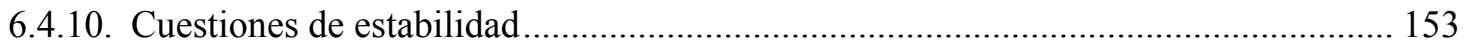

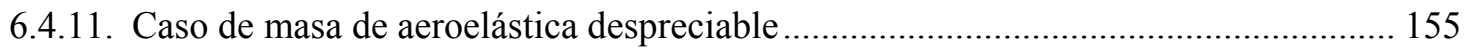

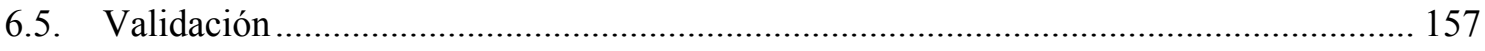

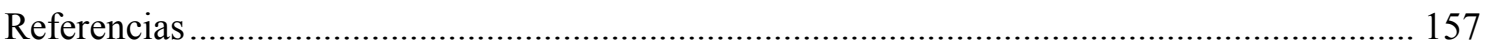

\section{Capítulo 7. Ejemplos}

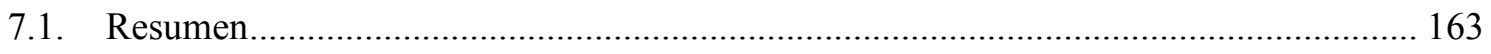

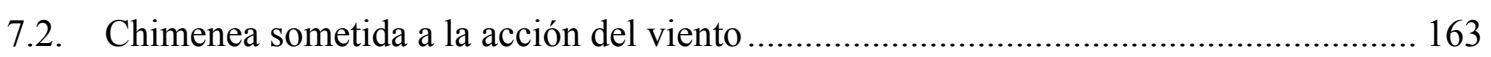

7.3. Estudio del número de planos de fluido en la respuesta transversal de un mástil de

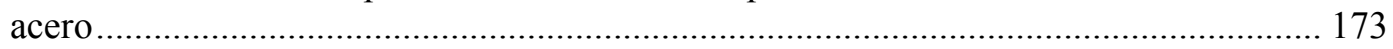

7.4. Mástil de acero. Estudio del fenómeno de lock-in ........................................................ 183

7.5. Mástil de acero. Estudio de la amplificación del coeficiente de arrastre .......................... 195

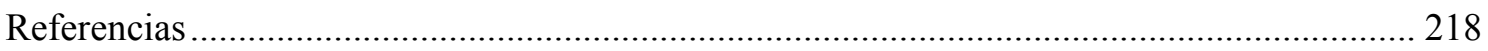

\section{Capítulo 8. Conclusiones y Líneas Futuras}

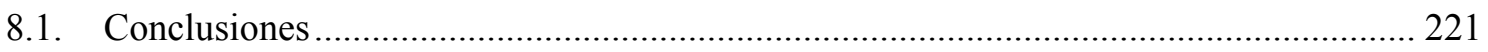

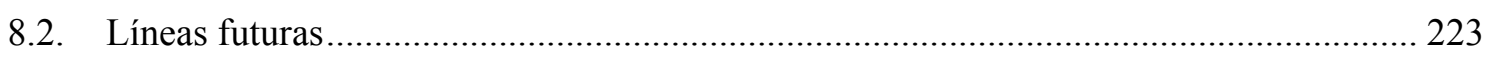


Anexos

Anexo A. Derivadas de los vectores unitarios 227

Anexo B. Derivadas de las componentes del radio vector ............................................................ 229

Anexo C. Centro de esfuerzos cortantes y área sectorial .......................................................... 231

Anexo D. Ecuaciones de compatibilidad de una lámina ............................................................. 235

Anexo E. Ecuaciones de equilibro de una lámina ..................................................................... 239

Anexo F. Ecuaciones de comportamiento de un laminado ....................................................... 245

Anexo G. Ejemplos. Modelo de Barras................................................................................. 253

G.1. Ejemplo 1. Sección en doble T y en T....................................................................... 253

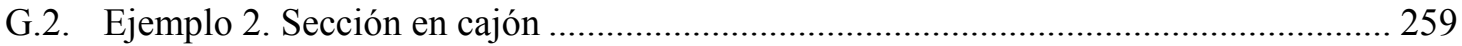

G.2.1. Configuración antisimétrica (CUS) ..................................................................... 260

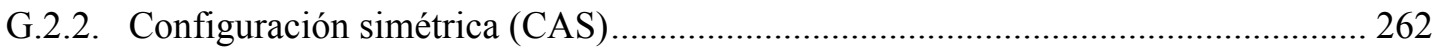

Anexo H. Filosofía de implementación. Núcleo de resolución................................................... 265

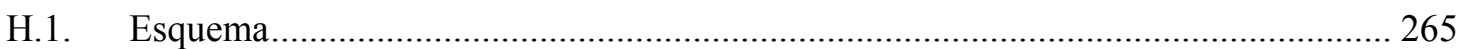

H.1.1. Esquema predictor-corrector estático ………..................................................... 265

H.1.2. Esquema predictor-corrector de Newmark.............................................................. 266

H.2. Ensamblador-Solver (BuilderAndSolver) ............................................................. 267

H.2.1. ResidualBasedElminationBuilderAndSolver ........................................................ 268

H.2.2. ExtLoadBasedElminationBuilderAndSolver ........................................................ 268

H.2.3. ExtLoadBasedDiagonalSpringBuilderAndSolver .................................................. 269

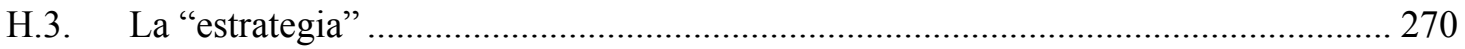

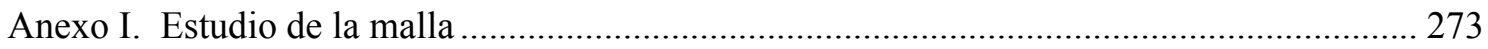

Anexo J. Amplificación del coeficiente de arrastre ................................................................. 283

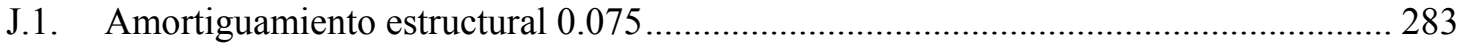

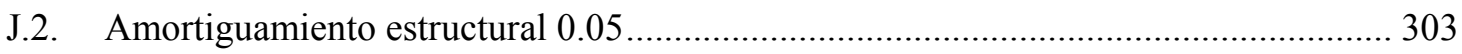

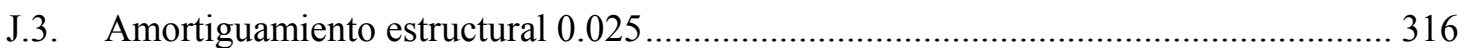

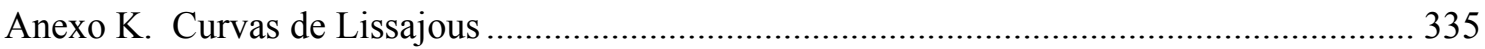

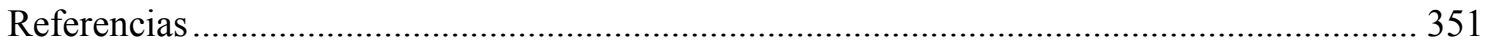


Capítulo 1

\section{Introducción}





\subsection{Presentación del tema en su contexto}

Las vibraciones inducidas por el desprendimiento de torbellinos en estructuras tales como chimeneas, mástiles, torres o puentes son un problema de interés práctico para muchos campos de la ingeniería. Aunque raramente pueden llegar a provocar el colapso inmediato de la estructura, con el tiempo pueden reducir su vida útil por fatiga.

En el caso de chimeneas o mástiles, el viento es un tipo de acción que induce flexión sobre este tipo de estructuras. Comportamientos anómalos caracterizados por vibraciones excesivas debidas al viento han sido descritos por varios autores $[1,2,3]$. En el caso de torres metálicas, en general ligeras y con bajo amortiguamiento, las vibraciones son lo suficientemente fuertes y frecuentes como para causar un acúmulo de daño en la estructura. Las torres de hormigón son, en general, más rígidas que las metálicas y en los dos casos los esfuerzos originados debido a la acción del viento son determinantes en el proyecto.

Se distinguen dos tipos de acciones generadas por el viento: aerodinámicas y aeroelásticas. Las acciones denominadas aerodinámicas están caracterizadas por la incidencia del viento sobre la estructura de forma que las fuerzas resultantes no dependen del movimiento de la misma. Por otra parte, las acciones aeroelásticas ocurren cuando el viento incide sobre una estructura en movimiento dando lugar a una interacción entre los movimientos de la estructura y el fluido, generando fuerzas dependientes del movimiento de la estructura. La interacción fluido-estructura puede ocasionar oscilaciones de carácter divergente o no, en función de las llamadas fuerzas auto-excitadas.

Los fenómenos aeroelásticos más importantes descritos en ingeniería de estructuras según Simiu y Scanlan [4] son el desprendimiento de torbellinos (vortex shedding), el galope transversal (galloping), el galope inducido por una estela (wake galloping), el flameo (flutter) y el bataneo (buffeting).

El fenómeno aeroelástico ocasionado por la generación y desprendimiento de torbellinos se debe a la separación del flujo de aire por la presencia de un obstáculo y se caracteriza por el desprendimiento periódico de torbellinos con sentido de rotación alternado llamados vórtices de Von Kármán. El mencionado desprendimiento de torbellinos genera unas fuerzas transversales a la dirección del flujo de aire sobre la estructura cuyo sentido se va alternando, las cuales son la causa de las vibraciones transversales típicas de este fenómeno aeroelástico. Una particularidad del desprendimiento de torbellinos es el fenómeno de lock-in $[5,6,7,8]$ que se produce cuando la frecuencia a la que se desprenden los torbellinos está próxima a la frecuencia natural de la 
estructura en la dirección transversal al flujo de aire. En esta situación, el desprendimiento de torbellinos pasa a estar controlado por la frecuencia mecánica y la amplitud de la oscilación es máxima, pero autolimitada.

El galope transversal genera movimientos de gran amplitud en la dirección normal al flujo de aire, con frecuencias menores a las que se producirían en el caso de desprendimiento de torbellinos. Este fenómeno es característico de estructuras esbeltas con secciones transversales rectangulares o en forma de D, así como de cables de tendido eléctrico con hielo adherido. El galope inducido por una estela tiene lugar cuando existen dos obstáculos próximos de tal forma que uno de los cuerpos se encuentra en la estela del otro. Debido a la estela de torbellinos que genera el cuerpo aguas arriba del flujo de aire, el segundo cuerpo recibe una corriente incidente cuya intensidad y sentido variarán con el tiempo. Este fenómeno es típico de las catenarias de ferrocarril y de las líneas de transporte de energía eléctrica en las que los cables discurren agrupados en conjuntos formados por dos, cuatro, seis o más cables situados en paralelo y separados por espaciadores mecánicos en la dirección transversal.

La inestabilidad aeroelástica provocada por el flameo se produce a partir de una cierta velocidad crítica de viento cuando las fuerzas que el flujo de aire provoca sobre la estructura, en combinación con los movimientos de la propia estructura, dan lugar a amortiguamientos negativos en la estructura de tal manera que los movimientos de la misma se van amplificando hasta que, debido al elevado nivel de tensiones que alcanza el material, se produce el colapso, como en el ejemplo clásico del Puente de Tacoma [9].

La vibración por bataneo es aquella que se produce por las turbulencias u otras perturbaciones de la corriente no producidas por el obstáculo que las sufre, distinguiéndose dos tipos de bataneo: el generado por la propia turbulencia de la corriente incidente (que puede dar lugar a cargas cuasiestáticas o dinámicas) y el debido a las perturbaciones causadas por algún otro obstáculo próximo situado corriente arriba del obstáculo en consideración; este último tipo de bataneo se conoce en la literatura como bataneo de estela o interferencia.

Dependiendo del tipo de estructura, los fenómenos citados anteriormente pueden ocurrir aisladamente o asociados con otros, como por ejemplo, las vibraciones por flexión de torres, son provocadas por el desprendimiento periódico de torbellinos y por la turbulencia del viento. El problema en el que se centrará este trabajo se refiere al caso específico de vibraciones aeroelásticas en estructuras esbeltas de sección circular como son las chimeneas industriales o los mástiles.

En mástiles o chimeneas de sección circular variable o constante, la formación de torbellinos se desarrolla en regiones o tramos a lo largo de la altura de la torre, donde las frecuencias de 
desprendimiento o presiones sobre la superficie de la estructura son equivalentes. Esas regiones se denominan células de torbellinos y son importantes en la aparición de las oscilaciones de la estructura.

\subsection{Motivación}

Es necesario disponer de herramientas numéricas que permitan simular el comportamiento vibratorio de ciertas estructuras industriales (chimeneas, cables, etc.) o civiles (mástiles, puentes, etc.). De esta manera, en la etapa de diseño se podrá evaluar la respuesta y en caso necesario dimensionar adecuadamente para que no aparezcan problemas a lo largo de la vida útil.

Otro escenario aparece cuando en estructuras existentes se detectan desplazamientos o vibraciones inadmisibles que habrá que limitar o mitigar. En este caso, para chimeneas, edificios y otras estructuras similares, puede ser eficiente incorporar dispositivos pasivos de absorción de vibraciones denominados TMD (tuned mass damper). Para evaluar a priori la mejora obtenida con estos sistemas no es suficiente realizar una simulación dinámica del conjunto estructura-TMD bajo excitaciones conocidas (fuerzas armónicas, vibraciones libres, etc.) u otras que simulen la acción del viento (desprendimiento de torbellinos a una determinada frecuencia, respuesta tras un golpe de viento, etc.). Con esta forma de proceder la eficiencia esperada de estos sistemas es mayor que la cuantificable tras su puesta en servicio (experimentalmente), donde el viento interactúa con el conjunto estructural. Es importante disponer de aplicaciones de simulación de la interacción entre el fluido y la estructura para poder sintonizar adecuadamente los parámetros (masa, rigidez y amortiguamiento) de los sistemas de absorción de vibraciones. El elevado esfuerzo de cálculo necesario para realizar estas simulaciones en 3D hace aún hoy en día inviable este tipo de estudios. Con propuestas simplificadas como la incluida en esta Tesis Doctoral se pretende conseguir resultados útiles para aplicaciones industriales como la comentada, o para explicar otros fenómenos relacionados con la interacción del viento con estructuras flexibles.

En definitiva, la falta de conocimiento respecto al fenómeno físico de interacción fluidoestructura, la necesidad de modelos estructurales adecuados, la demanda de datos de alta calidad a escala real, la necesidad de desarrollar técnicas de análisis de datos para comprender mejor los resultados y la demanda de dispositivos eficaces de supresión de vibraciones por parte de la industria constituyen aspectos que requieren de herramientas numéricas como la desarrollada en este trabajo. 


\subsection{Estado de los conocimientos sobre el tema}

El interés desde un punto de vista práctico de las vibraciones inducidas por el desprendimiento de torbellinos ha dado lugar a un gran número de estudios, muchos de los cuales han sido analizados en los trabajos de Sarpkaya [10], Griffin y Ramberg [11], Berman [12], Parkinson [13]; en el capítulo de un libro por Anagnostopoulos [14]; en libros por Blevins [15], Naudascher y Rockwell [16], Simiu y Scanlan [4], y Sumer y Fredsфe [17], y recientemente por Williamson y Govardhan $[18,19]$.

A pesar de que existen en la bibliografía un gran número de estudios sobre cilindros rígidos apoyados elásticamente bajo vibración libre y forzada donde sólo se permite el movimiento en la dirección transversal al flujo, hay pocos artículos en los que también se permita la vibración del cuerpo en línea con el flujo, se estudie la dinámica de estructuras en voladizo, cilindros apoyados, cables o cuerpos atados. Como ejemplo clásico de sistemas sometidos a vibraciones inducidas por el desprendimiento de torbellinos, se considera un cilindro rígido apoyado elásticamente cuyo movimiento queda restringido a la dirección transversal al flujo. En función del movimiento del cuerpo, si es controlado o si bien la vibración ocurre por la acción del fluido, se pueden establecer diferentes modelos para el desprendimiento de torbellinos. Williamson y Roshko [20] estudiaron modelos de desprendimiento de torbellinos para un cilindro sometido a un movimiento sinusoidal en un amplio rango de amplitudes y longitudes de onda, definieron un conjunto de regiones para los distintos modos de desprendimiento de torbellinos e introdujeron una nueva terminología para cada modo. Cada ciclo de desprendimiento de torbellinos comprende torbellinos simples (S) y parejas de torbellinos $(\mathrm{P})$, dando lugar a modelos tales como los modos 2S, 2P y P + S (Figura 1.1), los cuales son los principales modos de desprendimiento de torbellinos cerca de la región fundamental de lock-in, la cual se estudiará con detenimiento en este trabajo.

Brika y Laneville $[21,22]$ fueron los primeros que probaron la existencia del modo 2P para la vibración libre usando un cable dentro de un túnel de viento. Afirmaron que los modos $2 \mathrm{~S}$ y $2 \mathrm{P}$ pueden ser fácilmente reconocibles y confirmaron la explicación dada por Williamson y Roshko [20] para el ciclo de histéresis en términos de cambio en el modelo de desprendimiento de torbellinos. La Figura 1.2(d) muestra la visualización de estos modos por medio de la utilización de humo. Encontraron una clara correspondencia entre el modo $2 \mathrm{~S}$ y el tramo inicial de la respuesta, $\mathrm{y}$ el modo $2 \mathrm{P}$ y el tramo inferior. 


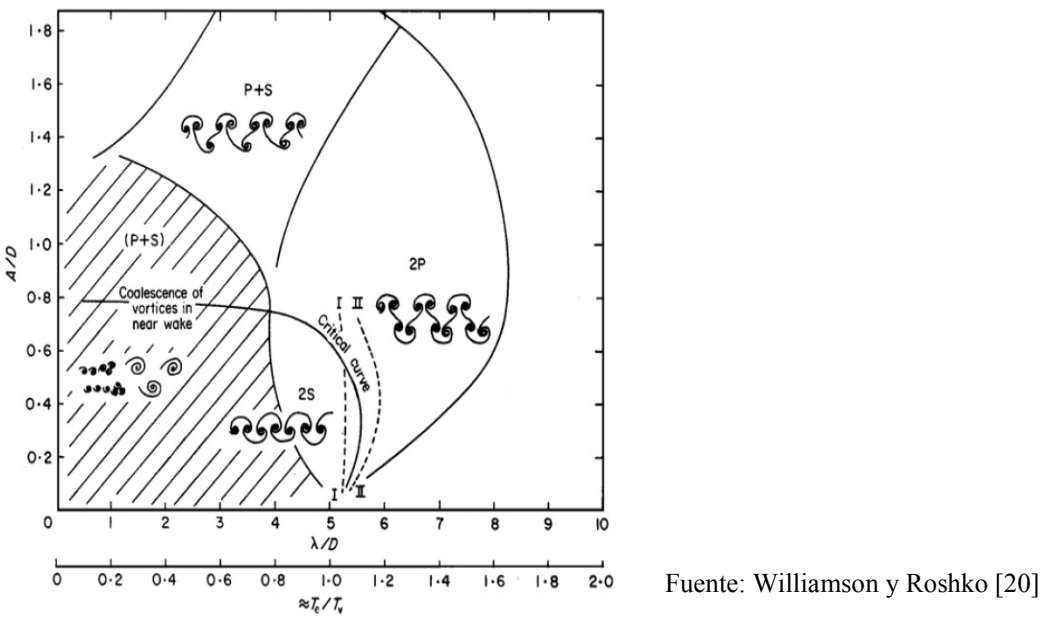

Figura 1.1. Mapa de regiones de los modos de desprendimiento de torbellinos

El fenómeno de lock-in o sincronización (Blevins [23], Sumer y Fredsфe [17]) de forma tradicional significa que a medida que la velocidad del fluido se incrementa, una vez alcanzado un cierto valor de la velocidad, la frecuencia de desprendimiento de torbellinos $\left(n_{s}\right)$ llega a estar muy próxima a la frecuencia natural $\left(n_{e}\right)$ de la estructura con lo que las dos frecuencias se sincronizan dentro de un rango de velocidades. La frecuencia de desprendimiento de vórtices y la frecuencia de oscilación permanecen próximas a la frecuencia natural de forma que la relación $f^{*}=n_{s} / n_{e}$ está próxima a la unidad. Sin embargo, para cuerpos ligeros sumergidos en agua, el cuerpo vibra a una frecuencia más alta, $f^{*}=1.4$ (Khalak y Williamson [24]).
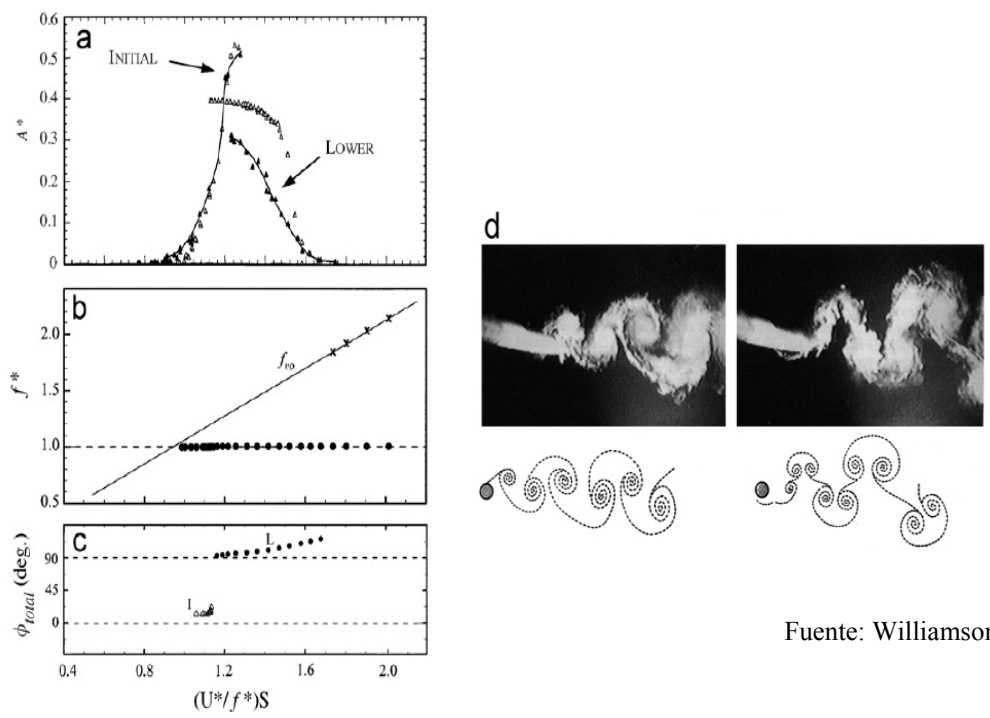

Fuente: Williamson y Govardhan [19]

Figura 1.2. Vibración libre de un cilindro elásticamente apoyado 
Para poder predecir las vibraciones inducidas por el desprendimiento de los torbellinos se ha creado una base de datos muy completa de fuerzas experimentales, obteniéndose éstas por medio de ensayos con cilindros sometidos a una oscilación sinusoidal controlada en el seno de una corriente libre. Varios investigadores, incluyendo a Bishop y Hassan [25], Mercier [26], Sarpkaya [27], Staubli [28], Gopalkrishnan [29], y mas recientemente Hover et al.[30, 31], Sheridan et al. [32], y Carberry et al. [33, 34, 35, 36], han medido las fuerzas en cuerpos sometidos a un movimiento armónico simple y multifrecuencia. Hover et al. [30, 31], en colaboración con el grupo de investigación de Michael Triantafyllou del MIT Towing Tank, desarrollaron un experimento ingenioso y extremadamente versátil, en concreto, se trata de un nuevo retroalimentador de fuerza "virtual cable testing apparatus". Las contribuciones más recientes de este grupo se han centrado en trabajar con números de Reynolds altos. El grupo de trabajo conjunto de Rockwell de la Universidad de Lehigh y John Sheridan de la Universidad de Monash ha llevado a cabo un gran número de medidas a partir de la vibración controlada de cilindros, dando lugar a numerosos artículos interesantes (Sheridan et al. [32] y Carberry et al. [33, 34, 35, 36]).

En el trabajo experimental llevado a cabo por Moe y Wu [37] al cilindro se le permitía vibrar en la dirección transversal al flujo $(Y)$ y también en línea con el mismo $(X)$. La relación de masas (cociente entre la masa de la estructura que vibra y la masa del fluido desplazado, $m^{*}$ ) y las frecuencias naturales se eligieron de tal manera que tuviesen valores diferentes en la dirección transversal al flujo y en la dirección paralela. Bajo estas condiciones, los estudios demostraron un amplio rango de sincronización, similar a los estudios en los que sólo se considera el movimiento en la dirección transversal al flujo, pero sin evidencias de diferentes tramos en la respuesta. Jeon y Gharib [38] adoptaron un nuevo planteamiento de tal forma que el cilindro, en el seno de una corriente fluida, era forzado a moverse en la dirección $Y$ y en la $X$ bajo movimientos impuestos por las expresiones

$$
\begin{aligned}
& x(t)=A_{X} \sin (2 \omega t+\theta) \\
& y(t)=A_{Y} \sin (\omega t)
\end{aligned}
$$

Fijaron como ángulos de fase los valores específicos $\theta=0^{\circ}$ y $\theta=-45^{\circ}$. Uno de los resultados más interesantes del estudio de Jeon y Gharib fue que incluso pequeños desplazamientos en la dirección $X\left(A_{X} / A_{Y}=20 \%\right)$ pueden inhibir la aparición del modo 2P en la formación de torbellinos.

En la mayoría de los casos prácticos, las estructuras cilíndricas tienen la misma relación de masas y la misma frecuencia natural en la dirección paralela al flujo $(X)$ y en la dirección transversal $(Y)$. Los ensayos llevados a cabo por Leyva et al. [39] y Jauvtis y Williamson [40, 41, 
42] han demostrado que incluso para bajas relaciones de masas (hasta $m^{*}=6$ ) el hecho de que el cuerpo pueda oscilar en la dirección paralela al flujo afecta muy poco al movimiento en la dirección transversal. Este resultado es importante porque indica que todo el conocimiento que se tiene sobre las vibraciones inducidas por el desprendimiento de torbellinos en cuerpos a los que sólo se les permite el movimiento en la dirección transversal, es aplicable al caso de dos grados de libertad. Sin embargo, para relaciones de masas por debajo de $m^{*}=6$ se produce un cambio importante en la interacción fluido-estructura. Aparece un nuevo tramo en la respuesta con un significativo incremento en la magnitud del movimiento según la dirección $X$, el cual fue definido por Jauvtis y Williamson [41, 42] como tramo "súper alto" (Figura 1.3). Esta respuesta se corresponde con un nuevo modo de formación de torbellinos, el cual comprende un trío de torbellinos formándose cada mitad de ciclo, el cual ha sido definido como modo $2 \mathrm{~T}$ siguiendo la terminología introducida por Williamson y Roshko [20].
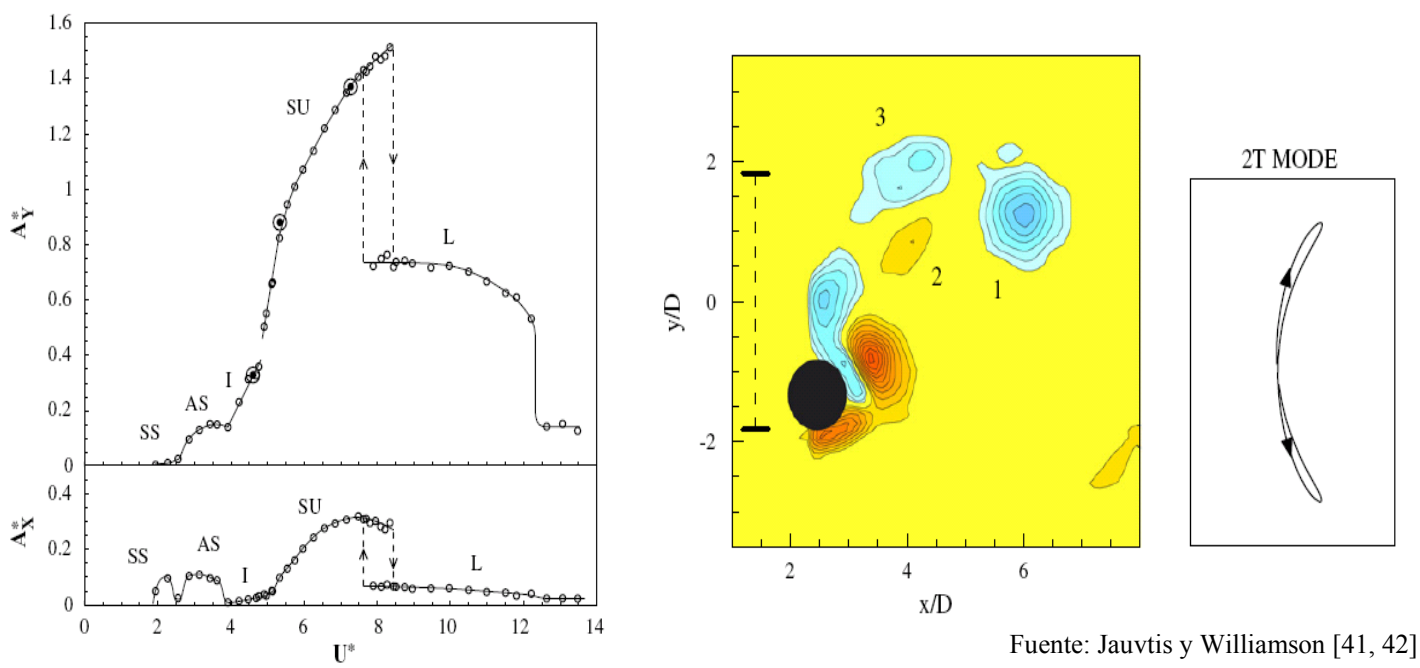

Figura 1.3. Descubrimiento de un tramo "súper alto" de alta amplitud

En general, a medida que los problemas son más reales desde un punto de vista práctico, más complejos resultan. No obstante, muchos de los fenómenos descubiertos para el problema tipo del cilindro apoyado elásticamente se pueden trasladar a estructuras más complejas. Por ejemplo, para el caso estructuras flexibles en voladizo, el trabajo de Pesce y Fujarra [43] y Fujarra et al. [44] indica que existe un tramo inicial en la amplitud de la respuesta similar al de un cilindro elásticamente apoyado. Techet et al. [45] descubrieron un modo 2S-2P, que era combinación de los modos $2 \mathrm{~S}$ y $2 \mathrm{P}$, en la formación de torbellinos para diferentes secciones a lo largo de la longitud de un cilindro de sección variable sometido a una vibración transversal controlada (Figura 1.4). 


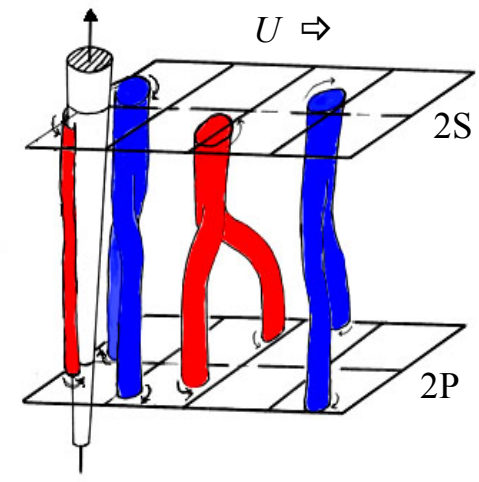

Fuente: Techet et al. [45]

Figura 1.4. Modo híbrido 2S-2P en un cilindro de sección variable

Las vibraciones inducidas por desprendimiento de torbellinos en el caso de cilindros apoyados también muestran tramos similares en la respuesta a los existentes para estructuras en voladizo como pusieron de manifiesto los trabajos de Balasubramanian et al. [46] y Weiss y Szewczyk [47]. En estos estudios el movimiento del cilindro estaba restringido a la dirección transversal, sin embargo, recientemente Flemming y Williamson [48] estudiaron la respuesta de un cilindro apoyado el cual tenía permitido el movimiento en la dirección transversal y paralela al flujo. Finalmente, Flemming y Williamson descubrieron un nuevo modo formado por dos torbellinos que giran a la vez y se forman cada medio ciclo, denominado modo $2 \mathrm{C}$, para las estructuras más ligeras que estudiaron.

Kim Vandiver en el MIT ha emprendido numerosos estudios de campo y ensayos de laboratorio relativos a la dinámica de cables (Vandiver [49]; Vandiver y Jong [50]) y ha desarrollado el conocido programa SHEAR7 para el estudio de las vibraciones inducidas por el desprendimiento de torbellinos en cables (Vandiver [51]) el cual actualmente está basado en datos experimentales obtenidos a partir de cilindros cortos. Similares experimentos han sido llevados a cabo también por Marcollo y Hinwood [52] utilizando un cable sumergido en el seno de una corriente uniforme. Trianyafyllou y Grosenbaugh [53] fueron capaces de calcular exitosamente la dinámica de cables con un modelo empírico de la fuerza de sustentación tratándola como una función lineal de la amplitud.

Algunos modelos analíticos 2D empleados para el análisis de las vibraciones inducidas por el desprendimiento de torbellinos describen la fuerza debida al desprendimiento de los torbellinos como una forma sinusoidal usando coeficientes y parámetros de ajuste y considerando que la respuesta en esa región es básicamente armónica [54]. Otros autores hacen uso de la teoría de vibraciones aleatorias para evaluar la respuesta de la estructura, con una fuerza de desprendimiento 
escrita en términos de espectro de banda larga o estrecha, como una función del tipo Gaussiana. Se encuadra en este caso el modelo de Vickery y Clark [55], aplicado a torres, y usado por otros investigadores [56] para su comparación con resultados experimentales obteniéndose resultados satisfactorios.

También se han desarrollado modelos semi-empíricos para el estudio las fuerzas debidas al desprendimiento de torbellinos en cilindros, teniendo en cuenta la correlación espacial de esas fuerzas, las cuales dependen de la amplitud, y tratando el fenómeno como un proceso aleatorio, estacionario, de banda estrecha, para números altos de Reynolds [57].

La naturaleza de las vibraciones auto-excitadas por el desprendimiento de torbellinos sugiere que el comportamiento del fluido también pueda ser modelado como un oscilador no lineal. Esta idea fue inicialmente propuesta por Bishop y Hassan [58] y seguida por otros investigadores [59, $60,61,62]$ usando modelos donde el coeficiente de sustentación (para cilindros dispuestos horizontalmente) o de fuerza lateral (para cilindros dispuestos verticalmente) satisface una ecuación del tipo van der Pol, la cual está acoplada a la ecuación de movimiento de la estructura en términos de la respuesta en velocidad. Algunos modelos evalúan puntos extremos de la región de lock-in para flujo bidimensional [63]. Otros modelos introducen una variable que recoge el movimiento del fluido para describir los efectos provocados por el desprendimiento de torbellinos [64].

Considerando la idea de un oscilador auto-excitado, Scanlan [4] presentó dos modelos empíricos para simular la fuerza de desprendimiento de torbellinos en cuerpos rígidos: uno lineal en el cual consideraba parámetros de excitación, de amortiguamiento y de rigidez, siendo utilizado este modelo por otros investigadores [65, 66], y otro no lineal con parámetros de la fuerza aeroelástica dependientes de la velocidad y del desplazamiento de la estructura. Los modelos de Vickery y Basu [67, 68, 69] y Paluch et al. [70] toman como partida la ecuación de van der Pol. Skop y Balasubramanian [71], y Skop y Luo [72] realizaron numerosos modelos para simular el desprendimiento de torbellinos a lo largo de la longitud del cilindro y de la dinámica estructural de cilindros para flujo uniforme y no uniforme usando también un oscilador del tipo van der Pol, con un término de acoplamiento proporcional a la velocidad del cuerpo. En un metódico artículo Facchinetti et al. [73] llegaron a la conclusión de que el término de acoplamiento óptimo ha de tener en cuenta la aceleración del cuerpo, en lugar del desplazamiento o la velocidad.

Modelos más recientes [74] toman como punto de partida el modelo empírico no lineal desarrollado por Scanlan [4] combinándolo con el modelo oscilador acoplado de Blevins [64, 75], y con aplicación de las técnicas de análisis modal. 
A diferencia de los modelos analíticos, la metodología puramente numérica basada en la Mecánica de Fluidos Computacional (CFD) y empleada para resolver problemas aeroelásticos en el ámbito de la ingeniería de estructuras tiene como característica la ausencia de requerimientos de datos cuyo origen sea experimental.

Los primeros estudios numéricos del flujo de aire alrededor de un cilindro fueron llevados a cabo en 1969 por Son y Hanratty [76]. A su vez, Braza et al. [77] emplearon en 1986 el método de los volúmenes finitos para analizar el desprendimiento de torbellinos en cilindros con un número de Reynolds inferior a 1000. Por otro lado, Leconite y Piquet aplicaron diferencias finitas en los casos de flujo estacionario y no estacionario. En el año 1993 Dawes [78] empleó el método de los volúmenes finitos para resolver el mismo problema. Utilizó un método adaptativo incorporando la capacidad de refinar la malla en función de la solución obtenida, logrando así una solución computacionalmente económica. Kalktsis [79] utilizó el método de los elementos espectrales para llevar a cabo un estudio computacional de las fuerzas que actúan sobre un cilindro oscilando armónicamente en dirección perpendicular a un flujo uniforme. Autores como Anagnostopoulos [80, 81], Jan y Sheu [82] y Mittal et al. [8, 83, 84] han empleado el método de los elementos finitos para analizar el fenómeno de desprendimiento de torbellinos. Dicho método es el que se empleará en esta Tesis Doctoral.

El grupo de George Karniadakis de la Universidad de Brown ha llevado a cabo amplios estudios computacionales. Las investigaciones de este grupo parten del análisis del flujo laminar alrededor de un cable sometido a vibración libre (Blackburn y Karniadakis [85]). El potencial de un análisis computacional tridimensional se puso de manifiesto en la simulación que hicieron Newman y Karniadakis [86, 87], donde mostraron las diferentes estelas de torbellinos formadas en los nodos y antinodos de un cable sometido a una vibración de onda estacionaria (Figura 1.5).

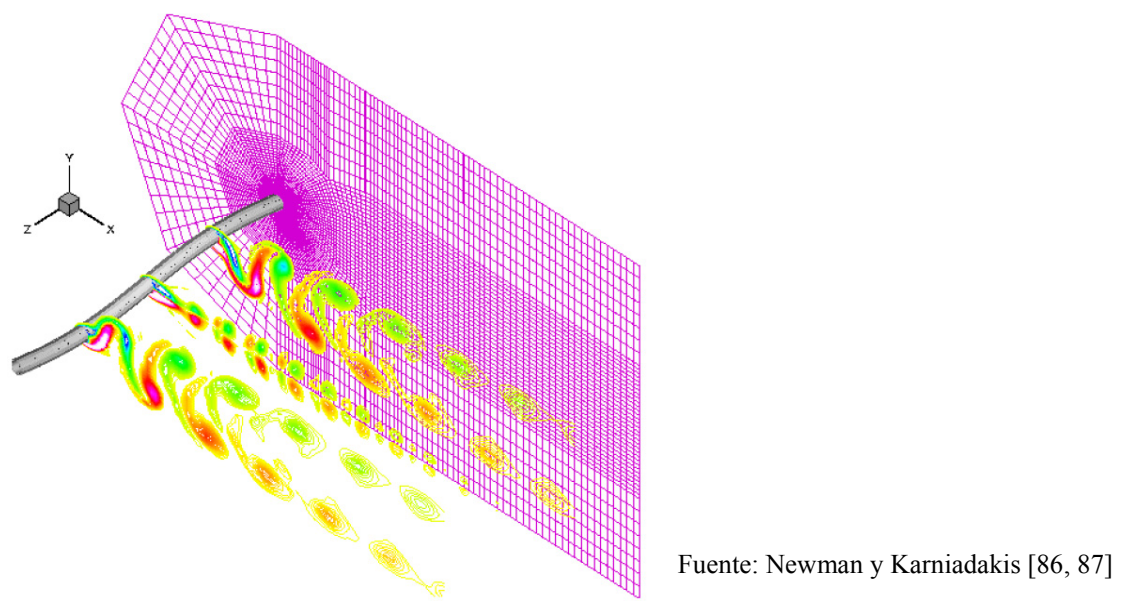

Figura 1.5. Análisis 3D de la vibración inducida por el desprendimiento de torbellinos de un cable 
Lucor et al. [88] estudiaron las vibraciones inducidas por el desprendimiento de torbellinos en cilindros flexibles muy esbeltos (esbeltez $>500$ ) sometidos a flujos tangenciales uniformes y exponenciales. Observaron dislocaciones de torbellinos similares a las visualizadas en experimentos realizados sobre cilindros inmóviles, las cuales pueden causar una sustancial modulación en las fuerzas de sustentación.

Willden y Graham [89] llevaron a cabo una exitosa simulación "quasi-3D”. En su simulación, resolvían un fluido 2D para distintas posiciones a lo largo de la longitud del cilindro elásticamente apoyado y las soluciones para cada plano se relacionaban hidrodinámicamente a través de una representación mallada tridimensional a gran escala de los torbellinos. El modelo estructural que acoplaban al fluido despreciaba el amortiguamiento estructural.

Otros investigadores como Rodi [90] en 1997 estudiaron el fenómeno de desprendimiento de torbellinos en cuerpos no aerodinámicos. Steggel y Rockliff [91] emplearon a su vez el método de los vórtices discretos. Utilizando ese mismo método, Larsen [92] y Larsen y Walther [93] calcularon para diferentes secciones transversales parámetros aerodinámicos como los coeficientes de sustentación y de arrastre o el número de Strouhal.

Otros autores como Lopes, Cunha y Simoes [94] emplean la metodología numérica para analizar el fenómeno de lock-in y su relación con las condiciones de utilización de estructuras esbeltas con el fin de limitar los niveles de vibración. Para analizar el flujo de aire y su acción sobre la estructura estos investigadores emplean un método basado en la técnica de los volúmenes finitos mientras que el comportamiento dinámico de la estructura es calculado mediante el método de los elementos finitos.

\subsection{Modelos numéricos}

A continuación se muestra una breve descripción de las características de diferentes métodos numéricos utilizados en la simulación de problemas de interacción fluido-estructura.

\section{- CENAERO}

Durante los últimos años CENAERO [95] ha desarrollado una plataforma de simulación de problemas de interacción fluido-estructura por medio del acoplamiento entre el programa Argo [96] de CFD y el programa de elementos finitos Samcef Mecano de análisis estructural a través del programa MpCCI (Mesh based parallel Code Coupling Interface) [97, 98]. 
El solver Argo de CFD se basa en una descomposición del dominio para resolver las ecuaciones de Navier-Stokes en 3D. Dado que se trata de un esquema implícito, en cada paso de tiempo se debe resolver un sistema de ecuaciones no lineales. Para ello utiliza un método de Newton inexacto basado en un algoritmo de Newton-GMRES por diferencias finitas. Samcef [99] es un programa de elementos finitos de carácter general para el análisis de estructuras y transferencia de calor.

El solver del fluido se acopla al solver de la estructura por medio del programa MpCCI para realizar simulaciones aeroelásticas. MpCCI es un programa que proporciona una interfaz independiente para el acoplamiento de diferentes códigos de simulación. Es capaz de intercambiar diferentes datos (desplazamientos, fuerzas, etc.) entre las mallas del fluido y la estructura a lo largo de la interfaz fluido-estructura. Ofrece interpolaciones lineales y bilineales, conservativas y noconservativas cuando las mallas no son compatibles, lo cual suele ser habitual en el caso de aplicaciones aeroelásticas. Esta herramienta de simulación fue utilizada por Fitschy et al. [100] para la optimización estructural del ala de una avioneta a partir de las respuestas aeroelásticas.

\section{- ANSYS}

ANSYS [101] es un programa de análisis y diseño basado en el método de cálculo por elementos finitos. El programa ANSYS CFX de simulación fluido-dinámica permite realizar análisis estacionarios y/o transitorios de flujo y transferencia de calor, permitiendo la interacción fluido-estructura gracias a la conexión con ANSYS.

La solución ANSYS FSI es una herramienta que permite realizar simulaciones de problemas de interacción fluido-estructura. En el caso de acoplamiento en una sola dirección, el solver del fluido y el de la estructura se ejecutan de forma independiente utilizando los resultados de una simulación como cargas o condiciones de contorno de una segunda simulación. Los datos se transfieren en una sola dirección. Este nivel de acoplamiento de la mecánica de fluidos computacional es posible entre ANSYS (versión Profesional o superior) y el solver CFX.

Para los casos en los cuales la estructura se deforma considerablemente es necesario realizar un análisis de interacción fluido-estructura con acoplamiento en dos direcciones. El solver iterativo ANSYS Multi-field permite la simulación de problemas de interacción fluido-estructura acoplados en dos direcciones con una transmisión de datos bidireccional. Este programa constituye el marco de trabajo y proporciona el acoplamiento entre el solver del fluido (ANSYS CFX) y el de la estructura (ANSYS Mechanical), los cuales se ejecutan simultáneamente. Se pueden realizar análisis estacionarios y/o transitorios con movimiento/deformación de la geometría de la estructura. Estas son algunas de las características de este programa: 
- El problema estructural y el dominio fluido son tratados como campos diferentes con modelos y mallas independientes.

- Los datos entre los dos sistemas se intercambian a través de la interfaz fluido-estructura.

- La solución del fluido y de la estructura se puede dividir entre dos ordenadores diferentes.

- No-linealidad material y geométrica.

Chen et al. [102] utilizaron esta aplicación para realizar una simulación numérica a escala real de las vibraciones inducidas por el desprendimiento alternado de torbellinos en las tuberías utilizadas para transportar petróleo desde el fondo del mar (risers).

El programa ANSYS CFX también se puede acoplar a código desarrollado in-house por investigadores, universidades, etc. a través del programa MpCCI para simular la respuesta de estructuras civiles sometidas a cargas de viento. En el trabajo de Miana et al. [103] se emplea la herramienta MpCCI para acoplar el programa de mecánica de fluidos computacional FLUENT [104] con el programa de elementos finitos ABAQUS [105] y simular el problema de interacción fluido-estructura en un cilindro oscilante.

\section{- SolidWorks}

SolidWorks [106] es un programa de diseño asistido por ordenador (CAD) que cuenta con diferentes herramientas para la validación de los diseños. Una de esas herramientas es SolidWorks Flow Simulation, la cual permite el análisis de flujo externo de líquidos y gases alrededor de cuerpos rígidos.

Respecto al estudio de las vibraciones inducidas por el desprendimiento de torbellinos en las tuberías utilizadas para transportar petróleo desde el fondo del mar, en mayo de 2003 varios grupos de investigadores desarrollaron 11 modelos numéricos para predecir desplazamientos, curvaturas y frecuencias una vez habían concluido las mediciones en laboratorio de las vibraciones inducidas por el desprendimiento de torbellinos en un modelo de tubería [107]. Estos métodos numéricos se pueden dividir en tres grupos.

El primero estaría formado por los códigos Norsk Hydro, USP, DeepFlow y VIVIC. Estos cuatro códigos usan las técnicas de la mecánica de fluidos computacional para calcular el flujo 2D alrededor de una tubería en diferentes planos horizontales distribuidos a lo largo de su longitud (strip theory approach). Bajo este planteamiento, la única comunicación entre los flujos en diferentes planos es a través del movimiento de la tubería, cuya posición se actualiza en cada paso 
de tiempo en respuesta a la fuerza instantánea inducida por el flujo en cada plano. El segundo grupo estaría compuesto por dos códigos, Orcina Vortex Tracking y Orcina Wake Oscillator, los cuales usan el mismo el planteamiento que los anteriores, pero adoptan métodos más pragmáticos para calcular la fuerza en cada plano. Estos dos grupos de códigos operan en el dominio del tiempo. Los códigos del tercer grupo (VIVA, VIVANA, VICoMo, SHEAR7 y ABAVIV) usan de diversas maneras los datos obtenidos de mediciones realizadas sobre cilindros rígidos sometidos a vibraciones inducidas por el desprendimiento de torbellinos o forzadas para identificar la amplitud del modo (o el rango de modos) más probable de ser excitado. En muchos de estos modelos no se considera la posibilidad de calcular la respuesta de la tubería en la dirección del flujo. En el trabajo de Chaplin et al. [108] se puede encontrar una descripción más detallada de todos estos modelos numéricos.

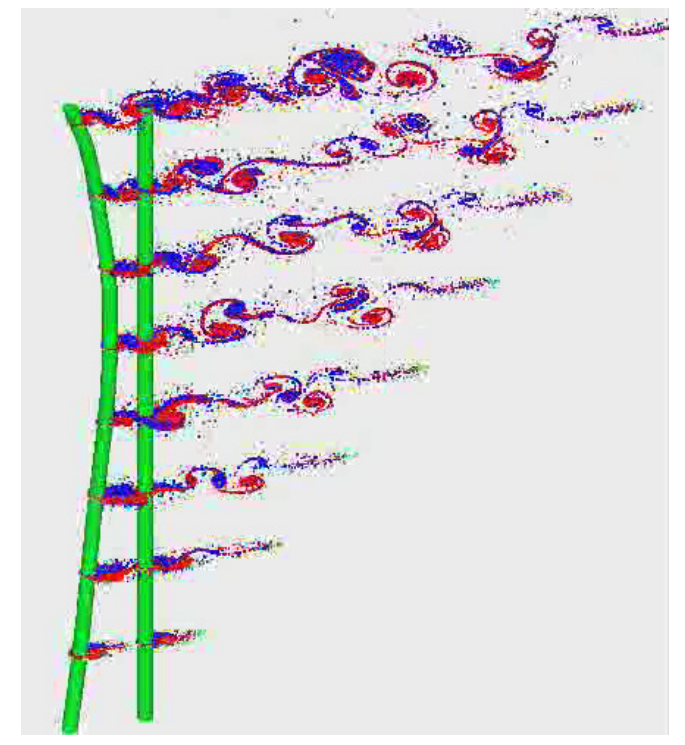

Fuente: Kendon [109]

Figura 1.6. Simulación en VIVIC de la vibración inducida por el desprendimiento de torbellinos en dos tuberías

\section{- NekTar}

NekTar [110] es un programa desarrollado en la Universidad de Brown (Newman y Karniadakis [111]) que permite una simulación numérica directa (DNS) del flujo alrededor de cilindros rígidos o flexibles, acoplado al correspondiente modelo estructural. Hace uso de dos formulaciones diferentes para tener en cuenta el movimiento del dominio: un sistema de coordenadas fijo al contorno y una formulación arbitrariamente Lagrangiana-Euleriana. Evangelinos et al. [112] utilizaron este programa para determinar la distribución de fuerzas sobre un cilindro flexible sujeto a vibraciones inducidas por el desprendimiento de torbellinos. 


\section{- DEEPSEA Engineering and Management}

DeepSea [113] ha desarrollado su propio código CFD para el estudio de diferentes proyectos, entre los que destaca el estudio de las vibraciones inducidas por el desprendimiento de torbellinos en las tuberías utilizadas para el transporte de petróleo desde el fondo marino. Esta herramienta fue utilizada por Stavropoulos et al. [114] para evaluar el comportamiento hidrodinámico de este tipo de tuberías. Los resultados hidrodinámicos obtenidos se pueden utilizar como datos de entrada en ciertas aplicaciones de análisis de las vibraciones inducidas por el desprendimiento de torbellinos en el dominio de la frecuencia, como por ejemplo SHEAR7.

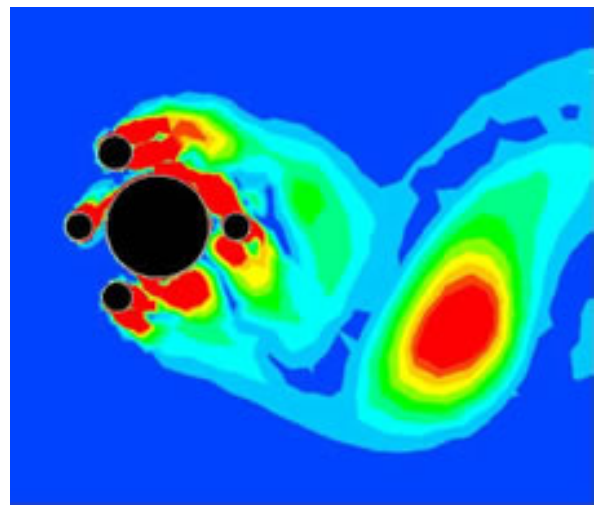

Fuente: DeepSea [113]

Figura 1.7. Simulación de la vibración de las tuberías satélites próximas a la tubería principal con DeepSea

\section{- Principia}

Principia [115] ha desarrollado junto con el Instituto Francés del Petróleo (IFP) el programa de elementos finitos DeepLines. Este programa dispone del módulo DeepVIV que permite simular las vibraciones inducidas por el desprendimiento de torbellinos en las tuberías utilizadas para el transporte del petróleo desde el fondo del mar, realizar pre-análisis estáticos y modales, identificar las zonas de lock-in y la respuesta en amplitud de la tubería y estudiar la variación del coeficiente de arrastre.

La amplitud de la vibración y la fatiga resultante se obtienen a partir de un cálculo modal de la respuesta a una corriente estacionaria. La estructura se define con elementos cable, viga o barra. En primer lugar se realiza un análisis estático para determinar la posición de equilibrio, a continuación se lleva a cabo un análisis modal y finalmente se hace el cálculo de las vibraciones inducidas por el desprendimiento de torbellinos. Existe la posibilidad de utilizar los resultados almacenados en una base de datos de un análisis modal anterior, lo cual resulta de gran utilidad cuando se excita un elevado número de nodos. Una descripción más detallada de este programa se puede encontrar en el trabajo de Le Cunff et al. [116]. 


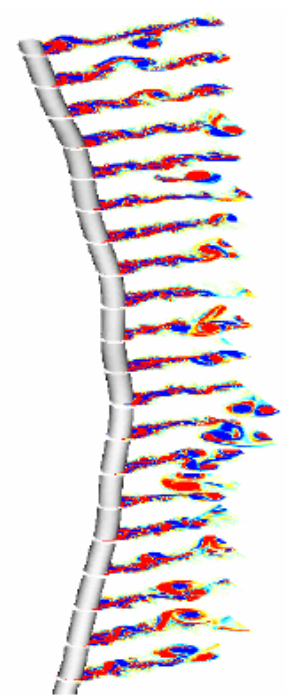

Fuente: Le Cunff [116]

Figura 1.8. Simulación del desprendimiento de torbellinos en una tubería con el programa DeepVIV

\section{- PFLOW}

PFLOW [117] es un programa de interacción-fluido estructura desarrollado en el Centro Internacional de Métodos Numéricos en Ingeriría (CIMNE) basado en el método de las partículas finitas (Particle Finite Element Method, PFEM). Este método es una clase particular de la formulación Lagrangiana que trata de resolver los problemas en los que existe interacción entre fluidos y sólidos de una manera unificada. PFEM trata los nodos de la malla del fluido y del sólido como partículas que pueden desplazarse libremente e incluso separase del dominio principal del fluido, como por ejemplo, el efecto de las gotas de agua.

\subsection{Objetivos y aportaciones originales}

El principal objetivo de este trabajo es desarrollar un método simplificado que permita simular ciertos fenómenos propios de interacción entre un determinado fluido y la estructura sobre la que incide, más concretamente se estudian las vibraciones inducidas por el desprendimiento alternado de torbellinos en estructuras esbeltas.

Este estudio se realizará dentro del contexto de la Mecánica de Fluidos Computacional y respecto a los fenómenos aeroelásticos causados por el desprendimiento de torbellinos se prestará especial interés al fenómeno de lock-in y al de amplificación del coeficiente de arrastre. 
El método desarrollado para abordar este estudio resulta novedoso. De forma simplificada la propuesta consiste en no resolver el fluido en 3D, sino solamente en algunos planos transversales a la estructura a lo largo de su longitud. Aunque la estructura se modela con elementos monodimensionales, en cada plano se considera la forma concreta de la sección transversal de la estructura y sobre ella se calculan las presiones con las cuales se determinan, tras integración e interpolación, las cargas por unidad de longitud que actúan sobre la estructura.

\subsection{Contenido}

En el primer capítulo se presenta el tema en su contexto dejando constancia de la importancia que tienen las vibraciones inducidas por el desprendimiento de torbellinos en estructuras tales como chimeneas o mástiles. También se realiza una descripción de los trabajos desarrollados por otros autores respecto al tema en cuestión y se indican los objetivos específicos de esta Tesis Doctoral.

En el segundo capítulo se realiza una descripción teórica del flujo alrededor de un cilindro. Se describe la variación de los coeficientes de sustentación y de arrastre y se introduce el número de Strouhal. Posteriormente, se describe el fenómeno aeroelástico de lock-in mostrando resultados experimentales de otros autores y se introduce el concepto de células de vórtices para el estudio de las vibraciones debidas al desprendimiento de torbellinos en torres o chimeneas sometidas a la acción del viento. Finalmente, se describe el fenómeno, también aeroelástico, de amplificación del coeficiente de arrastre aportando resultados obtenidos experimentalmente por otros autores.

En el tercer capítulo se presenta un modelo monodimensional para el análisis de barras de sección de pared delgada. La formulación hace uso de los modelos de Navier-Bernoulli y Vlasov para describir la flexión y la torsión, respectivamente, a nivel de barra, y del modelo de LoveKirchhoff para plantear las relaciones de comportamiento a nivel de lámina. Este modelo tiene carácter general y contempla la posibilidad de que las barras estén constituidas por materiales compuestos.

En el cuarto capítulo se aborda el problema de la dinámica estructural, con especial interés en el rango no-lineal. Aunque existen tanto técnicas explícitas como implícitas para resolver el problema, sólo se considerarán los algoritmos implícitos.

El quinto capítulo se centra en la resolución de las ecuaciones de Navier-Stokes para flujo incompresible. En la primera parte se describen las ecuaciones de conservación que gobiernan los fenómenos de la fluidodinámica, en una segunda parte se aborda la resolución práctica de las 
ecuaciones utilizando métodos de paso fraccionado y finalmente, se estudia el movimiento de la malla.

En el sexto capítulo se estudia el problema de interacción fluido-estructura. El primer apartado contiene una descripción del problema y de las principales estrategias computacionales usadas para su resolución, además de una breve revisión del estado de los conocimientos. En el segundo apartado se describe el esquema computacional utilizado en este trabajo, el cual integra las etapas de la resolución estructural en la formulación de paso fraccionado del fluido. En el apartado siguiente se analiza el error local que introduce este planteamiento teniendo en cuenta las etapas de resolución del fluido. Finalmente, en el último apartado se muestra un estudio de la estabilidad del esquema.

En el séptimo capítulo se muestran los resultados obtenidos para las diferentes simulaciones que se han realizado. Se trata de aplicar el método descrito en los capítulos anteriores a modelos de estructuras esbeltas sometidas a cargas de viento para estudiar su comportamiento vibratorio. En el primer ejemplo se muestra la respuesta de una chimenea de acero de $90 \mathrm{~m}$ sometida a la acción del viento, a continuación se analiza la influencia del número de planos de fluido utilizados para modelar la acción del viento en la respuesta transversal de un mástil de acero de $34 \mathrm{~m}$ de altura de sección constante y finalmente se estudian los fenómenos de lock-in y de amplificación del coeficiente de arrastre para esa misma estructura.

Por último, en el octavo capítulo se muestran las conclusiones extraídas de este trabajo y se establecen las líneas futuras de investigación.

\section{Referencias}

1 Battista R. C., “Avaliação da Estabilidade da Estrutura do Poste Metálico para Telecomunicações”, PEC-4317, Fundación COPPETEC, Universidad Federal de Rio de Janeiro, Rio de Janeiro, 2003.

${ }^{2}$ Koten H. Van, "Vortex Excitation of Steel Chimneys", Wind Effects on Buildings and Structures, Riera \& Davenport (eds.), Balkema, Rotterdam, pp. 209-219, 1998.

${ }^{3}$ Blessmann J., "Acidentes Causados pelo Vento", 4a Edición, Editorial de la Universidad Federal del Rio Grande do Sul, 2001.

${ }^{4}$ Simiu, E., y Scanlan, R.H., Wind effects on structures, fundamental and applications to design (3rd ed.), John Wiley and Sons Inc., NY, 1996. 
${ }^{5}$ Cheng L., Zhou Y., Zhang M. M., "Perturbed interaction between vortex shedding and induced vibration”, Journal of Fluids and Structures, 17 (7), pp. 887-901, 2003.

${ }^{6}$ Singh S. P., Mittal S., "Vortex-induced oscillations at low Reynolds numbers: Hysteresis and vortex-shedding modes", Journal of Fluids and Structures, 20 (8), pp. 1085-1104, 2005.

${ }^{7}$ Yokoi Y. y Kamemoto K., "Vortex shedding from an oscillating circular cylinder in a uniform flow”, Experimental Thermal and Fluid Science, 8 (2), pp. 121-127, 1994.

${ }^{8}$ Prasanth T. K., Behara S., Singh S. P., Kumar R. y Mittal S., "Effect of blockage on vortexinduced vibrations at low Reynolds numbers" Journal of Fluids and Structures, 22 (6-7), pp. 865876, 2006.

${ }^{9}$ Billah K. Y. y Scanlan R. H., "Resonance, Tacoma Narrows Bridge Failure, and Undergraduate Physics Textbooks", The American Journal of Physics, 59 (2), pp. 118-124, 1991,

${ }^{10}$ Sarpkaya, T., "Vortex-induced oscillations", ASME Journal of Applied Mechanics. 46, pp. 241$258,1979$.

${ }^{11}$ Griffin, O.M., Ramberg, S.E., "Some recent studies of vortex shedding with application to marine tubular and risers", Trans. ASME Journal of Energy Resources Technology. 104, pp. 2-13, 1982.

${ }^{12}$ Bearman, P.W., "Vortex shedding from oscillating bluff bodies". Annual Review of Fluid Mechanics. 16, pp. 195-222, 1984.

${ }^{13}$ Parkinson, G.V., "Phenomena and modelling of flow-induced vibrations of bluff bodies", Prog. Aerosp. Sci. 26, 169-224, 1989.

${ }^{14}$ Anagnostopoulos, P., Flow-Induced Vibrations in Engineering Practice, WIT Press, Ashurst, UK, 2002.

${ }^{15}$ Blevins, R.D., Flow-Induced Vibrations, Van Nostrand Reinhold, New York, 1990

${ }^{16}$ Naudascher, E., Rockwell, D., Flow-Induced Vibrations: An Engineering Guide, Balkema, Rotterdam, Netherlands, 1994.

${ }^{17}$ Sumer, B.M. y Fredsфe, J., Hydrodynamics Around Cylindrical Structures, World Scientific, Singapore, 1997.

${ }^{18}$ Williamson, C.H.K., Govardhan, R., "Vortex-induced vibrations". Annual Review of Fluid Mechanics, 36, pp. 413-455, 2004.

19 Williamson, C.H.K., Govardhan, R., "A brief review of recent results in vortex induced vibrations", Journal of Wind Engineering and Industrial Aerodynamics, 96 (6-7), pp. 713-735, 2008.

${ }^{20}$ Williamson, C.H.K. y Roshko, A., "Vortex formation in the wake of an oscillating cylinder", Journal of Fluids and Structures, 2, pp. 355-381, 1988. 
${ }^{21}$ Brika, D., Laneville, A., "Vortex-induced vibrations of a long flexible circular cylinder", Journal of Fluid Mechanics, 250, pp. 481-508, 1993.

${ }^{22}$ Brika, D., Laneville, A., "An experimental study of the Aeolian vibrations of a flexible circular cylinder at different incidences", Journal of Fluids and Structures, 9, pp. 371-391, 1995.

${ }^{23}$ Blevins, R.D., Flow-Induced Vibrations, Van Nostrand Reinhold, NewYork, 1990

${ }^{24}$ Khalak, A., Williamson, C.H.K., "Investigation of there lative effects of mass and damping in vortex-induced vibration of a circular cylinder", Journal of Wind Engineering and Industrial Aerodynamics, 69-71, pp. 341-350, $1997 \mathrm{~b}$.

${ }^{25}$ Bishop, R.E.D., Hassan, A.Y., "The lift and drag forces on a circular cylinder oscillating in a flowing fluid", Proceedings of the Royal Society of London. Series, A277, pp. 51-75, 1964

${ }^{26}$ Mercier, J.A., Large amplitude oscillations of a circular cylinder in a low speed stream, Ph.D. Thesis, Stevens Institute of Technology, Hoboken, NJ., 1973.

${ }^{27}$ Sarpkaya, T., "Fluid forces on oscillating cylinders", ASCE Journal of the Waterway, Port, Coastal and Ocean Division, 104, pp. 275-290, 1978.

${ }^{28}$ Staubli,T., "Calculation of the vibration of an elastically mounted cylinder using experimental data from forced oscillation”, ASME Journal of Fluids Engineering, 105, pp. 225-229, 1983.

${ }^{29}$ Gopalkrishnan, R., Vortex-induced forces on oscillating bluff cylinders, Ph.D., Thesis, MIT, Cambridge, MA, 1993.

${ }^{30}$ Hover, F., Davis, J.T., Triantafyllou, M.S., "Vortex-induced vibration of marine cables: experiments using force feedback", Journal of Fluids and Structures, 11, pp. 307-326, 1997.

${ }^{31}$ Hovver, F.S., Techet, A.H., Triantafyllou, M.S., "Forces on oscillating uniform and tapered cylinders in cross flow", Journal of Fluid Mechanics, 363, pp. 97-114, 1998.

${ }^{32}$ Sheridan, J., Carberry, J., Lin, J.C., Rockwell, D., "On the near wake topology of an oscillating cylinder", Journal of Fluids and Structures, 12, pp. 215-220, 1998.

${ }^{33}$ Carberry, J., Sheridan, J., Rockwell, D.O., "Wake modes of an oscillating cylinder", Journal of Fluids and Structures, 15 (3), pp. 523-532, 2001.

${ }^{34}$ Carberry, J., Sheridan, J., Rockwell, D.O., "Controlled oscillations of a cylinder: a new wake state", Journal of Fluids and Structures, 17 (2), pp. 337-343, 2003.

${ }^{35}$ Carberry, J., Sheridan, J., Rockwell, D.O., "Wake states and response branches of forced and freely oscillating cylinder”, European Journal of Mechanics, B23 (1), pp. 89-97, 2004.

${ }^{36}$ Carberry, J., Sheridan, J., Rockwell, D.O., "Controlled oscillations of a cylinder: forces and wake modes", Journal of Fluid Mechanics, 538, 31-69, 2005.

${ }^{37}$ Moe, G., Wu, Z.J., "The lift force on a cylinder vibrating in a current". ASME Journal of Offshore Mechanics and Arctic Engineering, 112, pp. 297-303, 1990. 
${ }^{38}$ Jeon, D., Gharib, M., "On circular cylinders undergoing two-degree-of-freedom forced motions", Journal of Fluids and Structures, 15:3-43-4, pp. 533-541, 2001.

${ }^{39}$ Leyva, J., Rockwell, D.O., Jauvtis, N., Williamson, C.H.K., "A comparative study of $X Y$ vortexinduced vibration of a circular cylinder", Journal of Fluids and Structures, 2007.

${ }^{40}$ Jauvtis, N., Williamson, C.H.K., "Vortex-induced vibration of a cylinder with two degrees of freedom", Journal of Fluids and Structures, 17, pp. 1035-1042, 2003 a.

${ }^{41}$ Jauvtis, N., Williamson, C.H.K., "A high-amplitude $2 \mathrm{~T}$ mode of vortex formation, and the effects of nonharmonic forcing in vortex-induced vibration", European Journal of Mechanics, B 23, pp. 107-115, 2003b.

42 Jauvtis, N., Williamson, C.H.K., "The effects of two degrees of freedom on vortex-induced vibration”, Journal of Fluid Mechanics, 509, pp. 23-62, 2003c.

${ }^{43}$ Pesce, C.P., Fujarra, A.L.C., "Vortex-induced vibrations and jump phenomenon: experiments with a clamped flexible cylinder in water", International Journal of Offshore and Polar Engineering, 10, pp. 26-33, 2000.

${ }^{44}$ Fujarra, A.L.C., Pesce, C.P., Flemming, F., Williamson, C.H.K., "Vortex-induced vibration of a flexible cantilever", Journal of Fluids and Structures, 15, 651-658, 2001.

${ }^{45}$ Techet, A.H., Hover, F.S., Triantafyllou, M.S., "Vortical patterns behind a tapered cylinder oscillating transversely to a uniform flow”, Journal of Fluid Mechanics, 363, pp. 79-96, 1998.

${ }^{46}$ Balasubramanian, S., Skop, R.A., Haan, F.L., Szewczyk, A.A., "Vortex-excited vibrations of uniform pivoted cylinders in uniform and shear flow", Journal of Fluids and Structures, 14, 65-85, 2000 .

${ }^{47}$ Weiss, L.G., Szewczyk, A.A., "An experimental investigation of some three-dimensional effects of pivoted circular cylinders". In: Ziada, S., Staubli, T. (Eds.), Flow Induced Vibration. Balkema, Rotterdam, Netherlands, pp. 75-83, 2000.

${ }^{48}$ Flemming, F., Williamson, C.H.K., "Vortex-induced vibrations of a pivoted cylinder", Journal of Fluid Mechanics, 522, pp. 215-252, 2005.

49 Vandiver, J.K., "Dimensionless parameters important to the prediction of vortex-induced vibration of long flexible cylinders in ocean currents", Journal of Fluids and Structures, 7, pp. 423455, 1993.

${ }^{50}$ Vandiver, J.K., Jong, J.Y., "The relationship between in-line and cross-flow vortex-induced vibration of cylinders", Journal of Fluids and Structures, 1, pp. 381-399, 1987.

${ }^{51}$ Vandiver, J.K., SHEAR7 User Guide. Department of Ocean Engineering, MIT, Cambridge, MA, 2003. 
${ }^{52}$ Marcollo, H., Hinwood, J.B., "Vortex-induced vibration of a long flexible cylinder in uniform flow with both forcing and response", Hourigan, K., Leweke, T., Thompson, M.C., Williamson, C.H.K. (Eds.), Conference on Bluff Body Wakes and Vortex-Induced Vibrations, 17-20 December 2002, Port Douglas, Australia, pp. 219-223, 2002.

53 Triantafyllou, M.S., Grosenbaugh, M.A., "Prediction of vortex-induced vibrations in sheared flows", Bearman, P.W. (Ed.), Flow-Induced Vibration. Balkema, Rotterdam, Netherlands, pp. 73$82,1995$.

${ }^{54}$ Kwok, C.S., "Wind-Induced Lock-In Excitation of Tall Structures", Journal of the Structural Division, Proceedings of the American Society of Civil Engineers, 107 (ST1), pp. 57-72, 1981.

${ }^{55}$ Vickery, B.J. y Clark, A.W., "Lift or Across-Wind Response of Tapered Stacks", Journal of the Structural Division, Actas de la ASCE, 8634 (ST1), pp. 1-20, 1972.

${ }^{56}$ Hansen, S. O., "Cross-Wind Vibrations of a 130-m Tapered Concrete Chimney”, Journal of Wind Engineering and Industrial Aerodynamics, 8, pp. 145-155, 1981.

${ }^{57}$ Blevins, R.D. y Burton, T.E., "Fluid Forces Induced by Vortex Shedding", Journal of Fluids Engineering, 95, pp. 19-24, 1976.

${ }^{58}$ Bishop, R.E.D., y Hassan, A.Y., "The Lift and Drag Forces on a Circular Cylinder in a Flowing Field”, Actas de la Real Sociedad (London), Series A, 277, pp. 51-75, 1964.

${ }^{59}$ Hartlen, R.T., y Curie, I.G., "Lift-Oscillator of Vortex-Induced Vibration", Journal of the Engineering Mechanics Division, Actas de la ASCE, 96, pp. 577-591, 1970.

${ }^{60}$ Skop, R.A., y Griffin O.M., "A Model for the Vortex-Excited Resonant Response of Bluff Cylinders", Journal of Sound and Vibration, 27 (2), 225-233, 1973.

${ }^{61}$ Griffin, O.M., Skop R.A., y Kopmann, G.H., "The Vortex-Excited Resonant Vibrations of Circular Cylinders”, Journal of Sound and Vibration, 31 (2), pp. 235-249, 1973.

${ }^{62}$ Skop, R.A. y Griffin, O.M., "On a Theory for the Vortex-Excited Oscillations of Flexible Cylindrical Structures”, Journal of Sound and Vibration, 41 (2), pp. 263-274, 1975.

${ }^{63}$ Dowell Earl H. y Ilgamov M., Studies in Nonlinear Aeroelasticity, Springer-Verlag, New York, 1988.

${ }^{64}$ Iwan, W.I. y Blevins, R.D., “A Model for Vortex Induced Oscillation of Structures”, Transactions of the ASME, Journal of Applied Mechanics, 41, pp. 581-586, 1974.

${ }^{65}$ Datta, T.K., y Jain, A.K., “An Analytical Study of the Across-Wind Response of Cylinders Due to Vortex Shedding”, Engineering Structural, 9, 1987.

${ }^{66}$ Gade, R.H., Bosh, H.R., y Poldony, W.Jr., "Recent Aerodynamic Studies of Long-Span Bridges", Journal of the Structural Division, Actas de la ASCE, 102 (ST7), pp. 1299-1315, 1976. 
${ }^{67}$ Vickery, B.J., y Basu, R.I., “Across-Wind Vibrations of Structures of Circular Cross-Section. Part I. Development of a Mathematical Model for Two-Dimensional Conditions". Journal of Wind Engineering and Industrial Aerodynamics, 12, pp. 49-73, 1983.

${ }^{68}$ Basu R. I. \& Vickery B. J., “Across-Wind Vibrations of Structures of Circular Cross-Section Part II Development of a Mathematical Model for Full-Scale Application”. Journal of Wind Engineering and Industrial Aerodynamics, 12, pp. 75-97, 1983.

${ }^{69}$ Vickery, B.J., y Basu, R.I., "Simplified Approaches to the Evaluation of the Across-Wind Response of Chimneys", Journal of Wind Engineering and Industrial Aerodynamics, 14, pp. 153$166,1983$.

${ }^{70}$ Paluch, M., Riera J. D. y Iturrioz, I., "Determinación de la Respuesta Dinámica de una Torre de Transmisión-Estudio Comparativo", Anais XXVIII Jornadas Sul-Americanas de Engenharia Estrutural, São Carlos, pp. 675-684, 1997.

${ }^{71}$ Skop, R.A., Balasubramanian, S., "A new twist on an old model for vortex-excited vibrations". Journal of Fluids and Structures, 11, pp. 395-412, 1997.

${ }^{72}$ Skop, R.A., Luo, G., "An inverse-direct method for predicting the vortex-induced vibrations of cylinders in uniform and nonuniform flows", Journal of Fluids and Structures, 15, pp. 867-884, 2001.

${ }^{73}$ Facchinetti, M.L., DeLangre, E., Biolley, F., "Vortex-induced travelling waves along a cable", European Journal of Mechanics, B23, pp. 199-208, 2004.

74 Pinheiro, M.A.S., Vibrações Aeroelásticas em Torres Esbeltas, Tesis Doctoral, Universidad Federal de Río de Janeiro, 2004.

${ }^{75}$ Blevins, R. D., Flow Induced Vibration, Van Nostrand Reinhold Company, New York. 1977.

${ }^{76}$ Son J. S. y Hanratty T. J., "Numerical Solution for the Flow around a Cylinder at Reynolds Number of 40, 200, 500”. Journal of Fluid Mechanics, 35, pp. 369-386, 1969.

${ }^{77}$ Braza M., Chassaing P. y Minh H. H. "Numerical Study and Physical Analysis of the Pressure and Velocity fields in the near Wake of a Circular Cylinder", Journal of Fluid Mechanics, 165, pp. 79-130, 1986.

${ }^{78}$ Dawes, W.N., "Simulating unsteady turbomachinery flows on unstructured meshes which adapt both in time and space". International Gas Turbine and Aeroengine Congress and Exposition, Cincinnati, Ohio, 1993.

${ }^{79}$ Kalktsis, L, Triantafyllou, G.S., Ozbas, M., "Excitation, inertia, and drag forces on a cylinder vibrating transversely to a steady flow", Journal of Fluids and Structures, 23 (1) pp. 1-21, 2007.

${ }^{80}$ Anagnostopoulos, P., "Computer-aided Flow Visualization and Vorticity Balance in the laminar Wake of a Circular Cilinder", Journal of Fluids and Structures, 11, pp. 33-72, 1997. 
${ }^{81}$ Anagnostopoulos, P., "Numerical study of the flow past a cylinder excited transversely to the incident stream. Part 1: Lock-in zone, hydrodynamic forces and wake geometry", Journal of Fluids and Structures, 14 (6), pp. 819-851, 2000.

82 Jan, Y.J., y Sheu, T.W.H., "Finite element analysis of vortex shedding oscillations from cylinders in the straight channel", Computational Mechanics, 33 (2), pp. 81-94, 2004.

${ }^{83}$ Mittal, S., y Kumar, V., "Finite element study of vortex-induced cross-flow and in-line oscillations of a circular cylinder at low Reynolds numbers", International Journal for Numerical Methods in Fluids, 31(7), pp. 1087-1120, 1999.

${ }^{84}$ Prasanth, T.K. y Mittal, S., "Vortex-induced vibrations of a circular cylinder at low Reynolds numbers", Journal of Fluid Mechanics, 594, pp. 463-491, 2008.

${ }^{85}$ Blackburn, H., Karniadakis, G.E., "Two and three-dimensional simulations of vortex-induced vibration of a circular cylinder", Third International Offshore Polar Engineering Conference, 3, pp. 715-720, 1993.

${ }^{86}$ Newman, D.J., Karniadakis, G.E., "Simulations of flow over a flexible cable: comparison of forced and flowinduced vibration", Journal of Fluids and Structures, 10, pp. 439-453, 1996.

${ }^{87}$ Newman, D.J., Karniadakis, G.E., "Simulations of flow past a freely vibrating cable" Journal of Fluid Mechanics, 344, pp. 95-136, 1997.

${ }^{88}$ Lucor, D., Imas, L., Karniadakis, G.E., "Vortex dislocations and force distribution of long flexible cylinders subjected to sheared flows". Journal of Fluids and Structures, 15, pp. 641-650, 2001.

${ }^{89}$ Willden, R.H.J., Graham, J.M.R., "Numerical prediction of VIV on long flexible circular cylinders", Journal of Fluids and Structures, 15, pp. 659-669, 2001.

${ }^{90}$ Rodi, W., "Comparison of LES and RANS calculations of the flow around bluff bodies", Journal of Wind Engineering and Industrial Aerodynamics, 69-71, pp. 55-75, 1997.

${ }^{91}$ Steggel, N. y Rockliff, N., "Simulations of the Effects of Body Shape on Lock-in Characteristics in Pulsating Flow by the Discrete Vortex Method", Journal of Wind Engineering and Industrial Aerodynamics, 69-71, pp. 317-329, 1997.

${ }^{92}$ Larsen, A., "Advances in Aeroelastic Analyses of Suspension and Cable-stayed Bridges", Journal of Wind Engineering and Industrial Aerodynamics, 74-76, pp. 73-90, 1998.

${ }^{93}$ Larsen, A. y Walther, J.H., "Discrete Vortex Simulation of Flow around Five generic Bridge Deck Sections", Journal of Wind Engineering and Industrial Aerodynamics, 77-78, pp. 591-602, 1998. 
${ }^{94}$ Lopes, A. V., Cunha, A., y Simoes, L.M.C., "Modelo Computacional de Análise Aeroelástica das Condiçoes de Utilizaçao de Estructuras Esbeltas", Congresso de Metodos Computacionais em Engenharia, Lisboa, Portugal, 2004.

${ }^{95} \mathrm{http} / / / \mathrm{www} . c e n a e r o . b e /$

${ }^{96} \mathrm{http}: / /$ www.cenaero.be/Page_Generale.asp?DocID=15338\&la=1\&langue=EN

${ }^{97} \mathrm{http}: / /$ www.mpcci.de/

${ }^{98}$ Wolf, K., MpCCI - a geenral coupling library for multidisciplinary simulation, Worshop on Scalable Solver Software Multiscale Coupling and Computational Earth Science, 2001.

${ }^{99} \mathrm{http}: / / \mathrm{www}$. samcef.com/

${ }^{100}$ Fitschy, L., Thirifay, F., Wyart, E., Pierret, S., y Geuzaine, P., "Structural optimization of a small aircraft wing from aeroelastic responses", Fluid Structure Interaction and Moving Boundary Problems Iv, New Forest, England 92, pp. 173-181, 2007.

${ }^{101} \mathrm{http}: / / \mathrm{www}$. ansys.com/

${ }^{102}$ Chen, Z.S., Kim, W.J., Yu, D.J., Choi J.S. y Hong, S., "Numerical Simulation of a Large-scale Riser with Vortex-induced Vibration”, ISOPE PACOMS, King Mongkut, Bangkok, Universidad de Tecnología Thonburi, 2000.

${ }^{103}$ Miana, M., Valdes, J.R., Royo, J., Jimenez, M.A., "Validation of MpCCI by simulation of a free vertical oscillating cylinder", MpCCI Forum, 2008.

${ }^{104} \mathrm{http}: / /$ www.fluent.es/

${ }^{105} \mathrm{http}: / / \mathrm{www} \cdot$ simulia.com/

${ }^{106} \mathrm{http}: / /$ www.solidworks.es/

${ }^{107}$ Chaplin, J.R., Bearman, P.W., Huera Huarte, F.J., Pattenden, R.J., "Laboratory measurements of vortex-induced vibrations of a vertical tension riser in a stepped current", Journal of Fluids and Structures, 21, pp. 3-24, 2005.

${ }^{108}$ Chaplin, J.R., Bearman, P.W., et al.,"Blind predictions of laboratory measurements of vortexinduced vibrations of a tension riser", Journal of Fluids and Structures, 21, pp. 25-40, 2005.

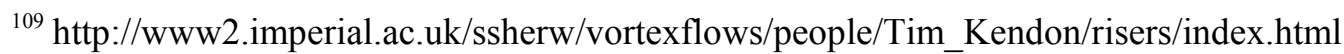
${ }^{110} \mathrm{http} / / / \mathrm{www} . \mathrm{cfm}$. brown.edu/crunch/nektar.html

${ }^{111}$ Newman, D., y Karniadikis, G.E., "A direct numerical simulation study of flow past a freely vibrating cable", Journal of Fluid Mechanics, 344, pp. 95-136, 1997.

${ }^{112}$ Evangelinos, C., Lucor, D. y Karniadakis, G.E., "Dns-derived force distribution on flexible cylinders subject to vortex-induced vibration", Journal of Fluids and Structures, 14, pp. 429-440, 2000.

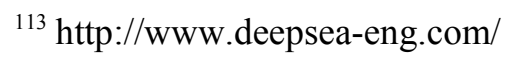


${ }^{114}$ Stavropoulos, M., Charlesworth, D. y Dixon, M., "The Application of CFD for Vortex Induced Vibration Analysis of Marine Risers in Projects", International Conference on Computational Methods in Marine Engineering, MARINE 2005, CIMNE Ed., Oslo, Noruega, 2005.

${ }^{115} \mathrm{http} / / / w w w . p r i n c i p i a . f r / e n g /$ logiciels/deeplines/

${ }^{116}$ Le Cunff, C., Biolley, F., Fontaine, E., Etienne, S. y Facchinetti, M.L., "Vortex-Induced Vibrations of Risers: Theoretical, Numerical and Experimental Investigation”, Oil \& Gas Science and Technology -Rev. IFP, 51 (1), pp. 59-69, 2002.

${ }^{117} \mathrm{http}: / / \mathrm{www}$. cimne.upc.es/pfem/intro.asp 
Capítulo 2

\section{Fenómenos Aeroelásticos}





\subsection{Resumen}

En este capítulo se realiza una descripción teórica del flujo alrededor de un cilindro. Se describe la variación de los coeficientes de sustentación y de arrastre y se introduce el número de Strouhal. Posteriormente, se describe el fenómeno aeroelástico de lock-in o de sincronización mostrando resultados experimentales de otros autores y se introduce el concepto de células de vórtices para el estudio de las vibraciones inducidas por el desprendimiento alternado de torbellinos en torres o chimeneas sometidas a la acción del viento. Finalmente, se describe el fenómeno, también aeroelástico, de amplificación del coeficiente de arrastre aportando resultados obtenidos de forma experimental por otros autores.

\subsection{Flujo alredor de un cilindro}

El flujo alrededor de un cilindro es uno de los problemas clásicos de la Mecánica de Fluidos. En principio, la geometría circular sugiere la existencia de un patrón de flujo estacionario y claramente simétrico, lo cual se cumple en el caso de valores del número de Reynolds muy bajos: cualquier perturbación que se introduzca aguas arriba es amortiguada por las fuerzas viscosas. Sin embargo, para números de Reynolds más grandes, las perturbaciones no pueden ser neutralizadas, lo cual conlleva importantes fenómenos periódicos aguas abajo del cilindro, conocidos como “desprendimiento de vórtices" (vortex shedding).

\subsubsection{Regímenes de flujo}

Cuando un cuerpo no aerodinámico se expone a una corriente de fluido de velocidad $U$, las presiones en la superficie del cuerpo varían de modo alternado a lo largo del tiempo, conforme se ilustra en la Figura 2.1 [1].

En la medida en que los torbellinos se desprenden de manera alternada en cada lado del cilindro provocan fuerzas periódicas sobre el cuerpo. Si el cilindro tiene permitido el movimiento en la dirección transversal a la que actúa la corriente de fluido, esas fuerzas periódicas interactúan con el movimiento del cilindro dando lugar a las vibraciones inducidas por el desprendimiento de torbellinos. En general, estas fuerzas se expresan en términos adimensionales por medio de coeficientes de fuerza de la forma 


$$
C=\frac{F(t)}{\frac{1}{2} \rho U^{2} d}
$$

donde $F(t)$ es la fuerza periódica que actúa sobre el cilindro, $\rho$ es la densidad del fluido y $d$ es el diámetro del cilindro. Nótese que el coeficiente de fuerza $C$ dependerá del tiempo, $C(t)$.
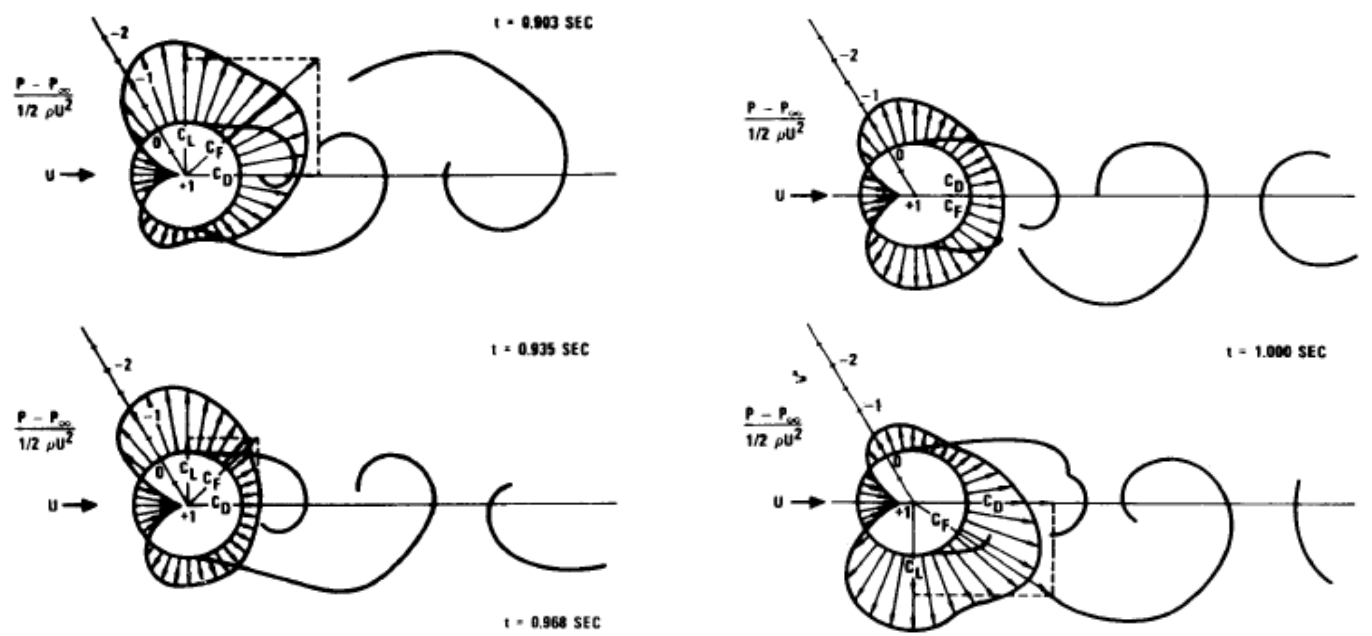

Figura 2.1. Campo de presiones en la superficie de un cilindro

Fuente: Pinheiro [2]

El arrastre y la sustentación se definen como las componentes paralela y normal, respectivamente, a la velocidad relativa de aproximación, de la fuerza ejercida sobre un cuerpo por el fluido en movimiento a su alrededor. La acción dinámica del fluido en movimiento relativo respecto al cuerpo es la que desarrolla el arrastre y la sustentación.

Para una sección circular, el coeficiente de arrastre $C_{D}$, y el de sustentación $C_{L}$, varían en el tiempo tal como se muestra en la Figura 2.2, la cual muestra los resultados obtenidos mediante la mecánica de fluidos computacional en el caso del cilindro considerado como obstáculo. Notar que el valor medio del coeficiente de sustentación es nulo, $C_{L}=0$, mientras que el valor medio del coeficiente de arrastre es distinto de cero, $C_{D} \neq 0$. La frecuencia de la componente de sustentación viene dada por la frecuencia de desprendimiento de los torbellinos y su valor se corresponde con la mitad del valor de la frecuencia de la componente de arrastre, es decir, el cuerpo vibra el doble de rápido en la dirección paralela al flujo, con una frecuencia doble que en la dirección perpendicular, sin embargo, la magnitud de la amplitud de las vibraciones en la dirección paralela es pequeña comparada con la que ocurre en la dirección perpendicular siendo, por lo tanto, éstas últimas las más importante. Finalmente, se utiliza $\tilde{C}_{D}$ y $\tilde{C}_{L}$ para designar los valores RMS (raíz cuadrada del valor cuadrático medio) de los coeficientes de arrastre y sustentación respectivamente. 
Ni siquiera para los casos más sencillos (sección circular, obstáculo, flujo laminar, etc.) existen soluciones analíticas capaces de describir estos fenómenos dada la complejidad del problema.
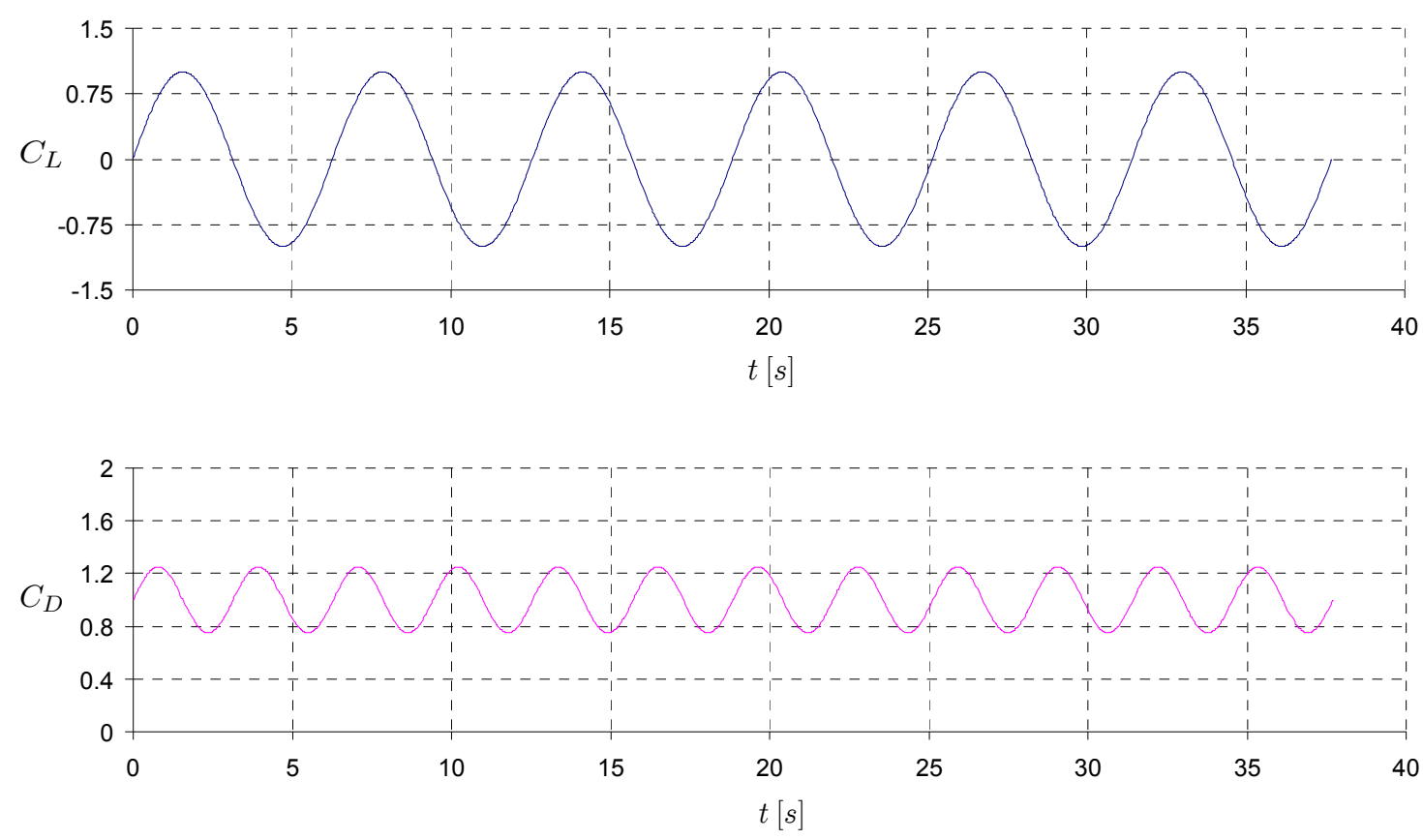

Figura 2.2. Variación con el tiempo del coeficiente de arrastre y de sustentación

La formación de torbellinos en un cilindro circular depende del número de Reynolds, Re. Los principales regímenes de desprendimiento de torbellinos se muestran en la Figura 2.3 [3].

Para valores bajos del número de Reynolds ( $R e<5$ ), el fluido pasa alrededor del cilindro sin que haya desprendimiento de vórtices. Cuando el número de Reynolds varía entre 5 y 40 , el fluido se separa formando un par de vórtices estacionarios justo en la parte posterior del cilindro con una configuración inestable, de manera que una pequeña perturbación daría lugar a una configuración alternada de desprendimiento de vórtices periódica [4].

El desprendimiento alternado de torbellinos se denomina en la literatura como calle de vórtices de Von Kármán (Von Kármán vortex street) y tiene lugar para valores del número de Reynolds comprendidos entre 40 y 150 (Figura 2.4).

Para valores del número de Reynolds entre 150 y 300 se produce una transición del régimen laminar al régimen turbulento y para la franja de 300 a $3 \cdot 10^{5}$, la estela es completamente turbulenta. En esta franja de valores del número de Reynolds, los vórtices se desprenden con una frecuencia bien definida. 
Cuando el número de Reynolds se sitúa entre $3 \cdot 10^{5}$ y $3.5 \cdot 10^{6}$ se produce una nueva transición, el punto de separación se mueve hacia atrás y la estela se hace más estrecha y desorganizada. En este rango el desprendimiento de vórtices se produce para una banda ancha de frecuencias y de forma no armónica. Para números de Reynolds mayores de $3.5 \cdot 10^{6}$ se reestablece la calle de vórtices turbulenta.
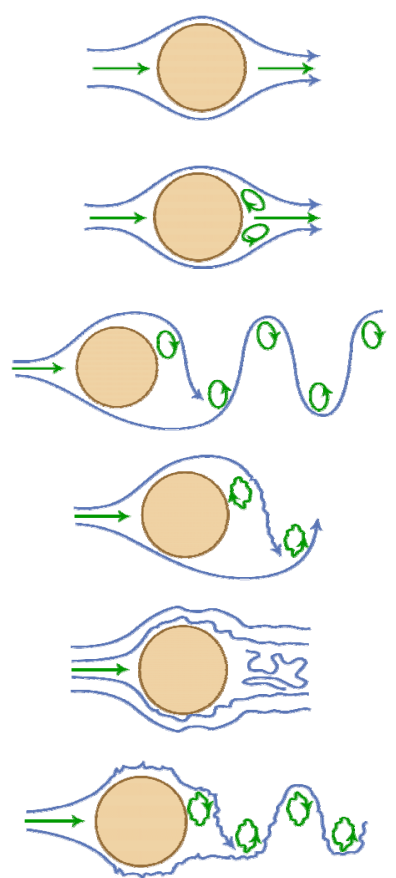

$\underline{R e}<5$ Flujo sin desprendimiento de vórtices

$\underline{5<R e<40}$ Se forman un par de vórtices estacionarios en la parte posterior del cilindro

$40<R e<150$ Desprendimiento alternado de vórtices en régimen laminar

$150<R e<300$ Transición del régimen laminar al turbulento $300<R e<3 \cdot 10^{5}$ Desprendimiento alternado de vórtices en régimen turbulento

$\underline{3 \cdot 10^{5}<R e<3 \cdot 10^{6}}$ La transición de flujo laminar a turbulento ocurre en la capa límite, la estela se hace más estrecha y desorganizada

$\operatorname{Re}>3 \cdot 10^{6}$ Reestablecimiento del desprendimiento alternado de vórtices en régimen turbulento

Figura 2.3. Regímenes de flujo alrededor de un cilindro circular

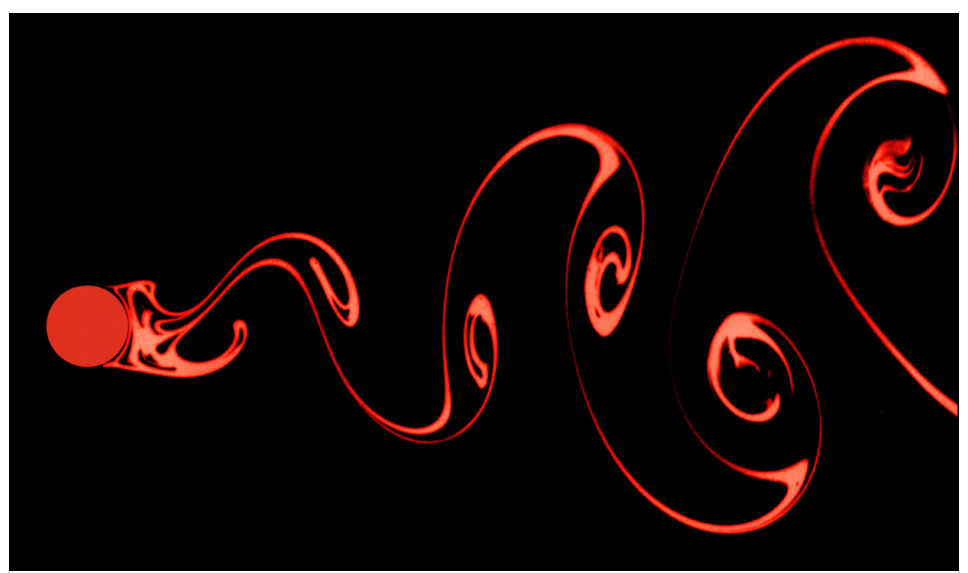

Fuente: Techet [5]

Figura 2.4. Calle de vórtices de Von Kármán $(R e=150)$ 


\subsubsection{Variación de los coeficientes de fuerza en función del número de Reynolds}

En la Figura 2.5 se muestra la variación del coeficiente de arrastre en función del número de Reynolds $[6,7]$. Cabe destacar en particular la drástica reducción del coeficiente de arrastre (drag crisis) cuando $R e \approx 2.5 \cdot 10^{5}$. Esta región se denomina región crítica [8] y corresponde a un estado en el cual la transición de flujo laminar a flujo turbulento ocurre en la capa límite que se forma en la superficie del cilindro. El punto de separación de la capa límite se desplaza hacia la parte posterior del cilindro y la estela se hace más estrecha, reduciéndose el valor del coeficiente de arrastre de forma considerable.

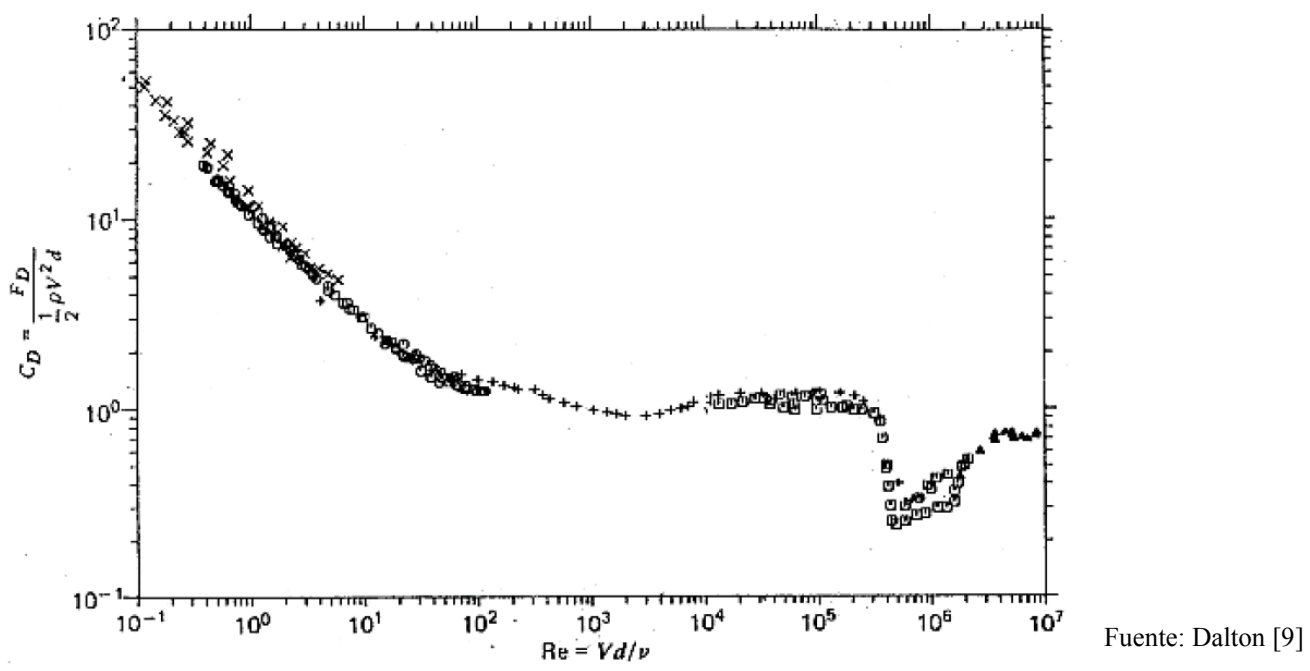

Figura 2.5. Variación del $C_{D}$ en función del número de $R e$

La rugosidad relativa de la superficie del cilindro juega un papel muy importante en la definición del perfil de la curva de variación del coeficiente de arrastre en función del número de Reynolds $[10,11]$, tal como se muestra en la Figura 2.6. Una rugosidad relativa elevada hace que la transición de la capa límite laminar a la turbulenta ocurra para números de Reynolds más bajos y provoca que la caída del coeficiente de arrastre se produzca antes. Sin embargo, esta caída es menos acusada cuanto menos lisa es la superficie del cilindro. Cuanto más lisa sea la superficie del cilindro, mayor será el descenso del coeficiente de arrastre, llegándose a reducir éste, en el caso de un cilindro liso, hasta el 50\%. 


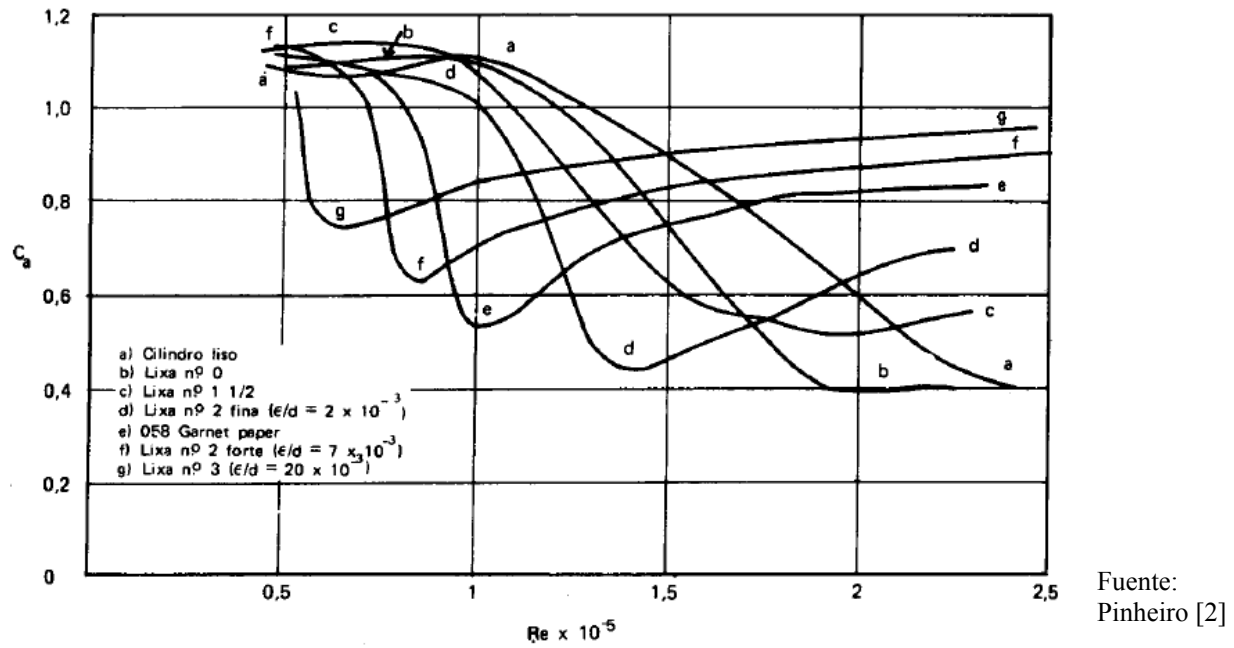

Figura 2.6. Influencia de la rugosidad sobre el $C_{D}$

El coeficiente de sustentación también varía en función del número de Reynolds tal como se aprecia en la Figura 2.7 [12], en la cual se han representado los valores RMS del coeficiente de sustentación a nivel de sección. Además de resultados experimentales también se recogen resultados numéricos $2 \mathrm{D}$ y $3 \mathrm{D}$.

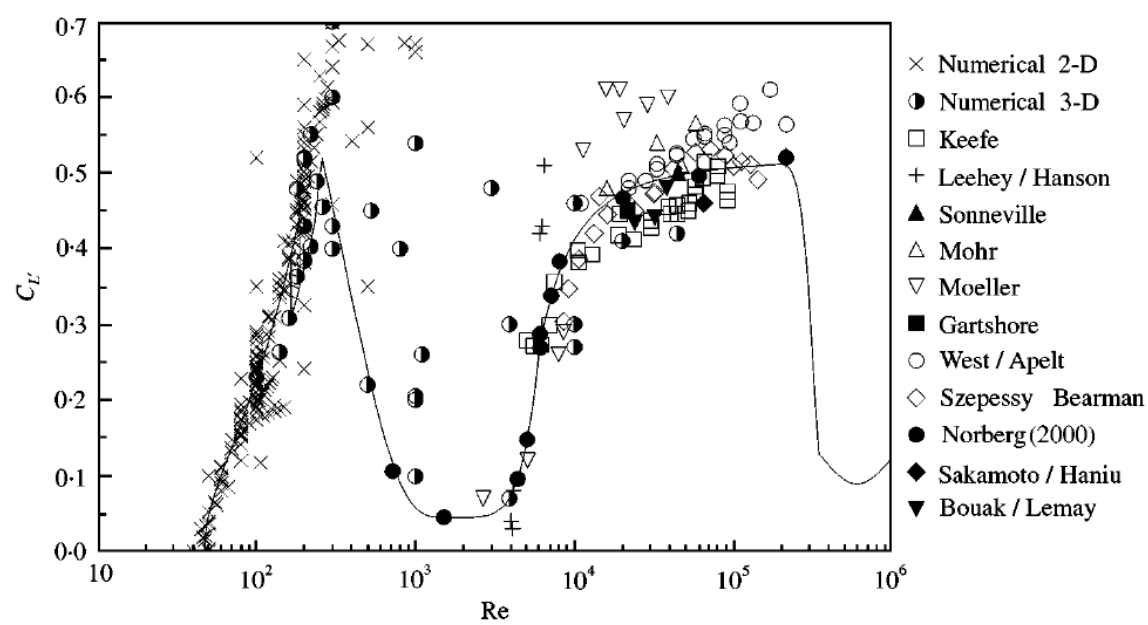

Figura 2.7. Valor RMS del coeficiente de sustentación frente al número de Reynolds

\subsubsection{Número de Strouhal}

Las vibraciones inducidas por el desprendimiento de torbellinos ocurren cuando los torbellinos se desprenden alternativamente desde lados opuestos de la estructura. Esto da lugar a una carga fluctuante perpendicular a la dirección del fluido. Cuando se forma un torbellino en un lado de la 
estructura, la velocidad del fluido se incrementa en el otro lado, y de acuerdo con la teoría de Bernoulli, esto da lugar a una reducción en la presión. De modo que la estructura estará sometida a una fuerza lateral tal como se muestra en la Figura 2.8. En la medida en que los torbellinos se desprenden alternativamente, primero desde un lado y luego desde el otro, se establece una fuerza lateral armónica con la misma frecuencia que la frecuencia de desprendimiento de los torbellinos.

La velocidad en la dirección del fluido $U_{1}$ de los torbellinos es aproximadamente $0.85 U$, donde $U$ es la velocidad de la corriente sin perturbar. Para una estructura que tiene impedido el movimiento, la distancia $l_{v}$ entre torbellinos en la misma dirección debe ser proporcional al ancho de la estructura $d$ perpendicular a la dirección del fluido. El tiempo transcurrido entre torbellinos es igual a la distancia $l_{v}$ dividida por la velocidad $U_{1}$ de los torbellinos. Esto significa que la frecuencia $n_{s}$ de la carga lateral causada por el desprendimiento de torbellinos es $U_{1} / l_{v}$, que es proporcional a $U / d$. El factor de proporcionalidad se llama número de Strouhal, St. Esta relación fue publicada por Strouhal en 1878 [13].

$$
n_{s}=S t \frac{U}{d}
$$

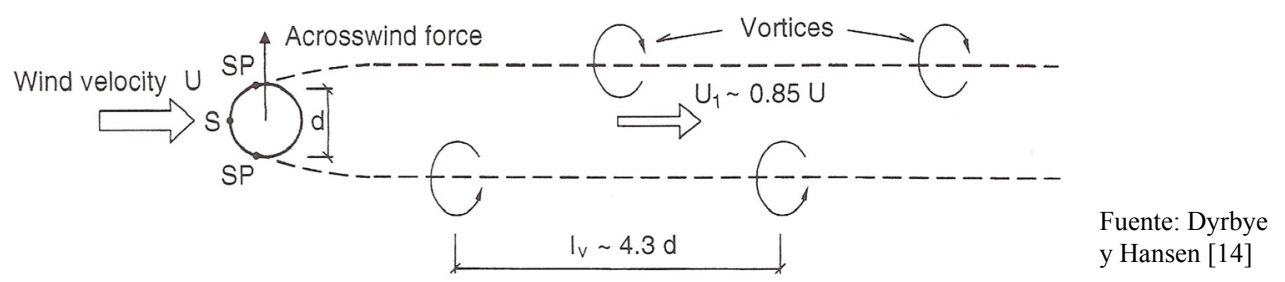

Figura 2.8. Desprendimiento alternado de torbellinos

En estructuras de ingeniería civil pueden ocurrir, bajo determinados vientos, grandes vibraciones si la frecuencia dominante del desprendimiento de torbellinos $n_{s}$, está próxima o coincide con una frecuencia natural de la estructura $n_{e}$, asociada a un modo de vibración en la dirección transversal al viento. Se define la velocidad crítica del viento $U_{c r i t}$, para $n_{s}=n_{e} \mathrm{y}$ tiene la siguiente expresión

$$
U_{\text {crit }}=n_{e} \frac{d}{S t}
$$

El número de Strouhal depende de la forma de la sección transversal de la estructura, de la rugosidad de la superficie, de la turbulencia del viento, del movimiento de la estructura (aeroelasticidad) y del número de Reynolds. En la Figura $2.9[15,16]$ se muestra la dependencia del número de Strouhal respecto del número de Reynolds. 


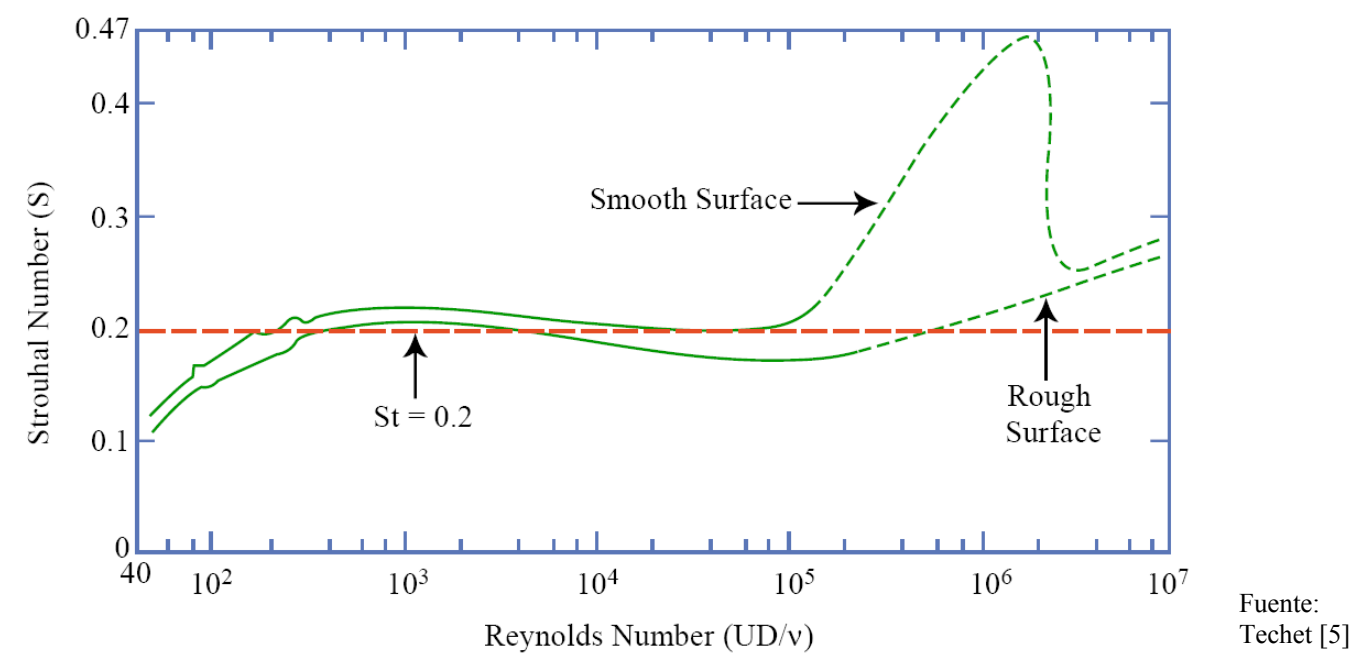

Figura 2.9. Número de Strouhal frente al número de Reynolds para cilindros circulares

\subsubsection{Turbulencia}

La velocidad del viento se puede expresar por medio de un vector con tres componentes

$$
V(t)=\{U+u(t), v(t), w(t)\}
$$

asociadas a la dirección longitudinal de la velocidad media del viento $\sin$ perturbar $U$, a la dirección lateral y vertical, siendo $u, v$ y $w$ las componentes de fluctuación del viento en las tres direcciones señaladas. La velocidad media del viento $U$, en estructuras "tipo" chimenea o mástil como las que se estudian en este trabajo, sólo depende de la altura respecto del suelo y las componentes que describen la fluctuación del viento pueden ser tratadas matemáticamente como procesos estacionarios estocásticos con valor medio nulo. Las componentes de fluctuación lateral $v, \mathrm{y}$ vertical $w$, dependen de las variaciones de la dirección del viento. En general, la velocidad media del viento $U$, y la componente de turbulencia $u$ en la dirección del viento son las más importantes ya que proporcionan las mayores contribuciones a la carga de viento que actúa sobre la estructura.

El efecto de la turbulencia en las fuerzas aerodinámicas y aeroelásticas depende de:

- La intensidad de la turbulencia $I_{u}$, definida por (2.5) siendo $\tilde{u}$ la desviación estándar de la componente longitudinal $u$

$$
I_{u}=\tilde{u} / U
$$


- Las escalas de turbulencia, que miden el tamaño medio de los remolinos de un flujo turbulento [17]

La escala longitudinal de turbulencia viene definida por

$$
L_{u}^{x}=\frac{1}{\tilde{u}^{2}} \int_{0}^{\infty} R_{u 1, u 2}(t) d t
$$

donde $R_{u 1, u 2}$ es la función de correlación cruzada entre los registros de velocidades del viento $u_{1} \mathrm{y}$ $u_{2}$ de dos puntos separados entre sí una cierta distancia en la dirección de la velocidad media del viento $U$.

Para estimar las escalas de turbulencia $L_{u}^{x}$ se recurre a mediciones experimentales, las cuales dependen de la altura respecto del suelo, de la rugosidad y del propio valor de la velocidad media del viento $U$. Como estimaciones para $L_{u}^{x}$ atmosférica se indican valores mínimos del orden de decenas de metros y valores máximos del orden de centenares de metros.

Fluctuaciones de la componente de turbulencia $u$ en la dirección del viento de gran escala dan lugar a una modificación lenta de la velocidad media del viento $U$, haciendo variar la frecuencia de desprendimiento de los torbellinos y el número de Strouhal.

Si la relación $L_{u}^{x} / d$ es grande, la fuerza lateral generada por el desprendimiento de torbellinos se ve modificada $[18,19]$. El espectro de potencia de esa fuerza tendrá un pico muy pronunciado para flujo con baja turbulencia, siendo prácticamente armónica la fuerza, pero a medida que la turbulencia aumenta, mayor es el ancho de banda a la vez que se reduce el valor del pico, tal como se observa en Figura 2.10. De forma que cuanto mayor es la turbulencia del viento, más bajo es el pico de la fuerza lateral y más ancho es su espectro.

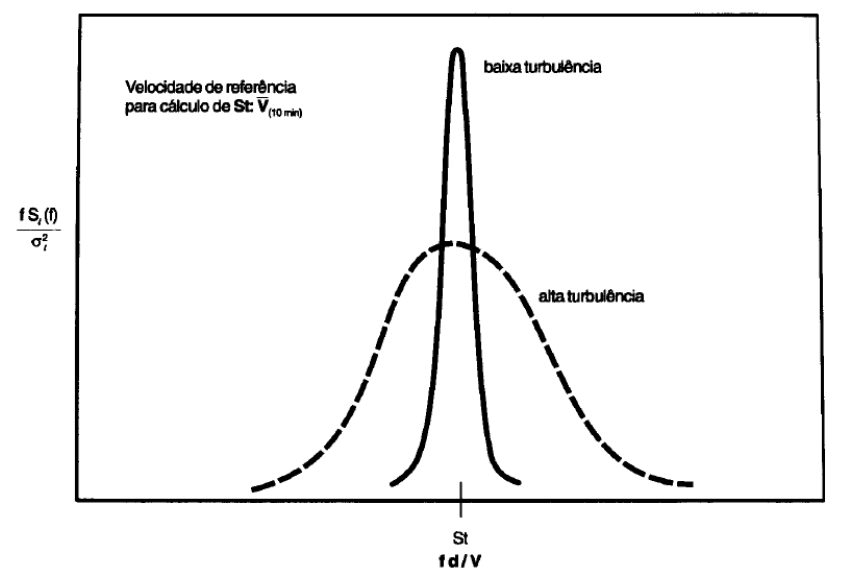

Fuente: Pinheiro [2]

Figura 2.10. Influencia de la turbulencia en el espectro de la fuerza lateral 
La turbulencia de pequeña escala influye en el coeficiente de arrastre, en concreto, en la región crítica en la cual ocurre la caída brusca del coeficiente, tal como se muestra en la Figura 2.11 [20], en la cual se observa que a medida que la turbulencia se incrementa disminuye el número de Reynolds crítico.

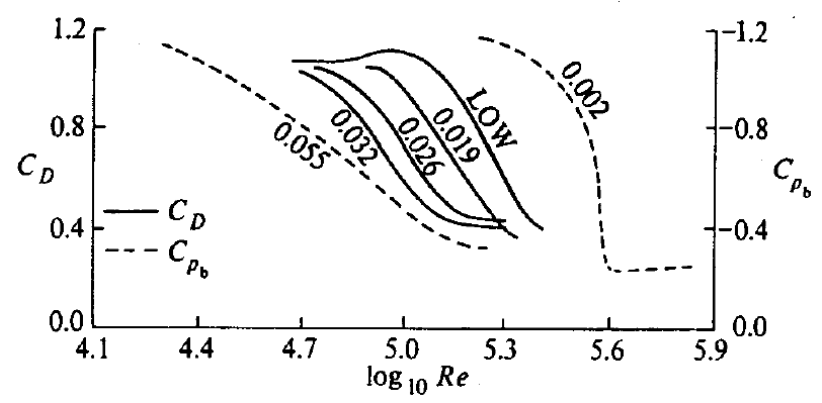

Fuente: Pinheiro [2]

Figura 2.11. Influencia de la turbulencia en el $C_{D}$ para un cilindro circular

\subsubsection{Interacción fluido-estructura. Fenómeno de lock-in}

El flujo aguas abajo de un cuerpo situado perpendicularmente a una corriente incidente uniforme se caracteriza por el desprendimiento alternado y periódico de torbellinos: la denominada calle de torbellinos de Von Kármán. El desprendimiento de torbellinos genera sobre el cuerpo fuerzas laterales periódicas que son la causa de las vibraciones laterales experimentadas por muchas estructuras esbeltas cualquiera que sea su sección, aunque el fenómeno es más apreciable en obstáculos de sección circular.

Para el caso más sencillo, se considerará que el cuerpo es un cilindro circular rígido inmerso en el seno de una corriente de aire con velocidad media uniforme, está elásticamente apoyado y posee amortiguamiento mecánico en la dirección perpendicular a la de incidencia del viento y tiene impedido el movimiento en la dirección paralela.

En estas condiciones, el desprendimiento de torbellinos hace que el cilindro se mueva periódicamente, si bien, la amplitud del moviendo será pequeña a menos que la frecuencia de desprendimiento de los torbellinos se aproxime o coincida con la frecuencia natural del cilindro. Cerca de esta frecuencia, la amplitud del movimiento se hace mayor y el cuerpo comienza a interaccionar con la corriente (fenómeno aeroelástico). Experimentalmente se observa que en ese punto la frecuencia mecánica del cuerpo controla el desprendimiento de torbellinos incluso cuando variaciones en la velocidad media de la corriente alejan la frecuencia nominal de Strouhal de la frecuencia natural del cilindro en un pequeño porcentaje. Este control del fenómeno por las fuerzas 
mecánicas es conocido como lock-in. En la teoría de sistemas dinámicos este fenómeno es conocido como sincronización.

En definitiva, existe una franja de velocidades de la corriente en la cual la frecuencia de desprendimiento de los torbellinos se sincroniza con la frecuencia natural del cuerpo. El fenómeno de lock-in se representa en la Figura 2.12, en la cual se aprecia que en la región de lock-in la frecuencia de desprendimiento de torbellinos es constante e igual a la frecuencia natural del cuerpo y no sigue una función lineal de la velocidad, tal como establece la ley de Strouhal (2.2).

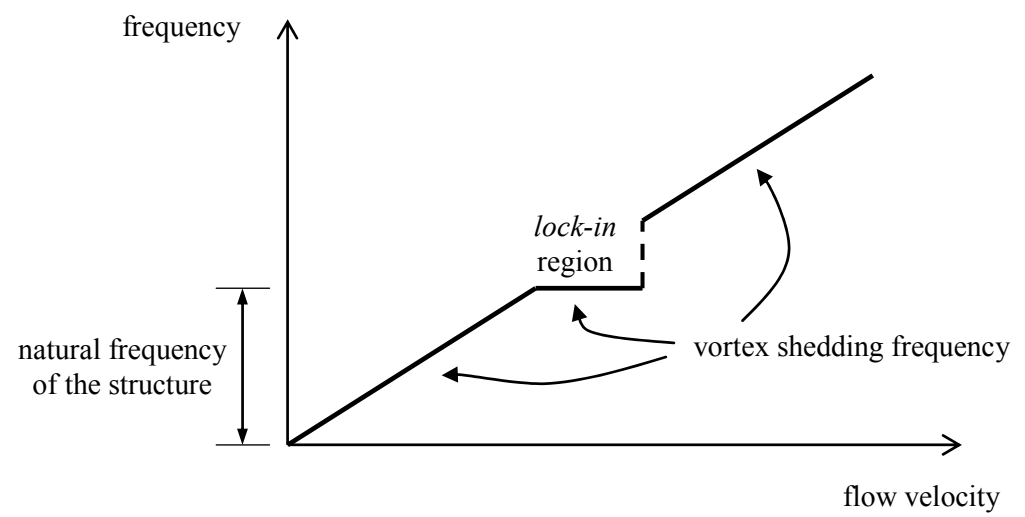

Figura 2.12. Evolución de la frecuencia de desprendimiento de torbellinos con la velocidad de la corriente

Las observaciones muestran que en la región de lock-in la amplitud de las oscilaciones alcanza los valores máximos y se corresponden con alguna fracción de la sección transversal del cuerpo, la cual raramente excede la mitad. En la Figura 2.13 se muestran los resultados experimentales obtenidos por Feng [21] para un cilindro circular rígido apoyado elásticamente con diferentes tasas de amortiguamiento.

La velocidad reducida $U_{r}$, se define como el cociente entre la velocidad media de la corriente incidente y el producto de la frecuencia natural del cuerpo por el diámetro característico

$$
U_{r}=\frac{U}{n_{e} d}
$$

En la Figura 2.14 se muestran los resultados experimentales de la oscilación de un cilindro circular rígido elásticamente apoyado antes, durante y después de la sincronización, junto con los correspondientes espectros de las oscilaciones, donde $f_{s}$ y $f_{n}$ son la frecuencia del desprendimiento de torbellinos y la frecuencia natural del cilindro respectivamente. Antes y después de la sincronización en la respuesta temporal del cilindro existen las dos frecuencias, mientras que 
durante la sincronización la respuesta sólo tiene una componente en frecuencia y ésta es la frecuencia natural del cilindro.

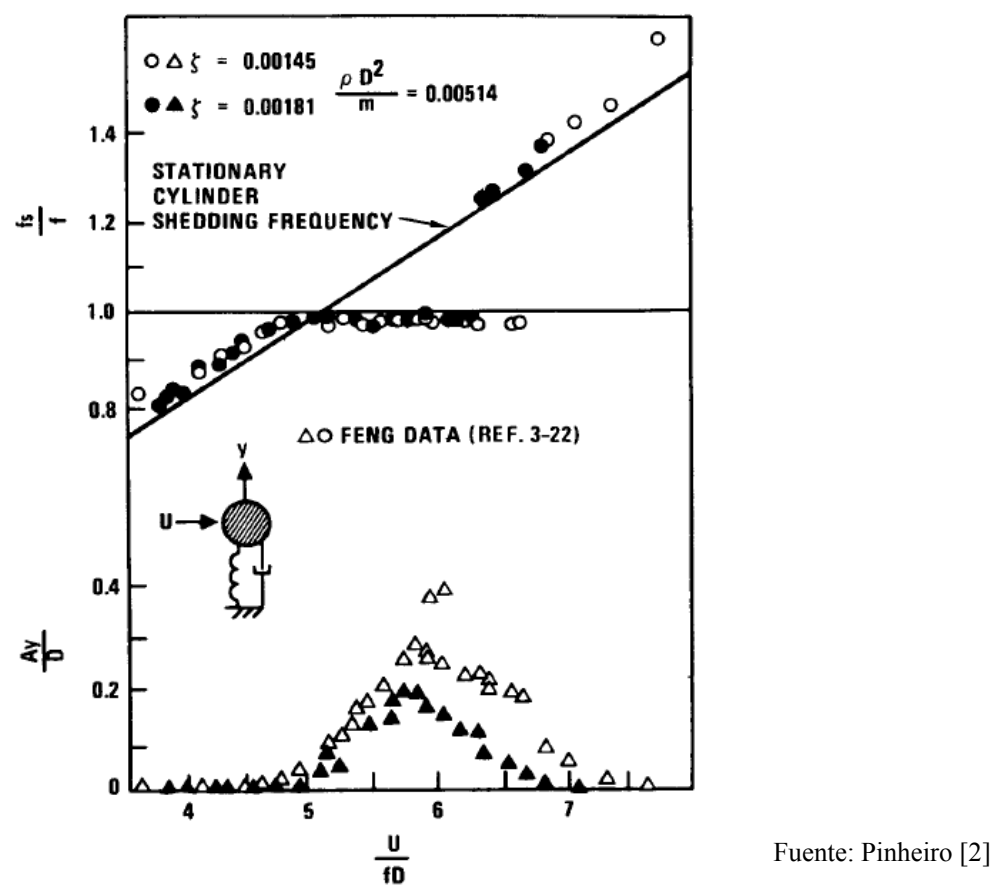

Figura 2.13. Resultados experimentales en frecuencia y amplitud para un cilindro circular rígido
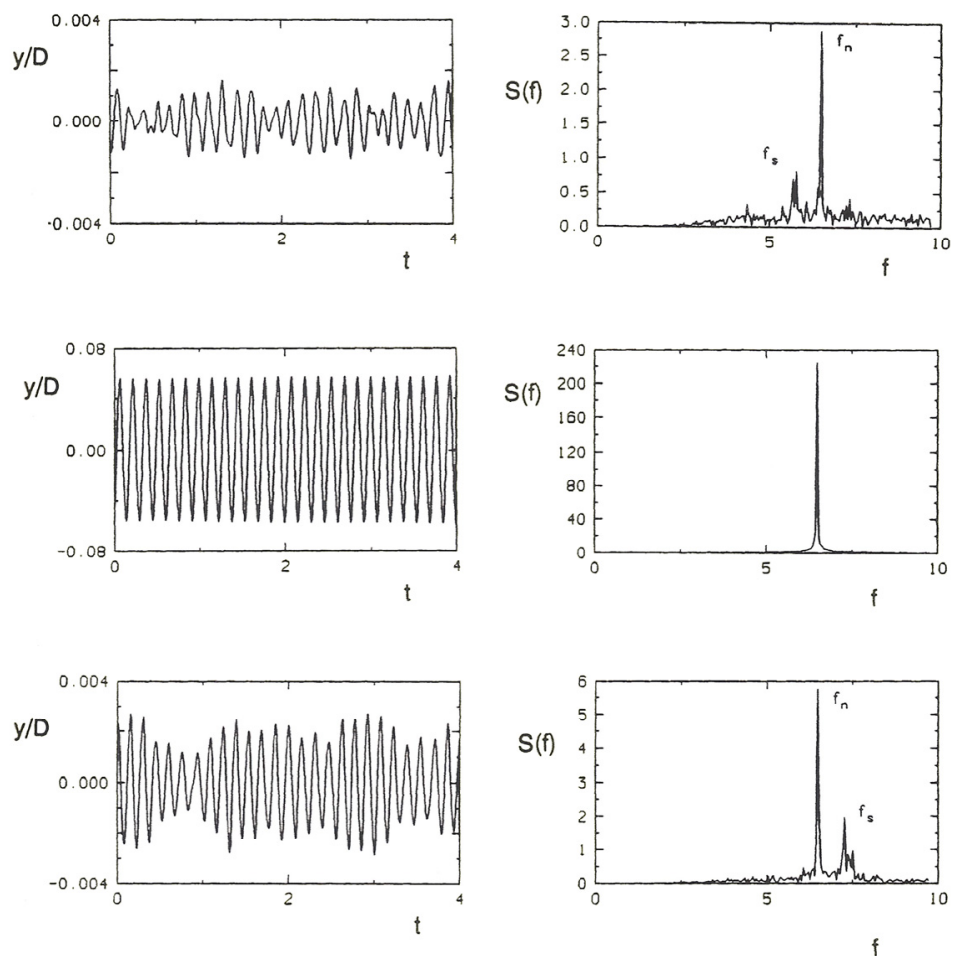

Fuente: Simiu y Scanlan [8]

Figura 2.14. Oscilación de un cilindro circular rígido elásticamente apoyado 


\subsubsection{Longitud de correlación}

La correlación espacial es una medida de los efectos tridimensionales del fluido en la estela que se forma aguas abajo del cilindro. Una correlación igual a la unidad corresponde a un flujo bidimensional, donde los torbellinos se desprenden uniformemente a lo largo de la longitud del cilindro y con la misma frecuencia. Multitud de experimentos se han realizado para determinar la influencia del movimiento del cuerpo en la correlación entre las cargas laterales que provoca el viento. Los resultados de una de estas investigaciones se muestran en la Figura 2.15 [18], en la cual se aprecia que un incremento en la amplitud de la oscilación incrementa la longitud de correlación.
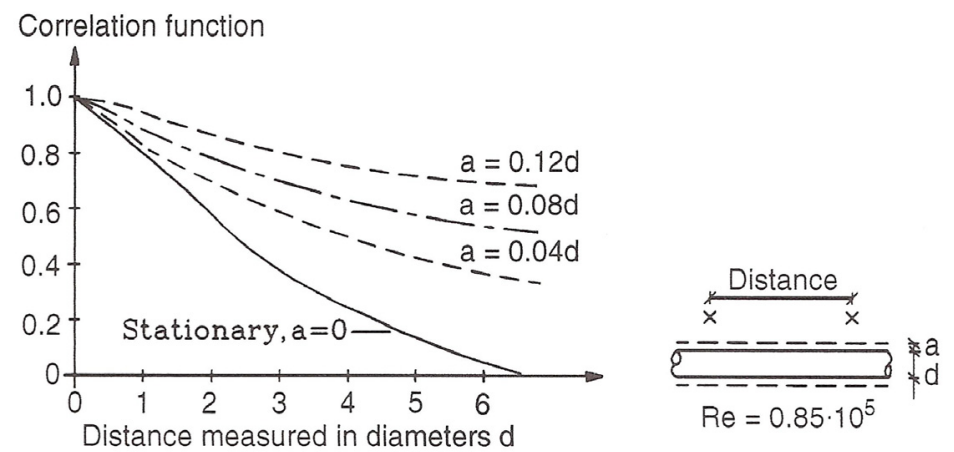

Fuente: Dyrbye y Hansen [14]

Figura 2.15. Influencia de la oscilación del cilindro sobre la correlación espacial del desprendimiento de torbellinos

Por otro lado, la turbulencia provoca una disminución de la correlación espacial entre las presiones laterales de acuerdo con los resultados experimentales de Vickery [22], los cuales son mostrados en la Figura 2.16.

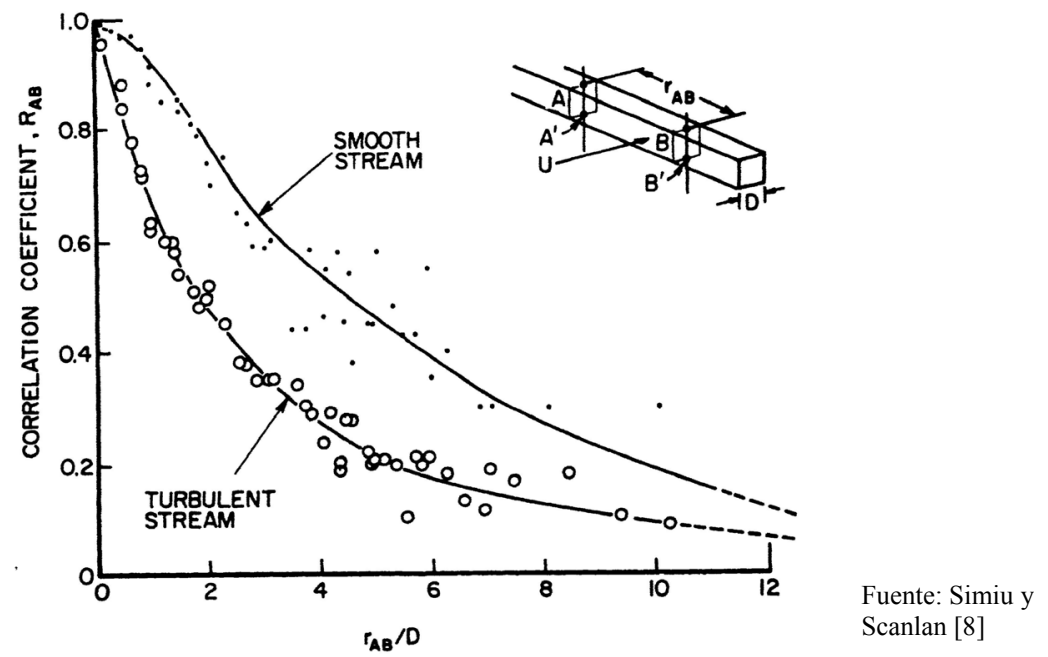

Figura 2.16. Correlación entre las cargas laterales a lo largo del eje de un paralelepípedo de sección cuadrada, $R e=10^{5}$ 
En definitiva, el fenómeno de lock-in tiene un efecto organizador en la formación de la estela, aumentando de forma considerable la correlación entre los torbellinos a lo largo del eje del cilindro en esa región.

\subsection{Acción del viento sobre torres y chimeneas}

El comportamiento de torres o chimeneas sometidas a la acción del viento presenta una mayor complejidad que el comportamiento del cilindro circular rígido elásticamente apoyado expuesto en secciones anteriores. Para el problema del cilindro, excepto cerca de los extremos, la corriente podía ser considerada como bidimensional, mientras que para el caso de torres o chimeneas existen distintos factores que introducen efectos tridimensionales, entre los cuales se citan, por ejemplo:

- La sección transversal de la estructura, en general, es variable.

- La estructura tiene dimensiones finitas y la corriente pasa por el extremo libre de la estructura.

- El viento presenta un perfil de velocidades variable a lo largo de la altura de la estructura, normalmente definido por una expresión exponencial o logarítmica.

- La turbulencia del viento.

\subsubsection{Células de vórtices}

De acuerdo con las observaciones experimentales, el desprendimiento alternado de torbellinos en cilindros [23, 24, 25, 26, 27] se desarrolla en células de vórtices. Estas células son tramos de una cierta longitud a lo largo de la extensión de la estructura en los cuales la frecuencia de desprendimiento de los torbellinos $n_{s}$, es constante y se puede expresar en función del número de Strouhal, un diámetro característico de la celda $d_{c e l}$, y un valor $U$ de la velocidad de la corriente

$$
n_{s}=S t \frac{U}{d_{c e l}}
$$

Esto mismo ocurre en torres y chimeneas de sección constante o variable sometidas a la acción del viento con un gradiente de velocidades a lo largo de la altura de la estructura tal como muestran los resultados experimentales de Vickery y Clark [28]. En su trabajo estudiaron el desprendimiento de torbellinos a lo largo de la altura de un modelo reducido de una chimenea flexible con sección 
linealmente variable. En la Figura 2.17 se muestran las frecuencias de desprendimiento de los torbellinos en función de la altura y para flujo laminar y turbulento. Para flujo laminar se observan con mayor claridad las regiones en las cuales la frecuencia de desprendimiento de los torbellinos es constante, configurando las llamadas células de torbellinos.

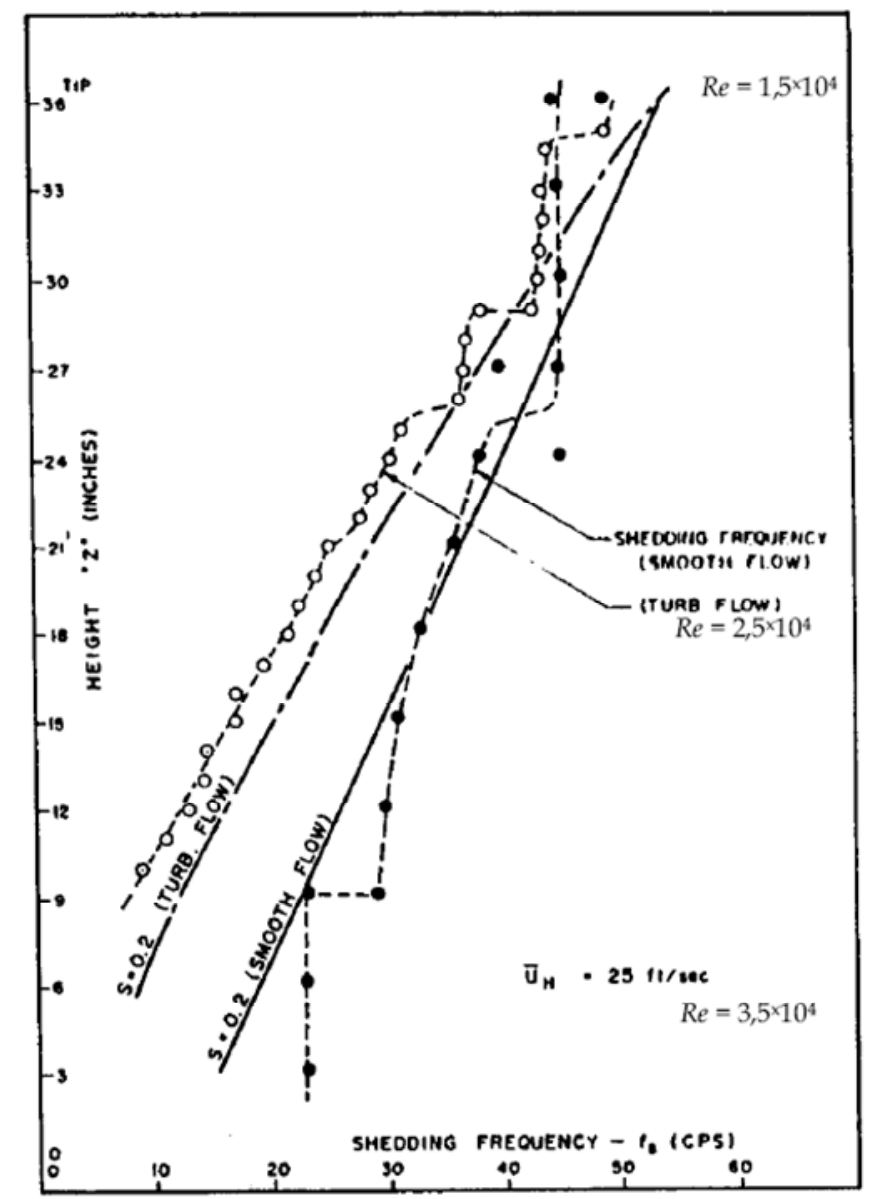

Fuente: Pinheiro [2]

Figura 2.17. Variación de la frecuencia de desprendimiento de los torbellinos con la altura

Aún no se conoce con precisión el mecanismo de formación de las células de torbellinos, sin embargo, se sabe que existen varios factores que influyen en la longitud de estas células, como son la relación entre la altura de la estructura y su diámetro, las condiciones de contorno, la rugosidad superficial de la estructura, la propia amplitud del desplazamiento, la cual tiene un efecto organizador de los torbellinos, además del gradiente de velocidades del viento que incide sobre la estructura. Diversos ensayos descritos en [18] para cilindros lisos y rugosos, con perfil de velocidad lineal, para régimen laminar y turbulento, indican que la longitud de las células se sitúa aproximadamente entre 3 y 10 diámetros. 


\subsubsection{Influencia del amortiguamiento estructural}

El aumento del amortiguamiento estructural disminuye la amplitud de las oscilaciones, tal como se aprecia en la Figura 2.18, en la cual se muestran los resultados experimentales obtenidos por Wootton [29] de la respuesta de una estructura en voladizo de sección transversal circular sometida a una corriente uniforme para diferentes valores del amortiguamiento estructural.

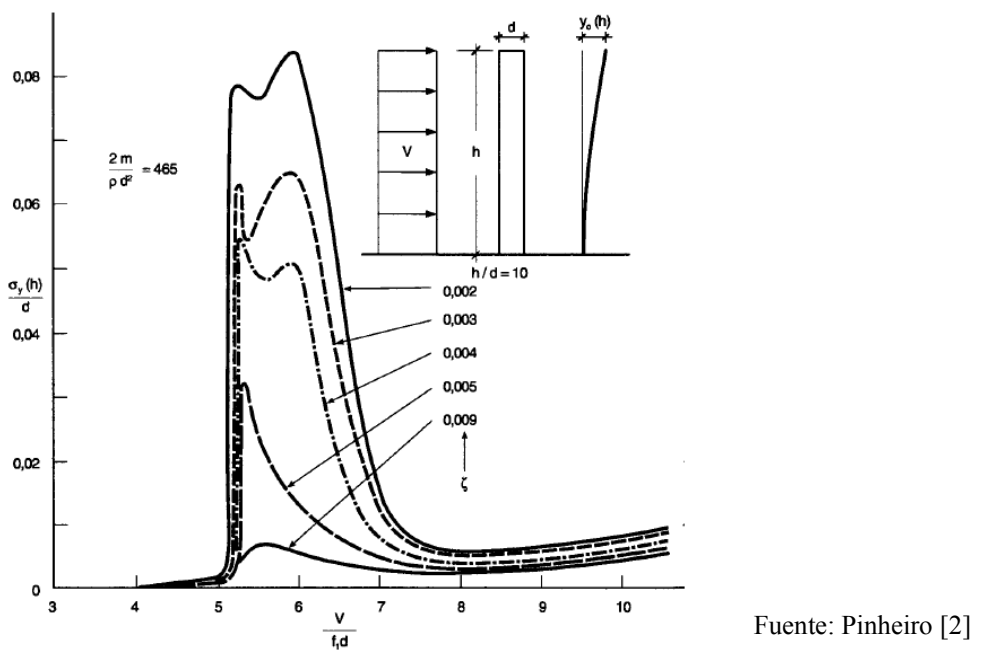

Figura 2.18. Respuesta de una estructura en voladizo de sección circular para diferentes valores del amortiguamiento estructural

La Figura 2.19 muestra los resultados calculados con el modelo analítico de Vickery y Basu [30] frente a los resultados experimentales obtenidos por Wootton [29] de las amplitudes de las oscilaciones correspondientes a las velocidades del flujo más desfavorables en función del amortiguamiento estructural para una estructura en voladizo. Se aprecia que el fenómeno de lock-in ocurre sólo para valores del amortiguamiento estructural pequeños. Cuando el amortiguamiento estructural es grande, se tiene una vibración forzada ocasionada por la carga lateral que induce el desprendimiento de torbellinos, existiendo una zona de transición entre medias.

\subsection{Amplificación del coeficiente de arrastre}

Es sabido que las vibraciones inducidas por el desprendimiento de torbellinos tienden a incrementar el valor del coeficiente de arrastre. Este incremento fue estudiado experimentalmente por Gopalkrishnan [31], Sarpkaya [32] y Bishop y Hassan [33] en cilindros sometidos a vibraciones forzadas con diferentes amplitudes en la dirección transversal a la corriente de fluido. 


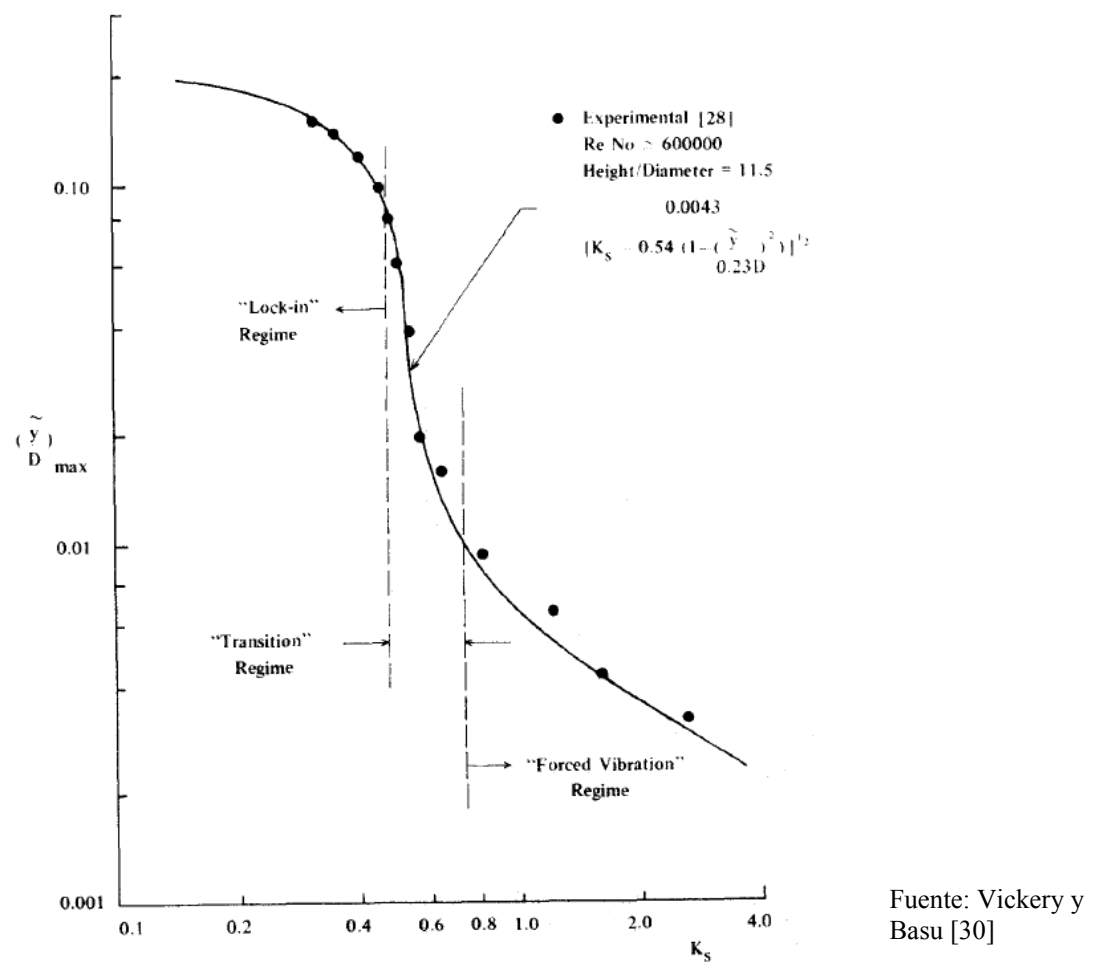

Figura 2.19. Respuesta experimental y estimada en flujo laminar de una estructura en voladizo en función del amortiguamiento estructural

Los resultados obtenidos por Gopalkrishnan [31] se muestran en la Figura 2.20 para $A_{y} / d=$ 0.75 y en la Figura 2.21 para $A_{y} / d=1.2$. Se puede observar que tanto un aumento en la velocidad del fluido como en la amplitud de la vibración del cilindro conducen a un incremento en el coeficiente de arrastre.

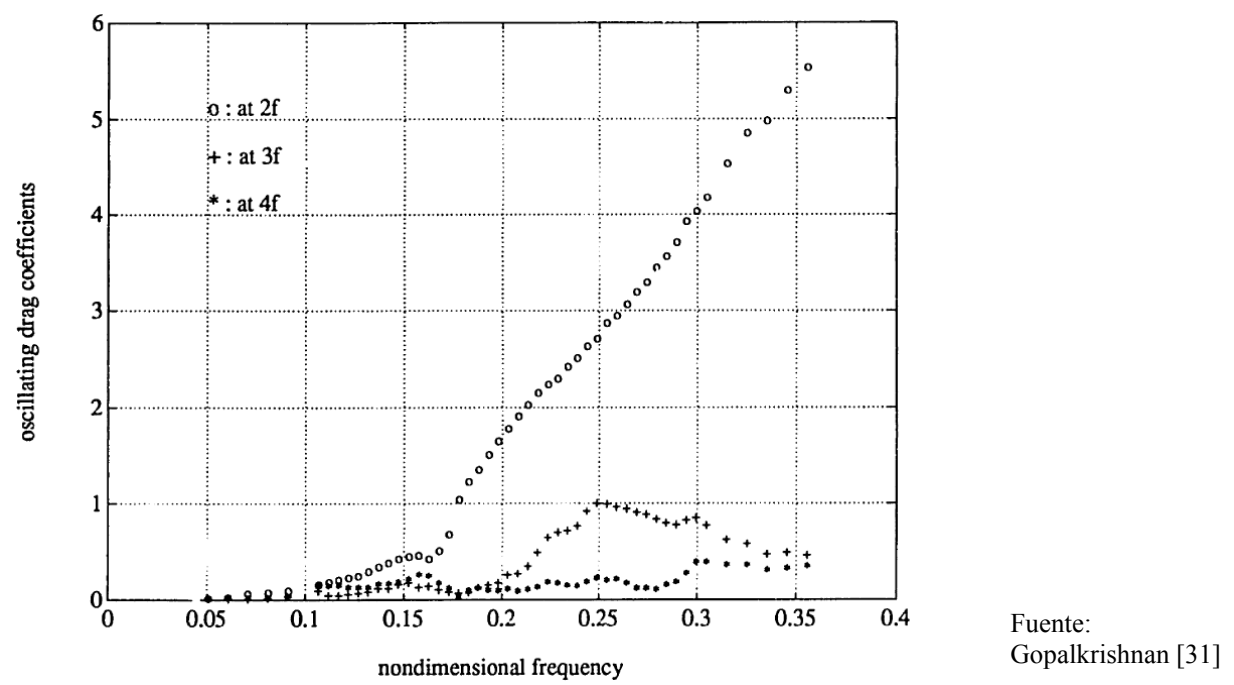

Figura 2.20. Amplificación del coeficiente de arrastre para $A_{y} / d=0.75$ 


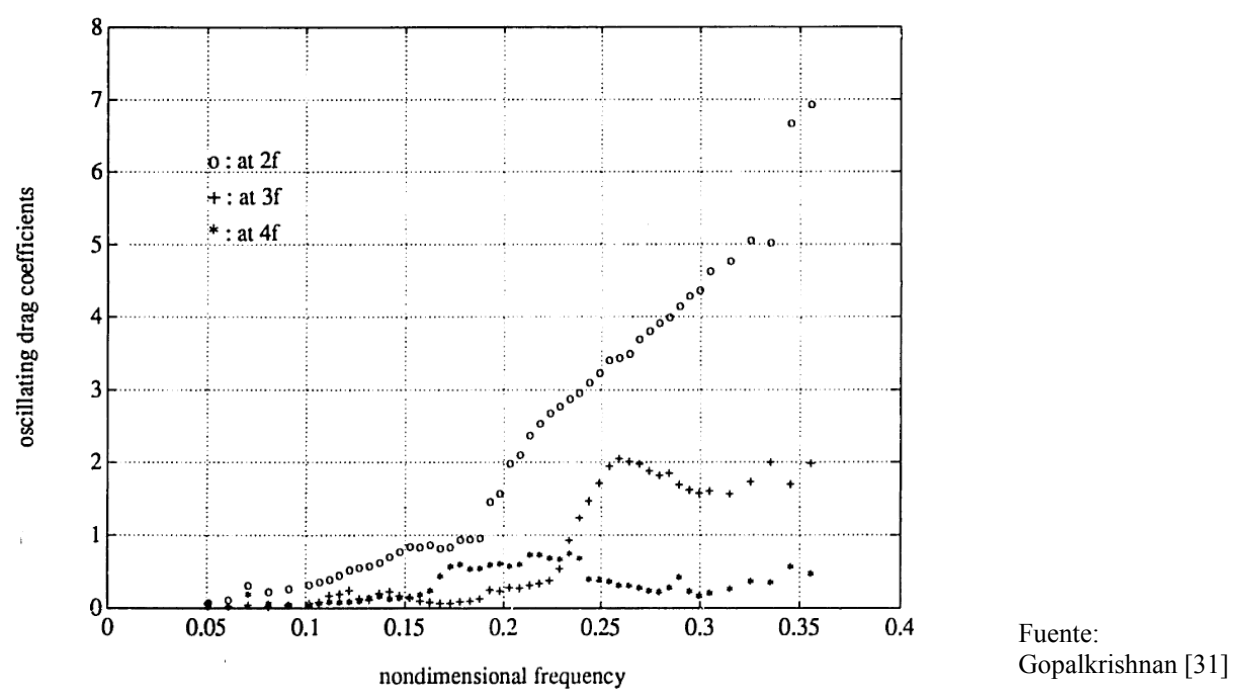

Figura 2.21. Amplificación del coeficiente de arrastre para $A_{y} / d=1.2$

La Figura 2.22 ilustra la amplificación del coeficiente de arrastre obtenida por Sarpkaya [32] y en la Figura 2.23 se muestran los resultados de Bishop y Hassan [33]. Se observa que la fuerza de arrastre se incrementa al incrementar la amplitud de la vibración. Esto está relacionado con el hecho de que la velocidad del movimiento del cilindro junto con la velocidad del fluido definen una velocidad relativa distinta que, en definitiva, es la que gobierna el comportamiento del fluido.

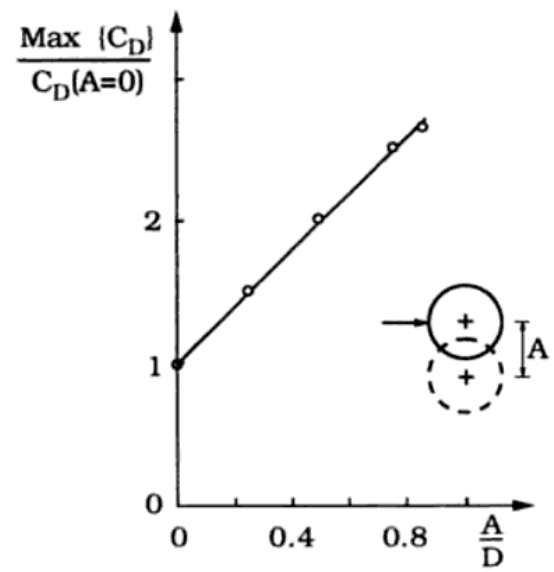

Fuente: Sumer y Fredsфe [34]

Figura 2.22. Amplificación del coeficiente de arrastre medio para $R e=5 \cdot 10^{3}-2.5 \cdot 10^{4}$ 


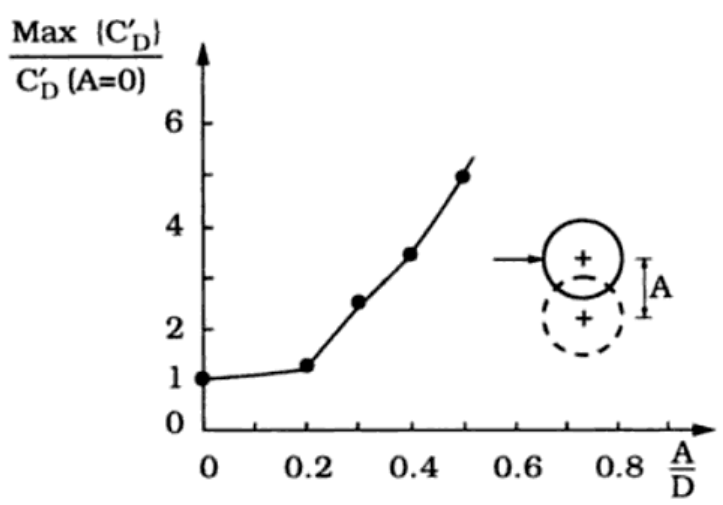

Fuente: Sumer y Fredsфe [34]

Figura 2.23. Amplificación del coeficiente de arrastre para $R e=1 \cdot 1 \cdot 10^{4} . C_{D}{ }^{\prime}$ representa $\left(C_{D}^{2}\right)^{1 / 2}$

\section{Referencias}

${ }^{1}$ Blevins, R.D., Flow Induced Vibration, Van Nostrand Reinhold Company, New York. 1977.

${ }^{2}$ Pinheiro, M.A.S., Vibrações Aeroelásticas em Torres Esbeltas, Tesis Doctoral, Universidad Federal de Rio de Janeiro, 2004.

${ }^{3}$ Lienhard, J.H., Synopsis of lift, drag and vortex frequency for rigid circular cylinders. College of Engineering Research Division, Techn. Extension Service, Universidad de Washington, 1966.

${ }^{4}$ Blessmann, J., Aerodinâmica das Construções. Editorial Sagra, 2 ${ }^{\text {a }}$ Ed., Puerto Alegre, 1990.

${ }^{5}$ Techet, A. H., 13.42 Lecture: Vortex Induced Vibrations, 2005.

${ }^{6}$ Singh, S.P. y Mittal, S., "Flow past a cylinder: shear layer instability and drag crisis", International Journal for Numerical Methods in Fluids, 47, pp. 75-98, 2005.

${ }^{7}$ Roshko. A., "Experiments on the flow past a circular cylinder at very high Reynolds number", Journal of Fluid Mechanics, 10, 345-356, 1961.

${ }^{8}$ Simiu, E., y Scanlan, R.H., Wind effects on structures, fundamental and applications to design (3rd ed.), John Wiley and Sons Inc., NY, 1996.

${ }^{9}$ Dalton, C., "Fundamentals of Vortex-Induced Vibration", Universidad de Houston.

${ }^{10}$ Blessmann, J., Aerodinâmica das Construções. Editorial Sagra, 2 2 Ed., Porto Alegre, 1990.

${ }^{11}$ Scruton, C., "Introduction to Wind Effects on Structures, Engineering Design Guides 40, Published for the Design Council, the British Standards Institution and the Council of Engineering Institutions, Oxford University Press, 1981.

${ }^{12}$ Norberg, C., "Flow around a circular cylinder: aspects of fluctuating lift", Journal of Fluids and Structures, 15 (3), pp. 459-469, 2001. 
${ }^{13}$ Strouhal, V., "Über eine besondere Art der Tonerregung”, Annals of Physics, 5, pp. 216-250, 1878.

${ }^{14}$ Dyrbye, C., y Hansen, S.O., Wind loads on structures, John Wiley \& Sons, Inc., 1997.

${ }^{15}$ Lienhard, J.H., "Synopsis of lift, drag, and vortex frequency data for rigid circular cylinder", Washington State Univ., Coll. Eng., Rept. No. 300, 1966.

${ }^{16}$ Achenbach, E., y Heinecke, E., "On vortex shedding from smooth and rough cylinders in the range of Reynolds numbers $6 \times 10^{3}-5 \times 10^{6 ”}$, Journal of Fluids Mechanics, 109, pp. 239-251, 1981 .

${ }^{17}$ Blessmann, J., "O Vento na Engenharia Estrutural". Editorial de la Universidad, Universidad Federal do Rio Grande do Sul. $1^{\text {a }}$ Ed., 1995.

${ }^{18}$ Blessmann, J., Introdução ao Estudo das Ações Dinâmicas do Vento, Puerto Alegre, Ed. de la Universidad, UFRGS, 1988.

${ }^{19}$ Lawson, T.V., Wind Effects on Buildings: Design Applications, Volume 1. Applied Science Publishers LTD, London, 1980.

${ }^{20}$ Scruton, C., "An Introduction to Wind Effects on Structures”. Engineering Design Guides 40, Published for the Design Council, the British Standards Institution and the Council of Engineering Institutions, Oxford University Press.

${ }^{21}$ Feng, C.C., The measurement of vortex-induced effects in flow past a stationary and oscillating circular cylinder and d-section cylinders, Master's Thesis, Universidad de British Columbia, Vancouver, Canada, 1968.

${ }^{22}$ Vickery, B.J., "Fluctuating Lift and Drag on a Long Cylinder of Square Cross-Section in a Smooth and in a Turbulent Stream”, Journal of Fluids Mechanics, 25 (3), pp. 481-494, 1966.

${ }^{23}$ Humphreys, J.S., "On a Circular Cylinder in a Steady wind at Transition Reynolds Numbers", Journal of Fluid Mechanics, 9, pp. 603-612, 1960.

${ }^{24}$ Stansby, P.K., "The Locking-on of Vortex Shedding Due to the Cross- Stream Vibration of Circular Cylinders in Uniform and Shear Flows", Journal of Fluid Mechanics, 74 (4), pp. 641-665, 1976.

${ }^{25}$ Balasubramanian, S., Hann Jr., F.L., Szewczyk, A.A. \& Skop, R.A., "On the Existence of a Critical Shear Parameter for Cellular Vortex Shedding from Cylinders in Nonuniform Flow", Journal of Fluids and Structures, 12, pp. 3-15, 1998.

${ }^{26}$ Mair, W.A., y Stansby, P. K., "Vortex Wakes of Bluff Cylinders in Shear Flow", Journal of Applied Mathematics, 28, pp. 519-540, 1975.

${ }^{27}$ Hsiao, F.B., y Chiang, C.H., "Experimental study of cellular shedding vortices behind a tapered circular cylinder”, Experimental Thermal and Fluid Science, 17 (3), pp. 179-188, 1998. 
${ }^{28}$ Vickery, B.J., y Clark, A.W., "Lift or Across-Wind Response of Tapered Stacks", Journal of the Structural Division, Proceedings of the American Society of Civil Engineers, 8634, ST 1, Janeiro, pp. 1-20, 1972.

${ }^{29}$ Wootton, L.R., "The Oscillations of Large Circular Stacks in Wind", Proceedings of the Institution of Civil Engineers., 42, pp. 573-598, 1969.

${ }^{30}$ Vickery, B.J., y Basu, R.I., “Across-Wind Vibrations of Structures of Circular Cross-Section. Part

I. Development of a Mathematical Model for Two-Dimensional Conditions". Journal of Wind Engineering and Industrial Aerodynamics, 12, pp. 49-73, 1983.

31 Gopalkrishnan, R., Vortex-induced forces on oscillating bluff cylinders, Tesis Doctoral, Massachusetts Institute of Technology and Woods Hole Oceanographic Institution, 1993.

${ }^{32}$ Sarpkaya, T., "Fluid Forces on Oscillating Cylinders", Journal of the Waterway Port Coastal and Ocean Division - ASCE, 104 (3), pp. 275-290, 1978.

${ }^{33}$ Bishop, R.E.D., y Hassan, A.Y., "The Lift and Drag Forces on a Circular Cylinder oscillating in a Flowing Fluid", Proceedings of the Royal Society of London. Series A, Mathematical and Physical Sciences, 277 (1368), pp. 32-50, 1964.

${ }^{34}$ Sumer, B.M. y Fredsфe, J., Hydrodynamics Around Cylindrical Structures, World Scientific, Singapore, 1997. 

Capítulo 3

\section{Formulación Teórica del Modelo de Barras}





\subsection{Resumen}

En este capítulo se presenta un modelo monodimensional para el análisis de barras de sección de pared delgada constituidas por materiales compuestos. La formulación hace uso de los modelos de Navier-Bernoulli y Vlasov para describir la flexión y la torsión, respectivamente, a nivel de barra, y del modelo de Love-Kirchhoff para plantear las relaciones de comportamiento a nivel de lámina. Como primer resultado, se consigue una matriz de rigidez de dimensiones $5 \times 5$ que relaciona los esfuerzos y las deformaciones monodimensionales a nivel de sección. Posteriormente, utilizando el Principio de los Trabajos Virtuales y las funciones de forma apropiadas se obtiene la matriz de rigidez de un elemento tipo barra. El método es aplicable a barras con secciones de forma arbitraria con cualquier esquema de laminado, tanto abiertas como cerradas.

\subsection{Introducción}

Jung, Nagaraj y Chopra [1] han descrito el estado del arte en lo referente a las técnicas para la obtención de modelos capaces de reproducir la respuesta de barras de sección de pared delgada constituidas por materiales compuestos ante diferentes estados de carga, poniendo de manifiesto la importancia de los acoplamientos elásticos y del alabeo de las secciones. Para caracterizar estos fenómenos se puede plantear el problema desde dos perspectivas diferentes.

Uno de estos planteamientos consiste en el Análisis Asintótico Variacional de la Sección Transversal de la Barra (VABS) [2, 3, 4, 5]. Esta herramienta permite dividir un problema general de elasticidad no lineal 3D en dos partes, una lineal 2D para analizar la sección transversal que permita obtener sus características y otra no-lineal 1D en la que se estudia la barra considerando las características de la sección antes obtenidas. Se trata de un modelo de elementos finitos en el que se desprecian los términos poco significativos, con la consiguiente pérdida de perspectiva física en la formulación.

La otra tendencia para el análisis de barras de materiales compuestos consiste en desarrollar modelos analíticos monodimensionales basados en la combinación de las teorías clásicas de barras, de láminas y de materiales compuestos. Estos modelos tienen la ventaja de ser simples y útiles para realizar diseños preliminares y para optimización. Su formulación permite plantear el problema tanto en desplazamientos como en fuerzas. El primero de los planteamientos ha sido aplicado por autores como Rehfield, Atilgan y Hodges [6], Smith y Chopra [7], Chandra y Chopra [8, 9], Jonson 
[10], Patil y Jonson [11], Zhang y Smith [12]. Consiste en plantear un campo de desplazamientos adecuado a nivel de lámina y a partir de él obtener la energía de deformación y la matriz de rigidez de la sección transversal de la barra. De otra parte, el planteamiento en fuerzas fue aplicado por Mansfield y Sobey [13] y por Libove [14] a barras de pared delgada de materiales compuestos de sección cerrada. En él se asume directamente un campo de tensiones a nivel de lámina.

Como alternativa a estos dos planteamientos extremos, Jung, Nagaraj y Chopra [15] desarrollaron un modelo mixto combinando ambos y lo aplicaron con éxito tanto a secciones abiertas como a cerradas. Estos autores plantearon el funcional de energía semi-complementaria de Reissner y obtuvieron una matriz de rigidez de dimensiones $7 \times 7$ (considerando la teoría de Timoshenko) por derivación de las ecuaciones que relacionan los desplazamientos generalizados a nivel de barra con las correspondientes fuerzas generalizas. Recientemente, Jung y Park [16] han aplicado este modelo mixto al estudio de secciones multicelulares.

En el presente capítulo se desarrolla un modelo analítico monodimensional para describir el comportamiento a flexión y a torsión de barras de sección de pared delgada constituidas por materiales compuestos. La metodología propuesta parte del modelo de lámina de Love-Kirchhoff para relacionar los esfuerzos con las deformaciones a nivel de lámina. En dicha relación se introducen las deformaciones generalizadas de barra correspondientes a los modelos de NavierBernoulli y Vlasov mediante consideraciones geométricas. A continuación, integrando los esfuerzos de lámina en la sección se obtienen los esfuerzos de barra en función de las deformaciones generalizadas de barra. Dicha relación se establece a través de una matriz de rigidez de dimensiones $5 \times 5$. Por último, utilizando el Principio de los Trabajos Virtuales y las funciones de forma apropiadas se obtiene la matriz de rigidez de un elemento tipo barra con siete grados de libertad en cada nodo, que son las tres traslaciones y las tres rotaciones respecto a cada eje, así como el alabeo. En definitiva, se consigue una matriz de rigidez que puede ser utilizada para el análisis de barras sometidas a flexión-torsión.

En el Anexo G se presentan dos ejemplos que ilustran la aplicabilidad de la técnica descrita. En el primer ejemplo se analizan dos secciones abiertas, una en doble $\mathrm{T}$ y otra en $\mathrm{T}$, y en el segundo se estudia una barra de sección en cajón rectangular bajo dos configuraciones de laminado diferentes, una con rigidez circunferencial uniforme (CUS) y otra con rigidez circunferencial asimétrica (CAS), para el estudio de los acoplamientos axil-torsión y flexión-torsión, respectivamente La técnica ha sido validada comparando los resultados obtenidos con los deducidos por otros autores. 


\subsection{Formulación teórica}

En la Figura 3.1.a se muestra la geometría de una barra de sección de pared delgada con forma arbitraria. En dicha barra se considerará un sistema de referencia global $O x y z$, con origen en una sección arbitraria. Para referir localmente las variables asociadas a los puntos de la sección se utilizará otro sistema de referencia $O^{\prime} x n s$ (Figura 3.1.b), con origen en un extremo libre si la sección es abierta y en un punto arbitrario de la línea media de la sección si ésta es cerrada. El eje local $O^{\prime} s$ es tangente a la línea media de la sección en el punto considerado, el eje $O^{\prime} x$ es paralelo al eje $O x$ global de la barra y la tercera dirección, definida por el eje $O^{\prime} n$, lleva la dirección del espesor, de modo que con los dos anteriores forme un triedro directo. Los vectores unitarios según los ejes coordenados serán $\vec{i}_{x}, \vec{j}_{y}$ y $\vec{k}_{z}$ en el sistema global y $\vec{i}, \vec{j}$ y $\vec{k}$ en el sistema local. Para describir el comportamiento de una rebanada genérica de barra, con longitud $d x$, se considerará la interacción entre elementos lámina de esa misma longitud y área $d x d s$.

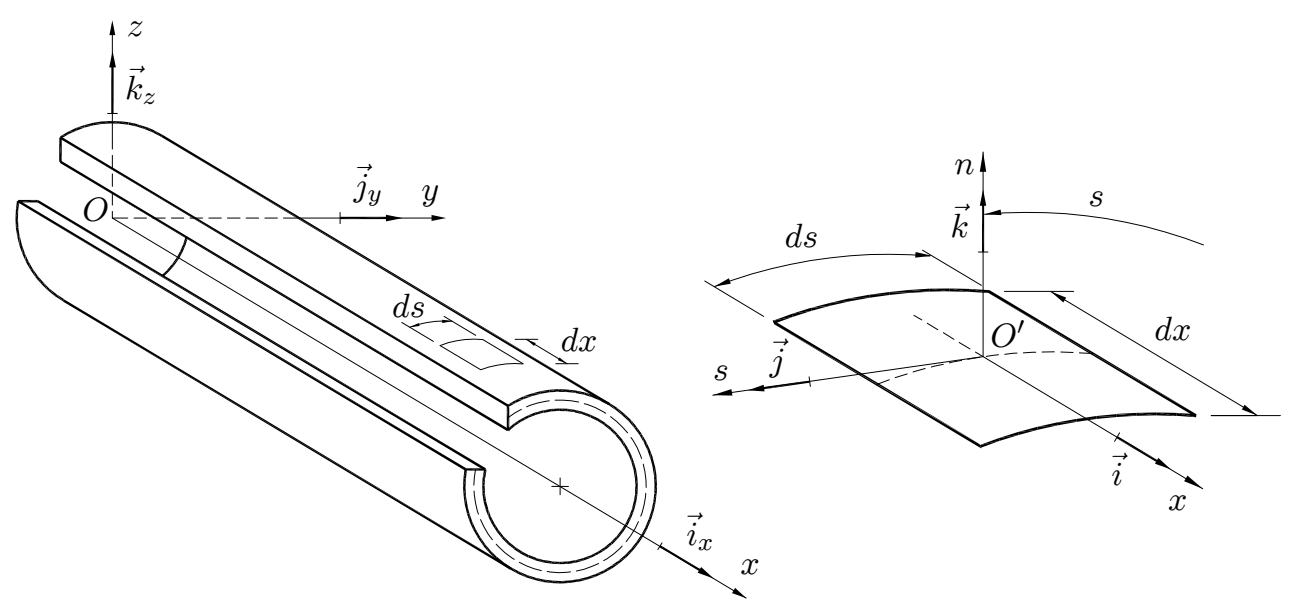

(a)

(b)

Figura 3.1. Sistemas de coordenadas

\subsubsection{Cinemática}

Tras la deformación, el desplazamiento de cualquier punto viene dado por

$$
\begin{aligned}
& \vec{D}(x, y, z)=\left(U(x)+\beta_{y}(x) z+\beta_{z}(x) y+U^{\Omega}(x, y, z)\right) \vec{i}_{x}+ \\
& \quad+\left(V(x)+V^{\phi}(x, y, z)\right) \vec{j}_{y}+\left(W(x)+W^{\phi}(x, y, z)\right) \vec{k}_{z}
\end{aligned}
$$


que consiste en una traslación de toda la sección dada por el vector $U \vec{i}_{x}+V \vec{j}_{y}+W \vec{k}_{z}$ más un movimiento $V^{\phi} \vec{j}_{y}+W^{\phi} \vec{k}_{z}$ adicional en el plano de la sección transversal que contiene al punto considerado debido al giro $\phi$ por torsión alrededor del eje $O x$, más sendos giros $\beta_{y}$ y $\beta_{z}$ alrededor de los ejes $O y$ y $O z$, respectivamente, a causa de la flexión, más un desplazamiento en la dirección axial $U^{\Omega}$ debido al alabeo.

Ese mismo desplazamiento se puede expresar en las coordenadas locales $(x, n, s)$ del elemento lámina

$$
\vec{d}(x, n, s)=u(x, n, s) \vec{i}+v_{t}(x, n, s) \vec{j}(s)+v_{n}(x, n, s) \vec{k}(s)
$$

Dado el pequeño espesor, se despreciará la deformación normal en la dirección de esa dimensión $\left(\varepsilon_{n n}=0 \rightarrow v_{n} \equiv v_{n}(x, s)\right)$. El campo de desplazamientos se puede expresar de la siguiente forma

$$
\vec{d}(x, n, s)=\left(u^{0}(x, s)+n \psi_{x}(x, s)\right) \vec{i}+\left(v_{t}^{0}(x, s)+n \psi_{s}(x, s)\right) \vec{j}(s)+v_{n}^{0}(x, s) \vec{k}(s)
$$

donde $\left\{u^{0}(x, s), v_{t}^{0}(x, s), v_{n}^{0}(x, s)\right\}$ son las componentes del desplazamiento del punto de coordenadas $(x, 0, s)$ (situado en el plano medio), Figura 3.2.

$$
\vec{d}^{0}(x, s)=\vec{d}(x, 0, s)=u^{0}(x, s) \vec{i}+v_{t}^{0}(x, s) \vec{j}(s)+v_{n}^{0}(x, s) \vec{k}(s)
$$

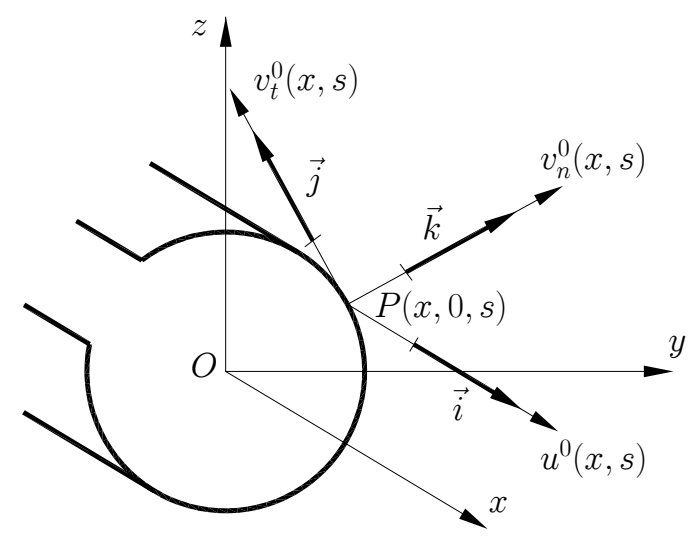

Figura 3.2. Plano medio del perfil

Se admitirá que los planos $x n$ y $n s$ de los elementos lámina se mantienen planos en la deformación. Con referencia a la Figura 3.3, $\psi_{x}$ es un giro del plano medio alrededor del eje $O^{\prime} s$, 
que provoca un desplazamiento en la dirección $x, \mathrm{y} \psi_{s}$ es un giro del plano medio alrededor del eje $O^{\prime} x$, que origina un desplazamiento en la dirección de $s$.
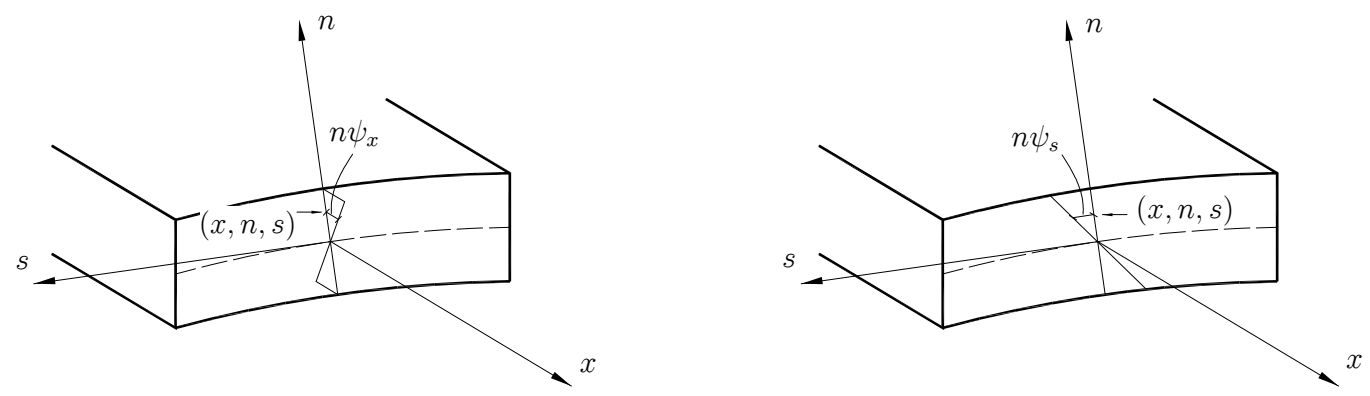

Figura 3.3. Giros $\psi_{x} \mathrm{y} \psi_{s}$

El campo de desplazamientos asumido se corresponde con el modelo de lámina de LoveKirchhoff.

$$
\vec{d}=\left\{\begin{array}{c}
u \\
v_{t} \\
v_{n}
\end{array}\right\}=\left\{\begin{array}{c}
u^{0}+n \psi_{x} \\
v_{t}^{0}+n \psi_{s} \\
v_{n}^{0}
\end{array}\right\}
$$

Teniendo en cuenta que en una lámina de Love-Kirchhoff no se produce deformación por cortante $\left(\omega_{x}=0, \omega_{s}=0\right)$, las rotaciones de la de la normal $\psi_{x}$ y $\psi_{s}$ han de coincidir con las rotaciones de las tangentes a las líneas coordenadas de la superficie de referencia $\Sigma$, denominadas $\varphi_{x} \mathrm{y} \varphi_{s}$ (Figura 3.4).

$$
\psi_{x}=\varphi_{x} \quad \psi_{s}=\varphi_{s}
$$

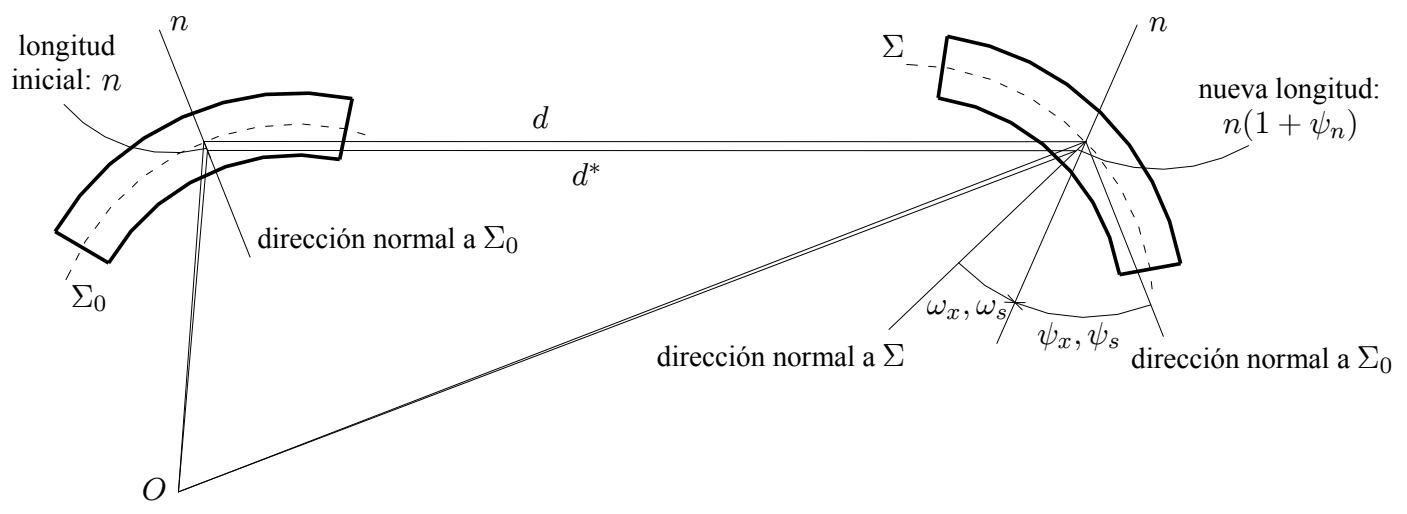

Figura 3.4. Cinemática 


\subsubsection{Ecuaciones de compatibilidad}

Como es sabido, en términos de ecuaciones de compatibilidad en coordenadas locales, el campo de deformaciones es (Anexo D)

$$
\begin{aligned}
& \left\{\varepsilon^{*}\right\}=\{\varepsilon\}+n\{\kappa\} \\
& \left\{\begin{array}{c}
\varepsilon_{x x} \\
\varepsilon_{s s} \\
\gamma_{x s} \\
\gamma_{x n} \\
\gamma_{s n}
\end{array}\right\}=\left\{\begin{array}{c}
u_{, x}^{0} \\
v_{t, s}^{0}+\frac{v_{n}^{0}}{a} \\
u_{, s}^{0}+v_{t, x}^{0} \\
v_{n, x}^{0}+\psi_{x} \\
v_{n, s}^{0}-\frac{v_{t}^{0}}{a}+\psi_{s}
\end{array}\right\} \\
& \left\{\begin{array}{c}
\kappa_{x x} \\
\kappa_{s s} \\
\kappa_{x s}
\end{array}\right\}=\left\{\begin{array}{c}
\psi_{x, x} \\
\psi_{s, s} \\
\psi_{x, s}+\psi_{s, x}
\end{array}\right\}
\end{aligned}
$$

donde $a$ es radio de curvatura de la lámina en el punto considerado y se ha usado la notación tensorial para indicar las derivaciones. Dado que no se considera la deformación por cortante, se tiene que

$$
\begin{gathered}
\gamma_{x n}=v_{n, x}^{0}+\psi_{x}=0 \Rightarrow \psi_{x}=-v_{n, x}^{0} \\
\gamma_{s n}=v_{n, s}^{0}-\frac{v_{t}^{0}}{a}+\psi_{s}=0 \Rightarrow \psi_{s}=-v_{n, s}^{0}+\frac{v_{t}^{0}}{a}
\end{gathered}
$$

De otra parte, las componentes $v_{t}^{0}$ y $v_{n}^{0}$ del desplazamiento de los puntos pertenecientes a la superficie de referencia se pueden expresar en función de las correspondientes componentes globales $V$ y $W$ del desplazamiento de un punto $P(x, 0, s)$ de la línea media y del desplazamiento adicional debido al giro $\phi$ por torsión adoptando la teoría de Navier-Bernoulli para la flexión y de Vlasov para la torsión.

$$
\begin{gathered}
v_{t}^{0}(x, s)=V(x) y(s)_{, s}+W(x) z(s)_{, s}+r(s) \phi(x) \\
v_{n}^{0}(x, s)=V(x) z(s)_{, s}-W(x) y(s)_{, s}-q(s) \phi(x) \\
\psi_{s}(x, s) \equiv \phi(x)
\end{gathered}
$$

donde $r$ y $q$ son las distancias existentes entre el centro de esfuerzos cortantes y las rectas tangente y normal, respectivamente, a la línea media de la sección en el punto considerado (Anexo B). 
En definitiva, las componentes tangencial y normal del desplazamiento de un punto cualquiera de la sección serán

$$
\begin{gathered}
v_{t}(x, n, s)=V(x) y(s)_{, s}+W(x) z(s)_{, s}+[r(s)+n] \phi(x) \\
v_{n}(x, n, s) \equiv v_{n}^{0}(x, s)=V(x) z(s)_{, s}-W(x) y(s)_{, s}-q(s) \phi(x)
\end{gathered}
$$

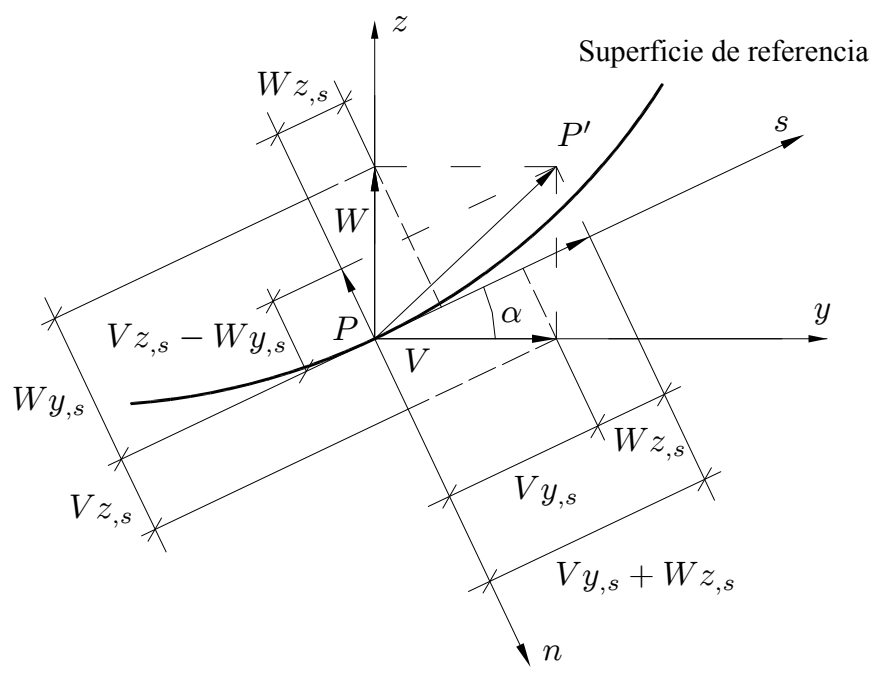

Figura 3.5. Desplazamiento del punto $P(x, 0, s)$ debido a la torsión

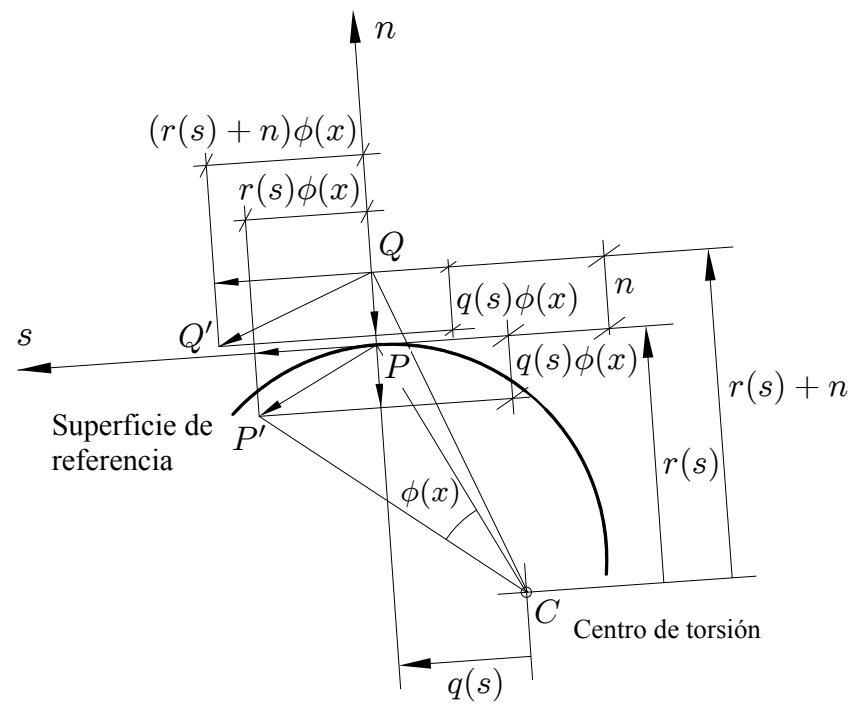

Figura 3.6. Desplazamiento del punto $Q(x, n, s)$ debido a la torsión 
Derivando adecuadamente las ecuaciones (3.11) y sustituyendo el resultado en (3.8) y (3.9), se obtienen las siguientes relaciones

Extensión de la superficie de referencia según la dirección $x, \varepsilon_{x x}$

$$
\varepsilon_{x x}=u_{, x}^{0}
$$

Extensión de la superficie de referencia según la dirección $s, \varepsilon_{s s}$

$$
\varepsilon_{s s}=v_{t, s}^{0}+\frac{v_{n}^{0}}{a}=0
$$

Variación angular, $\gamma_{x s}$

$$
\gamma_{x s}=u_{, s}^{0}+v_{t, x}^{0}=u_{, s}^{0}+V_{, x} y_{, s}+W_{, x} z_{, s}+r \phi_{, x}
$$

Pseudo-cambio de curvatura según la dirección $x, \kappa_{x x}$

$$
\kappa_{x x}=\psi_{x, x}=-v_{n, x x}^{0}=\beta_{z, x} z_{, s}-\beta_{y, x} y_{, s}+q \phi_{, x x}
$$

Pseudo-cambio de curvatura según la dirección $s, \kappa_{s s}$

$$
\kappa_{s s}=\psi_{s, s}=0
$$

Pseudo-torsión geodésica de la superficie media, $\kappa_{x s}$

$$
\kappa_{x s}=2 \phi_{, x}+\frac{1}{a}\left(\beta_{z} y_{, s}+\beta_{y} z_{, s}-r \phi_{, x}\right)
$$

En las ecuaciones anteriores, aún no ha sido deducido el desplazamiento axil $u^{0}$. Para su determinación es necesario integrar la expresión de la deformación angular $\gamma_{x s}$ entre el origen de la coordenada $s$ y el punto considerado en la línea media de la sección. De dicha integral se obtiene

$$
u^{0}(x, s)=U(x)+\beta_{z}(x) y(s)+\beta_{y}(x) z(s)-\phi(x){ }_{, x} \omega(s)
$$

estando $\omega$ definido como

$$
\omega=\int_{0}^{s} r d s
$$

Por tanto, El desplazamiento $u^{0}$ de cualquier punto del plano medio puede expresarse como la superposición de los siguientes movimientos axiles

- La traslación axil $U$ de la sección por la actuación de un esfuerzo axil, si lo hubiere.

- El desplazamiento $\beta_{z} y+\beta_{y} z$ en el que se traducen los giros alrededor de los ejes de la sección por la acción de los momentos flectores. 
- El desplazamiento axil asociado al alabeo por deformación angular $-\phi_{, x} \omega$.

Con ello, la ecuación (3.13) se transforma en

$$
\varepsilon_{x x}=U_{, x}+\beta_{z, x} y+\beta_{y, x} z-\phi_{, x x} \omega
$$

\subsubsection{Ecuaciones de equilibrio}

Según la teoría clásica de láminas, las ecuaciones de equilibrio de un elemento diferencial de lámina de pequeño espesor (se desprecian los esfuerzos de membrana $N_{s n}$ y $N_{x n}$ ) se muestran a continuación.

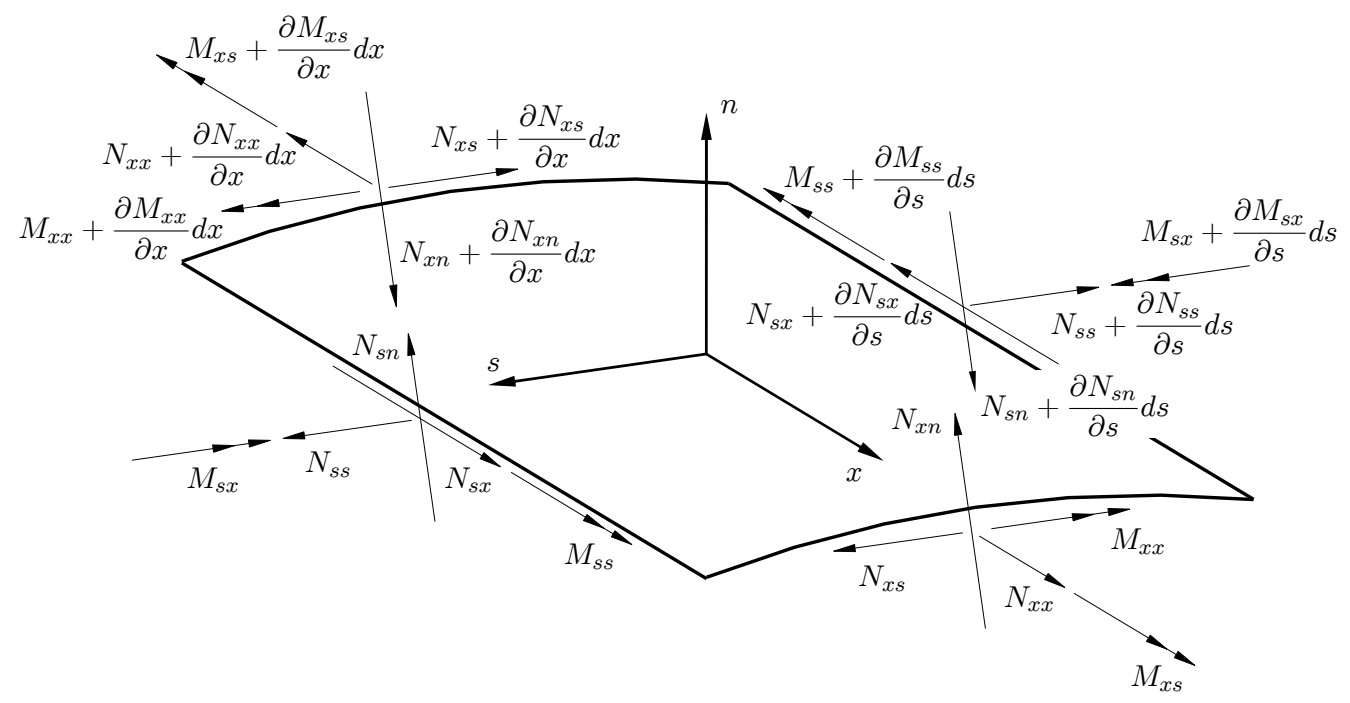

Figura 3.7. Esfuerzos de lámina

Imponiendo la condición de que la suma las fuerzas aplicadas al elemento debe ser nula, se obtiene la siguiente ecuación vectorial

$$
\frac{\partial}{\partial x}\left(N_{x x} \vec{i}+N_{x s} \vec{j}+N_{x n} \vec{k}\right)+\frac{\partial}{\partial s}\left(N_{s s} \vec{i}+N_{x s} \vec{j}+N_{s n} \vec{k}\right)=0
$$

La cual es equivalente a las siguientes dos ecuaciones escalares

$$
\begin{gathered}
N_{x x, x}+N_{x s, s}=0 \\
N_{x s, x}=0
\end{gathered}
$$

Actuando de forma análoga respecto a los momentos aplicados al elemento, se obtiene la siguiente ecuación vectorial 


$$
\frac{\partial}{\partial x}\left(\left(M_{x s}+s N_{x n}\right) \vec{i}-\left(M_{x x}-x N_{x n}\right) \vec{j}\right)+\frac{\partial}{\partial s}\left(\left(M_{s s}+s N_{s n}\right) \vec{i}-\left(M_{x s}-x N_{s n}\right) \vec{j}\right)=0
$$

La cual es equivalente a las ecuaciones escalares

$$
\begin{aligned}
& M_{x x, x}+M_{x s, s}=0 \\
& M_{x s, x}+M_{s s, s}=0
\end{aligned}
$$

En definitiva, las ecuaciones de equilibrio de un elemento diferencial de lámina son

$$
\begin{gathered}
N_{x x, x}+N_{x s, s}=0 \\
N_{x s, x}=0 \\
M_{x x, x}+M_{x s, s}=0 \\
M_{x s, x}+M_{s s, s}=0
\end{gathered}
$$

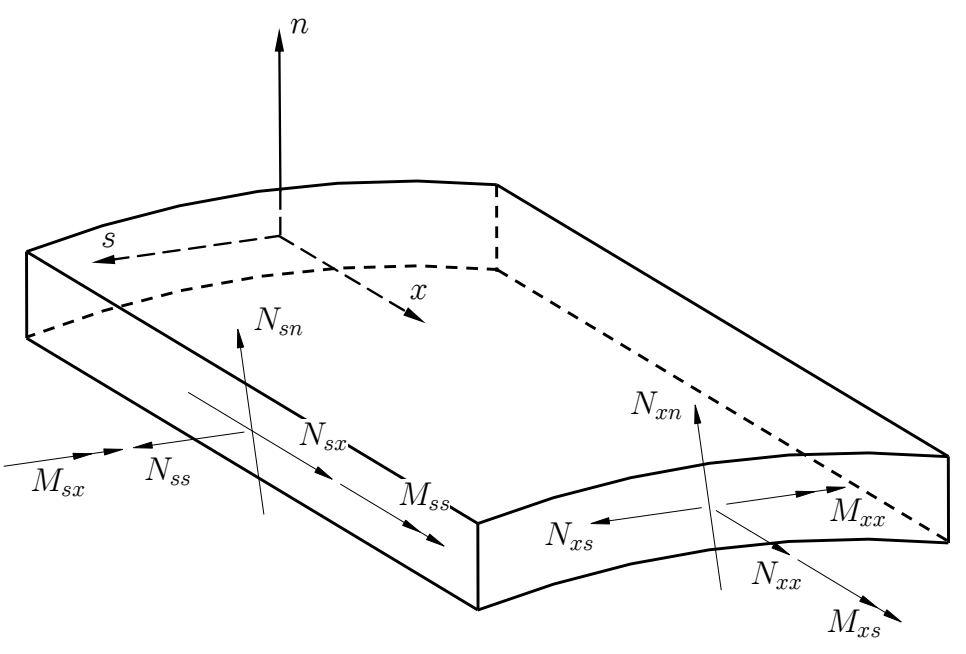

Figura 3.8. Esfuerzos sobre un elemento lámina

\subsubsection{Ecuaciones de comportamiento}

Con relación a la Figura 3.8, donde se muestran los sentidos positivos de los esfuerzos aplicados sobre un elemento diferencial de lámina, las ecuaciones de comportamiento de una lámina de material compuesto expresadas en forma matricial son

$$
\left\{\begin{array}{l}
N_{x x} \\
N_{s s} \\
N_{x s} \\
M_{x x} \\
M_{s s} \\
M_{x s}
\end{array}\right\}=\left[\begin{array}{llllll}
A_{11} & A_{12} & A_{16} & B_{11} & B_{12} & B_{16} \\
A_{12} & A_{22} & A_{26} & B_{12} & B_{22} & B_{26} \\
A_{16} & A_{26} & A_{66} & B_{16} & B_{26} & B_{66} \\
B_{11} & B_{12} & B_{16} & D_{11} & D_{12} & D_{16} \\
B_{12} & B_{22} & B_{26} & D_{12} & D_{22} & D_{26} \\
B_{16} & B_{26} & B_{66} & D_{16} & D_{26} & D_{66}
\end{array}\right]\left\{\begin{array}{c}
\varepsilon_{x x} \\
\varepsilon_{s s} \\
\gamma_{x s} \\
\kappa_{x x} \\
\kappa_{s s} \\
\kappa_{x s}
\end{array}\right\}
$$


siendo $A_{i j}, B_{i j}$ y $D_{i j}$ las rigideces de la lámina asociadas al esfuerzo axil, al acoplamiento flexiónaxil y a la flexión, respectivamente. Asumiendo, al igual que otros investigadores [15], que el esfuerzo $N_{s s}$ es despreciable frente al resto $\left(N_{s s}=0\right)$, se puede expresar la deformación $\varepsilon_{s s}$ en función del resto de deformaciones. Utilizando este resultado y reordenando, la expresión (3.27) se convierte en

$$
\left\{\begin{array}{c}
N_{x x} \\
M_{x x} \\
M_{x s} \\
\gamma_{x s} \\
\kappa_{s s}
\end{array}\right\}=\left[\begin{array}{ccccc}
A_{n \varepsilon} & A_{n \kappa} & A_{n \phi} & A_{n \gamma} & A_{n \tau} \\
A_{n \kappa} & A_{m \kappa} & A_{m \phi} & A_{m \gamma} & A_{m \tau} \\
A_{n \phi} & A_{m \phi} & A_{\phi \phi} & A_{\phi \gamma} & A_{\phi \tau} \\
-A_{n \gamma} & -A_{m \gamma} & -A_{\phi \gamma} & A_{\gamma \gamma} & A_{\gamma \tau} \\
-A_{n \tau} & -A_{m \tau} & -A_{\phi \tau} & A_{\gamma \tau} & A_{\tau \tau}
\end{array}\right]\left\{\begin{array}{c}
\varepsilon_{x x} \\
\kappa_{x x} \\
\kappa_{x s} \\
N_{x s} \\
M_{s s}
\end{array}\right\}
$$

En la expresión anterior, los tres primeros términos del vector de la derecha ya han sido expresados en función de las deformaciones generalizadas de barra (3.21), (3.16) y (3.18), sin embargo, los dos últimos términos aún no han sido calculados. Para ello se recurre a las ecuaciones de equilibrio de un elemento diferencial de lámina. A partir de las ecuaciones de equilibrio (3.25) se puede deducir que $N_{x s}$ consta de una parte constante y de otra que depende de la integral en $s$ de $N_{x x, x}$. Asimismo, $M_{s s}$ está formado por un término constante, otro que varía linealmente con $s$ y un tercero que depende de la integral en $s$ de $M_{x s, x}$. Despreciando los sumandos que dependen de las integrales en $s$, por ser función de las segundas derivadas de las deformaciones, se puede escribir

$$
\begin{gathered}
N_{x s}=N_{x s}^{0} \\
M_{s s}=M_{s s}^{0}+y M_{s s}^{y}+z M_{s s}^{z}
\end{gathered}
$$

Para obtener el valor de las constantes $N_{x s}^{0}, M_{s s}^{0}, M_{s s}^{y}$ y $M_{s s}^{z}$ se deben imponer las siguientes condiciones obtenidas al considerar la unicidad del campo de desplazamientos

$$
\begin{gathered}
\int_{S} \gamma_{x s} d s=2 A_{0} \phi_{, x} \\
\int_{S} \kappa_{x s} d s=0 \\
\int_{S} \kappa_{x s} y d s=0 \\
\int_{S} \kappa_{x s} z d s=0
\end{gathered}
$$

Finalmente, integrando los esfuerzos de lámina $N_{x x}, M_{x x}$ y $M_{x s}$ en la sección se obtienen los esfuerzos de barra en función de las deformaciones generalizadas de barra. Es decir, 


$$
\begin{gathered}
N=\int_{C} N_{x x} d s \\
M_{y}=\int_{C}\left[z N_{x x}-y_{, s} M_{x x}\right] d s \\
M_{z}=\int_{C}\left[y N_{x x}+z, s M_{x x}\right] d s \\
T_{s}=\int_{C}\left[2 M_{x s}+r M_{x s}\right] d s \\
M_{\omega}=\int_{C}\left[-\omega N_{x x}+q M_{x x}\right] d s
\end{gathered}
$$

donde $N$ es la fuerza axil, $M_{y}$ y $M_{z}$ son los momentos flectores alrededor de los ejes $O y$ y $O z$, respectivamente, $T_{s}$ es el momento torsor de St. Venant y $M_{\omega}$ es el bimomento de Vlasov. En la Figura 3.9 se han representado los sentidos positivos de los esfuerzos de barra.

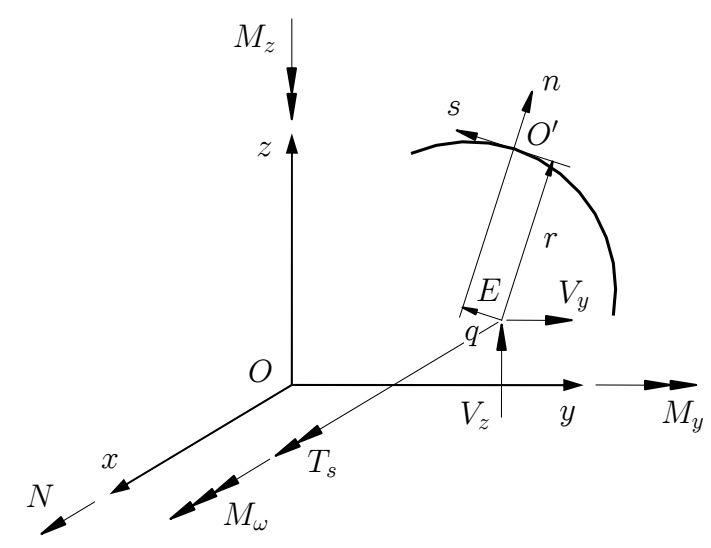

Figura 3.9. Esfuerzos de barra

La matriz de rigidez de la sección transversal de la barra, que relaciona los esfuerzos con las deformaciones de barra, se obtiene a partir de las ecuaciones (3.31) sin más que expresar en ellas los esfuerzos de lámina en función de las deformaciones de barra utilizando las expresiones (3.21), (3.16), (3.18), (3.28) y (3.29). Se trata de una matriz simétrica de dimensiones $5 \times 5$ que representa el modelo de Navier-Bernoulli respecto a flexión y de Vlasov en lo relativo a torsión

$$
\mathbf{F}=\overline{\mathbf{K}} \mathbf{q}
$$

siendo

$$
\begin{gathered}
\mathbf{F}^{T}=\left\{\begin{array}{lllll}
N & M_{y} & M_{z} & T_{s} & M_{\omega}
\end{array}\right\} \\
\mathbf{q}^{T}=\left\{\begin{array}{lllll}
U_{, x} & \beta_{y, x} & \beta_{z, x} & \phi_{, x} & \phi_{, x x}
\end{array}\right\}
\end{gathered}
$$




\subsection{Análisis mediante el método de los elementos finitos}

El sistema de ecuaciones que definen el comportamiento de un elemento tipo barra (beam) en el contexto de los elementos finitos se puede obtener aplicando el Principio de los Trabajos Virtuales

$$
\int_{V^{(e)}} \delta \varepsilon \sigma d V=\delta q^{(e)} f^{(e)}
$$

Para reproducir el comportamiento del elemento tipo barra se utilizan dos tipos de funciones de forma. Para el desplazamiento axil $U$ se emplea una función Lagrangiana de dos nodos

$$
\begin{gathered}
u(\xi)=N_{1}(\xi) u_{1}+N_{2}(\xi) u_{2} \\
N_{1}(\xi)=\frac{1}{2}(1-\xi) \quad N_{2}(\xi)=\frac{1}{2}(1+\xi)
\end{gathered}
$$

Las rotaciones $\beta_{y}$ y $\beta_{z}$ son interpoladas utilizando una función Hermítica de dos nodos de manera que se satisfaga la condición de continuidad $C^{1}$ en cada nodo del elemento. Finalmente, para aproximar el giro por torsión $\phi$ y sus derivadas se emplea también una función Hermítica de dos nodos

$$
\begin{array}{cl}
w(\xi)=N_{1}(\xi) w_{1}+N_{1}(\xi)\left(\frac{d w}{d \xi}\right)_{1} & +N_{2}(\xi) w_{2}+N_{2}(\xi)\left(\frac{d w}{d \xi}\right)_{2} \\
N_{1}(\xi)=\frac{1}{4}\left(2-3 \xi+\xi^{3}\right) & N_{2}(\xi)=\frac{1}{4}\left(2+3 \xi-\xi^{3}\right) \\
\bar{N}_{1}(\xi)=\frac{1}{4}\left(1-\xi-\xi^{2}+\xi^{3}\right) & \bar{N}_{2}(\xi)=\frac{1}{4}\left(-1-\xi+\xi^{2}+\xi^{3}\right)
\end{array}
$$

Así, cada uno de los dos nodos del elemento tiene asociados siete grados de libertad (con sus correspondientes esfuerzos), que son las tres traslaciones y las tres rotaciones respecto a cada eje, así como el alabeo. Es decir, cada elemento tiene un total de 14 grados de libertad. Llevando esta aproximación a la ecuación (3.34) se obtiene la relación

$$
\mathbf{F}_{\mathrm{g}}=\mathbf{K} \mathbf{g}_{\mathrm{g}}
$$

donde $\mathbf{K}$ es la matriz de rigidez del sistema de ecuaciones, $\mathbf{F}_{\mathrm{g}}$ es el vector de cargas, $\mathbf{y} \mathbf{g}_{\mathrm{g}}$ es el vector de desplazamientos generalizados.

El elemento tipo barra, cuyo desarrollo se ha expuesto anteriormente, ha sido implementado con éxito en el paquete de software Kratos [17, 18], con el cual se han obtenido los resultados, entre otros, que se exponen en el Anexo G. El amortiguamiento estructural es tenido en cuenta y se supone proporcional a la matriz de masa. 


\section{Referencias}

1 Jung, S.N., Nagaraj, V.T., y Chopra, I., "Assessment of Composite Rotor Blade Modeling Techniques”, Journal of the American Helicopter Society, 44 (3), pp. 188-205, 1999.

${ }^{2}$ Cesnik, C.E.S. y Hodges, D.H., "VABS: A New Concept for Composite Rotor Blade CrossSectional Modeling”, Journal of the American Helicopter Society, 42 (1), pp. 27-38, 1997.

3 Volovoi, V.V., y Hodges, D.H., "Single- and Multi-Cell Composite Thin-Walled Beams", American Institute of Aeronautics and Astronautics Journal, 40 (5), pp. 960-966, 2002.

${ }^{4}$ Yu, W., Volovoi, V.V., y Dewey, D.H., "Validation of the Variational Asymptotic Beam Sectional (VABS) Analysis", 40 (10), pp. 2105-2113, 2002.

${ }^{5}$ Yu, W., Hodges, D.H., Volovoi, V.V., y Fuchs, E.D., "A Generalized Vlasov Theory for Composite Beams”, Thin-Walled Structures, 43 (9), pp.1493-1511, 2005.

${ }^{6}$ Rehfield, L.W., Atilgan, A.R., y Hodges, D.H., "Nonclassical Behavior of Thin-Walled Composite Beams with Closed Cross Sections", Journal of the American Helicopter Society, 35 (2), pp. 42-51, 1990.

${ }^{7}$ Smith, E.C., y Chopra, I.,"Formulation and Evaluation of an Analytical Model for Composite Box-Beams", Journal of the American Helicopter Society, 36 (3), pp. 23-35, 1991.

${ }^{8}$ Chandra, R., y Chopra, I., "Experimental and Theoretical Analysis of Composite I Beams with Elastic Couplings", American Institute of Aeronautics and Astronautics Journal, 29 (12), pp. 2197 2206, 1991.

${ }^{9}$ Chandra, R., y Chopra, I., "Structural Response of Composite Beams and Blades with Elastic Couplings", Composites Engineering, 2 (5-7), pp. 347-374, 1992.

${ }^{10}$ Johnson, E.R., Vasiliev, V.V., y Vasiliev, D.V., “Anisotropic Thin-Walled Beams with Closed Cross-Sectional Contours", American Institute of Aeronautics and Astronautics Journal, 39 (12), pp. 2389-2393, 2001.

${ }^{11}$ Patil, M., y Johnson, E., 46th AIAA/ASME/ASCE/AHS/ASC Structures, Structural Dynamics and Materials Conference. 13th AIAA/ASME/AHS Adaptive Structures Conference. Austin, Texas, 2005

${ }^{12}$ Zhang, J., y Smith, E., "Structural Design and Optimization of Composite Blades for a Low Weight Rotor", The 2nd International Basic Research Conference on Rotorcraft Technology, Nanjing, China, 2005.

${ }^{13}$ Mansfield, E.H., y Sobey, A.J., "The Fiber Composite Helicopter Blade, Part I: Stiffness Properties, Part II: Prospects for Aeroelastic Tailoring”, Aeronautical Quarterly, 30 (2), pp. 413449, 1979. 
${ }^{14}$ Libove, C., "Stress and Rate of Twist in Single-Cell Thin-Walled Beams with Anisotropic Walls" American Institute of Aeronautics and Astronautics Journal, 26 (9), pp. 1107-1118, 1988.

${ }^{15}$ Jung, S.N., Nagaraj, V.T., y Chopra, I.,"Refined Structural Model for Thin- and Thick- Walled Composite Rotor Blades" American Institute of Aeronautics and Astronautics Journal, 40 (1), pp. 105-116, 2002.

${ }^{16}$ Jung, S.N., y Park, L.J., "Structural Behavior of Thin and Thick-Walled Composite Blades with Multi-Cell Sections", American Institute of Aeronautics and Astronautics Journal, 43 (3), pp. $572-$ 5812005 .

${ }^{17}$ CIMNE (International Center for Numerical Methods in Engineering), "KRATOS: Free Multiphysics Finite Element Method C++ Open Source Code", http://www.cimne.com/kratos/default.asp, 2002.

${ }^{18}$ Dadvand, P., Mora, J., Gonzalez, C., Arraez, A., Ubach, P.A., y Oñate. E., "KRATOS: An Object-Oriented Environment for Development of Multi-Physics Analysis Software", Proceedings of the WCCM V Fifth World Congress on Computational Mechanics, Vienna, Austria, 2002. 

Capítulo 4

\section{Problema Dinámico}





\subsection{Resumen}

En el presente capítulo se aborda el problema de la dinámica estructural, con especial interés en el rango no-lineal. Aunque existen tanto técnicas explícitas como implícitas para resolver el problema, sólo se considerarán los algoritmos implícitos.

\subsection{Introducción}

La solución de la ecuación de movimiento tiene un gran interés para el estudio de sistemas estructurales. Este problema ha sido estudiado desde los inicios del desarrollo del Método de los Elementos Finitos (MEF) e incluso desde antes, en lo referente a la resolución numérica de sistemas de Ecuaciones Diferenciales Ordinarias (EDO). Los primeros estudios se centraron principalmente en la precisión, conservando, por supuesto, la estabilidad. Recientemente, las propiedades disipativas de los métodos de integración en el dominio del tiempo han adquirido una gran importancia, fundamentalmente debido a cuestiones de estabilidad en el rango no-lineal.

La solución del problema dinámico consiste en la integración en el tiempo de un sistema de ecuaciones diferenciales de la forma

$$
\mathbf{M} \ddot{\mathbf{x}}+\mathbf{C} \dot{\mathbf{x}}+\mathbf{K x}=\mathbf{f}_{e x t}(\mathbf{x}, t)
$$

el cual se puede reescribir en forma residual de la siguiente manera

$$
\mathbf{M} \ddot{\mathbf{x}}+\mathbf{C} \dot{\mathbf{x}}-\mathbf{r}(\mathbf{x}, t)=\mathbf{0} \quad ; \quad \mathbf{r}(\mathbf{x}, t)=\mathbf{f}_{e x t}(\mathbf{x}, t)-\mathbf{f}_{\text {int }}(\mathbf{x}, t)
$$

Citando al Prof. Hughes [1], un buen esquema de integración en el tiempo debe estar caracterizado por

- ser capaz de comenzar por sí mismo (self-starting)

- precisión de segundo orden

- incondicionalmente estable

- presentar una disipación numérica controlable para las altas frecuencias

- requerir la solución de no más de un sistema de ecuaciones en cada paso de tiempo

La estabilidad incondicional y la precisión de segundo orden no requieren de comentario alguno. Por otro lado, el resto de las características han representado una línea de trabajo que ha influido notablemente en el desarrollo de los algoritmos numéricos durante los últimos años. 
Los métodos que son capaces de comenzar por sí mismos (self-starting) deben ser "locales" a un paso de tiempo dado y no hacer uso de un conjunto de datos históricos. Por otro lado, los métodos Lineales de Paso Múltiple utilizan un mayor conjunto de datos, lo cual hace que no cumplan esta "condición”. La necesidad de disipación numérica se describe en la sección siguiente. Finalmente, la última característica está relacionada con la dimensión del sistema a resolver: existen métodos de orden alto en los cuales se incrementa la dimensión del sistema a resolver, por ejemplo, escribiendo el sistema en términos de desplazamientos y velocidades.

Aunque las directrices dadas por el Prof. Hughes son ampliamente aceptadas, existen métodos eficaces que no satisfacen la primera o la última condición.

\subsection{La necesidad de disipación numérica}

Aunque los fenómenos disipativos pueden manifestarse debido a diferentes causas, en general, se acepta la hipótesis de la conservación de la energía para sistemas estructurales no amortiguados. Desde este punto de vista, la introducción de la "disipación numérica" puede ser considerada como una fuente de error innecesaria. Este argumento tiene cierta validez y de hecho representa el planteamiento correcto cuando el método de integración se aplica a la resolución de problemas teóricos. Sin embargo, cuando el método tiene que ser aplicado a un sistema de ecuaciones discretizado, como en nuestro caso, la discretización espacial es una fuente de error en sí misma. Este error llega a ser muy importante para los modos altos de la estructura [1,2] y puede ser considerado como una fuente de "ruido numérico". La introducción de un adecuado amortiguamiento numérico puede ser estudiada de manera que afecte de forma importante a los modos altos, sin alterar de forma razonable la precisión alcanzada en los modos más bajos (los cuales son más fiables). Esto constituye el fundamento base de los "Métodos Disipativos".

Esta característica se manifiesta también en el rango no-lineal. Lamentablemente, la prueba de estabilidad espectral y energética está basada en una descomposición modal y no necesariamente se sostiene en el dominio no-lineal. Citando las palabras del Prof. Belytschko [3] "Los resultados generales relativos a la estabilidad numérica están en gran parte basados en el análisis de sistemas lineales. Estos resultados son extrapolados a sistemas no-lineales por medio del estudio de modelos linealizados". Una condición suficiente para asegurar la estabilidad en presencia de no-linealidades viene dada por [4]

$$
\Delta U+\Delta T \leq W_{e x t}
$$


donde $\Delta U$ representa la variación de la energía de deformación durante un paso de tiempo, $\Delta T$ la variación de la energía cinética y $W_{e x t}$ el trabajo de las fuerzas externas.

De manera sorprendente, se observó que las propiedades disipativas introducidas para controlar el ruido en los modos altos garantizaban de manera eficaz la condición (4.3). Se las considera completamente necesarias, especialmente cuando existen no-linealidades geométricas importantes. En el trabajo desarrollado por Taylor [5] se proporciona un detallado estudio sobre diferentes algoritmos disipativos aplicados a este tipo de problemas. Este trabajo fue usado en la elección del algoritmo Bossak para su implementación en el código Kratos [6, 7].

\subsection{Análisis lineal}

El procedimiento para analizar las propiedades de los métodos de integración en el tiempo es ampliamente conocido hoy en día y puede encontrarse en cualquier texto sobre análisis de elementos finitos $[1,2,8]$. El planteamiento depende en gran medida de la suposición de linealidad y de superposición de efectos, y está basado en la posibilidad de descomponer cualquier sistema estructural lineal (sin amortiguamiento o proporcionalmente amortiguado) en una base de vectores propios generalizados. En líneas generales, la idea consiste en lo siguiente: un sistema no amortiguado libre (sin término de fuerzas) es de la forma

$$
\mathbf{M} \ddot{\mathbf{x}}+\mathbf{K x}=\mathbf{0}
$$

La matriz de masa es definida positiva y la matriz de rigidez es semidefinida positiva. Asumiendo una solución del tipo $\mathbf{x}=e^{i \omega t} \mathbf{v}$, sustituyendo esta solución en (4.4) y simplificando, resulta el siguiente problema de valores propios

$$
\left(\mathbf{K}-\omega^{2} \mathbf{M}\right) \mathbf{v}=\mathbf{0}
$$

Las propiedades de las matrices involucradas garantizan que existe una base de vectores propios. La ortogonalidad entre los vectores propios puede ser demostrada fácilmente y permite escribir cualquier solución posible como $\mathbf{x}(t)=y_{1}(t) \mathbf{v}_{1}+\ldots+y_{N}(t) \mathbf{v}_{N}$. Premultiplicando (4.4) por $\mathbf{v}_{i}^{T}$ se obtiene un conjunto de $N$ ecuaciones independientes entre sí del tipo

$$
\mathbf{v}_{i}^{T} \mathbf{M} \mathbf{v}_{i} \ddot{y}_{i}+\mathbf{v}_{i}^{T} \mathbf{K} \mathbf{v}_{i} y_{i}=0 \Rightarrow \ddot{y}_{i}+\omega_{i}^{2} y_{i}=0
$$

Por lo tanto, la solución completa puede ser obtenida resolviendo cada ecuación por separado para cada coordenada modal y posteriormente superponiendo los resultados. 
En definitiva, el resultado más importante es que la estabilidad puede ser estudiada sobre el sistema de ecuaciones de la forma (4.6): cuando se garantiza la estabilidad para todas las ecuaciones modales, la estabilidad del conjunto global también se garantiza.

El método iterativo se puede expresar de la siguiente manera

$$
\mathbf{v}_{n+1}=\mathbf{A} \mathbf{v}_{n}
$$

donde $\mathbf{A}$ es una matriz de amplificación y $\mathbf{v}$ es un vector que contiene la solución y sus derivadas.

Considerando dos soluciones distintas para un tiempo $n, \mathbf{v}_{n}^{a} \mathrm{y} \mathbf{v}_{n}^{b}$, la aplicación del algoritmo de integración para la determinación de la solución en el siguiente paso se corresponde con

$$
\begin{aligned}
& \mathbf{v}_{n+1}^{a}=\mathbf{A} \mathbf{v}_{n}^{a} \\
& \mathbf{v}_{n+1}^{b}=\mathbf{A} \mathbf{v}_{n}^{b}
\end{aligned}
$$

Restando ambas expresiones, se obtiene de forma inmediata

$$
\mathbf{v}_{n+1}^{a}-\mathbf{v}_{n+1}^{b}=\mathbf{A}\left(\mathbf{v}_{n}^{a}-\mathbf{v}_{n}^{b}\right)
$$

Si en particular, una de las dos soluciones fuera la exacta y la otra una aproximación, su diferencia correspondería con la definición de error. Considerando que la expresión (4.9) se mantiene recursivamente para cada paso de tiempo se puede comprobar fácilmente que el error viene definido por una expresión del tipo $\epsilon_{n}=\mathbf{A}^{n} \epsilon_{0}$. Por lo tanto, la condición necesaria para asegurar la estabilidad puede ser planteada a través de los valores propios de la matriz de amplificación. Esto conlleva que la condición necesaria y suficiente para la estabilidad es que el radio espectral debe ser menor o igual a 1 .

El grado de precisión obtenido en la solución puede ser evaluado a través del error de truncamiento local, definido como la diferencia entre la solución exacta en un instante de tiempo dado y la solución aproximada para un punto de partida común, es decir, es el error que se comete en un solo paso. La idea es que tanto la solución exacta como la aproximada puedan ser expresadas en su forma amplificada

$$
\begin{gathered}
\mathbf{v}_{n+1}^{e x}=\mathbf{A}^{e x} \mathbf{v}_{n} \\
\mathbf{v}_{n+1}^{\text {aprox }}=\mathbf{A}^{\text {aprox }} \mathbf{v}_{n}
\end{gathered}
$$

De manera que el error de truncamiento se puede expresar de la siguiente forma

$$
\mathbf{v}_{n+1}^{e x}-\mathbf{v}_{n+1}^{a p r o x}=\left(\mathbf{A}^{e x}-\mathbf{A}^{a p r o x}\right) \mathbf{v}_{n}
$$


De lo cual se concluye que el error de truncamiento local puede ser evaluado a través de la diferencia entre la matriz de amplificación exacta y otra aproximada.

\subsection{Solución exacta}

Según se ha indicado en el punto anterior, el estudio de la precisión del método requiere la definición de la matriz de amplificación exacta, la cual se puede obtener de una manera relativamente sencilla usando cualquier manipulador simbólico.

Su determinación está basada en la integración analítica de la ecuación (4.1) para fuerzas externas nulas y condiciones iniciales $\quad x(0)=x_{0} \quad ; \quad \dot{x}(0)=\dot{x}_{0}$

El estudio del problema general con amortiguamiento no presenta ninguna dificultad, sin embargo, los resultados que se obtienen son bastante extensos y no serán mostrados aquí. El problema no amortiguado, que en particular tiene una gran importancia en el estudio de la estabilidad, conduce a la definición de la matriz

$$
\left[\begin{array}{cc}
\cos (\omega x) & \frac{\sin (\omega x)}{\omega} \\
-\sin (\omega x) \omega & \cos (\omega x)
\end{array}\right]
$$

la cual tiene un papel fundamental en la evaluación de la precisión de la solución obtenida.

\subsection{Uso de métodos numéricos para la resolución de EDO de orden alto}

Métodos numéricos para la resolución de EDO de orden alto se encuentran disponibles como librerías en muchos y diferentes lenguajes de programación. Comúnmente estos paquetes se ejecutan para resolver sistemas de EDO de primer orden de la forma

$$
\mathbf{v}=\mathbf{f}(\mathbf{v}, t)
$$

Por lo tanto, para resolver la ecuación (4.6) es necesario expresarla como una EDO de primer orden. Esto se lleva a cabo introduciendo una variable auxiliar $y=\dot{x}$, con lo que se obtiene el siguiente sistema de EDO de primer orden 


$$
\left[\begin{array}{ll}
1 & 0 \\
0 & 1
\end{array}\right]\left\{\begin{array}{l}
\dot{x} \\
\dot{y}
\end{array}\right\}=\left[\begin{array}{cc}
0 & 1 \\
-\omega^{2} & 0
\end{array}\right]\left\{\begin{array}{l}
x \\
y
\end{array}\right\}
$$

Se puede concluir de forma inmediata que las librerías pueden ser aplicadas usando

$$
\mathbf{v}:=\left\{\begin{array}{l}
x \\
\dot{x}
\end{array}\right\} ; \mathbf{f}(\mathbf{v}, t):=\left\{\begin{array}{c}
\dot{x} \\
-\omega^{2} x
\end{array}\right\}
$$

\subsection{Método de Newmark}

El método de Newmark representa uno de los métodos numéricos de integración en el tiempo más populares en la dinámica estructural. Para un análisis lineal el método proporciona buenos resultados, y aunque puede utilizarse para análisis no-lineal, en presencia de grandes no-linealidades geométricas se sabe que conduce a resultados inestables, a no ser que el paso de tiempo se reduzca drásticamente.

Este método parte de la ecuación de equilibrio dinámico particularizada para el instante $t_{n+1}$

$$
\mathbf{M} \ddot{\mathbf{x}}_{n+1}+\mathbf{C} \dot{\mathbf{x}}_{n+1}+\mathbf{K} \mathbf{x}_{n+1}=\mathbf{f}_{n+1}^{e x t}
$$

En esta expresión los vectores desplazamiento, velocidad y aceleración son desconocidos, pero bajo determinadas hipótesis, el desplazamiento y la velocidad pueden expresarse en función de la aceleración $\ddot{\mathbf{x}}_{n+1} \mathrm{y}$ de los resultados de la etapa anterior, con lo que queda un único vector incógnita. Para expresar la velocidad y el desplazamiento en función de la aceleración, Newmark [9] utiliza las siguientes expresiones

$$
\begin{gathered}
\mathbf{x}_{n+1}=\mathbf{x}_{n}+\Delta t \dot{\mathbf{x}}_{n}+\Delta t^{2}\left(\frac{1-2 \beta}{2} \ddot{\mathbf{x}}_{n}+\beta \ddot{\mathbf{x}}_{n+1}\right) \\
\dot{\mathbf{x}}_{n+1}=\dot{\mathbf{x}}_{n}+\Delta t\left((1-\gamma) \ddot{\mathbf{x}}_{n}+\gamma \ddot{\mathbf{x}}_{n+1}\right)
\end{gathered}
$$

siendo $\gamma \mathrm{y} \beta$ dos parámetros cuyos valores caracterizan los distintos métodos de la familia.

La segunda expresión puede interpretarse como una corrección de la velocidad $\dot{x}_{n}$ obtenida mediante la integración de una aceleración interpolada linealmente en un punto determinado por el parámetro $\gamma$. Puede hacerse una interpretación análoga para la primera expresión.

Estas dos ecuaciones se pueden resolver para el campo de velocidades y aceleraciones de la siguiente forma 


$$
\begin{gathered}
\dot{\mathbf{x}}_{n+1}=\left(\mathbf{x}_{n+1}-\mathbf{x}_{n}\right)-\left(\frac{\gamma}{\beta}-1\right) \dot{\mathbf{x}}_{n}-\frac{\Delta t}{2}\left(\frac{\gamma}{\beta}-2\right) \ddot{\mathbf{x}}_{n} \\
\ddot{\mathbf{x}}_{n+1}=\frac{1}{\beta \Delta t^{2}}\left(\mathbf{x}_{n+1}-\mathbf{x}_{n}\right)-\left(\frac{1}{\beta \Delta t}\right) \dot{\mathbf{x}}_{n}-\left(\frac{1}{2 \beta}-1\right) \ddot{\mathbf{x}}_{n}
\end{gathered}
$$

La forma residual de la ecuación de equilibrio es

$$
\mathbf{M} \ddot{\mathbf{x}}+\mathbf{C} \dot{\mathbf{x}}-\mathbf{r}(\mathbf{x}, t)=\mathbf{0} ; \mathbf{r}(\mathbf{x}, t)=\mathbf{f}_{e x t}(\mathbf{x}, t)-\mathbf{K x}
$$

La solución exacta requiere su linealización y el empleo del método de Newton-Raphson. Para preservar la convergencia cuadrática del método Newton-Raphson se debe calcular la matriz tangente consistente. Definiendo un residuo "dinámico" como

$$
\psi\left(\mathbf{x}_{n+1}\right)=\mathbf{r}_{n+1}-\mathbf{M} \ddot{\mathbf{x}}_{n+1}-\mathbf{C} \dot{\mathbf{x}}_{n+1}
$$

el término tangente puede ser obtenido realizando la diferenciación $-\frac{\partial \psi}{\partial \mathbf{x}_{n+1}}$

$$
\mathbf{K}_{\mathrm{tan}}^{d y n}:=-\frac{\partial \psi}{\partial \mathbf{x}_{n+1}}=-\frac{\partial \mathbf{r}(\mathbf{x})_{n+1}}{\partial \mathbf{x}_{n+1}}+\frac{\partial \mathbf{M} \ddot{\mathbf{x}}_{n+1}}{\partial \mathbf{x}_{n+1}}+\frac{\partial \mathbf{C} \dot{\mathbf{x}}_{n+1}}{\partial \mathbf{x}_{n+1}}
$$

El primer término corresponde a la linealización de la contribución estática, que por definición es el operador tangente estático. La matriz de masa y de amortiguamiento pueden suponerse constantes, de forma que

$$
\begin{aligned}
\frac{\partial \ddot{\mathbf{x}}_{n+1}}{\partial \mathbf{x}_{n+1}} & =\frac{1}{\beta \Delta t^{2}} \mathbf{I} \\
\frac{\partial \dot{\mathbf{x}}_{n+1}}{\partial \mathbf{x}_{n+1}} & =\frac{\gamma}{\beta \Delta t} \mathbf{I}
\end{aligned}
$$

donde la matriz identidad I aparece ya que los grados de libertad en (4.18) están desacoplados. Finalmente, el tensor dinámico tangente es de la forma

$$
\mathbf{K}_{\mathrm{tan}}^{\text {dyn }}=\mathbf{K}_{\tan }+\frac{1}{\beta \Delta t^{2}} \mathbf{M}+\frac{\gamma}{\beta \Delta t} \mathbf{C}
$$

Las relaciones descritas permiten la definición de un modelo del tipo predictor-corrector para el algoritmo de Newmark, el cual es adecuado tanto para problemas lineales como no-lineales. El procedimiento se muestra resumido en la Tabla 4.1. Para problemas lineales la matriz tangente estática coincide con la matriz elástica. Se puede verificar fácilmente que el procedimiento de Newton-Raphson proporciona la solución "exacta" en un solo paso para problemas lineales.

El uso de las ecuaciones (4.18) y (4.20) para un tiempo $n$ y $n+1$ permiten, en el caso de problemas lineales, la determinación de la matriz de amplificación. Ésta se obtiene resolviendo las 
relaciones de equilibrio para las aceleraciones y substituyendo en las relaciones de diferencias finitas. Su determinación es descrita en detalle por el Prof. Hughes [1].

Se demuestra que el esquema es incondicionalmente estable si los parámetros $\gamma$ y $\beta$ cumplen las siguientes condiciones [9]

$$
\gamma \geq \frac{1}{2} ; \beta \geq \frac{1}{4}\left(\gamma+\frac{1}{2}\right)^{2}
$$

El grado de amortiguamiento artificial del esquema de integración viene determinado por el parámetro $\gamma$. Para un sistema sin amortiguamiento con un solo grado de libertad, que vibra libremente, los valores $\gamma=0.5$ y $\beta=0.25$, que habitualmente se utilizan, hacen que el amortiguamiento artificial introducido por el algoritmo sea nulo para cualquier paso de tiempo elegido. El valor de dicho amortiguamiento aumenta a medida que se incrementa el valor de $\gamma$ por encima de 0.5. Este incremento implica, al mismo tiempo, una peor precisión en los resultados, a menos que se elija un paso de tiempo muy pequeño.

- Predicción del desplazamiento como $\mathbf{x}_{n+1}=\mathbf{x}_{n}+\Delta t \dot{\mathbf{x}}_{n}$, llevar a cabo la actualización usando las expresiones (4.18)

- Calcular el residuo usando el desplazamiento predicho (4.20)

- Calcular la matriz tangente dinámica utilizando (4.21)

- $\quad$ Resolver $\mathbf{K}_{\tan }^{d y n} d \mathbf{x}=\psi$

- Corregir el desplazamiento como $\mathbf{x}_{n+1}^{i+1}=\mathbf{x}_{n+1}^{i}+d \mathbf{x}$, y a continuación actualizar las velocidades $\mathbf{y}$ aceleraciones

- Comprobar la convergencia, si no converge ir al paso 2 y repetir el bucle

Tabla 4.1. Diagrama de flujo para la implementación del método de Newmark

\subsection{Generalización alfa de la familia de Newmark}

Las ecuaciones en diferencias finitas que constituyen la base del método de Newmark pueden ser usadas de manera eficaz en el diseño de algoritmos disipativos de integración en el tiempo. El método alfa, también conocido como método de Hilber-Hughes-Taylor (HHT), y el método de Bossak representan dos buenos ejemplos de algoritmos disipativos de segundo orden de precisión obtenidos a partir de las ecuaciones en diferencias finitas de Newmark. Dichos métodos se basan en 
modificar la forma de la ecuación de equilibrio incluyendo un término de suavizado (regularizador) entre el paso de tiempo actual y un paso anterior para preservar los requisitos de consistencia.

Para el método de HHT la forma de la ecuación de equilibrio asumida es

$$
\mathbf{M} \ddot{\mathbf{x}}_{n+1}+\mathbf{C} \dot{\mathbf{x}}_{n+1+\alpha^{H}}-\mathbf{r}\left(\mathbf{x}_{n+1+\alpha^{H}}, t_{n+1+\alpha^{H}}\right)=\mathbf{0}
$$

donde $z_{n+1+\alpha^{H}}=\left(1+\alpha^{H}\right) z_{n+1}-\alpha^{H} z_{n}$ y los términos suavizados son las fuerzas residuales $\mathrm{y}$ las de amortiguamiento.

La elección de

$$
-\frac{1}{3}<\alpha^{H}<0 ; \quad \gamma=\frac{1-2 \alpha^{H}}{2} ; \quad \beta=\frac{\left(1-\alpha^{H}\right)^{2}}{4}
$$

garantiza una precisión de segundo orden y estabilidad incondicional. La máxima disipación se consigue para $\alpha^{H}=-1 / 3$.

El método de Bossak sigue un planteamiento similar, pero la modificación afecta exclusivamente al término relacionado con las fuerzas de inercia. La ecuación de equilibrio se expresa de la siguiente forma

$$
\mathbf{M} \ddot{\mathbf{x}}_{n+1-\alpha^{B}}+\mathbf{C} \dot{\mathbf{x}}_{n+1}-\mathbf{r}\left(\mathbf{x}_{n+1}, t_{n+1}\right)=\mathbf{0}
$$

Eligiendo

$$
\alpha^{B}<0 ; \quad \gamma=\frac{1}{2}-\alpha^{B} ; \quad \beta=\frac{\left(1-\alpha^{B}\right)^{2}}{4}
$$

se asegura una precisión de segundo orden y estabilidad incondicional.

Actualmente se considera que el método de HHT es más exacto cuando la disipación numérica es máxima, sin embargo, el método de Bossak presenta algunas ventajas a la hora de su implementación para problemas no-lineales.

\subsection{Métodos multipaso lineales}

Las técnicas multipaso lineales fueron introducidas por Felippa y Park [10,11] con el objetivo de proporcionar algoritmos disipativos precisos. Desde el punto de vista de su utilización práctica tienen poca importancia ya que su precisión está limitada por el Teorema Dahlquist [1], sin embargo, las técnicas introducidas para el análisis son útiles y merecen una descripción. 
En el curso publicado por el Prof. Felippa [12], relativo a la resolución de problemas de Interacción Fluido-Estructura (FSI), se puede encontrar una buena introducción al tema.

Dado un sistema de segundo orden de EDO de la forma (4.1), el primer paso para lograr una discretización multipaso es la reducción del sistema de ecuaciones a otro de primer orden. Una opción para hacer esto es la utilización de un vector auxiliar $\mathbf{y}=\mathbf{A M} \dot{\mathbf{x}}+\mathbf{B} \mathbf{x}$ donde $\mathbf{A}$ es una matriz cuadrada no singular y B es una matriz cuadrada. Derivando esta ecuación y sustituyendo en (4.1) se obtiene un sistema equivalente de primer orden

$$
\left[\begin{array}{cc}
\mathbf{A M} & \mathbf{0} \\
\mathbf{A C}-\mathbf{B} & \mathbf{I}
\end{array}\right]\left\{\begin{array}{c}
\dot{x} \\
\dot{\mathbf{y}}
\end{array}\right\}+\left[\begin{array}{cc}
\mathbf{B} & -\mathbf{I} \\
\mathbf{A K} & \mathbf{0}
\end{array}\right]\left\{\begin{array}{l}
\mathbf{x} \\
\mathbf{y}
\end{array}\right\}=\left\{\begin{array}{c}
\mathbf{0} \\
\mathbf{A f}
\end{array}\right\}
$$

Los métodos del tipo multipaso están basados en fórmulas en diferencias finitas, para un paso de tiempo $\Delta t$ constante, de la forma

$$
\begin{aligned}
\mathbf{x}_{n+1}+\sum_{i=1}^{m} \alpha_{i}^{x} \mathbf{x}_{n+1-i} & =\Delta t \sum_{i=0}^{m} \beta_{i}^{x} \dot{\mathbf{x}}_{n+1-i} \\
\mathbf{y}_{n+1}+\sum_{i=1}^{m} \alpha_{i}^{y} \mathbf{y}_{n+1-i} & =\Delta t \sum_{i=0}^{m} \beta_{i}^{y} \dot{\mathbf{y}}_{n+1-i}
\end{aligned}
$$

Ejemplos de distintos métodos multipaso se pueden obtener particularizando para diferentes matrices auxiliares A y B, o para los coeficientes indicados en (4.30). Sustituyendo (4.30) en (4.29) se obtiene la definición de la expresión de amplificación para el método de integración en el tiempo dado, que puede expresarse como

$$
\mathbf{A}_{0} \mathbf{v}_{n+1}+\mathbf{A}_{1} \mathbf{v}_{n}+\ldots+\mathbf{A}_{m} \mathbf{v}_{n+1-m}=\mathbf{0} ; \mathbf{v}_{i}=\left\{\begin{array}{l}
\mathbf{x}_{i} \\
\mathbf{y}_{i}
\end{array}\right\}
$$

Esta forma es interesante porque representa una generalización de la expresión de amplificación para los métodos de un solo paso.

El polinomio de amplificación se obtiene asumiendo una solución de la forma $\mathbf{v}_{k+1}=z \mathbf{v}_{k}$, donde $z$ es un factor de amplificación. La estabilidad del método se puede estudiar evaluando las raíces de dicho polinomio

$$
\operatorname{det}\left(z^{m} \mathbf{A}_{0}+z^{m-1} \mathbf{A}_{1}+\ldots+z^{0} \mathbf{A}_{m}\right)=0
$$

En general, calcular las raíces de este polinomio resulta tedioso, si bien, existen diferentes técnicas que simplifican el cálculo. Algunas de estás técnicas han sido descritas ampliamente por el Prof. Felippa [12]. 


\subsection{Otras Técnicas para abordar el problema de la precisión. Método de Ecuaciones Modificadas}

El planteamiento tradicional para el estudio de la precisión está basado en la estimación del error local de truncamiento, definido como el error introducido en cada paso de tiempo debido al método iterativo. Este planteamiento se conoce con el nombre de "análisis del error a prior" (Forward Error Analysis). Existen diferentes maneras de abordar el mismo problema, en particular el denominado "análisis del error a posteriori" (Backward Error Analysis). En este trabajo no se emplea el análisis del error a posteriori, sin embargo, resulta conveniente indicar alguna de sus particularidades.

El fundamento teórico de estos métodos consiste en lo siguiente: dada una ecuación en diferencias que describe la acción de un método de integración en el tiempo para una ecuación test, el método de análisis a posteriori busca la ecuación diferencial que si se resolviese exactamente reproduciría el comportamiento de la ecuación en diferencias original. Cuando esta operación es posible, la ecuación diferencial se puede resolver y comparar su solución con la solución analítica de la ecuación test. Esto permite la evaluación del error en cualquier instante de tiempo, lo cual resulta más conveniente que la evaluación del error de truncamiento. Una descripción más detallada de estos métodos se puede encontrar en el trabajo del Prof. Felippa [12].

\subsection{Filosofía de implementación en Kratos}

Incluso en un entorno estándar proporcionado por el MEF, cada planteamiento particular para la resolución de un problema dado tiene algunas características "favorables" que a menudo hacen necesario la implementación de técnicas de resolución peculiares con objeto de maximizar el rendimiento. Cuando un código es desarrollado para "un solo objetivo", generalmente, es posible tener en cuenta las necesidades particulares de la implementación para conseguir algunos objetivos específicos como maximizar la velocidad computacional o minimizar el uso de memoria.

Dependiendo de las primeras suposiciones adoptadas en el diseño, cada código muestra aspectos positivos y negativos en relación con problemas diferentes. En nuestro caso, cuando el mismo código tiene que tratar con campos de resolución diferentes no es posible hacer suposiciones rígidas sobre la "forma" que el problema dado asumirá, ya que la especialización en un campo concreto probablemente conlleve una resolución desfavorablemente en otro. Por lo tanto, la palabra clave para el diseño es "flexibilidad". 
Lamentablemente no es posible omitir los aspectos vinculados a la velocidad de cálculo y al uso eficaz de la memoria, por lo tanto, el reto consistirá en aportar la flexibilidad necesaria al código sin penalizar severamente estos aspectos.

Dada la naturaleza del problema, es posible considerar que usuarios diferentes pueden esperar cosas diferentes del mismo núcleo de resolución; "un recién llegado" puede demandar una estrategia de resolución estándar para comprobar la implementación de un elemento finito, sin embargo, un usuario "experto" podría estar interesado en la obtención de la máxima velocidad posible utilizando una formulación conocida. Por lo tanto, se puede aceptar que se requiere un grado de conocimiento diferente para realizar las dos tareas indicadas, respecto del primer usuario, se espera que conozca más bien poco o nada sobre los detalles de la implementación de la estrategia de resolución, mientras el segundo necesita un conocimiento y control total sobre la fase de resolución.

En definitiva, el desafío consiste en proporcionar unas pocas herramientas estándares de carácter general pero eficientes, permitiendo a usuarios avanzados la posibilidad hacer uso de ellas para el desarrollo de sus propias aplicaciones.

El trabajo parte de la consideración de que la mayoría de los problemas de elementos finitos tienen en común los siguientes puntos:

1. Cada elemento (y en definitiva cada nodo) lleva asociado unos "términos" característicos, por ejemplo, para el caso estructural serían la matriz de rigidez StiffnesMatrix, la matriz de masa MassMatrix, y la matriz de amortiguamiento DampingMatrix, junto con un vector de residuos ResidualVector.

2. Para cada elemento, los diferentes términos se unen para formar una matriz y un vector de residuos adecuados al esquema de resolución de interés (estático, dinámico,...).

3. Las contribuciones de cada elemento son ensambladas en una o más matrices globales.

4. Se forma un sistema lineal (ensamblado directamente las contribuciones de cada elemento o añadiendo matrices ya ensambladas), y se resuelve.

5. La base de datos debe ser actualizada teniendo en cuenta la naturaleza del problema.

6. Se comprueba la convergencia si fuera necesario.

Se puede ver como los pasos 2 y 5 están relacionados y deben ser consistentes el uno con el otro para la implementación de un esquema dado, a saber, un esquema "estático" o un esquema de integración en el tiempo. Por lo tanto, ambas etapas estarán organizadas conjuntamente en una sola 
clase, la cual contiene la naturaleza "física" del problema. Esta clase "pide" al elemento las características necesarias (etapa 1) y las combina para formar las contribuciones adecuadas.

Aparte de la comprobación de la convergencia, todas las otras etapas están estrechamente relacionadas con el manejo de los datos asociados a los elementos, la formación de un sistema lineal y la inversión de la matriz de dicho sistema. En general, el usuario no está interesado en los detalles de la implementación de estas operaciones, sin embargo, la mayor parte del esfuerzo computacional, probablemente, se invierte en la ejecución de las mismas. La introducción de un objeto "Ensamblador-Solver" BuilderAndSolver, exige realizar todas estas etapas, las cuales no se muestran al usuario final manteniendo un código simple y eficiente.

Cada una de las etapas descritas anteriormente demanda un acceso rápido a las matrices ensambladas y a los vectores, y puede implicar el manejo de datos en una forma más compleja que el clásico "double". El empleo de un lenguaje de bajo nivel como Fortran implicaría un gran esfuerzo a la hora hacer frente a esta situación, por ejemplo, si el tipo de dato o el formato de almacenamiento de las matrices fuesen cambiados, ello supondría reescribir gran cantidad de código. Por otro lado, las características del moderno lenguaje $\mathrm{C}++$ permiten un manejo eficiente del problema mediante el uso del patrón de diseño Meta-programming. Todos los objetos descritos son diseñados para ser implementados como patrones de diseño, pasando el tipo de dato y el formato de almacenamiento de la matriz como argumentos del patrón de diseño. Esto permite al compilador generar automáticamente en tiempo de compilación diferentes instalaciones de los objetos para las distintas combinaciones de los argumentos del patrón de diseño, permitiendo la máxima eficiencia posible y la mínima reescritura de código.

En el Anexo H se muestra una breve descripción del papel de cada uno de los objetos que constituyen el núcleo de resolución.

\subsection{Validación}

La validación del procedimiento descrito, tanto en el contexto lineal como no-lineal, se puede encontrar en la Tesis de Rossi [13], donde se eligió el problema del péndulo para llevar a cabo dicha validación. Se utilizó una malla no estructurada y altamente distorsionada para maximizar eventuales problemas relacionados con el comportamiento a altas frecuencias, y la densidad del muelle se estableció próxima a cero para poder comparar los resultados obtenidos con los analíticos para el péndulo idealizado. 
En lo que hace referencia a este trabajo, se estudió el comportamiento dinámico de estructuras constituidas por barras y modeladas utilizando el modelo monodimensional de barras descrito en el Capítulo 3. En la Figura 4.1 se muestra la respuesta dinámica de una barra de acero empotradalibre, obtenida con el software Kratos y utilizando el modelo monodimensional de barras, cuando se encuentra sometida a una carga concentrada constante normal a la directriz de la barra en el extremo libre. Junto a ella se muestra la respuesta que proporciona el software de elementos finitos COSMOS/M, así como también, la repuesta analítica calculada con el manipulador simbólico Mathematica. Se observa una plena coincidencia en los resultados.

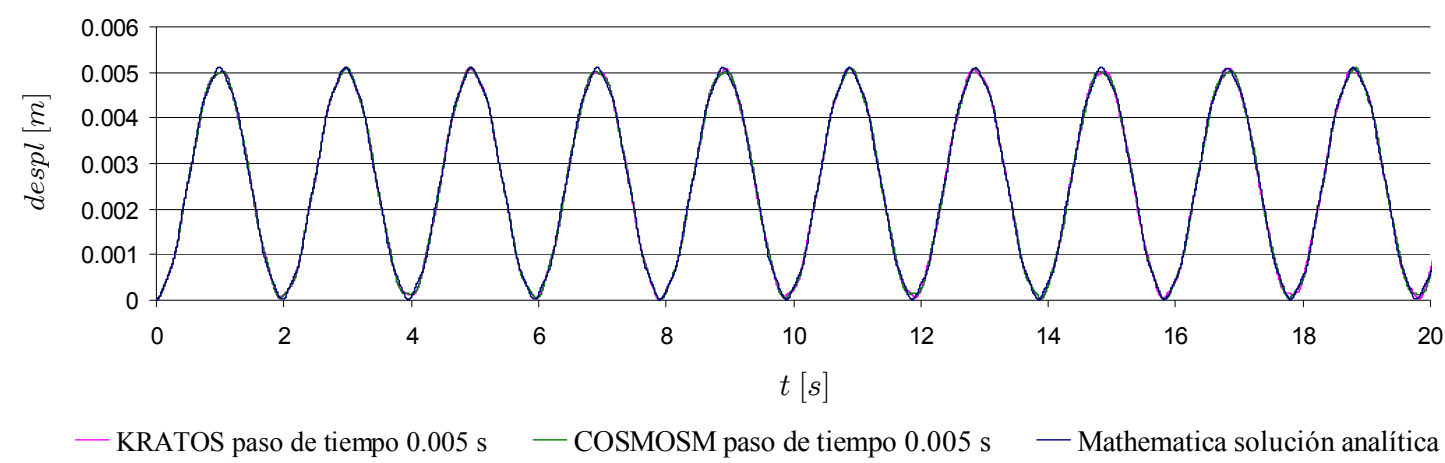

Figura 4.1. Respuesta dinámica de una barra empotrada-libre

En la Figura 4.2 se muestra la respuesta dinámica de una barra en voladizo para diferentes valores del amortiguamiento estructural, el cual se supone proporcional a la matriz de masa. La barra es de acero y se encuentra sometida a una carga concentrada constante normal a la directriz en el extremo libre, siendo su primera frecuencia natural $n_{e}=1 \mathrm{~Hz}$. En la Figura 4.3 se muestra el análisis FFT de la respuesta dinámica de la barra, y se puede observar que la estructura vibra a su frecuencia natural, tal como era de esperar.

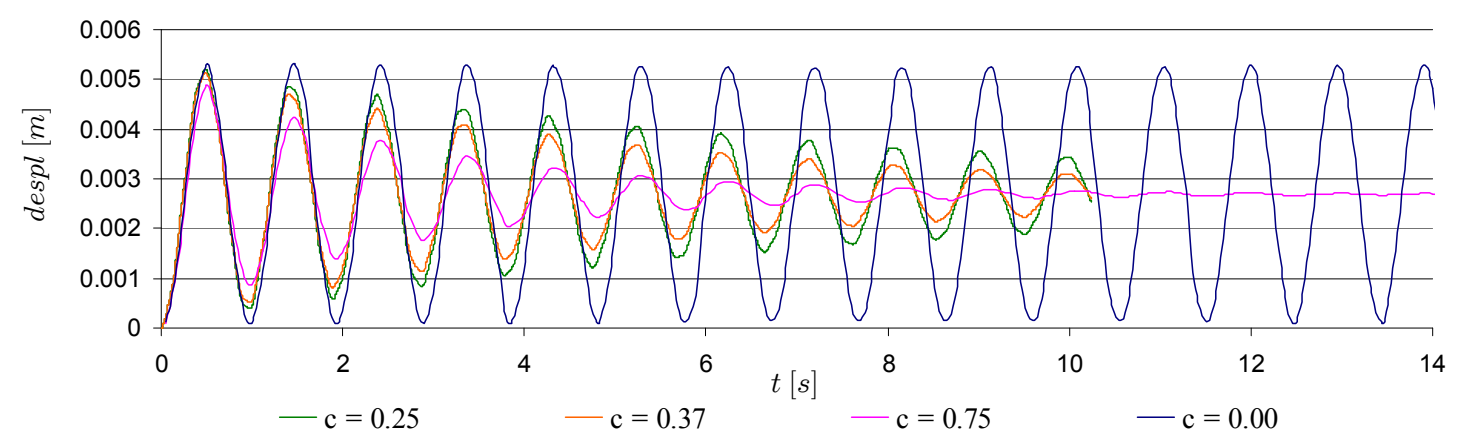

Figura 4.2. Respuesta de una barra en voladizo para diferentes valores del amortiguamiento estructural 


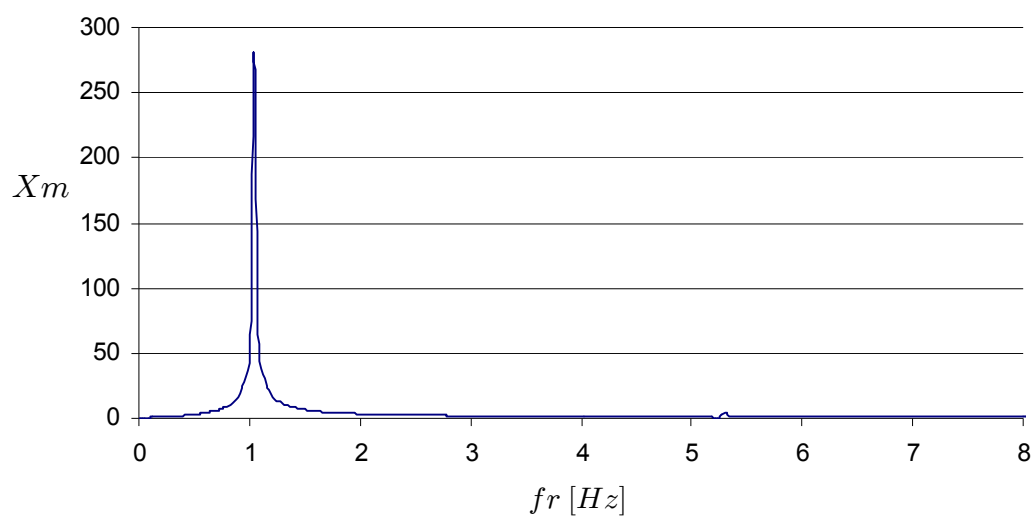

Figura 4.3. Análisis FFT de la respuesta dinámica

\section{Referencias}

${ }^{1}$ Hughes, T.J.R., "The Finite Element Method, Linear Static and Dynamic Finite Element Analysis", Dover, 2000.

2 Taylor, R.L., y Zienkiewicz, O.C., "The Finite Element Method: Volume 1, The basis", Butterworth Heinemann, 2000.

${ }^{3}$ Liu, W.K., Belytschko, T., y Moran, B., "Nonlinear Finite Elements for Continua and Structures", John Wiley and Sons, 2000.

${ }^{4}$ Schoeberle, D.F., Belytschko, T., "On the unconditional stability of an implicit algorithm for nonlinear structural dynamics”, Journal of Applied Mechanics, 42, pp. 865-869, 1975.

${ }^{5}$ Taylor, W., “Aplicaciones de algoritmos que conservan la energia-momentum en dinámica nolineal", Technical report, CIMNE (International Center for Numerical Methods in Engineering), Granada, 2004

${ }^{6}$ CIMNE, "KRATOS: Free Multi-physics Finite Element Method C++ Open Source Code", http://www.cimne.com/kratos/default.asp, 2002.

${ }^{7}$ Dadvand, P., Mora, J., Gonzalez, C., Arraez, A., Ubach, P.A., y Oñate, E., “KRATOS: An ObjectOriented Environment for Development of Multi-Physics Analysis Software", Proceedings of the WCCM V Fifth World Congress on Computational Mechanics, Vienna, Austria, 2002.

${ }^{8}$ Bathe, K.J., "Finite Element Procedures", Prentice-Hall, 1996.

${ }^{9}$ Newmark, N.M., "A method of computation for structural dynamics”, Journal of the Geotechnical and Geoenvironmental Engineering, ASCE, 85 (3), pp. 67-94, 1959. 
${ }^{10}$ Felippa, C.A., y Park, K.C., "Computational aspects of time integration procedures in structural dynamics, Part I: Implementation”, Journal of Applied Mechanics, 45, 595-602, 1978.

${ }^{11}$ Park, K.C., y Felippa, C. A., "Computational aspects of time integration procedures in structural Dynamics", Part II: Error propagation, Journal of Applied Mechanics, 45, 603-611, 1978.

${ }^{12}$ Felippa, C.A., "Fluid-Structure Interaction", http://www.colorado.edu/engineering/CAS/courses .d/FSI.d/Home.html, 2004.

${ }^{13}$ Rossi R., Light Weight Structures: Structural Analysis and Coupling Issues, Tesis Doctoral, Universidad de Bologna, 2005. 
Capítulo 5

Mecánica de Fluidos
Computacional 



\subsection{Resumen}

El presente capítulo se centra en la resolución de las ecuaciones de Navier-Stokes para flujo incompresible. En la primera parte se describen las ecuaciones de conservación de la masa y de la cantidad de movimiento que gobiernan los fenómenos de la fluidodinámica, en una segunda parte se aborda la resolución práctica de las ecuaciones a través de la utilización de métodos de paso fraccionado y finalmente, se estudia el movimiento de la malla.

\subsection{Introducción}

Desde sus inicios, el método de los elementos finitos experimentó un amplio éxito en su aplicación a problemas estructurales y de transmisión de calor. A pesar de sus ventajas frente a los métodos de diferencias finitas, en el campo de la simulación de la mecánica de fluidos computacional (CFD) no experimentó tal éxito. Las razones de esto se debían fundamentalmente a la estructura matemática del problema.

El método de Galerkin en elementos finitos, usado para la discretización de problemas estructurales, presenta un comportamiento óptimo para problemas de operadores autoadjuntos. Sin embargo, este comportamiento se pierde si el método se aplica a operadores que no son autoadjuntos, como sucede en los problemas en los que intervienen fluidos. En la práctica, la aplicación directa de las técnicas de Galerkin conduce a un comportamiento sub-difusivo, el cual se manifiesta en soluciones excesivamente oscilatorias cuando el término convectivo tiende a dominar. Este fenómeno se puede corregir introduciendo una viscosidad "artificial" adecuada, la cual suele depender de la dirección. Esta cuestión se aborda brevemente más adelante y se proporcionan algunas referencias para un tratamiento más exhaustivo.

Un segundo problema surge de la solución numérica del problema de flujo incompresible. El problema de incompresibilidad fue estudiado detalladamente en la mecánica estructural y condujo a la definición de la conocida condición de Babuska-Brezzi. En el presente capítulo se proporciona una descripción sobre las técnicas de estabilización modernas tanto para el problema de convección -difusión como para el de incompresibilidad.

Finalmente, se obtendrá la forma adecuada del problema de flujo incompresible y se describirá el procedimiento de paso fraccionario empleado para el desarrollo de los diferentes solvers de flujo de fluidos (flow solvers) usados en este trabajo. 


\subsection{Ecuaciones de conservación}

Para analizar el problema de Navier-Stokes, el primer paso es plantear la formulación matemática subyacente, y para ello, es conveniente referirse a las ecuaciones de conservación que gobiernan el problema.

En el caso de flujo incompresible son suficientes dos ecuaciones:

- Ecuación de Conservación de la Masa o Ecuación de Continuidad.

- Ecuación de Conservación de la Cantidad de Movimiento.

Para obtener la forma fuerte de estas ecuaciones son necesarios unos resultados matemáticos preliminares. El planteamiento de los teoremas y de las ecuaciones de conservación propuesto en este capítulo se basa en el trabajo de Liu, Belytschko y Moran [1].

- Teorema de la divergencia

$$
\int_{\Omega} \frac{\partial g_{i}}{\partial x_{i}} d \Omega=\int_{\Gamma} n_{i} g_{i} d \Gamma \quad \text { o } \quad \int_{\Omega} \nabla \cdot \mathbf{g} d \Omega=\int_{\Gamma} \mathbf{n} \cdot \mathbf{g} d \Gamma
$$

\section{- Teorema de Gauss}

$$
\int_{\Omega} \frac{\partial g_{i}}{\partial x_{j}} d \Omega=\int_{\Gamma} n_{j} g_{i} d \Gamma \quad \text { о } \quad \int_{\Omega} \nabla \mathbf{g} d \Omega=\int_{\Gamma} \mathbf{n} \otimes \mathbf{g} d \Gamma
$$

- Teorema de Green-Gauss

$$
\begin{gathered}
-\int_{\Omega} \omega \nabla^{2} \mathbf{u} d \Omega=-\int_{\Omega}(\nabla \omega \cdot \nabla \mathbf{u}-\nabla \cdot \omega \nabla \mathbf{u}) d \Omega= \\
=\int_{\Omega} \nabla \omega \cdot \nabla \mathbf{u} d \Omega-\int_{\Gamma} \omega(\mathbf{n} \cdot \nabla \mathbf{u}) d \Gamma
\end{gathered}
$$

- Derivada del determinante jacobiano

$$
j:=\operatorname{det}(\mathrm{F}) \rightarrow \frac{D j}{D t} \equiv \dot{j}=j \nabla \cdot \mathrm{v}
$$

- Teorema de transporte de Reynolds

$$
\frac{D}{D t} \int_{\Omega} f(\mathbf{x}, t) d \Omega=\int_{\Omega}\left(\frac{D f}{D t}+\nabla \cdot(f \mathbf{v})\right) d \Omega
$$




\subsubsection{Ecuación de conservación de la masa}

La masa contenida en un determinado dominio se puede expresar como

$$
m(\Omega)=\int_{\Omega} \rho(\mathbf{x}, t) d \Omega
$$

donde $\rho$ es la densidad del material. La conservación de la masa implica que ésta se conserva en cualquier dominio dado, es decir

$$
\frac{D m(\Omega(t))}{D t}=\frac{D}{D t} \int_{\Omega} \rho(\mathbf{x}, t) d \Omega=0
$$

Aplicando el teorema de Reynolds se llega a

$$
\int_{\Omega} \frac{D \rho}{D t}+\rho \nabla \cdot \mathbf{v} d \Omega=0
$$

La arbitrariedad del dominio asegura que para cualquier punto se cumple

$$
\frac{D \rho}{D t}+\rho \nabla \cdot \mathbf{v}=0
$$

En general, la densidad sigue una ecuación de estado del tipo $\rho(T, p)$. Para flujos isotérmicos se cumple la siguiente relación

$$
d \rho=\frac{\rho}{K} d p \rightarrow d \rho=\frac{1}{c^{2}} d p \rightarrow \frac{D \rho}{D t}=\frac{1}{c^{2}} \frac{D p}{D t}
$$

donde $K$ es el módulo de Bulk y $c$ es la velocidad de propagación de la onda.

Muchos fluidos reales bajo ciertas condiciones de flujo adoptan un comportamiento incompresible, en este caso, la dependencia de la densidad respecto del tiempo desaparece y la ecuación (5.9) se reduce a

$$
\nabla \cdot \mathbf{v}=0
$$

la cual se utiliza en el tratamiento de flujos incompresibles.

\subsubsection{Ecuación de conservación de la cantidad de movimiento}

La ecuación de conservación de la cantidad de movimiento relaciona las fuerzas que actúan sobre un determinado cuerpo con sus aceleraciones. Dado un dominio $\Omega$ con contorno $\Gamma$ sujeto a unas fuerzas de volumen $\mathbf{b}$ y a unas fuerzas de superficie $\mathbf{t}$, la fuerza total que actúa sobre el dominio se puede calcular como 


$$
\mathbf{f}(\Omega, t)=\int_{\Omega} \rho \mathbf{b}(\mathrm{x}, t) d \Omega+\int_{\Gamma} \mathbf{t}(\mathbf{x}, t) d \Gamma
$$

El momento lineal se define como

$$
\mathbf{p}_{\text {lin }}(\Omega, t):=\int_{\Omega} \rho(\mathbf{x}, t) \mathbf{v} d \Omega
$$

La segunda ley de Newton (conservación de la cantidad de movimiento en un medio continuo) es de la forma

$$
\frac{D \mathbf{p}_{l i n}(\Omega, t)}{D t}=\mathbf{f}(\Omega, t)
$$

Diferenciando la ecuación (5.13) y usando el teorema de Reynolds se llega a

$$
\frac{D \mathbf{p}_{l i n}(\Omega, t)}{D t}=\int_{\Omega}\left(\frac{D \rho \mathbf{v}}{D t}+\rho \mathbf{v} \nabla \cdot \mathbf{v}\right) d \Omega=\int_{\Omega}\left(\rho \frac{D \mathbf{v}}{D t}+\mathbf{v}\left(\frac{D \rho}{D t}+\rho \nabla \cdot \mathbf{v}\right)\right) d \Omega
$$

Teniendo en cuenta que la conservación de la masa implica (5.9), se obtiene

$$
\frac{D \mathbf{p}_{l i n}(\Omega, t)}{D t}=\int_{\Omega} \rho \frac{D \mathbf{v}}{D t} d \Omega
$$

Por otro lado, usando el teorema de la divergencia

$$
\int_{\Gamma} \mathbf{t} d \Gamma=\int_{\Gamma} \mathbf{n} \cdot \sigma d \Gamma=\int_{\Omega} \nabla \cdot \sigma d \Omega
$$

agrupando los términos convenientemente y teniendo en cuenta la arbitrariedad del dominio, se obtiene finalmente

$$
\rho \frac{D \mathbf{v}}{D t}=\nabla \cdot \sigma+\rho \mathbf{b}
$$

que es la forma fuerte de la ecuación de conservación de la cantidad de movimiento.

\subsection{Ecuaciones de comportamiento}

Una diferencia importante entre los fluidos y las estructuras está relacionada con la ley de comportamiento. Un fluido en reposo no puede soportar tensiones tangenciales, pero presenta un comportamiento viscoso cuando se deforma dinámicamente. Esto sugiere, por lo tanto, que la ley de comportamiento debe ser expresada como una función de la tasa de deformación en lugar de la 
deformación. De forma análoga a la definición de pequeñas deformaciones, la tasa de deformación se puede expresar como

$$
\dot{\varepsilon}_{i j}=\frac{1}{2}\left(\frac{\partial v_{i}}{\partial x_{j}}+\frac{\partial v_{j}}{\partial x_{i}}\right)
$$

En muchas situaciones de interés práctico el fluido se comporta como incompresible o cuasiincompresible. Asumiendo esta condición, resulta conveniente distinguir entre deformación volumétrica y desviadora para tratar por separado las presiones y las tensiones tangenciales. Para ello, se descompone el tensor de tensiones de Cauchy $\sigma_{i j}$ en una parte volumétrica

$$
p:=\frac{1}{3} \operatorname{Tr}(\sigma)=\frac{1}{3} \sigma_{i i}
$$

y otra parte desviadora

$$
\tau:=\sigma-p \mathbf{I} \rightarrow \tau_{i j}:=\sigma_{i j}-\delta_{i j} \frac{1}{3} \sigma_{i i}
$$

Para fluidos newtonianos, los cuales representan una categoría muy importante, las ecuaciones constitutivas se pueden expresar de la siguiente forma

$$
\tau=2 \mu\left(\dot{\varepsilon}-\frac{1}{3} \operatorname{Tr}(\dot{\varepsilon}) \mathbf{I}\right) \rightarrow \tau_{i j}=2 \mu\left(\dot{\varepsilon}_{i j}-\frac{1}{3} \dot{\varepsilon}_{i i}\right)
$$

Para flujo incompresible, que es el caso de interés para nuestras aplicaciones, no se establece ninguna ley de comportamiento para la presión, la cual se considera dada por la solución de elementos finitos. Haciendo uso de la ley de comportamiento viscoso (5.22), el tensor de tensiones de Cauchy puede expresarse como

$$
\sigma=\tau+p \mathbf{I}=2 \mu\left(\dot{\varepsilon}-\frac{1}{3} \operatorname{Tr}(\dot{\varepsilon}) \mathbf{I}\right)+p \mathbf{I}
$$

o directamente como función de las velocidades

$$
\sigma_{i j}=\mu\left(\frac{\partial u_{i}}{\partial x_{j}}+\frac{\partial u_{j}}{\partial x_{i}}\right)-\frac{2}{3} \mu \frac{\partial u_{k}}{\partial x_{k}} \delta_{i j}+p \delta_{i j}
$$

el cual se reduce para flujo incompresible $(\nabla \cdot \mathbf{v}=0)$ a

$$
\sigma_{i j}=\mu\left(\frac{\partial u_{i}}{\partial x_{j}}+\frac{\partial u_{j}}{\partial x_{i}}\right)+p \delta_{i j}
$$

Para este último caso, la notación puede simplificarse considerando que el gradiente de velocidades 


$$
\nabla \mathbf{v}:=\left[\begin{array}{lll}
\frac{\partial v_{1}}{\partial x_{1}} & \frac{\partial v_{1}}{\partial x_{2}} & \frac{\partial v_{1}}{\partial x_{3}} \\
\frac{\partial v_{2}}{\partial x_{1}} & \frac{\partial v_{2}}{\partial x_{2}} & \frac{\partial v_{2}}{\partial x_{3}} \\
\frac{\partial v_{3}}{\partial x_{1}} & \frac{\partial v_{3}}{\partial x_{2}} & \frac{\partial v_{3}}{\partial x_{3}}
\end{array}\right]
$$

se puede descomponer en una parte simétrica $\nabla^{s} \mathbf{v}$ y en otra antisimétrica $\nabla^{\omega} \mathbf{v}$

$$
\nabla^{s} \mathbf{v}:=\frac{1}{2}\left(\nabla \mathbf{v}+\nabla \mathbf{v}^{T}\right) \quad ; \quad \nabla^{\omega} \mathbf{v}:=\frac{1}{2}\left(\nabla \mathbf{v}+\nabla \mathbf{v}^{T}\right)
$$

Por lo tanto, la ecuación de comportamiento para flujo incompresible se puede reescribir de la siguiente forma

$$
\sigma=2 \mu \nabla^{s} \mathbf{v}-p \mathbf{I}
$$

\subsection{Ecuaciones de Navier-Stokes. Flujo incompresible}

Las ecuaciones de Navier-Stokes se pueden escribir substituyendo la ley de comportamiento (5.24) en las ecuaciones de conservación (5.9) y (5.18). En el marco de la ingeniería civil, la hipótesis de incompresibilidad está generalmente aceptada. A partir de ahora, por lo tanto, se particularizará para flujo incompresible, el cual se puede describir haciendo uso de las ecuaciones (5.11) y (5.28). Ello conduce a lo siguiente

$$
\rho \frac{D \mathbf{v}}{D t}=\nabla \cdot \sigma+\rho \mathbf{b}=\nabla \cdot 2 \mu \nabla^{s} \mathbf{v}-\nabla \cdot p \mathbf{I}+\rho \mathbf{b}
$$

Por otro lado, considerando la expresión

$$
\frac{D \mathbf{v}}{D t}=\frac{\partial \mathbf{v}}{\partial t}+(\mathbf{v} \cdot \nabla) \mathbf{v}
$$

e introduciendo la viscosidad cinemática $\nu=\mu / \rho$ y la presión cinemática $p$, definida como el cociente entre la presión y la densidad, se obtiene

$$
\frac{\partial \mathbf{v}}{\partial t}+(\mathbf{v} \cdot \nabla) \mathbf{v}=2 \nu \nabla \cdot \nabla^{s} \mathbf{v}-\nabla \cdot p \mathbf{I}+\mathbf{b}
$$

Teniendo en cuenta que

$$
\nabla \cdot p \mathbf{I}=\nabla p
$$


e introduciendo la definición de $\nabla^{s} \mathbf{v}$

$$
2 \nu \nabla \cdot \nabla^{s} \mathbf{v}=\nu \nabla \cdot \nabla \mathbf{v}+\nu \nabla \cdot \nabla \mathbf{v}^{T}=\nu \nabla^{2} \mathbf{v}+\nu \nabla(\nabla \cdot \mathbf{v})
$$

donde $\nabla^{2}$ representa el operador Laplaciano, la ecuación (5.31) se puede expresar como

$$
\frac{\partial \mathbf{v}}{\partial t}+(\mathbf{v} \cdot \nabla) \mathbf{v}-\nu \nabla^{2} \mathbf{v}-\nu \nabla(\nabla \cdot \mathbf{v})+\nabla p=\mathbf{b}
$$

La expresión (5.34) adopta la forma de la ecuación de la velocidad-presión tensión-divergencia (velocity-pressure stress-divergence equation). El empleo de la condición de incompresibilidad (5.11) permite obtener finalmente

$$
\begin{gathered}
\frac{\partial \mathbf{v}}{\partial t}+(\mathbf{v} \cdot \nabla) \mathbf{v}-\nu \nabla^{2} \mathbf{v}+\nabla p=\mathbf{b} \\
\nabla \cdot \mathbf{v}=0
\end{gathered}
$$

la cual representan la forma habitual usada para la discretización de las ecuaciones de NavierStokes.

La ventaja de esta forma sobre otras posibles opciones radica en que las componentes de la velocidad están desacopladas salvo para el término convectivo. Otra forma interesante se puede obtener substituyendo el término convectivo en (5.35) por

$$
(\mathbf{v} \cdot \nabla) \mathbf{v} \rightarrow(\mathbf{v} \cdot \nabla) \mathbf{v}+\frac{1}{2} \nabla \cdot \mathbf{v v}
$$

que es equivalente al primero siempre que la divergencia de la velocidad sea nula. Se denomina forma antisimétrica del término convectivo y presenta algunas ventajas en el estudio de la estabilidad.

\subsection{Condición de incompresibilidad}

Es interesante observar el papel de la presión en las ecuaciones de Navier-Stokes para flujo incompresible. La ecuación de conservación de la cantidad de movimiento adquiere la forma (5.35) bajo la hipótesis de que la condición de incompresibilidad se cumple en cualquier punto del dominio. La presión actúa como un multiplicador de Lagrange y hace que la velocidad cumpla la condición de divergencia nula, lo cual implica que la presión no puede estar gobernada por una ley de comportamiento. Por lo tanto, el papel de la presión es "ajustarse" para asegurar que el campo de velocidades satisface la condición de incompresibilidad. 
Cuando el problema se expresa como una función de $(\mathbf{v}, p)$ surgen problemas asociados a la capacidad de resolución eficaz de los sistemas resultantes. De hecho, a menudo sucede que el sistema a resolver está mal condicionado o incluso, es singular. Aunque esta cuestión se trata en diferentes libros, como por ejemplo [1, 2, 3, 4], a continuación se muestran algunos comentarios sobre el problema. Para ello, se considera la ecuación (5.35) particularizada para un estado estacionario y para el caso en el que las fuerzas dominantes son las viscosas. Este problema se denomina problema estacionario de Stokes o flujo de Stokes y está descrito por las ecuaciones

$$
\begin{gathered}
-\nu \nabla^{2} \mathbf{v}+\nabla p=\mathbf{b} \\
\nabla \cdot \mathbf{v}=0
\end{gathered}
$$

Aplicando el planteamiento de Galerkin estándar a la discretización del problema (5.37) se llega a la definición de un sistema discreto de ecuaciones de la forma

$$
\left[\begin{array}{cc}
\mathbf{K} & \mathbf{G} \\
\mathbf{G}^{T} & \mathbf{0}
\end{array}\right]\left\{\begin{array}{l}
\mathbf{u} \\
\mathbf{p}
\end{array}\right\}=\left\{\begin{array}{l}
\mathbf{f} \\
\mathbf{h}
\end{array}\right\}
$$

donde las contribuciones proporcionadas por la discretización de los diferentes términos se describen a continuación [3]

$$
-\nu \nabla^{2} \mathbf{v} \rightarrow \mathbf{K u} \quad ; \quad \nabla p \rightarrow \mathbf{G p} \quad ; \quad \nabla \cdot \mathbf{v} \rightarrow \mathbf{G}^{T} \mathbf{u}
$$

El sistema (5.39) sólo se puede resolver si su determinante es distinto de cero, lo cual sucede si $\operatorname{ker}(\mathbf{G})=\mathbf{0}[2]$, donde $\operatorname{ker}(\mathbf{G})$ se define según la siguiente expresión

$$
\operatorname{ker}(\mathbf{G}):=\{\mathbf{q} \mid \mathbf{G q}=\mathbf{0}\}
$$

Ladyzhenskaya, Babuska y Brezzi $[5,6,7]$ determinaron una condición de compatibilidad, conocida como condición de Ladyzhenskaya-Babuska-Brezzi (LBB), la cual se debe satisfacer por los espacios de interpolación para los campos de velocidad y presión con objeto de garantizar que se cumple la condición $\operatorname{ker}(\mathbf{G})=\mathbf{0}$, es decir, los campos de velocidad y de presión no pueden ser elegidos arbitrariamente. Esto conduce al desarrollo de diferentes familias de elementos finitos caracterizados por diferentes órdenes de interpolación para los campos de velocidad y presión [1, 4]. La utilización de estas familias de elementos permite la solución directa del problema (5.37).

El uso de diferentes órdenes de interpolación para la velocidad y la presión no resulta adecuado cuando el interés se centra tanto en las presiones como en las velocidades. Planteamientos modernos del problema están basados en el uso de interpolaciones de igual orden para la velocidad y la presión. La idea es imponer directamente la definición positiva de (5.37) modificando la forma débil y así evitar que el término diagonal resultante de la condición de incompresibilidad sea nulo. 
Esto se consigue, por ejemplo, a través de la utilización de técnicas de estabilización tales como Galerkin Least Squares (GLS) o Finite Increment Calculus [8] (FIC).

La presencia del término convectivo no modifica la situación. Para el problema estacionario de Navier-Stokes la presencia del término convectivo modifica (5.39) de la siguiente manera

$$
\left[\begin{array}{cc}
\mathbf{K}+\mathbf{C}(\mathbf{v}) & \mathbf{G} \\
\mathbf{G}^{T} & \mathbf{0}
\end{array}\right]\left\{\begin{array}{l}
\mathbf{u} \\
\mathbf{p}
\end{array}\right\}=\left\{\begin{array}{l}
\mathbf{f} \\
\mathbf{h}
\end{array}\right\}
$$

El término adicional $\mathbf{C}(\mathbf{v})$, procedente de la discretización del término convectivo $(\mathbf{v} \cdot \nabla) \mathbf{v}$, es no simétrico, no-lineal e introduce un nuevo reto en el proceso de resolución. Sin embargo, el uso del mismo planteamiento de estabilización permite resolver tanto inestabilidades debidas al problema de incompresibilidad como a la presencia del término convectivo.

\subsection{Estabilización del término convectivo}

En la sección anterior se describía brevemente el problema de incompresibilidad indicando que se pueden usar interpolaciones de igual orden para la velocidad y la presión cuando se elige una técnica de estabilización adecuada. El término convectivo introduce un problema similar. Cuando los efectos convectivos tienden a dominar sobre los efectos viscosos (flujos con números de Reynolds altos), planteamientos de elementos finitos no estabilizados conducen a una solución excesivamente oscilatoria que pronto pierde cualquier semejanza con la solución real del problema.

En el trabajo de Liu et al.[1] y de Huerta y Donea [3] se puede encontrar una descripción más detallada del problema. A continuación se expone un resumen del planteamiento utilizado.

De la misma manera que para el problema de incompresibilidad era conveniente estudiar el problema estacionario de Stokes en lugar de las ecuaciones completas de Navier-Stokes, las principales características del problema se pueden estudiar sobre una ecuación más simple. Para ello se parte del problema de convección-difusión, el cual está gobernado por una ecuación de la forma

$$
(\mathbf{a} \cdot \nabla) \mathbf{v}-\nu \nabla^{2} \mathbf{v}=s
$$

donde a representa la velocidad de convección y $s=s(\mathbf{x})$ es un término fuente volumétrico. La forma débil asociada es

$$
\int_{\Omega} \omega(\mathbf{a} \cdot \nabla) \mathbf{v} d \Omega-\int_{\Omega} \omega \nu \nabla^{2} \mathbf{v} d \Omega=\int_{\Omega} \omega s d \Omega
$$


donde $\omega$ es una función test que vale cero en el contorno en el cual se han impuesto condiciones tipo Dirichlet.

Usando el teorema de Green-Gauss (5.3) se obtiene lo siguiente

$$
\int_{\Omega} \omega(\mathbf{a} \cdot \nabla) \mathbf{v} d \Omega+\int_{\Omega} \nabla \omega \cdot \nu \nabla \mathbf{v} d \Omega=\int_{\Omega} \omega s d \Omega
$$

Aplicando el planteamiento estándar de Galerkin se llega a la definición de una ecuación discreta de la forma

$$
(\mathbf{C}+\mathbf{K}) \mathbf{u}=\mathbf{f}
$$

donde $\mathbf{C}$ y $\mathbf{K}$ se obtienen ensamblando las distintas contribuciones elementales

$$
\begin{gathered}
\mathbf{C}_{a b}^{e}=\int_{\Omega^{e}} N_{a}\left(\mathbf{a} \cdot \nabla N_{b}\right) d \Omega \\
\mathbf{K}_{a b}^{e}=\int_{\Omega^{e}} \nu \nabla N_{a} \cdot\left(\nabla N_{b}\right) d \Omega \\
\mathbf{f}_{a}^{e}=\int_{\Omega^{e}} \omega N_{a} s d \Omega
\end{gathered}
$$

El caso multidimensional es demasiado complejo para el tratamiento analítico. Sin embargo, es posible estudiar analíticamente la solución para el caso 1D sobre una malla regular con elementos lineales. La ecuación (5.41) en una dimensión es de la forma

$$
a \frac{\partial v}{\partial x}-\nu \frac{\partial^{2} v}{\partial x^{2}}=s
$$

Asumiendo un tamaño de elemento constante $h$, y un término fuente constante $s=1$, los operadores discretos son

$$
\begin{aligned}
\mathbf{C}^{e} & =\frac{a}{2}\left[\begin{array}{ll}
-1 & 1 \\
-1 & 1
\end{array}\right] \\
\mathbf{K}^{e} & =\frac{\nu}{h}\left[\begin{array}{cc}
1 & -1 \\
-1 & 1
\end{array}\right] \\
\mathbf{f}^{e} & =\left\{\begin{array}{l}
1 / 2 \\
1 / 2
\end{array}\right\}
\end{aligned}
$$

Ensamblando las diferentes contribuciones se llega a

$$
a \frac{v_{j+1}-v_{j-1}}{2 h}-\nu \frac{v_{j+1}-2 v_{j}+v_{j-1}}{h^{2}}=1
$$


Introduciendo el número de Peclet $P e=a h / 2 \nu$, la expresión anterior se puede reescribir como

$$
\frac{a}{2 h}\left(\frac{P e-1}{P e} v_{j+1}+\frac{2}{P e} v_{j}-\frac{P e+1}{P e} v_{j-1}\right)=1
$$

Bajo la hipótesis de que la ecuación, sujeta a condiciones de contorno homogéneas, se resuelve en un dominio 1D de longitud 1, la solución analítica es

$$
v(x)=\frac{1}{a}\left(x-\frac{1-\exp (\gamma x)}{1-\exp \gamma}\right) ; \gamma=\frac{a}{\nu}
$$

Teniendo en cuenta la solución analítica es posible encontrar la ecuación en diferencias que reproduciría exactamente dicha solución. Esta ecuación en diferencias es de la forma

$$
a \frac{v_{j+1}-v_{j-1}}{2 h}-(\nu+\bar{\nu}) \frac{v_{j+1}-2 v_{j}+v_{j-1}}{h^{2}}=1
$$

donde $\bar{\nu}$ es una difusión numérica adicional definida como

$$
\bar{\nu}=\left(\operatorname{coth} P e-\frac{1}{P e}\right) \nu P e
$$

Comparando (5.52) con (5.55) es evidente que la solución de Galerkin introduce una falsa difusión numérica negativa. El planteamiento estándar de Galerkin también se puede aplicar para la resolución de la siguiente ecuación

$$
a \frac{\partial v}{\partial x}-(\nu-\bar{\nu}) \frac{\partial^{2} v}{\partial x^{2}}=s
$$

Cuando el número de Peclet adopta valores superiores a la unidad la difusión efectiva puede caer por debajo de cero, dando lugar a una solución inestable. En la Figura 5.1 [4] se muestran ejemplos de soluciones numéricas para diferentes números de Peclet.

En definitiva, el comportamiento oscilatorio mostrado por la discretización espacial de Galerkin se puede corregir de manera eficaz añadiendo una difusión numérica adecuada. Esto lleva a la definición de los esquemas Streamline Upwind (SU), los cuales asumen, para el caso general, el tensor de difusión numérica siguiente

$$
\tilde{\nu}=\bar{\nu} \frac{\mathbf{a} \otimes \mathbf{a}}{\|\mathbf{a}\|^{2}}
$$

La correspondiente forma débil es

$$
\int_{\Omega} \omega(\mathbf{a} \cdot \nabla) \mathbf{v} d \Omega+\int_{\Omega} \nabla \omega \cdot(\nu \mathbf{I}-\tilde{\nu}) \cdot(\nabla \mathbf{v}) d \Omega=\int_{\Omega} \omega s d \Omega
$$


Las técnicas SU permiten una fácil implementación y aseguran una solución suave para problemas con números de Peclet altos. Sin embargo, el principal inconveniente que presentan estas técnicas es que la solución exacta de (5.41) no satisface exactamente la forma débil (5.59), lo cual conduce a problemas de precisión en situaciones complejas.

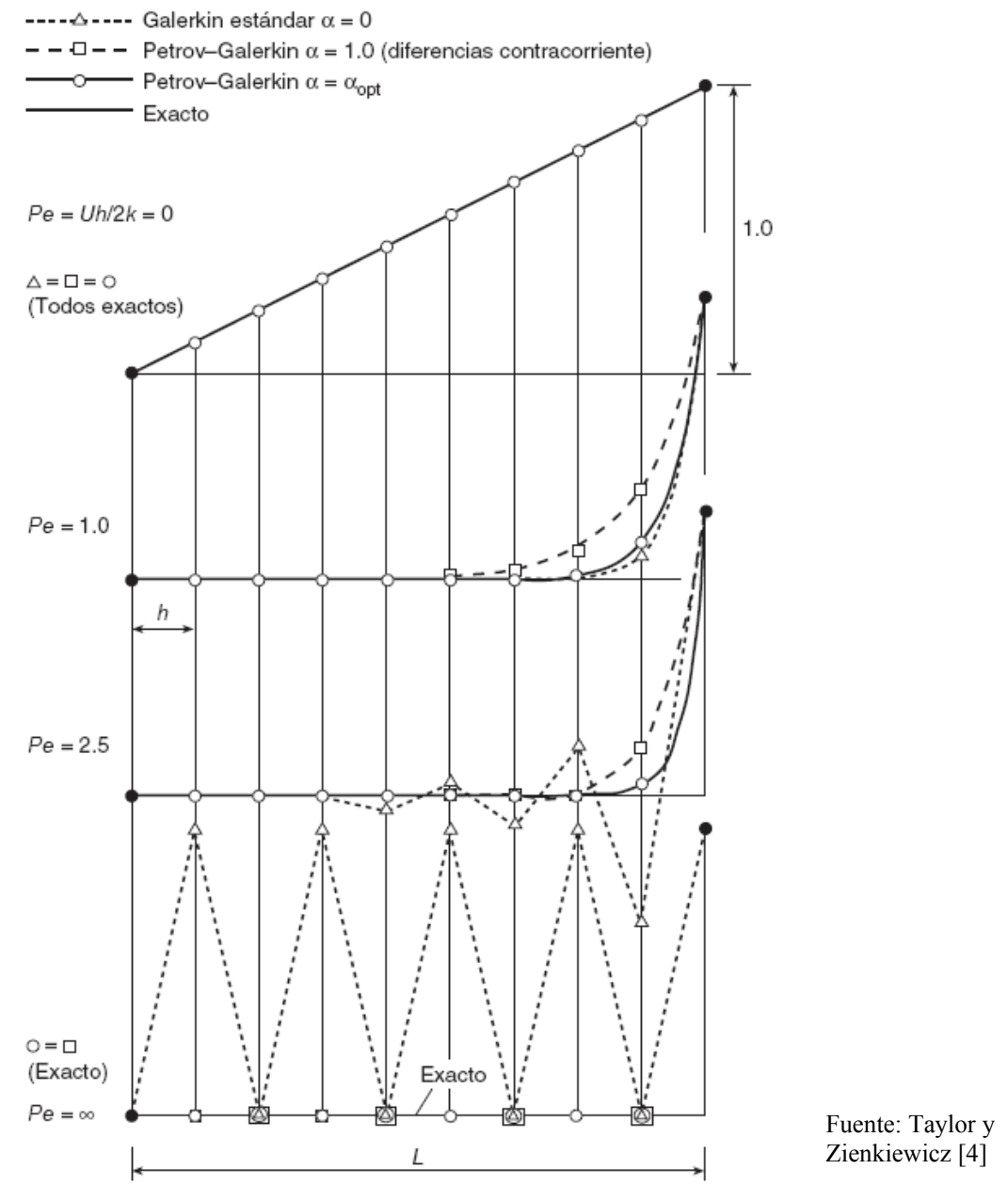

Figura 5.1. Resultados para el problema de convección-difusión en 1D usando diferentes técnicas de estabilización

\subsubsection{Técnicas de estabilización Streamline Upwind Petrov-Galerkin y Galerkin Least Squares}

La desventaja de las técnicas SU se puede superar introduciendo las formulaciones Streamline Upwind Petrov-Galerkin (SUPG) y Galerkin Least Squares (GLS). Estos métodos están basados en la definición de una forma débil modificada que se verifica por la solución exacta. La razón 
fundamental del éxito de estas técnicas radica en la definición de una forma residual que se hace cero de acuerdo con la solución exacta.

Definiendo el operador diferencial

$$
L(v)=(\mathbf{a} \cdot \nabla) \mathbf{v}-\nu \nabla^{2} \mathbf{v}
$$

el residuo se define como

$$
R(v)=L(v)-s
$$

Estas técnicas de estabilización utilizan una forma débil del tipo

$$
\int_{\Omega} R(v) d \Omega+\sum_{e} \int_{\Omega^{e}} P(\omega) \tau R(v) d \Omega^{e}=0
$$

donde $\tau$ es un parámetro de estabilización y $P(\omega)$ es un operador aplicado a la función test.

El método SUPG se obtiene haciendo

$$
P(\omega):=L(\omega)=(\mathbf{a} \cdot \nabla) \omega
$$

y el método GLS se obtiene para

$$
P(\omega):=L(\omega)=(\mathbf{a} \cdot \nabla) \omega-\nu \nabla^{2} \omega
$$

En ambos casos el operador $P(\omega)$ se toma como discontinuo a través de los elementos. Esto hace que los métodos SUPG y GLS sean prácticamente equivalentes para problemas lineales. La elección del parámetro de estabilización es muy importante para el éxito de la formulación. Según Codina [9], un valor apropiado para este parámetro es

$$
\tau=\left(\frac{2 a}{h}+\frac{4 \nu}{h^{2}}\right)^{-1}
$$

\subsubsection{Finite Increment Calculus}

Una alternativa para la obtención de formulaciones estabilizadas viene dada por el método Finite Increment Calculus (FIC). Este método se basa fundamentalmente en imponer las ecuaciones de conservación sobre dominios de tamaño finito en lugar de discretizar directamente la forma fuerte de estas ecuaciones. Esto conduce a la definición de ecuaciones de balance de orden alto, las cuales implícitamente contienen una contribución estabilizadora. Una explicación más detallada de la aplicación de este método a problemas de convección-difusión se puede encontrar en el trabajo de Oñate [10]. 
A continuación se proporciona un resumen del método. Dado un dominio 1D de longitud $\delta$ en el intervalo $(x-\delta, x)$, el balance de los términos convectivo y difusivo viene dado por

$$
\begin{gathered}
F_{\text {convectivo }}^{\text {out }}=a v \quad F_{\text {convectivo }}^{\text {in }}=a v-\delta a \frac{\partial v}{\partial x}+\frac{\delta^{2}}{2} \frac{\partial^{2} v}{\partial x^{2}}+O\left(\delta^{3}\right) \\
F_{\text {difusivo }}^{\text {out }}=-n u \frac{\partial v}{\partial x} \quad F_{\text {difusivo }}^{\text {in }}=-\nu \frac{\partial}{\partial x}\left(v-\delta \frac{v}{x}+\frac{1}{2} \frac{\delta^{2}}{2} \frac{\partial^{2} v}{\partial x^{2}}\right)+O\left(\delta^{3}\right) \\
\int s d x=\delta s-\frac{\delta^{2}}{2} \frac{\partial s}{\partial x}+O\left(\delta^{3}\right)
\end{gathered}
$$

La condición de equilibrio es

$$
\left(F_{\text {convectivo }}^{\text {in }}-F_{\text {convectivo }}^{\text {out }}\right)+\left(F_{\text {difusivo }}^{\text {in }}-F_{\text {difusivo }}^{\text {out }}\right)=\int s d x
$$

sustituyendo (5.66) en (5.67) y simplificando se obtiene finalmente la ecuación

$$
a \frac{\partial v}{\partial x}-\nu \frac{\partial^{2} v}{\partial x^{2}}+\frac{\delta}{2} \frac{\partial}{\partial x}\left(a \frac{\partial v}{\partial x}-\nu \frac{\partial^{2} v}{\partial x^{2}}\right)=s
$$

la cual conduce a un esquema estabilizado.

\subsubsection{Variational Multiscale Methods}

La idea original del método de estabilización de las sub-escalas o multi-escalas (sub-grid scales) fue inicialmente propuesta por Hughes [11] para resolver ecuaciones escalares del tipo convección-difusión.

La idea fundamental que introduce el método de las sub-escalas es que al definir una malla de elementos finitos quedan establecidos dos niveles de resolución; un nivel corresponde a la malla y será aproximado por elementos finitos y el otro, denominado sub-escala, que no podrá ser captado $[11,12]$. Si bien se admite que existe una componente de la solución que no puede ser resuelta, se debería aproximar al menos "el efecto" de la sub-escala sobre la componente que se resuelve numéricamente. De acuerdo con esto, la causa de diversos problemas de estabilidad numérica sería el no considerar este efecto en la formulación. En este contexto, considerando que además de la componente aproximada por la solución de elementos finitos $\overline{\mathbf{v}}$ existe una componente no resuelta $\mathbf{v}^{\prime}$, la solución exacta se puede expresar como $\mathbf{v}=\overline{\mathbf{v}}+\mathbf{v}^{\prime}$.

El solver de flujo de fluidos utilizado en este trabajo emplea el método de estabilización de las sub-escalas ortogonales descrito por Codina en [13]. 


\subsection{Métodos de paso fraccionado}

Como se indicó anteriormente, la discretización del problema de flujo incompresible conduce a la definición de un sistema discreto de ecuaciones de la forma (5.39) ó (5.40). Incluso cuando la resolución del sistema está garantizada, éste suele estar mal condicionado debido a la coexistencia de términos procedentes de la discretización de diferentes variables.

En un primer instante se podría pensar en la utilización de solvers directos para la resolución del problema, pero teniendo en cuenta que el tamaño real de los problemas CFD puede suponer cientos de miles, o incluso millones de grados de libertad (DOF), la utilización de estos solvers podría resultar ineficaz, o incluso imposible. Por otro lado, los solvers iterativos son sumamente eficientes en la resolución de sistemas no "stiff" de gran tamaño, sin embargo resultan poco robustos al tratar con sistemas mal condicionados. El éxito de los métodos de paso fraccionado depende en gran medida de su capacidad para dividir el problema en una serie de etapas, implicando cada una de ellas la inversión de un sistema bien condicionado. La aplicación de métodos de paso fraccionado en el contexto de los elementos finitos a la mecánica de fluidos es algo relativamente reciente, no obstante, en el trabajo de Huerta y Donea [3] se describen diferentes planteamientos del problema.

A continuación se describe brevemente el esquema de paso fraccionado propuesto por Codina [9] y que ha sido utilizado en el desarrollo de este trabajo. Se presenta en su forma básica, sin la introducción de ningún tipo de estabilización para el término de presión.

El punto de partida es la discretización espacial de las ecuaciones de Navier-Stokes (5.35) y (5.36). El sistema discreto de ecuaciones equivalente se puede obtener usando el método de Galerkin

$$
\begin{gathered}
\mathbf{M} \frac{\mathbf{V}^{n+1}-\mathbf{V}^{n}}{\delta t}+\mathbf{K}\left(\mathbf{V}^{n+\theta}\right) \mathbf{V}^{n+\theta}+\mathbf{G} \mathbf{P}^{n+1}=\mathbf{F}^{n+\theta} \\
\mathbf{D} \mathbf{V}^{n+1}=0
\end{gathered}
$$

donde $\mathbf{V}$ y $\mathbf{P}$ son los vectores de las velocidades y presiones nodales, respectivamente.

Las componentes de las matrices que aparecen en las ecuaciones anteriores son

$$
\begin{gathered}
\mathbf{M}_{i j}^{a b}=\int_{\Omega} N_{a} N_{b} d \Omega \\
\mathbf{K}\left(\mathbf{V}^{n+\theta}\right)_{i j}^{a b}=\int_{\Omega}\left(N_{a} \mathbf{v}^{n+\theta} \cdot \nabla N_{b}\right)+\frac{1}{2} N_{a}\left(\nabla \cdot \mathbf{v}^{n+\theta}\right) N_{b}+\nu \nabla N_{a} \cdot \nabla N_{b} d \Omega
\end{gathered}
$$




$$
\begin{gathered}
\mathbf{G}_{i j}^{a b}=-\int_{\Omega} \frac{\partial N_{a}}{\partial x_{i}} N_{b} d \Omega \\
\mathbf{F}_{i}^{a}=\int_{\Omega} N_{a} f_{i} d \Omega \\
\mathbf{D}=-\mathbf{G}^{T}
\end{gathered}
$$

El método de paso fraccionado se basa en la introducción de una variable auxiliar $\tilde{\mathbf{V}}^{n+1}$, la cual permite reescribir la derivada temporal como

$$
\frac{\mathbf{V}^{n+1}-\mathbf{V}^{n}}{\delta t}=\frac{\mathbf{V}^{n+1}-\tilde{\mathbf{V}}^{n+1}}{\delta t}+\frac{\tilde{\mathbf{V}}^{n+1}-\mathbf{V}^{n}}{\delta t}
$$

Substituyendo (5.76) en (5.69) se puede dividir la ecuación de la conservación de la cantidad de movimiento en dos

$$
\begin{gathered}
\mathbf{M} \frac{\tilde{\mathbf{V}}^{n+1}-\mathbf{V}^{n}}{\delta t}+\mathbf{K}\left(\mathbf{V}^{n+\theta}\right) \mathbf{V}^{n+\theta}+\gamma \mathbf{G} \mathbf{P}^{n}=\mathbf{F}^{n+\theta} \\
\mathbf{M} \frac{\mathbf{V}^{n+1}-\tilde{\mathbf{V}}^{n+1}}{\delta t}+\mathbf{G}\left(\mathbf{P}^{n+1}-\gamma \mathbf{P}^{n}\right)=0 \\
\mathbf{D} \mathbf{V}^{n+1}=0
\end{gathered}
$$

donde $\gamma$ es un parámetro numérico, cuyos valores de interés son 0 y 1 .

En este punto es necesario hacer la aproximación

$$
\mathbf{K}\left(\mathbf{V}^{n+\theta}\right) \mathbf{V}^{n+\theta} \approx \mathbf{K}\left(\tilde{\mathbf{V}}^{n+\theta}\right) \tilde{\mathbf{V}}^{n+\theta}
$$

donde $\tilde{\mathbf{V}}^{n+\theta}:=\theta \tilde{\mathbf{V}}^{n+1}+(1-\theta) \mathbf{V}^{n}$. Expresando $\mathbf{V}^{n+1}$ en términos de $\tilde{\mathbf{V}}^{n+1}$ usando (5.78) e introduciendo el resultado en (5.79), el conjunto de ecuaciones a resolver es

$$
\begin{gathered}
\mathbf{M} \frac{\tilde{\mathbf{V}}^{n+1}-\mathbf{V}^{n}}{\delta t}+\mathbf{K}\left(\tilde{\mathbf{V}}^{n+\theta}\right) \tilde{\mathbf{V}}^{n+\theta}+\gamma \mathbf{G} \mathbf{P}^{n}=\mathbf{F}^{n+\theta} \\
\delta t \mathbf{D} \mathbf{M}^{-1} \mathbf{G}\left(\mathbf{P}^{n+1}-\gamma \mathbf{P}^{n}\right)=\mathbf{D} \tilde{\mathbf{V}}^{n+1} \\
\mathbf{M} \frac{\mathbf{V}^{n+1}-\tilde{\mathbf{V}}^{n+1}}{\delta t}+\mathbf{G}\left(\mathbf{P}^{n+1}-\gamma \mathbf{P}^{n}\right)=0
\end{gathered}
$$

Notar que $\mathbf{D M}^{-1} \mathbf{G}$ representa una aproximación del operador Laplaciano. Para evitar tratar con esta matriz, se puede hacer la siguiente aproximación 


$$
\mathbf{D M}^{-1} \mathbf{G} \approx \mathbf{L}, \quad \text { con componentes } \mathbf{L}^{a b}=-\int_{\Omega} \nabla N_{a} \cdot \nabla N_{b} d \Omega
$$

Después de usar (5.80) y (5.84), el método de paso fraccionado asume finalmente la forma

- $\quad$ paso 1

$$
\mathbf{M} \frac{\tilde{\mathbf{V}}^{n+1}-\mathbf{V}^{n}}{\delta t}+\mathbf{K}\left(\mathbf{V}^{n+\theta}\right) \mathbf{V}^{n+\theta}+\gamma \mathbf{G} \mathbf{P}^{n}=\mathbf{F}^{n+\theta}
$$

- $\quad$ paso 2

$$
\delta t \mathbf{L}\left(\mathbf{P}^{n+1}-\gamma \mathbf{P}^{n}\right)=\mathbf{D} \tilde{\mathbf{V}}^{n+1}
$$

- $\quad$ paso 3

$$
\mathbf{M} \frac{\mathbf{V}^{n+1}-\tilde{\mathbf{V}}^{n+1}}{\delta t}+\mathbf{G}\left(\mathbf{P}^{n+1}-\gamma \mathbf{P}^{n}\right)=0
$$

El método de paso fraccionado descrito tiene muchas propiedades interesantes, en particular, posee una cierta estabilidad intrínseca incluso en ausencia de cualquier término de estabilización. En el trabajo de Codina [9] se puede encontrar un estudio completo sobre la estabilidad del esquema incluyendo el efecto de los términos de estabilización. En los trabajos de Codina y Badia $[14,15]$ se estudia la estabilidad de los esquemas de segundo orden de paso fraccionado estabilizados que actualmente están implementados en Kratos.

\subsection{Ejemplo. Flujo alrededor de un cilindro}

A continuación se resuelve el problema de flujo bidimensional alrededor de un cilindro circular utilizando el esquema que se ha presentado anteriormente. Se trata de una prueba clásica para evaluar el comportamiento de un algoritmo en la resolución de flujos incompresibles. El problema ha sido ampliamente estudiado y existen en la bibliografía numerosos resultados experimentales $[16,17]$ y numéricos $[18,19,20,21,22]$ con los que poder contrastar los resultados obtenidos.

El diámetro del cilindro es $0.5 \mathrm{~m}$ y las dimensiones del dominio del fluido se muestran en la Figura 5.2. Respecto a las condiciones de contorno, se impone una velocidad de entrada de fluido de valor $\left(v_{x}, 0\right)$ en el contorno $\Gamma_{u}$, en los contornos laterales $\Gamma_{w}$ se fija velocidad normal nula $v_{y}=$ 0 , en el contorno de salida $\Gamma_{d}$ se asigna la condición de salida sin tensión (traction-free boundary condition) y en el contorno del cilindro $S$ se fija la condición de no deslizamiento (no-slip condition). Inicialmente el campo de velocidades es nulo en todos los puntos salvo en la entrada. 


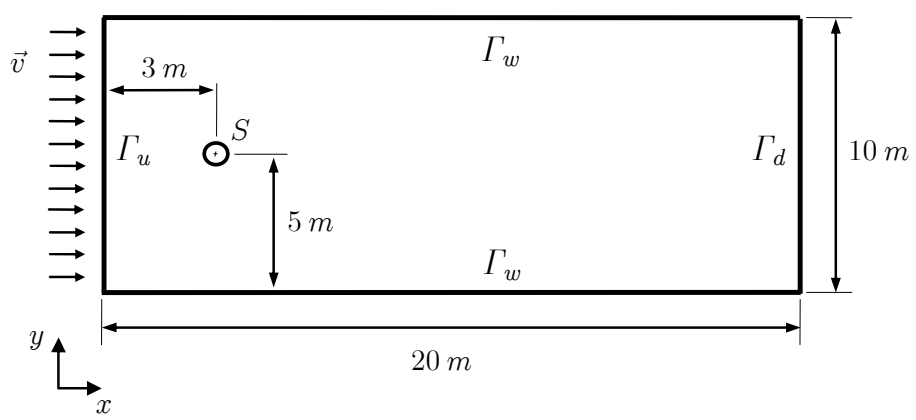

Figura 5.2. Dominio del problema de flujo bidimensional alrededor de un cilindro

El mallado del contorno el cilindro $S$ se realizó asignándole un tamaño característico de $0.05 \mathrm{~m}$ y el del dominio del fluido de acuerdo con el esquema de la Figura 5.3 concentrando un mayor número de elementos en el entorno más próximo al cilindro. La malla resultante tiene la forma que se muestra en la Figura 5.4.
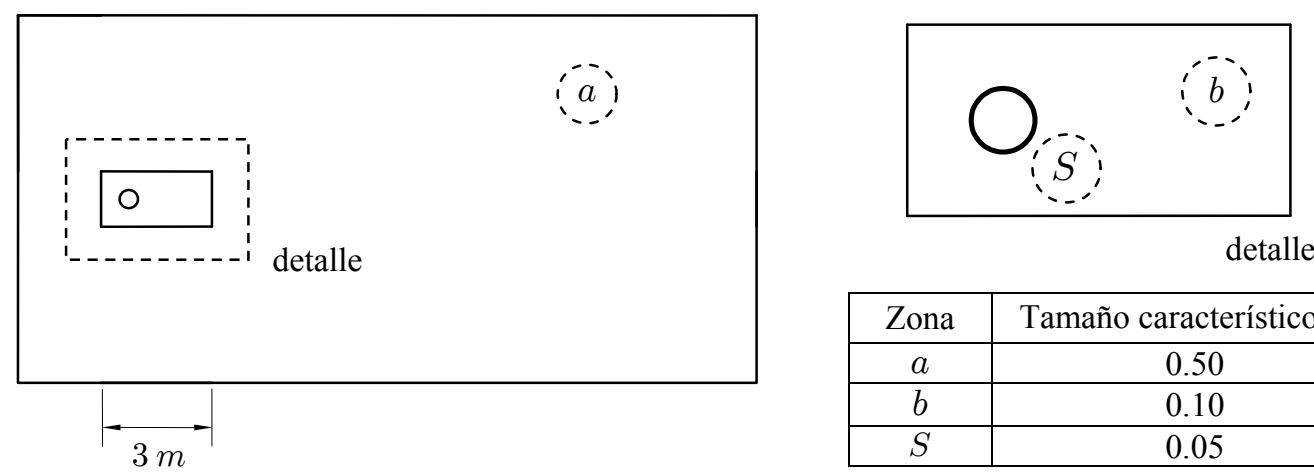

\begin{tabular}{|c|c|}
\hline Zona & Tamaño característico $[\mathrm{m}]$ \\
\hline$a$ & 0.50 \\
\hline$b$ & 0.10 \\
\hline$S$ & 0.05 \\
\hline
\end{tabular}

Figura 5.3. Esquema de mallado del dominio del fluido

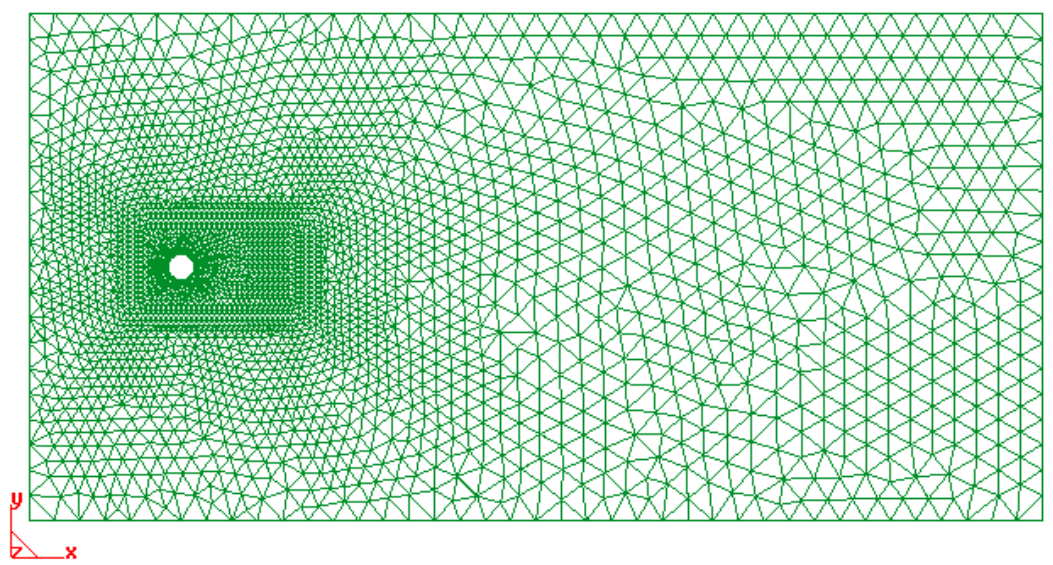

Figura 5.4. Malla del fluido 
Se llevaron a cabo varias simulaciones, asignando distintos valores a la componente de la velocidad $v_{x}$ de entrada del fluido, con objeto de obtener datos suficientes para representar la variación del número de Strouhal y del coeficiente de arrastre en función del número de Reynolds.

En la Figura 5.5 se muestra la dependencia del número de Strohual respecto al número de Reynolds observándose una buena correlación con los resultados de Lienhart [23] y Achenbach y Heinecke [24]. En la Figura 5.6 se ha representado el coeficiente de arrastre en función del número de Reynolds. Se aprecia una tendencia correcta respecto a los resultados experimentales [25, 26], aunque en el intervalo $10^{4} \leq R e \leq 10^{5}$ el algoritmo subestima ligeramente los valores del coeficiente de arrastre. Notar que no se capta la caída brusca del coeficiente de arrastre (drag crisis) que ocurre para $R e \approx 2.5 \cdot 10^{5}$.

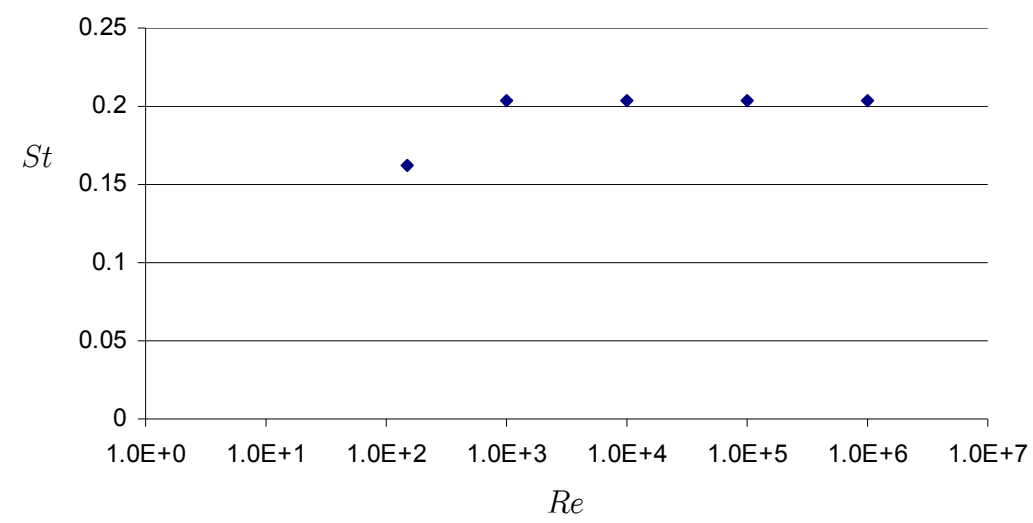

Figura 5.5. Variación del número de Strohual en función del número de Reynolds

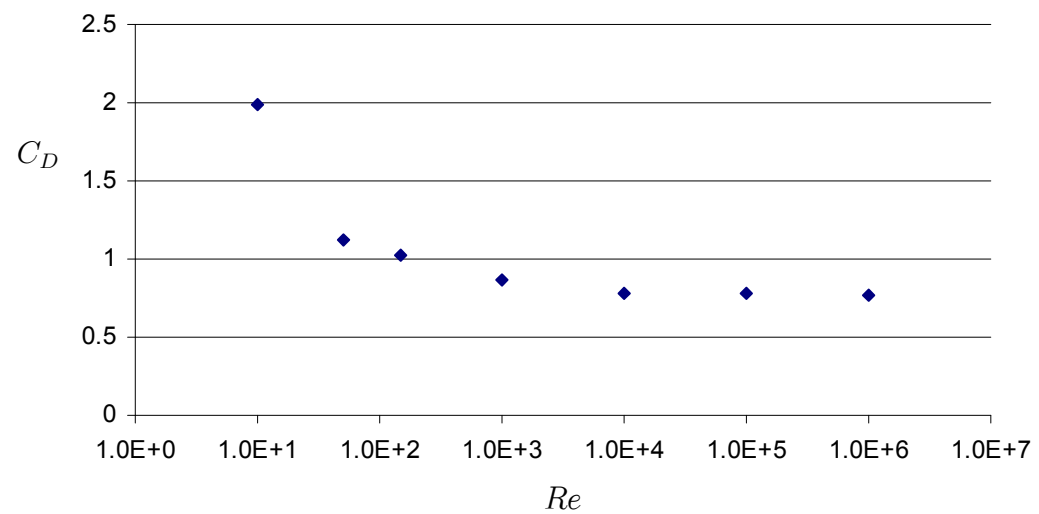

Figura 5.6. Variación del coeficiente de arrastre en función del número de Reynolds 
A modo de ejemplo, en la Figura 5.7 se muestra la variación en el tiempo del coeficiente de arrastre y del coeficiente de sustentación para $R e=150$ y en la Figura 5.8 para $R e=10^{4}$. Se observa en ambos casos que el coeficiente de sustentación presenta una mayor amplitud de oscilación siendo su valor medio cero, tal como era de esperar.

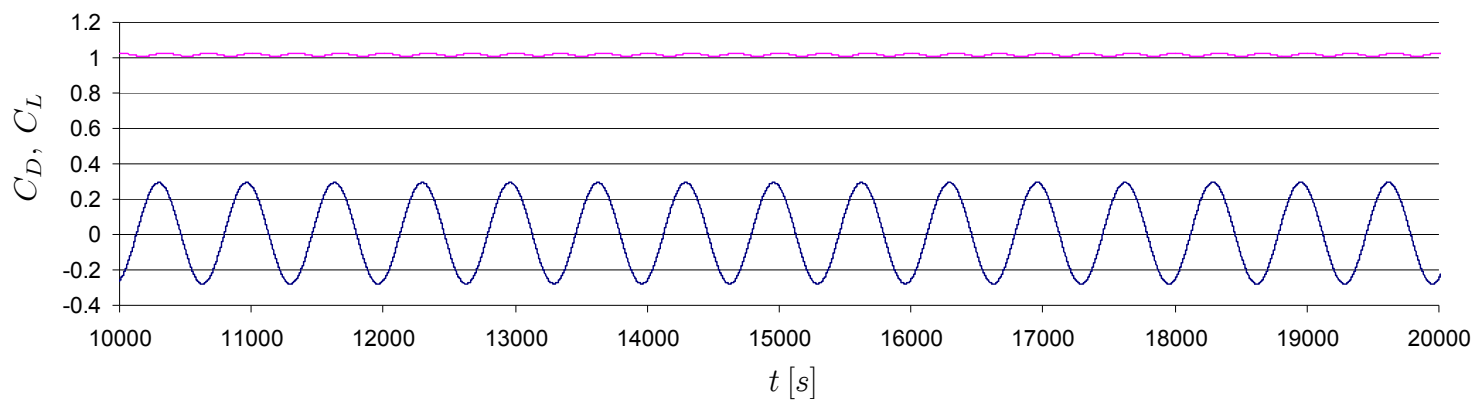

Figura 5.7. Variación en el tiempo de los coeficientes de arrastre y sustentación para $R e=150$

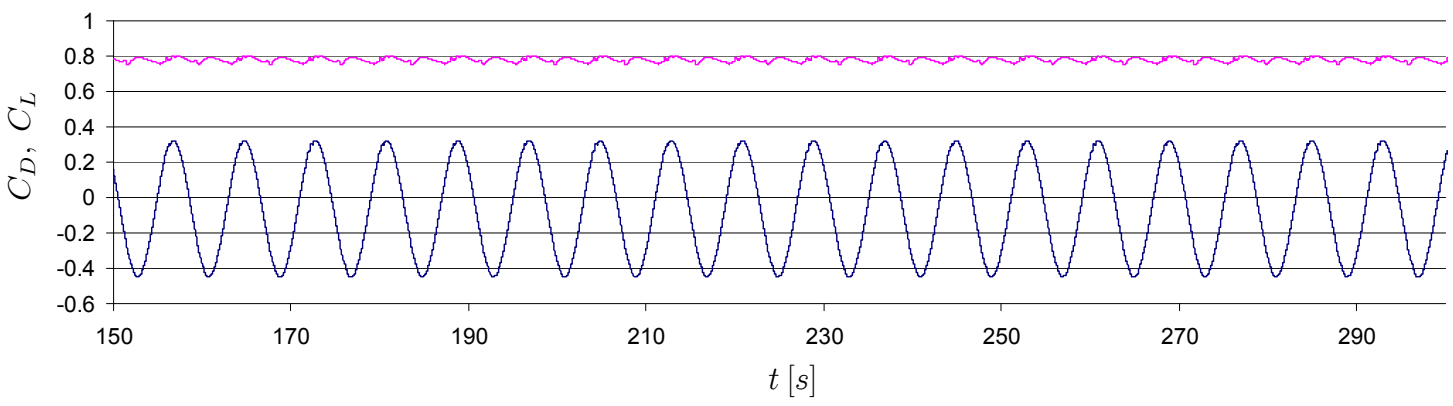

Figura 5.8. Variación en el tiempo de los coeficientes de arrastre y sustentación para $R e=10^{4}$

En la Figura 5.9 y en la Figura 5.10 se muestran los análisis en frecuencias de la oscilación del coeficiente de arrastre y de sustentación para $R e=150$ y $R e=10^{4}$, respectivamente. El análisis en frecuencias se realizado a partir de la transformada rápida de Fourier (FFT).
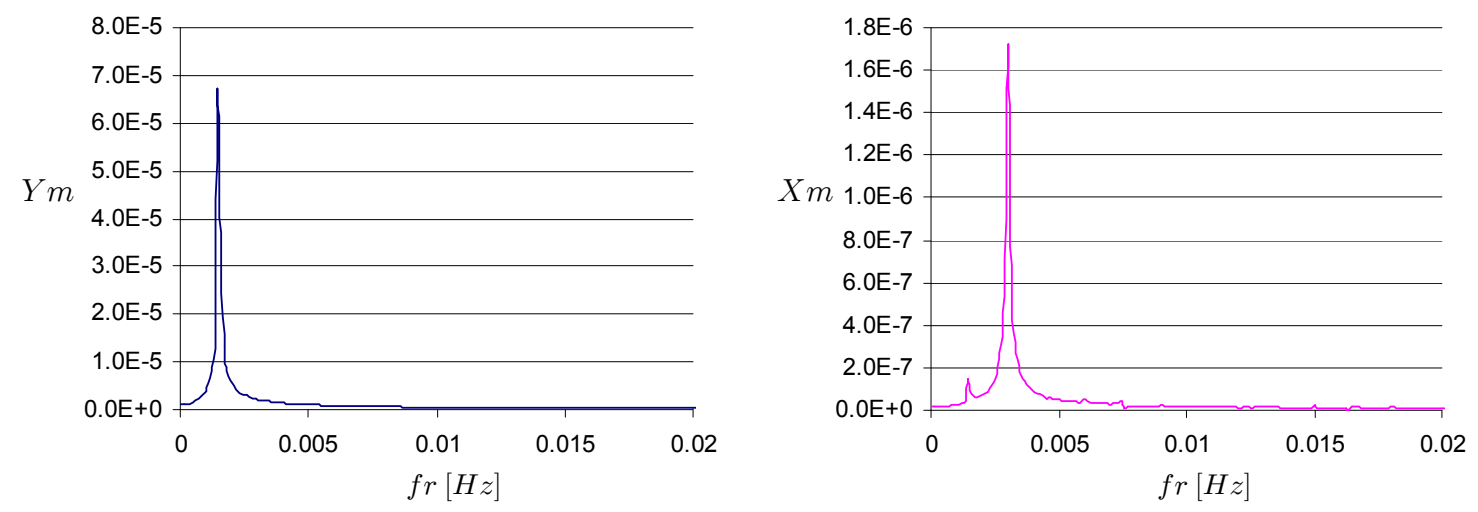

Figura 5.9.Análisis en frecuencias de los coeficientes de sustentación y de arrastre para $R e=150$ 

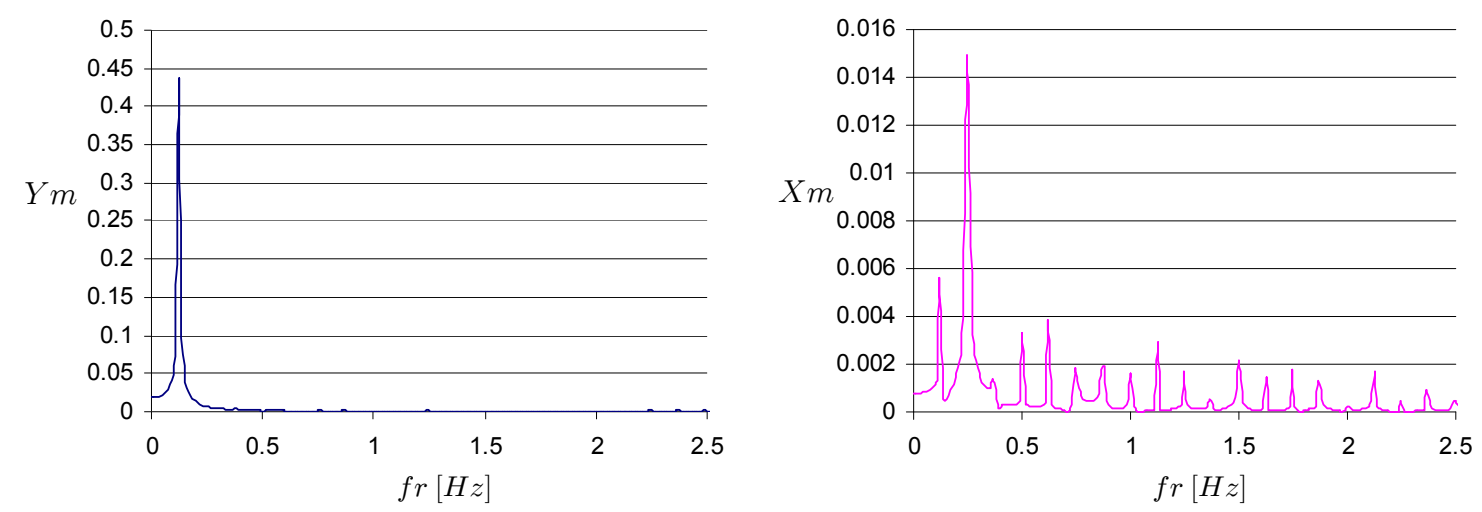

Figura 5.10. Análisis en frecuencias de los coeficientes de sustentación y de arrastre para $R e=10^{4}$

Finalmente, en la Tabla 1 se indican las frecuencias de oscilación para cada caso. Se comprueba que la frecuencia de vibración del coeficiente de sustentación, la cual coincide con la frecuencia de desprendimiento de los vórtices, es la mitad que la del coeficiente de arrastre.

\begin{tabular}{|l|c|c|}
\cline { 2 - 3 } \multicolumn{1}{c|}{} & $R e=150$ & $R e=10^{4}$ \\
\hline$C_{L}$ & $0.00147 \mathrm{~Hz}$ & $0.122 \mathrm{~Hz}$ \\
\hline$C_{D}$ & $0.00301 \mathrm{~Hz}$ & $0.244 \mathrm{Hzz}$ \\
\hline
\end{tabular}

Tabla 1. Frecuencias de oscilación de los coeficientes de arrastre y sustentación

\subsection{Formulación arbitrariamente Lagrangiana-Euleriana}

Los procedimientos de resolución presentados hasta aquí siguen un planteamiento puramente Euleriano, que es la opción natural en CFD. Sin embargo, para abordar la resolución de problemas acoplados de interacción fluido-estructura es necesario modificar los solvers de cálculo de fluidos para que puedan manejar mallas móviles. La formulación arbitrariamente Lagrangiana-Euleriana (ALE) proporciona el marco teórico necesario para tratar esta cuestión. La formulación ALE permite considerar el movimiento de la malla conforme el fluido interactúa con la estructura que se deforma. Una excelente presentación de las técnicas ALE se puede encontrar en los trabajos de Liu et al. [1], Huerta y Donea [3], y Stein et al. [27].

En la mecánica de los medios continuos es habitual definir dos variables $\mathrm{x}$ y $\mathbf{X}$ para describir el dominio espacial y el dominio material, respectivamente. Estas dos cantidades están relacionadas por una función de la forma 


$$
\phi:=(\mathbf{X}, t) \mapsto \phi(\mathbf{X}, t)=(\mathbf{x}, t)
$$

Por otro lado, las variables $\mathbf{x}$ y $\mathbf{X}$ pueden ser referidas a un tercer dominio el cual no coincida con el dominio espacial ni con el dominio material. Esta idea constituye la base de la formulación ALE. Desde un punto de vista práctico, el método parte de la definición de las funciones siguientes

$$
\begin{aligned}
& \Phi:=(\chi, t) \rightarrow \Phi(\chi, t)=(\mathbf{x}, t) \\
& \psi:=(\chi, t) \rightarrow \psi(\chi, t)=(\mathbf{X}, t)
\end{aligned}
$$

las cuales relacionan el dominio espacial y el dominio material con el dominio computacional $\chi$. La función $\phi$ se puede expresar como una composición de las funiones (5.89) y (5.90).

$$
\phi=\Phi \circ \psi^{-1}
$$

Considerando los gradientes de (5.88), (5.89) y (5.90) se puede obtener información útil

$$
\begin{gathered}
\frac{\partial \phi}{\partial(\mathbf{X}, t)}=\left[\begin{array}{cc}
\frac{\partial \mathbf{x}}{\partial \mathbf{X}} & \mathbf{v} \\
0 & 1
\end{array}\right] ; \quad \mathbf{v}:=\left.\frac{\partial \mathbf{x}}{\partial t}\right|_{\mathbf{X}} \\
\frac{\partial \Phi}{\partial(\chi, t)}=\left[\begin{array}{cc}
\frac{\partial \mathbf{x}}{\partial \chi} & \tilde{\mathbf{v}} \\
0 & 1
\end{array}\right] ; \quad \tilde{\mathbf{v}}:=\left.\frac{\partial \mathbf{x}}{\partial t}\right|_{\chi} \\
\frac{\partial \psi^{-1}}{\partial(\mathbf{X}, t)}=\left[\begin{array}{cc}
\frac{\partial \chi}{\partial \mathbf{X}} & \mathbf{w} \\
0 & 1
\end{array}\right] ; \quad \mathbf{w}:=\left.\frac{\partial \chi}{\partial t}\right|_{\mathbf{X}}
\end{gathered}
$$

Las distintas funciones están relacionadas a través de la operación de composición (5.91), por lo tanto, su gradiente sigue la regla

$$
\left[\begin{array}{cc}
\frac{\partial \mathbf{x}}{\partial \mathbf{X}} & \mathbf{v} \\
0 & 1
\end{array}\right]=\left[\begin{array}{cc}
\frac{\partial \mathbf{x}}{\partial \chi} & \tilde{\mathbf{v}} \\
0 & 1
\end{array}\right]\left[\begin{array}{cc}
\frac{\partial \chi}{\partial \mathbf{X}} & \mathbf{w} \\
0 & 1
\end{array}\right]
$$

Operando sobre la expresión anterior se puede verificar fácilmente que

$$
\mathbf{v}=\tilde{\mathbf{v}}+\frac{\partial \mathbf{x}}{\partial \chi} \mathbf{w}
$$

La ecuación (5.96) permite definir la velocidad convectiva c como

$$
\mathbf{c}:=\mathbf{v}-\tilde{\mathbf{v}}=\frac{\partial \mathbf{x}}{\partial \chi} \mathbf{w}
$$

que es la diferencia entre la velocidad del fluido y la velocidad de la malla. Una descripción más detallada de este concepto y otros asociados a la formulación ALE se pueden encontrar en [3]. 
Un desarrollo similar se puede aplicar a la derivada de cualquier función $f(\mathbf{X}, t)$. Es habitual en la mecánica computacional simplificar la notación usando el mismo símbolo para indicar la función equivalente operando desde un dominio diferente, por ejemplo, podemos utilizar $f(\mathbf{x}, t)$ para indicar $f(\phi(\mathbf{X}, t), t)$. Tomando como referencia el trabajo de Stein et al. [27], inicialmente se distinguirán las funciones operando desde dominios diferentes y sólo al final de la derivación se suprimirá la diferencia. Para ello se define

$$
\begin{gathered}
f^{* *}(\mathbf{X}, t):=f(\phi(\mathbf{X}, t), t) \\
f^{*}(\chi, t):=f(\phi(\mathbf{X}, t), t)=f^{* *}(\mathbf{X}, t)
\end{gathered}
$$

Usando la regla de la cadena se obtiene de forma inmediata

$$
\frac{\partial f^{* *}}{\partial t}=\frac{D f^{* *}}{D t}=\frac{\partial f}{\partial t}+\frac{\partial f}{\partial \mathbf{x}} \mathbf{v}
$$

que es la relación habitual entre las derivadas temporales materiales y espaciales.

Por otro lado, la relación entre $f^{* *} \mathrm{y} f^{*}$ es

$$
\frac{\partial f^{* *}}{\partial t}=\frac{D f^{* *}}{D t}=\frac{\partial f^{*}}{\partial t}+\frac{\partial f^{*}}{\partial \chi} \mathbf{w}
$$

Sustituyendo la definición de la velocidad convectiva c, se obtiene la expresión

$$
\left.\frac{\partial f}{\partial t}\right|_{\mathbf{X}}=\left.\frac{\partial f}{\partial t}\right|_{\chi}+\mathbf{c} \cdot \nabla f
$$

la cual permite reescribir las ecuaciones de conservación en el contexto de la formulación ALE de la siguiente manera

$$
\begin{gathered}
\frac{D \rho}{D t}+\rho \nabla \cdot \mathbf{v}=\left.\frac{\partial \rho}{\partial t}\right|_{\chi}+\mathbf{c} \cdot \nabla \rho+\rho \nabla \cdot \mathbf{v}=0 \\
\rho \frac{D \mathbf{v}}{D t}=\left.\rho \frac{\partial \mathbf{v}}{\partial t}\right|_{\chi}+\rho \mathbf{c} \cdot \nabla \mathbf{v}=\nabla \cdot \sigma+\rho \mathbf{b}
\end{gathered}
$$

Esto pone de manifiesto que las modificaciones con respecto a la formulación Euleriana son de poca importancia, por esta razón, a menudo la formulación ALE recibe el nombre de cuasiEuleriana. En la discretización para flujo incompresible el único cambio hace referencia a los términos convectivos, los cuales deben ser modificados substituyendo la velocidad Euleriana por la velocidad convectiva. Sin embargo, las consecuencias son importantes ya que la formulación ALE permite conservar las ventajas de la formulación Lagrangiana en el contorno acoplado conservando las características eulerianas en el dominio más alejado. 
Desde un punto de vista práctico, el problema de interacción fluido-estructura se puede formular como un sistema de tres campos: la estructura, el fluido y el movimiento de la malla. El movimiento de la estructura hace que la malla del fluido se mueva y la resolución del fluido se lleve a cabo sobre esta malla móvil. Intuitivamente, el movimiento de la malla coincide con el movimiento de la estructura en el contorno acoplado, donde la formulación ALE tiende al planteamiento Lagrangiano, mientras que la deformación del dominio disminuye con la distancia a la estructura, lo que hace que la formulación tienda al planteamiento Euleriano a medida que la distancia aumenta.

\section{Referencias}

${ }^{1}$ Liu, W.K., Belytschko, T., y Moran, B., Nonlinear Finite Elements for Continua and Structures, John Wiley and Sons, 2000.

${ }^{2}$ Hughes, T.J.R., The Finite Element Method, Dover, 2000.

${ }^{3}$ Huerta, A., y Donea, J., Finite Element Methods for Flow Problems, John Wiley and Sons, 2003.

${ }^{4}$ Taylor, R.L. y Zienkiewicz, O.C., "The Finite Element Method - Volume 3 - Fluid Dynamics", Butterworth Heinemann, 2000.

${ }^{5}$ O.A. Ladyzhenskaya, The Mathematical Theory of Viscous Incompressible Flows, Gordon and Breach, London, 1969.

${ }^{6}$ Babuska, I., The finite element method with Lagrange multipliers, Numerische Mathematik, 20, pp. 179-192, 1973.

${ }^{7}$ Brezzi, F., On the existence uniqueness and approximation of saddle point problems arising from Lagrange multipliers, RAIRO Ser. Anal. Numer., 8, pp. 129-151, 1974.

${ }^{8}$ Oñate, E., "Derivation of Stabilized equations for numerical solution of advective-difussive transport and fluid flow problems". Computer Methods in Applied Mechanics and Engineering, 151, pp. 233-265, 1998.

${ }^{9}$ Codina, R., "Pressure stability in fractional step finite element methods for incompressible flows", Journal of Computational Physics, 2001.

${ }^{10}$ Oñate, E., "Possibilities of finite calculus in computational mechanics", Technical report, CIMNE, 2001.

11 Hughes, T.J.R., "Multiscale phenomena: Green's functions, the Dirichlet-to-Neumann formulation, subgrid-scale models, bubbles and the origin of stabilized methods", Computer Methods in Applied Mechanics and Engineering, 127, pp. 387-401, 1995. 
${ }^{12}$ Hughes, T.J.R., Feijoo, G., Mazzei, L., Quincy, J.-B., "The variational multiscale method: a paradigm for computational mechanics, Computer Methods in Applied Mechanics and Engineering, 166, pp. 3-24, 1998.

${ }^{13}$ Codina, R., "Stabilization of incompressibility and convection through orthogonal sub-scales in finite element methods", Computer Methods in Applied Mechanics, 190, pp. 1579-1599, 2000.

${ }^{14}$ Codina, R., y Badia, S., "On some pressure segregation methods of fractional-step type for the finite element approximation of incompressible flow problems", Computer Methods in Applied Mechanics and Engineering, 195, pp. 2900-2918, 2006.

${ }^{15}$ Badia, S., y Codina, R., "Second order fractional step schemes for the incompressible NavierStokes equations. Inherent pressure stability and pressure stabilization”, Proceedings CD-ROM of the Sixth World Congress on Computational Mechanics in conjunction with the Second AsianPacific Congress on Computational Mechanics, paper 601, Beijing (China), 2004.

${ }^{16}$ Hamielec, A.E., y Raal, J.D., "Numerical studies of viscous flow around circular cylinders", Physics of Fluids, 12, pp. 11-17, 1969.

${ }^{17}$ Norberg, C. "Experimental investigation of the flow around a circular cylinder: influence of aspect ratio", Journal of Fluid Mechanics 258, pp. 287-316, 1994.

${ }^{18}$ Zdravkovich, M.M., Flow Around Circular Cylinders, Vol. 2 Applications, Oxford University Press, USA; 2003.

${ }^{19}$ Zdravkovich, M.M., Flow Around Circular Cylinders, Vol. 1 Fundamentals, Oxford University Press, USA; 1997.

${ }^{20}$ Sumer, B.M., Fredsфe, J., Hydrodynamics Around Cylindrical Structures, World Scientific, Singapore, 1997.

${ }^{21}$ Norberg, C., "Flow around a circular cylinder: Aspects of fluctuating lift", Journal of Fluids and Structures 15 (3-4), pp. 459-469, 2001

${ }^{22}$ Norberg, C., "Fluctuating lift on a circular cylinder: Review and new measurements", Journal of Fluids and Structures 17 (1), pp. 57-96, 2003.

${ }^{23}$ Lienhard, J.H., "Synopsis of lift, drag, and vortex frequency data for rigid circular cylinder", Washington State Univ., Coll. Eng., Rept. No. 300, 1966.

${ }^{24}$ Achenbach, E., y Heinecke, E., "On vortex shedding from smooth and rough cylinders in the range of Reynolds numbers $6 \times 10^{3}-5 \times 10^{6}$, Journal of Fluids Mechanics, 109, pp. 239-251, 1981.

${ }^{25}$ Singh, S.P., Mittal, S., "Flow past a cylinder: shear layer instability and drag crisis", International Journal for Numerical Methods in Fluids, 47, pp. 75-98, 2005.

${ }^{26}$ Roshko. A., "Experiments on the flow past a circular cylinder at very high Reynolds number", Journal of Fluid Mechanics, 10, 345-356, 1961. 
${ }^{27}$ Stein, E., Hughes, T.J.R., y De Borst, R., Encyclopedia of Computational Mechanics, Volumen 1, John Wiley and Sons, 2004. 
Capítulo 6

\section{Acoplamiento Fluido- Estructura}





\subsection{Resumen}

En el presente capítulo se estudia el problema de interacción fluido-estructura. El primer apartado contiene una descripción del problema y de las principales estrategias computacionales usadas para su resolución, además de una breve revisión del estado de los conocimientos. En el segundo apartado se describe el esquema computacional utilizado en este trabajo, el cual integra las etapas de la resolución estructural en la formulación de paso fraccionado del fluido. En el apartado siguiente se analiza el error local que introduce este planteamiento teniendo en cuenta las etapas de resolución del fluido. Finalmente, en el último apartado se muestra un estudio de la estabilidad del esquema tomando como referencia el trabajo de Piperno et al. [1,2].

\subsection{Introducción}

Durante los últimos años el Método de los Elementos Finitos ha experimentado un crecimiento exponencial que ha dado lugar a la definición de un conjunto de técnicas computacionales fiables para la resolución de problemas de muy diversa índole. Este desarrollo, junto con la cada vez mayor disponibilidad de potentes recursos informáticos, recientemente ha dado lugar a un creciente interés por los problemas acoplados. El trabajo de Park y Felippa [3] y de Felippa [4] proporciona una interesante introducción al problema junto con una revisión de las técnicas modernas utilizadas en el estudio de la estabilidad y precisión de los algoritmos.

A pesar de que se considera el problema de interacción estructura-estructura, el cual representa un caso límite de los sistemas acoplados, algunos de los resultados son generales y se pueden extrapolar a otros tipos de problemas.

Un gran número de problemas acoplados en los que intervienen dos campos se pueden expresar matemáticamente como un sistema de EDO de la forma

$$
\left[\begin{array}{ll}
\mathbf{M}_{x x} & \mathbf{M}_{x y} \\
\mathbf{M}_{y x} & \mathbf{M}_{y y}
\end{array}\right]\left\{\begin{array}{c}
\ddot{\mathbf{x}} \\
\ddot{\mathbf{y}}
\end{array}\right\}+\left[\begin{array}{ll}
\mathbf{C}_{x x} & \mathbf{C}_{x y} \\
\mathbf{C}_{y x} & \mathbf{C}_{y y}
\end{array}\right]\left\{\begin{array}{c}
\dot{\mathbf{x}} \\
\dot{\mathbf{y}}
\end{array}\right\}+\left[\begin{array}{ll}
\mathbf{K}_{x x} & \mathbf{K}_{x y} \\
\mathbf{K}_{y x} & \mathbf{K}_{y y}
\end{array}\right]\left\{\begin{array}{l}
\mathbf{x} \\
\mathbf{y}
\end{array}\right\}=\left\{\begin{array}{l}
\mathbf{f}_{x} \\
\mathbf{f}_{y}
\end{array}\right\}
$$

donde $\mathrm{x}$ e $\mathbf{y}$ representan las variables de estado de cada campo y los términos de las matrices proceden de la discretización espacial.

Los términos con índices combinados en (6.1) expresan la interacción o acoplamiento entre los campos. El problema acoplado en dos direcciones (two way coupling) surge cuando cada campo 
depende del otro, en términos matemáticos, cuando al menos uno de los términos " $x y$ " es distinto de cero y uno de los términos " $y x$ " también es no nulo. Por otro lado, si la formulación asume una forma del tipo

$$
\left[\begin{array}{cc}
\mathbf{M}_{x x} & \mathbf{0} \\
\mathbf{M}_{y x} & \mathbf{C}_{y y}
\end{array}\right]\left\{\begin{array}{c}
\ddot{\mathbf{x}} \\
\ddot{\mathbf{y}}
\end{array}\right\}+\left[\begin{array}{cc}
\mathbf{C}_{x x} & \mathbf{0} \\
\mathbf{C}_{y x} & \mathbf{C}_{y y}
\end{array}\right]\left\{\begin{array}{c}
\dot{\mathbf{x}} \\
\dot{\mathbf{y}}
\end{array}\right\}+\left[\begin{array}{cc}
\mathbf{K}_{x x} & \mathbf{0} \\
\mathbf{K}_{y x} & \mathbf{K}_{y y}
\end{array}\right]\left\{\begin{array}{l}
\mathbf{x} \\
\mathbf{y}
\end{array}\right\}=\left\{\begin{array}{c}
\mathbf{f}_{x} \\
\mathbf{f}_{y}
\end{array}\right\}
$$

describiendo una situación en la cual sólo el segundo campo depende del primero, se tiene el problema acoplado en una dirección (one way coupling). En último lugar, cuando los términos no diagonales en (6.1) son cero, cada campo es completamente independiente del otro y se tendría un problema desacoplado.

Asumiendo que todos los términos en la expresión (6.1) son explícitamente conocidos, el sistema de EDO se puede discretizar directamente en el tiempo dando lugar a la denominada formulación monolítica del problema acoplado. De este modo, el sistema acoplado de ecuaciones es tratado simultáneamente resolviéndose todas las variables al mismo tiempo. Aunque se consigue una alta precisión en la solución, el sistema que resulta generalmente es muy grande, lo cual supone un elevado coste computacional, y a menudo está mal condicionado debido a la coexistencia de términos que proceden de la descripción de problemas físicamente diferentes. Otra desventaja de este planteamiento es la pérdida de modularidad del software debido a la resolución simultánea de los dos campos.

Teniendo en cuenta estas circunstancias, a menudo resulta interesante introducir un esquema basado en la solución particionada del sistema de ecuaciones acopladas (6.1). El sistema se divide espacialmente en particiones (dominios) en función de consideraciones físicas o computacionales. La solución de cada campo progresa en el tiempo por separado y los efectos de interacción se justifican por la transmisión y la sincronización de las variables de estado acopladas.

Esta división puede ser algebraica (algebraic partitioning) o diferencial (differential partitioning). En la partición algebraica se discretiza espacialmente el sistema acoplado completo en primer lugar y después se divide, mientras que en la partición diferencial primero se realiza la división y posteriormente se discretiza por separado cada campo. La partición diferencial fue desarrollada originalmente para problemas de interacción fluido-estructura, en los cuales es fácil encontrar mallas no coincidentes. Se puede encontrar información más detallada sobre este planteamiento en el trabajo de Felippa et al. [5]. 
Este planteamiento da lugar a una redefinición del problema monolítico de la forma

$$
\left[\mathbf{H}^{1}\right]\left\{\mathbf{u}_{n+1}\right\}=\left\{\mathbf{g}_{n+1}\right\}-\left[\mathbf{H}^{2}\right]\left\{\mathbf{u}_{n+1}^{P}\right\} \quad ; \quad\left\{\mathbf{u}_{n+1}\right\}=\left\{\begin{array}{l}
\mathbf{x} \\
\mathbf{y}
\end{array}\right\}
$$

donde $\left[\mathbf{H}^{1}\right]+\left[\mathbf{H}^{2}\right]=\left[\mathbf{H}^{\text {monoltico }}\right]$ y $\left\{\mathbf{u}_{n+1}^{P}\right\}$ es una predicción.

$\mathrm{Si}\left[\mathbf{H}^{2}\right]=[\mathbf{0}]$ se tiene el sistema monolítico y el acoplamiento se resuelve implícitamente. Por otro lado, si $\left[\mathbf{H}^{2}\right]$ toma una forma del tipo

$$
\left[\mathbf{H}^{2}\right]=\left[\begin{array}{cc}
\mathbf{0} & \mathbf{H}_{12} \\
\mathbf{H}_{21} & \mathbf{0}
\end{array}\right] \rightarrow\left[\mathbf{H}^{1}\right]=\left[\begin{array}{cc}
\mathbf{H}_{11} & \mathbf{0} \\
\mathbf{0} & \mathbf{H}_{22}
\end{array}\right]
$$

el acoplamiento se resuelve de manera totalmente explícita.

La viabilidad de este planteamiento depende de las siguientes hipótesis:

- el sistema de ecuaciones debe ser lineal

- todos los coeficientes de la matriz han de ser conocidos

- posibilidad de definir un sistema test físicamente estable

En general, cuando se trata de problemas complejos de multifísica una o varias de estas hipótesis no se cumplen. En particular, para el problema de interacción entre estructuras flexibles y flujos de fluidos incompresibles (modelados usando las ecuaciones de Navier-Stokes), que es el caso de interés para nosotros, ninguna de las hipótesis anteriores puede ser aceptada.

Respecto a la hipótesis de linealidad del sistema para el fluido, simplemente no se sostiene debido a la estructura matemática del problema. Por otro lado, la segunda hipótesis es más delicada y requiere una explicación más detallada.

Dada la no linealidad del problema, los dos campos acoplados implican la definición de un sistema no lineal de ecuaciones de la forma

$$
\begin{aligned}
& f_{S}(x, y)=0 \\
& f_{F}(x, y)=0
\end{aligned}
$$

el cual puede ser linealizado usando el método de Newton-Raphson como

$$
\left[\begin{array}{cc}
\frac{\partial f_{S}}{\partial x} & \frac{\partial f_{S}}{\partial y} \\
\frac{\partial f_{F}}{\partial x} & \frac{\partial f_{F}}{\partial y}
\end{array}\right]\left\{\begin{array}{c}
d x \\
d y
\end{array}\right\}=\left\{\begin{array}{l}
-f_{S}\left(x^{k}, y^{k}\right) \\
-f_{F}\left(x^{k}, y^{k}\right)
\end{array}\right\}
$$


Los términos no diagonales de la matriz de rigidez tangente expresan la dependencia de un campo respecto de las variaciones del otro. Debido a la estructura matemática del problema fluido, cualquier perturbación sobre el contorno se propaga muy rápidamente (en el límite, de forma instantánea para fluidos realmente incompresibles) a todo el dominio fluido. El término $\partial f_{F} / \partial x$ no suele ser nulo y por lo tanto el cálculo explícito no resulta atractivo. Incluso si teóricamente fuese posible la linealización del campo fluido, por lo general no se realiza ya que suele ser preferible utilizar algún método de iteración del punto fijo para resolver las no linealidades. El cálculo del jacobiano en (6.6) implica una completa redefinición de la estrategia adoptada para el fluido, lo cual supone un importante inconveniente desde el punto de vista de la modularidad del software.

Respecto a la última hipótesis señalada, en general resulta difícil encontrar un sistema test adecuado, ya que la condición relativa a la estabilidad física no suele ser fácil de satisfacer en la práctica.

Para hacer frente a estas cuestiones existen dos planteamientos fundamentalmente. Uno de los planteamientos es puramente algebraico y está basado en técnicas iterativas de resolución del sistema (6.5) acercándose tanto como sea posible al comportamiento óptimo del sistema de Newton-Raphson (6.6), mientras que el otro se centra en técnicas de acoplamiento explícitas donde la convergencia a la solución acoplada se obtiene por ajuste de los valores estimados (predictors) y de los datos transferidos entre los dominios.

El primer planteamiento tiene su expresión más fácil en el método iterativo de Jacobi por bloques (block jacobi method) que consiste en la solución iterativa del sistema

$$
\begin{aligned}
& f_{S}\left(x^{k+1}, y^{k}\right)=0 \rightarrow y^{k+1} \\
& f_{F}\left(x_{\omega}^{k+1}, y^{k+1}\right)=0 \rightarrow x^{k+1}
\end{aligned}
$$

que puede ser modificado para incluir un paso de relajación del tipo $x_{\omega}^{k+1}=\omega x^{k+1}+(1-\omega) x^{k}$. Aunque esta técnica permite obtener resultados muy buenos en muchos casos (en otros casos los resultados pueden ser totalmente ineficaces), en general, presenta una convergencia muy lenta a la solución acoplada.

Otros planteamientos se basan en los métodos cuasi-Newton. Estos métodos reemplazan la matriz jacobiana en el método de Newton por una matriz de aproximación que se actualiza en cada iteración. Su desventaja radica en que se pierde la convergencia cuadrática de Newton al ser sustituida por una convergencia denominada superlineal. Destaca particularmente el trabajo de Wall y Mok [6] en el cual se propone la utilización del acelerador de Aitken para mejorar la convergencia del proceso iterativo. 
En términos generales, estas técnicas son ideales desde el punto de vista de la modularidad del software y, naturalmente, tienden a la solución de la formulación monolítica, de la cual toman las propiedades de estabilidad. Sin embargo, suelen presentar un gran coste computacional debido a la necesidad de resolver el sistema implícito con un alto grado de precisión.

Por otro lado, el segundo planteamiento se basa en un diseño meticuloso de la fase de predicción y de corrección para minimizar la energía no real que se introduce en el sistema. Farhat y Piperno llevaron a cabo trabajos pioneros sobre este tema y desarrollaron estrategias de resolución explícitas para aplicaciones aeronáuticas [1,2].

Dada la ausencia de una formulación matemática común para los dos dominios acoplados, el estudio de las propiedades de estabilidad de los diferentes métodos numéricos supone, en este caso, un importante reto. En esta línea, Piperno et al. [1] estudiaron un caso 1D para flujo compresible, mientras que en el trabajo de Piperno y Farhat [2] se planteaba un test simple para evaluar la tendencia de un método dado a la inestabilidad.

Farhat et al. [7] aplicaron las técnicas descritas a estructuras aeronáuticas obteniendo resultados satisfactorios. Por otro lado, ejemplos de aplicación de estas técnicas de resolución a estructuras flexibles en el campo de la ingeniería civil se pueden encontrar en los trabajos de Halfmann et al. [8] y Rank et al. [9].

\subsection{Esquema de acoplamiento}

En el desarrollo de un algoritmo de acoplamiento resulta muy importante la elección de la posición en el tiempo en la cual se desean plantear y resolver las ecuaciones de movimiento de la estructura y del fluido, es decir, la elección del dominio de integración. Cabe destacar que para preservar la coherencia con la formulación variacional subyacente, el dominio no debería ser modificado durante la ejecución del método de paso fraccionado, aunque pueda ser movido fuera del bloque de resolución del fluido.

El diseño del esquema de acoplamiento tiene en cuenta una serie de consideraciones:

- la solución estructural tiene menor coste computacional que la solución del fluido

- el esquema de integración en el tiempo asociado al problema estructural es al menos de segundo orden de precisión y por consiguiente mucho más preciso que el solver del fluido 
- se pueden seleccionar diferentes métodos de integración en el tiempo para el problema estructural

- generalmente el movimiento de la malla supone un coste computacional alto

- el usuario debe tener la posibilidad de elegir entre diferentes esquemas de actualización de la malla

La conclusión inmediata es que en el diseño de un esquema de acoplamiento las características particulares del esquema estructural y del algoritmo de movimiento de la malla no se deberían tener en cuenta. Por otro lado, el alto coste computacional del movimiento de la malla comparado con la resolución estructural sugiere minimizar los pasos de actualización de la malla y dedicar el tiempo a la resolución estructural en todos aquellos casos en los cuales se considere que esto puede mejorar la solución.

El solver del fluido usado en el presente trabajo está basado en el algoritmo Characteristic Based Split (CBS), el cual está descrito en los trabajos [10, 11, 12, 13, 14]. Su esquema básico, excluyendo todos los términos de estabilización [13, 15], adopta la forma

- paso 1

$$
\mathbf{M} \tilde{\mathbf{V}}_{n+1}=\mathbf{M V} \mathbf{V}_{n}-\delta t \mathbf{K}\left(\mathbf{C}_{n}\right) \mathbf{V}_{n}
$$

- paso 2

$$
\mathbf{L P}_{n+1}=-\frac{1}{\delta t} \mathbf{G}^{T} \tilde{\mathbf{V}}_{n+1}
$$

- $\quad$ paso 3

$$
\mathbf{M} \mathbf{V}_{n+1}=\mathbf{M} \tilde{\mathbf{V}}_{n+1}-\delta t \mathbf{G P}_{n+1}
$$

donde $\mathbf{C}=\mathbf{V}-\mathbf{V}_{\text {malla }}$. En el trabajo de Zienkiewicz y Taylor [16] se describe con sumo detalle el fundamento teórico del algoritmo, poniéndose de manifiesto claramente las ventajas de los métodos de paso fraccionado.

De manera muy simplificada, el algoritmo CBS se basa en el cálculo de una solución del campo de velocidad para presión constante en una primera etapa seguida posteriormente de una fase de corrección de los campos de velocidad y presión. En términos generales, este mismo planteamiento puede aplicarse al método de acoplamiento. Por lo tanto, se plantea un esquema de acoplamiento basado en los siguientes pasos: 
1. solución del problema estructural utilizando el método de integración en el tiempo escogido y una estimación inicial de la presión de la forma $\mathbf{P}_{n+1}^{\text {estimado }}=\mathbf{P}_{n+1}^{*}=\mathbf{P}_{n}$. En símbolos, $\mathbf{X}_{n+1_{S}}^{*}=\mathbf{X}_{n+1_{S}}^{*}\left(\mathbf{P}_{n+1}^{*}\right)$

2. mover fluido de manera acorde al movimiento de estructura $\rightarrow \mathbf{X}_{n+1}^{*}=\mathbf{X}_{n+1}^{*}\left(\mathbf{P}_{n+1}^{*}\right)$

3. paso $\mathrm{A} \rightarrow \tilde{\mathbf{V}}_{n+1}$

4. paso B $\rightarrow \mathbf{P}_{n+1}$, imponer las condiciones de contorno de la presión

5. “corregir" la solución estructural usando la presión final $\mathbf{X}_{n+1_{S}}=\mathbf{X}_{n+1_{S}}\left(\mathbf{P}_{n+1}\right)$

6. paso $\mathbf{C} \rightarrow \mathbf{V}_{n+1}$, imponer las condiciones de contorno de la velocidad

Por lo tanto, todas las ecuaciones del fluido se resuelven en la configuración del dominio "al final del paso". Cabe señalar el paralelismo entre este esquema y la división en etapas de la solución del fluido. En particular, el paso de predicción de la solución estructural aporta la configuración deformada a la que tiende el sistema estructural debido a la acción de la presión del fluido, que es el equivalente Lagrangiano (para un sólido) del primer paso de la solución del fluido.

Si no se realiza ninguna iteración, el esquema de acoplamiento es explícito y no se comprueba la convergencia. La diferencia entre el desplazamiento de la estructura predicho (paso 1) y el corregido introduce un error que se refleja en la determinación del campo de presiones, y por consiguiente en la posición "corregida" de la estructura. Este error se puede reducir iterando en el procedimiento descrito, sin embargo, tales iteraciones no resultan atractivas en la medida en que se elige un solver de fluido "explícito" (cuasi-explícito).

A continuación se valorarán las propiedades del esquema de acoplamiento propuesto. Para ello se compara la solución aproximada que proporciona el método con la solución "ideal" que se podría encontrar iterando los pasos descritos (asumiendo que tales iteraciones convergen). El análisis que se realiza es local en el tiempo, de forma que se puede asumir que tanto la solución acoplada "exacta" como la "calculada" comparten las mismas condiciones $\mathbf{X}_{n}, \mathbf{V}_{n}, \mathbf{P}_{n}$ al principio del paso de tiempo y la misma malla computacional inicial.

Para ello es necesario tener en cuenta la forma de los diferentes operadores implicados. En el trabajo de Park y Felippa [3] o de Zienkiewicz y Codina [10] se puede encontrar una información más detallada sobre la formulación del fluido. Para el caso de triángulos y tetraedros con integración sobre un sólo punto de Gauss, los diferentes términos se pueden describir como

$$
\mathbf{M}=\phi j
$$


donde $\phi$ es una matriz de factores agrupados (lumping factors) y $j:=\operatorname{det}(\mathbf{J})=\operatorname{det}\left(\frac{\partial x}{\partial X}\right)$

$$
\begin{gathered}
\mathbf{K}\left(\mathbf{c}^{n}\right)_{I i J j}=\mathbf{K}_{1}+\mathbf{K}_{2}+\mathbf{K}_{3} \\
\left(\mathbf{K}_{1}\right)_{I i J j}=N_{I}\left(\mathbf{C}_{n} \cdot \nabla_{\mathbf{x}}^{\mathbf{J}}\right) j \delta_{i j} \\
\left(\mathbf{K}_{2}\right)_{I i J j}=\left(\operatorname{Tr}\left(\nabla_{\mathbf{x}} \mathbf{C}_{n}\right)\right) N_{I} N_{J} j \delta_{i j} \\
\left(\mathbf{K}_{3}\right)_{I i J j}=\nu \nabla_{\mathbf{x}}^{\mathbf{I}} \cdot \nabla_{\mathbf{x}}^{\mathbf{J}} j \delta_{i j}
\end{gathered}
$$

donde se introduce el gradiente

$$
\left\{\nabla_{\mathbf{x}}^{\mathbf{a}}\right\}=\left\{\begin{array}{c}
\frac{\partial N_{a}}{\partial x_{1}} \\
\frac{\partial N_{a}}{\partial x_{2}} \\
\frac{\partial N_{a}}{\partial x_{3}}
\end{array}\right\}
$$

y finalmente

$$
\mathbf{G}_{I i J}=-\frac{\partial N_{I}}{\partial x_{i}} N_{J}
$$

\section{CASO "IDEAL"}

Se considerará como referencia la solución en la cual todas las condiciones de acoplamiento ajustan de forma coherente, es decir, la configuración del dominio que asegura el equilibrio de fuerzas y la compatibilidad de desplazamientos en la interfaz entre los diferentes dominios. Una cuestión importante es que más que referirnos a la solución "exacta", se considerará la solución que se obtendría después de un número infinito de iteraciones entre los dominios (bajo la hipótesis de que tal proceso iterativo converge). De esta manera, la solución "ideal” estará libre de cualquier error asociado al acoplamiento, pero se verá afectada por todas las fuentes de error propias de los solvers de cada campo.

Bajo esta hipótesis, la configuración de la estructura será de la forma $\mathbf{X}_{n+1}\left(\mathbf{V}_{n+1}, \mathbf{P}_{n+1}\right)$ y la del dominio del fluido será $\Omega=\Omega\left(\mathbf{X}\left(\mathbf{V}_{n+1}, \mathbf{P}_{n+1}\right)\right)$. Substituyendo la posición "exacta" de la estructura $\mathbf{X}_{n+1}\left(\mathbf{P}_{n+1}\right)$ en el esquema descrito anteriormente se obtiene 
- $\operatorname{paso} \mathrm{A}$

$$
\mathbf{M} \tilde{\mathbf{V}}_{n+1}=\mathbf{M} \mathbf{V}_{n}-\delta t \mathbf{K}\left(\mathbf{C}_{n}\right) \mathbf{V}_{n}
$$

- $\operatorname{paso} B$

$$
\mathbf{L} \mathbf{P}_{n+1}=-\frac{1}{\delta t} \mathbf{G}^{T} \tilde{\mathbf{V}}_{n+1}
$$

- $\operatorname{paso} \mathrm{C}$

$$
\mathbf{M V} \mathbf{V}_{n+1}=\mathbf{M} \tilde{\mathbf{V}}_{n+1}-\delta t \mathbf{G P}_{n+1}
$$

donde todas las matrices están definidas sobre el dominio $\Omega_{n+1}$, el cual representa la configuración “ideal” que el dominio adopta debido a la interacción.

\section{CASO "REAL"}

En el caso real la configuración final se desconoce a priori y se calcula usando un campo de presiones $\mathbf{P}_{n+1}^{*}$ estimado de la distribución de presiones final, la cual es desconocida. Esto conduce a una solución estructural $\mathbf{X}_{n+1 S}^{*}\left(\mathbf{P}_{n+1}^{*}\right)$ estimada y a una deformación posterior del dominio del fluido $\mathbf{X}_{n+1}^{*}\left(\mathbf{P}_{n+1}^{*}\right)$. El dominio de integración en el tiempo $t_{n+1}$, denominado $\Omega_{n+1}^{p}$, no coincide con el "ideal", por lo tanto, al hacer el análisis todos los términos calculados sobre esta malla se denotarán usando el superíndice ${ }^{p}$. De forma análoga al caso anterior, se obtienen las ecuaciones

- $\operatorname{paso} \mathrm{A}$

$$
\mathbf{M}^{p} \tilde{\mathbf{V}}_{n+1}^{p}=\mathbf{M}^{p} \mathbf{V}_{n}-\delta t \mathbf{K}^{p}\left(\mathbf{C}_{n}\right) \mathbf{V}_{n}
$$

- $\operatorname{paso} B$

$$
\mathbf{L}^{p} \mathbf{P}_{n+1}^{p}=-\frac{1}{\delta t} \mathbf{G}^{T p} \tilde{\mathbf{V}}_{n+1}^{p}
$$

- $\operatorname{paso} \mathrm{C}$

$$
\mathbf{M}^{p} \mathrm{~V}_{n+1}^{p}=\mathbf{M}^{p} \tilde{\mathbf{V}}_{n+1}^{p}-\delta t \mathbf{G}^{p} \mathbf{P}_{n+1}^{p}
$$

\section{Evaluación del error de acoplamiento}

El estudio del error de acoplamiento se puede realizar evaluando la diferencia entre la solución acoplada aproximada y la exacta. Haciendo la diferencia entre las ecuaciones (6.17) y (6.20) se obtiene

$$
\mathbf{M} \tilde{\mathbf{V}}_{n+1}^{e r r}=-\delta t \mathbf{K}^{e r r}\left(\mathbf{C}_{n}\right) \mathbf{V}_{n}-\mathbf{M}^{e r r}\left(\mathbf{V}_{n+1}^{p}-\mathbf{V}_{n}\right)
$$


donde se han introducido los símbolos $a^{e r r}=a-a^{p}$ tanto para matrices como para vectores, con el fin de indicar la diferencia entre un término calculado sobre la configuración de malla exacta y ese mismo término pero calculado sobre la configuración aproximada.

La ecuación (6.23) muestra claramente la dependencia del error de acoplamiento respecto del error que se comete en la determinación de las matrices. A continuación se mostrará la relación entre este error y el error cometido en la predicción de la solución estructural. Cuando la predicción se realiza avanzando en el tiempo y manteniendo la presión constante, como sucede en nuestro caso, se puede verificar fácilmente que (asumiendo al menos precisión de segundo de orden para el solver estructural)

$$
\begin{gathered}
\mathbf{X}_{n+1}^{p}-\mathbf{X}_{n+1}=O\left(\delta t^{2}\left(\mathbf{P}_{n+1}-\mathbf{P}_{n}\right)\right) \\
\mathbf{V}_{n+1}^{p}-\mathbf{V}_{n+1}=O\left(\delta t\left(\mathbf{P}_{n+1}-\mathbf{P}_{n}\right)\right)
\end{gathered}
$$

lo cual muestra que la predicción tiene una precisión de tercer orden.

El jacobiano de las coordenadas es de la forma

$$
\mathbf{J}_{i j}=\frac{\partial x_{I}}{\partial \xi_{j}}=\mathbf{x}_{I i} \frac{\partial N_{I}}{\partial \xi_{j}} \rightarrow\left(\mathbf{J}_{i j}-\mathbf{J}_{i j}^{p}\right)=\left(\mathbf{x}_{I i}-\mathbf{x}_{I i}^{p}\right) \frac{\partial N_{I}}{\partial \xi_{j}}
$$

Teniendo en cuenta que cada componente de la inversa de un tensor depende de todas las componentes del tensor original

$$
\mathbf{J}_{i j}^{-1}=\mathbf{J}_{i j}^{-1}\left(J_{11}, J_{12}, \ldots, J_{k k}\right)
$$

y utilizando del desarrollo en serie de Taylor, se puede concluir de forma inmediata que

$$
\mathbf{J}_{i j}^{*-1}-\mathbf{J}_{i j}^{-1}=\frac{\partial J^{-1}}{\partial J_{i j}} d J_{i j}
$$

Este resultado permite deducir que $\mathbf{J}_{i j}^{-1}-\mathbf{J}_{i j}^{p-1}=O\left(\delta t^{3}\right)$, así como también los resultados análogos $j-j^{p}=O\left(\delta t^{3}\right), \mathrm{y} \mathbf{J}^{-1} j-\mathbf{J}^{p-1} j^{p}=O\left(\delta t^{3}\right)$. Estos resultados son muy importantes para el análisis que se realizará posteriormente ya que permitirán asignar un valor al orden del error introducido por los diferentes términos.

Substituyendo en (6.10) se obtiene

$$
\mathbf{M}^{\text {err }}=\phi\left(j-j^{p}\right)=O\left(\delta t^{3}\right)
$$


Por otro lado, introduciendo el vector

$$
\left\{\nabla_{\xi}^{\mathbf{a}}\right\}=\left\{\begin{array}{c}
\frac{\partial N_{a}}{\partial \xi_{1}} \\
\frac{\partial N_{a}}{\partial \xi_{2}} \\
\frac{\partial N_{a}}{\partial \xi_{3}}
\end{array}\right\}
$$

es fácil verificar que

$$
\left\{\nabla_{\mathbf{x}}^{\mathbf{a}}\right\}=\left[\mathbf{J}^{-1}\right]\left\{\nabla_{\xi}^{\mathbf{a}}\right\}
$$

lo que permite encontrar la relación

$$
\nabla_{\mathbf{x}}^{\mathbf{I}} j-\nabla_{\mathbf{x}}^{\mathbf{I} p} j^{p}=\left(j \mathbf{J}^{-1}-j^{p} \mathbf{J}^{p-1}\right) \nabla_{\xi}^{\mathbf{I}}=O\left(\delta t^{3}\right)
$$

y a su vez proporcionar una estimación del error para los términos de la matriz $\mathbf{K}$

$$
\begin{gathered}
\left(\mathbf{K}_{1}\right)_{I i J j}^{e r r}=N_{I}\left(\mathbf{C}_{n} \cdot\left(\nabla_{\mathbf{x}}^{\mathbf{J}} j-\nabla_{\mathbf{x}}^{\mathbf{J} p} j^{p}\right)\right) \delta_{i j}=O\left(\delta t^{3}\right) \\
\left(\mathbf{K}_{2}\right)_{I i J j}^{e r r}=\left(\operatorname{Tr}\left(j J^{-1} \frac{\partial \mathbf{C}_{n}}{\partial \xi}-j^{p} J^{p-1} \frac{\partial \mathbf{C}_{n}}{\partial \xi}\right)\right) N_{I} N_{J} \delta_{i j}=O\left(\delta t^{3}\right) \\
\left(\mathbf{K}_{3}\right)_{I i J j}^{e r r}=\nu\left(\nabla_{\mathbf{x}}^{\mathbf{I}} \cdot \nabla_{\mathbf{x}}^{\mathbf{J}} j-\nabla_{\mathbf{x}}^{\mathbf{I} p} \cdot \nabla_{\mathbf{x}}^{\mathbf{J} p} j^{p}\right) \delta_{i j}= \\
=\nu\left(\nabla_{\mathbf{x}}^{\mathbf{I}}-\nabla_{\mathbf{x}}^{\mathbf{I} p}\right) \cdot \nabla_{\mathbf{x}}^{\mathbf{J}} j \delta_{i j}+\nu \nabla_{\mathbf{x}}^{\mathbf{I} p} \cdot\left(\nabla_{\mathbf{x}}^{\mathbf{J}} j-\nabla_{\mathbf{x}}^{\mathbf{J} p} j^{p}\right) \delta_{i j}=O\left(\delta t^{3}\right)
\end{gathered}
$$

De forma análoga se puede demostrar que la misma estimación es válida para $\mathbf{L}^{e r r}$ y $\mathbf{G}^{e r r}$.

Si bien esta estimación se cumple a nivel de elemento, las matrices globales se obtienen ensamblando las diferentes contribuciones elementales sin que se vea alterado el orden del error. Por lo tanto, substituyendo en (6.23) se puede comprobar que

$$
\mathbf{M} \tilde{\mathbf{V}}_{n+1}^{e r r}=\delta t O\left(\delta t^{3}\right)+O\left(\delta t^{3}\right) O(\delta t)=O\left(\delta t^{4}\right)
$$

lo cual indica que el error en $\tilde{\mathbf{V}}_{n+1}$ debido a la aproximación en la estimación es de cuarto orden en el tiempo.

Haciendo la diferencia entre las expresiones (6.18) y (6.21) se obtiene la expresión

$$
\mathbf{L} \mathbf{P}_{n+1}^{e r r}=-\frac{1}{\delta t} \mathbf{G}^{T e r r} \tilde{\mathbf{V}}_{n+1}^{e r r}-\mathbf{L}^{e r r} \mathbf{P}_{n+1}^{p}=\frac{O\left(\delta t^{3}\right)}{\delta t}+O\left(\delta t^{3}\right)=O\left(\delta t^{2}\right)
$$

la cual nos muestra que el error en la presión es de segundo orden en el tiempo. 
Finalmente, considerando (6.19) y (6.22) se llega a la expresión

$$
\begin{gathered}
\mathbf{M V}_{n+1}^{e r r}=-\delta t \mathbf{G} \mathbf{P}_{n+1}^{e r r}-\delta t \mathbf{G}^{e r r} \mathbf{P}_{n+1}^{p}+\mathbf{M} \tilde{\mathbf{V}}_{n+1}^{e r r}-\mathbf{M}^{e r r}\left(\mathbf{V}_{n+1}^{p}-\tilde{\mathbf{V}}_{n+1}^{p}\right)= \\
=O\left(\delta t^{3}\right)+O\left(\delta t^{4}\right)+O\left(\delta t^{4}\right)+O\left(\delta t^{4}\right)
\end{gathered}
$$

que da una estimación del error en la velocidad al final del paso.

Surge una cuestión relativa a las condiciones de contorno en velocidad utilizadas. Si la velocidad estructural predicha se impone en el contorno acoplado, el error estará gobernado por el error en el valor predicho de la velocidad estructural, que es de segundo orden en el tiempo. Esta situación se puede mejorar (al menos cuando el comportamiento estructural está acoplado sólo por medio de la presión) siguiendo el método de paso fraccionado descrito e imponiendo el valor corregido de la velocidad sobre el contorno acoplado.

En cualquier caso, el término de fuerzas calculado que actúa sobre la estructura difiere a lo sumo en un término de segundo orden respecto del "exacto", con lo que la posición final de la estructura diferirá a lo sumo en un término de cuarto orden respecto de la que se podría obtener utilizando un esquema de iteración.

Sin embargo, cabe destacar que el orden de precisión del esquema de acoplamiento en su conjunto viene determinado por el orden de su parte "más débil". El método de paso fraccionado es sólo de primer orden de precisión, con lo que el esquema de acoplamiento en su conjunto es a lo sumo de primer orden.

\subsection{Análisis del esquema de acoplamiento}

Hasta ahora se ha probado que el método de paso fraccionado acoplado propuesto converge a la solución del problema acoplado, y que al hacerlo, el error es de segundo orden en el tiempo y la precisión del solver del fluido no se ve afectada. Este resultado es válido para cualquier esquema de resolución estructural con precisión de segundo orden, pero sólo para el caso particular de la estrategia de resolución del fluido adoptada.

Ahora es necesario asegurar que todo el procedimiento es estable, en otras palabras, que los errores introducidos por la aproximación no se adicionan en el tiempo y estropean la solución. Para ello se estudiarán las propiedades del método evaluando su comportamiento en modelos simples.

Lamentablemente no se cuenta con un sistema de ecuaciones estándar para el problema de interés, de forma que se procederá según lo propuesto por Piperno y Farhat $[1,2]$ y se evaluará el 
comportamiento del esquema de acoplamiento en un caso especial (físicamente relevante). Después se elegirá una ecuación tipo (haciendo uso de consideraciones análogas) y se estudiará la estabilidad y la precisión utilizando un planteamiento clásico.

\subsubsection{Comprobación de la estabilidad}

Es sabido que los problemas de interacción fluido-estructura (FSI) pueden mostrar un comportamiento inestable con soluciones divergentes, lo cual puede constituir un problema para los métodos estándar de investigación. Se puede comprobar que aunque cada campo presente oscilaciones importantes, la energía total del sistema acoplado necesariamente se conserva (bajo algunas condiciones). Esto implica que la energía perdida por uno de los campos se transfiere al otro a través del contorno común [1]. En el trabajo de Farhat y Piperno [2] se muestra que en el diseño de un procedimiento alternado (staggered procedure), en el cual las ecuaciones del fluido y las de la estructura se integran alternativamente en el tiempo, un aspecto muy importante está relacionado con la descripción de este intercambio de energía.

La idea es que el error que surge de forma natural debido al procedimiento alternado se refleja en una diferencia entre la energía transmitida desde uno de los campos y la absorbida por el otro. Si estos errores en la energía se suman en el tiempo, la estrategia alternada seleccionada tiene una fuerte tendencia a la inestabilidad, por otro lado, si el error se mantiene pequeño en el tiempo, el procedimiento alternado puede ser considerado como estable.

La intuición sugiere que la solución acoplada tendrá una forma sinusoidal con el fluido actuando como un término de fuerza con una frecuencia similar a la de la estructura y con un cierto desfase. En línea con esta idea Farhat y Piperno [2] propusieron un problema test para evaluar la tendencia de una estrategia dada a la inestabilidad. En definitiva, se trata de evaluar el comportamiento del método ante la resolución de la ecuación escalar

$$
\ddot{x}+\omega^{2} x=\frac{p_{0}}{m} \sin (\omega t+\phi)
$$

la cual representa un sistema lineal de masa $m$ y frecuencia natural $\omega$. Por lo tanto, la idea es que procedimientos alternados que no funcionen de forma correcta para este supuesto, es decir, que tiendan a ser inestables, probablemente no se comporten de manera satisfactoria bajo condiciones más generales.

En el trabajo de referencia, la evaluación de la estabilidad del esquema de acoplamiento se lleva a cabo teniendo en cuenta la elección particular de los métodos de integración del fluido y de la 
estructura y asumiendo que la solución estructural es de la forma $u_{0}=\sin (\omega t)$. Esto permite concluir que algunas opciones son preferibles a otras respecto a las fórmulas utilizadas para la predicción (predictor) y el avance en el tiempo (time advancement formula).

En este trabajo no se hace ninguna suposición respecto al método de integración en el tiempo utilizado. Por lo tanto, se considera que la presión varia de forma sinusoidal $u_{0}=\sin (\omega t+\phi)$ y se asume que el desplazamiento de la estructura resuelve exactamente la correspondiente ecuación diferencial (lo cual es coherente con la hipótesis de exactitud de los solvers de cada campo) Aunque esto es ligeramente diferente de lo que se hace en el artículo de referencia, en el cual el movimiento de la estructura está prescrito y la presión se calcula consecuentemente, el planteamiento propuesto en esta trabajo es muy similar al del artículo.

En nuestro método, la malla del fluido se mueve de acuerdo a la estimación estructural mientras que la estructura está sujeta a una fase de corrección. Los dos campos comparten la presión, lo que significa que la solución del fluido en el contorno avanza en el tiempo como

$$
p(t), x^{*}(t) \rightarrow p(t+h), x^{*}(t+h)
$$

y la de la estructura como

$$
p(t), x(t) \rightarrow p(t+h), x(t+h)
$$

donde $h$ representa el tamaño de paso usado.

El valor predicho del desplazamiento estructural viene dado por la solución de la ecuación diferencial

$$
\ddot{\mathbf{x}}_{\text {aux }}(t)+\omega^{2} x_{\text {aux }}(t)=\frac{p_{\text {estimado }}}{m}
$$

para las condiciones iniciales

$$
\dot{x}_{\text {aux }}(0)=v_{\text {in }} \quad ; \quad x_{\text {aux }}(0)=x_{\text {in }}
$$

con lo que el valor del desplazamiento predicho de la estructura (sobre el cual se mueve la malla del fluido) se puede expresar como

$$
x_{1}^{*}=x^{*}(t)=x_{\text {aux }}(h)
$$

con $v_{i n}=\dot{x}(t-h) ; x_{i n}=x(t-h) ; p_{\text {estimado }}=p_{0} \sin (\omega(t-h)+\phi)$ para el valor al principio del paso, $\mathrm{y}$

$$
x_{2}^{*}=x^{*}(t+h)=x_{\text {aux }}(t+h)
$$

con $v_{\text {in }}=\dot{x}(t) ; x_{\text {in }}=x(t) ; p_{\text {estimado }}=p_{0} \sin (\omega(t)+\phi)$ para el valor al final del paso. 
Se puede calcular el trabajo en el lado del fluido como

$$
\mathrm{W}_{\text {fluido }}(t, h)=\frac{x_{2}^{*}-x_{1}^{*}}{h} \int_{t}^{t+h} p_{0} \sin (\omega y+\phi) d y
$$

y el trabajo en el lado de la estructura como

$$
\mathrm{W}_{\text {estructura }}(t, h)=\frac{x(t+h)-x(t)}{h} \int_{t}^{t+h} p_{0} \sin (\omega y+\phi) d y
$$

donde se usa la misma regla para el cálculo del trabajo con objeto de asegurar que la diferencia es cero cuando el valor predicho y corregido coinciden para la estructura.

Se define una función para describir su diferencia de la siguiente manera

$$
\mathbf{W}_{\text {dif }}(t, h)=\mathbf{W}_{\text {fluido }}(t, h)-\mathbf{W}_{\text {estructura }}(t, h)
$$

Esta función permite obtener toda la informaciones que se necesita y en particular

1. el orden de la "aproximación local" para un tiempo dado como una función del tamaño de paso

2. el orden de la diferencia del trabajo "total", el cual se obtiene sumando todas las contribuciones locales

$$
t=k h \quad ; \quad \mathbf{W}_{d i f}^{T O T}=\sum_{k=0}^{n} \mathbf{W}_{d i f}(k h, h)
$$

Estos resultados se pueden obtener una vez se conocen las expresiones de $\mathbf{W}_{d i f}^{T O T}$ y $\mathbf{W}_{d i f}(t, h)$ por medio del desarrollo en serie de Taylor [17]

- el error local para cualquier tiempo $t$ es $O\left(h^{4}\right)$ y tiene la forma

$$
\mathbf{W}_{d i f}(t, h)=-\frac{p_{0}^{2}}{6 m} \sin (\phi)^{2} \omega^{2} h^{4}+O\left(h^{5}\right)
$$

- el error total (después del desarrollo en serie de Taylor) tiene la forma

$$
\mathbf{W}_{\text {dif }}^{T O T}(t, h)=\sum_{k=0}^{n} \mathbf{W}_{\text {diff }}(k h, h)=-\frac{p_{0}^{2}}{6 m} \sin (\phi)^{2} \omega^{2}(n+1) h^{4}+O\left(h^{5}\right)
$$

Se observa que el error total es de orden cuarto. Aplicando el mismo procedimiento al caso básico alternado se obtendría una dependencia del error respecto de $h^{2}$. Notar que el problema test propuesto no debe ser considerado como una prueba de estabilidad, no obstante su capacidad para 
distinguir correctamente entre procedimientos alternados fiables y poco fiables se puso de manifiesto en el trabajo de Farhat y Piperno [2].

Cabe destacar que también se obtienen resultados muy similares cuando la frecuencia de la carga es diferente a la frecuencia natural de la estructura. Resulta muy fácil verificar, introduciendo el parámetro $\beta=\Omega_{\text {carga }} / \omega_{\text {estructura }}$, que

$$
\begin{gathered}
\mathbf{W}_{\text {dif }}(t, h)=-\frac{p_{0}^{2}}{6 m} \sin (\phi)^{2} \omega^{2} \beta^{2} h^{4}+O\left(h^{5}\right) \\
\mathbf{W}_{\text {dif }}^{T O T}(n)=\sum_{k=0}^{n} \mathbf{W}_{d i f}(k h, h)=-\frac{p_{0}^{2}}{6 m} \sin (\phi)^{2} \omega^{2} \beta^{2}(n+1) h^{4}+O\left(h^{5}\right)
\end{gathered}
$$

Se comprueba que el error está gobernado exclusivamente por la frecuencia de la carga y no por la frecuencia de la estructura.

\subsubsection{Algunos comentarios relativos a la estabilidad y a la precisión}

La estabilidad del esquema de acoplamiento se puede estudiar utilizando un planteamiento más tradicional una vez que se ha elegido un sistema de ecuaciones adecuado. La elección de tal sistema lamentablemente no es unívoca ya que el comportamiento del fluido es, en general, sumamente complejo. En la práctica, se suele eludir la solución del fluido y la interacción entre la estructura y el fluido se describe a través del intercambio de una fuerza de presión entre el fluido y la estructura. Tal fuerza de presión suele ser modelada expresando el campo de presiones del fluido como

$$
p=A \dot{x}+B x
$$

donde los coeficientes $A$ y $B$ generalmente dependen de la frecuencia del movimiento y suelen ser de origen experimental.

De esta forma, una carga puede ser considerada en el dominio de frecuencia, pero debe ser transformada para aplicaciones en el dominio del tiempo. La utilización de estos datos para el análisis en el dominio del tiempo es un tema de investigación [18] y no será tratado aquí. Sin embargo, el interés de este resultado radica en que esta transformación da lugar a la definición de un término dependiente de la aceleración, denominado masa aeroelástica (aeroelastic mass). Este término se hace más importante en la medida en que la densidad del fluido se aproxima a la de la estructura. Sin embargo, para los casos de interés en ingeniería civil a menudo es posible asumir que la masa aeroelástica es pequeña o incluso despreciable en comparación con la masa estructural. 
Es sabido que una elección cuidadosa de las constantes aeroelásticas puede conducir a una excelente descripción de las características más importantes de la acción del fluido sobre la estructura. Por lo tanto, para una velocidad y desplazamiento estructural dados, un buen modelo del fluido debería producir sobre la estructura una fuerza que pudiera ser aproximada con una ecuación como la descrita de manera muy precisa. Esto sugiere la posibilidad de estudiar la estabilidad a través del sistema de ecuaciones tipo

$$
\begin{gathered}
\mathbf{M} \ddot{\mathbf{x}}+\mathbf{C} \dot{\mathbf{x}}+\mathbf{K} \mathbf{x}=\mathbf{p} \\
\mathbf{M}_{a e} \ddot{\mathbf{x}}+\mathbf{C}_{a e} \dot{\mathbf{x}}+\mathbf{K}_{a e} \mathbf{x}=\mathbf{p}
\end{gathered}
$$

El fundamento teórico utilizado para estudiar las propiedades de los diferentes algoritmos de acoplamiento se puede exponer de forma bastante simple: los procedimientos que "se comportan bien" para un sistema test de la forma (6.55), con parámetros $\mathbf{K}_{a e} \mathbf{C}_{a e}$ ( $\mathbf{y} \mathbf{M}_{a e}$ si no fuese despreciable) físicamente adecuados, son idóneos para describir correctamente el efecto del fluido sobre la estructura. En otras palabras, es de esperar que procedimientos aproximados que resuelvan exactamente el sistema test muestren un comportamiento satisfactorio en la simulación de problemas de interacción fluido-estructura para todos los casos en los que se considera como válido un modelo de la forma $\mathbf{M}_{a e} \ddot{\mathbf{x}}+\mathbf{C}_{a e} \dot{\mathbf{x}}+\mathbf{K}_{a e} \mathbf{x}$ para objetivos de diseño.

La solución exacta del sistema (6.55) es

$$
\mathbf{M} \ddot{\mathbf{x}}_{n+1}+\mathbf{C} \dot{\mathbf{x}}_{n+1}+\mathbf{K} \mathbf{x}_{n+1}=\mathbf{M}_{a e} \ddot{\mathbf{x}}_{n+1}+\mathbf{C}_{a e} \dot{\mathbf{x}}_{n+1}+\mathbf{K}_{a e} \mathbf{x}_{n+1}
$$

la cual describe, en forma diferencial, el comportamiento del problema acoplado.

De otra parte, la aplicación del procedimiento alternado al mismo sistema conduce a la ecuación diferencial

$$
\mathbf{M} \ddot{\mathbf{x}}_{n+1}+\mathbf{C} \dot{\mathbf{x}}_{n+1}+\mathbf{K} \mathbf{x}_{n+1}=\mathbf{M}_{a e} \ddot{\mathbf{x}}_{n+1}^{p}+\mathbf{C}_{a e} \dot{\mathbf{x}}_{n+1}^{p}+\mathbf{K}_{a e} \mathbf{x}_{n+1}^{p}
$$

que representa una aproximación de la ecuación (6.56).

Nuestro interés se centra exclusivamente en la evaluación de las propiedades de (6.57) para un predictor determinado. La discretización en el tiempo de (6.56) y (6.57) introduce un error asociado al esquema de integración en el tiempo utilizado. Es importante observar que este error no tiene ningún interés para nosotros ya que es intrínseco a la integración en el tiempo y no depende del acoplamiento, por consiguiente, nos referiremos a la ecuación en tiempo discreto "exacta" en la evaluación del error de truncamiento. En definitiva, se trata de describir "cómo de mala" es la solución aproximada en comparación con la "exacta". 
Antes continuar es conveniente hacer un breve comentario acerca de las propiedades deseables para un esquema de integración en el tiempo acoplado. Citando al Prof. Felippa [4], un buen esquema de integración en el tiempo debería:

1. preservar la estabilidad de modelos matemáticos estables

2. manifestar la inestabilidad de modelos matemáticos inestables

Mientras que una extrapolación de las directrices dadas por el Prof. Hughes [19] sugiere que "la disipación numérica controlable para los modos altos" y "la estabilidad incondicional" son muy deseables.

A continuación se mostrará que el método propuesto es estable en las condiciones en las cuales el sistema test es estable, y que reproduce el comportamiento de la solución acoplada exacta cuando el sistema es físicamente inestable. Además, se evaluará el amortiguamiento "efectivo" mostrando las condiciones en las cuales el método aproximado está más o menos amortiguado que el exacto.

\subsubsection{Análisis del algoritmo de acoplamiento de paso fraccionado}

En párrafos anteriores se describió el método que se desea estudiar y se mostró su relación con el algoritmo CBS. Al aplicar el algoritmo propuesto al sistema tipo se obtiene lo siguiente:

1. resolución del sistema

$$
\mathbf{M} \ddot{\mathbf{x}}_{n+1}^{p}+\mathbf{C} \dot{\mathbf{x}}_{n+1}^{p}+\mathbf{K} \mathbf{x}_{n+1}^{p}=\eta \mathbf{p}_{n}+\phi \mathbf{p}_{n-1}
$$

2. cálculo de la presión del fluido

$$
\mathbf{p}_{n+1}=\mathbf{C}_{a e} \dot{\mathbf{x}}_{n+1}^{p}+\mathbf{K}_{a e} \mathbf{x}_{n+1}^{p}
$$

3. cálculo de la posición final de la estructura resolviendo

$$
\mathbf{M} \ddot{\mathbf{x}}_{n+1}+\mathbf{C} \dot{\mathbf{x}}_{n+1}+\mathbf{K} \mathbf{x}_{n+1}=\mathbf{p}_{n+1}
$$

Notar que el término del lado derecho de (6.58) incluye dos pasos previos. El caso descrito en párrafos anteriores puede ser retomado haciendo $\eta=1 \mathrm{y} \phi=0$.

Con objeto de simplificar los siguientes desarrollos, la masa y la rigidez aeroelásticas se expresarán (sin pérdida de generalidad) como un ratio de los correspondientes parámetros estructurales y los términos asociados a la rigidez y al amortiguamiento estructural también se expresarán de forma adecuada. 


$$
\begin{gathered}
\mathbf{K}=\omega^{2} ; \quad \mathbf{K}_{a e}=\beta \mathbf{K} \\
\mathbf{C}=2 \xi \omega ; \quad \mathbf{C}_{a e}=2 \xi_{a e} \omega \\
\mathbf{M}=1 \quad ; \quad \mathbf{M}_{a e}=\alpha \mathbf{M}
\end{gathered}
$$

Sin pérdida de generalidad, para el estudio de las propiedades de la solución se asumirá un método de integración en el tiempo de la forma

$$
\mathbf{y}_{n+1}=A \mathbf{y}_{n}+\mathbf{L}_{n} \mathbf{p}_{n}+\mathbf{L}_{n+1} \mathbf{p}_{n+1} \quad ; \quad \mathbf{y}_{n}=\left\{\begin{array}{c}
\mathbf{x}_{n} \\
\dot{\mathbf{x}}_{n}
\end{array}\right\}
$$

Los resultados que se muestran seguidamente implicarán manipulaciones puramente algebraicas de estas ecuaciones.

\subsubsection{Solución discreta del problema acoplado exacto}

El primer paso consiste en la definición de la solución de referencia, la cual se obtiene resolviendo de forma exacta el problema discreto asociado a (6.56).

Definiendo los vectores auxiliares

$$
\mathbf{G}=\{\mathbf{K}, \mathbf{C}\} \quad ; \quad \mathbf{G}_{a e}=\left\{\mathbf{K}_{a e}, \mathbf{C}_{a e}\right\}
$$

es posible expresar la presión como

$$
\mathbf{p}_{n+1}=\mathbf{M}_{a e} \ddot{\mathbf{x}}_{n+1}+\mathbf{G}_{a e} \mathbf{y}_{n+1} ; \mathbf{p}_{n}=\mathbf{M}_{a e} \ddot{\mathbf{x}}_{n}+\mathbf{G}_{a e} \mathbf{y}_{n}
$$

La aceleración puede ser calculada a partir de $\mathbf{y}_{n+1}$ imponiendo el equilibrio

$$
\mathbf{M} \ddot{\mathbf{x}}_{n+1}=\mathbf{p}_{n+1}-\mathbf{G y}_{n+1}
$$

Sustituyendo el resultado anterior en (6.66) se obtiene

$$
(1-\alpha) \mathbf{p}_{n+1}=\left(\mathbf{G}_{a e}-\alpha \mathbf{G}\right) \mathbf{y}_{n+1}
$$

Las expresiones (6.64) y (6.68) se pueden expresar en forma matricial como

$$
\left[\begin{array}{cc}
\mathbf{I} & -\mathbf{L}_{n+1} \\
\alpha \mathbf{G}-\mathbf{G}_{a e} & 1-\alpha
\end{array}\right]\left(\begin{array}{c}
\mathbf{y}_{n+1} \\
\mathbf{p}_{n+1}
\end{array}\right)=\left[\begin{array}{cc}
\mathbf{A} & \mathbf{L}_{n} \\
0 & 0
\end{array}\right]\left\{\begin{array}{l}
\mathbf{y}_{n} \\
\mathbf{p}_{n}
\end{array}\right\}
$$

o bien, en forma compacta como

$$
\mathbf{z}_{n+1}=\mathbf{A}_{e x} \mathbf{z}_{n}
$$

donde 


$$
\mathbf{A}_{e x}:=\left[\begin{array}{cc}
\mathbf{I} & -\mathbf{L}_{n+1} \\
\alpha \mathbf{G}-\mathbf{G}_{a e} & 1-\alpha
\end{array}\right]^{-1}\left[\begin{array}{cc}
\mathbf{A} & \mathbf{L}_{n} \\
0 & 0
\end{array}\right] ; \mathbf{z}_{n+1}:=\left\{\begin{array}{l}
\mathbf{y}_{n+1} \\
\mathbf{p}_{n+1}
\end{array}\right\}
$$

\subsubsection{Solución aproximada del problema acoplado}

En el punto anterior se expresó la solución exacta en función de una matriz de amplificación, la cual relaciona los desplazamientos, velocidades y presiones en un paso con los del paso anterior. Un desarrollo similar se puede llevar a cabo a partir del método de paso fraccionado descrito. Con objeto de facilitar el desarrollo, resulta útil distinguir entre una primera fase de predicción y las sucesivas correcciones.

\section{Fase de predicción}

En el primer paso (predicción) la presión se calcula como

$$
\mathbf{p}_{n+1}^{I}=\eta \mathbf{p}_{n}+\phi \mathbf{p}_{n-1}
$$

Teniendo en cuenta el método de integración en el tiempo de la estructura (6.64) y considerando que ésta se encuentra sometida a la presión predicha, la predicción estructural que se obtiene es

$$
\mathbf{y}_{n+1}^{I}=\mathbf{A} \mathbf{y}_{n}+\mathbf{L}_{n} \mathbf{p}_{n}+\mathbf{L}_{n+1} \mathbf{p}_{n+1}^{I}
$$

Agrupando las dos ecuaciones anteriores se obtiene

$$
\left[\begin{array}{cc}
\mathbf{I} & -\mathbf{L}_{n+1} \\
0 & 1
\end{array}\right]\left(\begin{array}{l}
\mathbf{y}_{n+1}^{I} \\
\mathbf{p}_{n+1}^{I}
\end{array}\right)=\left[\begin{array}{cc}
\mathbf{A} & \mathbf{L}_{n} \\
0 & \eta
\end{array}\right]\left\{\begin{array}{l}
\mathbf{y}_{n} \\
\mathbf{p}_{n}
\end{array}\right\}+\left[\begin{array}{ll}
\mathbf{0} & \mathbf{0} \\
0 & \phi
\end{array}\right]\left(\begin{array}{l}
\mathbf{y}_{n-1} \\
\mathbf{p}_{n-1}
\end{array}\right)
$$

Introduciendo las matrices auxiliares

$$
\mathbf{A}_{1}^{I}:=\mathbf{C}\left[\begin{array}{cc}
\mathbf{A} & \mathbf{L}_{n} \\
0 & \eta
\end{array}\right] ; \mathbf{A}_{2}^{I}:=\mathbf{C}\left[\begin{array}{cc}
\mathbf{0} & \mathbf{0} \\
0 & \phi
\end{array}\right]
$$

con

$$
\mathbf{C}:=\left[\begin{array}{cc}
\mathbf{I} & -\mathbf{L}_{n+1} \\
0 & 1
\end{array}\right]^{-1}=\left[\begin{array}{cc}
\mathbf{I} & -\mathbf{L}_{n+1} \\
0 & 1
\end{array}\right]
$$

la predicción se puede expresar como

$$
\mathbf{z}_{n+1}^{I}=\mathbf{A}_{1}^{I} \mathbf{z}_{n}+\mathbf{A}_{2}^{I} \mathbf{z}_{n-1}
$$




\section{Fase de corrección}

El primer paso de la fase de corrección es el cálculo del campo de presiones corregido. Para incluir esto en el modelo simplificado es necesario calcular las aceleraciones predichas. Imponiendo el equilibrio se tiene

$$
\mathbf{M} \ddot{\mathbf{x}}_{n+1}^{I}=\mathbf{p}_{n}^{I}-\mathbf{G} \mathbf{y}_{n+1}^{I}
$$

con lo que el valor corregido de la presión se puede expresar como

$$
\mathbf{p}_{n+1}^{I I}=\mathbf{M}_{a e} \ddot{\mathbf{x}}_{n+1}^{I}+\mathbf{G}_{a e} \mathbf{y}_{n+1}^{I}=\alpha \mathbf{p}_{n+1}^{I}+\left(\mathbf{G}_{a e}-\alpha \mathbf{G}\right) \mathbf{y}_{n+1}^{I}
$$

mientras que la corrección estructural adopta la forma

$$
\mathbf{y}_{n+1}^{I I}=\mathbf{A} \mathbf{y}_{n}+\mathbf{L}_{n} \mathbf{p}_{n}+\mathbf{L}_{n+1} \mathbf{p}_{n+1}^{I I}
$$

Esta expresión se puede reescribir en función de una serie de matrices de amplificación

$$
\mathbf{z}_{n+1}^{I I}=\mathbf{B}_{0} \mathbf{z}_{n+1}^{I}+\mathbf{B}_{1} \mathbf{z}_{n}+\mathbf{B}_{2} \mathbf{z}_{n-1}
$$

donde

$$
\begin{gathered}
\mathbf{B}_{0}:=\mathbf{C}\left[\begin{array}{cc}
0 & 0 \\
\mathbf{G}_{a e}-\alpha \mathbf{G} & \alpha
\end{array}\right]=\left[\begin{array}{cc}
\mathbf{I} & \mathbf{L}_{n+1} \\
0 & 1
\end{array}\right]\left[\begin{array}{cc}
0 & 0 \\
\mathbf{G}_{a e}-\alpha \mathbf{G} & \alpha
\end{array}\right] \\
\mathbf{B}_{1}:=\mathbf{C}\left[\begin{array}{cc}
\mathbf{A} & \mathbf{L}_{n} \\
0 & 0
\end{array}\right]=\left[\begin{array}{cc}
\mathbf{I} & \mathbf{L}_{n+1} \\
0 & 1
\end{array}\right]\left[\begin{array}{cc}
\mathbf{A} & \mathbf{L}_{n} \\
0 & 0
\end{array}\right] \\
\mathbf{B}_{2}:=\mathbf{C}\left[\begin{array}{ll}
\mathbf{0} & \mathbf{0} \\
0 & 0
\end{array}\right]=\left[\begin{array}{ll}
\mathbf{0} & \mathbf{0} \\
0 & 0
\end{array}\right]
\end{gathered}
$$

La matriz $\mathbf{B}_{2}$ está vacía y sólo se incluye para una futura generalización. Substituyendo (6.77) en (6.81) se obtiene

$$
\mathbf{z}_{n+1}^{I I}=\mathbf{B}_{0}\left(\mathbf{A}_{1}^{I} \mathbf{z}_{n}+\mathbf{A}_{2}^{I} \mathbf{z}_{n-1}\right)+\mathbf{B}_{1} \mathbf{z}_{n}+\mathbf{B}_{2} \mathbf{z}_{n-1}
$$

la cual permite obtener la forma de amplificación final para el método de paso fraccionado

$$
\mathbf{z}_{n+1}^{I I}=\left(\mathbf{B}_{0} \mathbf{A}_{1}^{I}+\mathbf{B}_{1}\right) \mathbf{z}_{n}+\left(\mathbf{B}_{0} \mathrm{~A}_{2}^{I}+\mathbf{B}_{2}\right) \mathbf{z}_{n-1}
$$

Esta expresión se utilizará a continuación para estudiar la estabilidad y la precisión de la formulación propuesta. 


\subsubsection{Análisis del error de truncamiento}

Bajo la hipótesis de que la solución exacta es conocida en los pasos 1 al $n$, el error de truncamiento se puede obtener haciendo la diferencia entre la solución aproximada y la exacta. La forma de amplificación (6.86) obtenida en el punto anterior permite evaluar este error. Sin embargo, para realizar esta operación es necesario indicar que $\mathbf{y}_{n}$ y $\mathbf{p}_{n}$ no pueden estar prescritos independientemente ya que se asume que la solución exacta se utiliza como punto de partida. En general, sólo los desplazamientos y las velocidades están prescritos y la presión se calcula en consecuencia. Una relación de equilibrio dinámico necesariamente establece una relación entre los desplazamientos, velocidades y presiones en los pasos iniciales. En símbolos, esto implica que $\mathbf{z}_{n}$ se puede expresar explícitamente como

$$
\mathbf{z}_{n}=\mathbf{D y}_{n}
$$

con

$$
\mathbf{D}:=\left[\begin{array}{c}
\mathbf{I} \\
\frac{1}{1-\alpha}\left(\mathbf{G}_{a e}-\alpha \mathbf{G}\right)
\end{array}\right]
$$

Por otro lado, la definición de la matriz de amplificación exacta permite escribir

$$
\mathbf{z}_{n}^{e x}=\mathbf{A}_{e x} \mathbf{z}_{n-1}^{e x}
$$

Este resultado, junto con (6.87), permite expresar la solución exacta en el paso $n+1$ de la siguiente forma

$$
\mathbf{z}_{n+1}^{e x}=\mathbf{A}_{e x} \mathbf{A}_{e x} \mathbf{D} \mathbf{y}_{n-1}
$$

Siguiendo el mismo razonamiento, la solución aproximada en el paso dado es

$$
\mathbf{z}_{n+1}^{a p r}=\left(\left(\mathbf{B}_{0} \mathbf{A}_{1}^{I}+\mathbf{B}_{1}\right) \mathbf{A}_{e x}+\left(\mathbf{B}_{0} \mathbf{A}_{2}^{I}+\mathbf{B}_{2}\right)\right) \mathbf{D} \mathbf{y}_{n-1}
$$

Finalmente, haciendo la diferencia entre (6.90) y (6.91) es posible definir una matriz de error reducida, la cual proporciona toda la información necesaria relativa al orden de precisión.

$$
\mathbf{z}_{n+1}^{e x}-\mathbf{z}_{n+1}^{a p r}=\mathbf{E}^{r e d} \mathbf{y}_{n+1}
$$

con

$$
\mathbf{E}^{r e d}:=\left(\mathbf{A}_{e x} \mathbf{A}_{e x}-\left(\mathbf{B}_{0} \mathbf{A}_{1}^{I}+\mathbf{B}_{1}\right) \mathbf{A}_{e x}-\left(\mathbf{B}_{0} \mathbf{A}_{2}^{I}+\mathbf{B}_{2}\right)\right) \mathbf{D} \mathbf{y}_{n-1}
$$




\subsubsection{Integración en el tiempo de Newmark y exacta}

La matriz de error (6.93), junto las expresiones de los términos que aparecen en ella, es válida para cualquier método de integración en el tiempo que pueda ser expresado según la forma (6.64). Sin embargo, para estudiar sus propiedades es necesario escoger un esquema de integración en el tiempo en concreto y encontrar una expresión explícita de las matrices de amplificación.

La matriz de amplificación y los términos de fuerza del esquema de integración en el tiempo de Newmark son ampliamente conocidos y pueden ser encontrados, por ejemplo, en el trabajo del Prof. Hughes [19]. En el caso más general asumen la forma

$$
\mathbf{A}:=\mathbf{A}_{1}^{-1} \mathbf{A}_{2}
$$

con

$$
\begin{gathered}
\mathbf{A}_{1}:=\left[\begin{array}{cc}
1+h^{2} \beta \omega^{2} & 2 h^{2} \beta \xi \omega \\
h \gamma \omega^{2} & 1+2 h \gamma \xi \omega
\end{array}\right] \\
\mathbf{A}_{2}:=\left[\begin{array}{cc}
1-\frac{h^{2}}{2}(1-2 \beta) \omega^{2} & h(1-h(1-2 \beta) \xi \omega) \\
-h(1-\gamma) \omega^{2} & 1-2 h(1-\gamma) \xi \omega
\end{array}\right] \\
\mathbf{L}_{n}:=\mathbf{A}_{1}^{-1}\left[\begin{array}{c}
\frac{h^{2}}{2}\left((1-2 \beta) F_{n}+2 \beta F_{n+1}\right) \\
h\left((1-\gamma) F_{n}+\gamma F_{n+1}\right)
\end{array}\right]
\end{gathered}
$$

En lo sucesivo se considerará precisión de segundo orden, caso no disipativo. Asumiendo que las fuerzas varían linealmente dentro del paso de tiempo, es posible encontrar un resultado similar correspondiente a una integración en el tiempo exacta. En este caso, la matriz de amplificación es

$$
\mathbf{A}=\left[\begin{array}{cc}
\cos (\omega h) & \frac{\sin (\omega h)}{\omega} \\
-\sin (\omega h) \omega & \cos (\omega h)
\end{array}\right]
$$

y el término de fuerza es

$$
\mathbf{L}_{n}=\left[\begin{array}{c}
\frac{-(-\sin (\omega h)+\cos (\omega \mathrm{h}) \omega h)}{\omega^{3} h} \\
\frac{\sin (\omega h) \omega h-1+\cos (\omega h)}{\omega^{2} h}
\end{array}\right]
$$


La sustitución en (6.93) es sencilla, pero implica cálculos algebraicos tediosos. Aunque realizar esta operación con la ayuda de un manipulador simbólico no presenta ninguna dificultad en particular, incluso para el caso general, resulta interesante prestar atención al caso no amortiguado ya que éste conduce a resultados más simples. Se puede comprobar que lo resultados obtenidos también son válidos para el caso general amortiguado.

Un resultado importante es que la condición de consistencia requiere que se cumpla la relación $\eta+\phi=1$. Las opciones $\eta=1, \phi=0$ y $\eta=2, \phi=-1$ son atractivas y corresponden a precisión de primer y segundo orden para el campo de presiones predicho. El orden de esta predicción inicial determina el orden del esquema de integración en el tiempo en su conjunto.

La Tabla 6.1 contiene los primeros términos no nulos del desarrollo en serie de Taylor de la matriz de error. El análisis de estos resultados muestra de forma inmediata que las mismas estimaciones del error son válidas para el esquema de integración en el tiempo de Newmark y el exacto. Esta característica resulta muy atractiva ya que pone de manifiesto que se pueden considerar diferentes esquemas de integración en el tiempo sin que el error de acoplamiento se vea afectado.

Las propiedades del esquema de paso fraccionado acoplado dependen de los valores adoptados por la masa aeroelástica y el término de amortiguamiento. Para $\alpha<1$ y $\beta<1$ (de otra manera la solución no tendría sentido físicamente) se obtienen las siguientes desigualdades

$$
\frac{\alpha^{2}}{\alpha-1}<0 \quad ; \quad \frac{\beta-1}{\alpha-1}>0
$$

las cuales permiten obtener información relativa a las propiedades del amortiguamiento del método de paso fraccionado propuesto.

La exactitud de estos resultados fue evaluada utilizando el esquema de integración en el tiempo de Newmark para comprobar que las predicciones teóricas se ajustaban a los valores numéricos. El planteamiento numérico permite también la evaluación directa del orden de precisión. La Figura 6.1 y la Figura 6.2 corresponden al caso general [17] (incluyendo un amortiguamiento distinto de cero) y muestran la variación del error respecto al tamaño del paso de tiempo. Se puede ver claramente que el error dominante es el asociado a la presión, lo cual no es sorprendente ya que el término de presión depende de la aceleración.

Nótese que estas estimaciones también son válidas para el caso general. Si la masa aeroelástica es despreciable, el comportamiento del método de paso fraccionado mejora significativamente y el orden de precisión en su conjunto es 3. Utilizando el método de integración en el tiempo exacto se obtiene la siguiente matriz de error 


$$
E:=\left[\begin{array}{cc}
-\frac{1}{6} \xi \omega^{5}(-1+\beta)\left(\beta+4 \xi^{2}\right) h^{5} & -\frac{1}{3} \xi^{2} \omega^{4}\left(2 \beta+4 \xi^{2}-1\right) h^{5} \\
-\frac{1}{2} \xi \omega^{5}(-1+\beta)\left(\beta+4 \xi^{2}\right) h^{4} & -\xi^{2} \omega^{4}\left(2 \beta+4 \xi^{2}-1\right) h^{4} \\
-\xi \omega^{5}(-1+\beta)\left(\beta+\xi^{2}\right) h^{3} & -2 \xi^{2} \omega^{4}\left(2 \beta+4 \xi^{2}-1\right) h^{3}
\end{array}\right]
$$

PREDICCIÓN DE PRIMER ORDEN EN LA PRESIÓN $\eta=1 ; \phi=0$

Integración en el tiempo exacta

$$
E_{\text {ord }=1, i t=0}^{r e d ~}=\frac{\alpha^{2}}{\alpha-1} \frac{(\alpha-\beta)}{\alpha}\left[\begin{array}{cc}
\frac{1}{4} \frac{\beta-1}{\alpha-1} \omega^{4} h^{4} & -\frac{1}{6} \omega^{2} h^{3} \\
\frac{3}{4} \frac{\beta-1}{\alpha-1} \omega^{4} h^{3} & -\frac{1}{2} \omega^{2} h^{2} \\
\frac{3}{2} \frac{\beta-1}{\alpha-1} \omega^{4} h^{2} & -\omega^{2} h
\end{array}\right]
$$

Integración en el tiempo de Newmark

$$
E_{\text {ord }=1, i t=0}^{\text {red }}=\frac{\alpha^{2}}{\alpha-1} \frac{(\alpha-\beta)}{\alpha}\left[\begin{array}{cc}
\frac{3}{8} \frac{\beta-1}{\alpha-1} \omega^{4} h^{4} & -\frac{1}{4} \omega^{2} h^{3} \\
\frac{3}{4} \frac{\beta-1}{\alpha-1} \omega^{4} h^{3} & -\frac{1}{2} \omega^{2} h^{2} \\
\frac{3}{2} \frac{\beta-1}{\alpha-1} \omega^{4} h^{2} & -\omega^{2} h
\end{array}\right]
$$

PREDICCIÓN DE SEGUNDO ORDEN EN LA PRESIÓN $\eta=2 ; \phi=-1$

Integración en el tiempo exacta

$$
E_{\text {ord }=2, i t=0}^{r e d}=\frac{\alpha^{2}}{\alpha-1} \frac{(\alpha-\beta)}{\alpha} \frac{\beta-1}{\alpha-1}\left[\begin{array}{cc}
\frac{1}{6} \omega^{4} h^{4} & -\frac{1}{6} \omega^{4} h^{5} \\
\frac{1}{2} \omega^{4} h^{3} & -\frac{1}{2} \omega^{4} h^{4} \\
\omega^{4} h^{2} & -\omega^{4} h^{3}
\end{array}\right]
$$

Integración en el tiempo de Newmark

$$
E_{\text {ord }=2, i t=0}^{r e d}=\frac{\alpha^{2}}{\alpha-1} \frac{(\alpha-\beta)}{\alpha} \frac{\beta-1}{\alpha-1}\left[\begin{array}{cc}
\frac{1}{6} \omega^{4} h^{4} & -\frac{1}{6} \omega^{4} h^{5} \\
\frac{1}{2} \omega^{4} h^{3} & -\frac{1}{2} \omega^{4} h^{4} \\
\omega^{4} h^{2} & -\omega^{4} h^{3}
\end{array}\right]
$$

Tabla 6.1. Matriz de error 


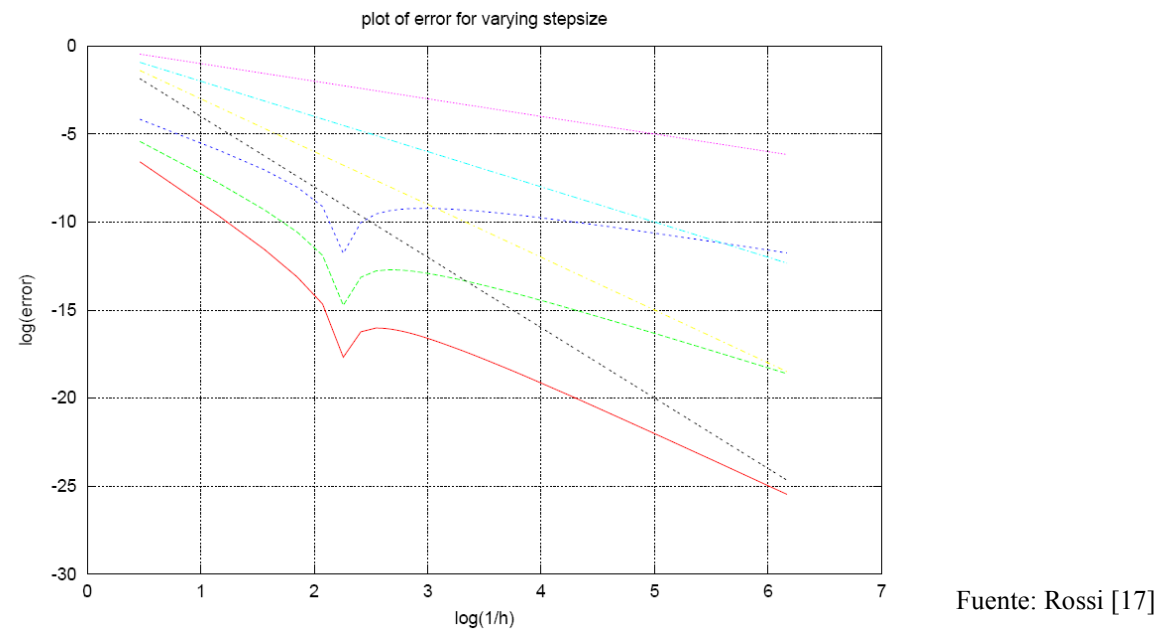

Figura 6.1. Esquema de paso fraccionado de primer orden. Gráfico del error logarítmico. PressureError: azul, DisplacementError: rojo, VelocityError: verde

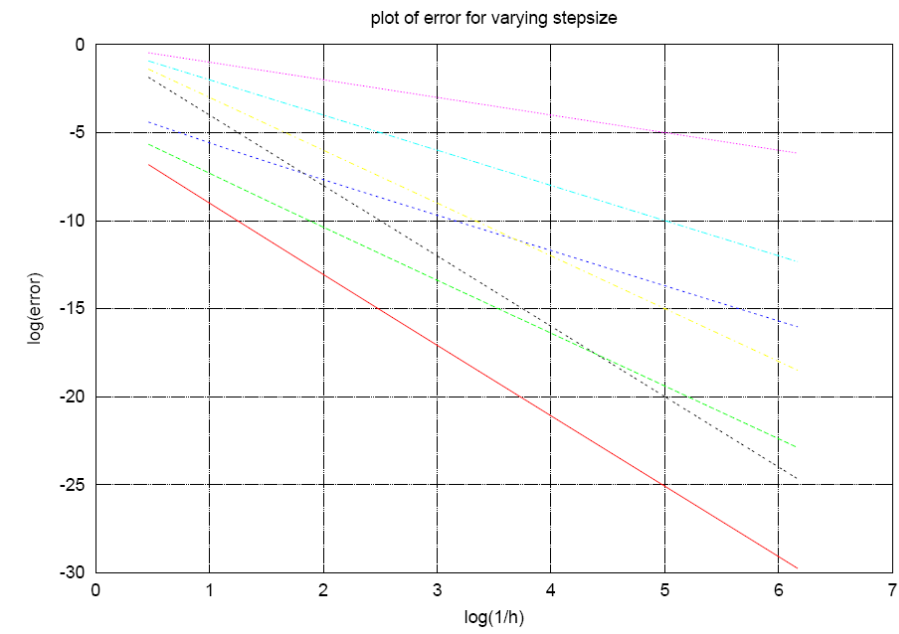

Fuente: Rossi [17]

Figura 6.2. Esquema de paso fraccionado de segundo orden. Gráfico del error logarítmico. PressureError: azul, DisplacementError: rojo, VelocityError: verde

\subsubsection{Técnicas iterativas}

La estabilidad y la precisión del método de paso fraccionado descrito pueden ser mejoradas adoptando una estrategia de iteración. Éste se puede expresar en símbolos modificando (6.81) de la siguiente manera

$$
\mathbf{z}_{n+1}^{i+1}=\mathbf{B}_{0} \mathbf{z}_{n+1}^{i}+\mathbf{B}_{1} \mathbf{z}_{n}+\mathbf{B}_{2} \mathbf{z}_{n-1}
$$

Esta expresión corresponde a un método de iteración de tipo Jacobi y puede no converger en algunas situaciones. Este problema ha sido ampliamente estudiado y normalmente se introduce un 
factor de relajación $\omega$ para solucionarlo. El valor óptimo de este parámetro de relajación se puede calcular por el método de prueba y el error o utilizando algún acelerador. Wall y Mok [6] abogan por el uso del acelerador de Aitken para problemas de interacción fluido-estructura.

Tomando como referencia el marco teórico propuesto, la matriz de error correspondiente a la aplicación iterativa de la etapa de corrección puede ser evaluada directamente. El resultado es simple e interesante. Siendo $\mathbf{E}^{\text {red }}$ la matriz de error reducida calculada para el método de paso fraccionado "básico" y que contiene los principales términos del desarrollo en serie del error, las relaciones siguientes son válidas para el error después de sucesivas iteraciones.

$$
\begin{gathered}
\mathbf{E}_{i t=0}^{r e d}=\frac{1}{\alpha} \mathbf{E}^{r e d} \text { (predicción explícita) } \\
\mathbf{E}_{i t=1}^{r e d}=\mathbf{E}^{r e d} \text { (final del método de paso fraccionado básico) } \\
\mathbf{E}_{i t=2}^{r e d}=\alpha \mathbf{E}^{r e d} \\
\mathbf{E}_{i t=3}^{r e d}=\alpha^{2} \mathbf{E}^{r e d}
\end{gathered}
$$

Por extrapolación se puede asumir

$$
\mathbf{E}_{i t=i}=\alpha^{i-1} \mathbf{E}
$$

Este resultado es importante ya que establece que la iteración simple de Jacobi no converge cuando $\alpha<-1$. Por lo tanto, para asegurar la convergencia será necesario utilizar un acelerador.

Por otro lado, a partir de la definición de error se obtiene

$$
\mathbf{y}_{n+1}^{i}-\mathbf{y}_{n+1}^{e x} \approx \alpha^{i-1} \mathbf{E y}_{n}
$$

Calculando la diferencia entre iteraciones sucesivas y premultiplicando por un cierto vector $\mathbf{v}^{T}$ se consigue

$$
\begin{aligned}
& \mathbf{v}^{T}\left(\mathbf{y}_{n+1}^{i}-\mathbf{y}_{n+1}^{i-1}\right) \approx \alpha^{i-2}(\alpha-1) \mathbf{v}^{T} \mathbf{E} \mathbf{y}_{n} \\
& \mathbf{v}^{T}\left(\mathbf{y}_{n+1}^{i-1}-\mathbf{y}_{n+1}^{i-2}\right) \approx \alpha^{i-3}(\alpha-1) \mathbf{v}^{T} \mathbf{E} \mathbf{y}_{n}
\end{aligned}
$$

Finalmente, el cociente entre ambas expresiones proporciona una estimación del coeficiente de masa aeroelástica

$$
\alpha=\frac{\mathbf{v}^{T}\left(\mathbf{y}_{n+1}^{i}-\mathbf{y}_{n+1}^{i-1}\right)}{\mathbf{v}^{T}\left(\mathbf{y}_{n+1}^{i-1}-\mathbf{y}_{n+1}^{i-2}\right)}
$$

Esta estimación resulta útil para la determinación del factor de relajación óptimo. 
Por definición, la relajación toma la forma

$$
\mathbf{y}^{*}=\omega \mathbf{y}^{i}+(1-\omega) \mathbf{y}^{i-1}
$$

Usando la definición del error (6.103) y simplificando se obtiene

$$
\mathbf{y}^{*} \approx \mathbf{y}^{e x}+(\omega+(\omega-1) \alpha) \mathbf{E y}_{n}
$$

que permite obtener el factor de relajación óptimo como

$$
\omega=\frac{1}{1+\alpha}=\frac{\mathbf{v}^{T}\left(\mathbf{y}_{n+1}^{i}-\mathbf{y}_{n+1}^{i-1}\right)}{\mathbf{v}^{T}\left(\mathbf{y}_{n+1}^{i}-\mathbf{y}_{n+1}^{i-2}\right)}
$$

Adoptando este factor de relajación se consigue (en el sistema test) un orden más alto de precisión en el tiempo.

Esta estrategia de aceleración consistente puede ser aplicada al modelo propuesto y comparada con la aceleración de Aitken. Cuando la masa aeroelástica es pequeña los resultados que proporcionan ambos métodos de aceleración son completamente comparables, sin embargo, cuando $\alpha<<-1$, la aceleración propuesta presenta una ventaja importante en la precisión. Esto se muestra en la Figura 6.3 y en la Figura 6.4.

Notar que en algunas condiciones el acelerador de Aitken encuentra la convergencia en sólo dos iteraciones, mientras el método de aceleración consistente propuesto requiere siempre tres pasos de resolución en el modelo. Cuando el acelerador de Aitken es forzado a realizar al menos tres iteraciones, los resultados de las dos estrategias son totalmente comparables.

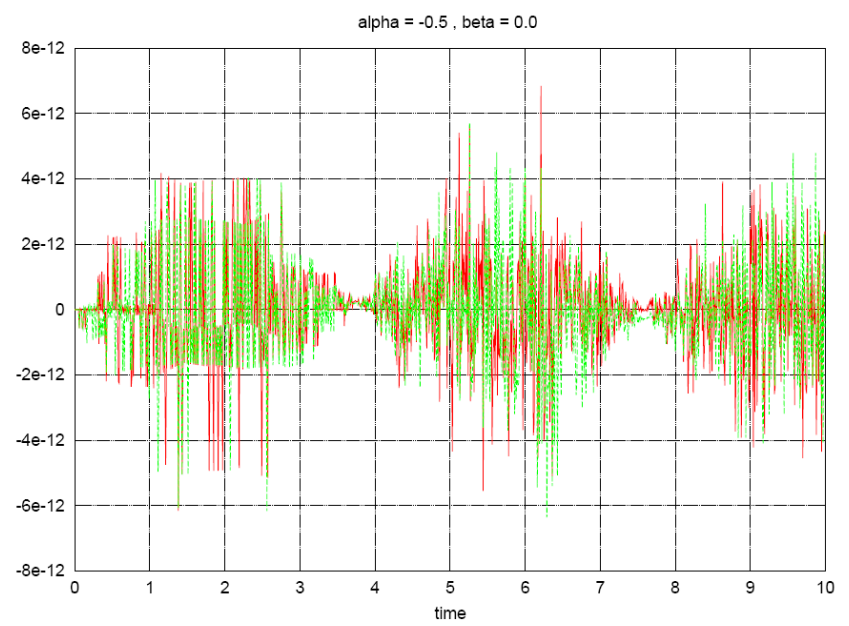

Fuente: Rossi [17]

Figura 6.3. Error con respecto a la solución exacta: Aceleración de Aitken - línea roja, "Aceleración consistente" - línea verde. $\alpha=0.5 ; \beta=0.0$ 


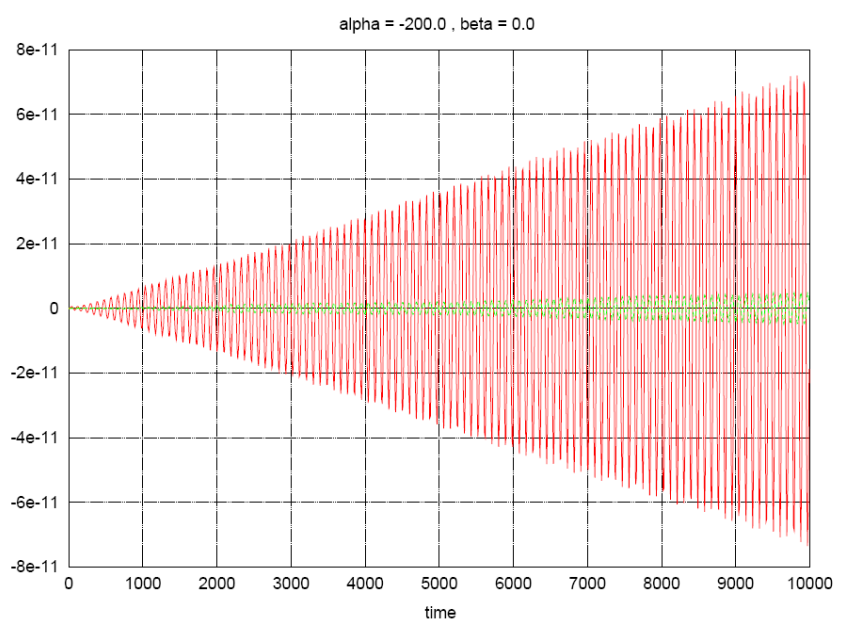

Fuente: Rossi [17]

Figura 6.4. Error con respecto a la solución exacta: Aceleración de Aitken - línea roja, “Aceleración Consistente" - línea verde. $\alpha=-200 ; \beta=0.0$

\section{Una forma iterativa del Método de Relajación Consistente}

Para aplicar el acelerador consistente propuesto a problemas con múltiples grados de libertad (MDOF) se debe expresar en una forma iterativa. Es necesario distinguir entre series no aceleradas y aceleradas para estudiar el factor de aceleración óptimo. Reescribiendo (6.101) en una forma más compacta, se tiene la serie no acelerada

$$
\mathbf{y}_{n+1}^{i+1}=\mathbf{C y}_{n+1}^{i}+\mathbf{D}
$$

donde $\mathbf{C}$ y $\mathbf{D}$ son dos operadores auxiliares lineales.

Teniendo en cuenta que los términos de la serie acelerada se representan como $\bar{y}$, y que los términos auxiliares como $\hat{y}$, se tiene

$$
\hat{\mathbf{y}}_{n+1}^{i+1}=\mathbf{C} \overline{\mathbf{y}}_{n+1}^{i}+\mathbf{D}
$$

La operación de relajación implica

$$
\overline{\mathbf{y}}_{n+1}^{i+1}=\omega_{i+1} \hat{\mathbf{y}}_{n+1}^{i+1}+\left(1-\omega_{i+1}\right) \overline{\mathbf{y}}_{n+1}^{i}
$$

de manera que los términos en la serie relajada son de la forma

$$
\overline{\mathbf{y}}_{n+1}^{i+1}=\omega_{i+1}\left(\mathbf{C} \overline{\mathbf{y}}_{n+1}^{i}+\mathbf{D}\right)+\left(1-\omega_{i+1}\right) \bar{y}_{n+1}^{i}
$$

Por otro lado, cualquiera de los términos en la serie relajada puede ser expresado como

$$
\overline{\mathbf{y}}_{n+1}^{i+1}=\mathbf{y}^{e x}+\overline{\mathbf{E}}^{i+1}
$$


Sustituyendo se obtiene

$$
\overline{\mathbf{y}}_{n+1}^{i+1}=\omega_{i+1}\left(\mathbf{C y} \mathbf{y}^{e x}+\mathbf{D}+\mathbf{C} \overline{\mathbf{E}}_{i}\right)+\left(1-\omega_{i+1}\right)\left(\mathbf{y}^{e x}+\overline{\mathbf{E}}_{i}\right)
$$

y teniendo en cuenta que $\mathbf{y}^{e x}=\mathbf{C y} \mathbf{y}^{e x}+\mathbf{D}$ se llega a

$$
\overline{\mathbf{E}}_{i+1}=\omega_{i+1} \mathbf{C} \overline{\mathbf{E}}_{i}+\left(1-\omega_{i+1}\right) \overline{\mathbf{E}}_{i}
$$

Este resultado es importante porque permite demostrar la dependencia lineal del error de la serie relajada respecto a los términos de la serie no relajada. Este resultado se deduce de forma inmediata observando que los primeros términos de la serie relajada coinciden con los correspondientes términos de la serie no relajada. Considerando (6.102) se obtiene

$$
\mathbf{C} \mathbf{E}_{i+1}=\alpha \mathbf{E}_{i+1} \Rightarrow \mathbf{C} \overline{\mathbf{E}}_{i+1}=\alpha \overline{\mathbf{E}}_{i+1}
$$

lo cual demuestra la relación

$$
\overline{\mathbf{E}}_{i+1}=\left(\alpha \omega_{i+1}+1-\omega_{i+1}\right) \overline{\mathbf{E}}_{i}
$$

Calculando el cociente entre la diferencia de la solución en pasos diferentes es posible obtener una estimación del coeficiente de masa aeroelástica como

$$
\alpha_{i+1} \approx \frac{\mathbf{v}^{T}\left(\overline{\mathbf{y}}_{n+1}^{i}-\overline{\mathbf{y}}_{n+1}^{i-1}\right)}{\mathbf{v}^{T}\left(\overline{\mathbf{y}}_{n+1}^{i-1}-\overline{\mathbf{y}}_{n+1}^{i-2}\right)}-\frac{1-\omega_{i}}{\omega_{i}}
$$

y estimar el parámetro de relajación óptimo como

$$
\omega_{i+1}=\frac{1}{1+\alpha_{i+1}}
$$

\section{Un comentario sobre la aplicación de técnicas de relajación a problemas MDOF}

Las técnicas de relajación descritas son aplicables a la resolución de problemas acoplados con MDOF. En particular, estos algoritmos fueron probados en el trabajo de Rossi [17] por medio de su aplicación a un voladizo flexible situado justo detrás de un cuerpo romo cuadrado, lo cual constituye una prueba clásica para este tipo formulaciones acopladas. Los resultados preliminares que se obtuvieron mostraron que en este caso no se obtenía ninguna ventaja significativa en la utilización del acelerador consistente o de Aitken respecto al método de iteración de punto fijo. 


\subsubsection{Uso de algoritmos disipativos para la integración estructural}

En muchas ocasiones la solución de problemas acoplados de interés real requiere la simulación de estructuras no lineales. Desde un punto de vista práctico, esto implica la utilización de solvers disipativos para la integración de las ecuaciones de movimiento.

En la Figura 6.5 se representa el resultado del esquema acoplado iterativo frente al obtenido para un planteamiento monolítico usando el mismo método de integración en el tiempo. Se observa que la solución iterativa presenta un falso amortiguamiento que no aparece cuando la solución se obtiene usando un algoritmo no disipativo. Este falso amortiguamiento es proporcional a la masa aeroelástica y desaparece cuando el paso de tiempo tiende a cero. Por lo tanto, la importancia de este efecto es pequeña en la medida en que la masa de la estructura es sensiblemente mayor que la del fluido circundante. Este efecto puede llegar a ser importante en el caso de fluidos de alta densidad.

Notar que la solución "exacta" se obtiene usando el mismo algoritmo de integración, por lo que el amortiguamiento que presenta la respuesta depende del algoritmo de acoplamiento utilizado.

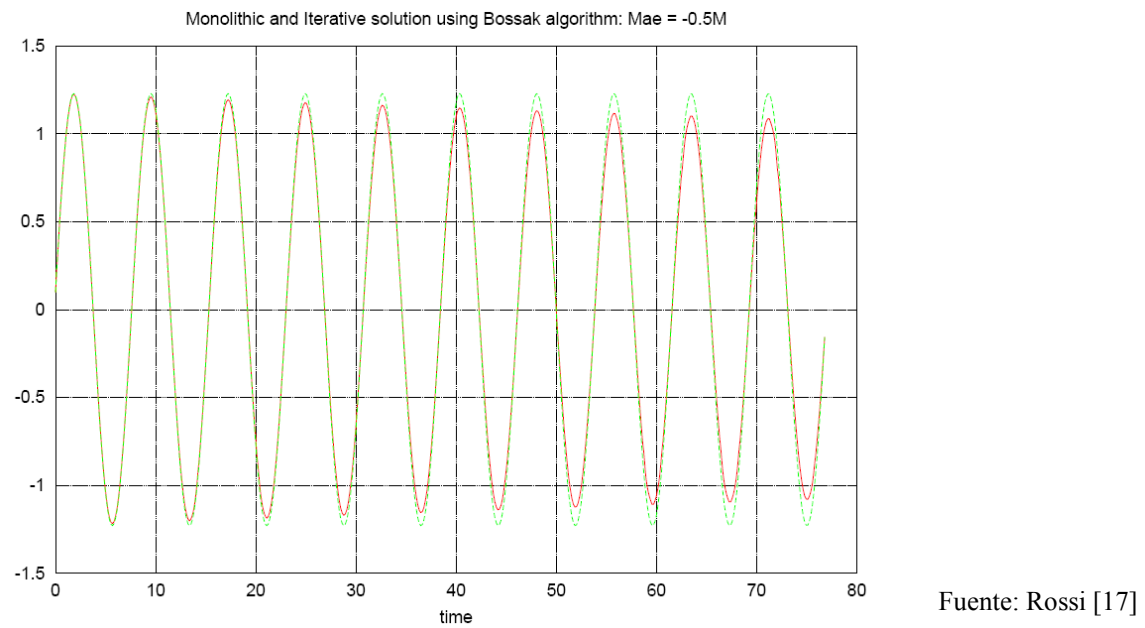

Figura 6.5. Comparación entre la solución exacta y la solución acoplada iterativa usando el algoritmo de Bossak. Parámetro de Bossak $=-0.1$, período dividido en 50 pasos

\subsubsection{Cuestiones de estabilidad}

Para completar el estudio es necesario estudiar la estabilidad del esquema acoplado propuesto. Considerando por simplicidad el método de paso fraccionado de primer orden (de un paso), la solución exacta del problema acoplado, incluyendo tanto las variables estructurales como la presión, viene dada por 


$$
\mathbf{z}_{n+1}^{e x}=\mathbf{A}_{e x} \mathbf{z}_{n}^{e x}
$$

mientras que la solución aproximada se puede expresar como

$$
\mathbf{z}_{n+1}=\mathbf{A}_{a p r} \mathbf{z}_{n}+\mathbf{E} \mathbf{z}_{n} ; \mathbf{E}:=\mathbf{A}_{a p r}-\mathbf{A}_{e x}
$$

donde se ha introducido una nueva matriz de error $\mathbf{E}$ que relaciona tanto las variables estructurales como la presión. Notar que las matrices de amplificación $\mathbf{A}_{e x}, \mathrm{y} \mathbf{A}_{a p r}$ son matrices cuadradas de orden 3 y no deben ser confundidas con las formas reducidas utilizadas en el estudio de la precisión.

La estabilidad del esquema esta controlada por el radio espectral de la matriz de amplificación. Para el método de paso fraccionado de un paso, los valores propios se pueden calcular analíticamente. Desafortunadamente, para el caso general los resultados son complejos y difíciles de manejar, sin embargo, a partir del desarrollo en serie de Taylor en $\Omega=\omega / h$ de los valores propios analíticos se puede obtener cierta información útil. Los principales términos de este desarrollo en serie son [17]

$$
\begin{gathered}
e_{1}=\frac{\alpha-1+i \Omega \sqrt{1-\alpha} \sqrt{1-2 \beta+1}}{\alpha-1} \\
e_{2}=a b s(\alpha) \\
e_{3}=\frac{\alpha-1-i \Omega \sqrt{1-\alpha} \sqrt{1-2 \beta+1}}{\alpha-1}
\end{gathered}
$$

Una condición necesaria para la estabilidad es que en el límite, con $\Omega=\omega / h$ tendiendo a cero, el radio espectral sea inferior o igual a uno. Este límite tiene la forma

$$
\rho_{\max }=\max (\|\alpha\|, 1)
$$

la cual pone de manifiesto que la estabilidad depende del problema y que el esquema acoplado propuesto es incondicionalmente inestable para $\alpha<-1$.

Cabe destacar que esta definición de estabilidad no es completamente apropiada para el problema de acoplamiento de interés. En problemas acoplados de interacción fluido-estructura son posibles tanto soluciones divergentes como convergentes. Esto implica que la matriz de amplificación exacta puede estar caracterizada por valores propios de valor mayor que uno. Cuando no se considerada el amortiguamiento el valor de referencia para el radio espectral es uno. Un valor mayor que uno (pero que tiende a uno cuando se disminuye el tamaño de paso de tiempo) puede ser aceptado, incluso si correspondiese a una solución numéricamente divergente. Sin embargo, cuando 
el sistema está caracterizado por $\alpha<-1$, incluso para pasos de tiempo pequeños, la solución es divergente y no se puede mejorar la situación reduciendo el paso de tiempo.

\subsubsection{Caso de masa de aeroelástica despreciable}

Como consideración final, es interesante considerar el comportamiento del algoritmo de acoplamiento para $\alpha$ tendiendo a cero. Cuando no se considera la masa aeroelástica las ecuaciones se simplifican considerablemente y la precisión se puede incrementar en un orden para el caso de predicción de segundo orden.

Si el amortiguamiento aeroelástico es cero, las ecuaciones se simplifican de manera importante y las condiciones de estabilidad se pueden estudiar analíticamente (para el caso de predicción de primer orden). Bajo estas condiciones, el esquema de acoplamiento es incondicionalmente estable si $\beta>-1$ y condicionalmente estable en caso contrario. Para $\beta<-1$ se puede comprobar que la condición de la estabilidad es

$$
h \leq \frac{2}{\omega \sqrt{-1-\beta}}
$$

Por otro lado, el caso general amortiguado conduce a resultados difíciles de manipular analíticamente. El radio espectral se puede expresar en este caso como $\rho\left(n_{d i v}, \xi_{a e}, \beta\right)$ donde $n_{\text {div }}=2 \pi / \Omega$ representa el número de pasos de tiempo utilizados para la discretización del período natural de la estrutura. Se puede obtener información útil relativa al comportamiento del método a partir de la representación gráfica del radio espectral como función de $\xi_{a e}$ y $\beta$ para diferentes valores crecientes del número de divisiones del periodo estructural.

En la Figura 6.6 se muestra que el radio espectral es siempre menor que uno cuando la constante de amortiguamiento aeroelástico es negativa. Se observa también que la región de estabilidad se extiende al rango de amortiguamiento positivo para valores muy pequeños del parámetro $n_{d i v}$. Esto sugiere que el amortiguamiento algorítmico (algorithmic damping) es alto cuando el número de divisiones es muy pequeño. Para valores grandes del número de divisiones las isolíneas de la representación gráfica del radio espectral son rectas y paralelas, lo cual significa que el amortiguamiento algorítmico adicional desaparece. Por otro lado, los gráficos de la Figura 6.7 muestran una medida del amortiguamiento algorítmico frente al amortiguamiento numérico e indican las áreas del plano $\xi_{a e} \beta$ donde el amortiguamiento algorítmico es mayor o menor que el del esquema de Newmark aplicado al caso exacto. 


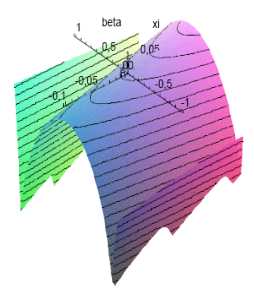

(a) $n_{\text {div }}=2$

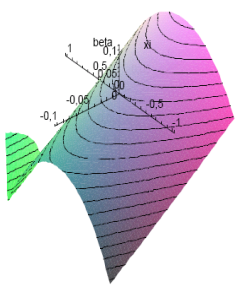

(b) $n_{\text {div }}=4$

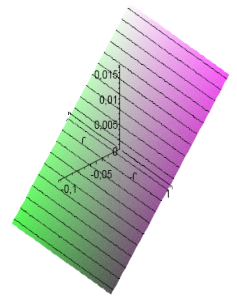

(e) $n_{\text {div }}=40$

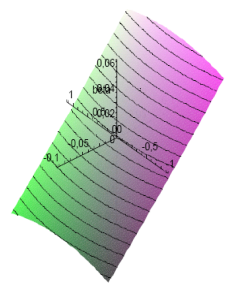

(c) $n_{\text {div }}=10$

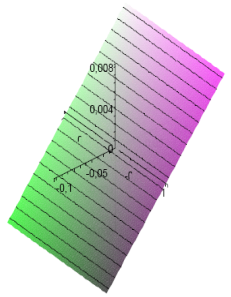

(f) $n_{d i v}=80$

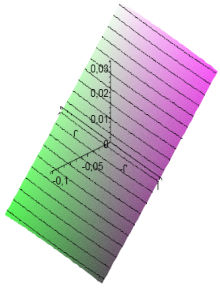

(d) $n_{d i v}=20$

Figura 6.6. Representación gráfica de $\rho_{\text {aprox }}-1$

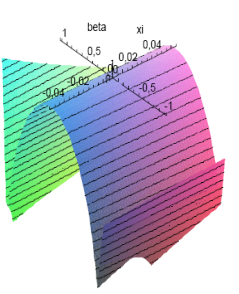

(a) $n_{\text {div }}=2$

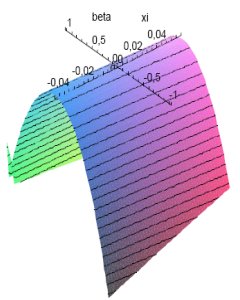

(b) $n_{\text {div }}=4$

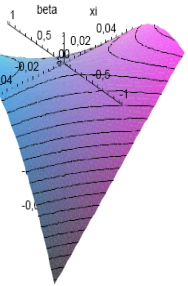

(e) $n_{d i v}=40$

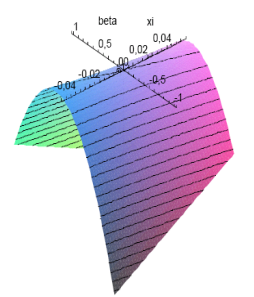

(c) $n_{\text {div }}=10$

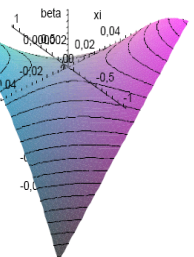

(f) $n_{\text {div }}=80$

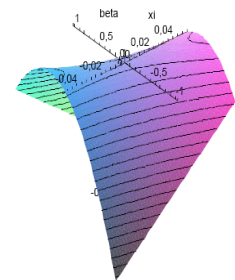

(d) $n_{\text {div }}=20$

Figura 6.7. Representación gráfica de $\frac{\rho_{\text {aprox }}-\rho_{\text {exact }}}{\rho_{\text {exact }}}$ 


\subsection{Validación}

El método numérico propuesto fue probado en diferentes ejemplos en el trabajo de Rossi [17]. Una prueba importante estaba constituida por un voladizo flexible situado detrás de un cuerpo romo cuadrado. La prueba fue realizada empleando tanto técnicas implícitas como explícitas y en la Figura 6.8 se muestran una serie de capturas de pantalla. El algoritmo explícito mostraba una tendencia a la inestabilidad aeorelástica por flameo (flutter), la cual se puede corregir iterando.
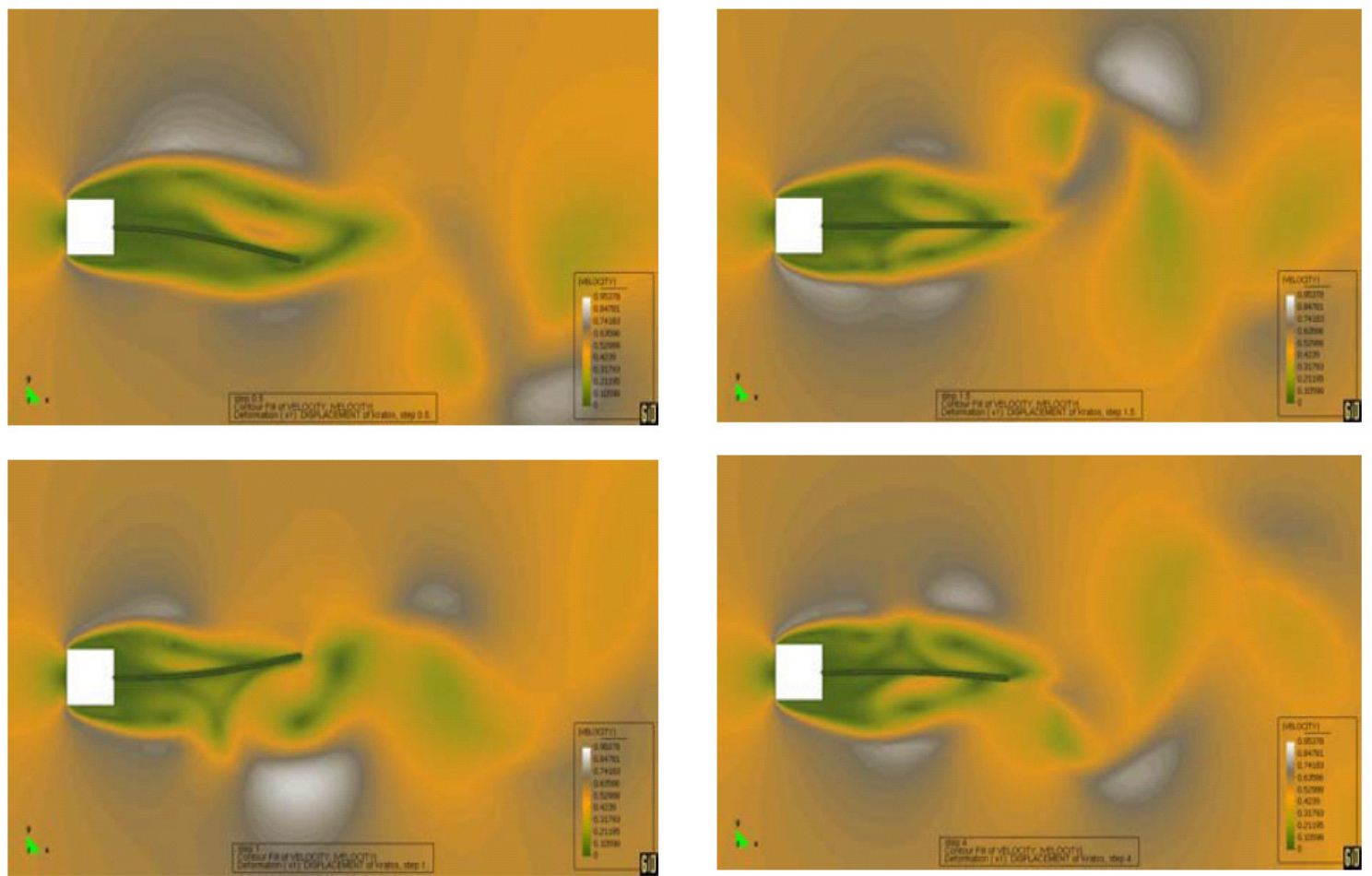

Fuente: Rossi [17]

Figura 6.8. Vista de la solución acoplada en diferentes pasos de la solución

\section{Referencias}

${ }^{1}$ Piperno, S., Farhat, C., y Larroutorou, B. "Partitioned procedures for the transient solution of coupled aeroelastic problems. Part 1: Model problem, theory and two dimensional applications", Computer Methods in Applied Mechanics and Engineering, 124, pp. 79-112, 1995.

${ }^{2}$ Piperno, S. y Farhat, C., "Partitioned procedures for the transient solution of coupled aeroelastic problems. Part 2: Energy transfer analysis and three dimensional applications", Computer Methods in Applied Mechanics and Engineering, 190, pp. 3147-3170, 2001. 
${ }^{3}$ Park, K.C., y Felippa, C., "Synthesis tools for structural dynamics and partitioned analysis of coupled systems", http://www.colorado.edu/engineering/CAS/courses.d/FSI.d/NATO-ARW.Felippa .paper.rev.pdf, 2004.

${ }^{4}$ Felippa, C., "FSI course: chapters 1-11", http://www.colorado.edu/engineering/CAS/courses.d/ FSI.d, 2004.

${ }^{5}$ Felippa, C.A.; Park., K.C.; Farhat, C., "Partitioned analysis of coupled mechanical systems", Computer Methods in Applied Mechanics and Engineering,190 (24-25), pp. 3247-3270, 2001.

${ }^{6}$ Wall, W.A., y Mok, D.P., "Partitioned analysis schemes for the transient interaction of incompressible lows and nonlinear flexible structures" In Trends in computational structural mechanics, Wall WA, Bletzinger KU, Schweizerhof K (eds). CIMNE: Barcelona, 689-698, 2001.

7 Farhat C.; Geuzaine P.; Brown G., "Application of a three-field non-linear fluid-structure formulation to the prediction of the aeroelastic parameters of an $\mathrm{f}-16$ fighter" Computers and Fluids, 32, pp. 3-29, 2003.

${ }^{8}$ Halfmann, A., Rank, E., Glück, M., Breuer, M., Durst. F., "A partitioned solution approach for the fluid-structure interaction of wind and thin walled structures", Technical report, TU Munchen, Universidad Erlangen-Nurnberg, 2000.

${ }^{9}$ Rank, E., Scholz, D., Halfmann, A., Glück, M., Breuer, M., Durst, F., "Fluid-structure interaction in civil engineering", In Second MIT conference on Computational Fluid and Solid Mechanics, 2003.

${ }^{10}$ Zienkiewicz, O.C., Codina, R., "A general algorithm for compressible and incompressible flow Part I. The split characteristic-based scheme”. International Journal for Numerical Methods in Fluids, 20, pp. 869-885, 1995.

${ }^{11}$ Zienkiewicz, O.C., Nithiarasu, P., Codina, R., Vazquez, M., Ortiz, P., "The characteristic-basedsplit procedure: an efficient and accurate algorithm for fluid problems", International Journal for Numerical Methods in Fluids; 31, pp. 359-392, 1999.

${ }^{12}$ Codina, R., "Pressure stability in fractional step finite element methods for incompressible flows", Journal of Computational Physics, 170 (1), pp. 112-140, 2001.

${ }^{13}$ Bugeda, G., Onate, E., Garcia, J., e Idelsohn, S.R., "A general stabilized formulation for incompressible fluid flow using finite calculus and the finite element method. In Towards a New Fluid Dynamics with its challenges in Aeronautics, Barcelona, 2002.

${ }^{14}$ Buscaglia, G.C., Codina, R., Blasco, J., y Huerta, A., "Implementation of a stabilized finite element formulation for the incompressible navier-stokes equations based on a pressure gradient projection”, International Journal for Numerical Methods in Fluids, 2001. 
${ }^{15}$ Oñate, E., "Possibilities of finite calculus in computational mechanics", Technical report, CIMNE, 2001.

${ }^{16}$ Zienkiewicz, O.C., y Taylor, R.L., "The Finite Element Method - Volume 3 - Fluid Dynamics, Butterworth Heinemann, 2000.

${ }^{17}$ Rossi, R., Light Weight Structures: Structural Analysis and Coupling Issues, Ph.D. Tesis, Universidad de Bologna, 2005.

${ }^{18}$ Lazzari, M., "Time domain modelling of aeroelastic bridge decks: a comparative study and an application", International Journal for Numerical Methods in Engineering, 62 (8), pp. 1064-1104, 2005.

${ }^{19}$ Hughes, T.J.R., "The Finite Element Method”, Dover, 2000. 

Capítulo 7

\section{Ejemplos}





\subsection{Resumen}

En este capítulo se muestran los resultados obtenidos para las diferentes simulaciones que se han realizado. Se trata de aplicar el método descrito en los capítulos anteriores a modelos de estructuras esbeltas sometidas a cargas de viento para estudiar su comportamiento vibratorio. La estructura se modela utilizando el modelo de barras descrito en el Capítulo 3, el fluido según el esquema expuesto en el Capítulo 5 y finalmente, al acoplamiento fluido-estructura se consigue a través del algoritmo de acoplamiento expuesto en el Capítulo 6. En el primer ejemplo se muestra la respuesta de una chimenea de acero de $90 \mathrm{~m}$ de altura sometida a la acción del viento, a continuación se estudia la influencia del número de planos de fluido utilizados para modelar la acción del viento en la respuesta transversal de un mástil de acero de $34 \mathrm{~m}$ de altura de sección constante y por último, se analiza el fenómeno de sincronización o de lock-in y la amplificación del coeficiente de arrastre para esa misma estructura. El análisis se realiza dentro del entorno de multifísica Kratos [1], el cual ha sido utilizado con éxito en la simulación de problemas de aeroeslasticidad en el trabajo de Rossi [2].

\subsection{Chimenea sometida a la acción del viento}

Según se expuso en el Capítulo 2, en el caso de chimeneas o torres sometidas a la acción del viento, el desprendimiento de torbellinos se desarrolla en células de torbellinos. Teniendo en cuenta esta hipótesis, parece lógico considerar que el flujo de aire en un punto de la estructura estará desacoplado del flujo que se desarrolla en otro punto de la misma, separados una cierta distancia. Esto sugiere la posibilidad de modelar la corriente de aire con un determinado número de planos 2D independientes entre sí, donde el fluido se resuelve por separado en cada plano. De esta manera, la acción del viento es modelada por medio de un cierto número de planos de fluido que actúan sobre ciertas secciones de la estructura a lo largo de su altura.

Conceptualmente, la solución en cada plano de fluido proporcionará una distribución de presiones sobre el contorno de la sección. Integrando sobre el contorno esa presión se obtiene una fuerza por unidad de longitud para dicha sección. A partir de estos valores discretos se interpola linealmente en cada tramo para obtener una ley de cargas por unidad de longitud distribuida sobre la estructura. La estructura se modela utilizando el modelo de barras descrito en el Capítulo 3 y su deformada establece la relación entre el movimiento de las diferentes secciones en las que actúa cada plano de fluido. 
La chimenea objeto de análisis es de acero, tiene una altura de $90 \mathrm{~m}$ y se encuentra situada dentro de una planta industrial en la provincia de Rayong (Tailandia). El cañón de la chimenea es una estructura lineal autosustentada por la sección inferior y con secciones transversales en forma de corona circular. El diámetro varía desde los $2.20 \mathrm{~m}$ en la parte superior hasta los $5.50 \mathrm{~m}$ en la base, tal como se muestra en la Figura 7.1. El espesor de la pared varía desde $22 \mathrm{~mm}$ en la base hasta $12 \mathrm{~mm}$ en el extremo libre según se indica en la Tabla 7.1. Respecto a las condiciones de contorno, se supondrá que está empotrada en la base y libre en la parte superior.

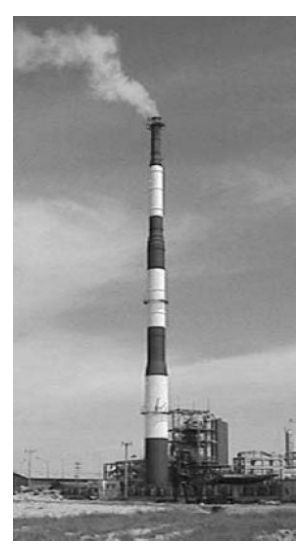

(a)

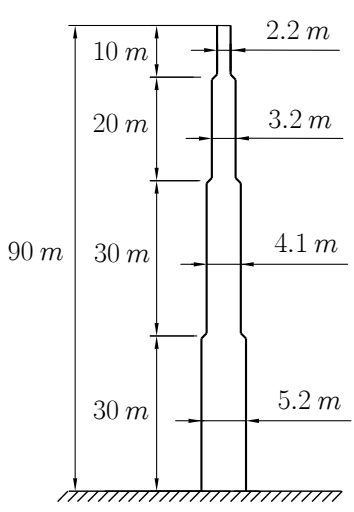

(b)

Figura 7.1. (a) Chimenea de $90 \mathrm{~m}$ en Rayong, (b) Dimensiones de la chimenea.

\begin{tabular}{|c|c|c|}
\hline Intervalo de altura $[\mathrm{m}]$ & Diámetro $[\mathrm{m}]$ & Espesor $[\mathrm{mm}]$ \\
\hline $0-30$ & 5.2 & 22 \\
\hline $30-60$ & 4.1 & 19 \\
\hline $60-80$ & 3.2 & 15 \\
\hline $80-90$ & 2.2 & 12 \\
\hline
\end{tabular}

Tabla 7.1. Datos de la chimenea

Las propiedades del acero son:

- densidad $\rho=7850 \mathrm{~kg} / \mathrm{m}^{3}$

- $\quad$ módulo de Young de elasticidad $E=2.1 \cdot 10^{11} \mathrm{~Pa}$

- $\quad$ módulo de cortadura $G=8.077 \cdot 10^{10} \mathrm{~Pa}$

El fluido es aire y sus propiedades son:

- viscosidad dinámica $\mu=1.810^{-5} \mathrm{Ns} / \mathrm{m}^{2}$

- densidad $\rho=1.21 \mathrm{~kg} / \mathrm{m}^{3}$ 
Antes de realizar el análisis acoplado se planteó el estudio de la chimenea sometida a una excitación tipo escalón de valor $1000 \mathrm{~N}$ aplicada en el extremo libre con objeto de caracterizar su primera frecuencia natural. Este dato servirá para calcular el paso de tiempo a utilizar en posteriores análisis y para realizar algunas comprobaciones. En la Figura 7.2 se ha representado la respuesta temporal de la chimenea y en la Figura 7.3 se muestra el análisis de Fourier de la misma. Se obtiene una primera frecuencia natural de $0.94 \mathrm{~Hz}$.

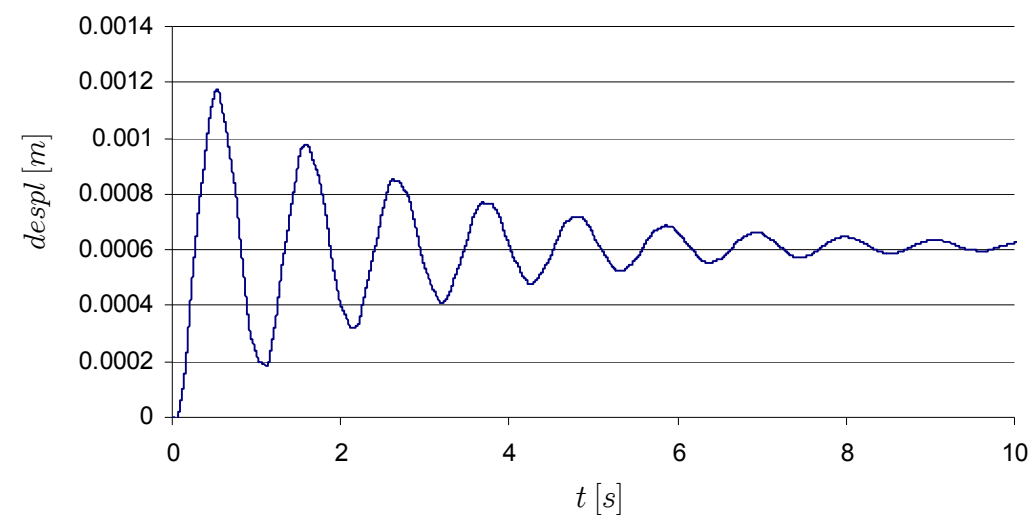

Figura 7.2. Respuesta temporal de la chimenea ante una excitación tipo escalón

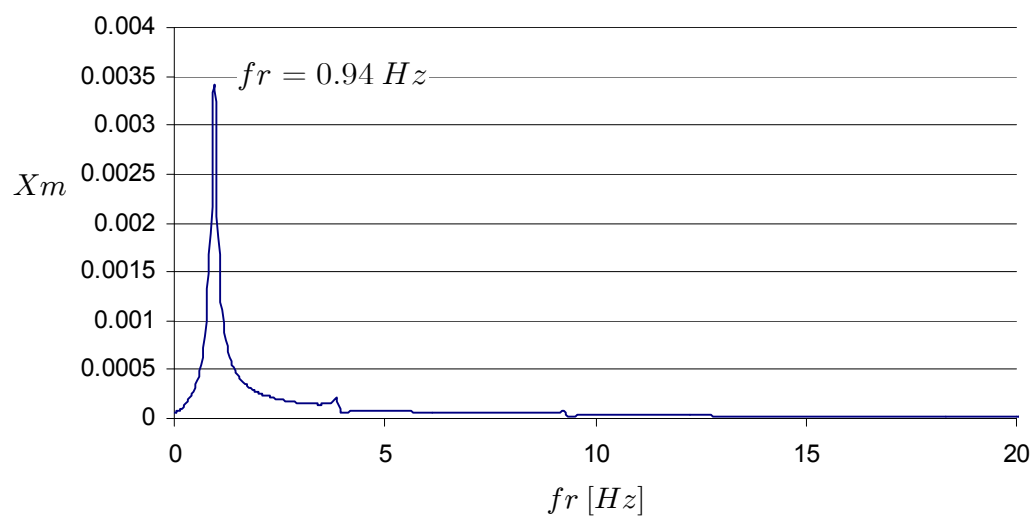

Figura 7.3. Análisis de Fourier de la respuesta temporal de la chimenea

Dado que la chimenea está constituida por cuatro tramos bien definidos con diferentes diámetros, el viento se modelará con cuatro planos de fluido situados a diferentes alturas $(30,60,80$ y $90 \mathrm{~m}$ de la base de la chimenea). Notar que según la ley de Strohual cada tramo tendrá asociada una frecuencia de desprendimiento de torbellinos diferente, tanto por la distribución del viento 
como por la variación del diámetro. La chimenea se considera que está orientada en la dirección del eje $O z$ y que el fluido circula en planos $O x y$. El modelo del problema se muestra en la Figura 7.4.

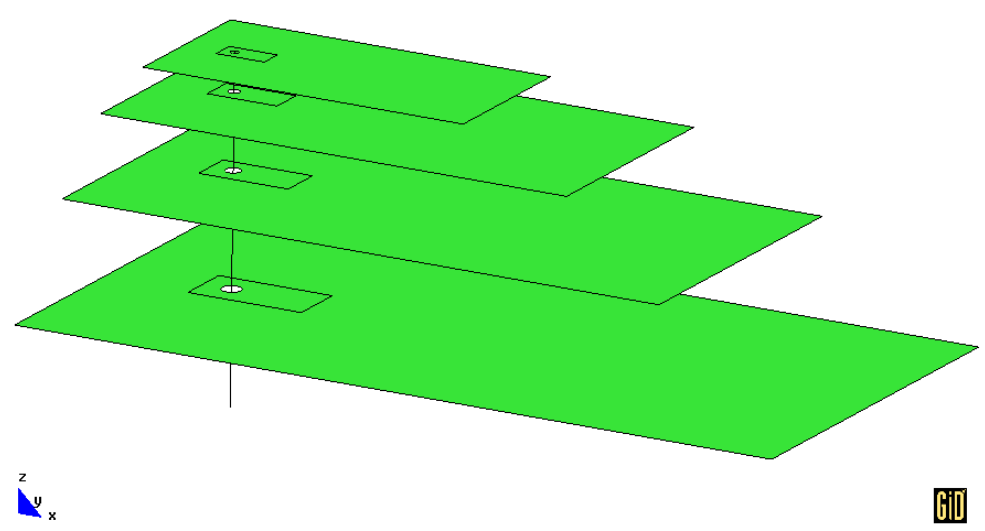

Figura 7.4. Modelo de la chimenea expuesta a la acción del viento

Al tener cada tramo de la chimenea una sección con un diámetro diferente, el dominio fluido asociado a cada sección varía con objeto de reducir el número de elementos finitos en cada plano de fluido y así también el coste computacional.

En la Figura 7.5 se muestra la geometría de uno de los planos de fluido considerados. Respecto a las condiciones de contorno, se impone una velocidad de entrada del fluido de valor $\left(v_{x}, 0\right)$ en el contorno $\Gamma_{u}$, en los contornos laterales $\Gamma_{w}$, se fija velocidad normal nula $v_{y}=0$, en el contorno de salida $\Gamma_{d}$, se asigna la condición de salida sin tensión (traction-free boundary condition) y en el contorno de la sección $S$, se fija la condición de no deslizamiento (no-slip condition). Inicialmente el campo de velocidades es nulo en todos los puntos salvo en la entrada.

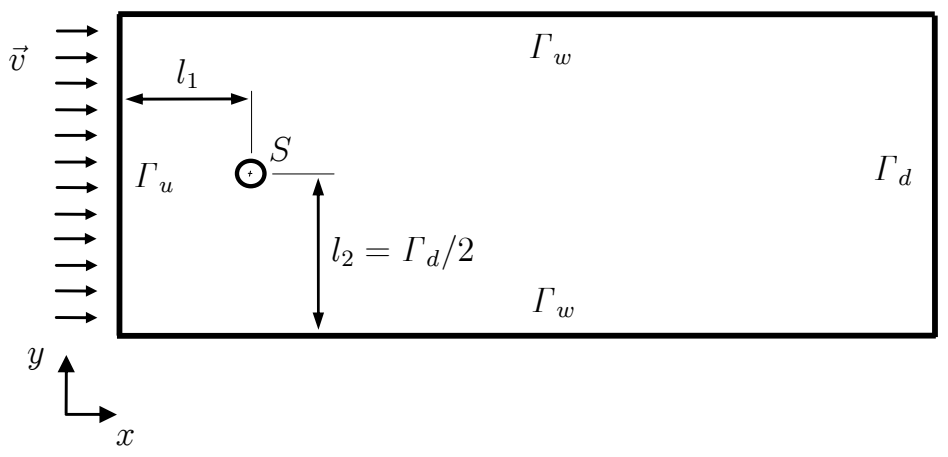

Figura 7.5. Geometría de un plano de fluido 
Se ha supuesto que el perfil de velocidades del viento viene dado por la expresión (7.1) [3]

$$
u=U_{r e f}\left(\frac{h}{h_{r e f}}\right)^{p}
$$

siendo $p$ el exponente del perfil del viento, $U_{r e f}$ la velocidad del viento a una altura de referencia $h_{\text {ref }}$ y $u$ la velocidad del viento a la altura $h$. La velocidad de entrada del fluido en cada plano se muestra en la Tabla 7.2 para $p=0.15, h_{r e f}=300 \mathrm{~m}$ y $U_{r e f}=15 \mathrm{~m} / \mathrm{s}$.

\begin{tabular}{|c|c|c|}
\hline $\boldsymbol{h}[m]$ & $\boldsymbol{v}_{\boldsymbol{x}}[\mathrm{m} / \mathrm{s}]$ & $\boldsymbol{R} \boldsymbol{e}$ \\
\hline 30 & 10.62 & $3.68 \mathrm{E}+06$ \\
\hline 60 & 11.78 & $3.22 \mathrm{E}+06$ \\
\hline 80 & 12.30 & $2.62 \mathrm{E}+06$ \\
\hline 90 & 12.52 & $1.84 \mathrm{E}+06$ \\
\hline
\end{tabular}

Tabla 7.2. Velocidades de entrada del viento

La chimenea fue mallada con cuatro elementos tipo barra y se asumió un amortiguamiento estructural proporcional a la matriz de masa, siendo el coeficiente de proporcionalidad 0.75. El dominio del fluido estaba constituido por 29694 elementos triangulares de superficie y las características de la geometría y del mallado de cada plano de fluido se indican a continuación en la Tabla 7.3 y Figura 7.6.

- Sección de diámetro $2.2 \mathrm{~m}$

\begin{tabular}{|c|c|c|c|}
\hline \multicolumn{2}{|c|}{ Longitud $[m]$} & \multicolumn{2}{c|}{ Tamaño característico $[m]$} \\
\hline$\Gamma_{w}$ & 88 & $a$ & 2.20 \\
\hline$\Gamma_{d}$ & 44 & $b$ & 0.44 \\
\hline$l_{1}=l_{3}$ & 13.2 & $S$ & 0.10 \\
\hline
\end{tabular}

- Sección de diámetro $4.1 \mathrm{~m}$

\begin{tabular}{|c|c|c|c|}
\hline \multicolumn{2}{|c|}{ Longitud $[m]$} & \multicolumn{2}{c|}{ Tamaño característico $[m]$} \\
\hline$\Gamma_{w}$ & 164 & $a$ & 4.10 \\
\hline$\Gamma_{d}$ & 82 & $b$ & 0.82 \\
\hline$l_{1}=l_{3}$ & 24.6 & $S$ & 0.19 \\
\hline
\end{tabular}

- Sección de diámetro $3.1 \mathrm{~m}$

\begin{tabular}{|c|c|c|c|}
\hline \multicolumn{2}{|c|}{ Longitud $[m]$} & \multicolumn{2}{c|}{ Tamaño característico $[m]$} \\
\hline$\Gamma_{w}$ & 128 & $a$ & 3.20 \\
\hline$\Gamma_{d}$ & 64 & $b$ & 0.64 \\
\hline$l_{1}=l_{3}$ & 19.2 & $S$ & 0.15 \\
\hline
\end{tabular}

- Sección de diámetro $5.2 \mathrm{~m}$

\begin{tabular}{|c|c|c|c|}
\hline \multicolumn{2}{|c|}{ Longitud $[m]$} & \multicolumn{2}{c|}{ Tamaño característico $[m]$} \\
\hline$\Gamma_{w}$ & 208 & $a$ & 5.20 \\
\hline$\Gamma_{d}$ & 104 & $b$ & 1.04 \\
\hline$l_{1}=l_{3}$ & 31.2 & $S$ & 0.24 \\
\hline
\end{tabular}

Tabla 7.3. Características geométricas y del mallado de los planos de fluido 

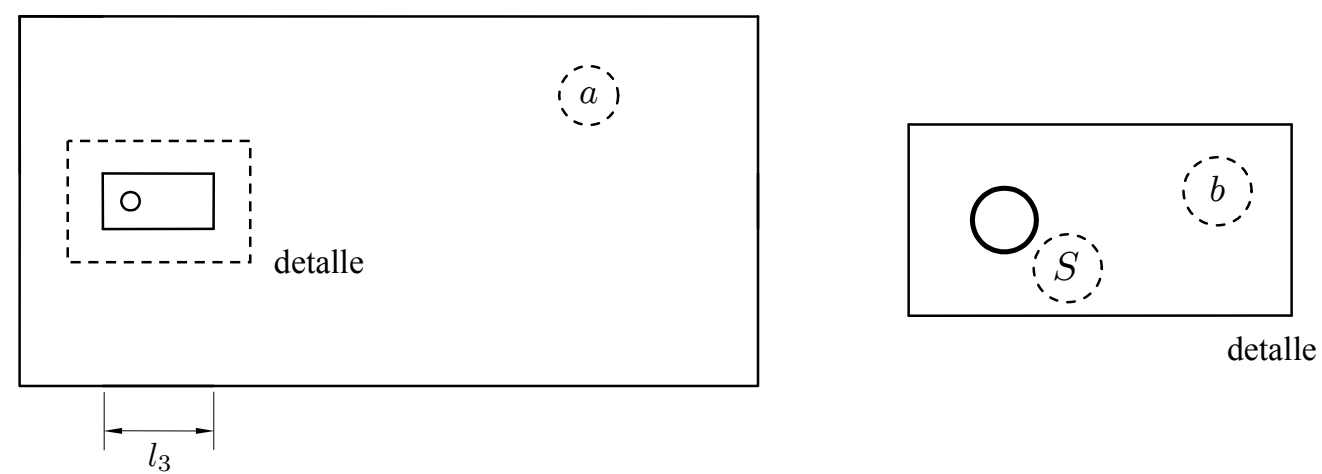

Figura 7.6. Características del mallado de un plano de fluido

Finalmente, en la Figura 7.7 se muestra la malla utilizada. En el anexo I se muestra una comparación de los resultados obtenidos con diferentes mallas.

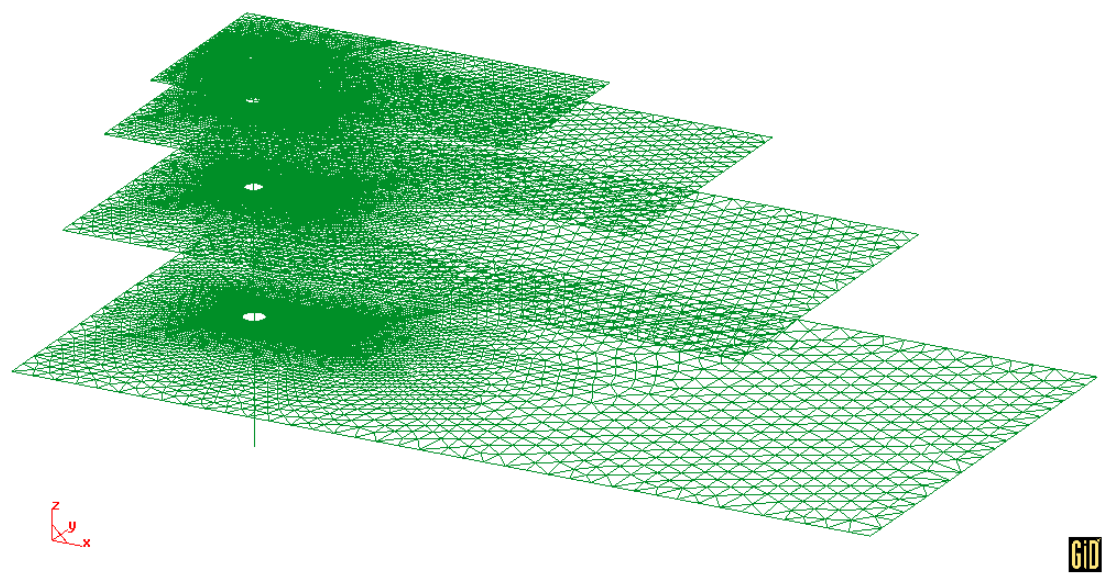

Figura 7.7. Malla del dominio del fluido

Dado que para encontrar la solución del problema acoplado es necesario resolver por separado el fluido y la estructura, se debe elegir un paso de tiempo adecuado para la resolución exitosa de ambos campos. Teniendo en cuenta la primera frecuencia natural de la chimenea, la velocidad de entrada del viento y el tamaño característico de los elementos de la malla del fluido se adoptó el valor de $0.002 s$ para el paso de tiempo.

La chimenea se somete a la acción del viento durante $40 \mathrm{~s}$. Transcurrido ese tiempo cesa la acción del viento y se estudia la respuesta libre de la estructura. Los resultados que se muestran a continuación corresponden a los desplazamientos del extremo libre de la chimenea según los ejes $O x$ (dirección paralela a la que actúa el viento) y $O y$ (dirección transversal a la que actúa el viento). 
En la Figura 7.8 se muestra el desplazamiento del extremo libre de la chimenea según el eje $O y$. Durante los primeros $40 \mathrm{~s}$ de estudio (desde $t=20 \mathrm{~s}$ a $t=60 \mathrm{~s}$ ) la chimenea vibra en esa dirección como consecuencia del desprendimiento alternado de torbellinos. Después de ese tiempo, la acción del fluido cesa y la chimenea vibra libremente hasta que recupera su posición de equilibrio. Nótese que la vibración en el eje $O y$ ocurre alrededor de la posición de equilibrio inicial.

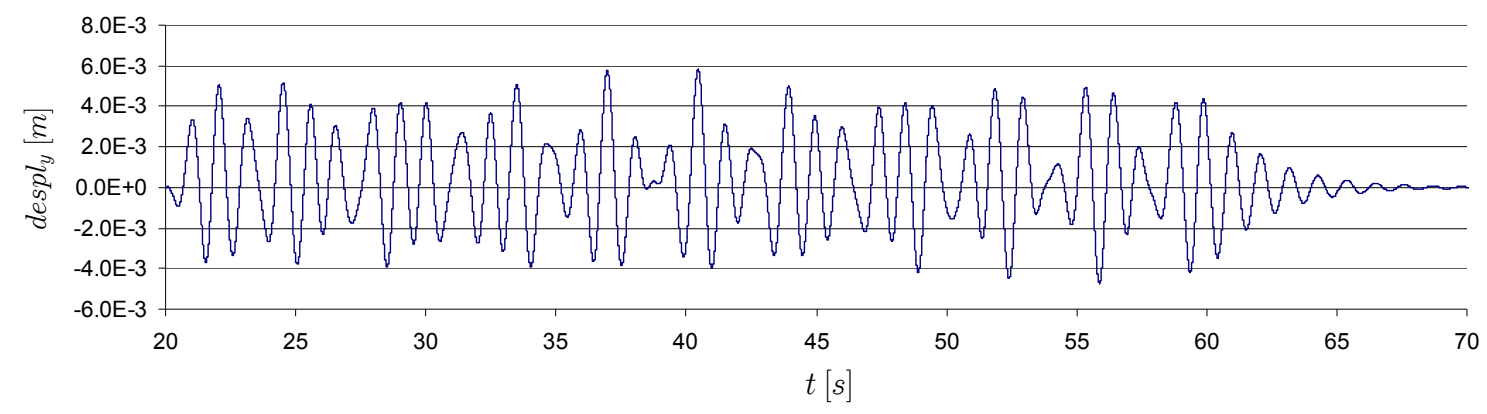

Figura 7.8. Vibración del extremo libre de la chimenea según el eje $O y$

El análisis FFT de la vibración forzada de la chimenea en el eje $O y$ entre $t=20 \mathrm{~s}$ y $t=60 \mathrm{~s}$ se muestra en la Figura 7.9. Se observa que la estructura vibra con varias frecuencias, predominando la frecuencia de $0.89 \mathrm{~Hz}$ sobre las frecuencias de $1.13,0.55$ y $1.62 \mathrm{~Hz}$.

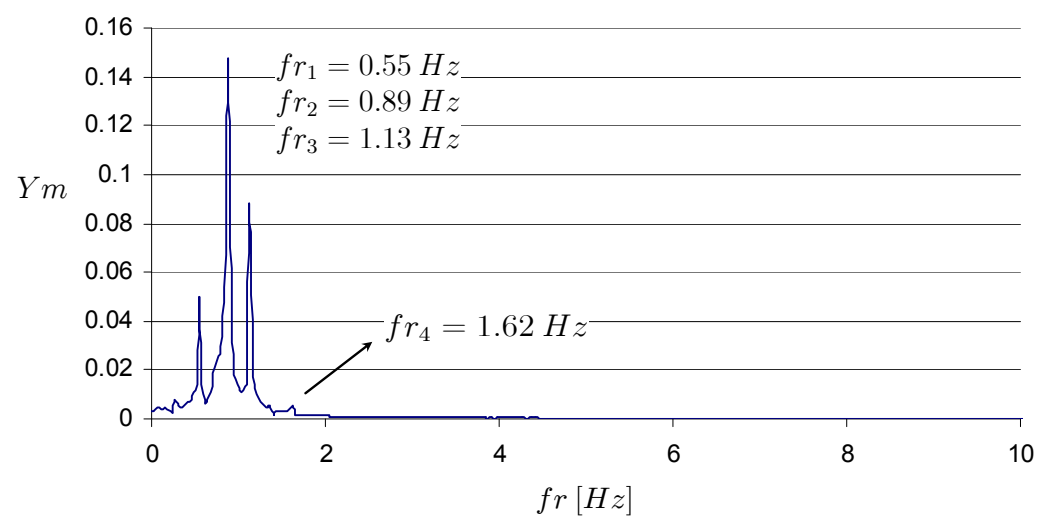

Figura 7.9. Análisis FFT de la vibración forzada de la chimenea en el eje $O y$

En la Figura $7.10 \mathrm{se}$ muestra el análisis FFT de la respuesta libre de la estructura entre $t=60 \mathrm{~s}$ y $t=70 \mathrm{~s}$. Se comprueba que vibra a su frecuencia natural de $0.94 \mathrm{~Hz}$. 


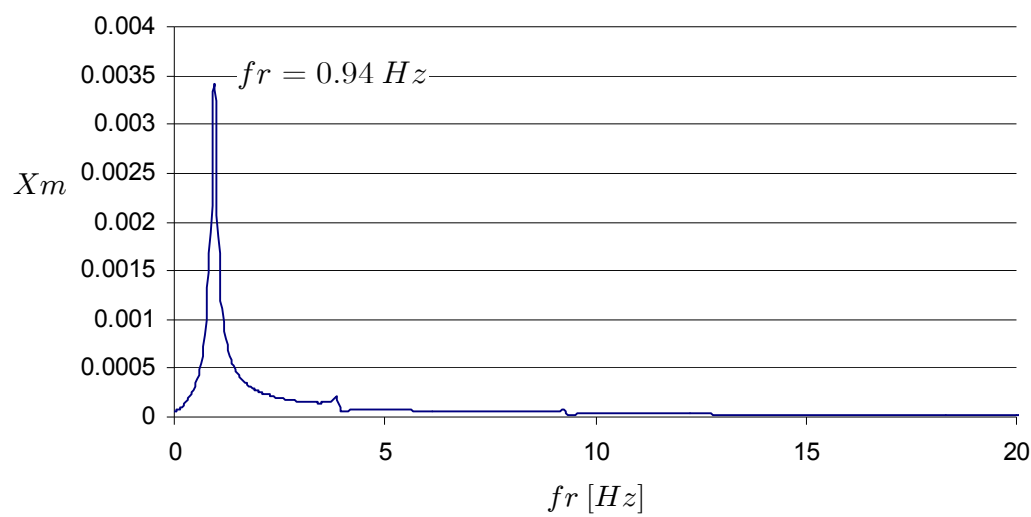

Figura 7.10. Análisis FFT de la respuesta libre de la chimenea en el eje $O y$

La variación del coeficiente de arrastre y de sustentación en el tiempo para la sección de la chimenea situada a $90 \mathrm{~m}$ de altura y con diámetro de $2.2 \mathrm{~m}$ se muestra en la Figura 7.11. En la Figura 7.12 se muestra el análisis FFT de la oscilación del coeficiente de sustentación y se puede observar que ésta ocurre a la frecuencia de $1.62 \mathrm{~Hz}$.

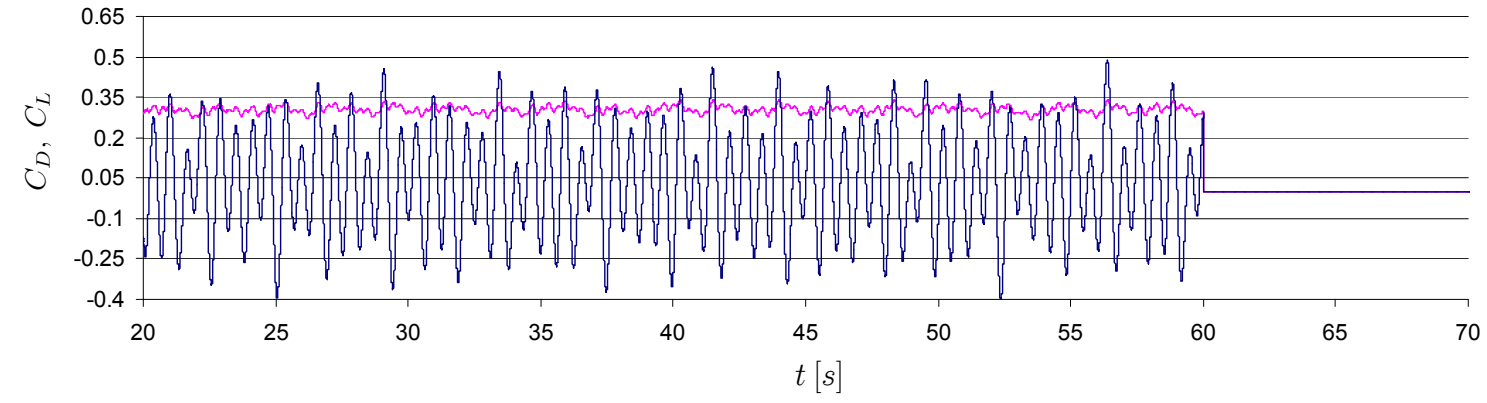

Figura 7.11. Variación del coeficiente de arrastre y de sustentación en el tiempo

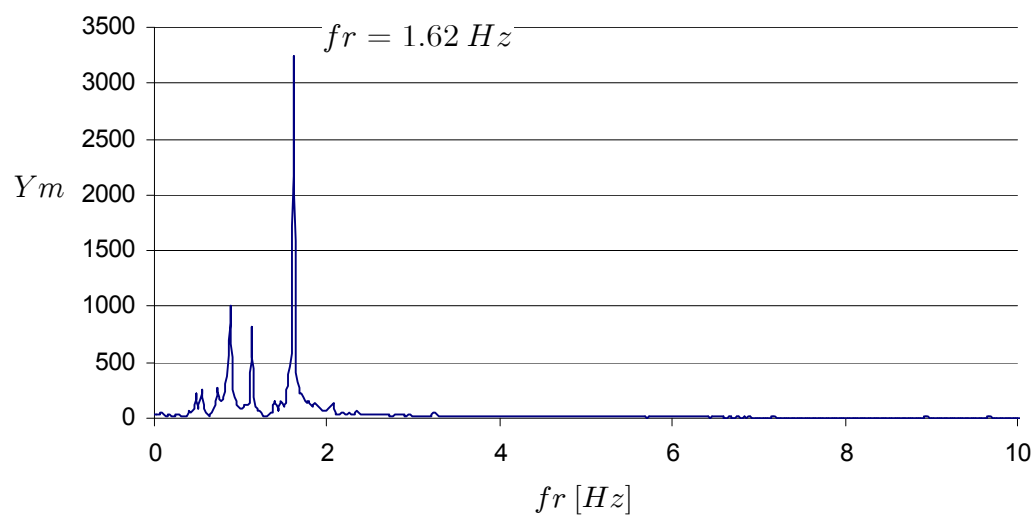

Figura 7.12. Análisis FFT de la oscilación de $C_{L}$ 
La frecuencia de oscilación del coeficiente de sustentación coincide con la frecuencia de desprendimiento de los torbellinos, de manera que aplicando la ley de Strouhal (7.2) se obtiene un número de Strouhal de 0.284, el cual está en sintonía con la Figura 2.8 del Capítulo 2 para $R e=1.84 \cdot 10^{6}$.

$$
S t=n_{s} \frac{d}{U}=1.62 \frac{2.2}{12.52}=0.284
$$

Por otro lado, el valor RMS del coeficiente de arrastre es 0.3 , el cual es coherente con la Figura 2.4 del Capítulo 2 para $R e=1.84 \cdot 10^{6}$.

De acuerdo con la ley de Strouhal y asumiendo que el número de Strouhal es 0.28, las frecuencias teóricas de la excitación causada por el desprendimiento periódico de torbellinos se muestran en la Tabla 7.4 para las distintas secciones de la chimenea.

\begin{tabular}{|c|c|c|c|}
\hline Diámetro $[\mathrm{m}]$ & Velocidad $[\mathrm{m} / \mathrm{s}]$ & Frec. de excitación $[\mathrm{Hz}]$ & Frec. de la respuesta $[\mathrm{Hz}]$ \\
\hline 5.2 & 10.62 & 0.58 & 0.55 \\
\hline 4.1 & 11.78 & 0.81 & 0.89 \\
\hline 3.2 & 12.30 & 1.09 & 1.13 \\
\hline 2.2 & 12.52 & 1.60 & 1.62 \\
\hline
\end{tabular}

Tabla 7.4. Frecuencias de excitación y de la respuesta

Comparando las frecuencias de excitación con las de la vibración transversal de la estructura (Figura 7.9), se observa que existe una buena sintonía entre ellas. Cabe destacar que en la oscilación del coeficiente de sustentación existen las frecuencias $0.55,0.89$ y $1.13 \mathrm{~Hz}$, las cuales no coinciden exactamente con ninguna de las frecuencias de excitación debido a la interacción entre el movimiento de la estructura y el dominio del fluido.

En la Figura 7.13 se muestra la vibración del extremo libre de la estructura según el eje $O x$. Inicialmente (entre $t=20 \mathrm{~s}$ y $t=25 \mathrm{~s}$ aproximadamente) la chimenea vibra con una amplitud mayor hasta que alcanza la nueva posición de equilibrio respecto a la cual prácticamente no vibra. Cuando cesa la acción del viento $(t>60 \mathrm{~s})$ la chimenea tiende a recuperar la posición de equilibrio inicial. Finalmente, en la Figura 7.14 se muestra el análisis FFT de la vibración forzada y de la vibración libre de la chimenea en el eje $O x$. Se comprueba que tanto en la vibración forzada como en la vibración libre predomina la frecuencia natural, observándose un pico en la vibración forzada a la frecuencia de $0.27 \mathrm{~Hz}$ correspondiente a la oscilación del coeficiente de arrastre.

Resulta atractivo representar las curvas de Lissajous de la trayectoria que describen las diferentes secciones de la chimenea. En la Figura 7.15 se muestra la trayectoria descrita por las 
distintas secciones entre $t=25 \mathrm{~s}$ y $t=60 \mathrm{~s}$ y en la Figura 7.16 se ha representado la respuesta libre para $t>60 \mathrm{~s}$ En el Anexo K se muestran este tipo de curvas para un mástil de $34 \mathrm{~m}$ de altura.

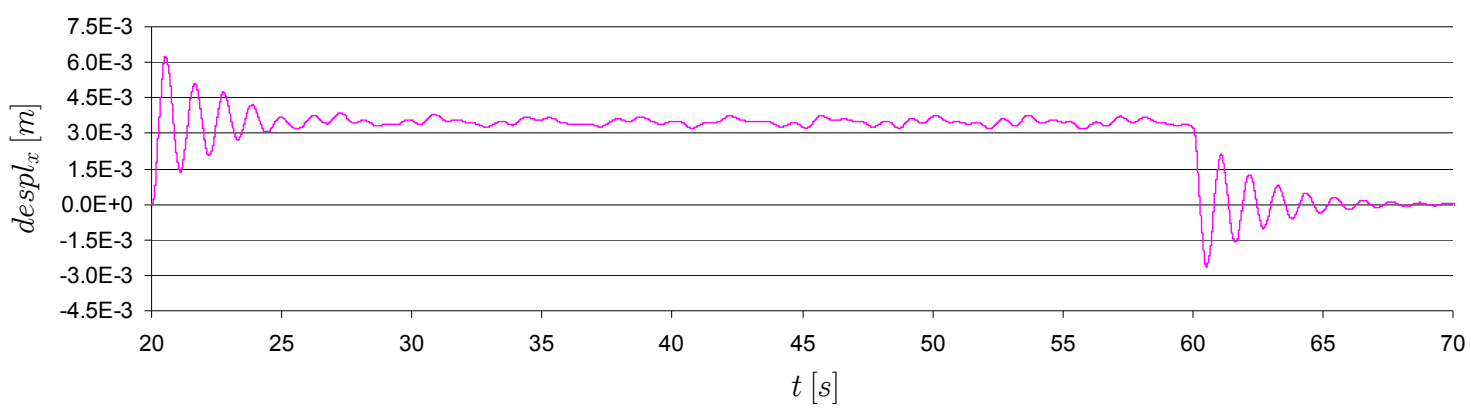

Figura 7.13. Vibración del extremo libre de la chimenea según el eje $O x$

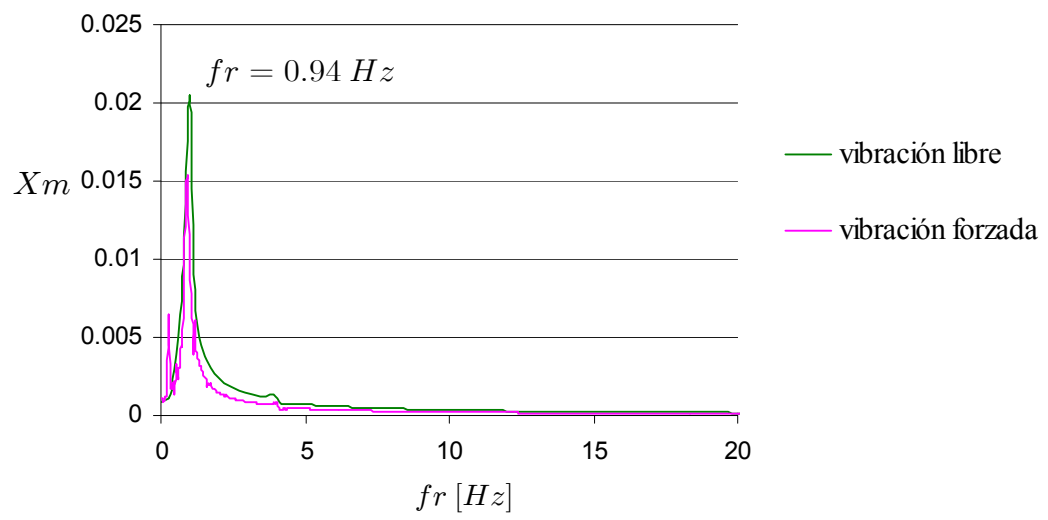

Figura 7.14. Análisis FFT de la respuesta libre de la chimenea en el eje $O x$

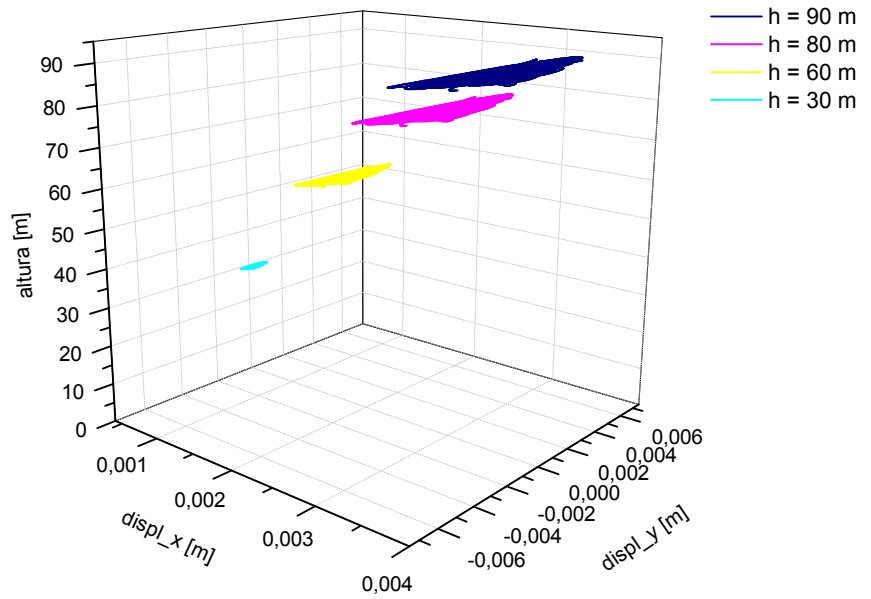

Figura 7.15. Curvas de Lissajous de la trayectoria de la chimenea entre $t=25 \mathrm{~s}$ y $t=60 \mathrm{~s}$ 


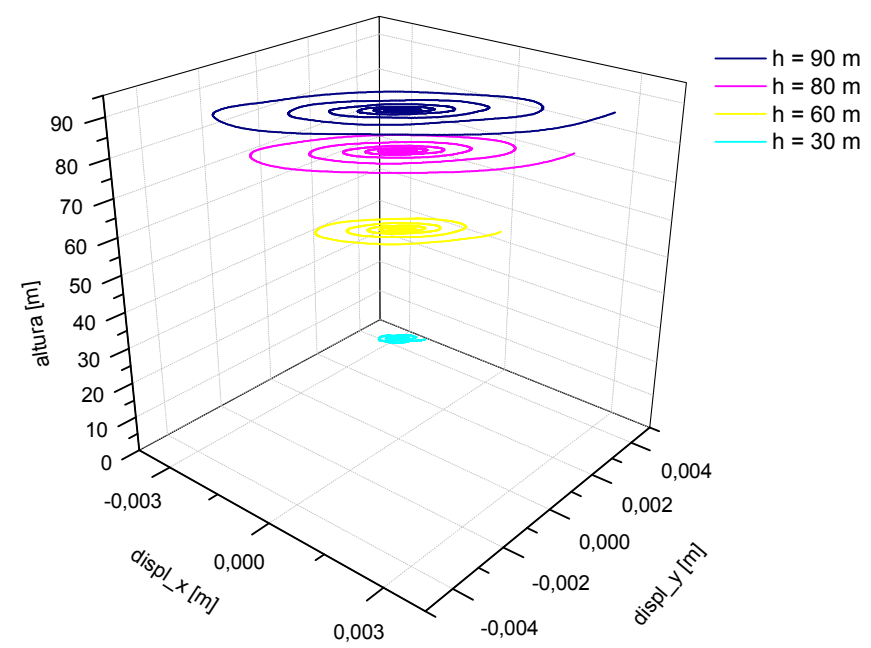

Figura 7.16. Curvas de Lissajous de la trayectoria de la chimenea para $t>60 \mathrm{~s}$

\subsection{Estudio del número de planos de fluido en la respuesta transversal de un mástil de acero}

Se pretende estudiar la influencia del número de planos de fluido utilizados para modelar la acción del viento en la respuesta de una estructura en la dirección transversal a la que actúa éste. Para ello se comparará la respuesta de un mástil de sección circular de acero para los casos en los que el viento es discretizado por medio de 4,6 y 8 planos.

Para simplificar se ha considerado que la sección es constante. La altura del mástil es $34 \mathrm{~m}$, el diámetro $0.5 \mathrm{~m}$ y el espesor $4.8 \mathrm{~mm}$. Las propiedades del material son:

- densidad $\rho=7772 \mathrm{~kg} / \mathrm{m}^{3}$

- módulo de Young de elasticidad $E=2.1 \cdot 10^{11} \mathrm{~Pa}$

- módulo de cortadura $G=8.077 \cdot 10^{10} \mathrm{~Pa}$

Respecto a las condiciones de contorno, se considera que el mástil está empotrado en la base y libre en la parte superior.

La estructura fue mallada con 4, 6 y 8 elementos tipo barra en función del número de planos utilizados para modelar el viento respectivamente y se asumió un amortiguamiento estructural proporcional a la matriz de masa, siendo el coeficiente de proporcionalidad 0.1 . 
Con el fin de determinar la primera frecuencia natural de la estructura se realizó un estudio dinámico de la misma. En la Figura 7.17 se muestra la respuesta dinámica de la estructura ante una excitación tipo escalón de $10 \mathrm{~N}$ aplicada en el extremo libre y en la Figura 7.18 se ha representado el análisis FFT de la respuesta dinámica correspondiente al movimiento en modo 1 de flexión con la frecuencia de $0.43 \mathrm{~Hz}$, que será la primera frecuencia natural buscada.

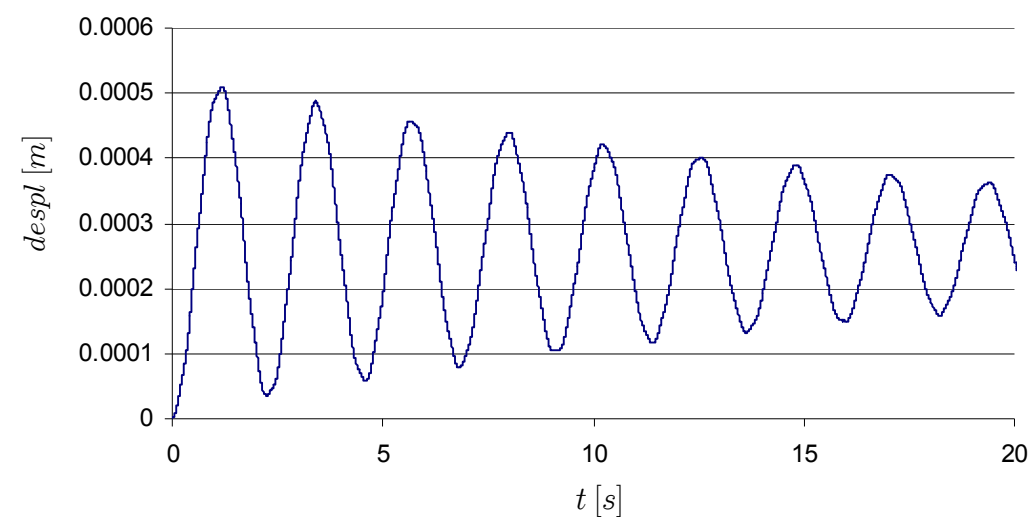

Figura 7.17. Respuesta dinámica de la estructura

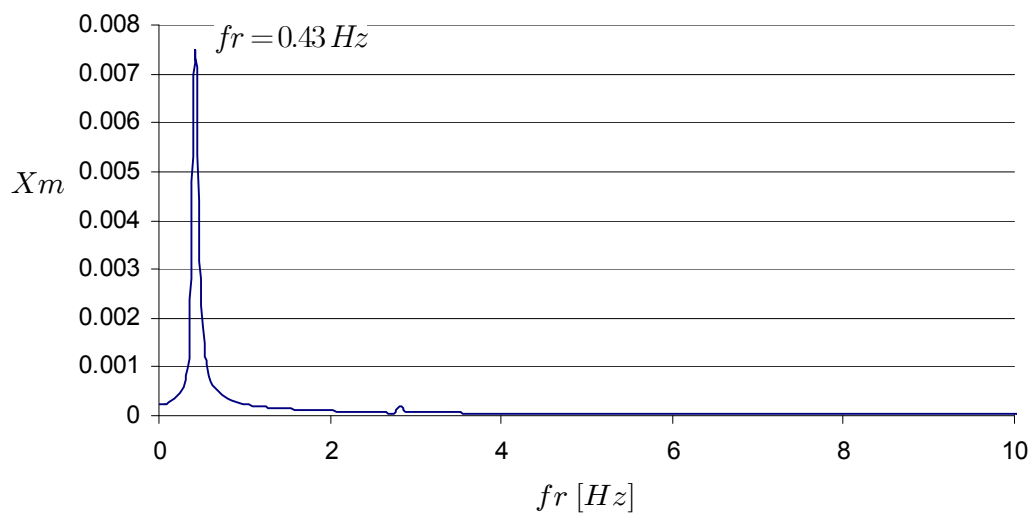

Figura 7.18. Análisis FFT de la respuesta dinámica de la estructura

El fluido es aire y sus propiedades son:

- viscosidad dinámica $\mu=1.810^{-5} \mathrm{Ns} / \mathrm{m}^{2}$

- densidad $\rho=1.21 \mathrm{~kg} / \mathrm{m}^{3}$

De forma análoga al ejemplo anterior, el fluido se supondrá actuando en planos perpendiculares a la directriz de la estructura situados a distintas alturas en cada caso de estudio según se muestra en la Tabla 7.11. 


\begin{tabular}{|c|c|}
\hline Número de planos & Altura respecto a la base $[m]$ \\
\hline 4 & $34,25,17,8.5$ \\
\hline 6 & $34,28.25,22.6,16.95,11.30,5,65$ \\
\hline 8 & $34,29.75,25,21.25,17,12.75,8.5,4.25$ \\
\hline
\end{tabular}

Tabla 7.5. Posición de los planos de fluido

En la Figura 7.19 se muestran los distintos modelos de la estructura sometida a la acción viento para los casos en los éste es modelado con 4, 6 y 8 planos. Notar que todos los planos de fluido son iguales.

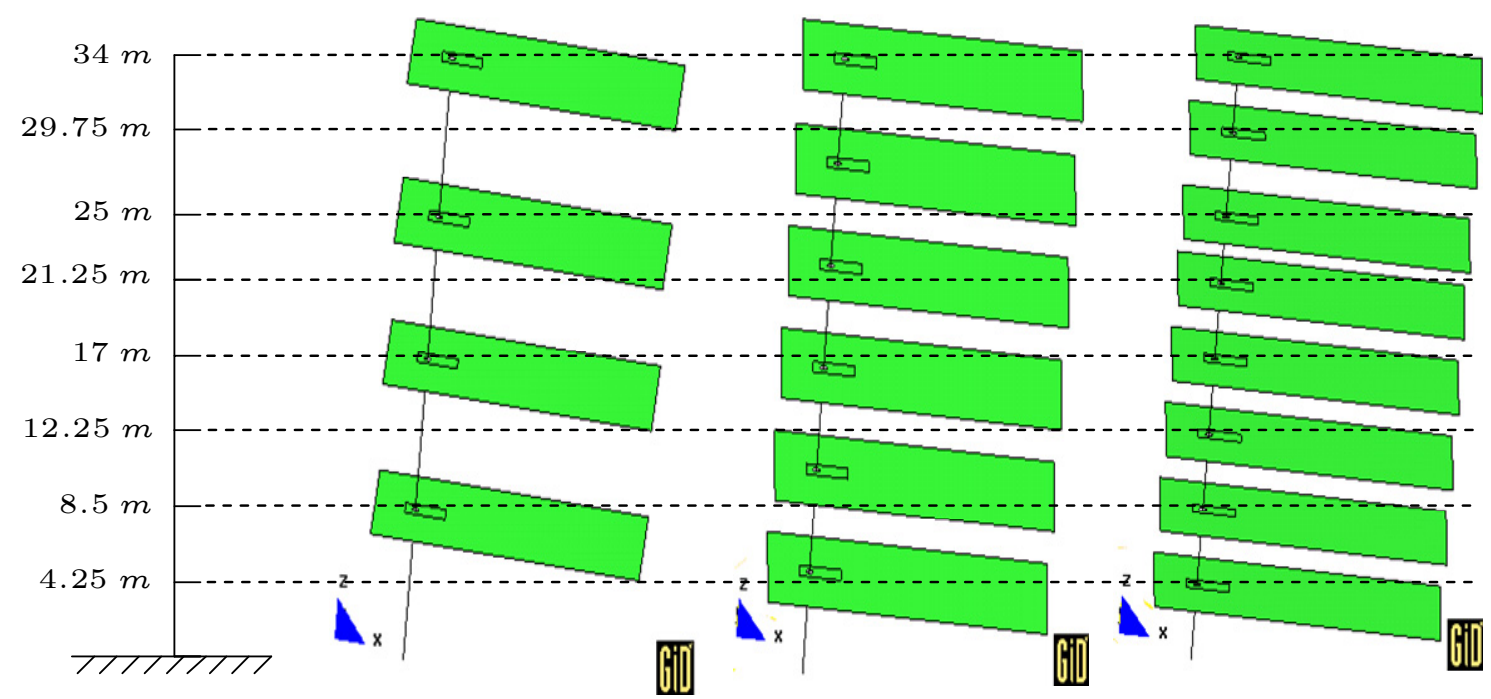

Figura 7.19. Modelo de la estructura sometida a la acción del viento

Las dimensiones del dominio del fluido se muestran en la Figura 7.20. Respecto a las condiciones de contorno, se impone una velocidad de entrada del fluido de valor $\left(v_{x}, 0\right)$ en el contorno $\Gamma_{u}$, en los contornos laterales $\Gamma_{w}$, se fija velocidad normal nula $v_{y}=0$, en el contorno de salida $\Gamma_{d}$, se asigna la condición de salida sin tensión (traction-free boundary condition) y en el contorno del cilindro $S$, se fija la condición de no deslizamiento (no-slip condition). Inicialmente el campo de velocidades es nulo en todos los puntos excepto en la entrada.

El mallado del contorno el cilindro $S$ se realizó asignándole un tamaño de 0.05 , y el del dominio del fluido de acuerdo con el esquema de la Figura 7.21, concentrando un mayor número de elementos en el entorno más próximo al cilindro. La malla resultante estaba constituida por 5846 elementos triangulares de superficie y tiene la forma que se muestra en la Figura 7.22. 


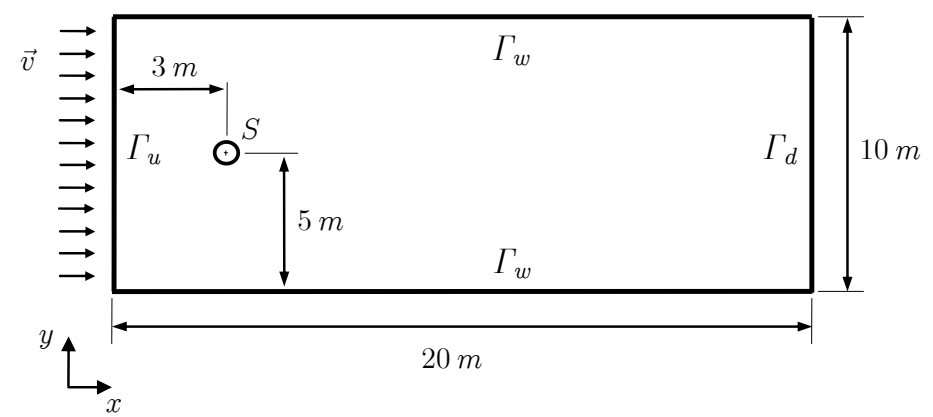

Figura 7.20. Dominio del fluido
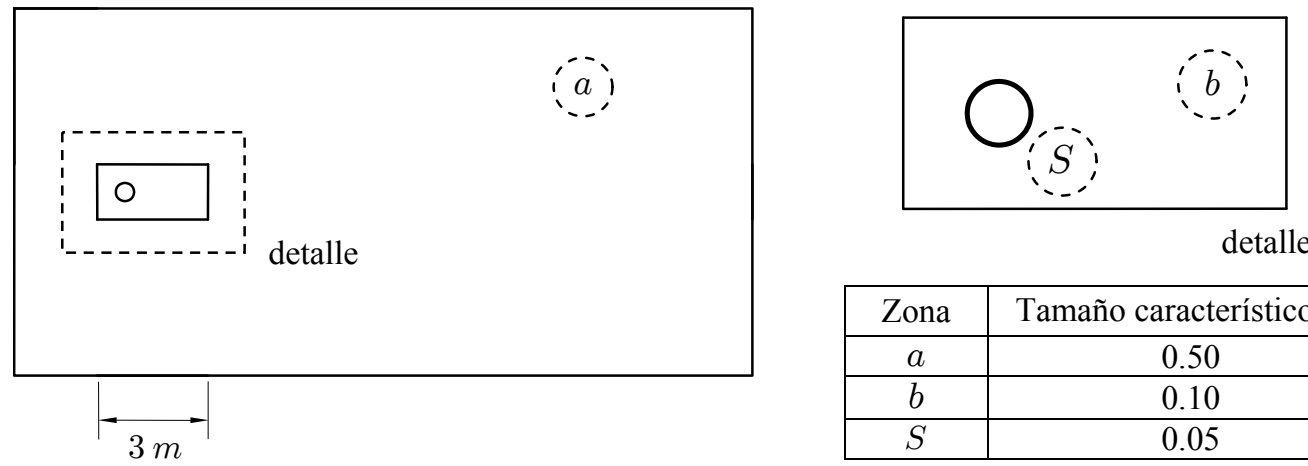

\begin{tabular}{|c|c|}
\hline Zona & Tamaño característico $[m]$ \\
\hline$a$ & 0.50 \\
\hline$b$ & 0.10 \\
\hline$S$ & 0.05 \\
\hline
\end{tabular}

Figura 7.21. Esquema de mallado del dominio del fluido

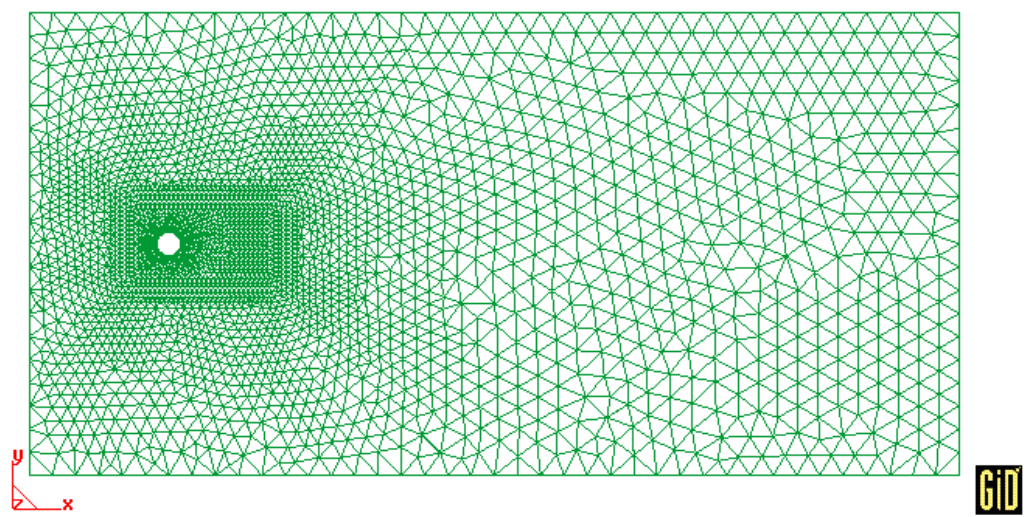

Figura 7.22. Mallado del dominio del fluido

Se ha supuesto un perfil de velocidades del viento exponencial de acuerdo con la expresión (7.1). La velocidad de entrada del fluido en cada plano se muestra en la Tabla 7.6, Tabla 7.7 y Tabla 7.8 para los casos estudiados con 8,6 y 4 planos respectivamente siendo $p=0.15$ y $h_{r e f}=34 \mathrm{~m}$. 


\begin{tabular}{|c|c|c|c|c|c|c|c|c|c|c|c|}
\hline \multirow{10}{*}{$\begin{array}{l}\frac{\mathscr{E}}{0} \\
\frac{\tilde{\sigma}}{2} \\
\infty\end{array}$} & $\begin{array}{c}\text { Altura } \\
{[\mathrm{m}]}\end{array}$ & 34.00 & 29.75 & 25.00 & 21.25 & 17.00 & 12.75 & 8.50 & 4.25 & $U_{r}$ & $\begin{array}{l}\boldsymbol{U}_{\boldsymbol{r} e f} \\
{[\mathrm{~m} / \mathrm{s}]}\end{array}$ \\
\hline & \multirow{9}{*}{$\boldsymbol{v}_{\boldsymbol{x}}[\mathrm{m} / \mathrm{s}]$} & 2.88 & 2.82 & 2.75 & 2.68 & 2.60 & 2.49 & 2.34 & 2.11 & 13.0 & 2.88 \\
\hline & & 2.43 & 2.38 & 2.32 & 2.26 & 2.19 & 2.10 & 1.97 & 1.78 & 11.0 & 2.43 \\
\hline & & 1.55 & 1.52 & 1.48 & 1.44 & 1.40 & 1.34 & 1.26 & 1.13 & 7.0 & 1.55 \\
\hline & & 1.33 & 1.30 & 1.27 & 1.24 & 1.20 & 1.15 & 1.08 & 0.97 & 6.0 & 1.33 \\
\hline & & 1.22 & 1.20 & 1.17 & 1.14 & 1.10 & 1.05 & 1.00 & 0.89 & 5.5 & 1.22 \\
\hline & & 1.11 & 1.09 & 1.06 & 1.03 & 1.00 & 0.96 & 0.90 & 0.81 & 5.0 & 1.11 \\
\hline & & 1.00 & 0.98 & 0.95 & 0.93 & 0.90 & 0.86 & 0.81 & 0.73 & 4.5 & 1.00 \\
\hline & & 0.90 & 0.88 & 0.86 & 0.84 & 0.81 & 0.78 & 0.73 & 0.66 & 4.0 & 0.90 \\
\hline & & 0.77 & 0.75 & 0.74 & 0.72 & 0.69 & 0.66 & 0.63 & 0.56 & 3.5 & 0.77 \\
\hline
\end{tabular}

Tabla 7.6. Velocidades de entrada del fluido para 8 planos

\begin{tabular}{|c|c|c|c|c|c|c|c|c|c|}
\hline \multirow{10}{*}{ 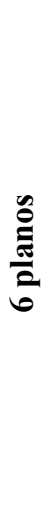 } & $\begin{array}{c}\text { Altura } \\
{[\mathrm{m}]}\end{array}$ & 34.00 & 28.25 & 22.60 & 16.95 & 11.30 & 5.65 & $U_{r}$ & $\begin{array}{l}\boldsymbol{U}_{\text {ref }} \\
{[\mathrm{m} / \mathrm{s}]}\end{array}$ \\
\hline & \multirow{9}{*}{$\boldsymbol{v}_{\boldsymbol{x}}[\mathrm{m} / \mathrm{s}]$} & 2.88 & 2.80 & 2.71 & 2.598 & 2.44 & 2.20 & 13.0 & 2.88 \\
\hline & & 2.43 & 2.36 & 2.29 & 2.19 & 2.06 & 1.86 & 11.0 & 2.43 \\
\hline & & 1.55 & 1.51 & 1.46 & 1.40 & 1.31 & 1.18 & 7.0 & 1.55 \\
\hline & & 1.33 & 1.29 & 1.25 & 1.20 & 1.13 & 1.02 & 6.0 & 1.33 \\
\hline & & 1.22 & 1.19 & 1.15 & 1.10 & 1.03 & 0.93 & 5.5 & 1.22 \\
\hline & & 1.11 & 1.08 & 1.04 & 1.00 & 0.94 & 0.85 & 5.0 & 1.11 \\
\hline & & 1.00 & 0.97 & 0.94 & 0.90 & 0.85 & 0.76 & 4.5 & 1.00 \\
\hline & & 0.90 & 0.88 & 0.85 & 0.81 & 0.76 & 0.69 & 4.0 & 0.90 \\
\hline & & 0.77 & 0.75 & 0.72 & 0.69 & 0.65 & 0.59 & 3.5 & 0.77 \\
\hline
\end{tabular}

Tabla 7.7. Velocidades de entrada del fluido para 6 planos

\begin{tabular}{|c|c|c|c|c|c|c|c|}
\hline \multirow{10}{*}{ 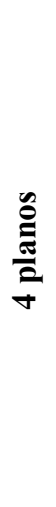 } & $\begin{array}{c}\text { Altura } \\
{[\mathrm{m}]}\end{array}$ & 34.00 & 25.00 & 17.00 & 8.50 & $\boldsymbol{U}_{r}$ & $\begin{array}{l}\boldsymbol{U}_{r e f} \\
{[\mathrm{~m} / \mathrm{s}]}\end{array}$ \\
\hline & \multirow{9}{*}{$\boldsymbol{v}_{\boldsymbol{x}}[\mathrm{m} / \mathrm{s}]$} & 2.88 & 2.75 & 2.60 & 2.34 & 13.0 & 2.88 \\
\hline & & 2.43 & 2.32 & 2.19 & 1.97 & 11.0 & 2.43 \\
\hline & & 1.55 & 1.48 & 1.40 & 1.26 & 7.0 & 1.55 \\
\hline & & 1.33 & 1.27 & 1.20 & 1.08 & 6.0 & 1.33 \\
\hline & & 1.22 & 1.17 & 1.10 & 1.00 & 5.5 & 1.22 \\
\hline & & 1.11 & 1.06 & 1.00 & 0.90 & 5.0 & 1.11 \\
\hline & & 1.00 & 0.95 & 0.90 & 0.81 & 4.5 & 1.00 \\
\hline & & 0.90 & 0.86 & 0.81 & 0.73 & 4.0 & 0.90 \\
\hline & & 0.77 & 0.74 & 0.69 & 0.63 & 3.5 & 0.77 \\
\hline
\end{tabular}

Tabla 7.8. Velocidades de entrada del fluido para 4 planos 
A modo de ejemplo, en la Figura 7.23 se ha representado el perfil de velocidades para $U_{r}=7$.

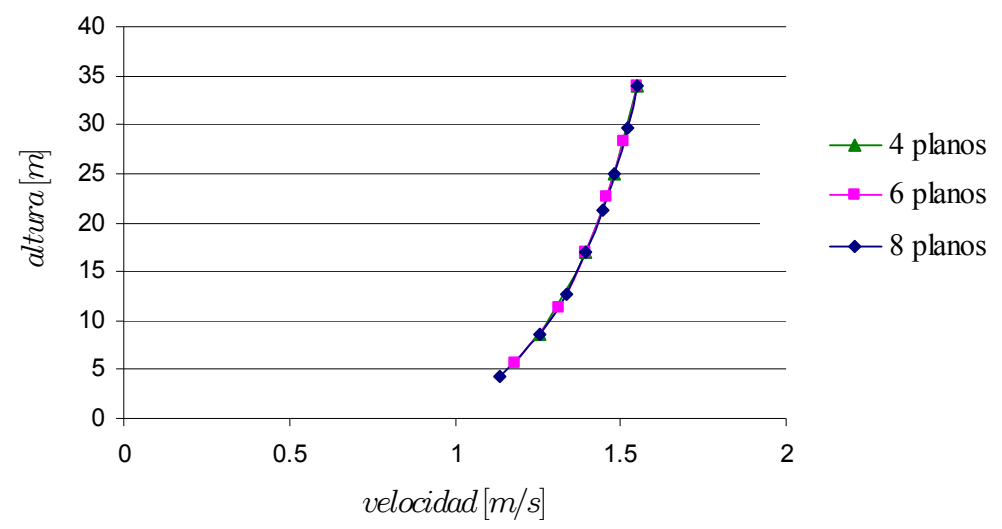

Figura 7.23. Perfil de velocidades para el caso $U_{r}=7$

De la misma manera que ocurría en el ejemplo anterior, es necesario elegir un paso de tiempo adecuado para la resolución exitosa del problema acoplado. Teniendo en cuenta la primera frecuencia natural de la estructura, la velocidad de entrada del viento y el tamaño característico de los elementos de la malla del fluido se adoptó como valor del paso de tiempo $0.005 s$ para todos los casos estudiados.

En la Tabla 7.9 se muestran los resultados numéricos relativos al desplazamiento transversal del extremo libre de la estructura para 8, 6 y 4 planos y en la Figura 7.24 y Figura 7.25 se han representado gráficamente esos resultados. Notar que en la Figura 7.24 se han utilizado dos representaciones gráficas, una en escala normal y otra en escala logarítmica, para una mayor claridad en la representación de los resultados.

\begin{tabular}{|c|c|c|c|}
\hline $\boldsymbol{U}_{\boldsymbol{r}}$ & $\mathbf{8}$ planos, $\boldsymbol{A}_{\boldsymbol{y}} / \boldsymbol{d}$ & $\mathbf{6 ~ p l a n o s}, \boldsymbol{A}_{\boldsymbol{y}} / \boldsymbol{d}$ & $\mathbf{4}$ planos, $\boldsymbol{A}_{\boldsymbol{y}} / \boldsymbol{d}$ \\
\hline 3.5 & 0.001434 & 0.001416 & 0.001422 \\
\hline 4.0 & 0.003208 & 0.003245 & 0.003104 \\
\hline 4.5 & 0.006870 & 0.006616 & 0.007340 \\
\hline 5.0 & 0.014792 & 0.014905 & 0.015006 \\
\hline 5.5 & 0.008091 & 0.008837 & 0.006762 \\
\hline 6.0 & 0.004335 & 0.004282 & 0.004273 \\
\hline 7.0 & 0.002433 & 0.002438 & 0.002297 \\
\hline 11.0 & 1.138332 & 1.090476 & 0.991824 \\
\hline 13.0 & 2.015796 & 1.932944 & 1.791256 \\
\hline
\end{tabular}

Tabla 7.9. Respuesta transversal de la estructura en desplazamientos 

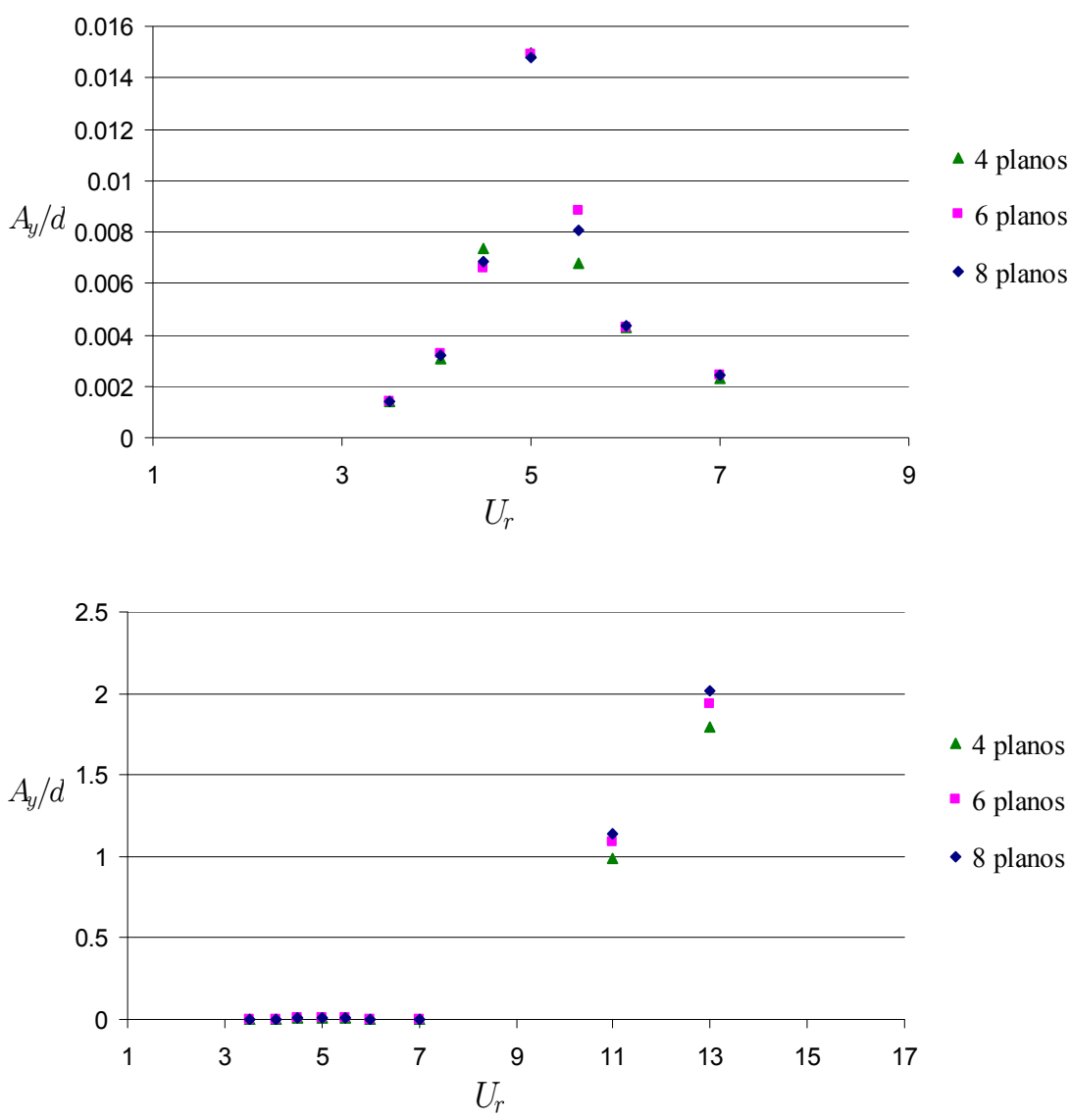

Figura 7.24. Respuesta transversal de la estructura en desplazamientos

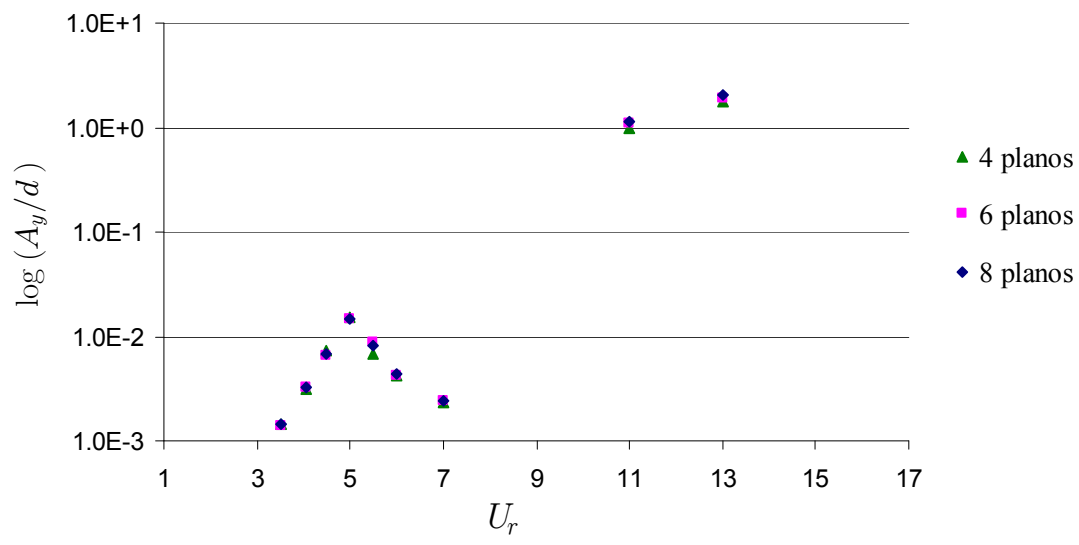

Figura 7.25. Respuesta transversal de la estructura en desplazamientos en escala logarítmica

En la Tabla 7.10 se indican numéricamente las frecuencias asociadas al movimiento transversal de la estructura y a la excitación correspondiente al plano de fluido que actúa en el extremo libre de la estructura. 


\begin{tabular}{|c|c|c|c|c|c|c|c|c|c|c|}
\hline \multirow[b]{2}{*}{$U_{r}$} & \multirow[b]{2}{*}{ Re } & \multicolumn{3}{|c|}{8 planos } & \multicolumn{3}{|c|}{6 planos } & \multicolumn{3}{|c|}{4 planos } \\
\hline & & $\begin{array}{c}\boldsymbol{n}_{s} \text { despl. } \\
{[H z]}\end{array}$ & $\begin{array}{c}\boldsymbol{n}_{s} \mathbf{F y} \\
{[H z]}\end{array}$ & $S t$ & $\begin{array}{c}\boldsymbol{n}_{s} \text { despl. } \\
{[H z]}\end{array}$ & $\begin{array}{c}\boldsymbol{n}_{s} \mathbf{F y} \\
{[H z]}\end{array}$ & $S t$ & $\begin{array}{c}\boldsymbol{n}_{s} \text { despl. } \\
{[H z]}\end{array}$ & $\begin{array}{c}\boldsymbol{n}_{\boldsymbol{s}} \mathbf{F y} \\
{[H z]}\end{array}$ & $S t$ \\
\hline 3.5 & $5.16 \mathrm{E}+04$ & 0.31 & 0.32 & 0.21 & 0.31 & 0.32 & 0.21 & 0.31 & 0.32 & 0.21 \\
\hline 4.0 & $4.43 \mathrm{E}+04$ & 0.37 & 0.37 & 0.21 & 0.37 & 0.37 & 0.21 & 0.37 & 0.37 & 0.21 \\
\hline 4.5 & $4.06 \mathrm{E}+04$ & 0.41 & 0.41 & 0.21 & 0.40 & 0.41 & 0.21 & 0.39 & 0.41 & 0.21 \\
\hline 5.0 & $3.69 \mathrm{E}+04$ & 0.42 & 0.45 & 0.20 & 0.42 & 0.45 & 0.20 & 0.44 & 0.46 & 0.20 \\
\hline 5.5 & $3.32 \mathrm{E}+04$ & 0.43 & 0.51 & 0.21 & 0.43 & 0.51 & 0.21 & 0.49 & 0.51 & 0.21 \\
\hline 6.0 & $2.99 \mathrm{E}+04$ & 0.54 & 0.56 & 0.21 & 0.54 & 0.56 & 0.21 & 0.53 & 0.56 & 0.21 \\
\hline 7.0 & $5.90 \mathrm{E}+04$ & 0.63 & 0.65 & 0.21 & 0.63 & 0.65 & 0.21 & 0.62 & 0.65 & 0.21 \\
\hline 11.0 & $9.59 \mathrm{E}+04$ & 0.43 & 0.43 & 0.09 & 0.43 & 0.43 & 0.09 & 0.43 & 0.43 & 0.09 \\
\hline 13.0 & $1.11 \mathrm{E}+05$ & 0.43 & 0.43 & 0.07 & 0.43 & 0.43 & 0.07 & 0.43 & 0.43 & 0.07 \\
\hline
\end{tabular}

Tabla 7.10. Respuesta en frecuencias de la estructura

Finalmente, en la Figura 7.26 se muestra gráficamente la relación entre la frecuencia del movimiento transversal y la frecuencia natural de la estructura y en la Figura 7.27 la relación entre la frecuencia de excitación asociada al plano de fluido que actúa en el extremo libre de la estructura y la frecuencia natural de la misma.

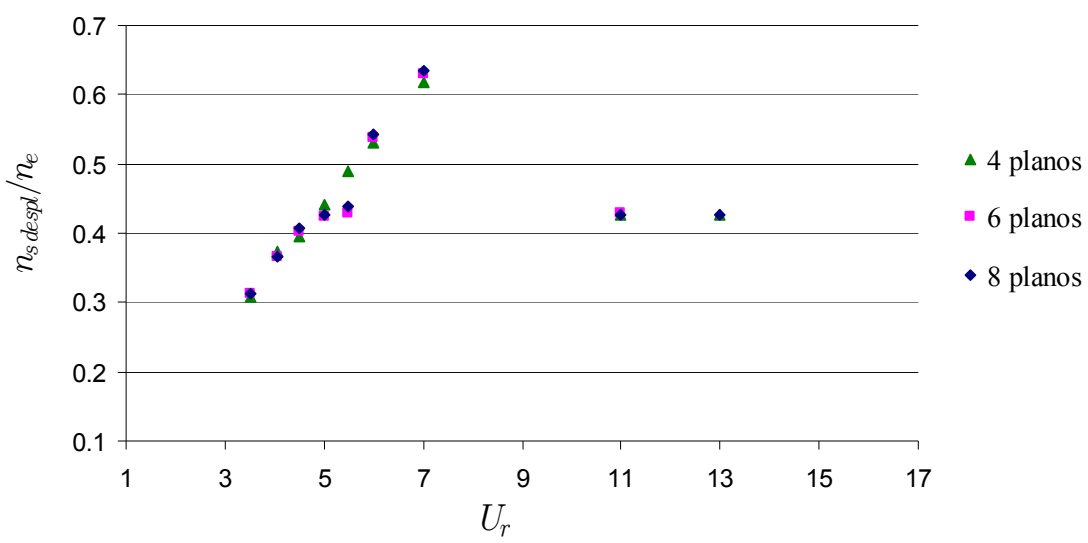

Figura 7.26. Relación entre la frecuencia del moviendo transversal y la frecuencia natural

Comparando los resultados obtenidos para 4, 6 y 8 planos se pone de manifiesto una buena similitud entre los resultados obtenidos tanto en desplazamientos como en frecuencias, observándose una convergencia en los resultados. Se puede concluir que la acción del viento se puede modelar con un número relativamente pequeño de planos de fluido. 


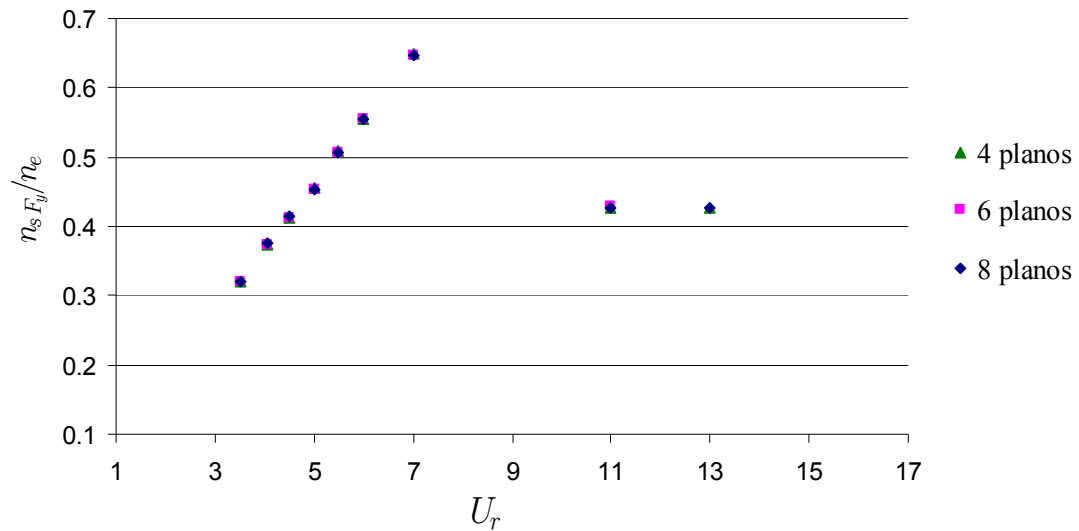

Figura 7.27. Relación entre la frecuencia de excitación en el extremo libre y la frecuencia natural

A modo de ejemplo, en la Figura 7.28 y en la Figura 7.29 se muestra la vibración de la estructura en la dirección transversal y en la dirección en la que actúa el viento para $U_{r}=5.5$, respectivamente, siendo en este caso en el que mayor discrepancia se observa entre los resultados. Esto es debido a la cercanía entre la frecuencia de excitación correspondiente al plano $Z=12.75 \mathrm{~m}$ en el caso de 8 planos y $Z=11.30 \mathrm{~m}$ en el caso de 6 planos (aplicando la ley de Strouhal para $S t=$ 0.21 se obtienen las frecuencias 0.44 y $0.43 \mathrm{~Hz}$, respectivamente) y la frecuencia natural de la estructura $(0.43 \mathrm{~Hz})$. En el caso de 4 planos, las frecuencias de excitación no están tan próximas a la frecuencia natural de la estructura. En la Figura 7.30 se muestra la variación con el tiempo del coeficiente de arrastre y del coeficiente de sustentación.

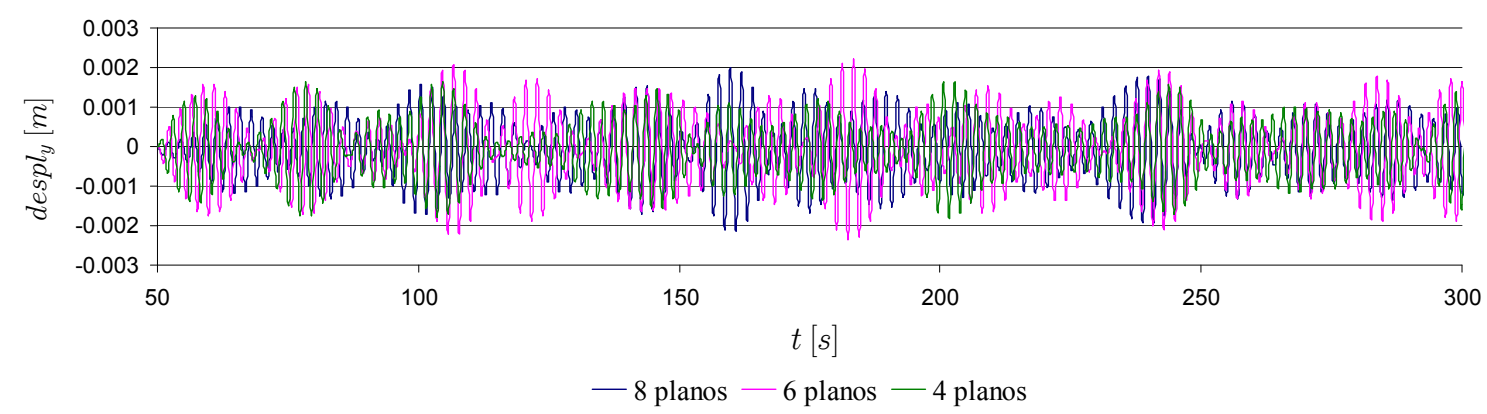

Figura 7.28. Vibración de la estructura en la dirección transversal para $U_{r}=5.5$

Finalmente, en la Figura 7.31 se ha representado la vibración transversal de la estructura y en la Figura 7.32 en la dirección del viento para $U_{r}=7$. En la Figura 7.33 se muestra la variación con el tiempo del coeficiente de arrastre y del coeficiente de sustentación. 


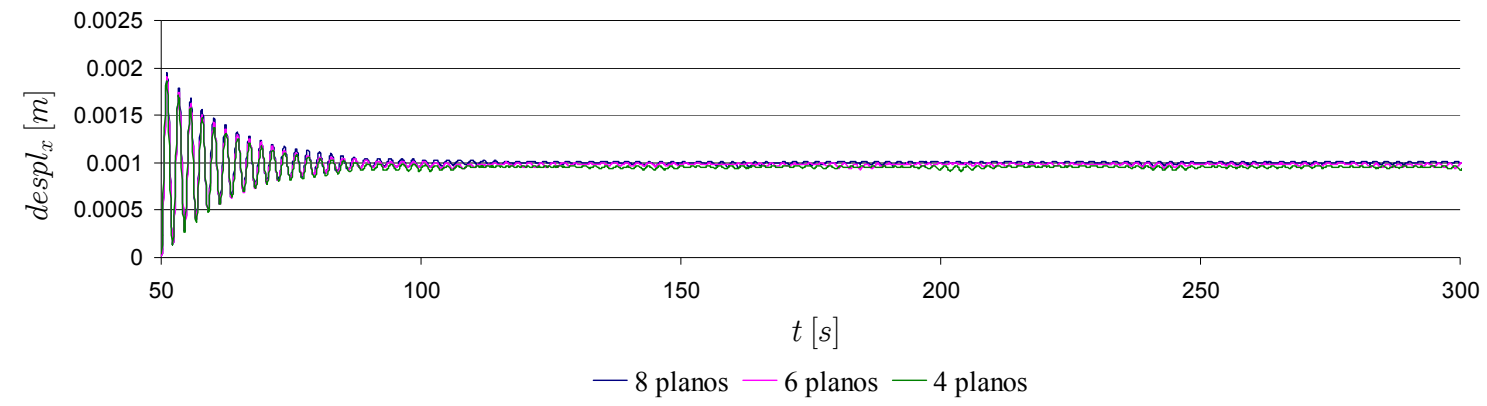

Figura 7.29. Vibración de la estructura en la dirección del viento para $U_{r}=5.5$

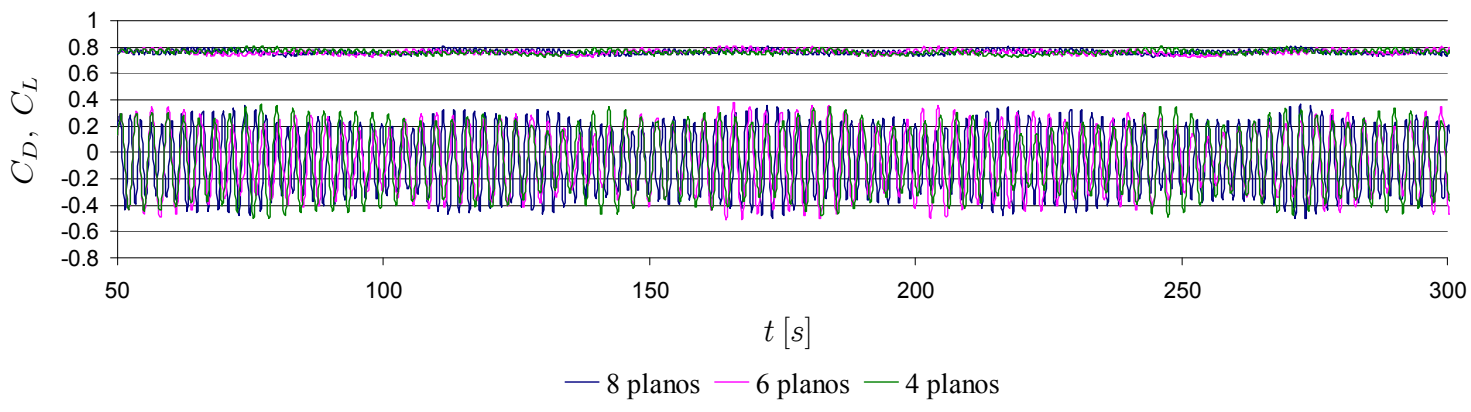

Figura 7.30. Oscilación del coeficiente de arrastre y del coeficiente de sustentación $U_{r}=5.5$

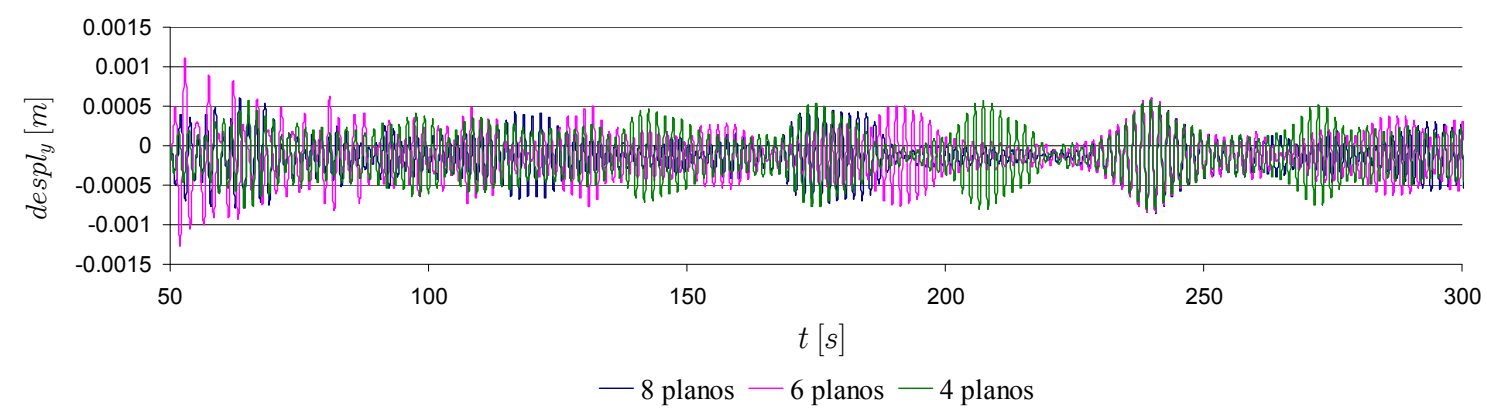

Figura 7.31. Vibración de la estructura en la dirección transversal $U_{r}=7$

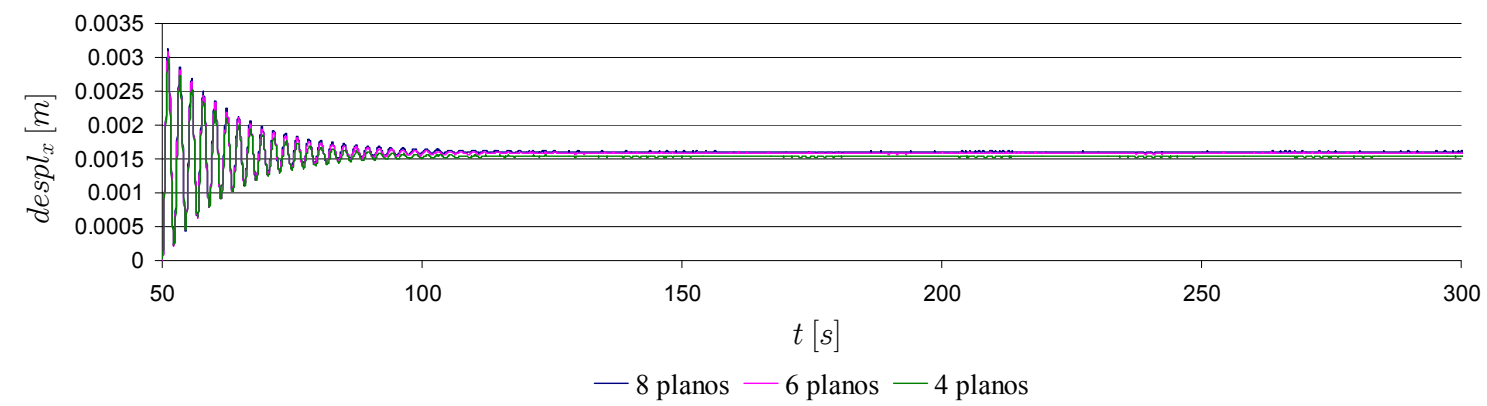

Figura 7.32. Vibración de la estructura en la dirección del viento $U_{r}=7$ 


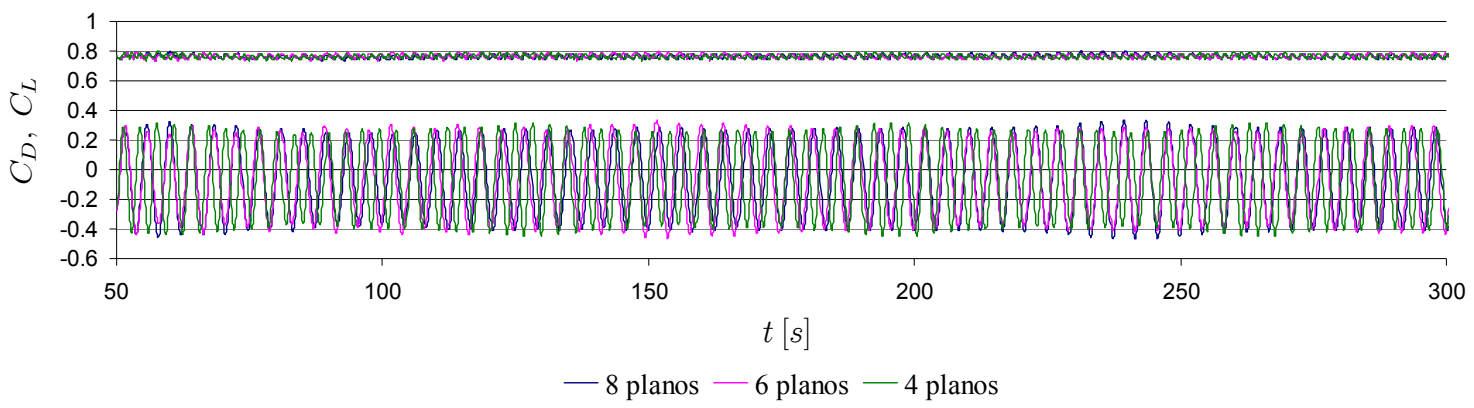

Figura 7.33. Oscilación del coeficiente de arrastre y del coeficiente de sustentación $U_{r}=7$

A la vista de estas gráficas se comprueba que la respuesta tanto en la dirección transversal a la que actúa el viento como en la dirección paralela son muy similares con independencia del número de planos utilizados para "discretizar" el viento.

En los siguientes puntos del capítulo que nos ocupa se estudiarán los fenómenos aeroelásticos de lock-in y de amplificación del coeficiente de arrastre considerando la misma estructura de sección circular y $34 \mathrm{~m}$ de altura, si bien, con objeto de simplificar el cálculo y centrarnos en el estudio de estos fenómenos, sólo se considerará un plano de fluido actuando en el extremo libre.

\subsection{Mástil de acero. Estudio del fenómeno de lock-in}

De acuerdo con lo expuesto en el Capítulo 2 (Figura 2.18) el amortiguamiento estructural tiene una fuerte influencia en el fenómeno de lock-in o de sincronización, de manera que este fenómeno sólo ocurre para valores pequeños del amortiguamiento estructural. A continuación se estudia este fenómeno aeroelástico para la misma estructura que la descrita en el punto anterior sometida a la acción del viento y considerando diferentes valores del amortiguamiento estructural.

La primera frecuencia natural de la estructura es $0.43 \mathrm{~Hz}$ y tanto para la estructura como para el fluido las condiciones de contorno son las indicadas en el punto anterior así como también la malla del fluido utilizada. La estructura fue mallada con cuatro elementos tipo barra. De forma análoga al ejemplo anterior, el fluido se supondrá actuando en planos, si bien, en este caso sólo se considerará un único plano perpendicular a la directriz de la estructura situado su extremo libre, tal como se muestra en la Figura 7.34.

Para estudiar la dependencia del fenómeno de sincronización respecto al amortiguamiento estructural se estudiaron varios casos con diferentes valores del coeficiente de amortiguamiento 
estructural. A su vez, para cada uno de los casos estudiados, se adoptaron valores distintos de la componente de la velocidad $v_{x}$ de entrada del fluido con objeto de obtener datos suficientes para representar la variación de la frecuencia de desprendimiento de los torbellinos y la amplitud de la respuesta vibratoria de la estructura en función de la velocidad reducida.
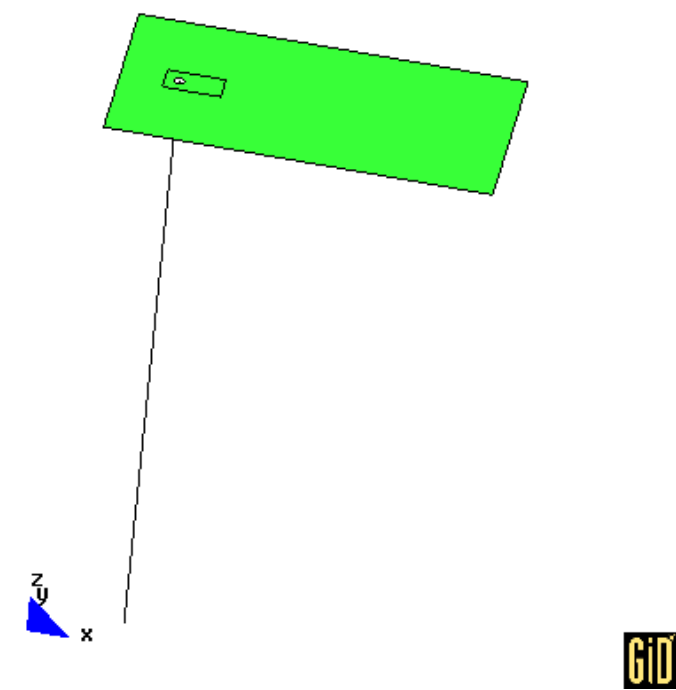

Figura 7.34. Modelo de la estructura sometida a la acción del viento

Al igual que sucedía en los ejemplos anteriores es necesario definir un paso de tiempo adecuado para la correcta resolución del problema estructural y del fluido. Para ello se debe tener en cuenta la frecuencia natural de la estructura, el tamaño característico de los elementos de la malla del fluido y la velocidad del flujo de aire. En definitiva, se tomó como valor del paso de tiempo $0.005 \mathrm{~s}$.

\section{Caso 1. Coeficiente de amortiguamiento estructural 0.075}

En la Figura 7.35 se ha representado la variación de la frecuencia de vibración de la estructura en la dirección transversal y de la frecuencia de desprendimiento de los torbellinos divididas por la frecuencia natural de la estructura, $n_{e}=0.43 \mathrm{~Hz}$, frente a la velocidad reducida. Se observa que el fenómeno de sincronización no se produce para el rango de velocidades estudiado y esto es debido a que el amortiguamiento estructural es elevado.

En la Figura 7.36 se muestra la respuesta en amplitud de la vibración de la estructura frente a la velocidad reducida. Se puede ver que en el entorno próximo a $U_{r}=5$ se obtienen las mayores amplitudes, ya que para esa velocidad reducida la frecuencia de desprendimiento de los torbellinos está muy próxima a la frecuencia natural de la estructura. 


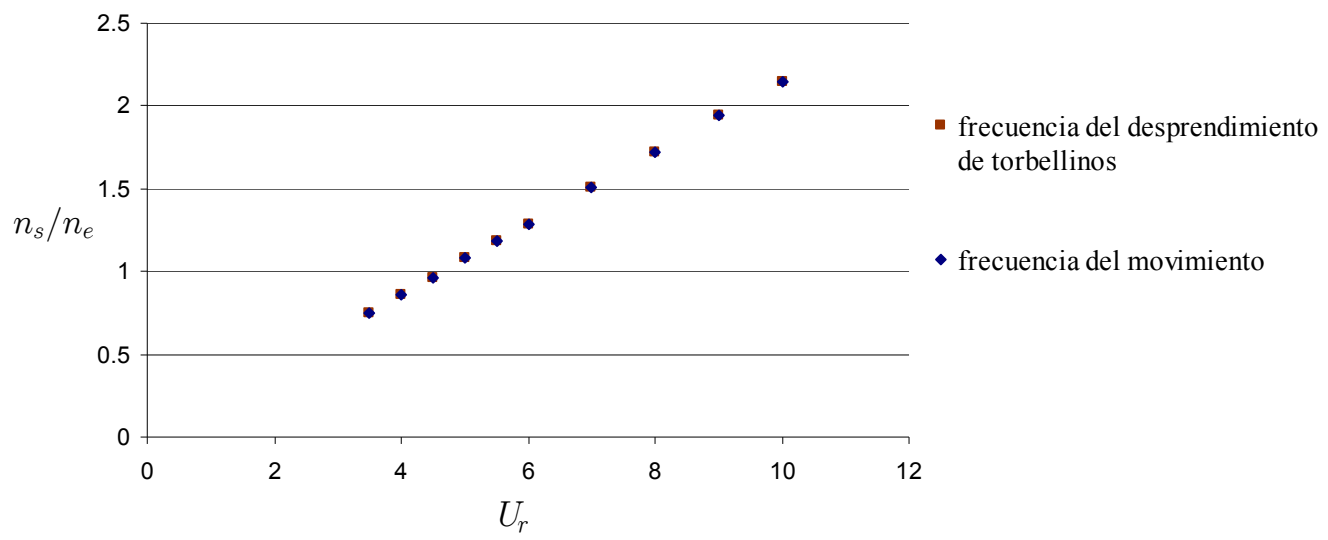

Figura 7.35. Respuesta en frecuencias frente a la velocidad reducida

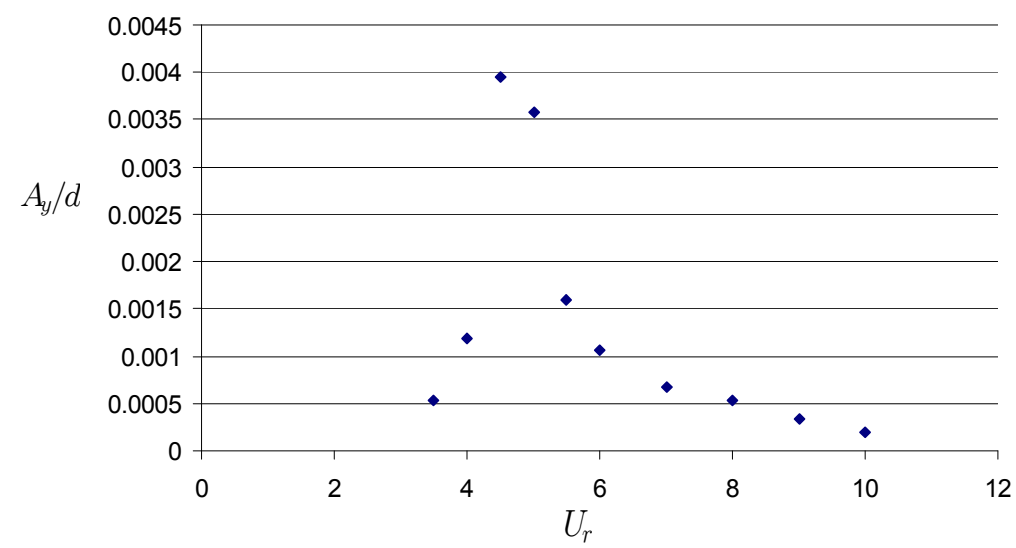

Figura 7.36. Respuesta en amplitud frente a la velocidad reducida

En la Tabla 7.11 se muestran los resultados obtenidos en frecuencias y amplitud para diferentes velocidades reducidas. También se proporcionan los valores obtenidos del número de Strouhal y del coeficiente de arrastre.

\begin{tabular}{|c|c|c|c|c|c|c|c|c|c|}
\hline $\boldsymbol{U}_{\boldsymbol{r}}$ & $\boldsymbol{R} \boldsymbol{e}$ & $\boldsymbol{S t}$ & $\boldsymbol{C}_{\boldsymbol{D}}$ & $\begin{array}{c}\text { fr. despl. } \\
{[\mathrm{Hz}]}\end{array}$ & $\boldsymbol{n}_{\boldsymbol{s}} / \boldsymbol{n}_{\boldsymbol{e}}$ & $\boldsymbol{A}_{\boldsymbol{y}}[\mathrm{m}]$ & $\boldsymbol{A}_{\boldsymbol{y}} / \boldsymbol{d}$ & $\begin{array}{c}\text { fr. despr. } \\
\text { torb. }[\mathrm{Hz}]\end{array}$ & $\boldsymbol{n}_{\boldsymbol{s}} / \boldsymbol{n}_{\boldsymbol{e}}$ \\
\hline 10.0 & $7.38 \mathrm{E}+04$ & 0.21 & 0.77 & 0.92 & 2.15 & $9.59 \mathrm{E}-05$ & $1.92 \mathrm{E}-04$ & 0.92 & 2.15 \\
\hline 9.0 & $6.65 \mathrm{E}+04$ & 0.21 & 0.78 & 0.84 & 1.94 & $1.70 \mathrm{E}-04$ & $3.40 \mathrm{E}-04$ & 0.84 & 1.94 \\
\hline 8.0 & $5.90 \mathrm{E}+04$ & 0.21 & 0.77 & 0.74 & 1.72 & $2.66 \mathrm{E}-04$ & $5.32 \mathrm{E}-04$ & 0.74 & 1.72 \\
\hline 7.0 & $5.16 \mathrm{E}+04$ & 0.21 & 0.77 & 0.65 & 1.51 & $3.33 \mathrm{E}-04$ & $6.65 \mathrm{E}-04$ & 0.65 & 1.51 \\
\hline 6.0 & $4.43 \mathrm{E}+04$ & 0.21 & 0.77 & 0.55 & 1.29 & $5.34 \mathrm{E}-04$ & $1.07 \mathrm{E}-03$ & 0.55 & 1.29 \\
\hline 5.5 & $4.06 \mathrm{E}+04$ & 0.21 & 0.77 & 0.51 & 1.19 & $8.00 \mathrm{E}-04$ & $1.60 \mathrm{E}-03$ & 0.51 & 1.19 \\
\hline 5.0 & $3.69 \mathrm{E}+04$ & 0.21 & 0.77 & 0.46 & 1.08 & $1.79 \mathrm{E}-03$ & $3.57 \mathrm{E}-03$ & 0.46 & 1.08 \\
\hline 4.5 & $3.32 \mathrm{E}+04$ & 0.21 & 0.78 & 0.42 & 0.97 & $1.98 \mathrm{E}-03$ & $3.96 \mathrm{E}-03$ & 0.42 & 0.97 \\
\hline 4.0 & $2.99 \mathrm{E}+04$ & 0.21 & 0.79 & 0.37 & 0.86 & $5.95 \mathrm{E}-04$ & $1.19 \mathrm{E}-03$ & 0.37 & 0.86 \\
\hline 3.5 & $2.58 \mathrm{E}+04$ & 0.21 & 0.77 & 0.32 & 0.75 & $2.63 \mathrm{E}-04$ & $5.27 \mathrm{E}-04$ & 0.32 & 0.75 \\
\hline
\end{tabular}

Tabla 7.11. Resultados obtenidos para amortiguamiento estructural 0.075 
A modo de ejemplo, en la Figura 7.37 se ha representado la vibración de la estructura en la dirección transversal a la que actúa el viento y en la Figura 7.38 en la misma dirección en la que actúa el viento para $U_{r}=6$.

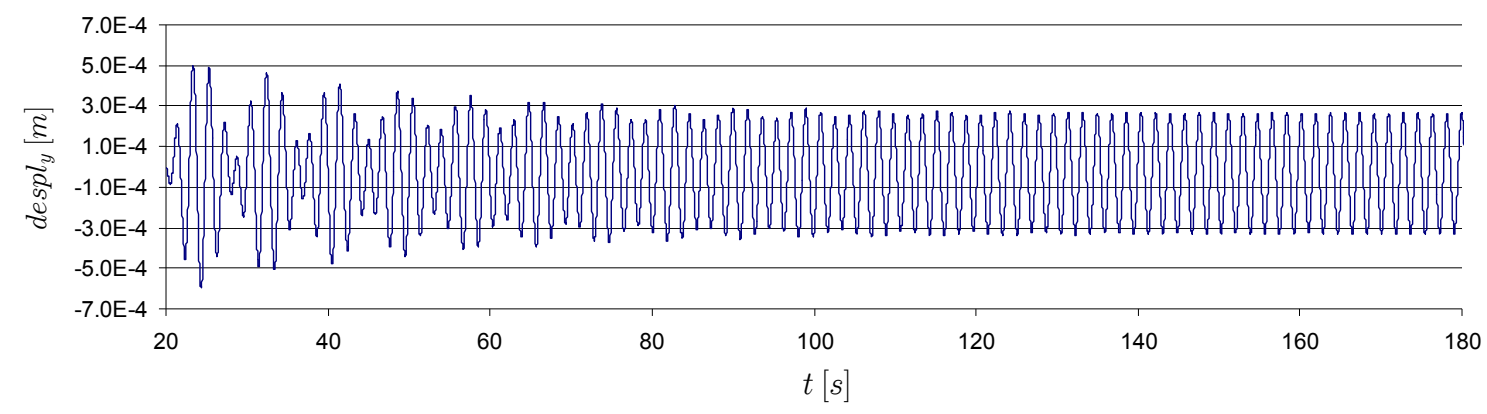

Figura 7.37. Vibración de la estructura en la dirección transversal

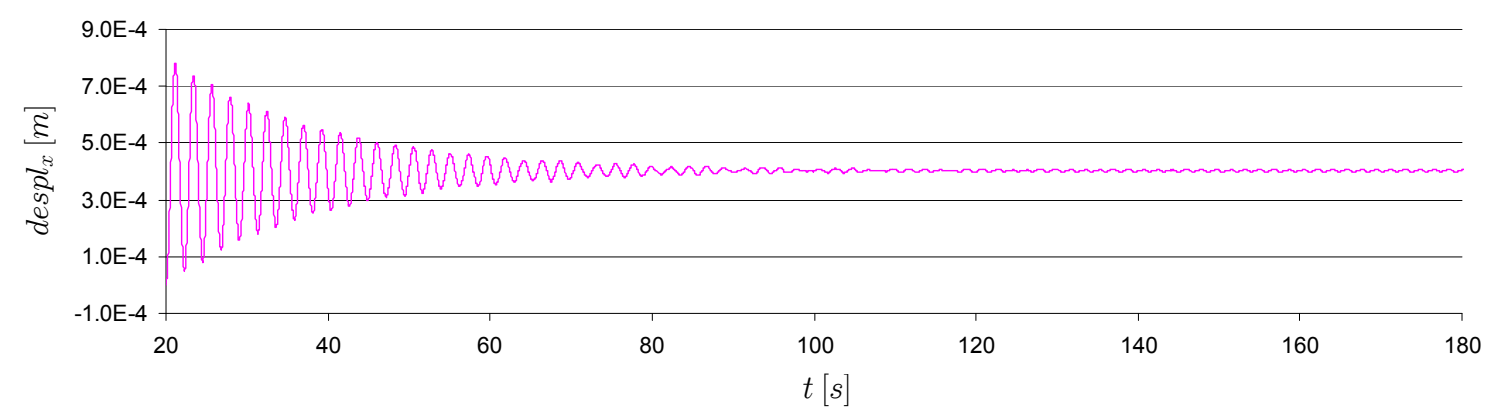

Figura 7.38. Vibración de la estructura en la dirección del viento

Finalmente, en la Figura 7.39 se muestra la variación con el tiempo del coeficiente de arrastre y del coeficiente de sustentación para $U_{r}=6$.

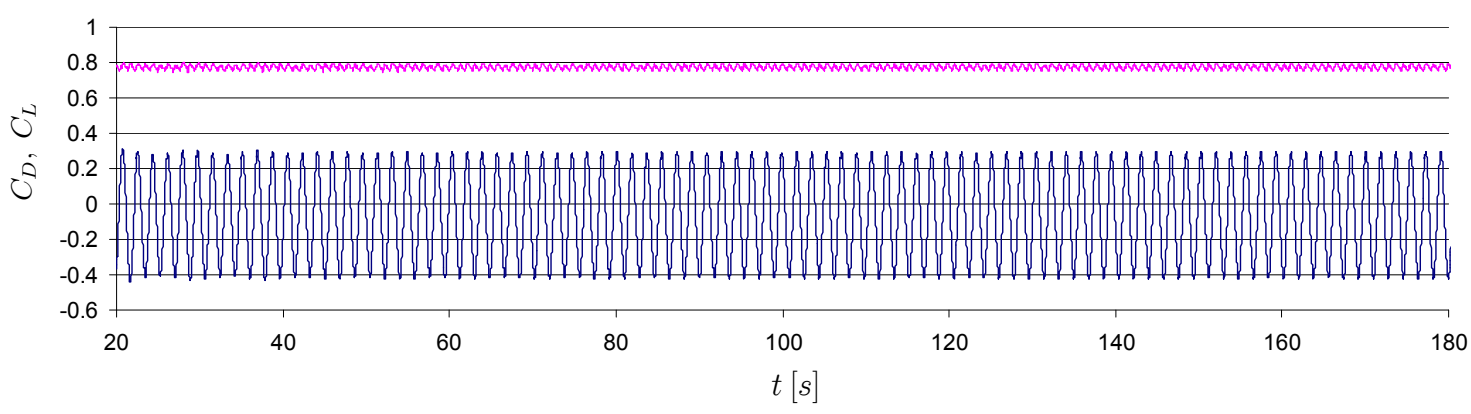

Figura 7.39. Oscilación del coeficiente de arrastre y del coeficiente de sustentación 


\section{Caso 2. Coeficiente de amortiguamiento estructural 0.05}

De la misma manera que en el caso anterior, en la Figura 7.40 se muestra la respuesta en frecuencias frente a la velocidad reducida. Se puede observar que el fenómeno de sincronización no se produce para el rango de velocidades estudiado debido a que el amortiguamiento estructural permanece elevado. En la Figura 7.41 se muestra la respuesta en amplitud observándose las mayores amplitudes en el entorno próximo a $U_{r}=5$.

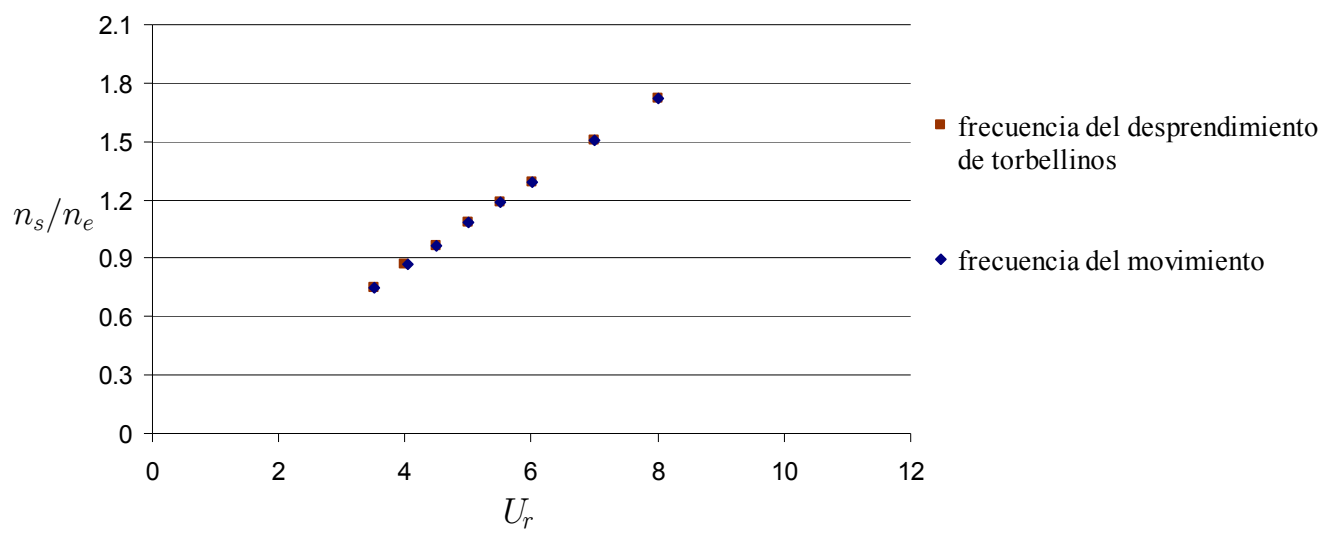

Figura 7.40. Respuesta en frecuencias frente a la velocidad reducida

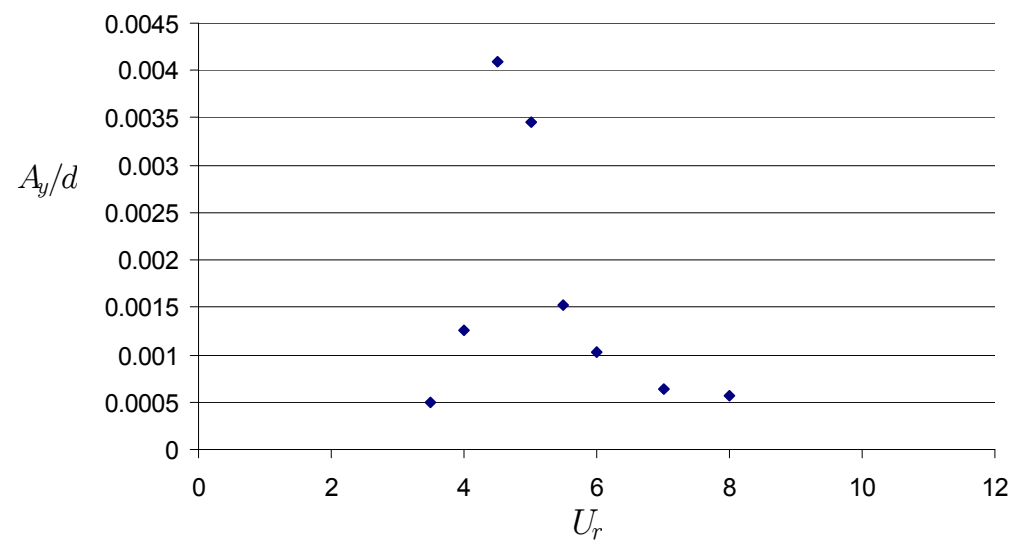

Figura 7.41. Respuesta en amplitud frente a la velocidad reducida

A modo de ejemplo, en la Figura 7.42 y en la Figura 7.43 se muestra la vibración de la estructura en la dirección transversal y en la dirección en la que actúa el viento para $U_{r}=5.5$, respectivamente. La variación del coeficiente de arrastre y del coeficiente de sustentación se ha representado en la Figura 7.44. 


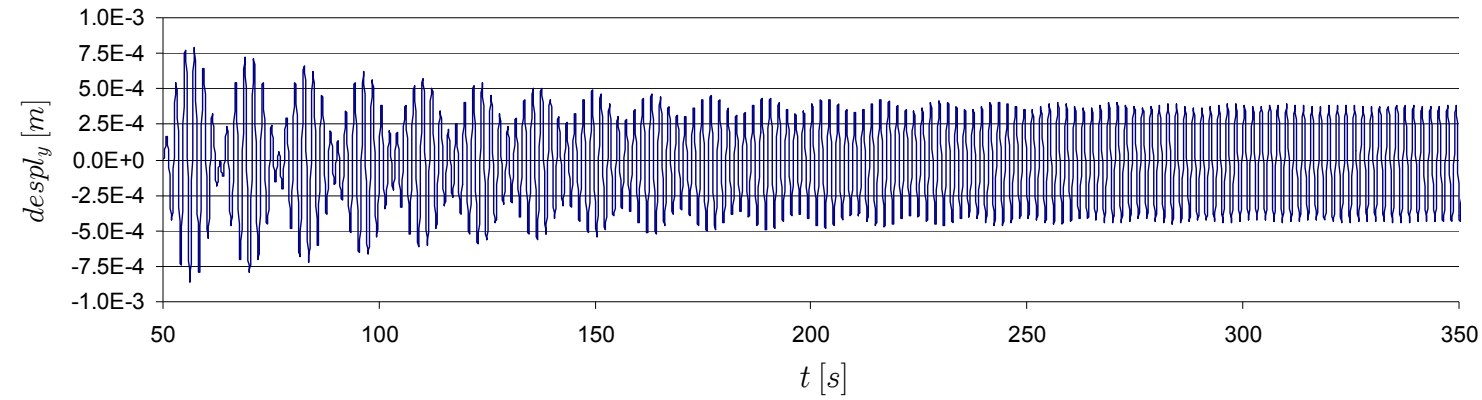

Figura 7.42. Vibración de la estructura en la dirección transversal

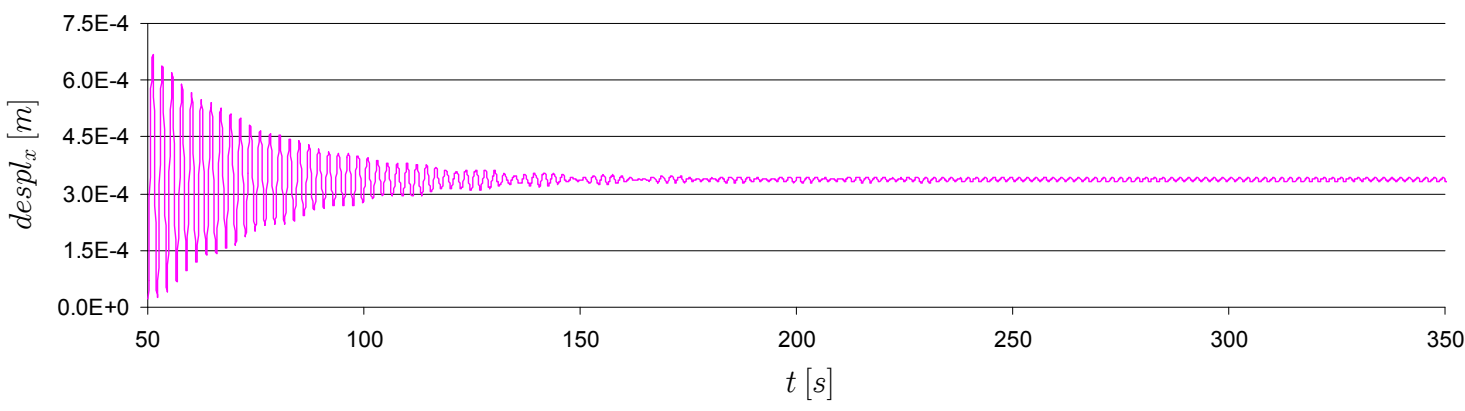

Figura 7.43. Vibración de la estructura en la dirección del viento

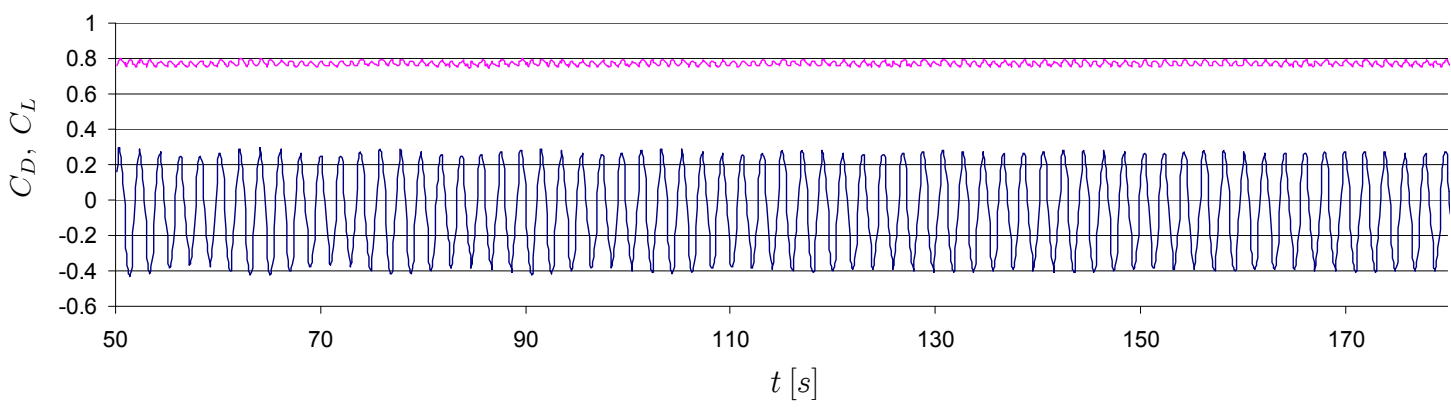

Figura 7.44. Variación del coeficiente de arrastre y del coeficiente de sustentación

En la Tabla 7.12 se resumen los resultados obtenidos para amortiguamiento estructural 0.05 .

\begin{tabular}{|c|c|c|c|c|c|c|c|c|c|}
\hline$U_{\boldsymbol{r}}$ & $\boldsymbol{R} \boldsymbol{S}$ & $\boldsymbol{S t}$ & $\boldsymbol{C}_{\boldsymbol{D}}$ & $\begin{array}{c}\text { fr. despl. } \\
{[\mathrm{Hz}]}\end{array}$ & $\boldsymbol{n}_{\boldsymbol{s}} / \boldsymbol{n}_{\boldsymbol{e}}$ & $\boldsymbol{A}_{\boldsymbol{y}}[\mathrm{m}]$ & $\boldsymbol{A}_{\boldsymbol{y}} / \boldsymbol{d}$ & $\begin{array}{c}\text { fr. despr. } \\
\text { torb. }[H z]\end{array}$ & $\boldsymbol{n}_{\boldsymbol{s}} / \boldsymbol{n}_{\boldsymbol{e}}$ \\
\hline 8.0 & $5.90 \mathrm{E}+04$ & 0.21 & 0.77 & 0.74 & 1.72 & $2.84 \mathrm{E}-04$ & $5.68 \mathrm{E}-04$ & 0.74 & 1.72 \\
\hline 7.0 & $5.16 \mathrm{E}+04$ & 0.21 & 0.77 & 0.65 & 1.51 & $3.16 \mathrm{E}-04$ & $6.32 \mathrm{E}-04$ & 0.65 & 1.51 \\
\hline 6.0 & $4.43 \mathrm{E}+04$ & 0.21 & 0.77 & 0.56 & 1.29 & $5.11 \mathrm{E}-04$ & $1.02 \mathrm{E}-03$ & 0.56 & 1.29 \\
\hline 5.5 & $4.06 \mathrm{E}+04$ & 0.21 & 0.77 & 0.51 & 1.19 & $7.64 \mathrm{E}-04$ & $1.53 \mathrm{E}-03$ & 0.51 & 1.19 \\
\hline 5.0 & $3.69 \mathrm{E}+04$ & 0.21 & 0.77 & 0.46 & 1.08 & $1.72 \mathrm{E}-03$ & $3.45 \mathrm{E}-03$ & 0.46 & 1.08 \\
\hline 4.5 & $3.32 \mathrm{E}+04$ & 0.21 & 0.78 & 0.41 & 0.96 & $2.05 \mathrm{E}-03$ & $4.09 \mathrm{E}-03$ & 0.41 & 0.96 \\
\hline 4.0 & $2.99 \mathrm{E}+04$ & 0.21 & 0.78 & 0.37 & 0.87 & $6.32 \mathrm{E}-04$ & $1.26 \mathrm{E}-03$ & 0.37 & 0.87 \\
\hline 3.5 & $2.58 \mathrm{E}+04$ & 0.21 & 0.77 & 0.32 & 0.75 & $2.51 \mathrm{E}-04$ & $5.03 \mathrm{E}-04$ & 0.32 & 0.75 \\
\hline
\end{tabular}

Tabla 7.12. Resultados obtenidos para amortiguamiento estructural 0.05 


\section{Caso 3. Coeficiente de amortiguamiento estructural 0.025}

En la Figura 7.45 se muestra la respuesta en frecuencias frente a la velocidad reducida y en la Figura 7.46 la respuesta en amplitud. Se observa que para este caso aparece el fenómeno de sincronización y por lo tanto, la ley de Strouhal no se cumple en un cierto rango de velocidades.

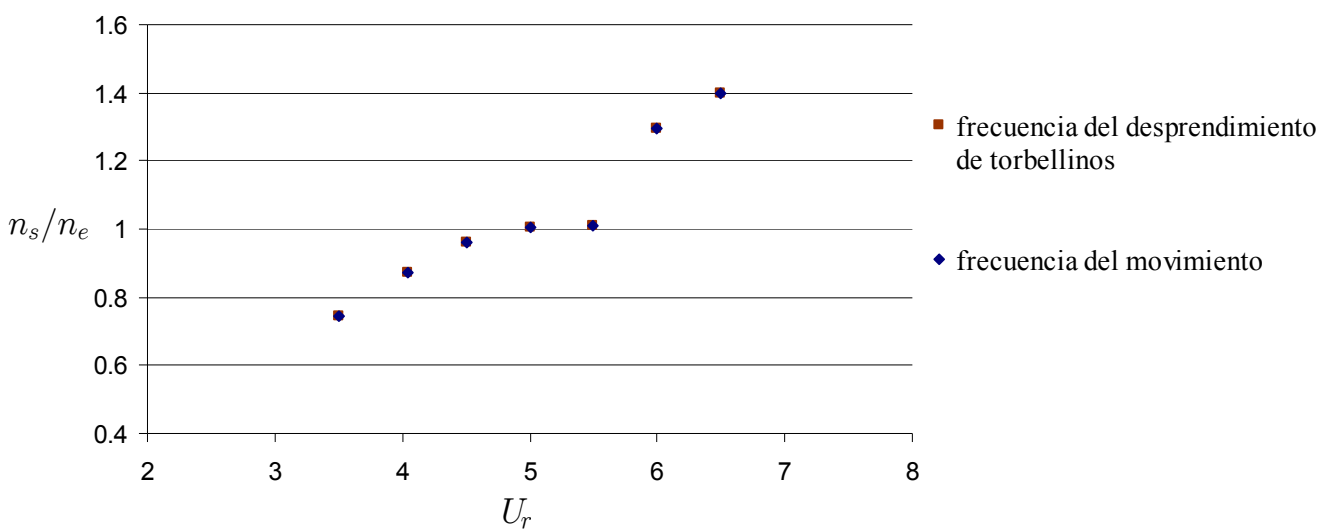

Figura 7.45. Respuesta en frecuencias frente a la velocidad reducida

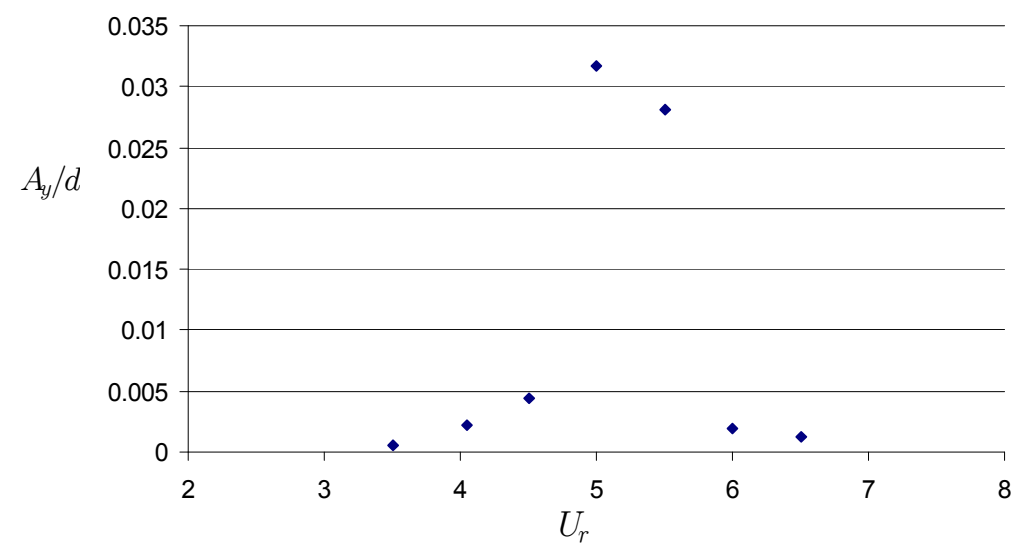

Figura 7.46. Respuesta en amplitud frente a la velocidad reducida

El fenómeno de lock-in se puede apreciar en la Figura 7.47, en la cual se ha representado la variación del coeficiente de sustentación respecto al tiempo para $U_{r}=5.5$. La frecuencia de oscilación de este coeficiente viene dada por la frecuencia a la que se produce el desprendimiento de los torbellinos. Al principio del estudio, hasta $t=800 \mathrm{~s}$ aproximadamente, la frecuencia a la que ocurre el desprendimiento de los torbellinos es $0.51 \mathrm{~Hz}$ (Figura 7.48), la cual se corresponde a la que se obtiene aplicando la ley de Strouhal para $S t=0.21$ de acuerdo a (7.3) 


$$
n_{s}=S t \frac{U}{d}=0.21 \frac{1.22}{0.5}=0.51 \mathrm{~Hz}
$$

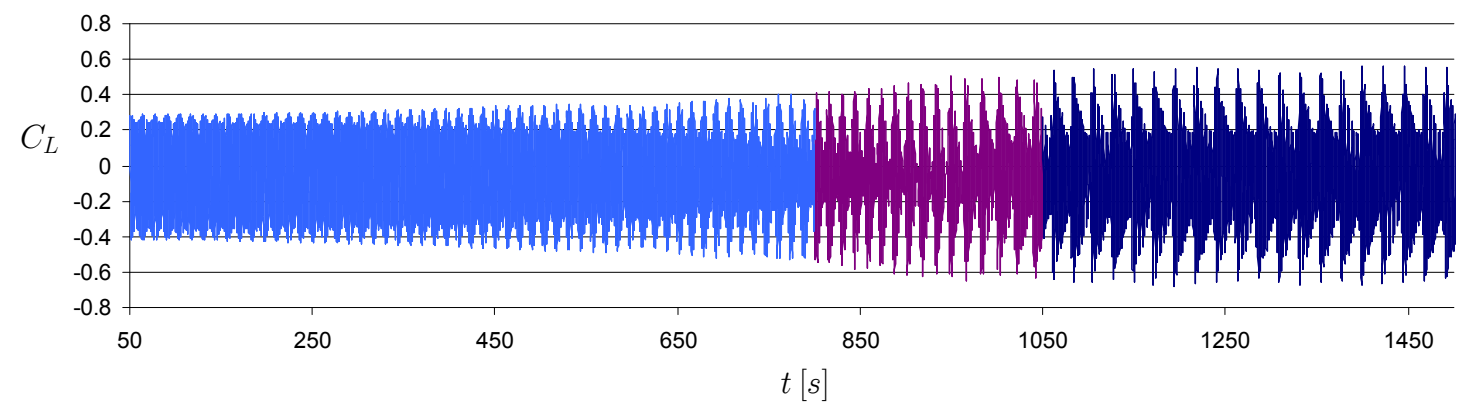

Figura 7.47. Variación del coeficiente de sustentación

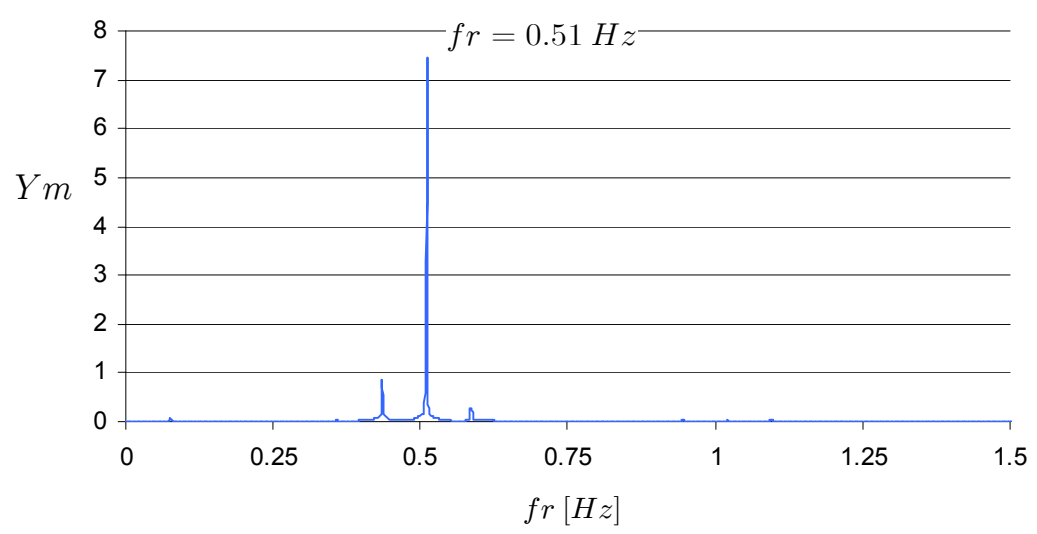

Figura 7.48. Análisis FFT de la oscilación del $C_{L}$ para $t<800 \mathrm{~s}$

Cuando $t$ se sitúa entre $800 \mathrm{~s}$ y $1050 \mathrm{~s}$ aproximadamente, la frecuencia del coeficiente de sustentación comienza a cambiar y se sincroniza con la frecuencia natural de la estructura. Es en este intervalo de tiempo cuando ocurre la sincronización entre las dos frecuencias (Figura 7.49).

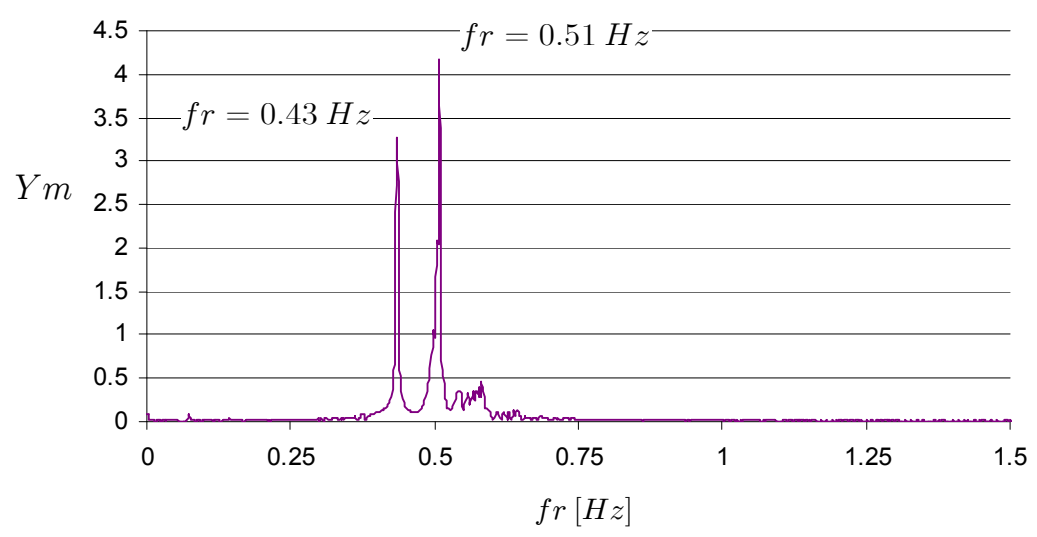

Figura 7.49. Análisis FFT de la oscilación del $C_{L}$ en el intervalo $800<t<1050 \mathrm{~s}$ 
A partir de $t=1050 \mathrm{~s}$ aproximadamente, la frecuencia a la que se desprenden los torbellinos es igual a la frecuencia natural de la estructura $n_{e}=0.43 \mathrm{~Hz}$ (Figura 7.50), lo cual implica que la frecuencia de desprendimiento de los torbellinos pasa a estar controlada por la frecuencia natural de la estructura.

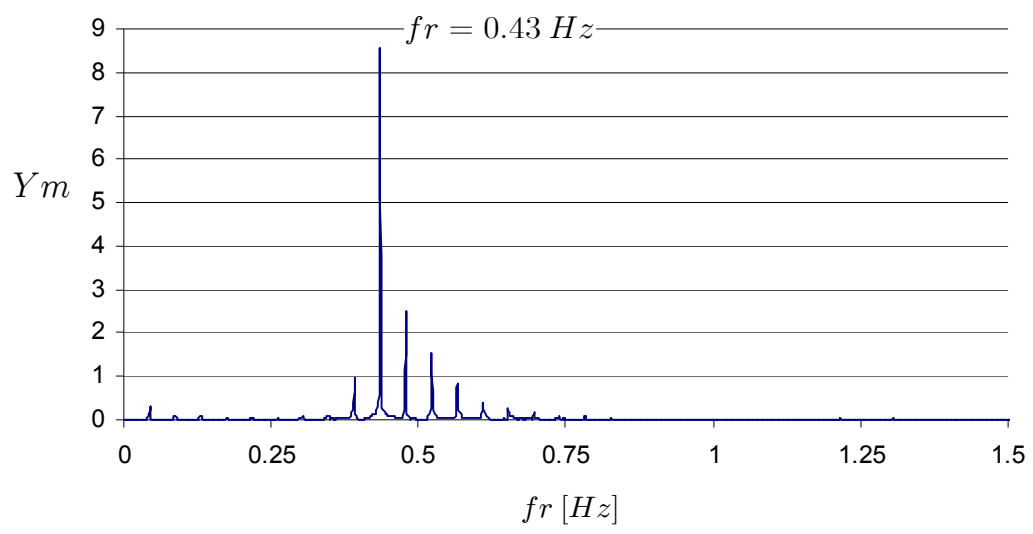

Figura 7.50. Análisis FFT de la oscilación del $C_{L}$ para $t>1050 \mathrm{~s}$

Notar que en el intervalo de tiempo en el que tiene lugar la sincronización entre las dos frecuencias la amplitud de la vibración de la estructura en la dirección transversal a la que actúa el viento comienza a incrementarse de forma considerable, tal como se muestra en la Figura 7.51. Se observa que la amplitud crece hasta un cierto valor y permanece constante una vez ha alcanzado dicho valor, lo cual pone de manifiesto el carácter autolimitado de estas vibraciones [4].

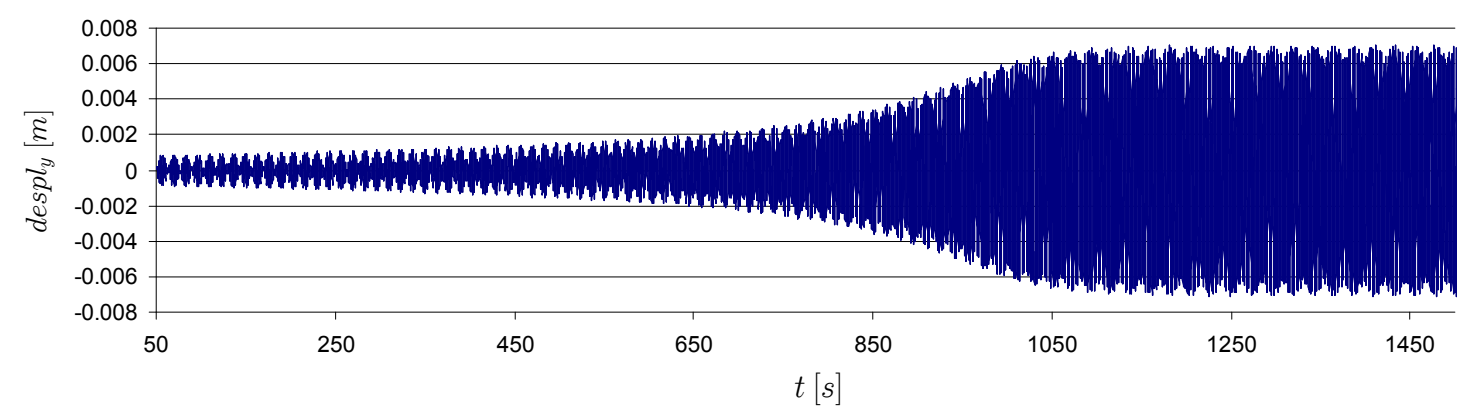

Figura 7.51. Vibración de la estructura en la dirección transversal

En la Figura 7.52 se muestra el análisis FFT de la vibración de la estructura en la dirección transversal. Se puede ver que la estructura vibra a su frecuencia natural de $0.43 \mathrm{~Hz}$, tal como era de esperar. 


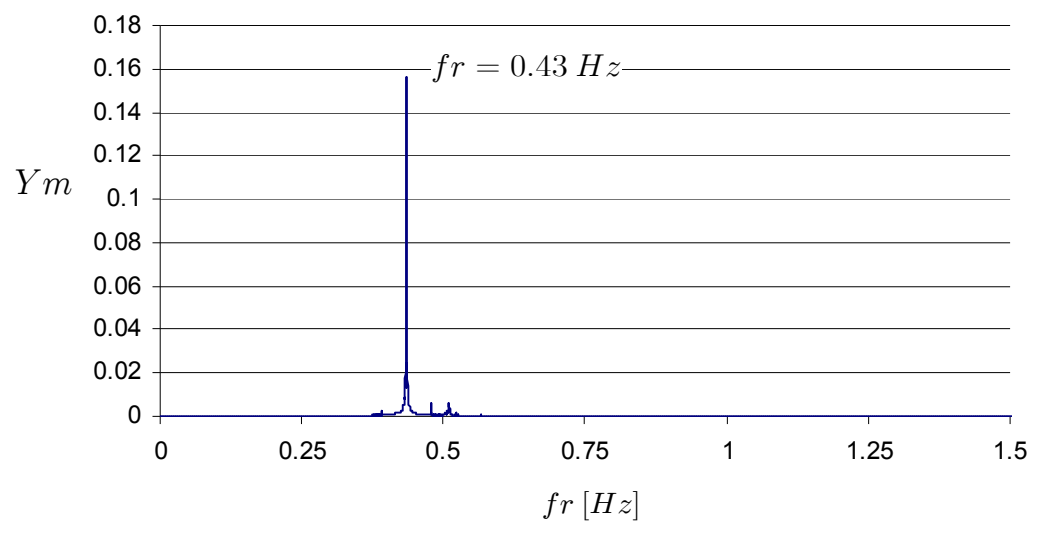

Figura 7.52. Análisis FFT de la vibración de la estructura en la dirección transversal

En la Figura 7.53 se muestra la vibración de la estructura en la dirección del viento y en la Figura 7.54 la variación con el tiempo del coeficiente de arrastre. Se aprecia un incremento en la amplitud de la vibración en la región de sincronización debido al incremento en la amplitud de la oscilación del coeficiente de arrastre.

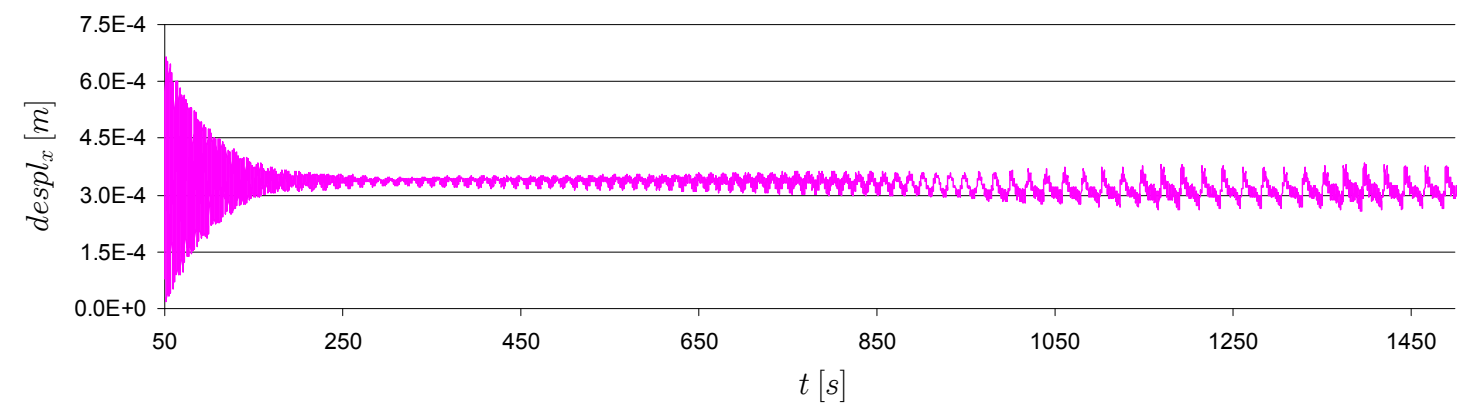

Figura 7.53. Vibración de la estructura en la dirección del viento

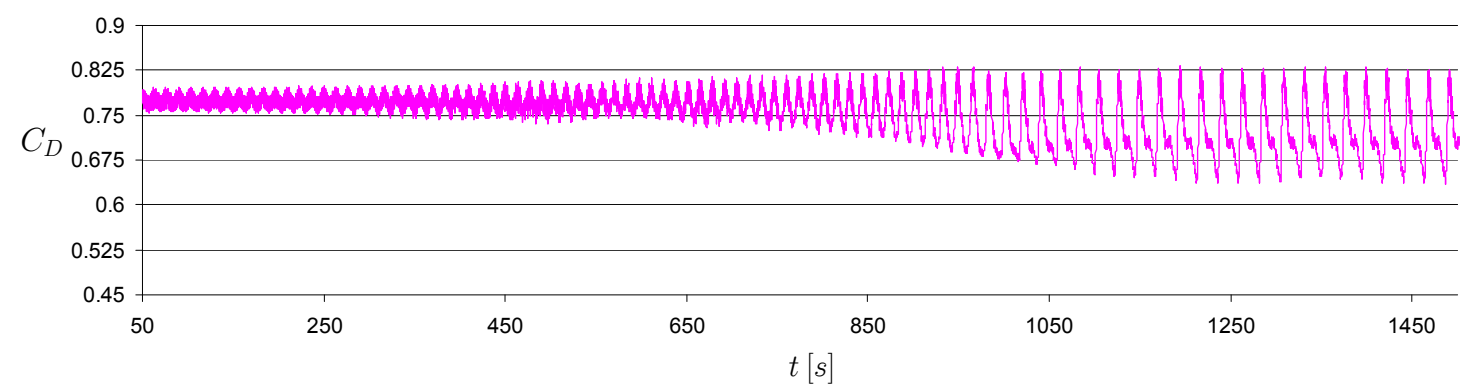

Figura 7.54. Oscilación del coeficiente de arrastre 
A continuación, para destacar el fenómeno de lock-in se mostrarán los resultados obtenidos en desplazamientos y en coeficientes de fuerza para $U_{r}=4$ y $U_{r}=6.5$.

- $U_{r}=4$

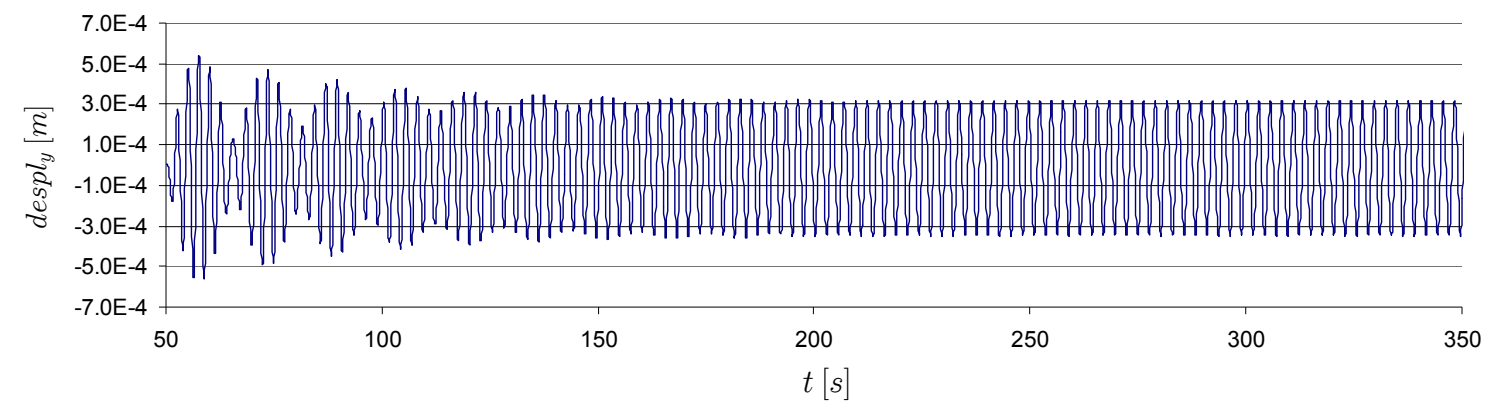

Figura 7.55. Vibración de la estructura en la dirección transversal para $U_{r}=4$

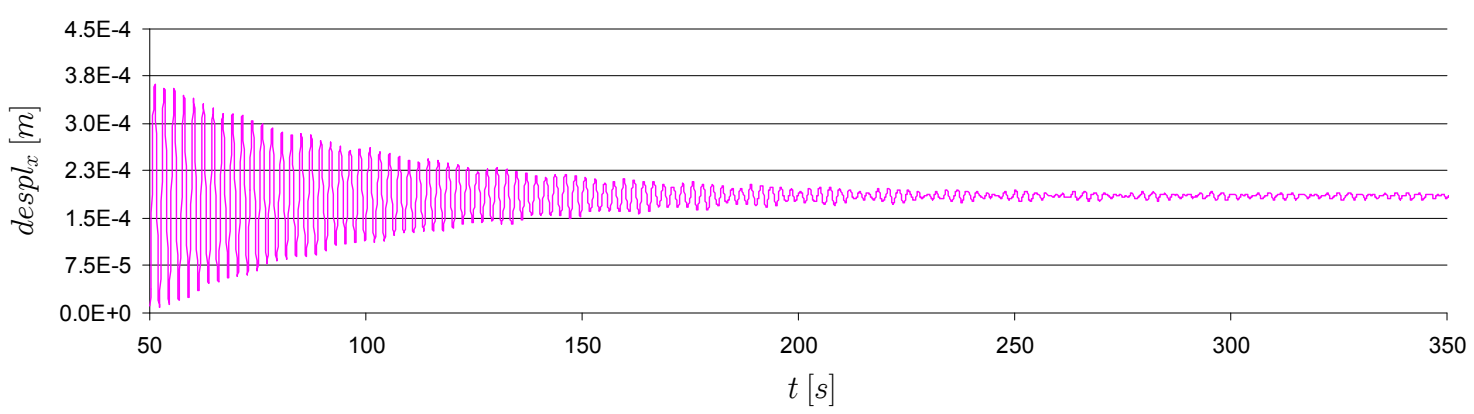

Figura 7.56. Vibración de la estructura en la dirección del viento para $U_{r}=4$

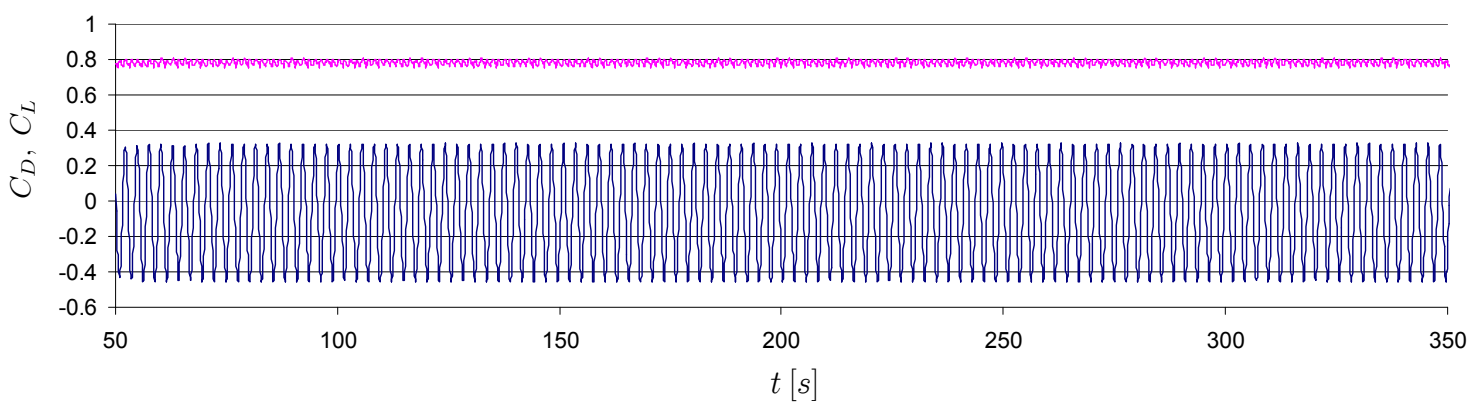

Figura 7.57. Oscilación del coeficiente de arrastre y del coeficiente de sustentación para $U_{r}=4$ 
- $U_{r}=6.5$

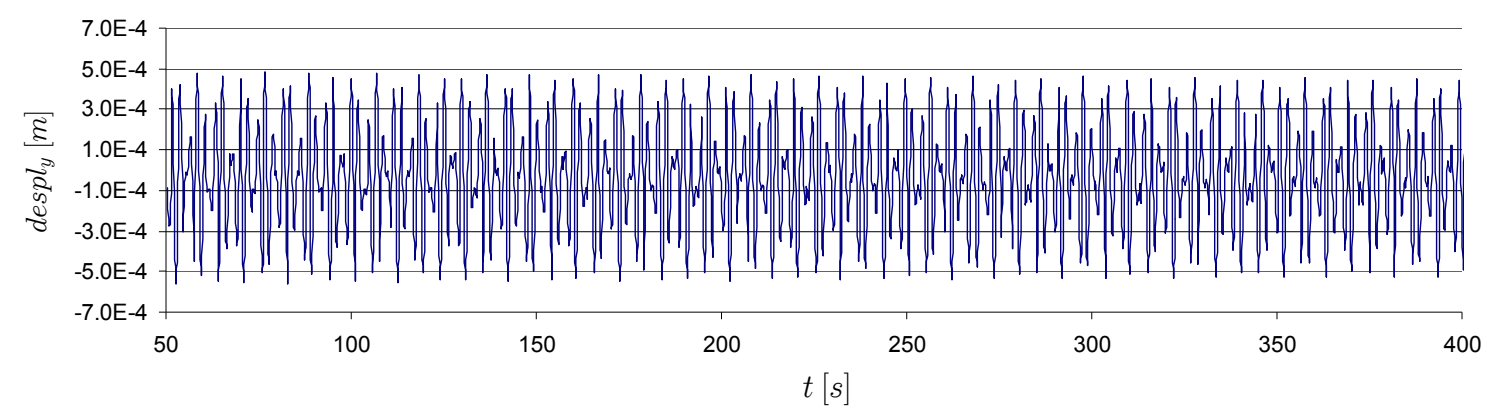

Figura 7.58. Vibración de la estructura en la dirección transversal para $U_{r}=6.5$

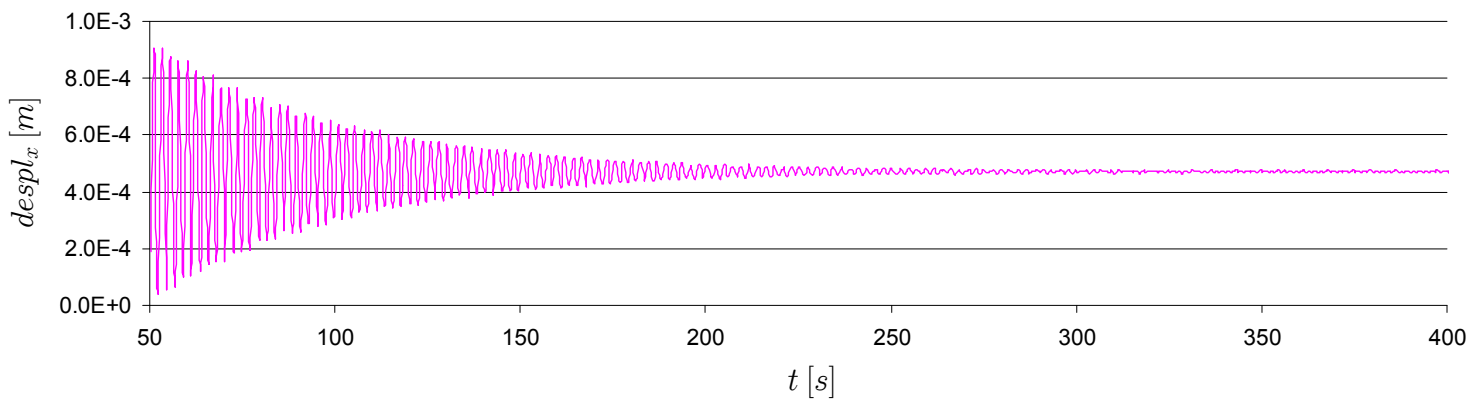

Figura 7.59. Vibración de la estructura en la dirección del viento para $U_{r}=6.5$

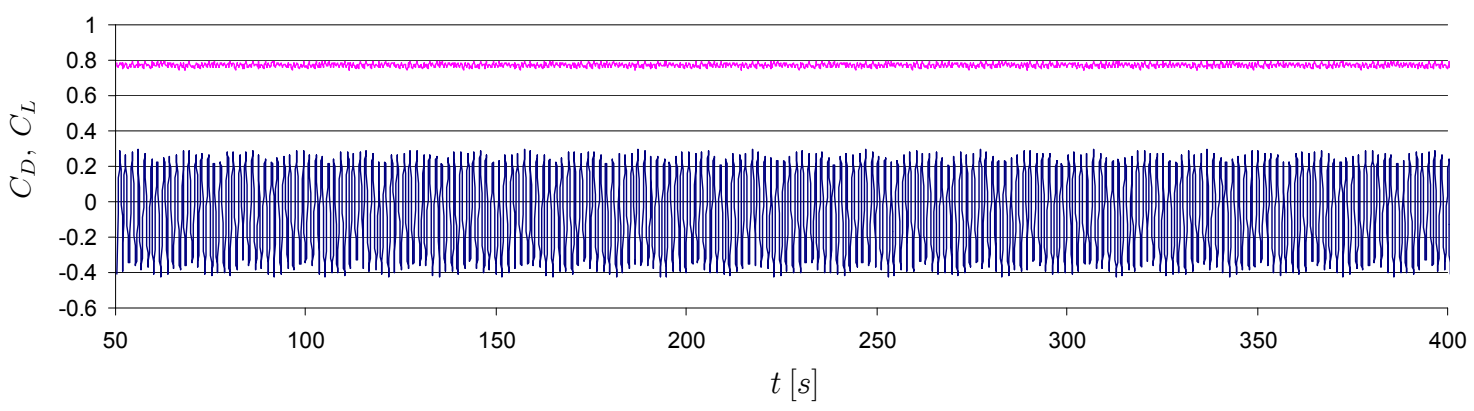

Figura 7.60. Oscilación del coeficiente de arrastre y del coeficiente de sustentación para $U_{r}=6.5$

Finalmente, en la Tabla 7.13 se muestra un resumen de los resultados obtenidos para amortiguamiento estructural 0.025 y en la Figura 7.61 se muestra una comparación de las respuestas en amplitud de los distintos casos de amortiguamiento estructural estudiados. Se observa que las mayores amplitudes se consiguen para amortiguamiento estructural 0.025 en la región de lock-in. 


\begin{tabular}{|c|c|c|c|c|c|c|c|c|c|}
\hline $\boldsymbol{U}_{\boldsymbol{r}}$ & $\boldsymbol{R} \boldsymbol{S}$ & $\boldsymbol{S t}$ & $\boldsymbol{C}_{\boldsymbol{D}}$ & $\begin{array}{c}\text { fr. despl. } \\
{[\mathrm{Hz}]}\end{array}$ & $\boldsymbol{n}_{\boldsymbol{s}} / \boldsymbol{n}_{\boldsymbol{e}}$ & $\boldsymbol{A}_{\boldsymbol{y}}[\mathrm{m}]$ & $\boldsymbol{A}_{\boldsymbol{y}} / \boldsymbol{d}$ & $\begin{array}{c}\text { fr. despr. } \\
\text { torb. }[\mathrm{Hz}]\end{array}$ & $\boldsymbol{n}_{\boldsymbol{s}} / \boldsymbol{n}_{\boldsymbol{e}}$ \\
\hline 6.50 & $4.79 \mathrm{E}+04$ & 0.21 & 0.77 & 0.60 & 1.40 & $6.28 \mathrm{E}-04$ & $1.26 \mathrm{E}-03$ & 0.60 & 1.40 \\
\hline 6.00 & $4.43 \mathrm{E}+04$ & 0.21 & 0.77 & 0.56 & 1.29 & $9.44 \mathrm{E}-04$ & $1.89 \mathrm{E}-03$ & 0.56 & 1.29 \\
\hline 5.50 & $4.06 \mathrm{E}+04$ & 0.18 & 0.74 & 0.43 & 1.01 & $1.41 \mathrm{E}-02$ & $2.82 \mathrm{E}-02$ & 0.43 & 1.01 \\
\hline 5.00 & $3.69 \mathrm{E}+04$ & 0.20 & 0.78 & 0.43 & 1.01 & $1.59 \mathrm{E}-02$ & $3.17 \mathrm{E}-02$ & 0.43 & 1.01 \\
\hline 4.50 & $3.32 \mathrm{E}+04$ & 0.21 & 0.79 & 0.41 & 0.96 & $2.20 \mathrm{E}-03$ & $4.40 \mathrm{E}-03$ & 0.41 & 0.96 \\
\hline 4.05 & $2.99 \mathrm{E}+04$ & 0.21 & 0.78 & 0.37 & 0.87 & $1.08 \mathrm{E}-03$ & $2.17 \mathrm{E}-03$ & 0.37 & 0.87 \\
\hline 3.50 & $2.58 \mathrm{E}+04$ & 0.21 & 0.77 & 0.32 & 0.75 & $2.52 \mathrm{E}-04$ & $5.03 \mathrm{E}-04$ & 0.32 & 0.75 \\
\hline
\end{tabular}

Tabla 7.13. Resultados obtenidos para amortiguamiento estructural 0.025

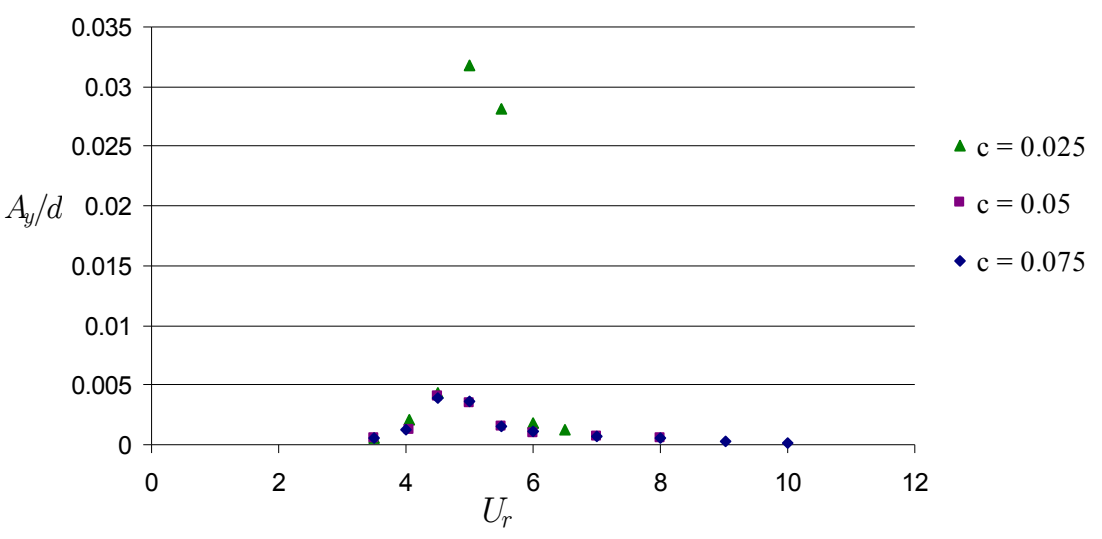

Figura 7.61. Respuesta en amplitud para diferentes valores del amortiguamiento estructural

\subsection{Mástil de acero. Estudio de la amplificación del coeficiente de arrastre}

A continuación se estudia el fenómeno aeroelástico de amplificación del coeficiente de arrastre utilizando el modelo descrito en la sección anterior para la misma estructura de $34 \mathrm{~m}$ de altura. Tanto para la estructura como para el fluido las condiciones de contorno son las indicadas en el punto anterior así como también las mallas utilizadas.

En la Figura 7.63 a la Figura 7.67 se muestran la respuesta en frecuencias y amplitud (en escala normal y logarítmica) para diferentes valores del amortiguamiento estructural. Se observa que a partir de una cierta velocidad la frecuencia de desprendimiento de los torbellinos se sincroniza con la frecuencia natural de la estructura y la amplitud de la vibración transversal de la estructura se incrementa notablemente. El valor de la velocidad para la cual ocurre esta situación es menor cuanto menor es el amortiguamiento estructural. 


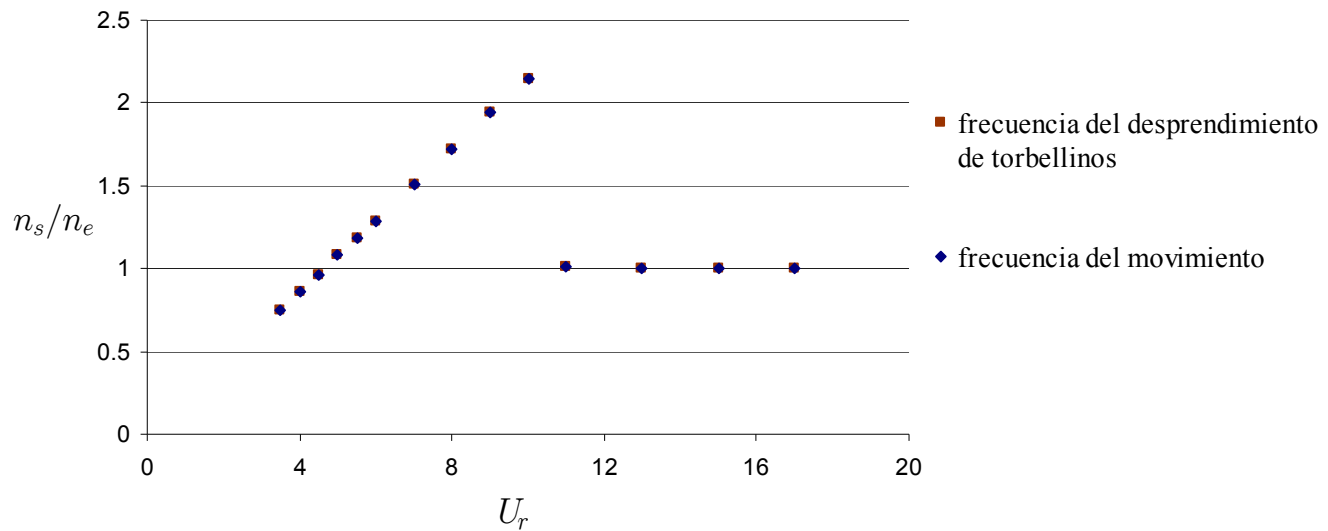

Figura 7.62. Respuesta en frecuencias para amortiguamiento estructural 0.075
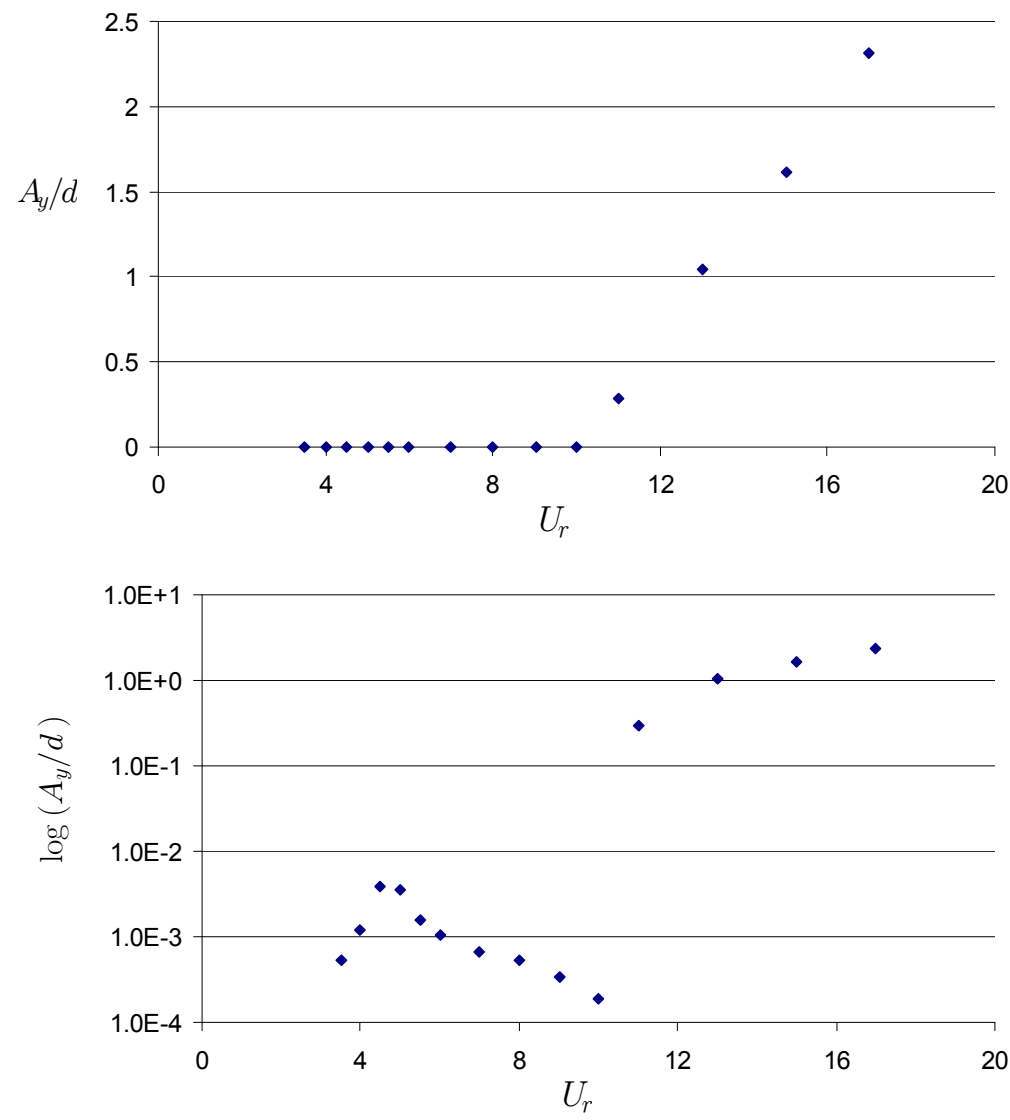

Figura 7.63. Respuesta en amplitud para amortiguamiento estructural 0.075 


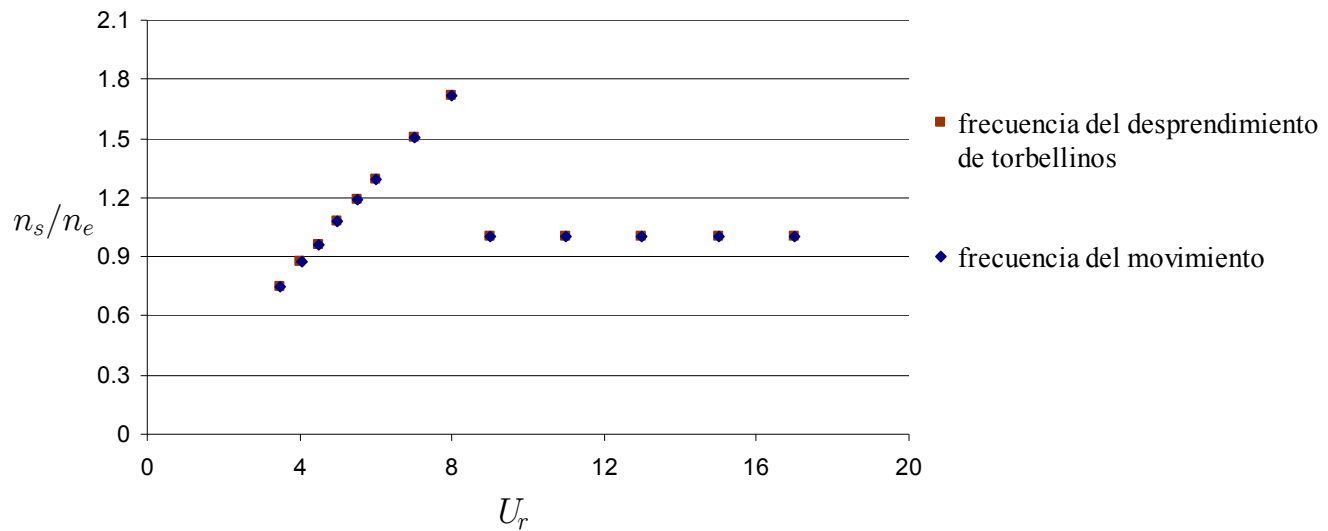

Figura 7.64. Respuesta en frecuencias para amortiguamiento estructural 0.05
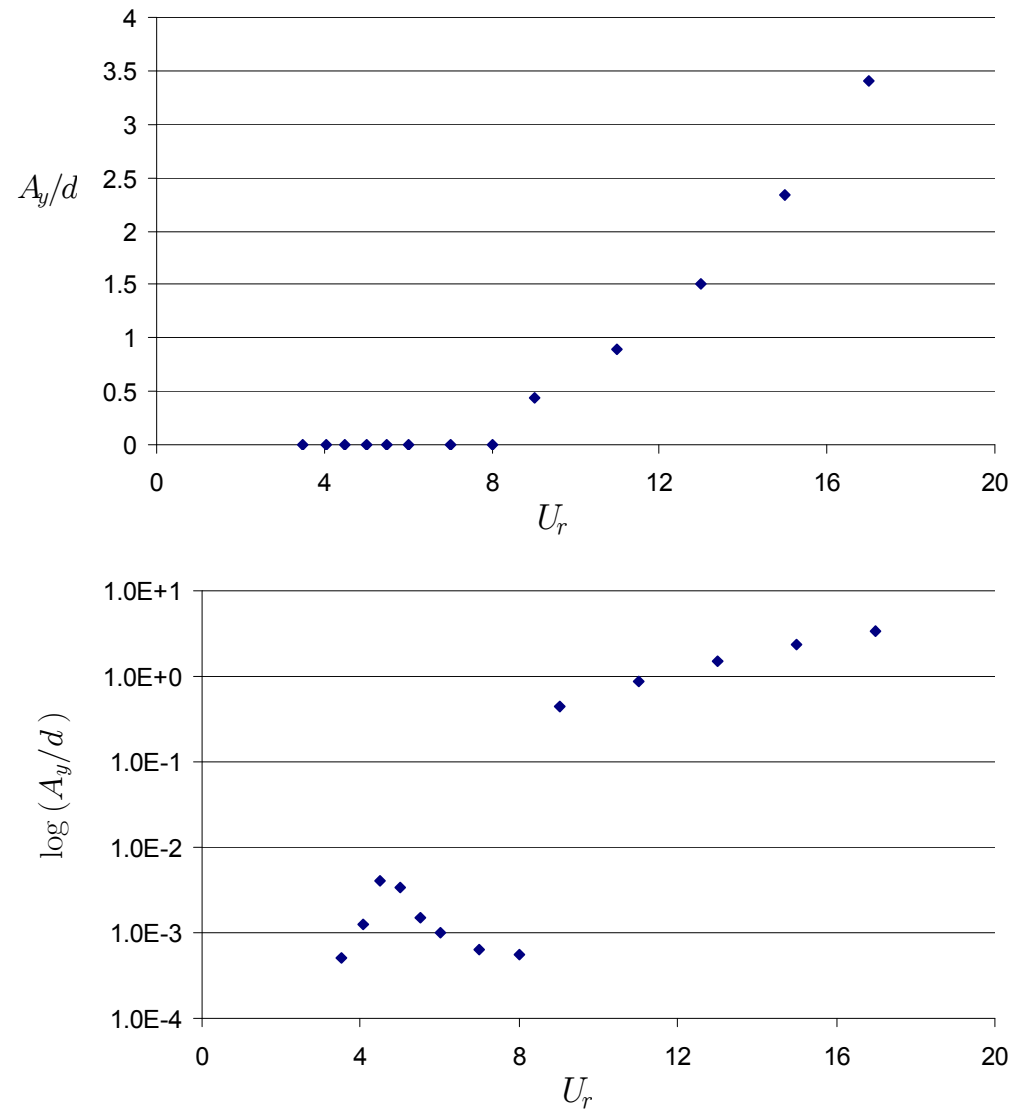

Figura 7.65. Respuesta en amplitud para amortiguamiento estructural 0.05 


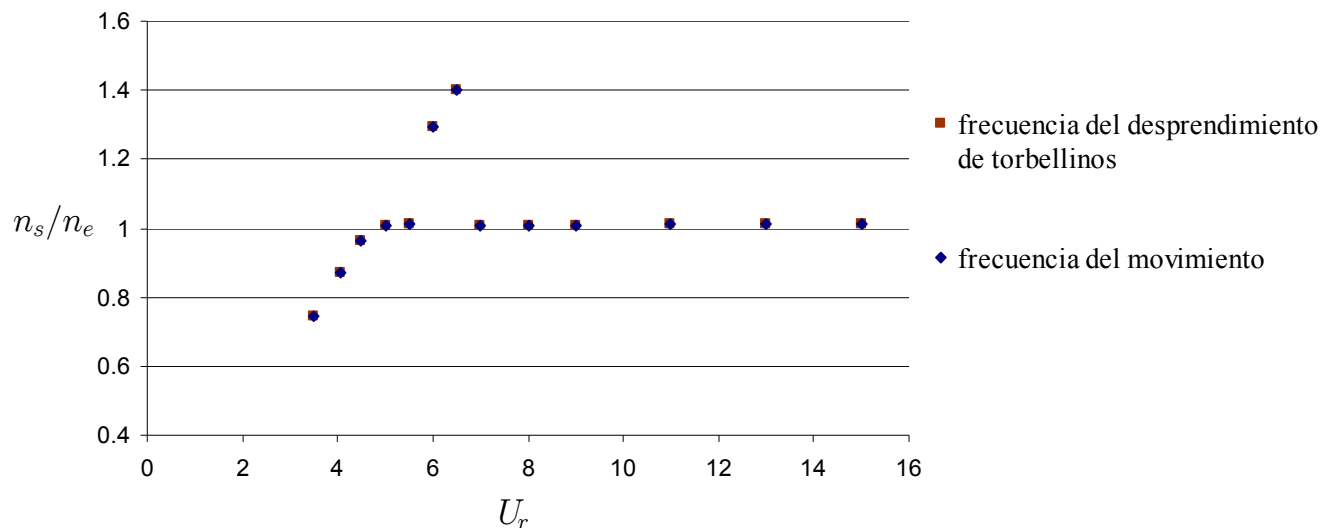

Figura 7.66. Respuesta en frecuencias para amortiguamiento estructural 0.025
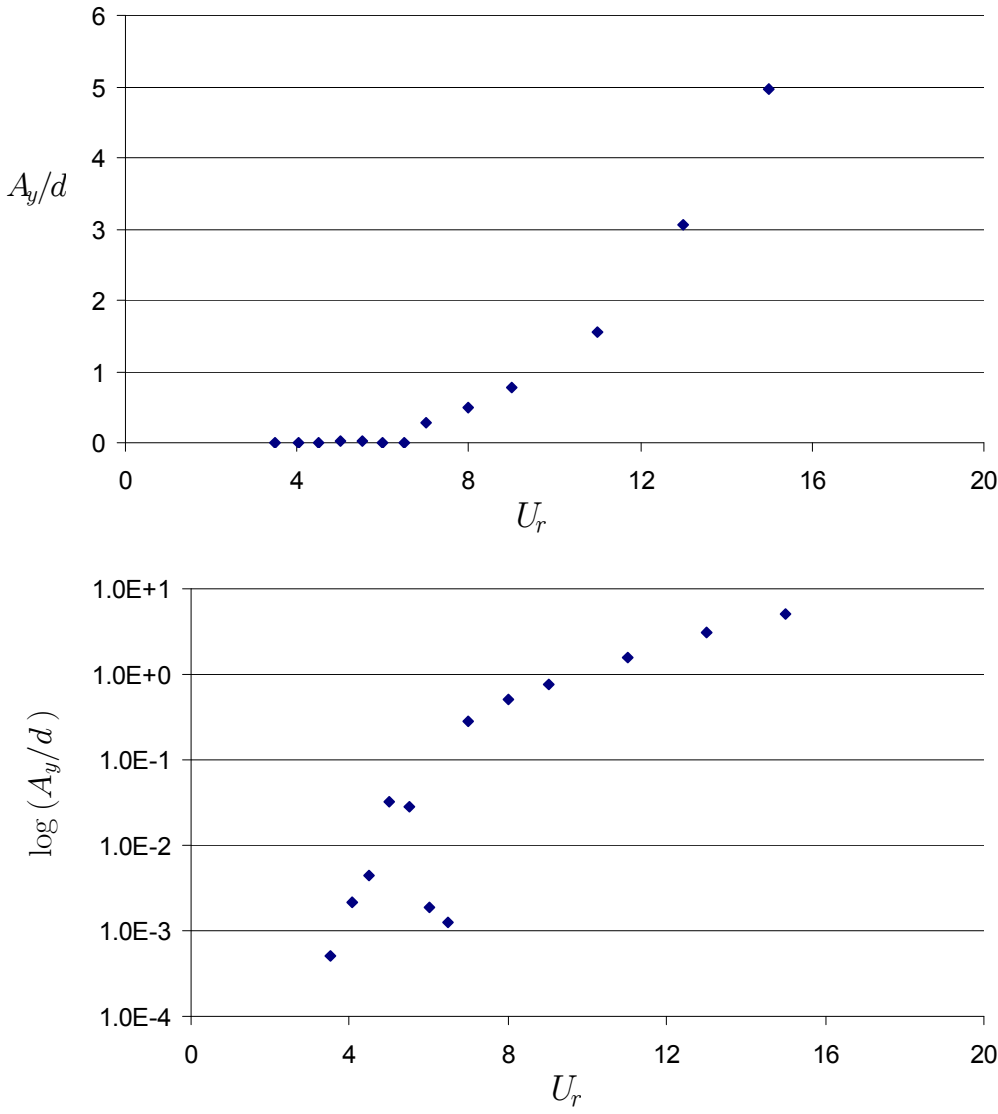

Figura 7.67. Respuesta en amplitud para amortiguamiento estructural 0.025 
Notar que en el caso de amortiguamiento estructural 0.025 (Figura 7.66 y Figura 7.67) existe una región de velocidades en la cual se produce el fenómeno de lock-in. Superado ese rango de velocidades se cumple la ley de Strouhal hasta que la velocidad alcanza un cierto valor y se produce de nuevo la sincronización entre la frecuencia de desprendimiento de los torbellinos y la de la estructura.

Fujarra et al. [5] estudiaron las vibraciones inducidas por el desprendimiento de torbellinos en cilindros flexibles en voladizo. Entre los resultados que se derivan de su estudio, cabe destacar en el que se mostraba que a partir de un cierto valor de la velocidad $\left(U_{r}=12\right.$, de acuerdo con sus ensayos) la amplitud de las vibraciones en la dirección transversal a la de la corriente de fluido aumentaba de forma considerable, y ello sucedía fuera del rango habitual de velocidades $\left(U_{r}=4-\right.$ 8) en el que ocurre el fenómeno de lock-in (Figura 7.68).

Este comportamiento se atribuye al acoplamiento entre el movimiento de la estructura en la dirección transversal y el que ocurre en la misma dirección en la que actúa el fluido, el cual se hace importante y por lo tanto no se puede despreciar. También se observa que la frecuencia de la vibración en la dirección transversal es exactamente igual a la mitad de la frecuencia de la vibración en la dirección de la corriente de fluido.

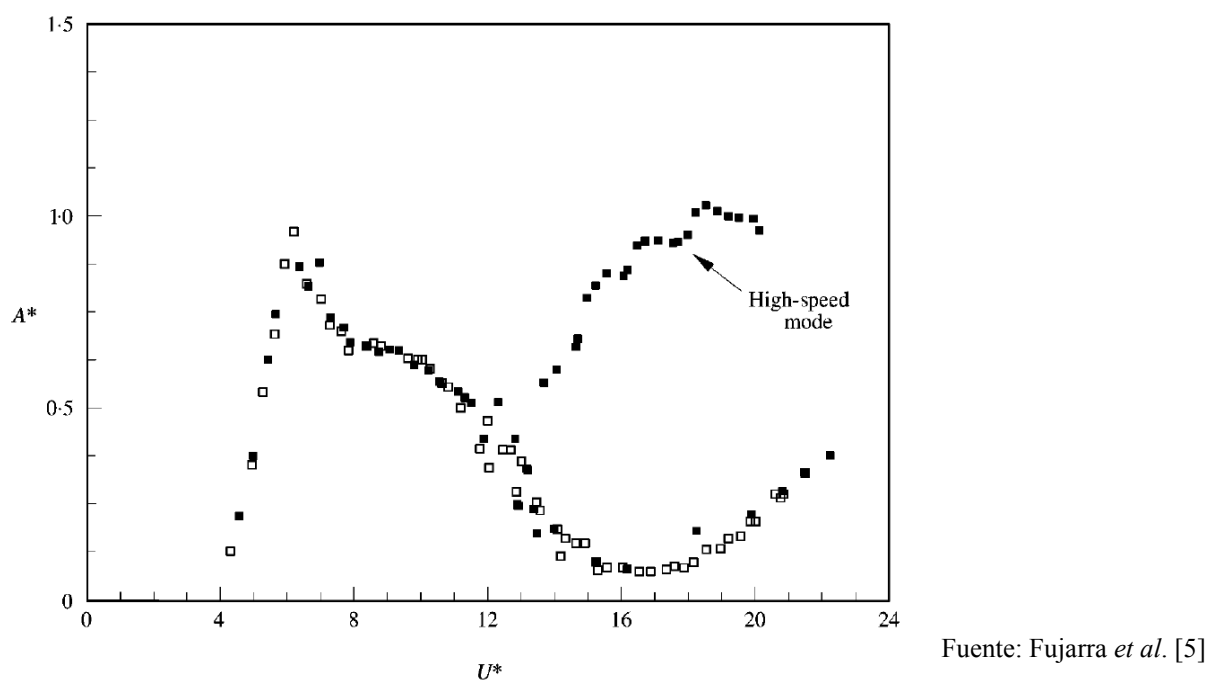

Figura 7.68. Respuesta en amplitud de un cilindro flexible en voladizo

Una respuesta similar se puede encontrar también en el trabajo de Kitagawa et al. [6] para velocidades elevadas $\left(U_{r}=14-17\right)$, aunque en este caso las amplitudes del movimiento en la dirección transversal eran bastante más pequeñas. En definitiva, los resultados mostrados por Fujarra et al. y Kitagawa et al. guardan una cierta relación con los obtenidos en este trabajo. 


\section{Caso 1. Amortiguamiento estructural 0.075}

Para ilustrar la amplificación del coeficiente de arrastre se estudiará el caso de $U_{r}=13$. En la Figura 7.69 se muestra la variación con el tiempo del coeficiente de sustentación y en la Figura 7.70 la del coeficiente de arrastre. A medida que transcurre el tiempo de análisis se observa que la amplitud de la oscilación de ambos coeficientes se incrementa de forma considerable hasta alcanzar un cierto valor, el cual permanece constante en el tiempo. Esto sucede cuando se produce la sincronización entre la frecuencia de desprendimiento de los torbellinos, la cual coincide con la frecuencia del coeficiente de sustentación, y la frecuencia natural de la estructura.

Al principio del análisis, para $t<250 \mathrm{~s}$, la oscilación del coeficiente de sustentación ocurre a la frecuencia de $1.2 \mathrm{~Hz}$ (Figura 7.71), la cual coincide con la que se obtiene a partir de la ley de Strouhal (7.4). La sincronización entre las dos frecuencias se produce entre $t=250 \mathrm{~s}$ y $t=350 \mathrm{~s}$ aproximadamente (Figura 7.72). Al final del análisis, para $t>350 \mathrm{~s}$, la frecuencia del coeficiente de sustentación coincide con la frecuencia natural de la estructura $n_{e}=0.43 \mathrm{~Hz}$ (Figura 7.73).

$$
n_{s}=S t \frac{U}{d}=0.21 \frac{2.88}{0.5}=1.2 \mathrm{~Hz}
$$

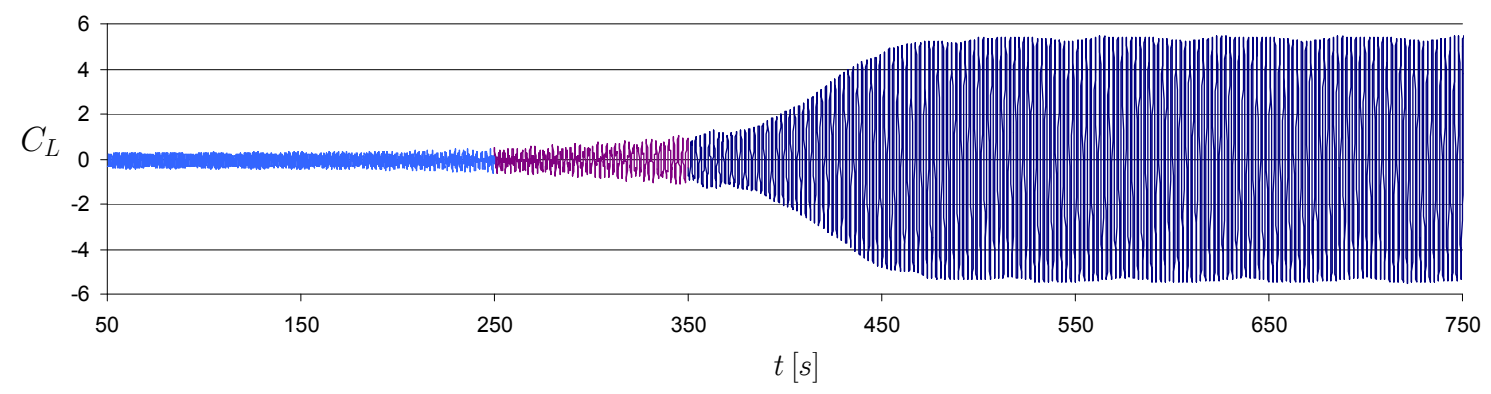

Figura 7.69. Oscilación del coeficiente de sustentación para amortiguamiento estructural 0.075

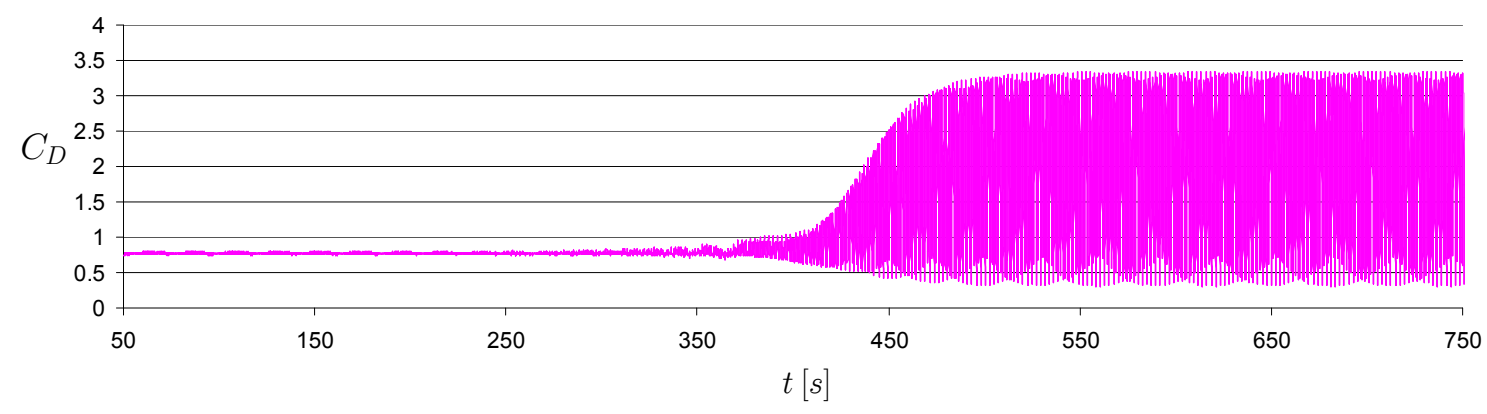

Figura 7.70. Oscilación del coeficiente de arrastre para amortiguamiento estructural 0.075 


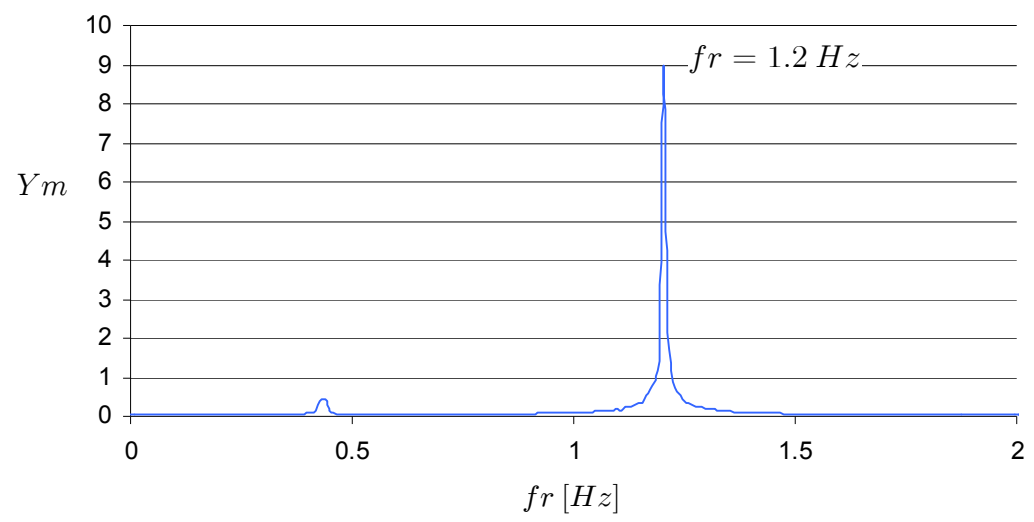

Figura 7.71. Análisis FFT de la oscilación del coeficiente de sustentación para $t<250 \mathrm{~s}$

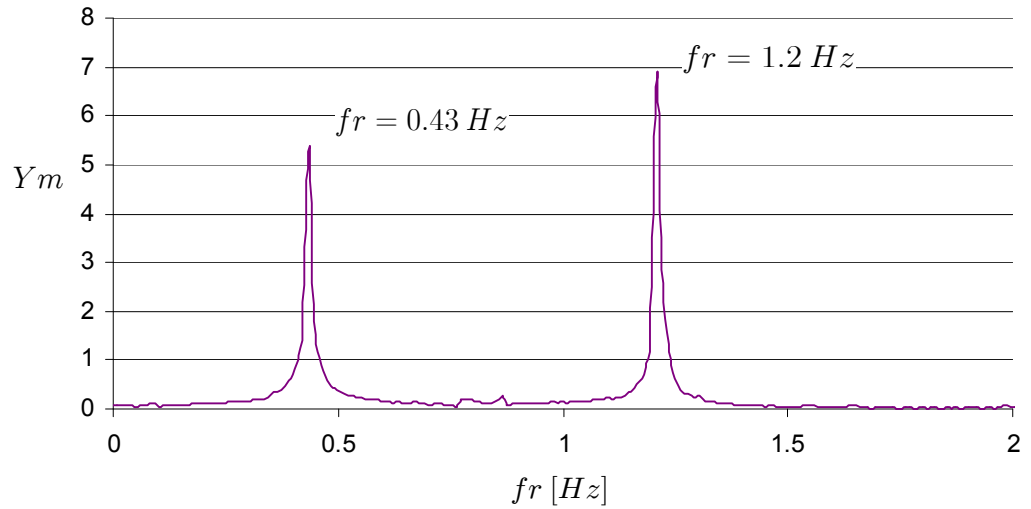

Figura 7.72. Análisis FFT de la oscilación del coeficiente de sustentación en el intervalo $250<t<350 \mathrm{~s}$

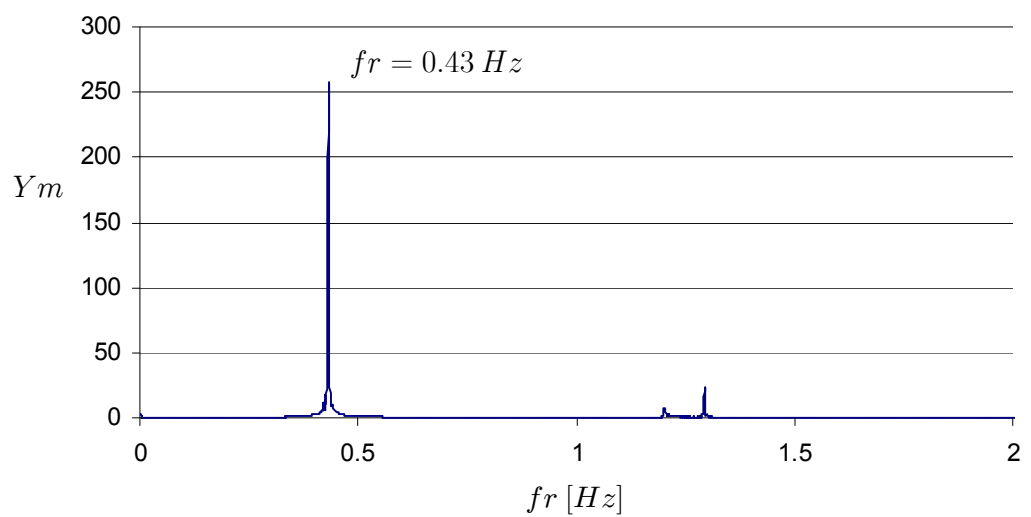

Figura 7.73. Análisis FFT de la oscilación del coeficiente de sustentación para $t>350 \mathrm{~s}$ 
Notar que el coeficiente de sustentación siempre oscila en torno a 0 , sin embargo, el coeficiente de arrastre pasa a oscilar en torno a un valor mayor una vez que se ha producido la sincronización de las frecuencias, es decir, ocurre la amplificación del coeficiente de arrastre. Al principio del análisis, para $t<350 \mathrm{~s}$, el valor RMS del coeficiente de arrastre es $0.77 \mathrm{y}$ al final del análisis, para $t>500 \mathrm{~s}$, dicho valor es 2.33 .

En la Figura 7.74 se muestra el análisis FFT de la oscilación del coeficiente de arrastre y se comprueba que ésta tiene lugar a la frecuencia de $0.86 \mathrm{~Hz}$, que es justo el doble de la frecuencia a la que oscila el coeficiente de sustentación.

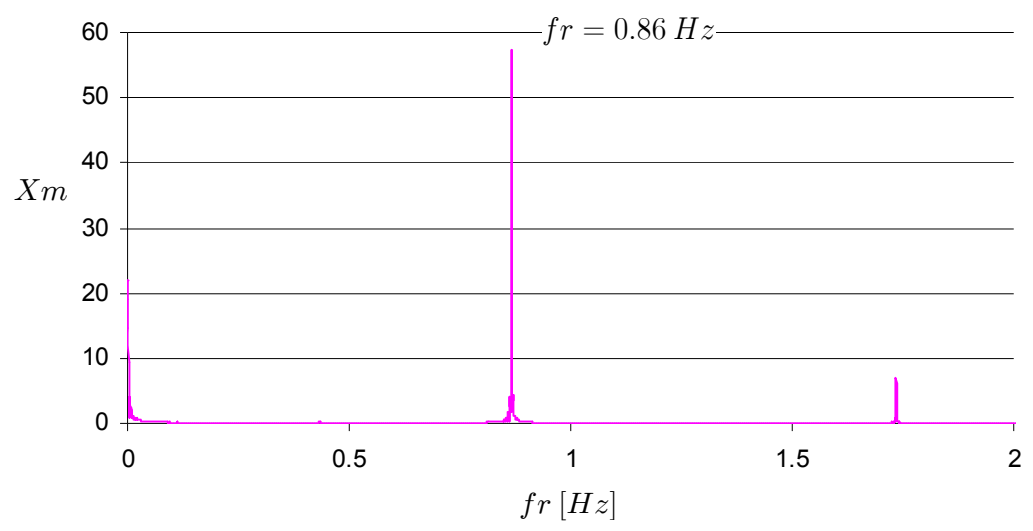

Figura 7.74. Análisis FFT de la oscilación del coeficiente de arrastre

En la Figura 7.75 se ha representado la vibración de la estructura en la dirección transversal y en la Figura 7.76 en la dirección del flujo. Se puede apreciar que a partir de que se produce la sincronización de las frecuencias la amplitud de la vibración de la estructura en ambas direcciones se incrementa, si bien, en mayor medida en la vibración transversal. También se observa que la estructura en la dirección del flujo pasa a vibrar respecto a una nueva posición de equilibrio como consecuencia de la amplificación que experimenta el coeficiente de arrastre.

En la Figura 7.77 se muestra el análisis FFT de la vibración de la estructura en la dirección transversal y en la dirección del flujo. En la dirección transversal se observa que la estructura vibra a su frecuencia natural $n_{e}=0.43 \mathrm{~Hz}$. En la dirección en la que actúa el aire, al principio del análisis la estructura vibra a su frecuencia natural hasta que alcanza la nueva posición de equilibrio, en torno a la cual prácticamente no vibra. A partir del momento en el que se produce la sincronización entre la frecuencia de desprendimiento de los torbellinos y la frecuencia natural de la estructura, ésta vibra respecto a una nueva posición de equilibrio a la frecuencia de $0.86 \mathrm{~Hz}$, que es exactamente igual al doble de la frecuencia de la vibración en la dirección transversal, $0.43 \mathrm{~Hz}$. Esto pone de 
manifiesto la fuerte correlación que existe entre el movimiento de la estructura en la dirección transversal y en la dirección paralela al flujo una vez que se han sincronizado las frecuencias.

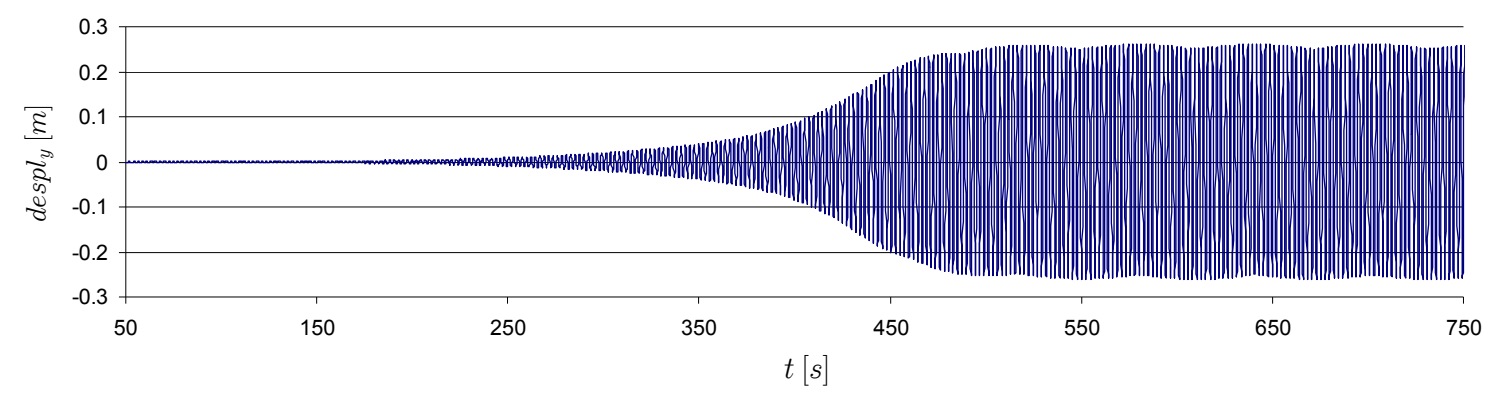

Figura 7.75. Vibración de la estructura en la dirección transversal para amortiguamiento estructural 0.075

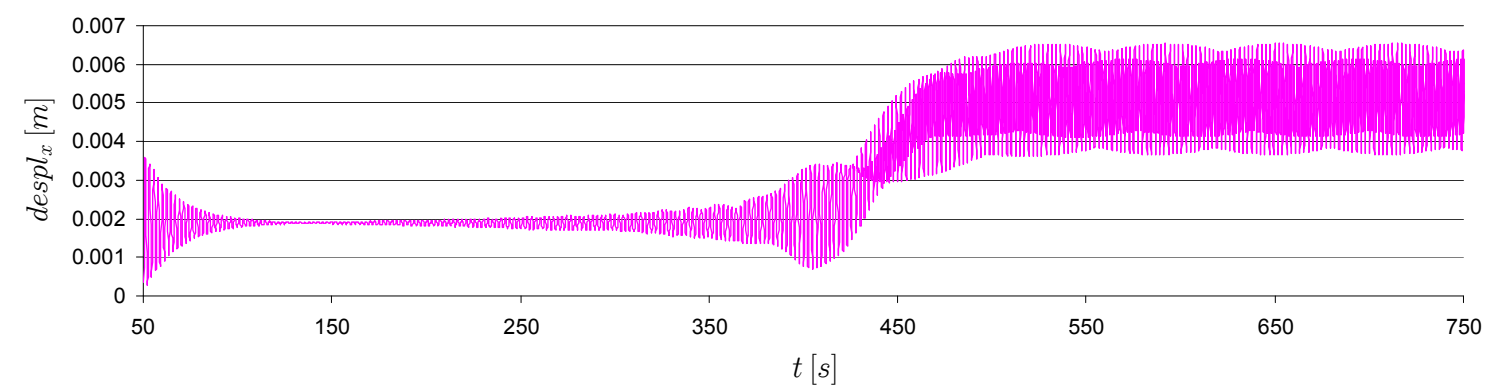

Figura 7.76. Vibración de la estructura en la dirección del flujo para amortiguamiento estructural 0.075

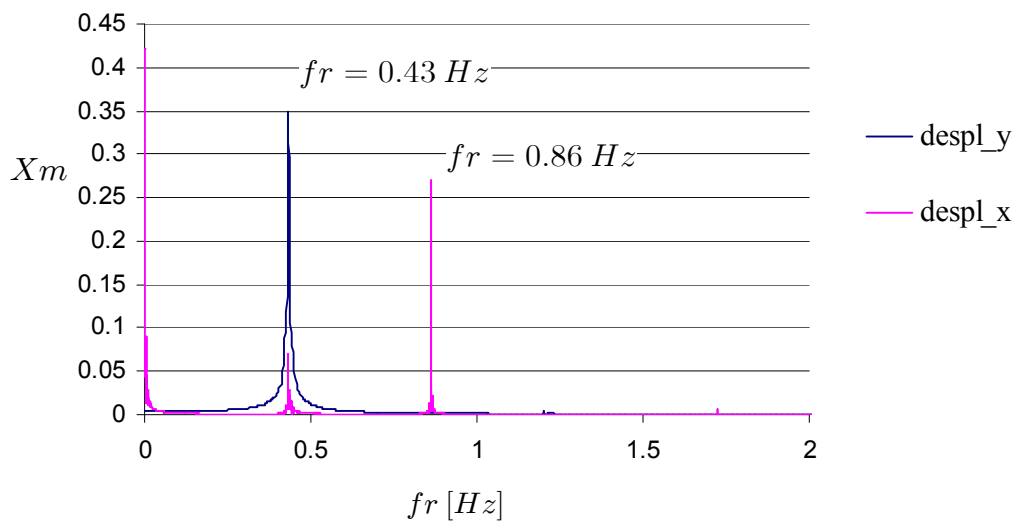

Figura 7.77. Análisis FFT de la vibración de la estructura

En la Figura 7.78 se ha representado la componente de la velocidad en la dirección transversal del extremo libre de la estructura y en la Figura 7.79 la componente de velocidad en la dirección del flujo. Finalmente, en la Figura 7.80 se muestra el módulo de la velocidad del extremo libre de la estructura y en la Figura 7.81 se ha representado el análisis FFT de las componentes de la velocidad y de su módulo. 


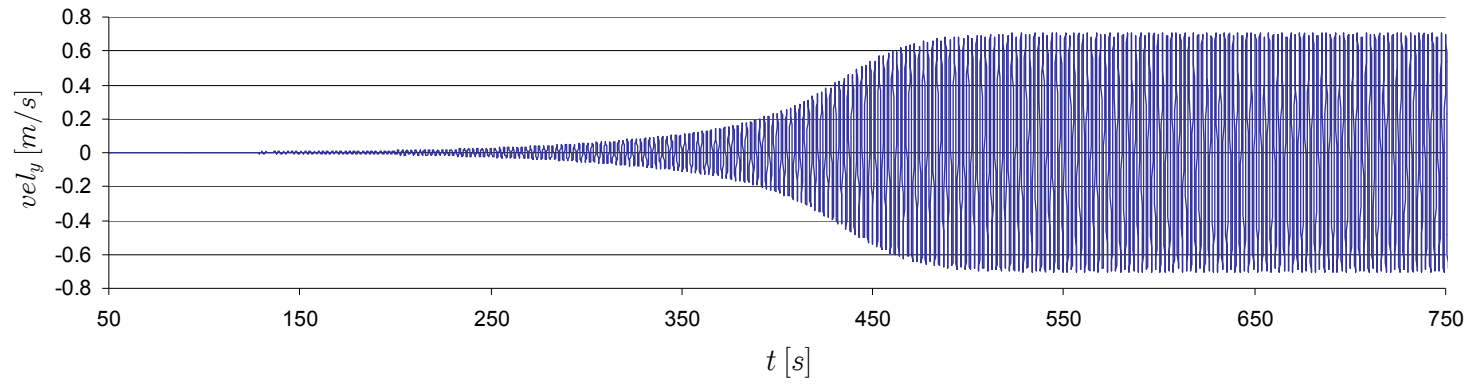

Figura 7.78. Componente transversal de la velocidad para amortiguamiento estructural 0.075

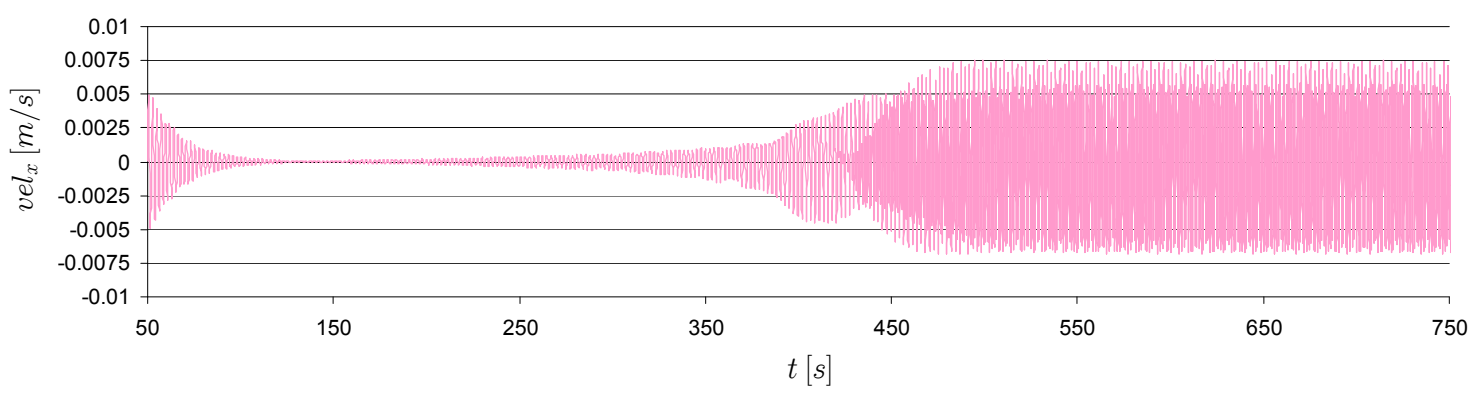

Figura 7.79. Componente de la velocidad en la dirección del flujo para amortiguamiento estructural 0.075

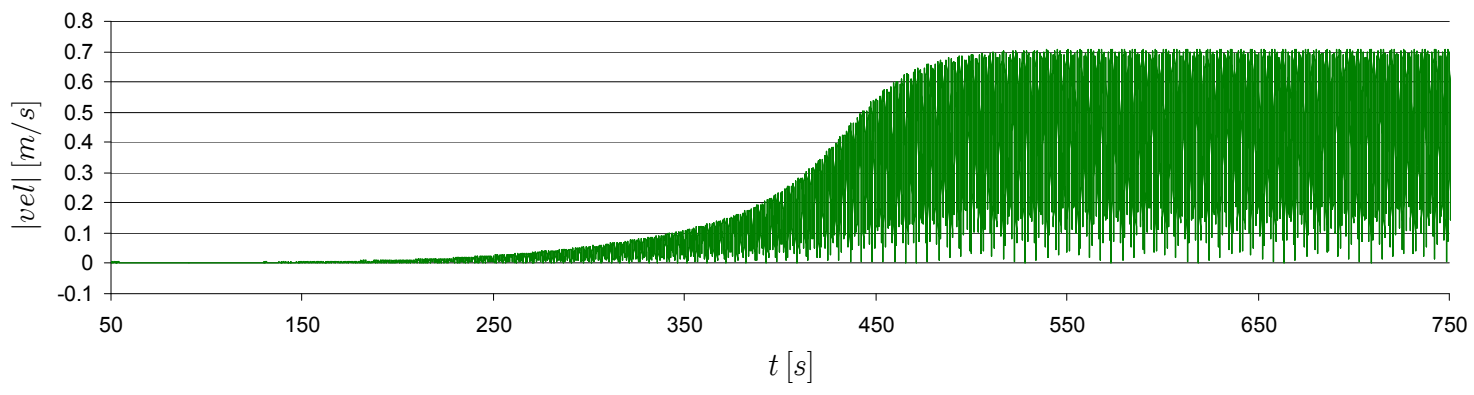

Figura 7.80. Módulo de la velocidad para amortiguamiento estructural 0.075

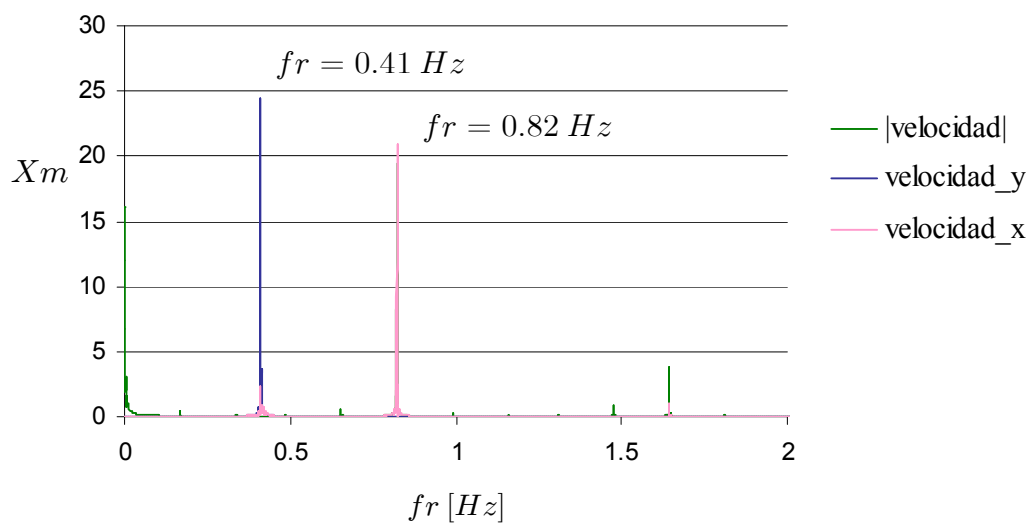

Figura 7.81. Análisis FFT de la velocidad del extremo libre de la estructura 
En la Tabla 7.14 se muestra un resumen de los resultados obtenidos para el caso de amortiguamiento estructural 0.075 . Se distinguen dos zonas de estudio, al principio del análisis, cuando aún no se ha producido la sincronización de las frecuencias y al final del análisis, cuando las frecuencias ya se han sincronizado. El resto de las gráficas se muestran en el punto J.1 del Anexo J.

\begin{tabular}{|c|c|c|c|c|c|c|c|c|c|}
\hline \multirow[b]{2}{*}{$U_{r}$} & \multirow[b]{2}{*}{$\begin{array}{c}\boldsymbol{U} \\
{[\mathrm{m} / \mathrm{s}]}\end{array}$} & \multirow[b]{2}{*}{ Re } & \multirow[b]{2}{*}{$A_{y} / d$} & \multicolumn{3}{|c|}{ Al principio del análisis } & \multicolumn{3}{|c|}{ Al final del análisis } \\
\hline & & & & $\begin{array}{c}\text { RMS } \\
\text { despl }_{x} \\
{[\mathrm{~m}]}\end{array}$ & $\begin{array}{c}\text { RMS } \\
|\mathbf{v e l}| \\
{[\mathrm{m} / \mathrm{s}]}\end{array}$ & $\tilde{C}_{D}$ & $\begin{array}{c}\text { RMS } \\
\text { despl }_{x} \\
{[m]}\end{array}$ & $\begin{array}{c}\text { RMS } \\
|\mathbf{v e l}| \\
{[\mathrm{m} / \mathrm{s}]}\end{array}$ & $\tilde{C}_{D}$ \\
\hline 7.0 & 1.55 & $2.57 \mathrm{E}+04$ & $6.65 \mathrm{E}-04$ & $5.52 \mathrm{E}-04$ & & 0.77 & $5.52 \mathrm{E}-04$ & & 0.77 \\
\hline 6.0 & 1.33 & $2.97 \mathrm{E}+04$ & $1.07 \mathrm{E}-03$ & 4.07E-04 & & 0.79 & 4.07E-04 & & 0.79 \\
\hline 5.5 & 1.22 & $3.33 \mathrm{E}+04$ & $1.60 \mathrm{E}-03$ & $3.42 \mathrm{E}-04$ & & 0.78 & $3.42 \mathrm{E}-04$ & & 0.78 \\
\hline 5.0 & 1.11 & $3.70 \mathrm{E}+04$ & $3.57 \mathrm{E}-03$ & $2.80 \mathrm{E}-04$ & & 0.77 & $2.80 \mathrm{E}-04$ & & 0.77 \\
\hline 4.5 & 1.00 & $4.07 \mathrm{E}+04$ & $3.96 \mathrm{E}-03$ & $2.32 \mathrm{E}-04$ & & 0.77 & $2.32 \mathrm{E}-04$ & & 0.77 \\
\hline 4.0 & 0.89 & $4.43 \mathrm{E}+04$ & 1.19E-03 & $1.83 \mathrm{E}-04$ & & 0.77 & $1.83 \mathrm{E}-04$ & & 0.77 \\
\hline 3.5 & 0.77 & $5.17 \mathrm{E}+04$ & $5.27 \mathrm{E}-04$ & $1.38 \mathrm{E}-04$ & & 0.77 & $1.38 \mathrm{E}-04$ & & 0.77 \\
\hline 8.0 & 1.77 & $5.90 \mathrm{E}+04$ & $5.32 \mathrm{E}-04$ & 7.23E-04 & & 0.77 & 7.23E-04 & & 0.77 \\
\hline 9.0 & 2.00 & $6.67 \mathrm{E}+04$ & $3.40 \mathrm{E}-04$ & $9.16 \mathrm{E}-04$ & & 0.78 & $9.16 \mathrm{E}-04$ & & 0.78 \\
\hline 10.0 & 2.21 & $7.37 \mathrm{E}+04$ & $1.92 \mathrm{E}-04$ & $1.11 \mathrm{E}-03$ & & 0.77 & $1.11 \mathrm{E}-03$ & & 0.77 \\
\hline 11.0 & 2.43 & $8.10 \mathrm{E}+04$ & $2.90 \mathrm{E}-01$ & $1.36 \mathrm{E}-03$ & 0.0013 & 0.77 & $1.45 \mathrm{E}-03$ & 0.1492 & 0.84 \\
\hline 13.0 & 2.88 & $9.60 \mathrm{E}+04$ & $1.04 \mathrm{E}+00$ & $1.91 \mathrm{E}-03$ & 0.0037 & 0.77 & $5.16 \mathrm{E}-03$ & 0.5001 & 2.33 \\
\hline 15.0 & 3.32 & $1.11 \mathrm{E}+05$ & $1.61 \mathrm{E}+00$ & $2.55 \mathrm{E}-03$ & 0.0045 & 0.77 & $8.60 \mathrm{E}-03$ & 0.7739 & 2.96 \\
\hline 17.0 & 3.76 & $1.25 \mathrm{E}+05$ & $2.31 \mathrm{E}+00$ & $3.29 \mathrm{E}-03$ & 0.0058 & 0.77 & $1.33 \mathrm{E}-02$ & 1.1091 & 3.61 \\
\hline 19.0 & 4.20 & $1.40 \mathrm{E}+05$ & $3.15 \mathrm{E}+00$ & $4.13 \mathrm{E}-03$ & 0.0067 & 0.77 & $1.97 \mathrm{E}-02$ & 1.5127 & 4.31 \\
\hline
\end{tabular}

Tabla 7.14. Resultados obtenidos para al caso de amortiguamiento estructural 0.075

En la Figura 7.82 se ha representado el valor RMS del coeficiente de arrastre en función de la velocidad reducida y en la Figura 7.83 en función de la amplitud de la vibración de la estructura en la dirección transversal en escala normal y logarítmica. Se observa que a partir de un cierto valor de la velocidad del flujo y de la amplitud de la vibración transversal de la estructura se produce la amplificación del coeficiente de arrastre.

Debido a la amplificación del coeficiente de arrastre el desplazamiento de la estructura en la dirección en la que actúa el flujo se incrementa. Esto se puede comprobar en la Figura 7.84, en la cual se ha representado el valor RMS del desplazamiento del extremo libre de la estructura frente a la velocidad reducida. 


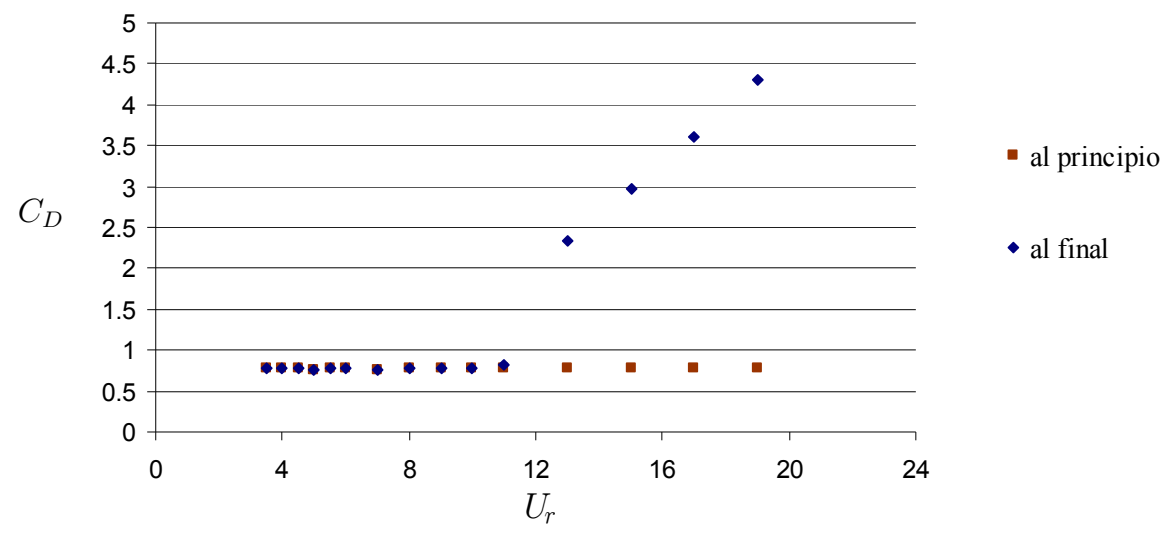

Figura 7.82. Valor RMS del coeficiente de arrastre frente a la velocidad reducida
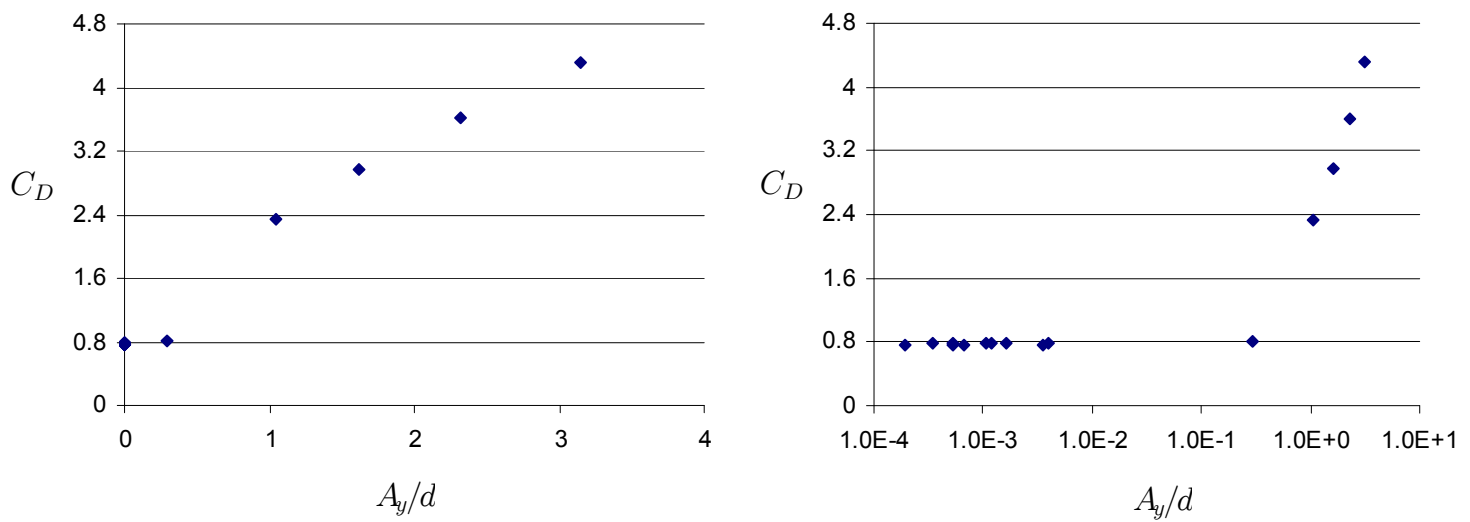

Figura 7.83. Valor RMS coeficiente de arrastre frente a la amplitud de la vibración de la estructura en la dirección transversal

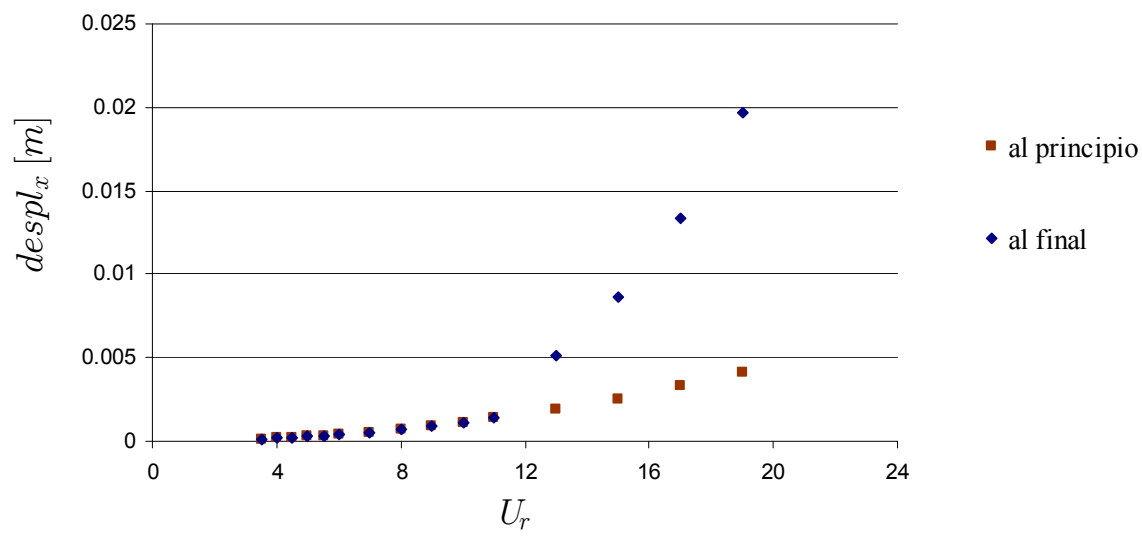

Figura 7.84. Valor RMS del desplazamiento en la dirección del flujo del extremo libre de la estructura 


\section{Caso 2. Amortiguamiento estructural 0.025}

De forma análoga al caso anterior, a continuación se estudia el caso de $U_{r}=9$. La oscilación del coeficiente de sustentación se muestra en la Figura 7.85 y la del coeficiente de arrastre en la Figura 7.86. Se aprecia que a medida que transcurre el tiempo la amplitud de la oscilación de ambos coeficientes aumenta notablemente hasta alcanzar un cierto valor, el cual permanece constante en el tiempo, de la misma manera que ocurría en el caso anterior.

Al principio del análisis, para $t<150 \mathrm{~s}$, la oscilación del coeficiente de sustentación ocurre a la frecuencia de $0.84 \mathrm{~Hz}$ (Figura 7.87), la cual coincide con la que se obtiene al aplicar la ley de Strouhal (7.5)

$$
n_{s}=S t \frac{U}{d}=0.21 \frac{2.0}{0.5}=0.84 \mathrm{~Hz}
$$

La sincronización de la frecuencia de desprendimiento de los torbellinos con la frecuencia natural de la estructura ocurre, en este caso, entre $t=150 \mathrm{~s}$ y $t=250 \mathrm{~s}$ aproximadamente (Figura 7.88). Al final del análisis, para $t>250 \mathrm{~s}$, la frecuencia del coeficiente de sustentación coincide con la frecuencia natural de la estructura $n_{e}=0.43 \mathrm{~Hz}$ (Figura 7.89).

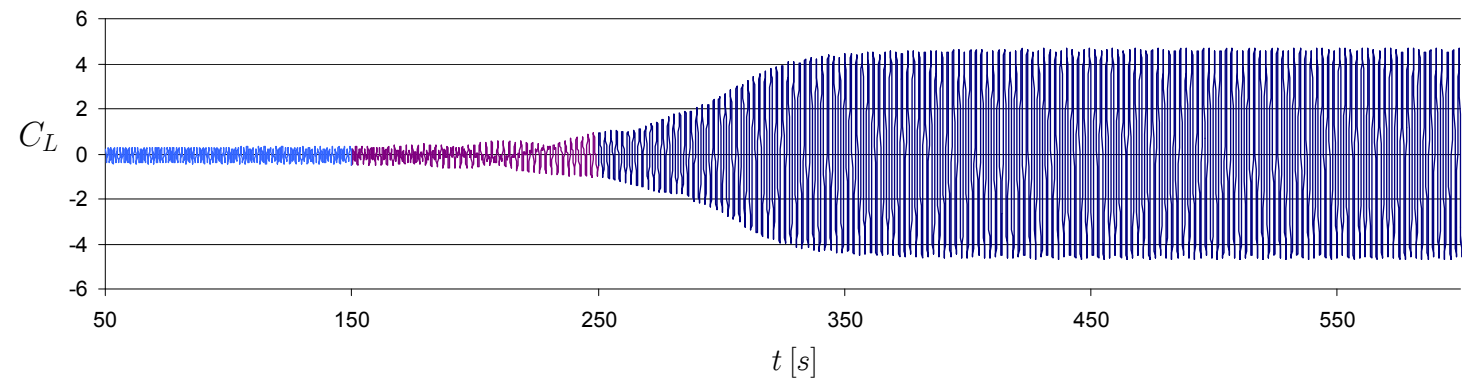

Figura 7.85. Oscilación del coeficiente de sustentación para amortiguamiento estructural 0.025

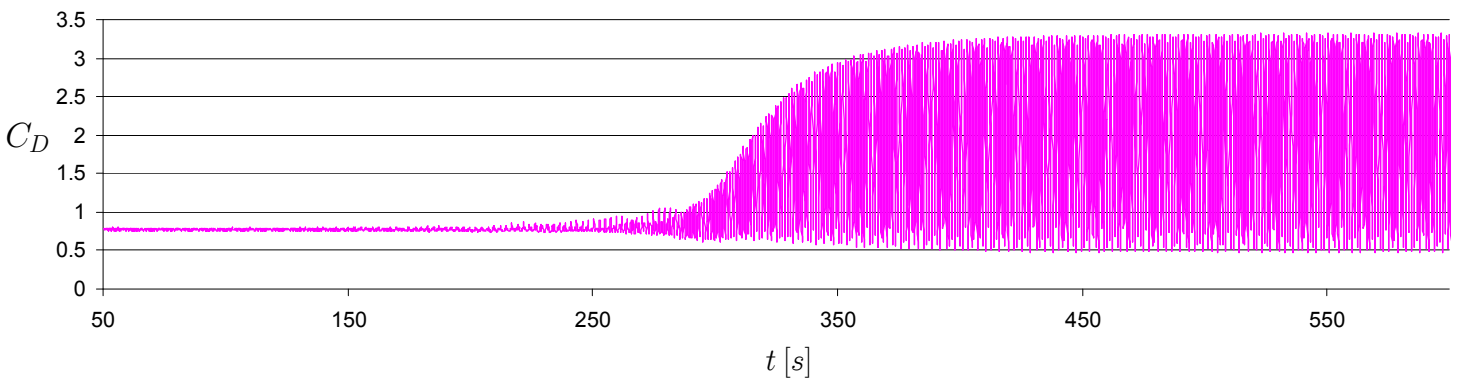

Figura 7.86. Oscilación del coeficiente de arrastre para amortiguamiento estructural 0.025 


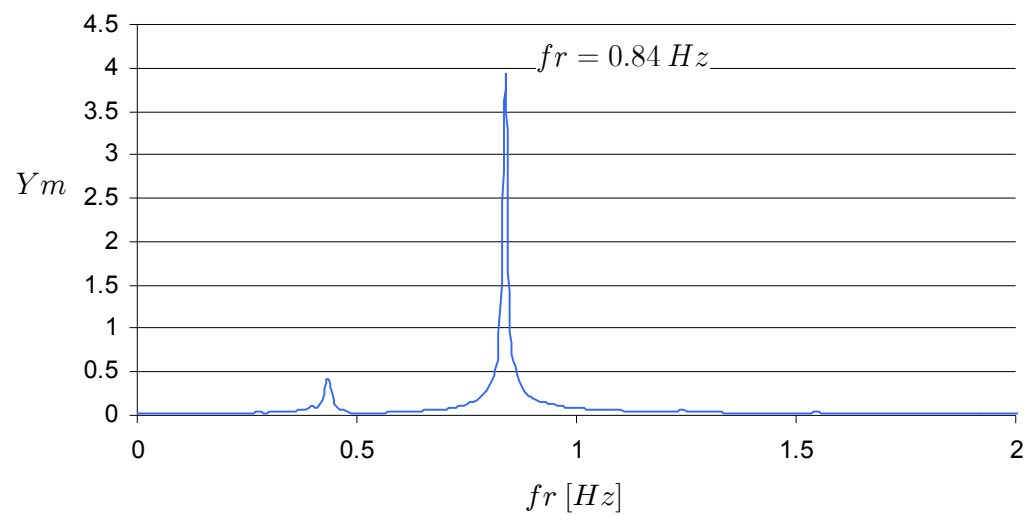

Figura 7.87. Análisis FFT de la oscilación del coeficiente de sustentación para $t<150 \mathrm{~s}$

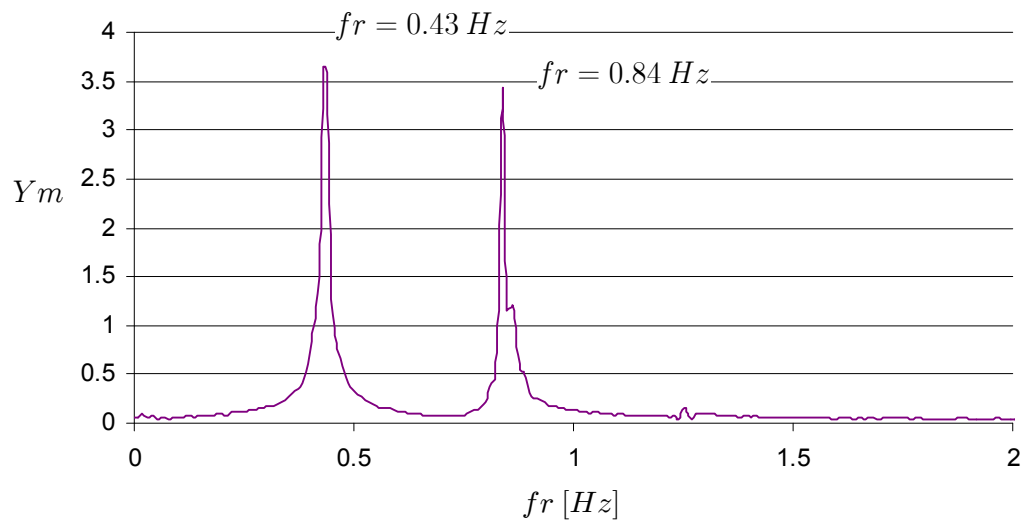

Figura 7.88. Análisis FFT de la oscilación del coeficiente de sustentación en el intervalo $150<t<250 \mathrm{~s}$

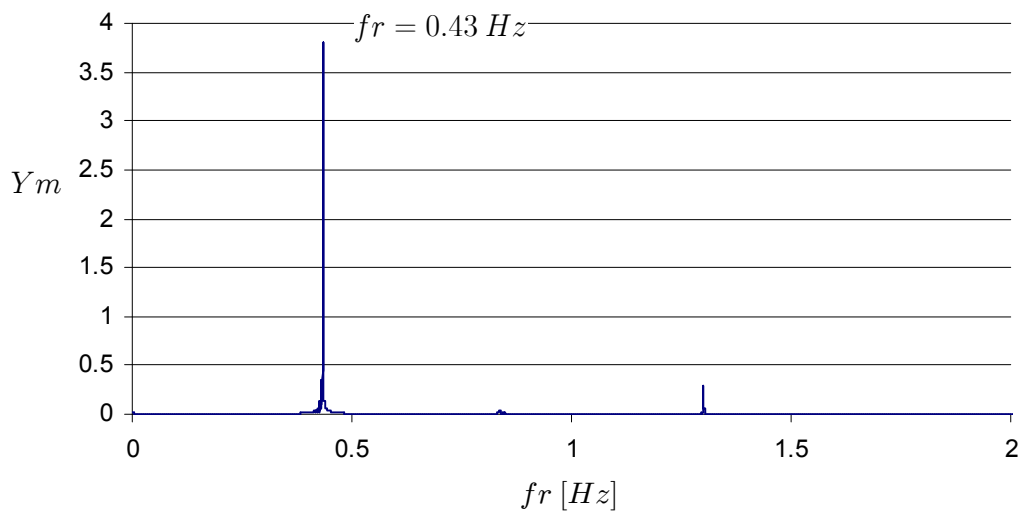

Figura 7.89. Análisis FFT de la oscilación del coeficiente de sustentación para $t>250 \mathrm{~s}$ 
Cabe destacar que si bien el coeficiente de sustentación oscila en torno a 0 , el coeficiente de arrastre pasa a oscilar respecto a un valor mayor, de la misma manera que ocurría en el ejemplo anterior. Al principio del análisis, para $t<250 \mathrm{~s}$, el valor RMS del coeficiente de arrastre es $0.77 \mathrm{y}$ después de que se produce la sincronización de las frecuencias el valor RMS de dicho coeficiente es 2.3. En la Figura 7.90 se muestra el análisis FFT de la oscilación del coeficiente de arrastre y se comprueba que oscila a una frecuencia de $0.86 \mathrm{~Hz}$, que es justo el doble de la del coeficiente de sustentación.

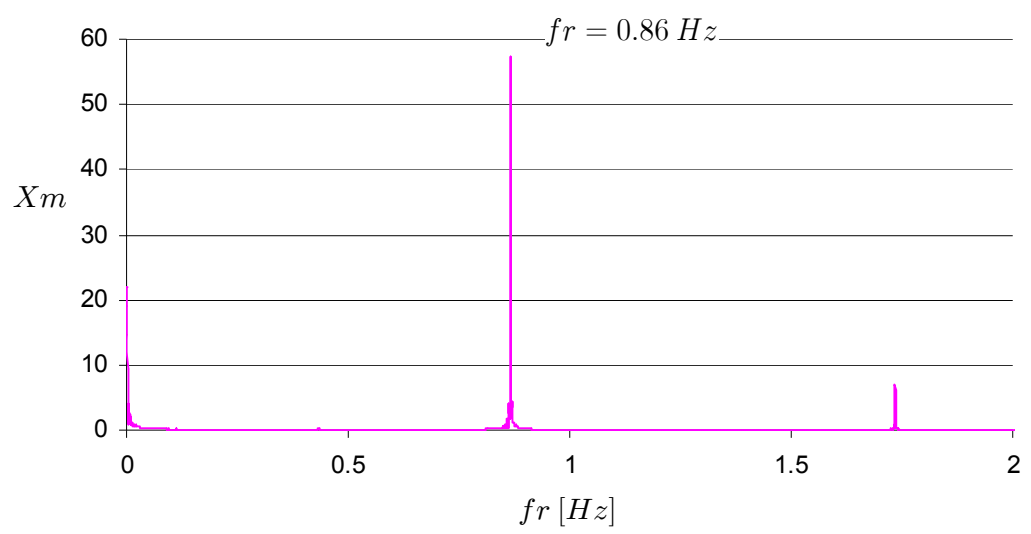

Figura 7.90. Análisis FFT de la oscilación del coeficiente de arrastre

En la Figura 7.91 se muestra la vibración de la estructura en la dirección transversal y en la Figura 7.92 en la dirección del flujo. Vuelve a suceder lo mismo que ocurría en el caso anterior, es decir, a partir de que se produce la sincronización de las frecuencias, la amplitud del movimiento en ambas direcciones aumenta y además, en la dirección del flujo, la estructura pasa a vibrar respecto a una nueva de posición de equilibrio debido a la amplificación del coeficiente de arrastre.

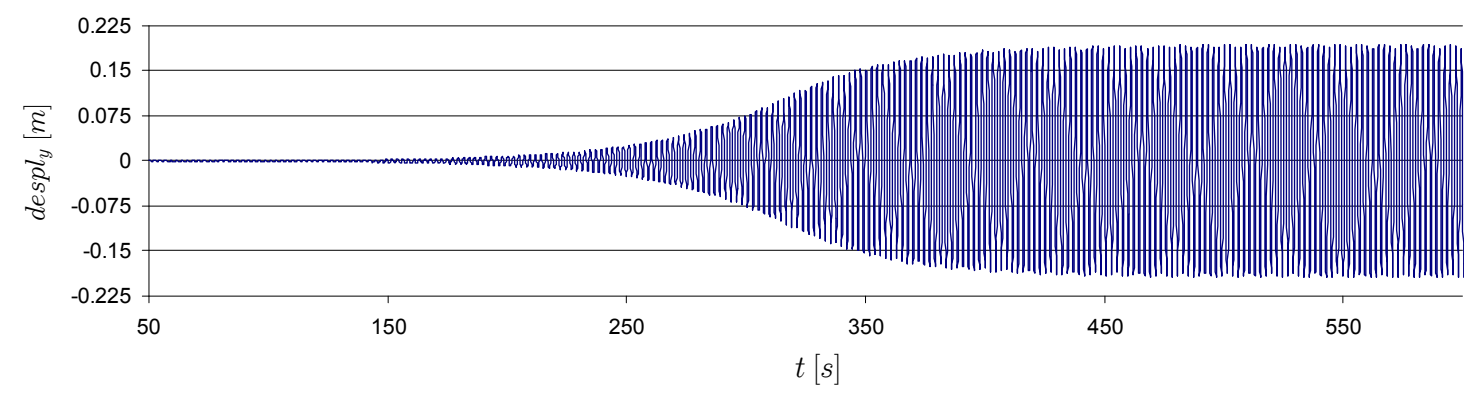

Figura 7.91. Vibración de la estructura en la dirección transversal para amortiguamiento estructural 0.025 


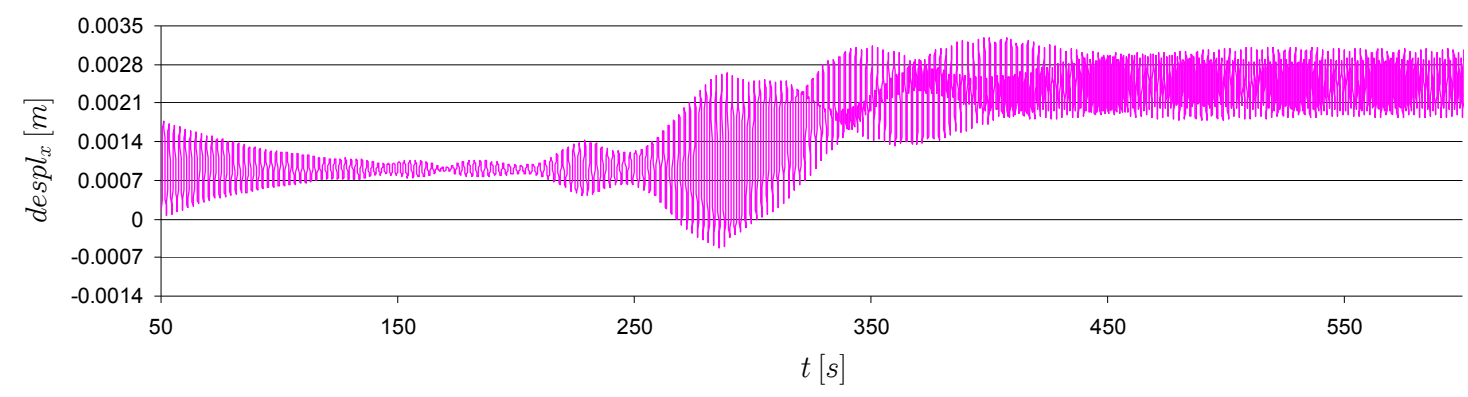

Figura 7.92. Vibración de la estructura en la dirección del flujo para amortiguamiento estructural 0.025

En la Figura 7.93 se muestra el análisis FFT de la vibración de la estructura en la dirección transversal y en la dirección del flujo. Se observa que en la dirección transversal la estructura vibra a su frecuencia natural $n_{e}=0.43 \mathrm{~Hz}$, y que en la dirección del flujo vibra a una frecuencia de 0.86 $H z$, que es justo el doble de la frecuencia de la vibración transversal de la estructura.

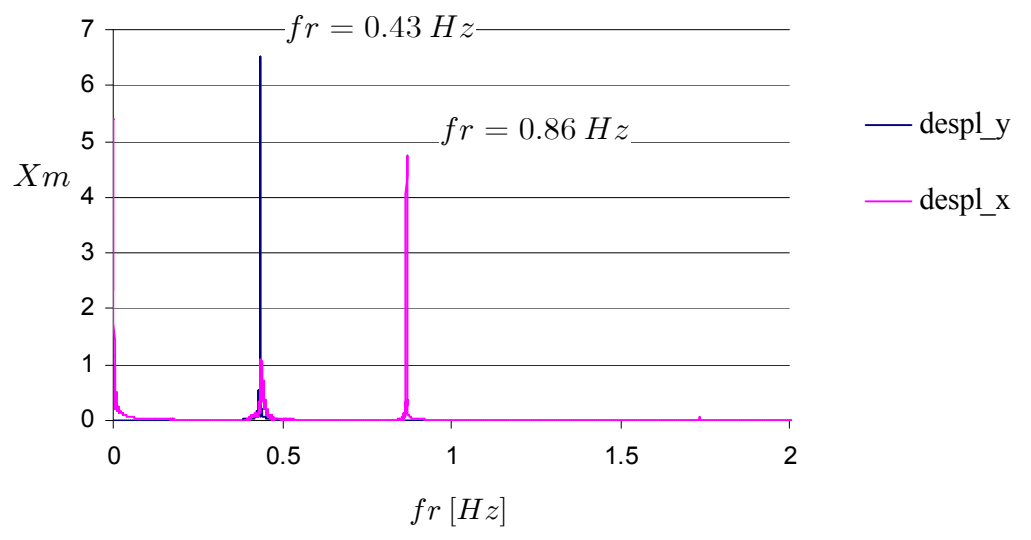

Figura 7.93. Análisis FFT del movimiento de la estructura

En la Figura 7.94 se ha representado la componente de la velocidad en la dirección transversal del extremo libre de la estructura y en la Figura 7.95 la componente de la velocidad en la dirección paralela al flujo.

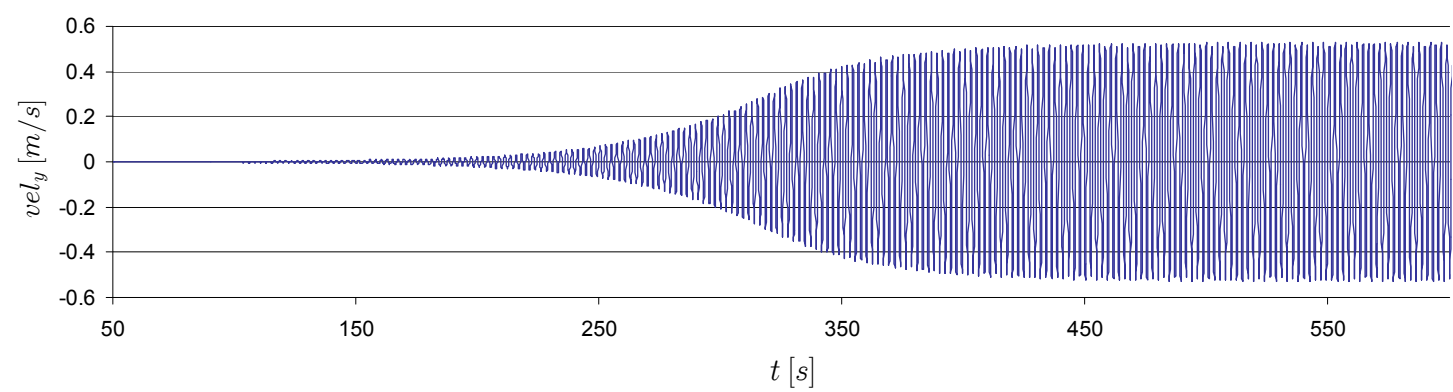

Figura 7.94. Componente transversal de la velocidad para amortiguamiento estructural 0.025 


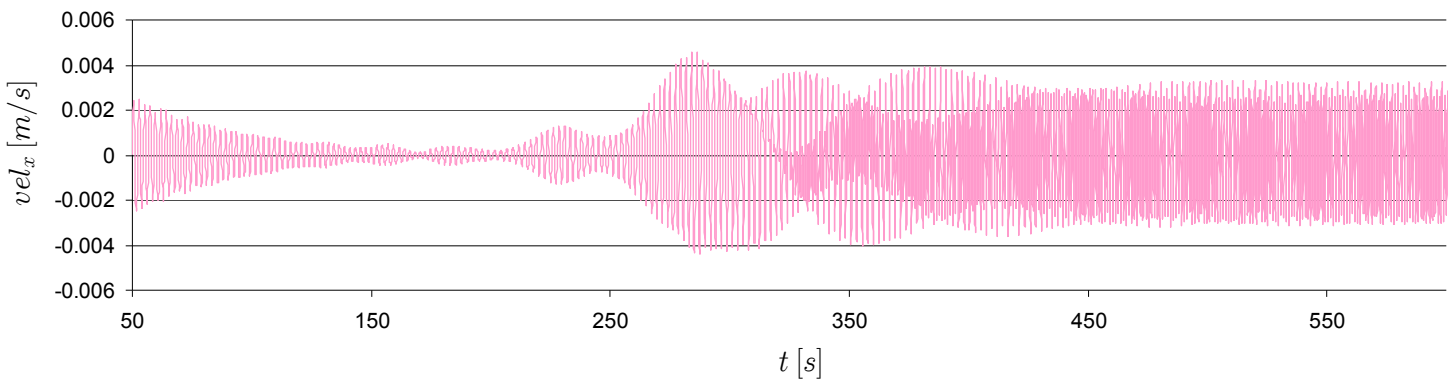

Figura 7.95. Componente de la velocidad en la dirección del flujo para amortiguamiento estructural 0.025

Finalmente, en la Figura 7.96 se muestra el módulo de la velocidad del extremo libre de la estructura y en la Figura 7.97 el análisis FFT de las componentes de la velocidad y de su módulo.

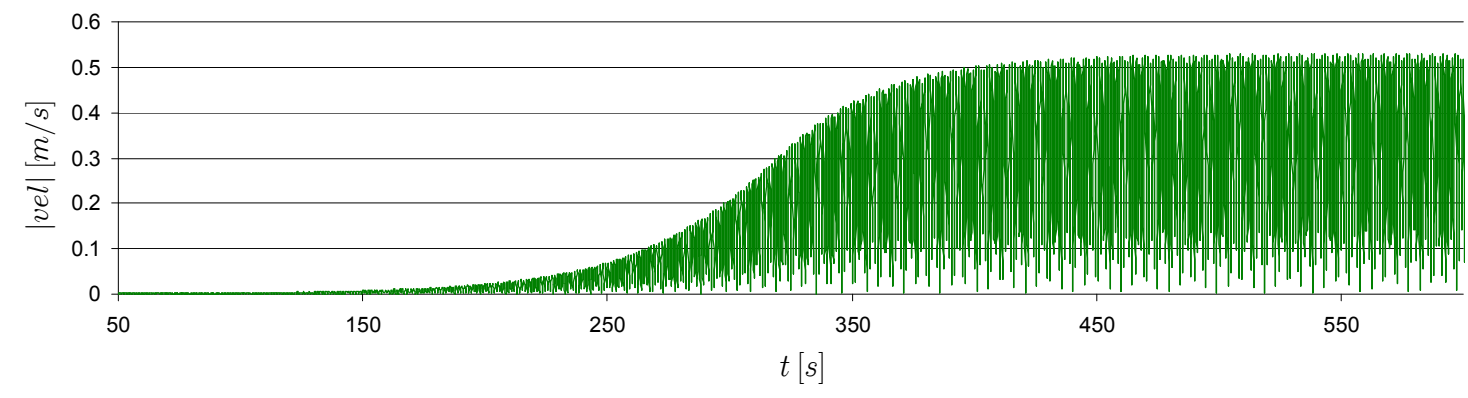

Figura 7.96. Módulo de la velocidad para amortiguamiento estructural 0.025

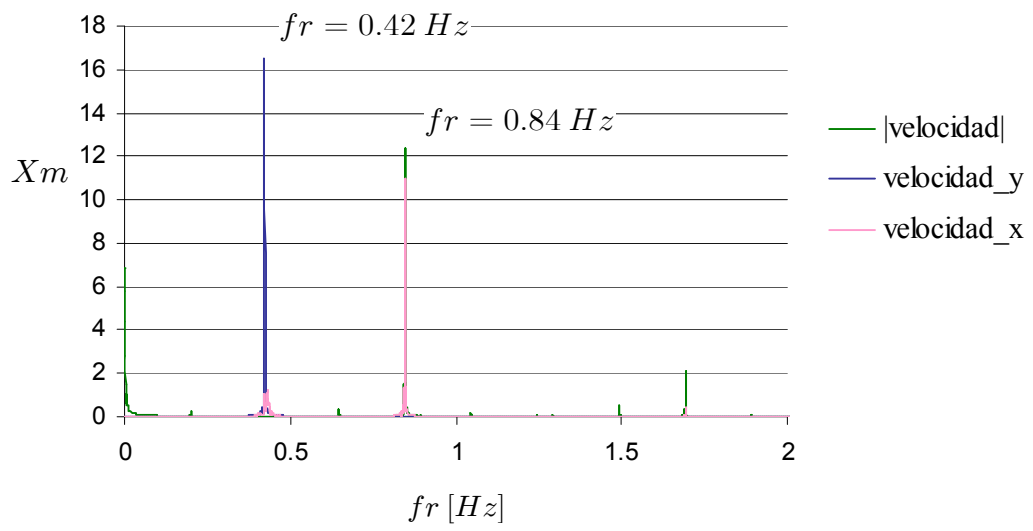

Figura 7.97. Análisis FFT de la velocidad del extremo libre de la estructura

En la Tabla 7.15 se resumen los resultados obtenidos para el caso de amortiguamiento estructural 0.025. Al igual que en el caso anterior, se distinguen dos zonas de estudio, al principio del análisis, cuando aún no se ha producido la sincronización de las frecuencias y al final del análisis, cuando las frecuencias ya se han sincronizado. El resto de las gráficas se muestran en el apartado J.3 del Anexo J. 


\begin{tabular}{|c|c|c|c|c|c|c|c|c|c|}
\hline \multirow[b]{2}{*}{$U_{r}$} & \multirow[b]{2}{*}{$\begin{array}{c}\boldsymbol{U} \\
{[\mathrm{m} / \mathrm{s}]}\end{array}$} & \multirow[b]{2}{*}{$R e$} & \multirow[b]{2}{*}{$A_{y} / d$} & \multicolumn{3}{|c|}{ al principio del análisis } & \multicolumn{3}{|c|}{ al final del análisis } \\
\hline & & & & $\begin{array}{c}\text { RMS } \\
\operatorname{despl}_{x} \\
{[m]}\end{array}$ & $\begin{array}{c}\text { RMS } \\
|\mathbf{v e l}| \\
{[\mathrm{m} / \mathrm{s}]}\end{array}$ & $\tilde{C}_{D}$ & $\begin{array}{c}\text { RMS } \\
\text { despl }_{x} \\
{[m]}\end{array}$ & $\begin{array}{l}\text { RMS } \\
|\mathbf{v e l}| \\
{[\mathrm{m} / \mathrm{s}]}\end{array}$ & $\tilde{C}_{D}$ \\
\hline 3.5 & 0.77 & $2.57 \mathrm{E}+04$ & $5.03 \mathrm{E}-04$ & $1.37 \mathrm{E}-04$ & & 0.77 & $1.37 \mathrm{E}-04$ & & 0.77 \\
\hline 4.1 & 0.90 & $3.00 \mathrm{E}+04$ & $2.17 \mathrm{E}-03$ & $1.87 \mathrm{E}-04$ & & 0.78 & $1.87 \mathrm{E}-04$ & & 0.78 \\
\hline 4.5 & 1.00 & $3.33 \mathrm{E}+04$ & $4.40 \mathrm{E}-03$ & $2.32 \mathrm{E}-04$ & & 0.79 & $2.32 \mathrm{E}-04$ & & 0.79 \\
\hline 5.0 & 1.11 & $3.70 \mathrm{E}+04$ & $3.17 \mathrm{E}-02$ & $2.86 \mathrm{E}-04$ & & 0.78 & $2.86 \mathrm{E}-04$ & & 0.78 \\
\hline 5.5 & 1.22 & $4.07 \mathrm{E}+04$ & $2.82 \mathrm{E}-02$ & $3.24 \mathrm{E}-04$ & & 0.74 & $3.24 \mathrm{E}-04$ & & 0.74 \\
\hline 6.0 & 1.33 & $4.43 \mathrm{E}+04$ & $1.89 \mathrm{E}-03$ & 4.05E-04 & & 0.77 & $4.05 \mathrm{E}-04$ & & 0.77 \\
\hline 6.5 & 1.44 & $4.80 \mathrm{E}+04$ & $1.26 \mathrm{E}-03$ & $4.72 \mathrm{E}-04$ & & 0.77 & $4.72 \mathrm{E}-04$ & & 0.77 \\
\hline 7.0 & 1.55 & $5.17 \mathrm{E}+04$ & $2.83 \mathrm{E}-01$ & $5.68 \mathrm{E}-04$ & 0.0014 & 0.77 & $9.09 \mathrm{E}-04$ & 0.1363 & 1.33 \\
\hline 8.0 & 1.77 & $5.90 \mathrm{E}+04$ & $4.97 \mathrm{E}-01$ & 7.33E-04 & 0.0023 & 0.77 & $1.63 \mathrm{E}-03$ & 0.2390 & 1.88 \\
\hline 9.0 & 2.00 & $6.67 \mathrm{E}+04$ & 7.76E-01 & $9.60 \mathrm{E}-04$ & 0.0025 & 0.77 & $2.48 \mathrm{E}-03$ & 0.3741 & 2.30 \\
\hline 11.0 & 2.43 & $8.10 \mathrm{E}+04$ & $1.55 \mathrm{E}+00$ & $1.46 \mathrm{E}-03$ & 0.0035 & 0.77 & $4.84 \mathrm{E}-03$ & 0.7491 & 3.17 \\
\hline 13.0 & 2.88 & $9.60 \mathrm{E}+04$ & $3.05 \mathrm{E}+00$ & $2.00 \mathrm{E}-03$ & 0.0042 & 0.77 & $1.06 \mathrm{E}-02$ & 1.4740 & 5.14 \\
\hline 15.0 & 3.32 & $1.11 \mathrm{E}+05$ & $4.97 \mathrm{E}+00$ & $2.75 \mathrm{E}-03$ & 0.0055 & 0.77 & $2.11 \mathrm{E}-02$ & 2.4067 & 7.78 \\
\hline
\end{tabular}

Tabla 7.15. Resultados obtenidos para al caso de amortiguamiento estructural 0.025

En la Figura 7.98 se muestra el valor RMS del coeficiente de arrastre en función de la velocidad reducida y en la Figura 7.99 en función de la amplitud de la vibración de la estructura en la dirección transversal en escala normal y logarítmica. Se observa que a partir de un cierto valor de la velocidad del flujo y de la amplitud de la vibración transversal de la estructura se produce la amplificación del coeficiente de arrastre. En la Figura 7.100 se muestra que el desplazamiento en la dirección del flujo del extremo libre de la estructura se incrementa como consecuencia de la amplificación del coeficiente de arrastre.

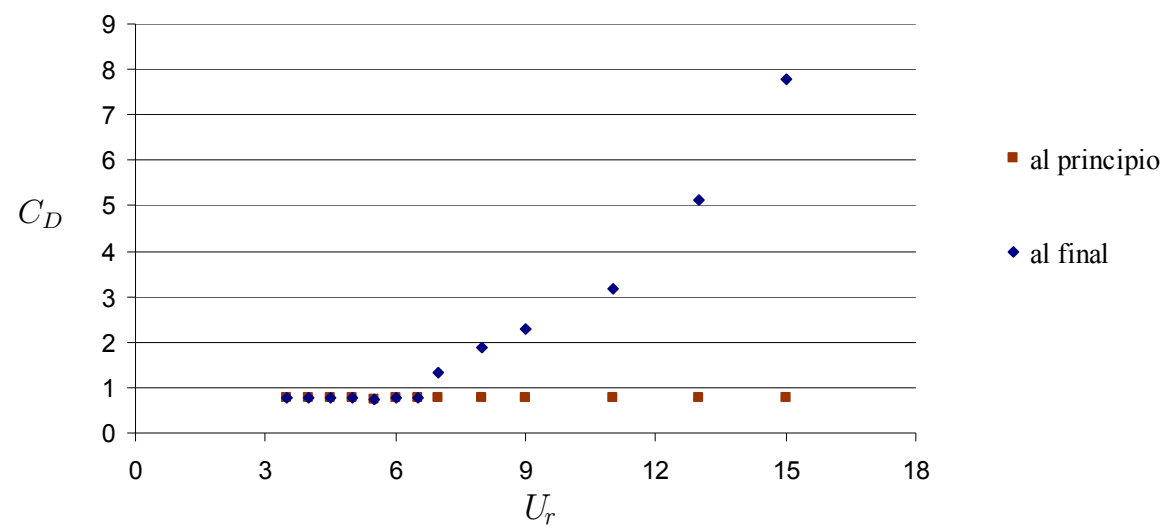

Figura 7.98. Valor RMS del coeficiente de arrastre frente a la velocidad reducida 

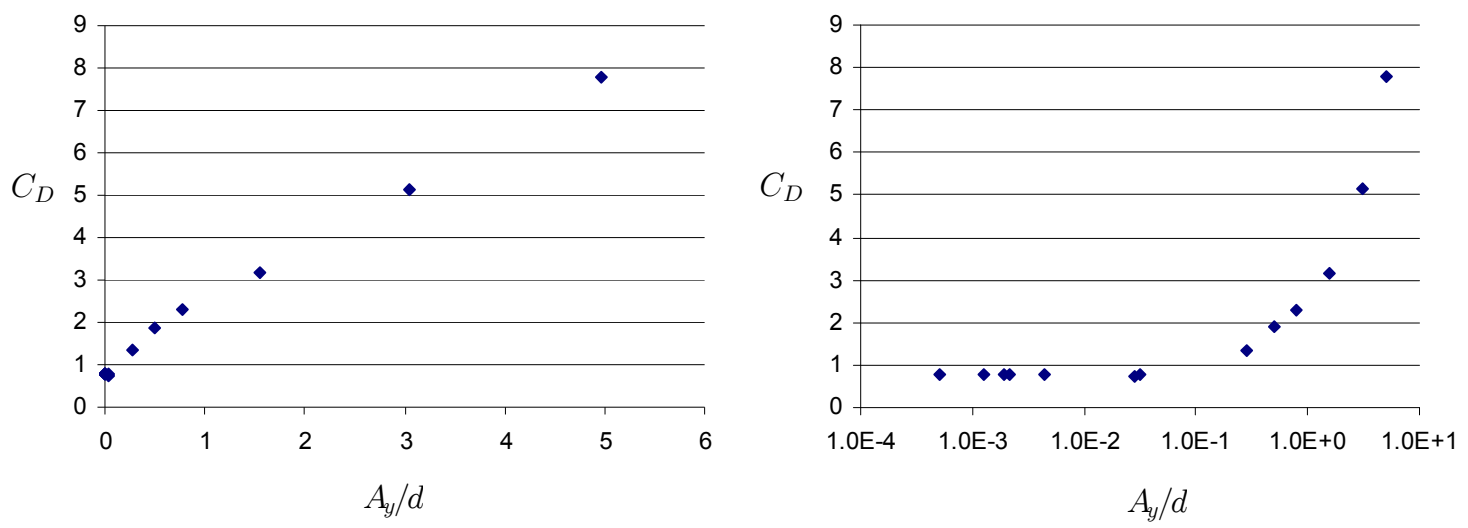

Figura 7.99. Valor RMS coeficiente de arrastre frente a la amplitud de la vibración de la estructura en la dirección transversal

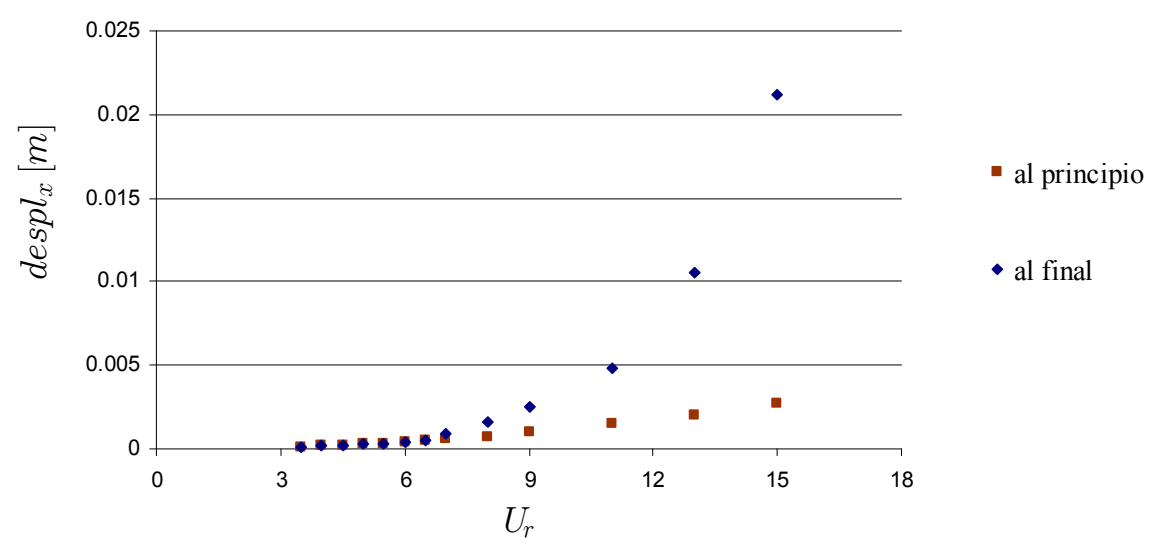

Figura 7.100. Valor RMS del desplazamiento en la dirección del flujo del extremo libre de la estructura

\section{Caso 3. Amortiguamiento estructural 0.05}

Dado que el comportamiento es análogo al de los casos estudiados anteriormente, en este caso sólo se muestran las gráficas relativas al valor RMS del coeficiente de arrastre frente a la velocidad reducida (Figura 7.101) y frente a la amplitud del movimiento en la dirección transversal (Figura 7.102). También se muestra la representación gráfica del incremento del desplazamiento en la dirección del flujo debido a la amplificación del coeficiente de arrastre frente a la velocidad reducida (Figura 7.103). El resto de las gráficas se muestran en el apartado J.2 del Anexo J. 


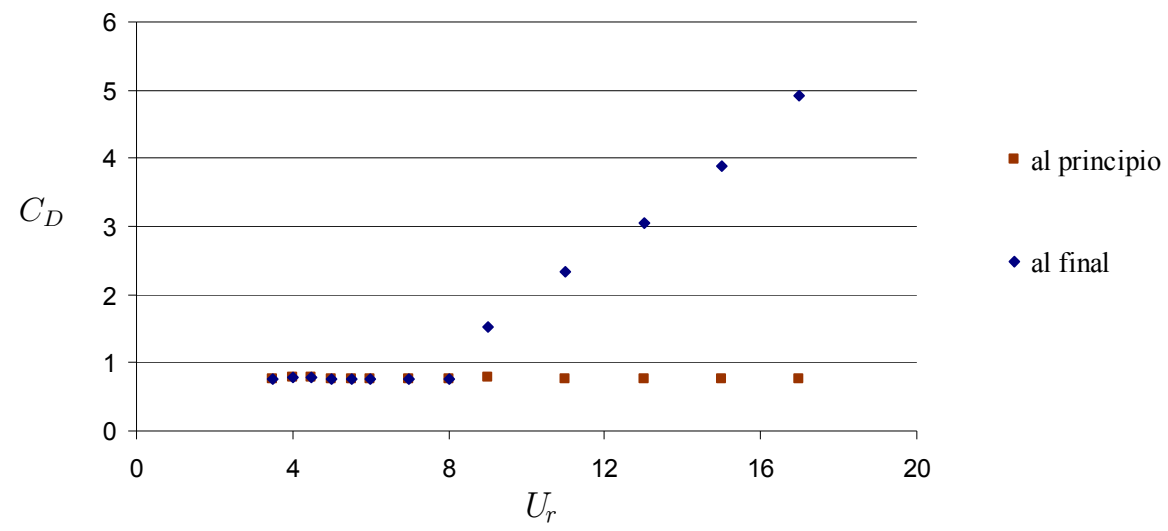

Figura 7.101. Valor RMS del coeficiente de arrastre frente a la velocidad reducida
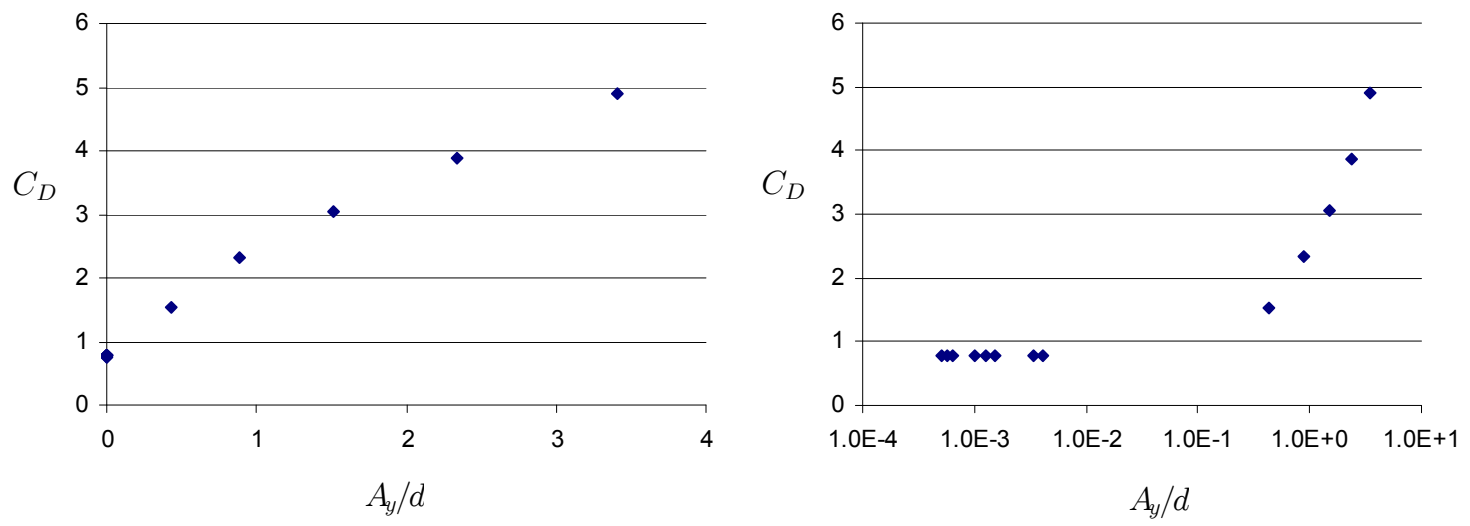

Figura 7.102. Valor RMS coeficiente de arrastre frente a la amplitud de la vibración de la estructura en la dirección transversal

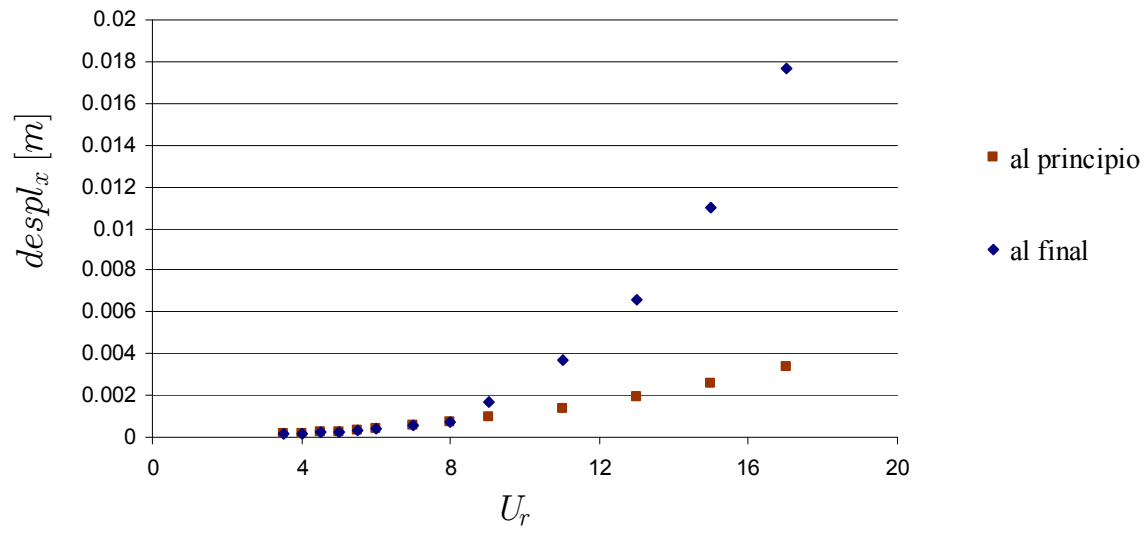

Figura 7.103. Valor RMS del desplazamiento en la dirección del flujo del extremo libre de la estructura 
Para finalizar este estudio se muestra una comparación entre los resultados obtenidos para cada caso estudiado en función del grado de amortiguamiento estructural.

En la Figura 7.104 se muestra el valor RMS del coeficiente de arrastre frente a la velocidad reducida. Se observa que la amplificación del coeficiente de arrastre ocurre antes, esto es, para valores más pequeños de la velocidad, cuanto menor es el amortiguamiento estructural que presenta la estructura.

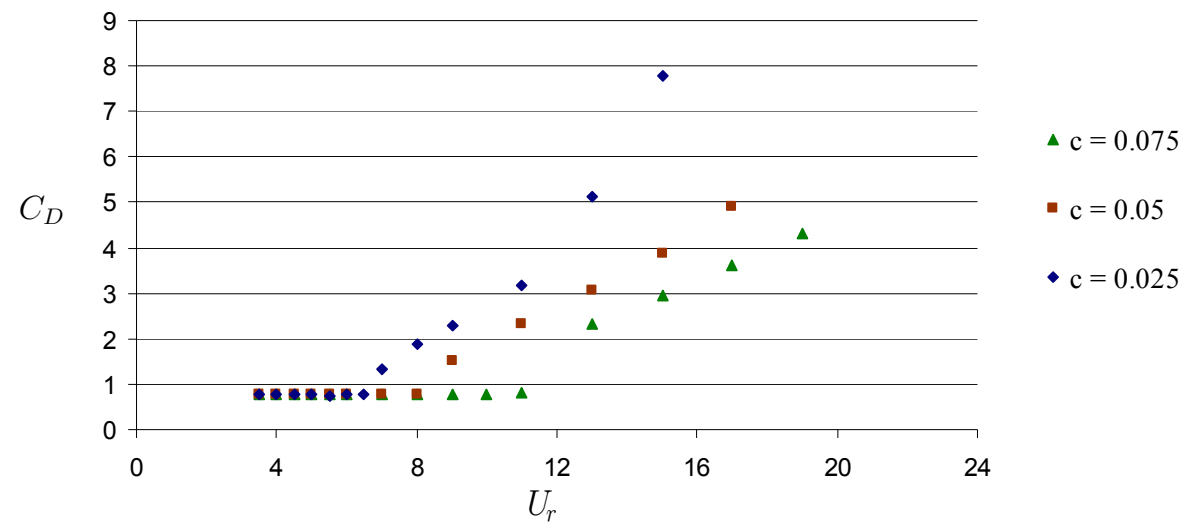

Figura 7.104. Valor RMS del coeficiente de arrastre frente a la velocidad reducida

El valor RMS del coeficiente de arrastre frente a la amplitud de la vibración transversal de la estructura en escala normal y logarítmica se ha representado en la Figura 7.105. Se puede apreciar que la amplificación del coeficiente de arrastre ocurre a partir de un cierto valor de la amplitud de la vibración transversal de la estructura con independencia del grado de amortiguamiento estructural, y que el incremento del valor del coeficiente de arrastre depende de la amplitud del movimiento transversal de la estructura.
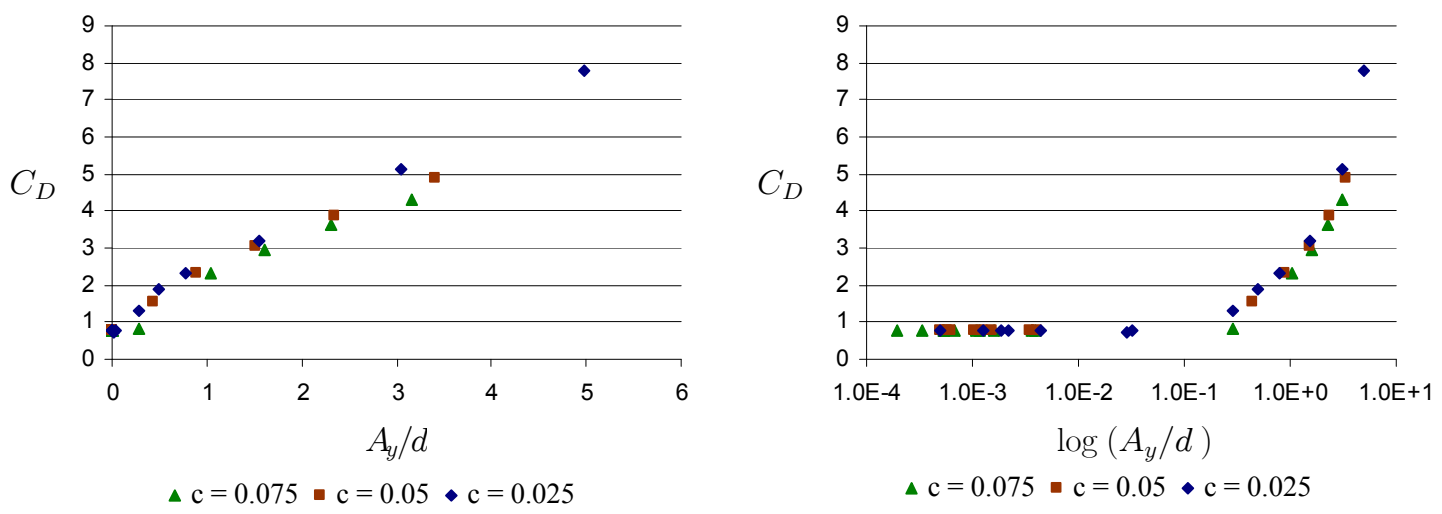

Figura 7.105. Valor RMS del coeficiente de arrastre frente a la amplitud de la vibración transversal 
En la Figura 7.106 se ha representado el desplazamiento en la dirección del flujo del extremo libre de la estructura en función de la velocidad reducida. Se observa un incremento notable del desplazamiento al producirse la amplificación del coeficiente de arrastre y que este incremento es mayor cuanto menor es el grado de amortiguamiento estructural.

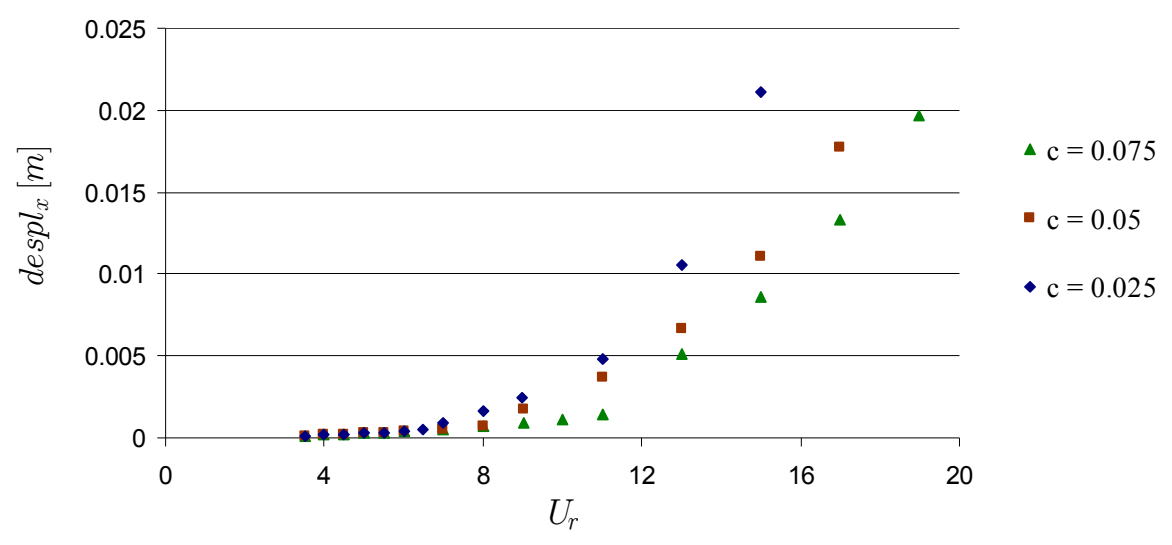

Figura 7.106. Valor RMS del desplazamiento del extremo libre de la estructura en la dirección del flujo

Respecto al coeficiente de sustentación, en la Figura 7.107 se muestra la variación de la amplitud de la oscilación de dicho coeficiente frente a la velocidad reducida. Se puede apreciar un incremento considerable de la amplitud a partir del momento en el que la frecuencia de desprendimiento de los torbellinos y la frecuencia natural de la estructura se sincronizan y tiene lugar la amplificación del coeficiente de arrastre. Este incremento ocurre a velocidades más pequeñas y es mayor cuanto menor es el grado de amortiguamiento estructural.

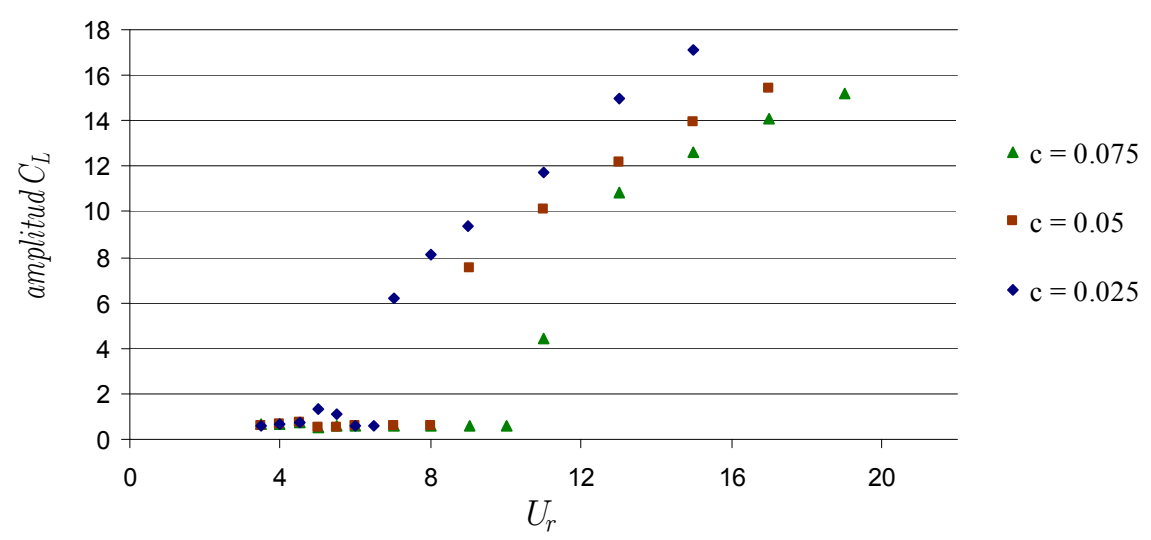

Figura 7.107. Amplitud del coeficiente de sustentación frente a al velocidad reducida 
Notar que en el caso de amortiguamiento estructural 0.025 existe un rango de velocidades en el cual las frecuencias se sincronizan pero no ocurre la amplificación del coeficiente de arrastre. En ese rango de velocidades también tiene lugar un incremento considerable de la amplitud de la oscilación del coeficiente de sustentación, tal como se muestra en la Figura 7.108. En ella se ha representado la amplitud del coeficiente de sustentación para el rango de velocidades en el que no se produce la amplificación del coeficiente de arrastre para cada caso de amortiguamiento estructural estudiado.

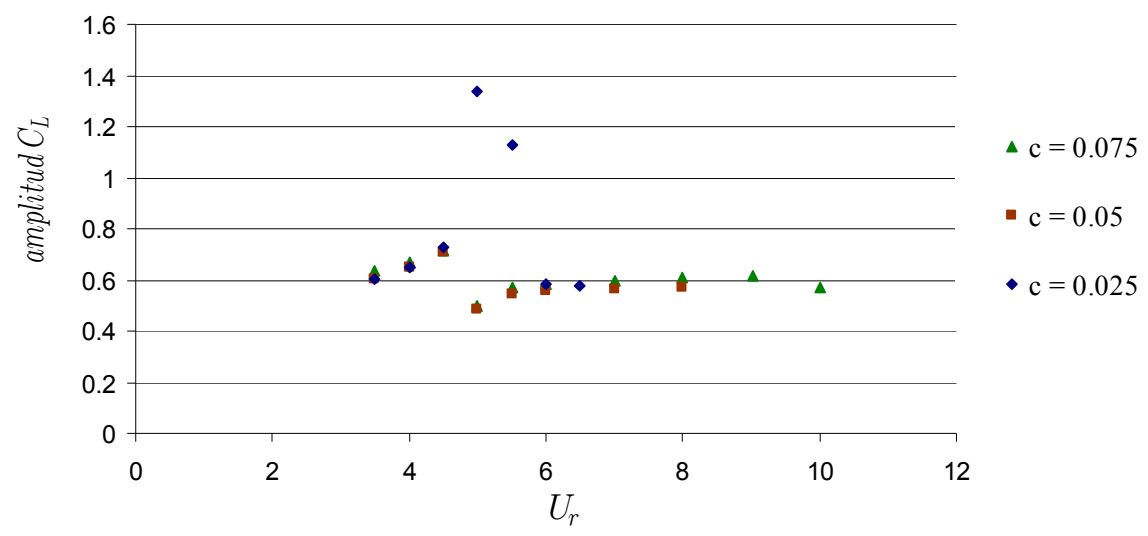

Figura 7.108. Amplitud del $C_{L}$ cuando no hay amplificación del $C_{D}$

Finalmente, En la Figura 7.109 se muestra la amplitud de la vibración transversal de la estructura en escala normal y logarítmica frente a la velocidad reducida. Se observa que cuanto menor es el amortiguamiento estructural mayor es la amplitud del movimiento transversal de la estructura.
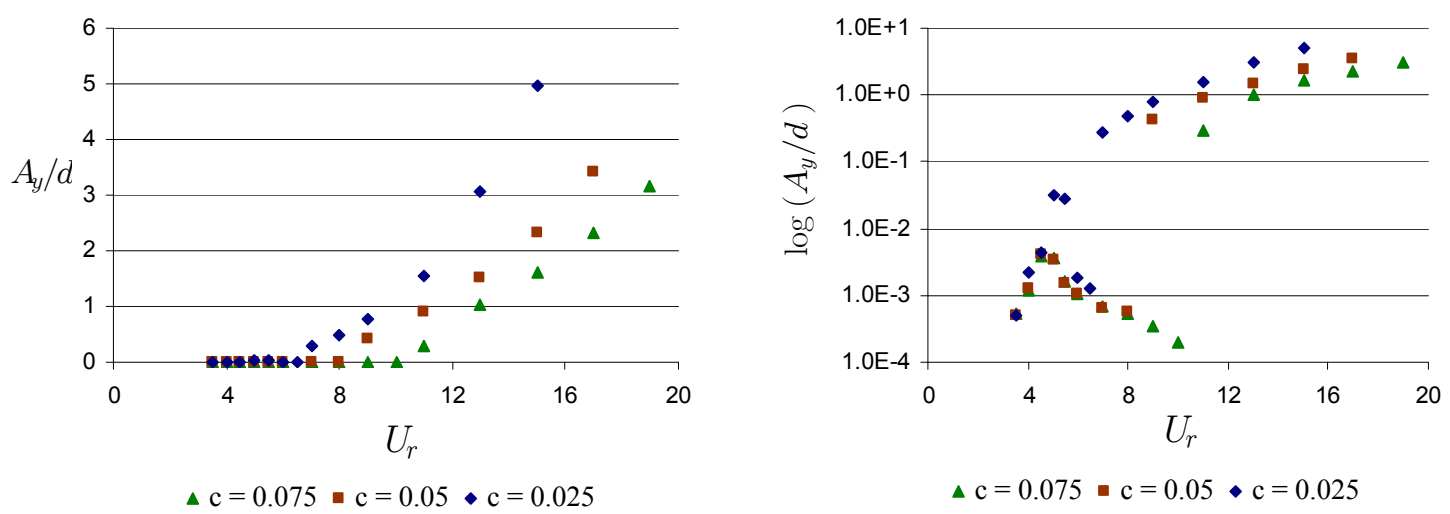

Figura 7.109. Respuesta en amplitud de la estructura 
En la representaciones gráficas anteriores se aprecia con claridad la relación entre la amplitud de la oscilación del coeficiente de sustentación y la amplitud de la vibración transversal de la estructura en el rango de velocidades en el que no hay amplificación del coeficiente de arrastre y en el rango de velocidades en el que si se produce. En definitiva, en función de que se establezca una fuerte correlación entre el movimiento de la estructura en la dirección transversal y en la dirección del flujo (conlleva la amplificación del coeficiente de arrastre) o no, se pueden distinguir dos zonas de comportamiento muy diferentes para una misma estructura.

\section{Referencias}

${ }^{1}$ http://kratos.cimne.upc.es/kratoswiki/index.php/Main_Page

${ }^{2}$ Rossi, R., Light Weight Structures: Structural Analysis and Coupling Issues, Ph.D. Tesis, Universidad de Bologna, 2005.

${ }^{3} \mathrm{http}: / /$ www6.uniovi.es/gma/gauss1_3.htm

${ }^{4}$ D'Asdia, P., y Noè, S., "Vortex induced vibration of reinforced concrete chimneys: in situ experimentation and numerical previsions", Journal of Wind Engineering and Industrial Aerodynamics, 74-76, pp. 765-776, 1998.

${ }^{5}$ Fujarra, A.L.C., Pesce, C.P., Flemming, F., y Williamson, C.H.K., "Vortex-induced vibration of a flexible cantilever". Journal of Fluids and Structures, 15, pp. 651-658, 2001.

${ }^{6}$ Kitagawa, T., Fujino, Y., y Kimura, K., "Effects of free end condition on end-cell induced vibration", Journal of Fluids and Structures, 13, pp. 499-518, 1999. 
Capítulo 8

\section{Conclusiones y Líneas Futuras}





\subsection{Conclusiones}

Se ha desarrollado una herramienta simplificada numérica para la simulación de problemas de interacción fluido-estructura. Para ello fue necesario desarrollar un modelo estructural e implementarlo en el código de Kratos, junto con el interfaz entre el solver del fluido y el de la estructura. Esta herramienta se ha aplicado al estudio de las vibraciones inducidas por el desprendimiento alternado de torbellinos en estructuras esbeltas, si bien, también podría aplicarse a la simulación de otros fenómenos aeroelásticos.

Respecto al planteamiento estructural, se ha formulado un modelo analítico monodimensional de propósito general que permite describir el comportamiento a flexión y a torsión de barras de material compuesto de pared delgada. El método es aplicable a barras con secciones de forma arbitraria con cualquier esquema de laminado, tanto abiertas como cerradas. El modelo ha sido validado con los resultados aportados por otros investigadores obteniéndose una buena correlación.

Respecto a la mecánica de fluidos computacional, en el presente trabajo se ha hecho uso de las técnicas numéricas y algoritmos implementados en Kratos para resolver y analizar el flujo del fluido. Se resuelven las ecuaciones de Navier-Stokes para flujo 2D incompresible.

Se ha conseguido simular con el método simplificado de interacción fluido-estructura propuesto la respuesta vibratoria forzada de una chimenea de $90 \mathrm{~m}$ de altura sometida a la acción del viento, así como también la respuesta libre cuando cesa esta acción. Se ha representado gráficamente la vibración de la chimenea en el tiempo en la dirección de incidencia del viento y en la transversal, además de la variación en el tiempo de los coeficientes de sustentación y de arrastre. Los resultados obtenidos han sido satisfactorios, dada la gran aproximación que se da entre éstos y los deducidos teóricamente.

Se ha estudiado la influencia del número de planos de fluido utilizados para modelar la acción del viento en la respuesta transversal de un mástil de $34 m$ de altura. Dado que la velocidad del viento varia con la altura y la sección de la estructura también puede variar, cada plano de fluido tendrá asociada una determinada frecuencia de excitación en función de la ley de Strouhal. Por lo tanto, resulta decisiva la elección del número de planos y la posición de cada uno de ellos a la hora de modelar la acción del viento.

Por otro lado, también se han podido simular con éxito los fenómenos aeroelásticos de lock-in o de sincronización y de amplificación del coeficiente de arrastre causados por el desprendimiento alternado de torbellinos en un mástil de $34 m$ de altura de sección constante. 
La variación de la frecuencia de desprendimiento de los torbellinos y la amplitud de la respuesta vibratoria de la estructura en la dirección transversal al flujo se han representado gráficamente en función de la velocidad reducida para diferentes valores del coeficiente de amortiguamiento estructural de la estructura, pudiéndose observar la influencia de este parámetro en la aparición del fenómeno de sincronización. Se muestran gráficas de la respuesta vibratoria del mástil a lo largo del tiempo en la dirección del flujo y en la transversal, observándose cambios en la frecuencia de desprendimiento de los torbellinos en un cierto rango de velocidades.

La variación del coeficiente de arrastre en función de la velocidad reducida y de la amplitud de la vibración transversal de la estructura se ha representado gráficamente para distintos valores del amortiguamiento estructural, observándose el fenómeno de amplificación de dicho coeficiente. También se muestra gráficamente la amplificación de la amplitud de la oscilación del coeficiente de sustentación para distintos valores del amortiguamiento estructural. Se puede concluir que, si bien desde un punto de vista clásico se ha considerado que los coeficientes de arrastre y de sustentación tienen valores constantes para una sección dada, al considerar el problema de interacción fluidoestructura se demuestra que éstos pueden variar y no ser constantes. Se muestran gráficas de la variación temporal del coeficiente de sustentación y de arrastre.

Finalmente, se han representado las curvas de Lissajous de la trayectoria que describen diferentes secciones de la estructura observándose gráficamente el acoplamiento entre el movimiento en la dirección del flujo y en la dirección transversal para ciertos valores de la velocidad reducida y del amortiguamiento estructural. A partir de la observación de este tipo de curvas se puede deducir el grado de interacción entre el fluido y la estructura identificando posibles fenómenos aeroelásticos como el lock-in o la amplificación del coeficiente de arrastre.

Adicionalmente, se ha encontrado un modo de vibración a alta velocidad con una respuesta en amplitud grande, el cual se sitúa fuera del rango principal de sincronización, y es debido al fuerte acoplamiento entre la vibración de la estructura en la dirección del flujo y la vibración en la dirección transversal. La frecuencia de la vibración en la dirección del flujo de este modo de alta velocidad es el doble de la frecuencia de la vibración transversal, también de acuerdo con resultados experimentales.

Este trabajo ofrece nuevas oportunidades de validación de las fuerzas inducidas por el desprendimiento alternado de torbellinos calculadas por medio de la teoría de la mecánica de fluidos computacional acoplada a un modelo estructural, así como también de la respuesta vibratoria de la estructura. Desde el punto de vista computacional resulta evidente la necesidad de mejorar los códigos actuales, no solo aquellos basados en la mecánica de fluidos computacional 
sino también los modelos empíricos basados en datos experimentales. Los métodos numéricos de la mecánica de fluidos computacional son especialmente interesantes debido a su potencial, junto con los modelos estructurales de elementos finitos, para simular comportamientos reales de ciertos elementos estructurales. Esto es una demanda del sector industrial (industria de la construcción, del petróleo, energética, etc.) a fin de diseñar sistemas más fiables y robustos.

En definitiva, se trata de una herramienta simplificada que reproduce cualitativamente y, en la medida de lo posible, cuantitativamente el comportamiento de estructuras esbeltas sometidas a un flujo de fluido. En los ejemplos planteados, el viento se ha modelado con un número relativamente pequeño de planos de fluido y suficientemente espaciados entre sí, ya que el modelo no contempla los posibles fenómenos de transporte que pudieran ocurrir entre capas próximas de viento que harían que los planos no pudiesen considerarse independientes. Los posibles efectos $3 \mathrm{D}$ que puedan ocurrir en el borde del extremo libre de la estructura tampoco son captados con este modelo.

\subsection{Líneas futuras}

Dentro de las simplificaciones adoptadas, una primera línea de trabajo futuro estaría constituida por la simulación de diferentes estructuras y situaciones:

- Chimeneas con aletas longitudinales uniformes.

- Cables sometidos al viento o a corrientes.

- Tablero de un puente.

- Aerogenerador.

- Otras construcciones o edificios altos.

- Tuberías de transporte del petróleo desde el fondo del mar (risers).

- Interacción entre dos o más estructuras alineadas en la dirección del flujo, en la dirección transversal o a tresbolillo.

- Incorporar a la simulación modelos de dispositivos pasivos de absorción de vibraciones (TMD) en mástiles o chimeneas.

El método, tal y como está planteado, exige gran capacidad de cálculo, sobre todo en la resolución del fluido en cada plano. Estos cálculos CFD son independientes en cada plano y podrían 
realizarse en ordenadores distintos, es decir, el método permite la paralelización. Para aprovechar este aspecto computacional debe adaptarse adecuadamente el código.

Otra línea de trabajo tendería a superar ciertas limitaciones de la herramienta numérica planteada:

- Introducir la formulación de grandes desplazamientos en el modelo estructural dado que en el modo de vibración a alta velocidad la amplitud del movimiento de la estructura en la dirección del flujo y en la transversal es importante.

- Actualizar el esquema numérico de resolución de las ecuaciones de Navier-Stokes.

- Estudiar la implementación de un modelo de turbulencia que acompañe a las ecuaciones de Navier-Stokes.

Aunque estas limitaciones fueran superadas, el modelo continuaría siendo simplificado en tanto que el flujo de fluido se seguiría modelando con planos transversales a la estructura separados entre sí una cierta distancia. El modelo no podría capturar los posibles fenómenos de transporte entre las capas de viento ni los efectos 3D en el borde extremo de la estructura.

Para solventar estas dificultades sería necesario recurrir a modelos 3D del problema de interacción fluido-estructura. 
Anexos

$\longrightarrow$ 



\section{Anexo A. Derivadas de los vectores unitarios}

Derivadas de los vectores unitarios según los ejes locales respecto a esos mismos ejes.

$$
\vec{i}_{, x}=0 \quad \vec{j}_{, x}=0 \quad \vec{k}_{, x}=0 \quad \vec{i}_{, s}=0 \quad \vec{j}_{, s}=-\frac{\vec{k}}{a} \quad \vec{k}_{, s}=\frac{\vec{j}}{a} \quad \vec{i}_{, n}=0 \quad \vec{j}_{, n}=0 \quad \vec{k}_{, n}=0
$$

Las expresiones de $\vec{j}_{s}$ y $\vec{k}_{s}$ se obtienen con la ayuda de la Figura A.1. Así, para una pequeñísima variación $\Delta s=a(s) \Delta \theta$

$$
\vec{k}(s)=\vec{k}(s+\Delta s) \cos (\Delta \theta)-\vec{j}(s+\Delta s) \sin (\Delta \theta)=\vec{k}(s+\Delta s)-\vec{j}(s+\Delta s) \Delta \theta
$$

donde se ha aproximado el seno por el ángulo y el coseno por la unidad, habida cuenta que $\Delta \theta$ es un ángulo muy pequeño. Reordenando y dividiendo por $\Delta s$ se obtiene

$$
\frac{\vec{k}(s+\Delta s)-\vec{k}(s)}{\Delta s}=\frac{\vec{j}(s+\Delta s) \Delta \theta}{a(s) \Delta \theta}=\frac{\vec{j}(s+\Delta s)}{a(s)}
$$

Calculando el límite de la expresión (A.3) se llega finalmente a

$$
\begin{aligned}
& \lim _{\Delta s \rightarrow 0} \frac{\vec{k}(s+\Delta s)-\vec{k}(s)}{\Delta s}=\lim _{\Delta s \rightarrow 0} \frac{\vec{j}(s+\Delta s)}{a(s)} \Rightarrow \frac{d \vec{k}(s)}{d s}=\frac{\vec{j}(s)}{a(s)} \\
& \vec{k}(s+\Delta s)
\end{aligned}
$$

Figura A.1.

Análogamente se tiene

$$
\begin{gathered}
\vec{j}(s)=\vec{k}(s+\Delta s) \sin (\Delta \theta)+\vec{j}(s+\Delta s) \cos (\Delta \theta)=\vec{k}(s+\Delta s) \Delta \theta+\vec{j}(s+\Delta s) \\
\lim _{\Delta s \rightarrow 0} \frac{\vec{j}(s+\Delta s)-\vec{j}(s)}{\Delta s}=\lim _{\Delta s \rightarrow 0} \frac{-\vec{k}(s+\Delta s)}{a(s)} \Rightarrow \frac{d \vec{j}(s)}{d s}=-\frac{\vec{k}(s)}{a(s)}
\end{gathered}
$$





\section{Anexo B. Derivadas de las componentes del radio vector}

Derivadas de las componentes $r(s)$ y $q(s)$ del radio vector $\vec{R}(s)$.

$$
q_{, s}(s)=1-\frac{r(s)}{a(s)} ; r_{, s}(s)=\frac{q(s)}{a(s)}
$$

En efecto, con referencia a la Figura A.2, donde $C$ define la posición del centro de esfuerzos cortantes de la sección

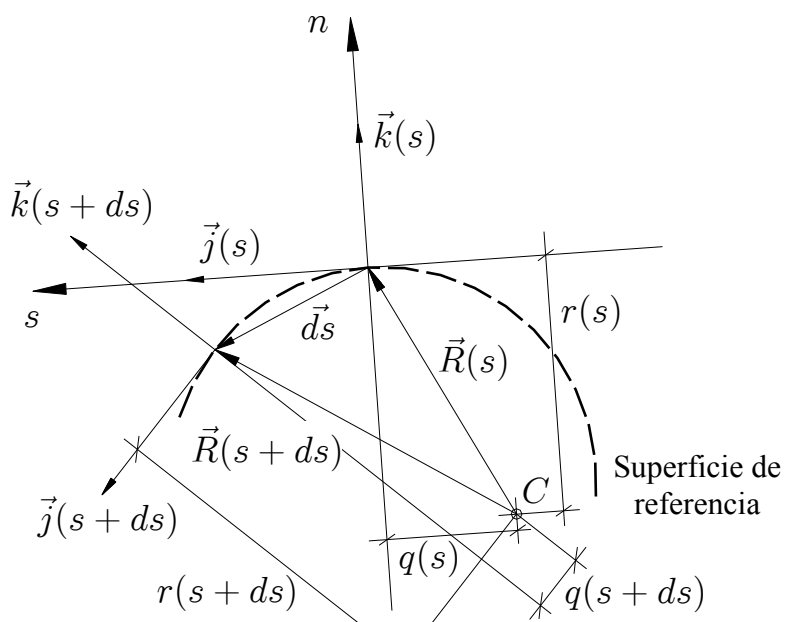

se tiene

Figura A.2.

$$
\begin{gathered}
\vec{R}=q \vec{j}+r \vec{k} \\
\frac{d \vec{R}}{d s}=q_{, s} \vec{j}+q \vec{j}_{, s}+r_{, s} \vec{k}+r \vec{k}_{, s}=q, s \vec{j}-q \frac{\vec{k}}{a}+r_{, s} \vec{k}+r \frac{\vec{j}}{a} \\
\vec{R}(s+d s)=\vec{R}(s)+d \vec{R}(s)=\left(q+\left(q_{, s}+\frac{r}{a}\right) d s\right) \vec{j}+\left(r+\left(r_{, s}-\frac{q}{a}\right) d s\right) \vec{k} \\
\vec{R}(s+d s)-\vec{R}(s)=\overrightarrow{d s} \Rightarrow\left(q, s+\frac{r}{a}\right) d s \vec{j}+\left(r_{, s}-\frac{q}{a}\right) d s \vec{k}=d s \vec{j} \\
\vec{j} \rightarrow\left(q_{, s}+\frac{r}{a}\right) s=d s \Rightarrow q, s=1-\frac{r}{a} \\
\vec{k} \rightarrow\left(r_{, s}-\frac{q}{a}\right) d s=0 \Rightarrow r_{, s}=\frac{q}{a}
\end{gathered}
$$





\section{Anexo C. Centro de esfuerzos cortantes y área sectorial}

Obtención del centro de esfuerzos cortantes y del área sectorial en secciones de pared delgada.

Para el cálculo del centro de esfuerzos cortantes y del área sectorial se tendrá en cuenta el espesor de la sección. El elemento diferencial de área se ha representado en la Figura A.3 y está definido por la expresión

$$
\left.\begin{array}{c}
d A=\left(1+\frac{n}{a}\right) d n d s=\mu d n d s \quad n \in[-e / 2, e / 2] \\
\left.\begin{array}{c}
d A=d n d s^{\prime}(n) \\
\frac{d s}{a}=\frac{d s^{\prime}(n)}{a+n} \rightarrow d s^{\prime}=\frac{a+n}{a} d s
\end{array}\right\} \\
d A=\mu d n d s
\end{array}\right\}
$$

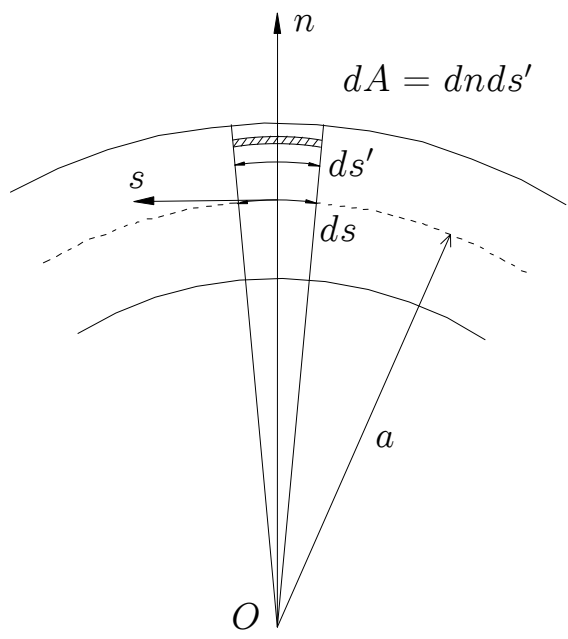

Figura A.3.

La siguiente expresión permite calcular el centro de esfuerzos cortantes $C$, y el área sectorial principal de la sección $\omega_{P}(n, s)$, a partir de un polo $A$

$$
\omega_{P}(n, s)=\omega_{A}(s)-\omega_{A}^{0}-n q_{A}(s)+z_{C} y(n, s)-y_{C} z(n, s)
$$

siendo $y_{C}$ y $z_{C}$ las coordenadas del centro de esfuerzos cortantes respecto del polo $A$.

Para un tramo genérico $k$ se tiene

$$
\omega_{A(k)}(s)=\int_{0}^{s} r_{A}(s) d s-\rho_{(k)} \alpha_{s(k)}(s)
$$

siendo

$$
\begin{gathered}
\alpha_{s(k)}(s)=\int_{0}^{s} \frac{d s}{e(s)} \\
\omega_{c(k)}(s)=\rho_{(k)} \alpha_{s(k)}(s)
\end{gathered}
$$


Por otro lado, el vector $\rho$ se obtiene a partir de la siguiente expresión

$$
\rho=2 \alpha^{-1} \Omega=\left\{\rho_{(i)}\right\}
$$

siendo

$\{\Omega\}$ el vector de áreas encerradas por la línea media de la sección

$\alpha_{i j}$ la matriz de coeficientes, definidos como sigue

$$
\alpha_{i i}=\int_{\partial \Omega_{i}} \frac{d s}{e(s)} \quad \alpha_{i j}=\int_{\Gamma_{i j}} \frac{d s}{e(s)}
$$

donde

$\partial \Omega_{i}$ define el conjunto de tramos que constituyen el contorno de la célula i-ésima

$\Gamma_{\mathrm{ij}}$ representa el tramo que separa las células $i$-ésima y j-ésima

$$
\alpha=\left(\begin{array}{ccccccc}
\alpha_{11} & -\alpha_{12} & 0 & 0 & 0 & . & . \\
-\alpha_{12} & \alpha_{22} & -\alpha_{23} & 0 & 0 & . & . \\
0 & -\alpha_{23} & \alpha_{33} & -\alpha_{34} & 0 & . & . \\
. & \cdot & \cdot & . & . & . & .
\end{array}\right)
$$

La constante torsional de la sección es

$$
J=J^{C}+J^{V}
$$

siendo

$J^{C}=4 \Omega^{T} \alpha^{-1} \Omega$

$J^{V}=\frac{1}{3} \sum_{i=1}^{n^{V}} \int_{0}^{l_{i}}\left(e_{i}(s)\right)^{3} d s$

$n^{V}$ el número de voladizos

Una vez obtenido $\omega_{A}(s)$, la constante $\omega_{A}^{0}$ se obtiene imponiendo la condición de que la distribución de las tensiones normales es autoequilibrada

$$
\int_{A} \omega_{P}(n, s) d A=0
$$

De forma que se obtiene la siguiente expresión para su cálculo

$$
\omega_{A}^{0}=\frac{1}{A}\left[\int_{0}^{S} \omega_{A}(s) e(s) d s-\int_{0}^{S} \int_{-e / 2}^{e / 2} n q_{A}(s) \mu d n d s\right]
$$


Con lo indicado hasta aquí se habría obtenido el término $\omega_{A}(s)-\omega_{A}^{0}-n q_{A}(s)$ del área sectorial principal, por lo que el siguiente paso será el cálculo del centro de esfuerzos cortantes. Para ello se utilizan las siguientes condiciones estáticas

$$
\begin{aligned}
& \int_{A} y_{G e}(n, s) \omega_{P}(n, s) d A=\int_{A} y_{G e}(n, s)\left(\omega_{A}(s)-\omega_{A}^{0}-n q_{A}(s)\right) d A+z_{C} I_{z}-y_{C} I_{y z}=0 \\
& \int_{A} z_{G e}(n, s) \omega_{P}(n, s) d A=\int_{A} z_{G e}(n, s)\left(\omega_{A}(s)-\omega_{A}^{0}-n q_{A}(s)\right) d A+z_{C} I_{y z}-y_{C} I_{y}=0
\end{aligned}
$$

Si se definen los productos de inercia sectoriales de la sección transversal referidos al polo como

$$
\begin{aligned}
& I_{y \omega_{A}}=\int_{A} z_{G e}(n, s)\left(\omega_{A}(s)-\omega_{A}^{0}-n q_{A}(s)\right) d A \\
& I_{z \omega_{A}}=\int_{A} y_{G e}(n, s)\left(\omega_{A}(s)-\omega_{A}^{0}-n q_{A}(s)\right) d A
\end{aligned}
$$

la resolución del sistema anterior conduce a

$$
y_{C}=\frac{I_{z} I_{y \omega_{A}}-I_{y z} I_{z \omega_{A}}}{I_{y} I_{z}-I_{y z}^{2}} \quad z_{C}=-\frac{I_{y} I_{z \omega_{A}}-I_{y z} I_{y \omega_{A}}}{I_{y} I_{z}-I_{y z}^{2}}
$$

Obtenido el centro de esfuerzos cortantes, el área sectorial principal de la sección es

$$
\omega_{P}(n, s)=\omega_{A}(s)-\omega_{A}^{0}-n q_{A}(s)+z_{C} y(n, s)-y_{C} z(n, s)
$$

Nótese que $q_{A}(s)$ es la componente tangencial del radio vector respecto del polo. Por lo tanto, desde el centro de esfuerzos cortantes se tiene

$$
\omega_{P}(n, s)=\omega(s)-\omega^{0}+n q(s)
$$

El módulo de alabeo de la sección se obtiene a partir de la siguiente fórmula

$$
I_{A}=\int_{A}\left(\omega_{P}(n, s)\right)^{2} d A
$$





\section{Anexo D. Ecuaciones de compatibilidad de una lámina}

Determinación de las ecuaciones de compatibilidad en la lámina de Love-Kirchhoff.

Con referencia a la Figura A.4

$$
\overrightarrow{A B}=d x \vec{i} \quad \overrightarrow{A C}=d s^{\prime} \vec{j} \quad \overrightarrow{A D}=d n \vec{k}
$$

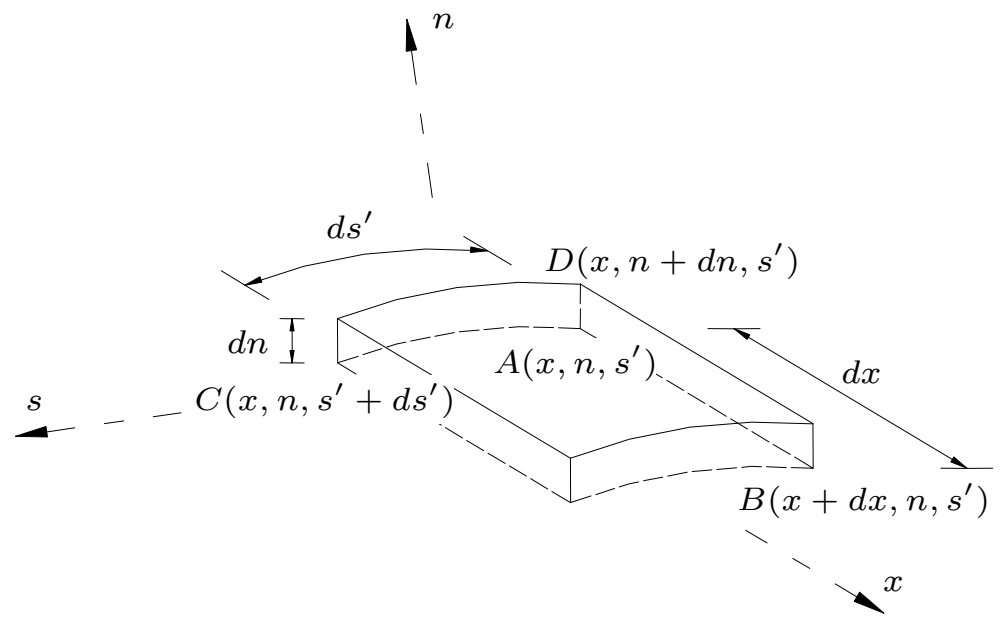

Figura A.4.

Dado que el desplazamiento del punto $A$ viene dado por

$$
\overrightarrow{A A^{\prime}}=\left(u^{0}+n \psi_{x}\right) \vec{i}+\left(v_{t}^{0}+n \psi_{s}\right) \vec{j}+v_{n}^{0} \vec{k}
$$

el de los puntos $B, C$ y $D$ será

$$
\begin{gathered}
\overrightarrow{B B^{\prime}}=\left(u^{0}+n \psi_{x}+\left(u_{, x}^{0}+n \psi_{x, x}\right) d x\right) \vec{i}+ \\
+\left(v_{t}^{0}+n \psi_{s}+\left(v_{t, x}^{0}+n \psi_{s, x}\right) d x\right) \vec{j}+\left(v_{n}^{0}+v_{n, x}^{0} d x\right) \vec{k} \\
\overrightarrow{C C^{\prime}}=\left(u^{0}+n \psi_{x}+\frac{1}{\mu}\left(u_{, s}^{0}+n \psi_{x, s}\right) d s^{\prime}\right) \vec{i}+ \\
+\left(v_{t}^{0}+n \psi_{s}+\frac{1}{\mu}\left(v_{t, s}^{0}+\frac{v_{n}^{0}}{a}+n \psi_{s, s}\right) d s^{\prime}\right) \vec{j}+\left(v_{n}^{0}+\frac{1}{\mu}\left(v_{n, s}^{0}-\frac{v_{t}^{0}}{a}-n \frac{\psi_{s}}{a}\right) d s^{\prime}\right) \vec{k} \\
\overrightarrow{D D^{\prime}}=\left(u^{0}+n \psi_{x}+\psi_{x} d n\right) \vec{i}+\left(v_{t}^{0}+n \psi_{s}+\psi_{s} d n\right) \vec{j}+v_{n}^{0} \vec{k}
\end{gathered}
$$

donde $\mu$ se ha obtenido de la siguiente forma (Figura A.5) 


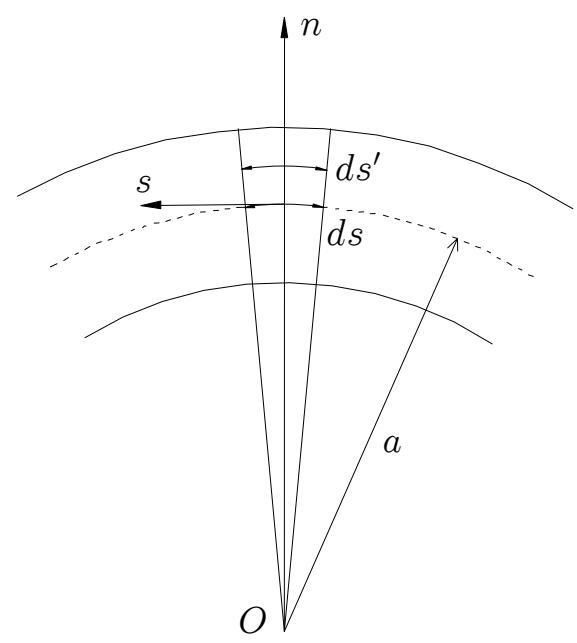

Figura A.5.

$$
\begin{gathered}
d A=\left.d n d s^{\prime}(n) \quad d s^{\prime}(n)\right|_{n=0}=d s \\
\frac{d s}{a}=\frac{d s^{\prime}(n)}{a+n} \rightarrow d s^{\prime}=\frac{a+n}{a} d s \\
d s^{\prime}=\left(1+\frac{n}{a}\right) d s=\mu d s
\end{gathered}
$$

A partir de la Figura A.6 se llega a las siguientes relaciones

$$
\begin{aligned}
& \left.\begin{array}{l}
\overrightarrow{A B}+\overrightarrow{B B^{\prime}}=\overrightarrow{A B^{\prime}} \\
\overrightarrow{A^{\prime} B^{\prime}}+\overrightarrow{A A^{\prime}}=\overrightarrow{A^{\prime}}
\end{array}\right\} \Rightarrow \overrightarrow{A^{\prime} B^{\prime}}=\overrightarrow{A B}+\overrightarrow{B B^{\prime}}-\overrightarrow{A A^{\prime}} \\
& \overrightarrow{A^{\prime} B^{\prime}}=\left(1+u_{, x}^{0}+n \psi_{x, x}\right) d x \vec{i}+\left(v_{t, x}^{0}+n \psi_{s, x}\right) d x \vec{j}+v_{n, x}^{0} d x \vec{k} \\
& \left.\begin{array}{l}
\overrightarrow{A C}+\overrightarrow{C C^{\prime}}=\overrightarrow{A C^{\prime}} \\
\overrightarrow{A^{\prime} C^{\prime}}+\overrightarrow{A A^{\prime}}=\overrightarrow{A C^{\prime}}
\end{array}\right\} \Rightarrow \overrightarrow{A^{\prime} C^{\prime}}=\overrightarrow{A C}+\overrightarrow{C C^{\prime}}-\overrightarrow{A A^{\prime}} \\
& \overrightarrow{A^{\prime} C^{\prime}}=\frac{1}{\mu}\left(u_{, s}^{0}+n \psi_{x, s}\right) d s^{\prime} \vec{i}+\left(1+\frac{1}{\mu}\left(v_{t, s}^{0}+\frac{v_{n}^{0}}{a}+n \psi_{s, s}\right)\right) d s^{\prime} \vec{j}+ \\
& +\frac{1}{\mu}\left(v_{n, s}^{0}-\frac{v_{t}^{0}}{a}-n \frac{\psi_{s}}{a}\right) d s^{\prime} \vec{k} \\
& \left.\begin{array}{l}
\overrightarrow{A D}+\overrightarrow{D D^{\prime}}=\overrightarrow{A D^{\prime}} \\
\overrightarrow{A^{\prime} D^{\prime}}+\overrightarrow{A A^{\prime}}=\overrightarrow{A D^{\prime}}
\end{array}\right\} \Rightarrow \overrightarrow{A^{\prime} D^{\prime}}=\overrightarrow{A D}+\overrightarrow{D D^{\prime}}-\overrightarrow{A A^{\prime}} \\
& \overrightarrow{A^{\prime} D^{\prime}}=\psi_{x} d n \vec{i}+\psi_{s} d n \vec{j}+d n \vec{k}
\end{aligned}
$$




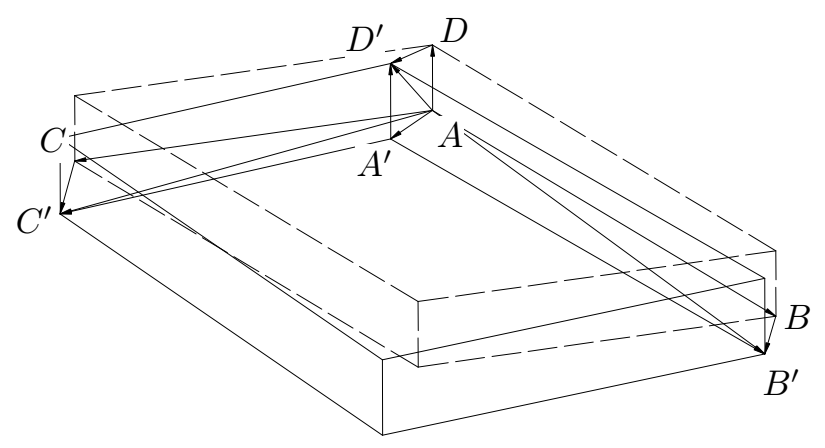

Figura A.6.

Por lo tanto, el tensor de deformaciones, en términos de ecuaciones de compatibilidad en coordenadas locales, es

$$
\left\{\varepsilon^{*}(x, n, s)\right\}=\left(\begin{array}{ccc}
u_{, x}^{0}+n \psi_{x, x} & \frac{1}{2}\left(v_{n, x}^{0}+\psi_{x}\right) & \frac{1}{2}\left(v_{t, x}^{0}+n \psi_{s, x}+\frac{1}{\mu}\left(u_{, s}^{0}+n \psi_{x, s}\right)\right) \\
\frac{1}{2}\left(v_{n, x}^{0}+\psi_{x}\right) & 0 & \frac{1}{2} \frac{1}{\mu}\left(v_{n, s}^{0}-\frac{v_{t}^{0}}{a}+\psi_{s}\right) \\
\frac{1}{2}\left(v_{t, x}^{0}+n \psi_{s, x}+\frac{1}{\mu}\left(u_{, s}^{0}+n \psi_{x, s}\right)\right) & \frac{1}{2} \frac{1}{\mu}\left(v_{n, s}^{0}-\frac{v_{t}^{0}}{a}+\psi_{s}\right) & \frac{1}{\mu}\left(v_{t, s}^{0}+\frac{v_{n}^{0}}{a}+n \psi_{s, x}\right)
\end{array}\right)
$$

donde se ha usado la notación tensorial para indicar las derivaciones.

Alternativamente, teniendo presente que en una lámina de Love-Kirchhoff no se produce deformación por cortante $\left(\omega_{x}=0, \omega_{s}=0\right)$ el campo de deformaciones también se puede expresar en función de las nuevas deformaciones generalizadas $\varepsilon_{x x}, \kappa_{x x}, \ldots$

$$
\begin{array}{ccl}
\varepsilon_{x x}^{*}=\varepsilon_{x x}+n \kappa_{x x} \quad \varepsilon_{s s}^{*}=\frac{\varepsilon_{s s}+n \kappa_{s s s}}{\mu} & \varepsilon_{n n}^{*}=0 \\
\gamma_{x s}^{*}=\varepsilon_{s x}+n \kappa_{s x}+\frac{\varepsilon_{x s}+n \kappa_{x s}}{\mu} \quad \gamma_{x n}^{*}=\omega_{x}=0 & \gamma_{s n}^{*}=\frac{1}{\mu} \omega_{s}=0
\end{array}
$$

Si la ecuación (D.12) se particulariza para la superficie de referencia de la lámina (basta hacer $n=0$ ), las componentes $\varepsilon_{x x}$ y $\varepsilon_{s s}$ aparecen como los alargamientos unitarios de la superficie de referencia en las direcciones coordenadas, $\mathrm{y} \gamma_{x s}=\varepsilon_{s x}+\varepsilon_{x s}$ proporciona la variación angular registrada por las mismas.

Deformaciones generalizadas de membrana

$$
\varepsilon_{x x}=u_{, x}^{0} \quad \varepsilon_{s s}=v_{t, x}^{0}+\frac{v_{n}^{0}}{a} \quad \gamma_{x s}=v_{t, x}^{0}+u_{, s}^{0}
$$


Deformaciones generalizadas de flexión

$$
\kappa_{x x}=\psi_{x, x} \quad \kappa_{x s}=\psi_{x, s} \quad \kappa_{s x}=\psi_{s, x} \quad \kappa_{s s}=\psi_{s, s}
$$

Teniendo en cuenta que en la teoría clásica de láminas se suele admitir que el escalar $\mu=(1+n / a)$ vale aproximadamente la unidad, la ecuación (D.11) queda de la siguiente forma

$$
\left\{\varepsilon^{*}(x, n, s)\right\}=\left(\begin{array}{ccc}
u_{, x}^{0}+n \psi_{x, x} & \frac{1}{2}\left(v_{n, x}^{0}+\psi_{x}\right) & \frac{1}{2}\left(v_{t, x}^{0}+n \psi_{s, x}+\left(u_{, s}^{0}+n \psi_{x, s}\right)\right) \\
\frac{1}{2}\left(v_{n, x}^{0}+\psi_{x}\right) & 0 & \frac{1}{2}\left(v_{n, s}^{0}-\frac{v_{t}^{0}}{a}+\psi_{s}\right) \\
\frac{1}{2}\left(v_{t, x}^{0}+n \psi_{s, x}+\left(u_{, s}^{0}+n \psi_{x, s}\right)\right) & \frac{1}{2}\left(v_{n, s}^{0}-\frac{v_{t}^{0}}{a}+\psi_{s}\right) & \left(v_{t, s}^{0}+\frac{v_{n}^{0}}{a}+n \psi_{s, x}\right)
\end{array}\right)
$$

que, a su vez, puede expresarse como suma de la matriz $\{\varepsilon\}$, la cual recoge las deformaciones de los puntos de coordenadas $(x, 0, s)$ correspondientes al plano medio, $\mathrm{y}$ las deformaciones que resultan de multiplicar por la coordenada $n$ (del punto en el que se calculan las deformaciones) la matriz $\{\kappa\}$ de curvaturas del plano medio en el punto $(x, 0, s)$.

$$
\left\{\varepsilon^{*}\right\}=\{\varepsilon\}+n\{\kappa\}
$$

siendo

$$
\begin{gathered}
\{\varepsilon\}=\{\varepsilon(x, 0, s)\}=\left(\begin{array}{ccc}
u_{, x}^{0} & \frac{1}{2}\left(v_{n, x}^{0}+\psi_{x}\right) & \frac{1}{2}\left(u_{, s}^{0}+v_{t, x}^{0}\right) \\
\frac{1}{2}\left(v_{n, x}^{0}+\psi_{x}\right) & 0 & \frac{1}{2}\left(v_{n, s}^{0}-\frac{v_{t}^{0}}{a}+\psi_{s}\right) \\
\frac{1}{2}\left(u_{, s}^{0}+v_{t, x}^{0}\right) & \frac{1}{2}\left(v_{n, s}^{0}-\frac{v_{t}^{0}}{a}+\psi_{s}\right) & \left(v_{t, s}^{0}+\frac{v_{n}^{0}}{a}\right)
\end{array}\right) \\
\{\kappa\}=\{\kappa(x, 0, s)\}=\left(\begin{array}{ccc}
\psi_{x, x} & 0 & \frac{1}{2}\left(\psi_{s, x}+\psi_{x, s}\right) \\
0 & 0 & 0 \\
\frac{1}{2}\left(\psi_{s, x}+\psi_{x, s}\right) & 0 & \psi_{s, s}
\end{array}\right)
\end{gathered}
$$

Las variables cinemáticas de los puntos del plano medio determinan por sí solas la cinemática de la lámina. 


\section{Anexo E. Ecuaciones de equilibro de una lámina}

Deducción de las ecuaciones de equilibrio de una lámina.

Se parte de un elemento diferencial $d x d s d n$ (Figura A.7)

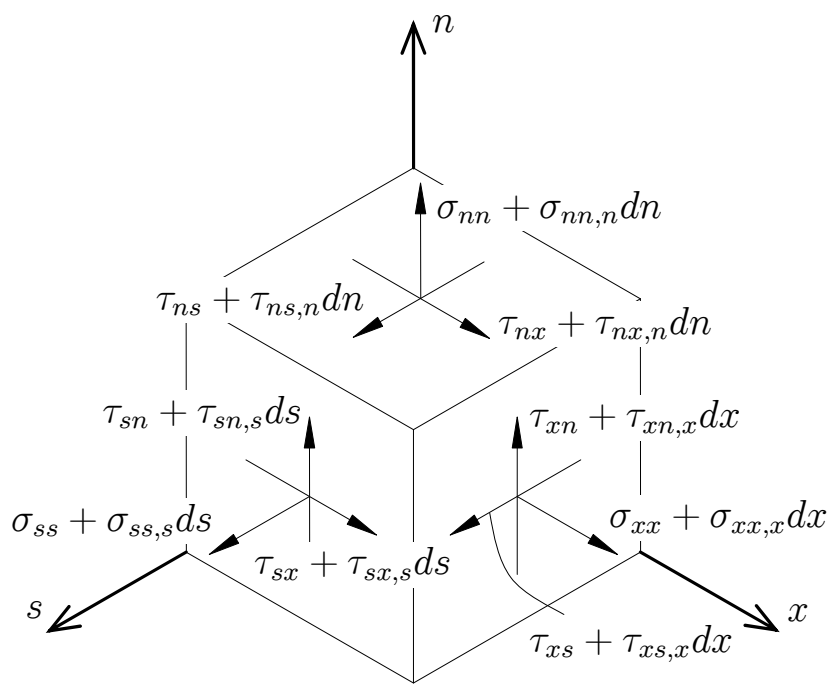

Figura A.7.

Puesto que el elemento diferencial debe estar en equilibrio, se tiene

$$
\begin{aligned}
& {\left[\frac{\partial}{\partial x}\left(\sigma_{x x} \vec{i}+\tau_{x s} \vec{j}+\tau_{x n} \vec{k}\right) d x\right] d s d n+\left[\frac{\partial}{\partial s}\left(\tau_{s x} \vec{i}+\sigma_{s s} \vec{j}+\tau_{s n} \vec{k}\right) d s\right] d x d n+} \\
& \quad+\left[\frac{\partial}{\partial n}\left(\tau_{n x} \vec{i}+\tau_{n s} \vec{j}+\sigma_{n n} \vec{k}\right) d n\right] d x d s+\left[\rho_{x} \vec{i}+\rho_{s} \vec{j}+\rho_{n} \vec{k}\right] d x d s d n=0
\end{aligned}
$$

donde $\rho_{i}$ son fuerzas por unidad de volumen.

Planteando el equilibrio según los ejes $O^{\prime} x, O^{\prime} s$ y $O^{\prime} n$ se obtiene respectivamente

$$
\begin{gathered}
\sigma_{x x, x}+\tau_{s x, s}+\tau_{n x, n}+\rho_{x}=0 \\
\tau_{x s, x}+\sigma_{s s, s}+\frac{\tau_{s n}}{a}+\tau_{n s, n}+\rho_{s}=0 \\
\tau_{x n, x}+\tau_{s n, s}-\frac{\sigma_{s s}}{a}+\sigma_{n n, n}+\rho_{n}=0
\end{gathered}
$$




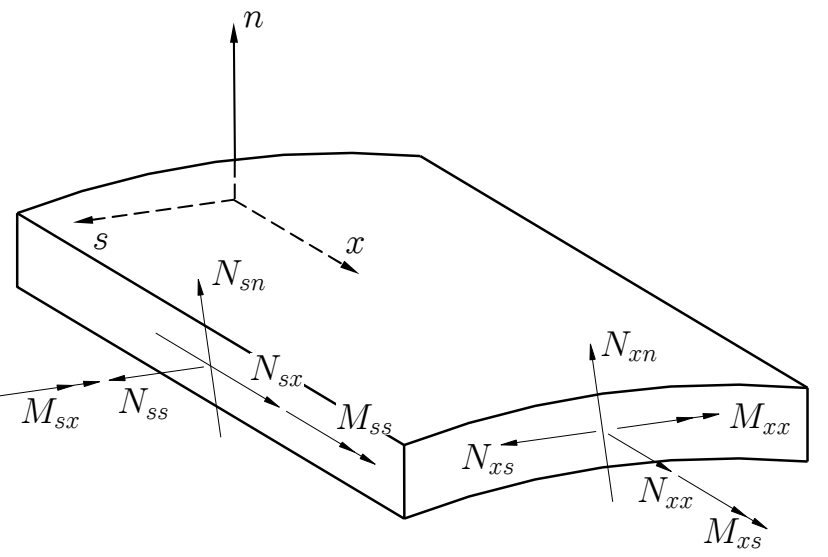

Figura A.8.

\section{Fuerzas por unidad de longitud}

A partir de la ecuación (E.2) se tiene

$$
\int_{-e / 2}^{e / 2} \frac{\partial}{\partial x} \sigma_{x x} d n+\int_{-e / 2}^{e / 2} \frac{\partial}{\partial s} \tau_{s x} d n+\int_{-e / 2}^{e / 2} \frac{\partial}{\partial n} \tau_{n x} d n+\int_{-e / 2}^{e / 2} \rho_{x} d n=0
$$

Admitiendo las siguientes definiciones (Figura A.8)

$$
\begin{gathered}
N_{x x}=\int_{-e / 2}^{e / 2} \sigma_{x x} d n \\
N_{x s}=\int_{-e / 2}^{e / 2} \tau_{s x} d n=N_{s x} \\
f_{x}=\int_{-e / 2}^{e / 2} d \tau_{n x}+\int_{-e / 2}^{e / 2} \rho_{x} d n=\tau_{n x}(e / 2)-\tau_{n x}(-e / 2)+\int_{-e / 2}^{e / 2} \rho_{x} d n
\end{gathered}
$$

se obtiene

$$
N_{x x, x}+N_{x s, s}+f_{x}=0
$$

Procediendo de forma análoga con la ecuación (E.3)

$$
\int_{-e / 2}^{e / 2} \frac{\partial}{\partial x} \tau_{x s} d n+\int_{-e / 2}^{e / 2} \frac{\partial}{\partial s} \sigma_{s s} d n+\int_{-e / 2}^{e / 2} \frac{\tau_{s n}}{a} d n+\int_{-e / 2}^{e / 2} \frac{\partial}{\partial n} \tau_{n s} d n+\int_{-e / 2}^{e / 2} \rho_{s} d n=0
$$

y admitiendo las siguientes definiciones (Figura A.8) 


$$
\begin{gathered}
N_{s s}=\int_{-e / 2}^{e / 2} \sigma_{s s} d n \\
N_{s n}=\int_{-e / 2}^{e / 2} \tau_{s n} d n \\
f_{s}=\int_{-e / 2}^{e / 2} d \tau_{n s}+\int_{-e / 2}^{e / 2} \rho_{s} d n=\tau_{n s}(e / 2)-\tau_{n s}(-e / 2)+\int_{-e / 2}^{e / 2} \rho_{s} d n
\end{gathered}
$$

se llega a

$$
N_{x s, x}+N_{s s, s}+\frac{N_{s n}}{a}+f_{s}=0
$$

Finalmente, para la ecuación (E.4)

$$
\int_{-e / 2}^{e / 2} \frac{\partial}{\partial x} \tau_{x n} d n+\int_{-e / 2}^{e / 2} \frac{\partial}{\partial s} \tau_{s n} d n-\int_{-e / 2}^{e / 2} \frac{\sigma_{s s}}{a} d n+\int_{-e / 2}^{e / 2} \frac{\partial}{\partial n} \sigma_{n n} d n+\int_{-e / 2}^{e / 2} \rho_{n} d n=0
$$

Admitiendo las siguientes definiciones (Figura A.8)

$$
\begin{aligned}
& N_{x n}=\int_{-e / 2}^{e / 2} \tau_{x n} d n \\
& f_{n}=\int_{-e / 2}^{e / 2} d \sigma_{n n}+\int_{-e / 2}^{e / 2} \rho_{n} d n=\sigma_{n n}(e / 2)-\sigma_{n n}(-e / 2)+\int_{-e / 2}^{e / 2} \rho_{n} d n
\end{aligned}
$$

se obtiene

$$
N_{x n, x}+N_{s n, s}-\frac{N_{s s}}{a}+f_{n}=0
$$

\section{Momentos por unidad de longitud}

A partir de la ecuación (E.2)

$$
\int_{-e / 2}^{e / 2} \frac{\partial \sigma_{x x}}{\partial x} n d n+\int_{-e / 2}^{e / 2} \frac{\partial \tau_{s x}}{\partial s} n d n+\int_{-e / 2}^{e / 2} \frac{\partial \tau_{n x}}{\partial n} n d n+\int_{-e / 2}^{e / 2} \rho_{x} n d n=0
$$

Teniendo en cuenta que

$$
\int_{-e / 2}^{e / 2} \frac{\partial \tau_{n x}}{\partial n} n d n=\int_{-e / 2}^{e / 2} \frac{\partial}{\partial n}\left(\tau_{n x} n\right) d n-\int_{-e / 2}^{e / 2} \tau_{x n} d n
$$


la ecuación (E.19) se transforma en

$$
\int_{-e / 2}^{e / 2} \frac{\partial \sigma_{x x}}{\partial x} n d n+\int_{-e / 2}^{e / 2} \frac{\partial \tau_{s x}}{\partial s} n d n+\int_{-e / 2}^{e / 2} \frac{\partial}{\partial n}\left(\tau_{n x} n\right) d n-\int_{-e / 2}^{e / 2} \tau_{x n} d n+\int_{-e / 2}^{e / 2} \rho_{x} n d n=0
$$

Admitiendo las siguientes definiciones (Figura A.8)

$$
\begin{gathered}
M_{x x}=\int_{-e / 2}^{e / 2} \sigma_{x x} n d n \\
M_{x s}=\int_{-e / 2}^{e / 2} \tau_{x s} n d n=M_{s x} \\
m_{x}=\int_{-e / 2}^{e / 2} \frac{\partial}{\partial n}\left(\tau_{n x} n\right) d n+\int_{-e / 2}^{e / 2} \rho_{x} n d n=\int_{-e / 2}^{e / 2} d\left(\tau_{n x} n\right)+\int_{-e / 2}^{e / 2} \rho_{x} n d n= \\
=\frac{e}{2}\left[\tau_{n x}(e / 2)+\tau_{n x}(-e / 2)\right]+\int_{-e / 2}^{e / 2} \rho_{x} n d n
\end{gathered}
$$

se obtiene

$$
M_{x x, x}+M_{x s, s}-N_{x n}+m_{x}=0
$$

Procediendo de forma análoga respecto a la ecuación (E.3)

$$
\int_{-e / 2}^{e / 2} \frac{\partial \tau_{s x}}{\partial x} n d n+\int_{-e / 2}^{e / 2} \frac{\partial \sigma_{s s}}{\partial s} n d n+\int_{-e / 2}^{e / 2} \frac{\tau_{s n}}{a} n d n+\int_{-e / 2}^{e / 2} \frac{\partial \tau_{n s}}{\partial n} n d n+\int_{-e / 2}^{e / 2} \rho_{s} n d n=0
$$

Teniendo en cuenta que

$$
\int_{-e / 2}^{e / 2} \frac{\partial \tau_{n s}}{\partial n} n d n=\int_{-e / 2}^{e / 2} \frac{\partial}{\partial n}\left(\tau_{n s} n\right) d n-\int_{-e / 2}^{e / 2} \tau_{s n} d n
$$

la ecuación (E.19) se transforma en

$$
\begin{gathered}
\int_{-e / 2}^{e / 2} \frac{\partial \tau_{s x}}{\partial x} n d n+\int_{-e / 2}^{e / 2} \frac{\partial \sigma_{s s}}{\partial s} n d n+\int_{-e / 2}^{e / 2} \frac{\tau_{s n}}{a} n d n+\int_{-e / 2}^{e / 2} \frac{\partial}{\partial n}\left(\tau_{n s} n\right) d n- \\
-\int_{-e / 2}^{e / 2} \tau_{n s} d n+\int_{-e / 2}^{e / 2} \rho_{s} n d n=0
\end{gathered}
$$

Admitiendo las siguientes definiciones (Figura A.8)

$$
M_{s s}=\int_{-e / 2}^{e / 2} \sigma_{s s} n d n
$$




$$
\begin{gathered}
m_{s}=\int_{-e / 2}^{e / 2} \frac{\partial}{\partial n}\left(\tau_{n s} n\right) d n+\int_{-e / 2}^{e / 2} \rho_{s} n d n=\int_{-e / 2}^{e / 2} d\left(\tau_{n s} n\right)+\int_{-e / 2}^{e / 2} \rho_{s} n d n= \\
=\frac{e}{2}\left[\tau_{n s}(e / 2)+\tau_{n s}(-e / 2)\right]+\int_{-e / 2}^{e / 2} \rho_{s} n d n
\end{gathered}
$$

y que

$$
\int_{-e / 2}^{e / 2} \frac{\tau_{s n}}{a} n d n=0 \quad\left(\vec{\tau}_{s n} \| \vec{n}\right)
$$

se obtiene

$$
M_{x s, x}+M_{s s, s}-N_{s n}+m_{s}=0
$$

En definitiva, las ecuaciones de equilibrio de un elemento diferencial de lámina son

$$
\begin{gathered}
N_{x x, x}+N_{x s, s}+f_{x}=0 \\
N_{x s, x}+N_{s s, s}+\frac{N_{s n}}{a}+f_{s}=0 \\
N_{x n, x}+N_{s n, s}-\frac{N_{s s}}{a}+f_{n}=0 \\
M_{x x, x}+M_{x s, s}-N_{x n}+m_{x}=0 \\
M_{x s, x}+M_{s s, s}-N_{s n}+m_{s}=0
\end{gathered}
$$





\section{Anexo F. Ecuaciones de comportamiento de un laminado}

Deducción de las ecuaciones de comportamiento de un laminado.

Además del sistema de referencia $O^{\prime} x n s$ se define el sistema de coordenadas cartesianas $O^{\prime} 123$, en el cual la dirección 1 coincide con la dirección de la fibra y las direcciones 2 y 3 son perpendiculares a la dirección de la misma según se muestra en la Figura A.9.

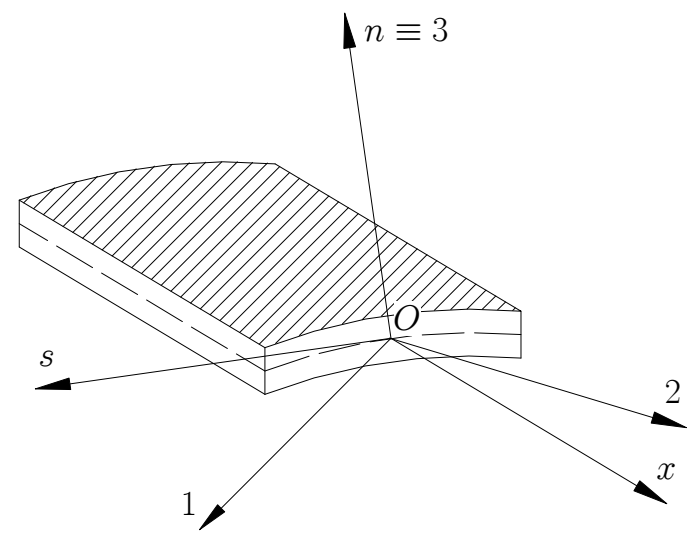

Figura A.9.

La matriz de rigidez de una lámina de material compuesto ortótropa bajo un estado de tensión plana $\left(\sigma_{3}=\tau_{13}=\tau_{23}=0\right)$ tiene la siguiente forma

$$
\begin{gathered}
\left\{\begin{array}{c}
\sigma_{1} \\
\sigma_{2} \\
\tau_{12}
\end{array}\right\}=\left[\begin{array}{ccc}
Q_{11} & Q_{12} & 0 \\
Q_{21} & Q_{22} & 0 \\
0 & 0 & Q_{66}
\end{array}\right]\left\{\begin{array}{c}
\varepsilon_{1} \\
\varepsilon_{2} \\
\gamma_{12}
\end{array}\right\} \\
\{\sigma\}_{1}=[Q]\{\varepsilon\}_{1}
\end{gathered}
$$

Para llevar acabo el cálculo de una estructura constituida por materiales compuestos se requiere una caracterización del material siguiendo la normativa correspondiente. En general, los coeficientes de la ecuación (F.1) no se pueden medir directamente en un laboratorio. Por otro lado, las constantes que pueden ser mediadas en un laboratorio se denominan constantes ingenieriles y son, entre otras, el módulo de Young, el módulo de cortadura y el coeficiente de Poisson. Esto hace necesario introducir la matriz de flexibilidad, en la cual se pueden expresar sus términos en función de dichas constantes ingenieriles de la forma que se indica a continuación. La relación matricial deformación-tensión (matriz de flexibilidad) de una lámina ortótropa está representada por la siguiente expresión 


$$
\left\{\begin{array}{c}
\varepsilon_{1} \\
\varepsilon_{2} \\
\gamma_{12}
\end{array}\right\}=\left[\begin{array}{ccc}
S_{11} & S_{12} & 0 \\
S_{21} & S_{22} & 0 \\
0 & 0 & S_{66}
\end{array}\right]\left\{\begin{array}{c}
\sigma_{1} \\
\sigma_{2} \\
\tau_{12}
\end{array}\right\}
$$

donde

$$
\begin{gathered}
S_{11}=\frac{1}{E_{1}} \quad S_{12}=-\frac{\nu_{12}}{E_{2}} \\
S_{21}=-\frac{\nu_{21}}{E_{1}} \quad S_{22}=\frac{1}{E_{2}} \\
S_{66}=\frac{1}{G_{12}}
\end{gathered}
$$

Las constantes ingenieriles $E_{1}$ y $E_{2}$ son los módulos de Young según las direcciones coordenadas. $E_{1}$ es el módulo axial (dirección de la fibra) y $E_{2}$ es módulo en la dirección transversal. Respecto a los coeficientes de Poisson $\nu_{i j}$, el primer subíndice se refiere a la dirección en la cual se aplica la tensión y el segundo subíndice corresponde a la dirección asociada a la deformación lateral. El módulo de cortadura $G_{i j}$, se define para tensiones tangenciales actuando en el plano $i-j$. Para una lámina de material compuesto unidireccional, $G_{12}$ es el módulo de cortadura axial en el plano ortogonal que contiene a las fibras.

Para expresar la ecuación (F.1) en el sistema de referencia $O^{\prime} x n s$ es necesario hacer una transformación de coordenadas (Figura A.10).

- vector de tensiones:

$$
\{\sigma\}_{1}=\left[T_{1}\right]\{\sigma\}_{x}
$$

donde la matriz de transformación $\left[T_{1}\right]$ es

$$
\left[T_{1}\right]=\left[\begin{array}{ccc}
m^{2} & n^{2} & 2 m n \\
n^{2} & m^{2} & -2 m n \\
-m n & m n & m^{2}-n^{2}
\end{array}\right]
$$

- vector de deformaciones

$$
\{\varepsilon\}_{1}=\left[T_{2}\right]\{\varepsilon\}_{x}
$$

donde la matriz de transformación $\left[T_{2}\right]$ es

$$
\left[T_{2}\right]=\left[\begin{array}{ccc}
m^{2} & n^{2} & m n \\
n^{2} & m^{2} & -m n \\
-2 m n & 2 m n & m^{2}-n^{2}
\end{array}\right]
$$




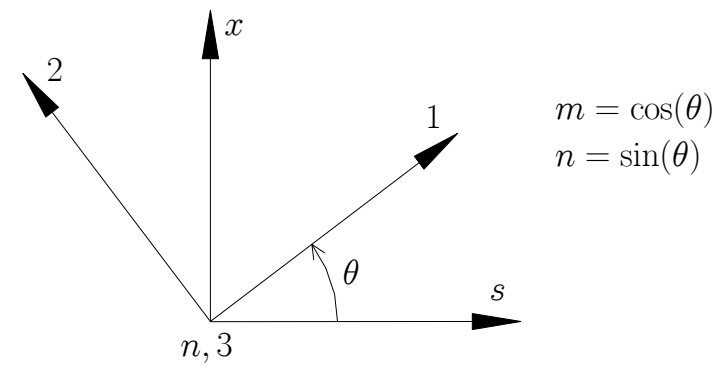

Figura A.10.

Manipulando adecuadamente las expresiones anteriores se tiene

$$
\{\sigma\}_{x}=\left[T_{1}\right]^{-1}[Q]\left[T_{2}\right]\{\varepsilon\}_{x}
$$

La ecuación (F.9) se puede reescribir como

$$
\begin{gathered}
\{\sigma\}_{x}=[\bar{Q}]\{\varepsilon\}_{x} \\
\left\{\begin{array}{c}
\sigma_{x x} \\
\sigma_{s s} \\
\tau_{x s}
\end{array}\right\}=\left[\begin{array}{lll}
\bar{Q}_{11} & \bar{Q}_{12} & \bar{Q}_{16} \\
\bar{Q}_{12} & \bar{Q}_{22} & \bar{Q}_{26} \\
\bar{Q}_{16} & \bar{Q}_{26} & \bar{Q}_{66}
\end{array}\right]\left\{\begin{array}{c}
\varepsilon_{x x} \\
\varepsilon_{s s} \\
\gamma_{x s}
\end{array}\right\}
\end{gathered}
$$

donde la matriz $[\bar{Q}]$ es

$$
[\bar{Q}]=\left[T_{1}\right]^{-1}[Q]\left[T_{2}\right]
$$

Los términos $\bar{Q}_{i j}$ de esta matriz se determinan a partir de las ecuaciones (F.6), (F.8) y (F.12)

$$
\begin{aligned}
& \bar{Q}_{11}=m^{4} Q_{11}+2 m^{2} n^{2}\left(Q_{12}+2 Q_{66}\right)+n^{4} Q_{22} \\
& \bar{Q}_{12}=m^{2} n^{2}\left(Q_{11}+Q_{22}-4 Q_{66}\right)+\left(m^{4}+n^{4}\right) Q_{12} \\
& \bar{Q}_{16}=m n\left[m^{2}\left(Q_{11}-Q_{12}-2 Q_{66}\right)+n^{2}\left(Q_{12}-Q_{22}+2 Q_{66}\right)\right] \\
& \bar{Q}_{22}=n^{4} Q_{11}+2 m^{2} n^{2}\left(Q_{12}+2 Q_{66}\right)+n^{4} Q_{22} \\
& \bar{Q}_{26}=m n\left[n^{2}\left(Q_{11}-Q_{12}-2 Q_{66}\right)+m^{2}\left(Q_{12}-Q_{22}+2 Q_{66}\right)\right] \\
& \bar{Q}_{44}=m^{2} Q_{44}+n^{2} Q_{55} \\
& \bar{Q}_{45}=m n\left(Q_{55}-Q_{44}\right) \\
& \bar{Q}_{55}=n^{2} Q_{44}+m^{2} Q_{55}
\end{aligned}
$$

Una lámina de material compuesto presenta espesores muy bajos $(0.1 \mathrm{~mm}$ a $1.5 \mathrm{~mm})$. Por lo tanto, prácticamente en la totalidad de los casos de estructuras de materiales compuestos, la lámina está integrada en un laminado constituido en general por $N$ láminas (Figura A.11). 


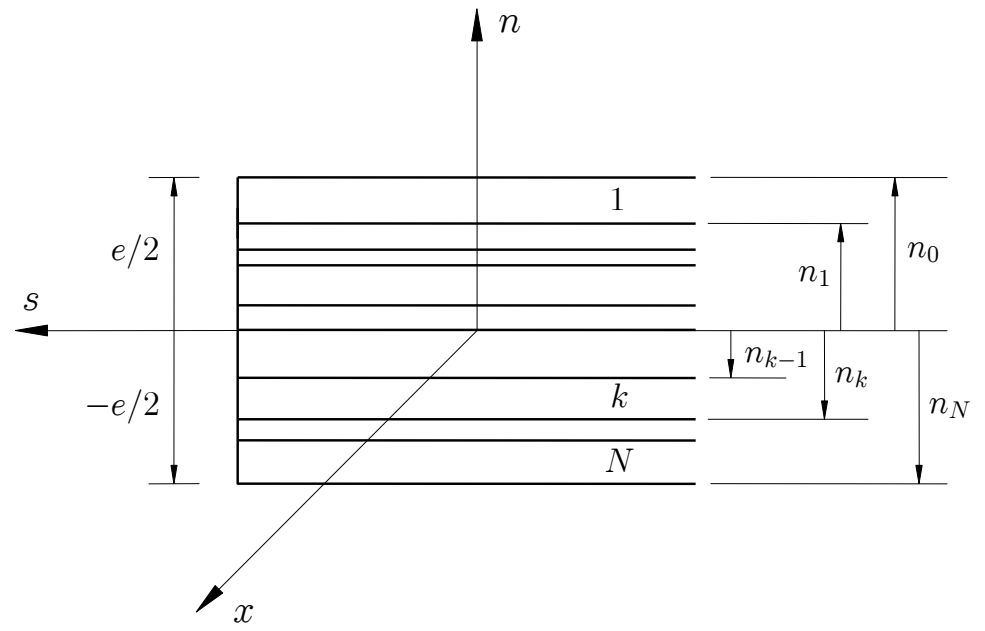

Figura A.11.

Se adoptan las siguientes hipótesis (teoría clásica de láminas)

- El laminado está constituido por un número finito de láminas perfectamente unidas.

- Cada lámina es un material homogéneo con propiedades efectivas conocidas.

- Las láminas individuales pueden ser isótropas, ortótropas o transversalmente isótropas.

- En cada lámina se asume estado de tensión plana.

- El laminado se deforma de acuerdo a la teoría de Love-Kirchhoff.

El campo de deformaciones de una lámina bajo estas hipótesis es

$$
\begin{gathered}
\left\{\varepsilon^{*}\right\}_{x}=\{\varepsilon\}_{x}+n\{\kappa\}_{x} \\
\left\{\begin{array}{c}
\varepsilon_{x x}^{*} \\
\varepsilon_{s s}^{*} \\
\gamma_{x s}^{*}
\end{array}\right\}=\left\{\begin{array}{l}
\varepsilon_{x x} \\
\varepsilon_{s s} \\
\gamma_{x s}
\end{array}\right\}+n\left\{\begin{array}{c}
\kappa_{x x} \\
\kappa_{s s} \\
\kappa_{x s}
\end{array}\right\}
\end{gathered}
$$

La tensión para cualquier cota- $n$ puede ser determinada sustituyendo (F.14) en (F.10)

$$
\{\sigma\}_{x}=[\bar{Q}]^{k}\left\{\varepsilon^{*}\right\}_{x}=[\bar{Q}]^{k}\{\varepsilon\}+n[\bar{Q}]^{k}\{\kappa\}
$$

En la Figura A.12 se muestran los sentidos positivos de los esfuerzos aplicados en un elemento diferencial de lámina. Las fuerzas por unidad de longitud $N_{x x}, N_{s s}$ y $N_{x s}$ se definen de la siguiente manera 


$$
\begin{aligned}
& N_{x x}=\int_{-e / 2}^{e / 2} \sigma_{x x} d n \\
& N_{s s}=\int_{-e / 2}^{e / 2} \sigma_{s s} d n \\
& N_{x s}=\int_{-e / 2}^{e / 2} \tau_{x s} d n
\end{aligned}
$$

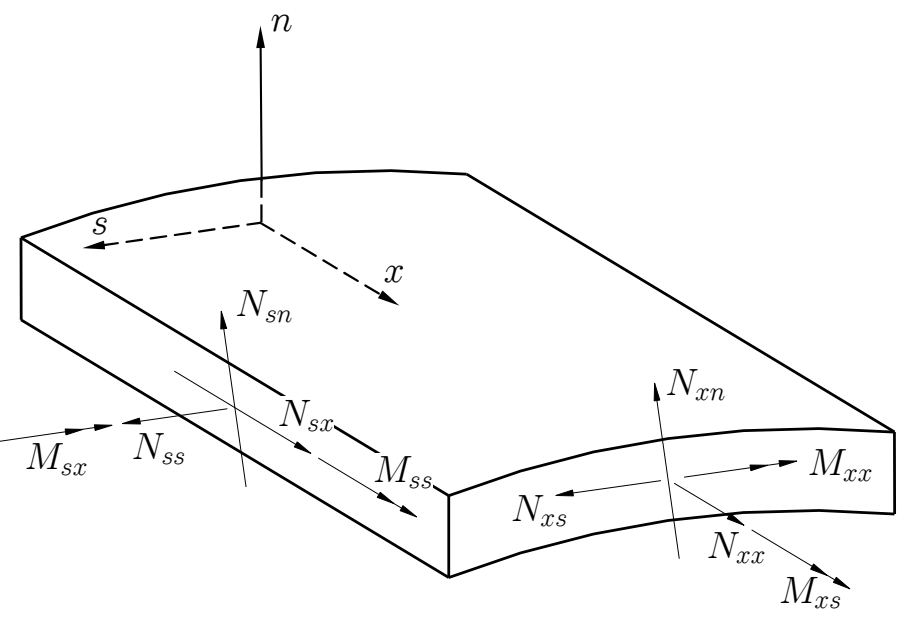

Figura A.12.

Las ecuaciones (F.17) se pueden expresar en forma condensada

$$
\{N\}=\int_{-e / 2}^{e / 2}\{\sigma\} d n
$$

Combinando las ecuaciones (F.16) y (F.18)

$$
\{N\}=\int_{-e / 2}^{e / 2}[\bar{Q}]^{k}\{\varepsilon\}_{x} d n+\int_{-e / 2}^{e / 2} n[\bar{Q}]^{k}\{\kappa\}_{x} d n
$$

Teniendo en cuenta que $\{\varepsilon\}$ y $\{\kappa\}$ son independientes de la coordenada $n$, la integral sobre el espesor del laminado se puede sustituir por un sumatorio de integrales sobre el espesor de cada una de las láminas que constituyen el laminado, entonces

$$
\{N\}=\sum_{k=1}^{N}\left(\int_{n_{k-1}}^{n_{k}}[\bar{Q}]^{k} d n\right)\{\varepsilon\}_{x}+\sum_{k=1}^{N}\left(\int_{n_{k-1}}^{n_{k}} n[\bar{Q}]^{k} d n\right)\{\kappa\}_{x}
$$

Esta ecuación se puede reescribir de la siguiente manera 


$$
\{N\}=[A]\{\varepsilon\}+[B]\{\kappa\}
$$

donde

$$
\begin{gathered}
{[A]=\sum_{k=1}^{N}[\bar{Q}]^{k}\left(n_{k}-n_{k-1}\right)} \\
{[B]=\frac{1}{2} \sum_{k=1}^{N}[\bar{Q}]^{k}\left(n_{k}^{2}-n_{k-1}^{2}\right)}
\end{gathered}
$$

Respecto a los momentos por unidad de longitud $M_{x x}, M_{s s}$ y $M_{x s}$, éstos se definen de la siguiente forma (Figura A.12)

$$
\begin{aligned}
& M_{x x}=\int_{-e / 2}^{e / 2} \sigma_{x x} n d n \\
& M_{s s}=\int_{-e / 2}^{e / 2} \sigma_{s s} n d n \\
& M_{x s}=\int_{-e / 2}^{e / 2} \tau_{x s} n d n
\end{aligned}
$$

En forma condensada

$$
\{M\}=\int_{-e / 2}^{e / 2}\{\sigma\} n d n
$$

Combinando las ecuaciones (F.16) y (F.25) se obtiene la siguiente expresión para los momentos por unidad de longitud

$$
\{M\}=[B]\{\varepsilon\}+[D]\{\kappa\}
$$

donde

$$
[D]=\frac{1}{3} \sum_{k=1}^{N}[\bar{Q}]^{k}\left(n_{k}^{3}-n_{k-1}^{3}\right)
$$

Las ecuaciones (F.21) y (F.26) pueden combinarse para obtener la ecuación fundamental de la teoría de materiales laminados

$$
\left\{\begin{array}{l}
N \\
M
\end{array}\right\}=\left[\begin{array}{ll}
A & B \\
B & D
\end{array}\right]\left\{\begin{array}{l}
\varepsilon \\
\kappa
\end{array}\right\}
$$

Esta ecuación se puede expresar en su forma expandida 


$$
\left\{\begin{array}{l}
N_{x x} \\
N_{s s} \\
N_{x s} \\
M_{s s} \\
M_{x s}
\end{array}\right\}=\left[\begin{array}{lllllll}
A_{11} & A_{12} & A_{16} & B_{11} & B_{12} & B_{16} \\
A_{12} & A_{22} & A_{26} & B_{12} & B_{22} & B_{26} \\
A_{16} & A_{26} & A_{66} & B_{16} & B_{26} & B_{66} \\
B_{11} & B_{12} & B_{16} & D_{11} & D_{12} & D_{16} \\
B_{12} & B_{22} & B_{26} & D_{12} & D_{22} & D_{26} \\
B_{16} & B_{26} & B_{66} & D_{16} & D_{26} & D_{66}
\end{array}\right]\left\{\begin{array}{c}
\varepsilon_{x x}^{0} \\
\varepsilon_{s s}^{0} \\
\gamma_{x s}^{0} \\
\kappa_{x x} \\
\kappa_{s s} \\
\kappa_{x s}
\end{array}\right\}
$$





\section{Anexo G. Ejemplos. Modelo de Barras}

Se presentan dos ejemplos que ilustran la aplicabilidad de la técnica descrita en el Capítulo 3. En el primero se analizan dos secciones abiertas, una en doble $\mathrm{T}$ y otra en $\mathrm{T}$, y el segundo se dedica al estudio de una sección en cajón bajo dos configuraciones de laminado diferentes, CUS y CAS.

\section{G.1. Ejemplo 1. Sección en doble T y en T}

La primera parte de este problema consiste en el estudio de una barra en voladizo de sección como la representada en la Figura A.13.a, cuyo extremo no empotrado tiene impedidos los desplazamientos de alabeo, aunque puede sufrir desplazamientos no axiles, incluidos giros por torsión. Estas condiciones cinemáticas suelen darse, por ejemplo, en determinadas palas de helicópteros, donde secciones como la representada en la mencionada Figura A.13.a constituyen el cuerpo resistente del elemento estructural ligero que une la pala al rotor. A efectos de comparación de algunos de los resultados presentados con los obtenidos por otros autores, se ha adoptado el sistema anglosajón de unidades, siendo la longitud de la pieza $30 \mathrm{in}$. Las dimensiones de la sección y los valores de las constantes elásticas del material (AS4/3501-6 grafito/epoxy) se especifican en la Tabla A.1. En cuanto a la orientación de las fibras de las láminas se refiere, los casos estudiados son los señalados en la Figura A.13.a, donde puede observarse que en el alma se disponen láminas intercaladas con fibras orientadas a $0^{\circ}$ y $90^{\circ}$, respectivamente.

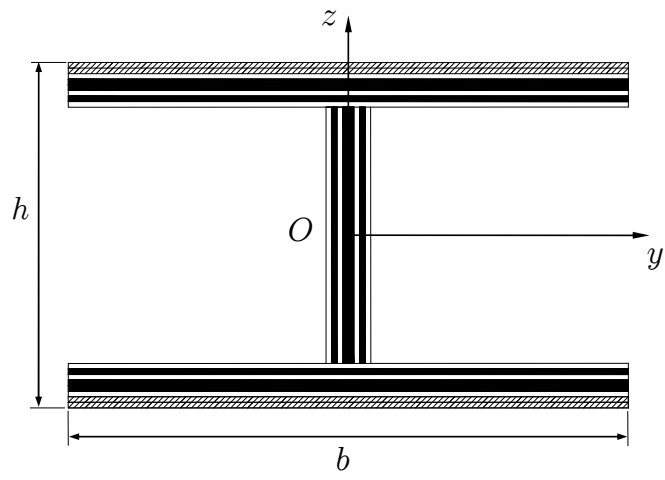

Esquema de laminado:

Alas: $\left[(0 / 90)_{2} /(90 / 0) / \theta_{2}\right]$

Alma: $\left[(0 / 90)_{2}\right]_{s}$

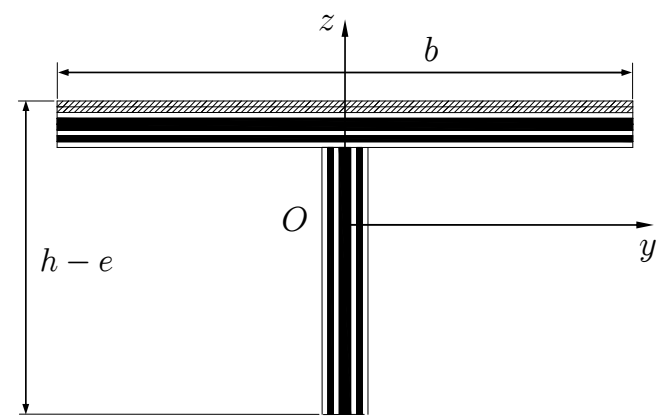

Esquema de laminado: Alas: $\left[(0 / 90)_{2} /(90 / 0) / \theta_{2}\right]$

Alma: $\left[(0 / 90)_{2}\right]_{s}$

Figura A.13. Secciones consideradas en el Ejemplo 1 


\begin{tabular}{|c|c|}
\hline Característica & Valor \\
\hline$E_{11}$ & $141.9 \mathrm{GPa}\left(20.5910^{6} \mathrm{psi}\right)$ \\
\hline$E_{22}$ & $9.78 \mathrm{GPa}\left(1.4210^{6} \mathrm{psi}\right)$ \\
\hline$G_{12}$ & $6.13 \mathrm{GPa}\left(0.8910^{6} \mathrm{psi}\right)$ \\
\hline$\nu_{12}$ & 0.42 \\
\hline Espesor de lámina $(e)$ & $0.127 \mathrm{~mm}(0.005 \mathrm{in})$ \\
\hline Canto $(h)$ & $12.7 \mathrm{~mm}(0.5 \mathrm{in})$ \\
\hline Ancho $(b)$ & $25.4 \mathrm{~mm}(1 \mathrm{in})$ \\
\hline Longitud de la barra $(L)$ & $762.12 \mathrm{~mm}(30 \mathrm{in})$ \\
\hline
\end{tabular}

Tabla A.1. Propiedades elásticas y geométricas del Ejemplo 1

Con objeto de caracterizar los acoplamientos elásticos, se han considerado dos estados de carga simples. El primero consiste en una carga unitaria concentrada normal a la directriz y el segundo con sólo un momento torsor de valor unidad, estando ambas cargas aplicadas en la sección extrema no empotrada.

Se comenzará exponiendo los resultados conseguidos para la sección en doble T (Figura A.13.a). En la Figura A.14 se muestra la variación del giro por flexión cuando se dispone una configuración simétrica del laminado con fibras orientadas a $15^{\circ}$ y se aplica una carga normal a la directriz. Junto a ella se muestra la curva obtenida por Jung et al. [1], a la cual se ajusta extraordinariamente, y los valores experimentales que derivan de los ensayos realizados por Chandra y Chopra [2].

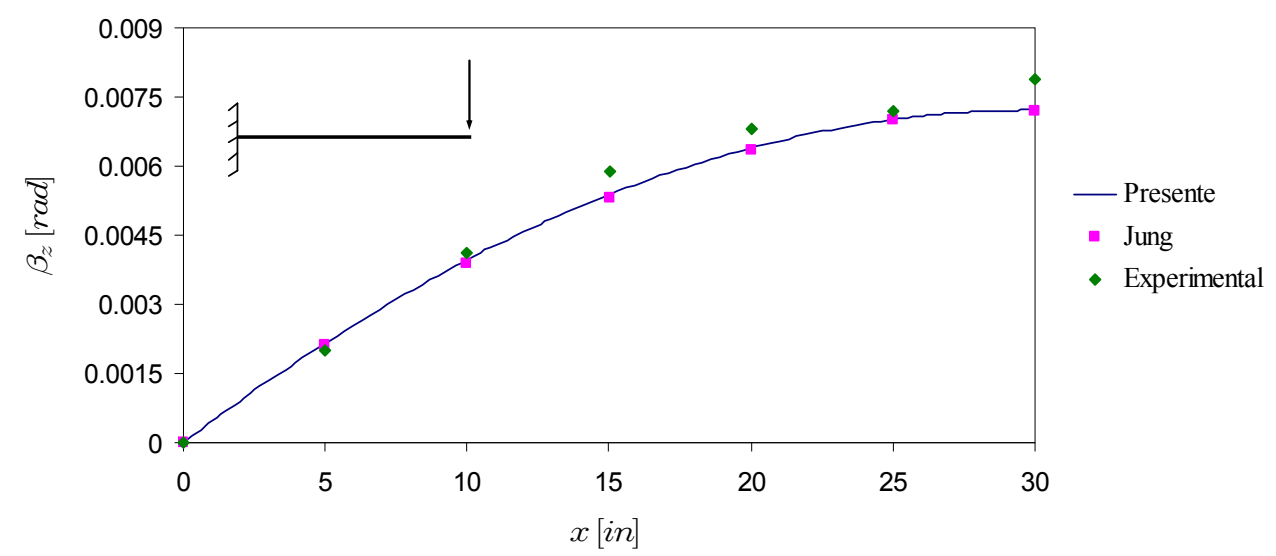

Figura A.14. Giros de flexión (doble T) 
Los giros por torsión se muestran en la Figura A.15. Se aprecia que los resultados coinciden con los deducidos por Jung et al. [1]. Sin embargo, el estudio experimental arroja valores algo mayores aunque en ambos casos siguen la misma evolución. En cuanto a las magnitudes, se tiene que el del giro inducido debido al acoplamiento flexión-torsión, originado por la orientación de las fibras y por el carácter simétrico de la configuración del laminado, es del orden de diez veces menor que en el problema de torsión.

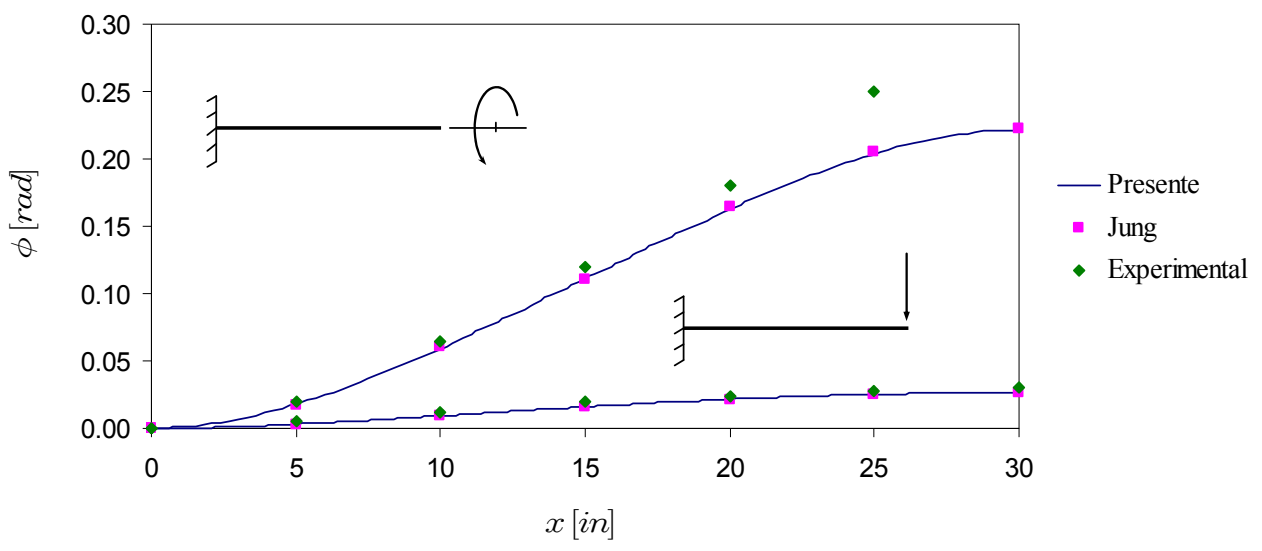

Figura A.15. Giros de torsión (doble T)

En la Figura A.16 y en la Figura A.17 se recogen la primera y la segunda derivada del giro inducido, respectivamente. En el problema de flexión, el alabeo crece rápidamente desde el empotramiento hasta la sección situada aproximadamente a un tercio de la longitud de la barra, para decrecer hasta anularse en el la sección exterior donde se han restringido los desplazamientos normales. Como es lógico, éstos son mucho mayores cuando la barra es sometida a torsión, resultando una ley simétrica como consecuencia de haber restringido los movimientos normales en el extremo no empotrado y de que la ley de giros presenta simetría puntual respecto a la sección central de la pieza.

Por su parte, en ambos casos, el bimomento decae desde el empotramiento hasta anularse en la sección donde el alabeo es máximo, con el consiguiente cambio de signo que afecta al resto de la pieza (Figura A.17). En consonancia con los resultados anteriores, la magnitud bimomento es menor en el problema de torsión inducida por flexión que en el de torsión, presentando simetría puntual en este caso la curva que lo define. El cambio de signo del mismo refleja un comportamiento cualitativo diferente respecto a la flexión local de las alas. 


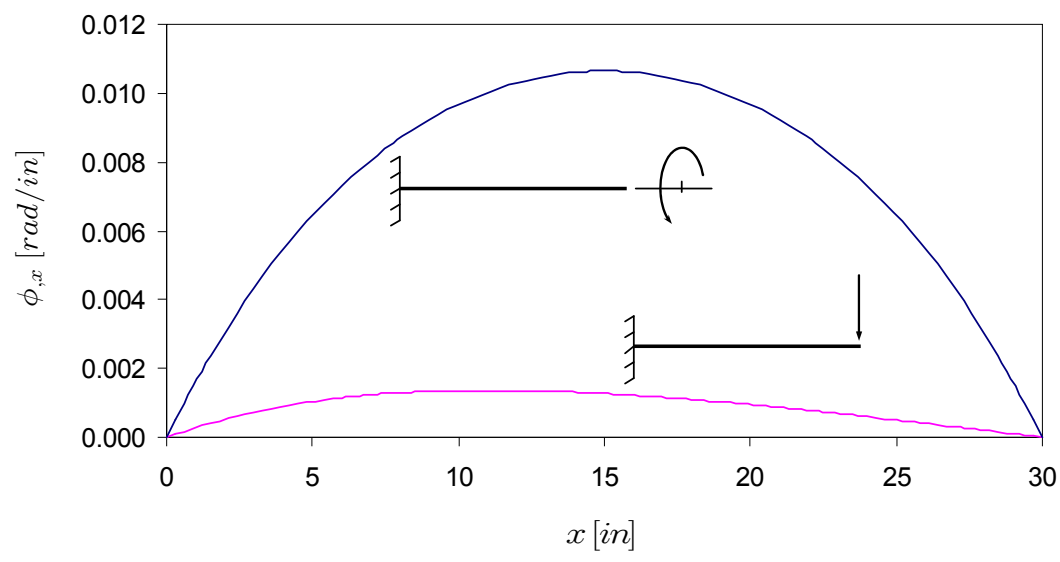

Figura A.16. Curvas de alabeo (doble T)

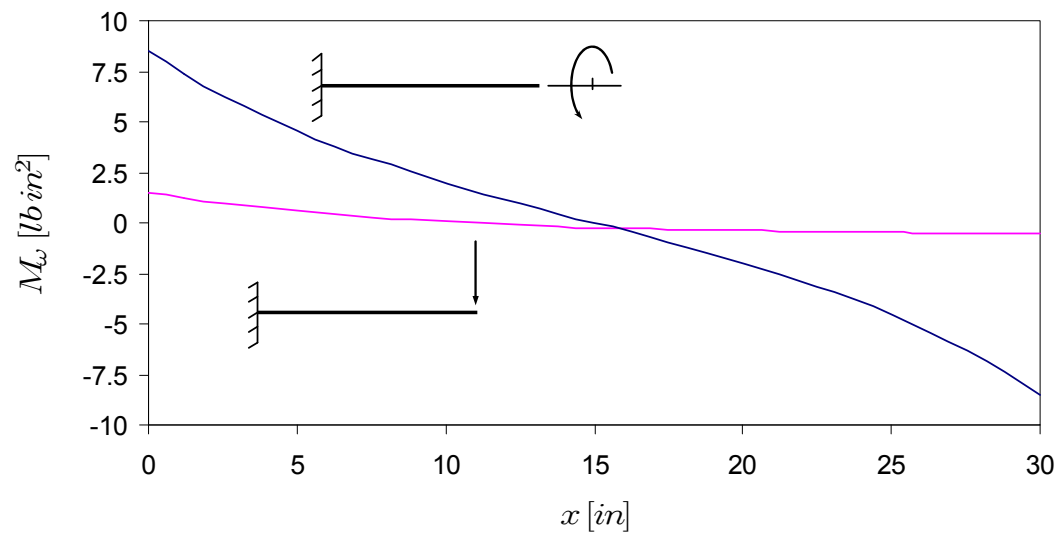

Figura A.17. Curvas de bimomento (doble T)

En la Figura A.18 se ha representado el valor del bimomento en el empotramiento para distintas orientaciones de las fibras del laminado cuando la barra está sometida a torsión y se permite el alabeo de la sección exterior. Se aprecia que la sección debe desarrollar mayores tensiones normales para hacer efectiva la restricción al alabeo cuando las fibras se orientan a $0^{\circ}$ y que el material debe esforzarse menos a medida que va aumentando la inclinación de las fibras. A $45^{\circ}$ se tiene un mínimo relativo y para inclinaciones mayores vuelve a crecer el bimomento. Esta conclusión es válida también cuando la configuración del laminado es antisimétrica.

A fin de conocer la influencia de la rigidez torsional en las variables de diseño de este tipo de barras, se ha estudiado también el caso en el que la sección de la barra se obtiene eliminando el ala inferior de la doble T (Figura A.13.b). En la Figura A.19 y en la Figura A.20 se muestran unas 
comparativas entre ambas secciones. En la primera se ha representado el giro por torsión para distintos ángulos de las fibras del laminado de las alas. Como puede apreciarse, en el perfil en T la torsión es prácticamente del tipo uniforme, mientras que para la doble T la torsión es del tipo no uniforme. Como consecuencia de ello, la ley de alabeo presenta la forma que se indica en la Figura A.20. Observando esta figura, junto con la anterior, se comprueba que el perfil en doble $\mathrm{T}$ trabaja mejor a torsión, pues tanto el giro como el alabeo son más pequeños.

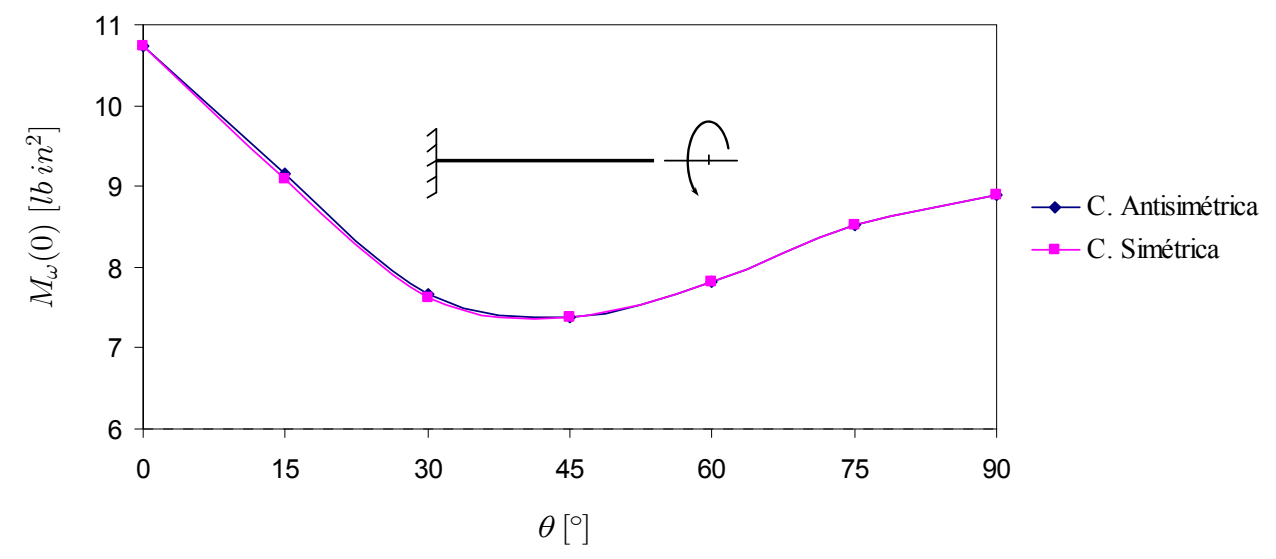

Figura A.18. Valor del bimomento en el empotramiento (doble T)

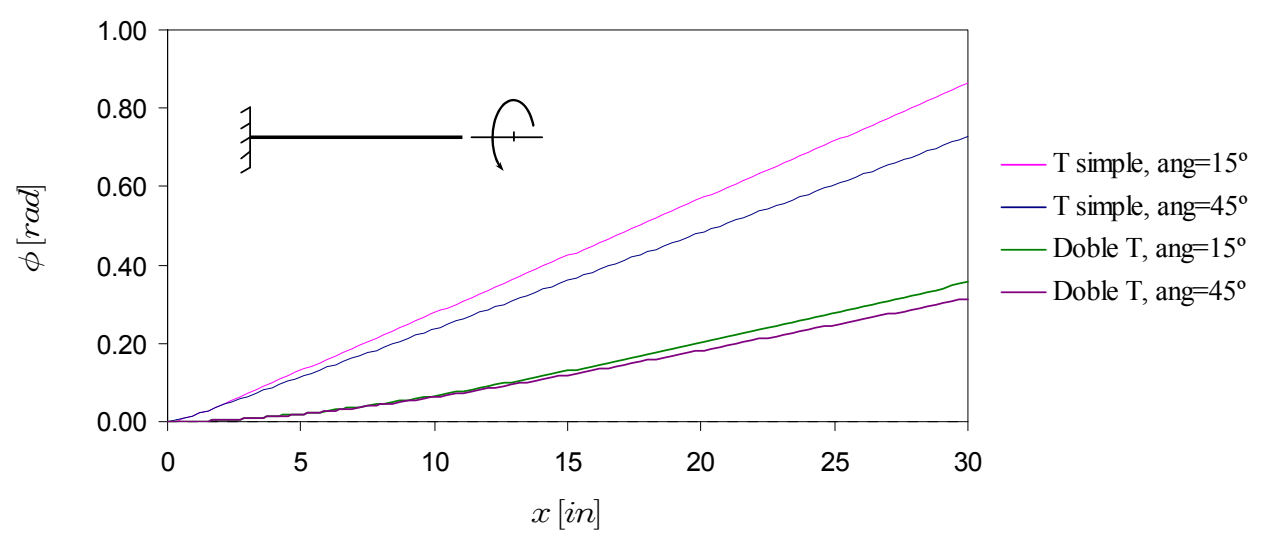

Figura A.19. Comparativa de giros por torsión

Por último, en la Figura A.21 y en la Figura A.22 se muestran, para el problema de torsión con los desplazamientos normales impedidos en la sección extrema libre y fibras orientadas a $15^{\circ}$, el alabeo y el bimomento de la barra con sección en $\mathrm{T}$, respectivamente. Al igual que sucede con la sección en doble T, la ley que define el alabeo es simétrica y la del bimomento presenta simetría puntal. 


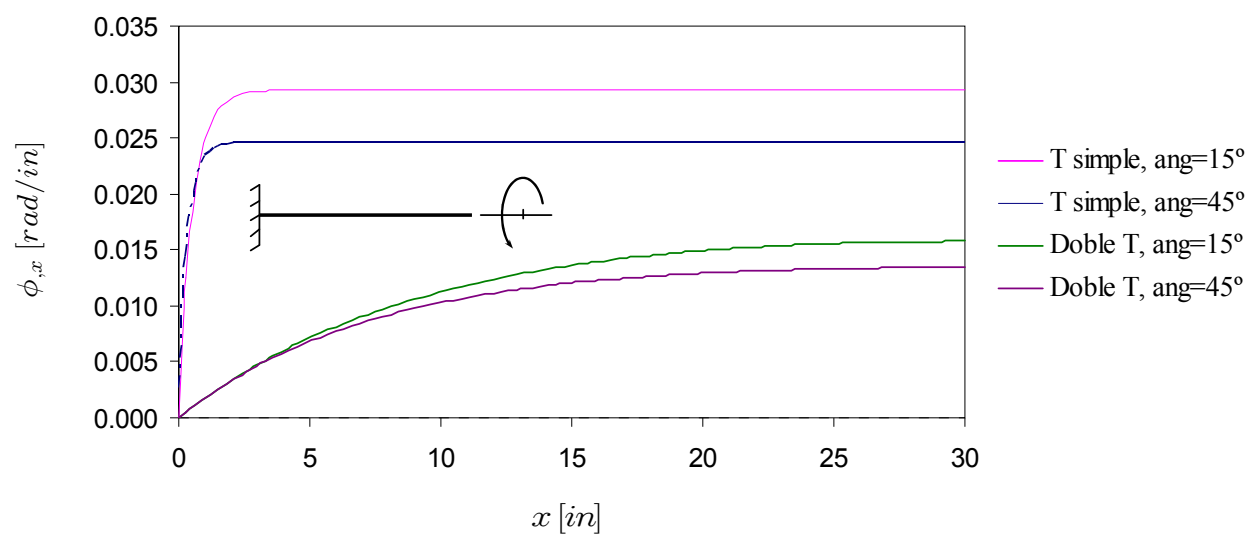

Figura A.20. Comparativa del alabeo

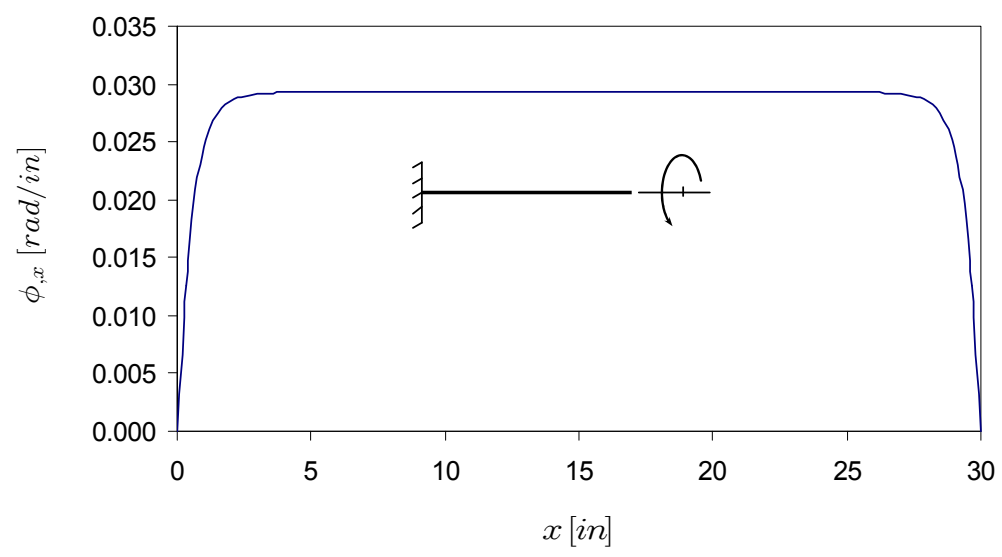

Figura A.21. Alabeo en el problema de torsión (sección en T)

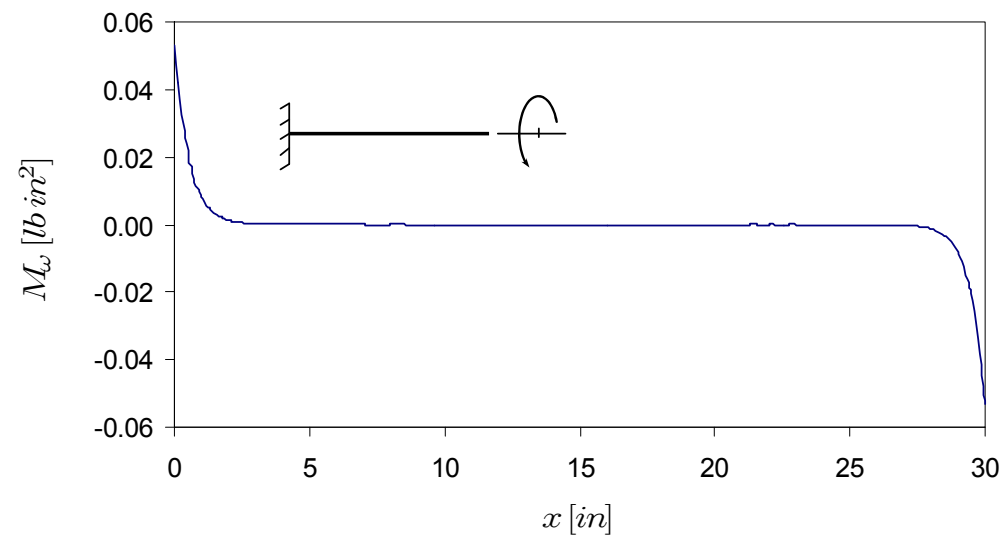

Figura A.22. Bimomento en el problema de torsión (sección en T) 


\section{G.2. Ejemplo 2. Sección en cajón}

Como segundo ejemplo, a continuación se estudia el comportamiento de una barra en voladizo con sección en cajón rectangular como la representada en la Figura A.14. Este tipo de sección también es ampliamente utilizada en la construcción de palas de helicópteros. El estudio se realiza tanto para la configuración simétrica (CAS, Figura A.23.a), en la cual existe un acoplamiento elástico flexión-torsión, como para la antisimétrica (CUS, Figura A.23.b), existiendo en este caso un acoplamiento elástico axil-torsión.

Los valores de las constantes elásticas del material (AS4/3501-6 grafito/epoxy) se especifican en la Tabla A.1. Las dimensiones de la sección son canto $h=13.64 \mathrm{~mm}(0,537 \mathrm{in})$, ancho $b=24.21 \mathrm{~mm}(0.953 \mathrm{in})$, espesor de lámina $e=0.127 \mathrm{~mm}(0.005 \mathrm{in})$ y longitud de la barra $L=762.12 \mathrm{~mm}(30 \mathrm{in})$.

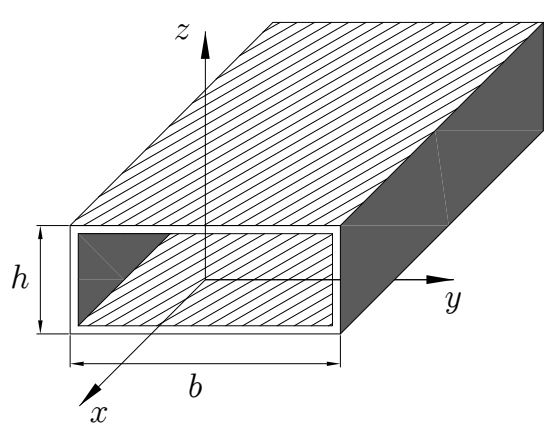

(a)

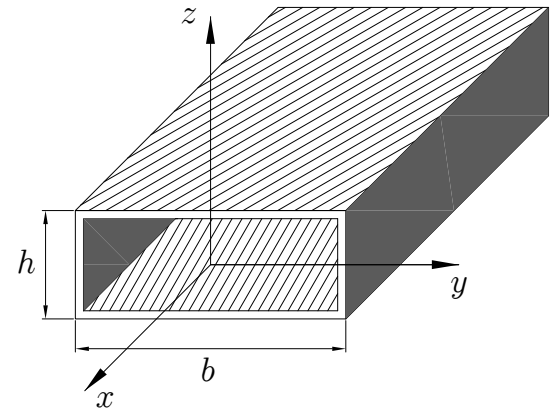

(b)

\begin{tabular}{|c|c|c|c|c|}
\hline \multicolumn{5}{|c|}{ Características del laminado } \\
\hline & \multicolumn{2}{|c|}{ Paredes horizontales } & \multicolumn{2}{c|}{ Paredes verticales } \\
\hline Configuración & Superior & Inferior & Izquierda & Derecha \\
\hline Simétrica & {$[15]_{6}$} & {$[15]_{6}$} & {$[15]_{6}$} & {$[15]_{6}$} \\
\hline Antisimétrica & {$[15]_{6}$} & {$[-15]_{6}$} & {$[15 /-15]_{6}$} & {$[-15 / 15]_{6}$} \\
\hline
\end{tabular}

Figura A.23. Secciones cajón correspondientes al Ejemplo 2

Con objeto de estudiar los acoplamientos elásticos se han estudiado tres estados simples de carga. En el primero de ellos se aplica una carga unitaria concentrada normal a la directriz de la barra en su extremo libre. En el segundo, la carga se aplica en la misma sección pero es paralela a dicha directriz. Por último, en el tercero se aplica un momento torsor, también de valor unidad y en el extremo libre de la barra. 


\section{G.2.1. Configuración antisimétrica (CUS)}

En la Figura A.24 se muestra la variación del giro por torsión cuando se aplica el momento torsor. Junto a ella se muestra la curva obtenida por Jung et al. [1], si bien se aprecia una pequeña discrepancia, esta puede ser debida a la diferente forma del cálculo del momento torsor de St. Venant. También se han representado los valores experimentales aportados por Chandra et al. [3]. La forma de la curva pone de manifiesto que la torsión es prácticamente del tipo uniforme.

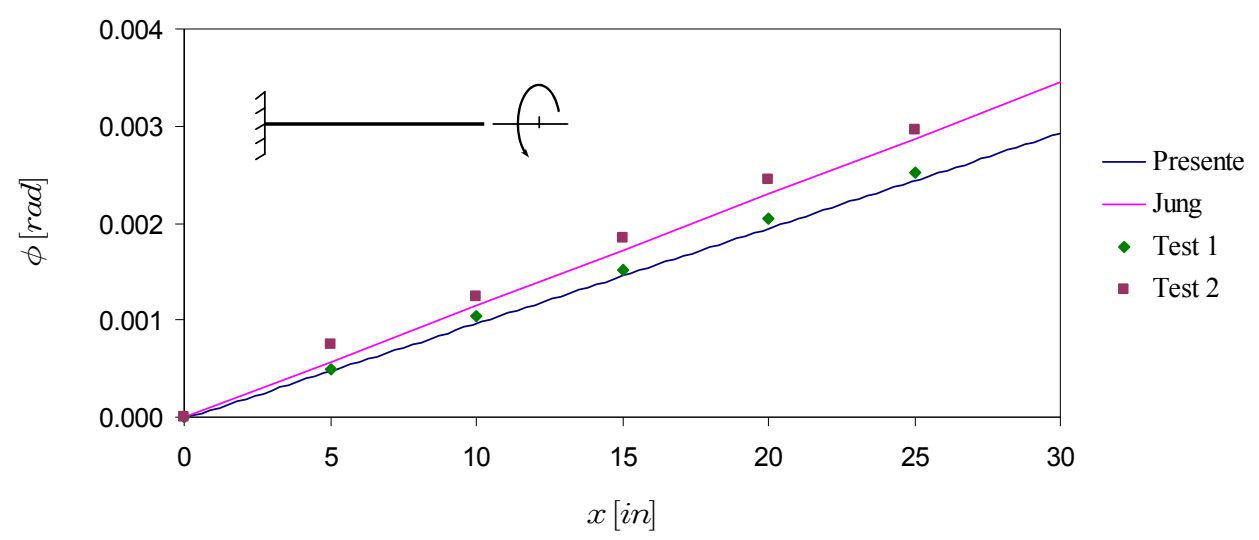

Figura A.24. Giro por torsión en el problema de torsión

El desplazamiento longitudinal originado como consecuencia de la carga de tracción aplicada en el extremo libre se representa en la Figura A.25, donde también se ha representado el desplazamiento longitudinal inducido por el acoplamiento elástico axil-torsión cuando se aplica el momento torsor. Como se aprecia, un torsor positivo produce acortamientos en la barra. Lógicamente, este resultado depende de la orientación de las fibras del laminado, llegando incluso a invertirse cuando se aumenta el ángulo del mismo en $90^{\circ}$. Además, en el caso que nos ocupa, el valor del acortamiento de la barra sometida a torsión es incluso cuatro veces mayor que el alargamiento producido por la carga de tracción.

En la Figura A.26 se muestra el giro inducido como consecuencia de los acoplamientos elásticos para el problema de tracción, así como también los resultados experimentales obtenidos por Chandra et al. [3] y la curva calculada a partir de la matriz de rigidez deducida por Jung et al. [1] para este mismo problema. Se vuelve a apreciar una pequeña diferencia entre los resultados. Asimismo, debido a la orientación del laminado que se ha utilizado, una carga de tracción produce giros negativos. 


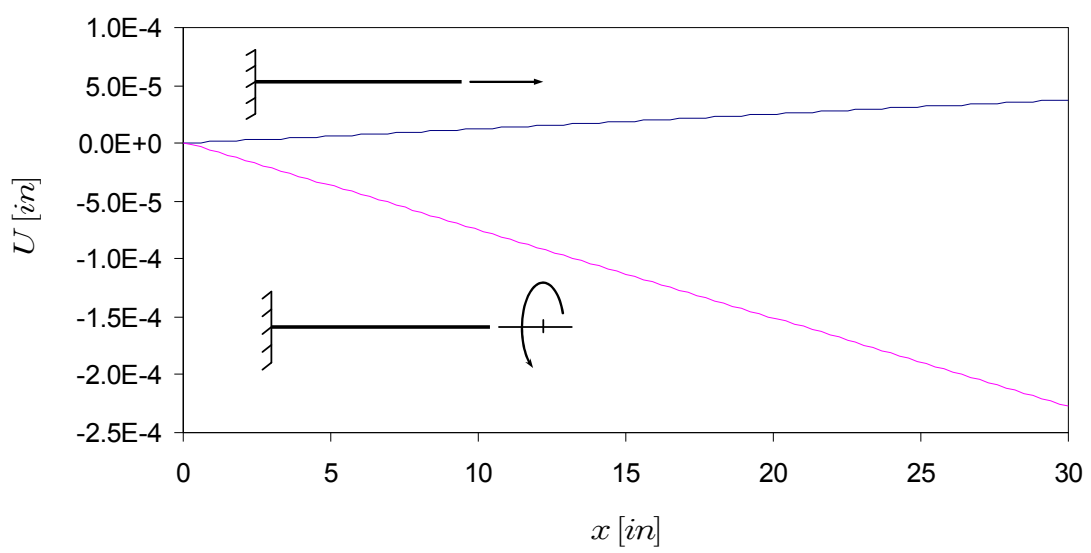

Figura A.25. Comparativa de desplazamientos longitudinales

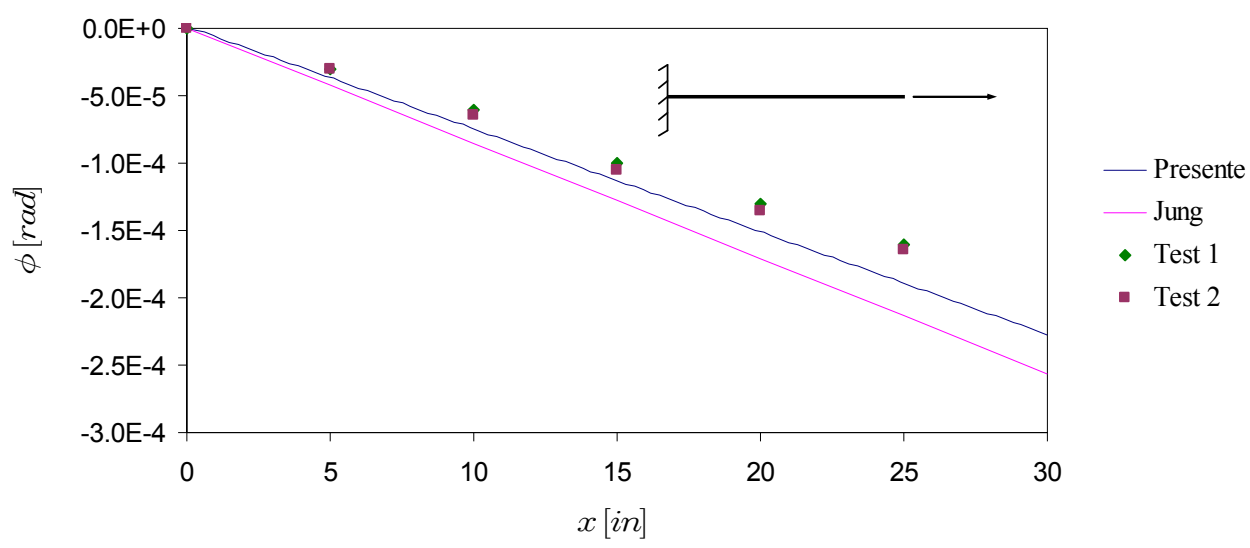

Figura A.26. Giro por torsión en el problema de tracción

Finalmente, en la Figura A.27 se representan los desplazamientos de alabeo para los problemas de torsión y de tracción. Dada la forma del giro alrededor del eje $O x$ de la barra, el alabeo es constante a partir de una sección muy próxima al empotramiento.

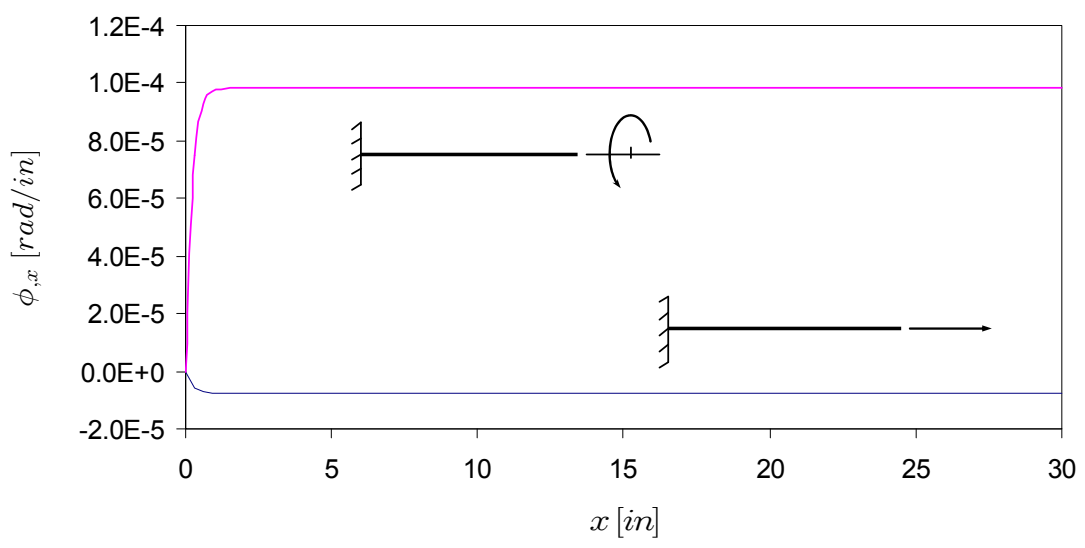

Figura A.27. Comparativa del alabeo 


\section{G.2.2. Configuración simétrica (CAS)}

En la Figura A.28 se muestra la variación del giro por flexión alrededor del eje $O z$, cuando la barra es sometida a una carga unitaria normal a la directriz en el extremo libre. Se ha representado también la curva obtenida por Jung et al. [1], así como los resultados derivados de los ensayos realizados por Chandra et al. [3]. La pequeña discrepancia existente entre los resultados puede ser debida a que en el presente análisis no se ha considerado la deformación por cortante.

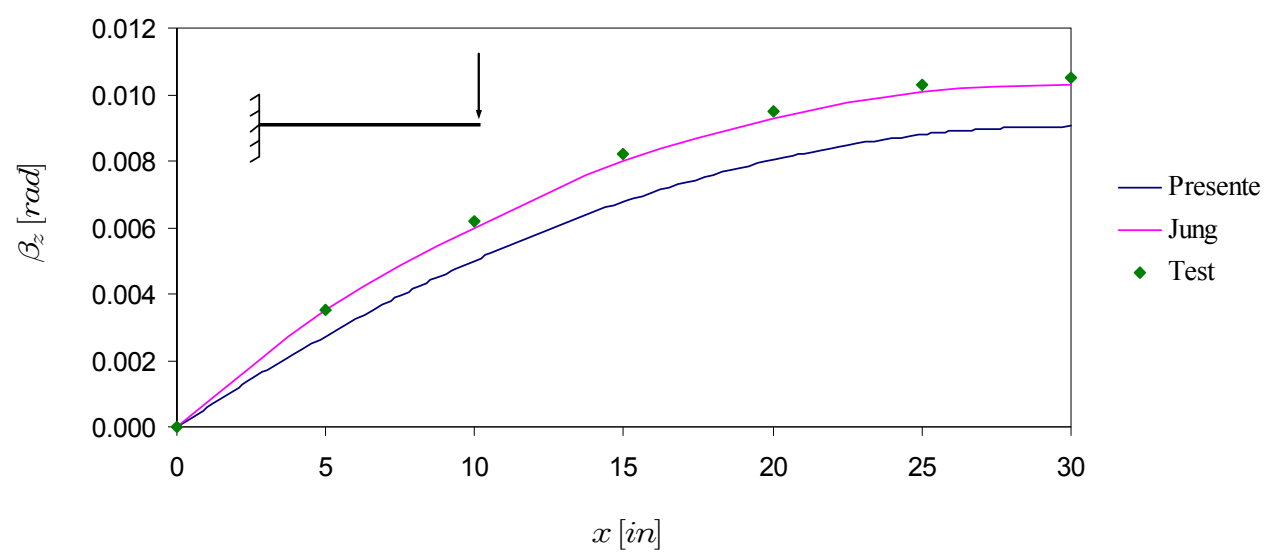

Figura A.28. Giro por flexión en el problema de flexión

Como consecuencia del acoplamiento elástico existente para esta configuración, se produce un giro inducido alrededor del eje $O x$, el cual se ha representado en la Figura A.29. Junto a la curva se ha representado la obtenida por Jung et al. [1] y los resultados derivados de los ensayos realizados por Chandra et al. [3], apreciándose una pequeña diferencia en los resultados, debido posiblemente a la diferencia en el cálculo del momento torsor uniforme.

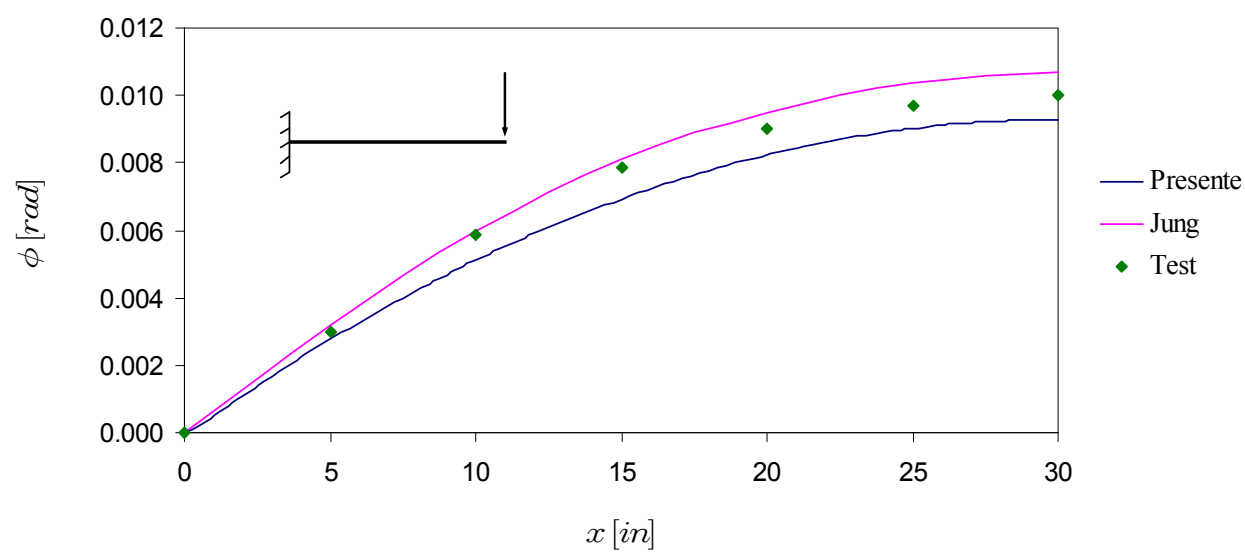

Figura A.29. Giro por torsión en el problema de flexión 
En la Figura A.30 se muestra el giro por torsión para el problema de torsión. Junto a la curva se han representado los resultados obtenidos experimentalmente por Chandra et al. [3], observándose una buena correlación entre los resultados. Se puede ver cómo la torsión es del tipo uniforme prácticamente.

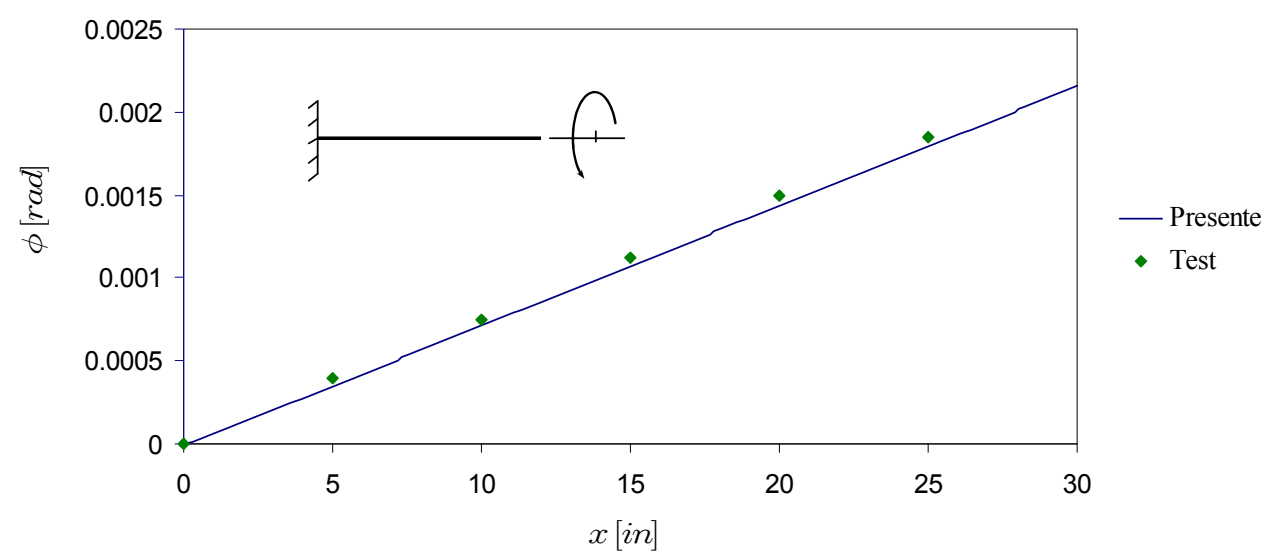

Figura A.30. Giro por torsión en el problema de torsión

Debido al acoplamiento elástico característico de este tipo de configuraciones, en el problema de torsión se ocasiona un giro inducido alrededor del eje $O z$. Esta ley de giros se muestra en la Figura A.31 así como también los resultados experimentales de Chandra et al. [3], poniéndose de manifiesto de nuevo una buena correlación entre los resultados.

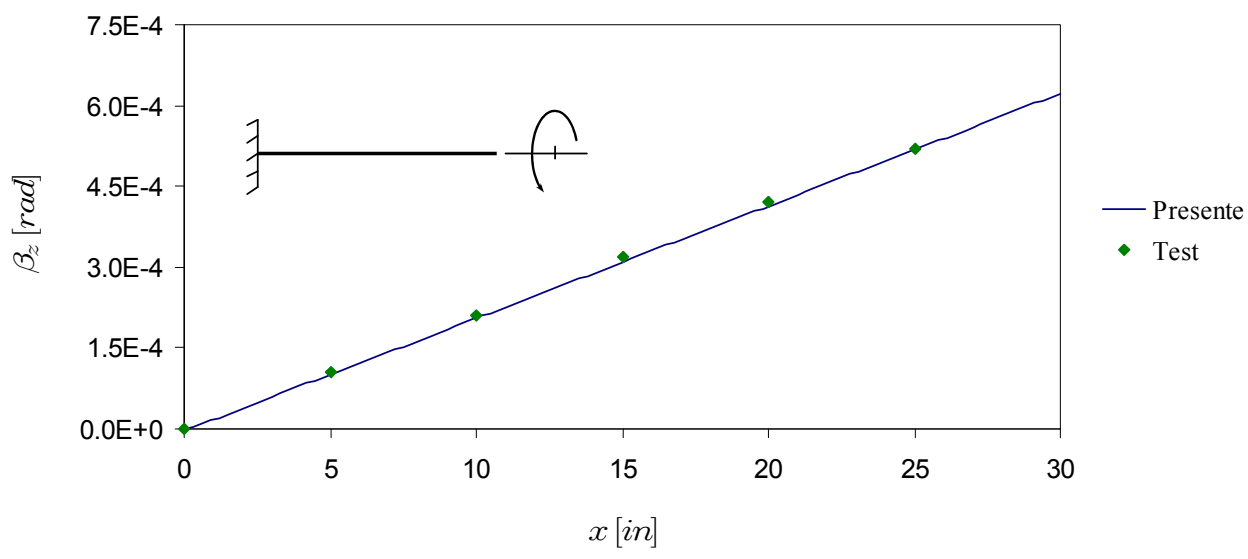

Figura A.31. Giro por flexión en el problema de torsión 
Para finalizar, en la Figura A.32 se ha representado el alabeo para los problemas de torsión y de flexión. El alabeo debido al momento torsor crece rápidamente para alcanzar un valor conste en una sección próxima al empotramiento, sin embargo, el alabeo inducido originado por la carga de flexión crece rápidamente hasta alcanzar su valor máximo (mayor que el debido al momento torsor) en las proximidades del empotramiento, para posteriormente decrecer hasta hacerse prácticamente nulo en el extremo libre.

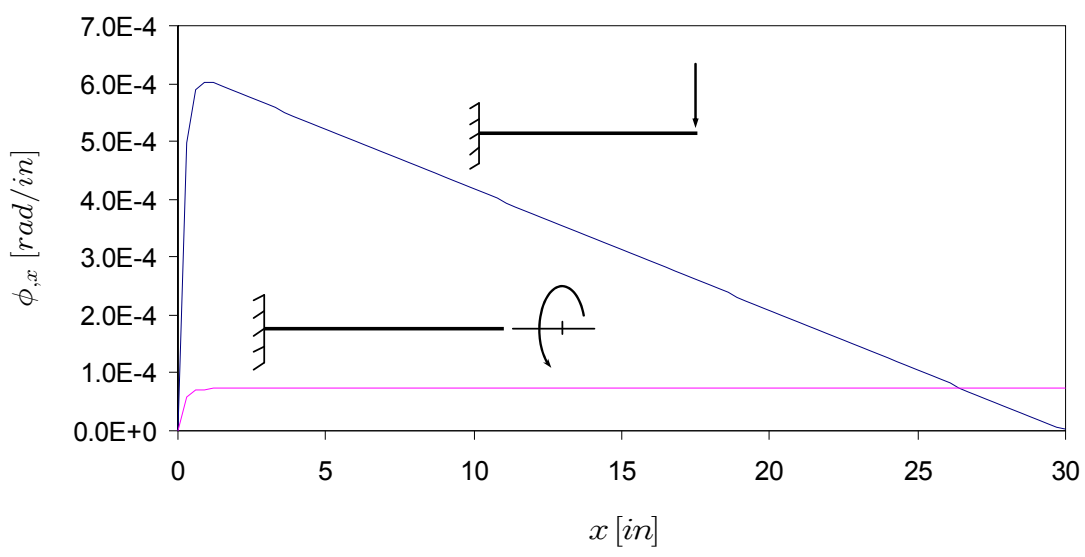

Figura A.32. Giro por flexión en el problema de torsión 


\section{Anexo H. Filosofía de implementación. Núcleo de resolución}

A continuación se muestra una breve descripción del papel de cada uno de los objetos que constituyen el núcleo de resolución.

\section{H.1. Esquema}

En la introducción del Capítulo 4 se explicó a grandes rasgos el problema y se hizo un breve comentario sobre los motivos que han llevado a un diseño como el que se expone a continuación. Se pretende describir el uso de la clase "esquema" aportando ejemplos para una mejor comprensión de la misma.

La discretización espacial de un determinado problema físico se considera realizada una vez que el dominio es discretizado utilizando una formulación de elementos finitos adecuada. En muchos casos la discretización del dominio del tiempo está claramente separada de la discretización del dominio espacial. En este planteamiento, el elemento describe el problema espacial y el esquema facilita la implementación del método de integración en el tiempo.

La mejor manera de describir el papel que desarrolla esta clase es a través de unos ejemplos, en concreto, la implementación de un esquema predictor-corrector estático y del esquema de Newmark.

\section{H.1.1. Esquema predictor-corrector estático}

En este caso el problema es puramente espacial. El esquema consigue de cada elemento la matriz de rigidez StiffnessMatrix, y el vector de residuos ResidualVector, y los devuelve sin modificarlos. Las funciones clave de este esquema son

- $\operatorname{Predict}(\ldots)$

$$
\mathbf{x}_{n+1}=\mathbf{x}_{n}
$$

- CalculateSystemContributions(...)

LHSContribution $=r$ CurrentElement $\rightarrow$ StiffnessMatrix $(. .$.

RHSContribution $=r$ CurrentElement $\rightarrow$ ResidualVector $(\ldots)$

- Update(...)

$$
\mathbf{x}_{n+1}^{i+1}=\mathbf{x}_{n+1}^{i}+d \mathbf{x} \quad(d \mathbf{x} \text { obtenido de la solución del sistema lineal })
$$


Surge la pregunta sobre "quién" pedirá al esquema estas contribuciones del sistema. Esto se mostrará mas adelante en la descripción del BuilderAndSolver.

\section{H.1.2. Esquema predictor-corrector de Newmark}

Este segundo caso es ligeramente más complejo. La dinámica se tiene en cuenta utilizando una aproximación implícita que conlleva una modificación tanto del término de la parte izquierda (LHS, Left Hand Side) como del término de la parte derecha (RHS, Right Hand Side) del problema. Incluso si el sistema se resuelve sólo para el campo de desplazamientos, la fase de actualización implica la modificación de su primera y segunda derivada en el tiempo. Por otro lado, no es necesario hacer cambios en el elemento, el cual puede proporcionar sin modificación alguna todos los términos demandados.

- $\operatorname{Predict}(. .$.

$$
\begin{aligned}
& \mathbf{x}_{n+1}=\mathbf{x}_{n}+\dot{\mathbf{x}}_{n} \Delta t \\
& \dot{\mathbf{x}}=\dot{\mathbf{x}}_{\text {old }} \\
& \ddot{\mathbf{x}}=\mathbf{0}
\end{aligned}
$$

- CalculateSystemContributions(...)

$$
\begin{aligned}
& \mathbf{K}=r \text { CurrentElement } \rightarrow \text { StiffnessMatrix }(\ldots) \\
& \mathbf{M}=\text { rCurrentElement } \rightarrow \text { MassMatrix }(\ldots) \\
& \mathbf{C}=r \text { CurrentElement } \rightarrow \text { DampMatrix }(\ldots) \\
& \mathbf{b}=r \text { CurrentElement } \rightarrow \text { ResidualVector }(\ldots) \\
& \text { LHSContribution }=\mathbf{K}+\frac{\gamma}{\beta \Delta t} \mathbf{C}+\frac{\gamma}{\beta \Delta t^{2}} \mathbf{M} \\
& \text { RHSContribution }=\mathbf{b}-\mathbf{M} \ddot{\mathbf{x}}-\mathbf{C} \dot{\mathbf{x}}
\end{aligned}
$$

- $\operatorname{Update}(. .$.

$$
\begin{aligned}
& \mathbf{x}_{n+1}^{i+1}=\mathbf{x}_{n+1}^{i}+d \mathbf{x} \\
& \dot{\mathbf{x}}_{n+1}^{i+1}=\frac{\gamma}{\beta \Delta t}\left(\mathbf{x}_{n+1}^{i+1}-\mathbf{x}_{n}\right)-\left(\frac{\gamma}{\beta}-1\right) \dot{\mathbf{x}}_{n}-\frac{\Delta t}{2}\left(\frac{\gamma}{\beta}-2\right) \ddot{\mathbf{x}}_{n} \\
& \ddot{\mathbf{x}}_{n+1}^{i+1}=\frac{\gamma}{\beta \Delta t^{2}}\left(\mathbf{x}_{n+1}^{i+1}-\mathbf{x}_{n}\right)-\frac{1}{\beta \Delta t} \dot{\mathbf{x}}_{n}-\left(\frac{\gamma}{2 \beta}-1\right) \ddot{\mathbf{x}}_{n}
\end{aligned}
$$

Se observa fácilmente que el mismo interfaz permite la descripción del comportamiento estático y dinámico, constituyendo la base de la definición de la clase esquema. 
Los ejemplos propuestos son simples pero dan una idea sobre la filosofía del planteamiento. El esquema constituye una "capa" entre la etapa de ensamblaje y el nivel elemental en el cual la dinámica está involucrada. Por lo tanto, el esquema debe combinar las contribuciones a nivel de elemento para permitir la aplicación de un método iterativo general. Los ejemplos mostrados proporcionan una idea de cómo pueden ser tratados problemas de mayor complejidad.

\section{H.2. Ensamblador-Solver (BuilderAndSolver)}

BuilderAndSolver es el objeto encargado de llevar a cabo todas las operaciones de ensamblaje así como la inversión del sistema lineal resultante. El hecho de agrupar conjuntamente la resolución y la etapa de ensamblaje se hizo con objeto de permitir, en un futuro, la paralelización del código, la cual debería implicar tanto la resolución del sistema lineal como la fase de ensamblaje.

Debido a sus características, BuilderAndSolver lleva asociadas las etapas de mayor coste computacional del conjunto del proceso de resolución. Esto claramente requiere ajustes a bajo nivel para asegurar un rendimiento alto. Un usuario no avanzado no necesita saber los detalles de la implementación de esta clase, sin embargo, es necesario que conozca el papel que juega este objeto dentro del código.

Es importante destacar que existen distintas posibilidades para llevar a cabo la etapa de ensamblaje. El punto crítico es que el mismo problema numérico se puede expresar en forma "predictor-corrector" usando un residuo, o en forma "total" usando un enfoque basado en la prescripción de las cargas externas. Cuando el problema se expresa en la forma predictor-corrector, las condiciones de Dirichlet se incluyen en el residuo implícitamente. Por otro lado, en el segundo caso el objeto BuilderAndSolver se encarga de las condiciones de Dirichlet sobre los nodos fijos. Los planteamientos ResidualBased proporcionan una "corrección" para una solución predicha y los ExtLoadBased devuelven la solución total. El convenio establecido para dar nombre a los objetos de esta clase tiene en cuenta esta diferencia.

Resulta útil aportar algunos ejemplos de implementaciones para mostrar algunas de las opciones disponibles en el código. En lo siguiente se considerará un sistema que tiene $n$ grados de libertad libres y $N$ grados de libertad totales. 


\section{H.2.1. ResidualBasedElminationBuilderAndSolver}

Este objeto está diseñado para resolver problemas de la forma

$$
\left[\begin{array}{cc}
{[\mathbf{K}]} & {\left[\mathbf{K}_{\mathrm{d}}\right]} \\
{\left[\mathbf{K}_{1}\right]} & {\left[\mathbf{K}_{2}\right]}
\end{array}\right]\left\{\begin{array}{c}
\{\Delta \mathbf{x}\} \\
\{\mathbf{0}\}
\end{array}\right\}=\left\{\begin{array}{c}
\{\mathbf{b}\} \\
\{-\mathbf{R}\}
\end{array}\right\}
$$

El sistema lineal se resuelve obteniéndose una corrección que es aplicada en la etapa de predicción. Notar que esta corrección es 0 en los nodos donde la solución está prescrita como una condición de Dirichlet.

A continuación se muestra una descripción "simbólica" de las operaciones realizadas por las funciones públicas

- BuildLHS(...) devuelve

$$
[\mathbf{A}]=[[\mathbf{K}]]_{n \times n}
$$

- BuildLHS-CompleteOnFreeRows(...) devuelve la matriz rectangular

$$
[\mathbf{A}]=\left[\begin{array}{ll}
{[\mathbf{K}]} & {\left[\mathbf{K}_{\mathrm{d}}\right]}
\end{array}\right]_{n \times N}
$$

- BuildLHS-Complete(...) devuelve la matriz singular

$$
[\mathbf{A}]=\left[\begin{array}{cc}
{[\mathbf{K}]} & {\left[\mathbf{K}_{d}\right]} \\
{\left[\mathbf{K}_{1}\right]} & {\left[\mathbf{K}_{2}\right]}
\end{array}\right]_{N \times N}
$$

- ApplyDirichletConditions() no hace nada, las condiciones de Dirichlet se tienen en cuenta implícitamente en el residuo

- BuildRHS() ensambla el vector $\{\mathbf{b}\}$ a partir de las contribuciones elementales

- CalculateReactions() calcula el vector de reacciones $\{-\mathbf{R}\}$ ensamblando las contribuciones elementales

En este caso, las condiciones de contorno se imponen eliminando (en realidad no ensamblando) los grados de libertad fijos, que es posiblemente la estrategia más eficiente para problemas incrementales (ResidualBased).

\section{H.2.2. ExtLoadBasedEIminationBuilderAndSolver}

Este objeto realiza las operaciones de ensamblaje y de resolución dado el vector de cargas externas. En este caso, el sistema lineal se resuelve obteniéndose la solución "total" en lugar de una corrección como en el caso anterior.

En este caso el problema es de la siguiente forma 


$$
\left[\begin{array}{cc}
{[\mathbf{K}]} & {\left[\mathbf{K}_{\mathrm{d}}\right]} \\
{\left[\mathbf{K}_{1}\right]} & {\left[\mathbf{K}_{2}\right]}
\end{array}\right]\left\{\begin{array}{c}
\{\mathbf{x}\} \\
\left\{\mathbf{x}_{\text {prescr }}\right\}
\end{array}\right\}=\left\{\begin{array}{c}
\left\{\mathbf{f}_{\text {ext }}\right\} \\
\{-\mathbf{R}\}
\end{array}\right\}
$$

Las operaciones realizadas por las funciones principales son

- BuildLHS(...) devuelve

$$
[\mathbf{A}]=[[\mathbf{K}]]_{n \times n}
$$

y construye dos matrices auxiliares

$$
\begin{gathered}
{\left[\mathbf{K}_{\mathrm{d}}\right]=\left[\left[\mathbf{K}_{d}\right]\right]_{n \times(N-n)}} \\
{[\mathbf{B}]=\left[\begin{array}{ll}
{\left[\mathbf{K}_{1}\right]} & \left.\left[\mathbf{K}_{2}\right]\right]_{(\mathrm{N}-n) \times N}
\end{array}\right.}
\end{gathered}
$$

las cuales se almacenan internamente y se utilizarán mientras se aplican las condiciones de contorno. La matriz $[\mathbf{B}]$ sólo se calcula si se requiere el cálculo de las reacciones.

- BuildLHS-CompleteOnFreeRows(...) devuelve la matriz rectangular

$$
[\mathbf{A}]=\left[\begin{array}{ll}
{[\mathbf{K}]} & {\left[\mathbf{K}_{\mathrm{d}}\right]}
\end{array}\right]_{n \times N}
$$

- BuildLHS-Complete(...) devuelve la matriz singular

$$
[\mathbf{A}]=\left[\begin{array}{cc}
{[\mathbf{K}]} & {\left[\mathbf{K}_{\mathrm{d}}\right]} \\
{\left[\mathbf{K}_{1}\right]} & {\left[\mathbf{K}_{2}\right]}
\end{array}\right]_{N \times N}
$$

- ApplyDirichletConditions() esta operación asume que la matriz auxiliar $\left[\mathbf{K}_{\mathrm{d}}\right]$ ha sido construida antes (de forma coherente con la matriz LHS) y devuelve

$$
\{\mathbf{b}\}=\left\{\mathbf{f}_{\text {ext }}\right\}-\left[\mathbf{K}_{d}\right]\left\{\mathbf{x}_{\text {prescr }}\right\}
$$

- BuildRHS() ensambla el vector $\left\{\mathbf{f}_{\text {ext }}\right\}$

- CalculateReactions() calcula el vector de reacciones haciendo

$$
\{\mathbf{R}\}=[\mathbf{B}]\left\{\begin{array}{c}
\{\mathbf{x}\} \\
\left\{\mathbf{x}_{\text {prescr }}\right\}
\end{array}\right\}
$$

Este planteamiento es muy eficiente, no obstante, al hacer uso de un cierto almacenamiento interno resulta menos flexible que el anterior. Por otro lado, este planteamiento representa una buena opción para problemas lineales, ya que permite imponer las condiciones fijas sobre el término RHS de un modo muy eficiente (sin tener que volver a ensamblar la matriz del sistema).

\section{H.2.3. ExtLoadBasedDiagonalSpringBuilderAndSolver}

También son posibles otros planteamientos para problema descrito en el punto anterior, en particular, resulta interesante evitar el almacenamiento interno. Una posible solución (menos precisa, pero eficaz) viene dada por el objeto ExtLoadBasedDiagonalSpring-BuilderAndSolver, el 
cual está basado en la idea de aplicar muelles equivalentes a los nodos fijos. La ventaja de este planteamiento radica en la posibilidad de manejar los contornos fijos sin requerir ningún tipo almacenamiento interno. Esto supone una pérdida de exactitud en la solución del sistema lineal y un peor acondicionamiento de la matriz del sistema.

En este caso, la forma asumida para el problema es

$$
\left[\begin{array}{cc}
{[\mathbf{K}]} & {\left[\mathbf{K}_{\mathrm{d}}\right]} \\
{\left[\mathbf{K}_{1}\right]} & {\left[\mathbf{K}_{2}\right]}
\end{array}\right]\left\{\begin{array}{c}
\{\mathbf{X}\} \\
\left\{\mathbf{K}_{\text {prescr }}\right\}
\end{array}\right\}=\left\{\begin{array}{c}
\left\{\mathbf{f}_{\text {ext }}\right\} \\
\{-\mathbf{R}\}
\end{array}\right\}
$$

Las operaciones realizadas por las funciones principales son

- BuildLHS(...) de vuelve la matriz singular

$$
[\mathbf{A}]=\left[\begin{array}{cc}
{[\mathbf{K}]} & {\left[\mathbf{K}_{\mathrm{d}}\right]} \\
{\left[\mathbf{K}_{1}\right]} & {\left[\mathbf{K}_{2}\right]}
\end{array}\right]_{N \times N}
$$

- BuildLHS-CompleteOnFreeRows(...) devuelve la matriz rectangular

$$
[\mathbf{A}]=\left[[\mathbf{K}] \quad\left[\mathbf{K}_{d}\right]\right]_{n \times N}
$$

- BuildLHS-Complete(...) funciona exactamente igual que la función BuildLHS(...)

- ApplyDirichletConditions() esta función modifica los términos LHS y RHS añadiendo un "pivote diagonal" a la diagonal (sobre los nodos fijos) y sustituyendo la correspondiente posición del término RHS con el valor fijo del grado de libertad.

- BuildRHS() ensambla el vector $\left\{\begin{array}{ll}\left\{\mathbf{f}_{\text {ext }}\right\} & \left\{\mathbf{f}_{\text {fijo }}\right\}\end{array}\right\}^{T}$

- CalculateReactions() calcula el vector de reacciones multiplicando la matriz del sistema por el vector solución

$$
\left\{\begin{array}{c}
\{\mathbf{0}\} \\
\{-\mathbf{R}\}
\end{array}\right\}=\left[\begin{array}{cc}
{[\mathbf{K}]} & {\left[\mathbf{K}_{\mathrm{d}}\right]} \\
{\left[\mathbf{K}_{1}\right]} & {\left[\mathbf{K}_{2}\right]}
\end{array}\right]\left\{\begin{array}{c}
\{\mathbf{x}\} \\
\left\{\mathbf{x}_{\text {prescr }}\right\}
\end{array}\right\}
$$

Este método resulta muy simple pero el tamaño del sistema que debe ser invertido es mayor que en los otros casos.

\section{H.3. La "estrategia"}

La estrategia es el objeto en el que se implementa el "orden de las llamadas" a las diferentes etapas de la resolución del problema. Todo el sistema de matrices y vectores debe ser almacenado en la estrategia, lo cual permite tratar con múltiples términos LHS y RHS. Ejemplos triviales de estrategias son la "estrategia lineal" y la "estrategia de Newton Raphson". En problemas lineales la implementación depende del método usado en el ensamblaje (ResidualBased o ExtLoadBased), y en 
problemas no lineales el planteamiento se basa en el residuo. Los puntos claves en el diseño de la estrategia son la flexibilidad y la capacidad de reutilización.

El interfaz básico, disponible directamente desde el archivo de entrada, viene dado por

- $\operatorname{Predict}(\ldots)$

- Solve(...)

- IsConverged(...)

- CalculateOutputData(...)

A continuación se muestran algunas funciones

- SetEchoLevel(int level) para fijar el comando "echo" facilitado por la estrategia

- $\quad$ nivel $=0$ : en silencio...

- $\quad$ nivel $=1$ : impresión del tiempo e informaciones básicas

- $\quad$ nivel $=2$ : impresión de datos del solver lineal

- $\quad$ nivel $=3$ : impresión de informaciones de depuración

- SetRebuildLevel(int level) este flag proporciona información sobre la frecuencia con la que la matriz del sistema tiene que ser ensamblada

- $\quad$ nivel $=0$ : construir la matriz de rigidez una vez

- $\quad$ nivel $=1$ : construir la matriz de rigidez al comienzo de cada paso de resolución

- $\quad$ nivel $=2$ : construir la matriz de rigidez en cada iteración

- SetMoveMeshFlag(bool flag) le dice a la estrategia si es necesario o no actualizar la posición de los nodos de la malla. Se debe fijar en 1 para problemas estructurales o de movimiento de malla, y en 0 para otros casos.

- SetBuilderAndSolver(Pointer NewBuilderAndSolver) permite modificar el ensamblador y el solver (para usuarios avanzados)

Este interfaz básico, complementado con otras funciones que se omiten aquí por brevedad, puede ser usado como componente básico en el desarrollo de métodos de paso fraccionado. Esto se puede realizar directamente desde el archivo de entrada definiendo adecuadamente las estrategias de resolución idóneas para cada una de las etapas, combinando, por ejemplo, fases de resolución lineales y no-lineales.

La implementación de las estrategias de acoplamiento depende en gran medida de este planteamiento. 



\section{Anexo I. Estudio de la malla}

Respecto al ejemplo de la chimenea de $90 \mathrm{~m}$ estudiado en el Capítulo 7, a continuación se estudian otras mallas para el dominio del fluido.

En la Figura A.33 se muestran las características de la geometría y del mallado de uno de los planos de fluido considerados. Se suponen las mismas condiciones de contorno, tanto para la estructura como para el fluido, que las utilizadas en el ejemplo de la chimenea. La malla de la estructura también es la misma, siendo el coeficiente de proporcionalidad del amortiguamiento estructural 0.75 . Respecto al perfil de velocidades de entrada del viento, se ha supuesto el mismo que el indicado en el ejemplo que nos ocupa.
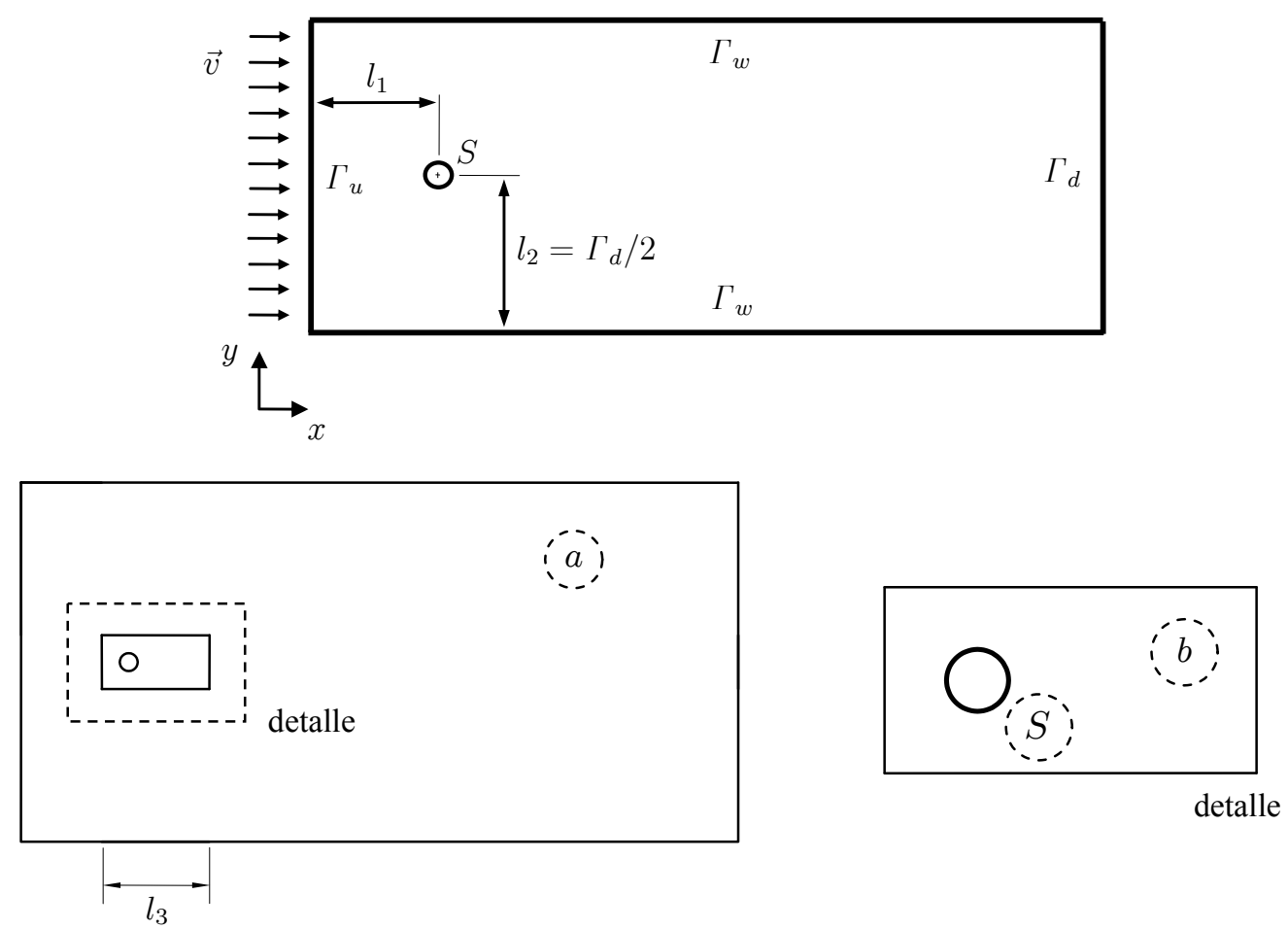

Figura A.33. Detalle de la geometría y del mallado de un plano de fluido

En las figuras que se muestran a continuación se ha representado la variación en el tiempo de los coeficientes de arrastre y de sustentación para la sección de la chimenea situada a $90 \mathrm{~m}$ de altura y con diámetro de $2.2 \mathrm{~m}$ así como también el análisis FFT de la oscilación del coeficiente de sustentación. El número de Reynolds en este caso es $1.84 \cdot 10^{6}$. 
Malla 1. 9348 elementos

- Sección de diámetro $2.2 \mathrm{~m}$

\begin{tabular}{|c|c|c|c|}
\hline \multicolumn{2}{|c|}{ Longitud $[m]$} & \multicolumn{2}{c|}{ Tamaño característico $[m]$} \\
\hline$\Gamma_{w}$ & 88 & $a$ & 2.20 \\
\hline$\Gamma_{d}$ & 44 & $b$ & 1.32 \\
\hline$l_{1}=l_{3}$ & 13.2 & $S$ & 0.60 \\
\hline
\end{tabular}

- Sección de diámetro $4.1 \mathrm{~m}$

\begin{tabular}{|c|c|c|c|}
\hline \multicolumn{2}{|c|}{ Longitud $[m]$} & \multicolumn{2}{c|}{ Tamaño característico $[m]$} \\
\hline$\Gamma_{w}$ & 164 & $a$ & 4.10 \\
\hline$\Gamma_{d}$ & 82 & $b$ & 2.46 \\
\hline$l_{1}=l_{3}$ & 24.6 & $S$ & 1.00 \\
\hline
\end{tabular}

Tabla A.2. Características geométricas y del mallado de los planos de fluido para la malla 1

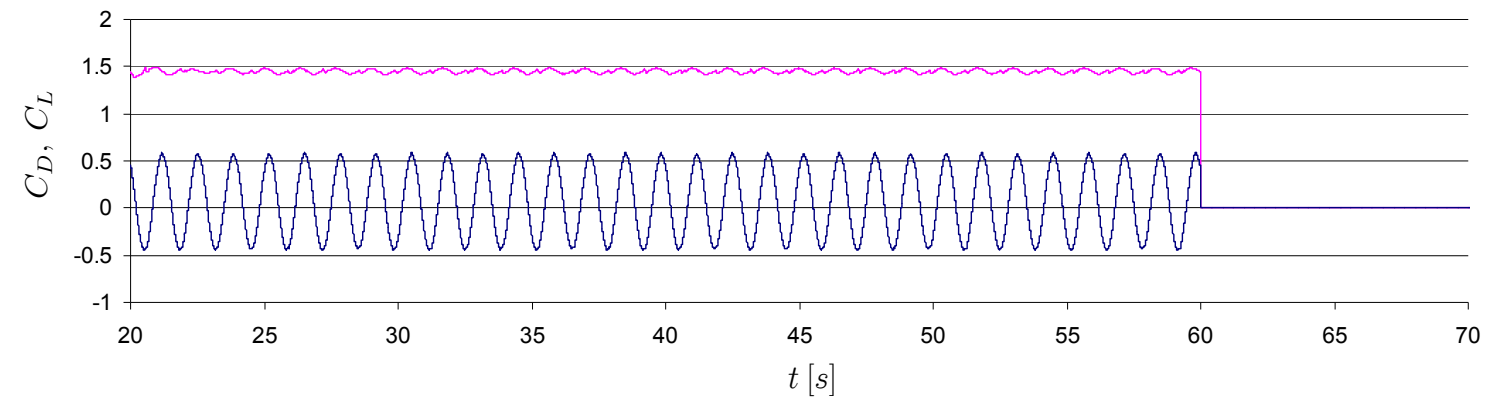

Figura A.34. Oscilación de los coeficientes de arrastre y de sustentación para la malla 1

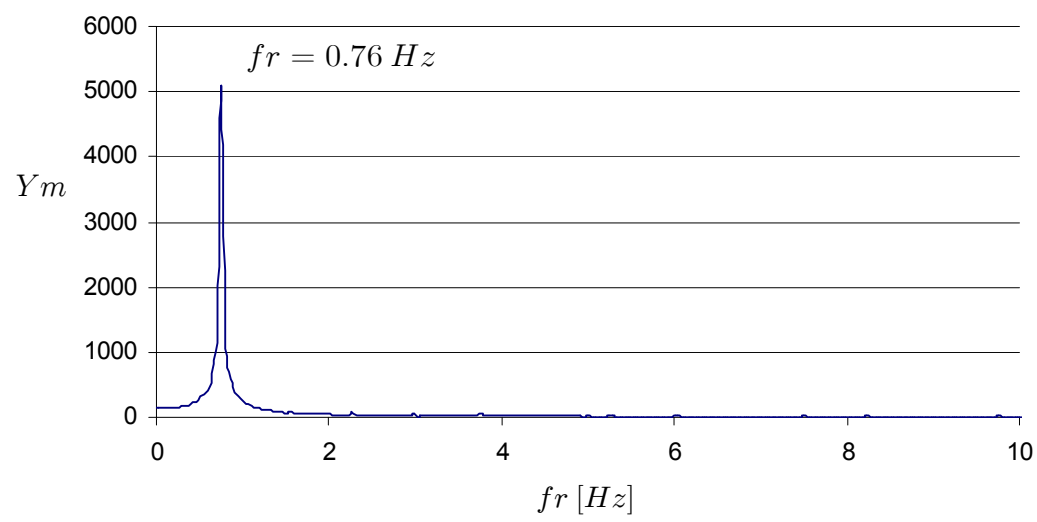

Figura A.35. Análisis FFT de la oscilación del coeficiente de sustentación para la malla 1 
Malla 2. 12792 elementos

- Sección de diámetro $2.2 \mathrm{~m}$

\begin{tabular}{|c|c|c|c|}
\hline \multicolumn{2}{|c|}{ Longitud $[m]$} & \multicolumn{2}{c|}{ Tamaño característico $[m]$} \\
\hline$\Gamma_{w}$ & 88 & $a$ & 2.20 \\
\hline$\Gamma_{d}$ & 44 & $b$ & 0.88 \\
\hline$l_{1}=l_{3}$ & 13.2 & $S$ & 0.38 \\
\hline
\end{tabular}

- Sección de diámetro $4.1 \mathrm{~m}$

\begin{tabular}{|c|c|c|c|}
\hline \multicolumn{2}{|c|}{ Longitud $[m]$} & \multicolumn{2}{c|}{ Tamaño característico $[m]$} \\
\hline$\Gamma_{w}$ & 164 & $a$ & 4.10 \\
\hline$\Gamma_{d}$ & 82 & $b$ & 1.64 \\
\hline$l_{1}=l_{3}$ & 24.6 & $S$ & 0.72 \\
\hline
\end{tabular}

Tabla A.3. Características geométricas y del mallado de los planos de fluido para la malla 2

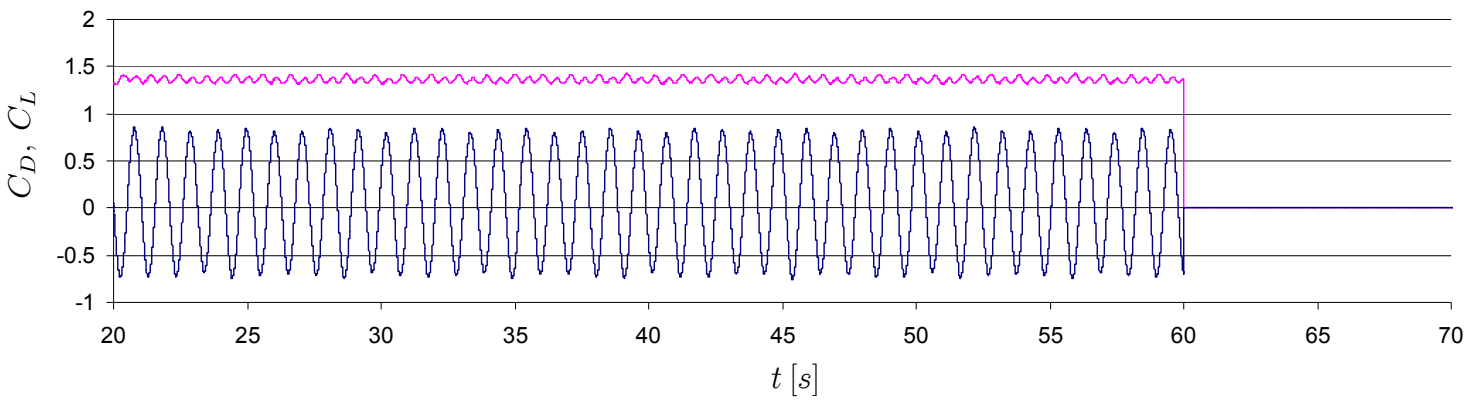

Figura A.36. Oscilación de los coeficientes de arrastre y de sustentación para la malla 2

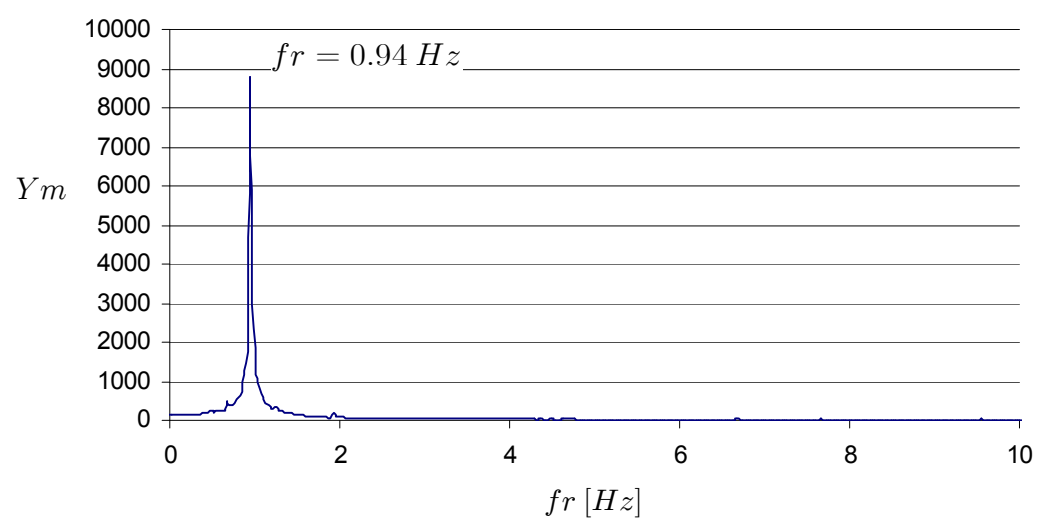

Figura A.37. Análisis FFT de la oscilación del coeficiente de sustentación para la malla 2
- Sección de diámetro $3.1 \mathrm{~m}$

\begin{tabular}{|c|c|c|c|}
\hline \multicolumn{2}{|c|}{ Longitud $[m]$} & \multicolumn{2}{c|}{ Tamaño característico $[m]$} \\
\hline$\Gamma_{w}$ & 128 & $a$ & 3.20 \\
\hline$\Gamma_{d}$ & 64 & $b$ & 1.28 \\
\hline$l_{1}=l_{3}$ & 19.2 & $S$ & 0.56 \\
\hline
\end{tabular}

- Sección de diámetro $5.2 \mathrm{~m}$

\begin{tabular}{|c|c|c|c|}
\hline \multicolumn{2}{|c|}{ Longitud $[m]$} & \multicolumn{2}{c|}{ Tamaño característico $[m]$} \\
\hline$\Gamma_{w}$ & 208 & $a$ & 5.20 \\
\hline$\Gamma_{d}$ & 104 & $b$ & 2.08 \\
\hline$l_{1}=l_{3}$ & 31.2 & $S$ & 0.91 \\
\hline
\end{tabular}


Malla 3. 22236 elementos

- Sección de diámetro $2.2 \mathrm{~m}$

\begin{tabular}{|c|c|c|c|}
\hline \multicolumn{2}{|c|}{ Longitud $[m]$} & \multicolumn{2}{c|}{ Tamaño característico $[m]$} \\
\hline$\Gamma_{w}$ & 88 & $a$ & 2.20 \\
\hline$\Gamma_{d}$ & 44 & $b$ & 0.44 \\
\hline$l_{1}=l_{3}$ & 13.2 & $S$ & 0.38 \\
\hline
\end{tabular}

- Sección de diámetro $4.1 \mathrm{~m}$

\begin{tabular}{|c|c|c|c|}
\hline \multicolumn{2}{|c|}{ Longitud $[m]$} & \multicolumn{2}{c|}{ Tamaño característico $[m]$} \\
\hline$\Gamma_{w}$ & 164 & $a$ & 4.10 \\
\hline$\Gamma_{d}$ & 82 & $b$ & 0.82 \\
\hline$l_{1}=l_{3}$ & 24.6 & $S$ & 0.72 \\
\hline
\end{tabular}

Tabla A.4. Características geométricas y del mallado de los planos de fluido para la malla 3

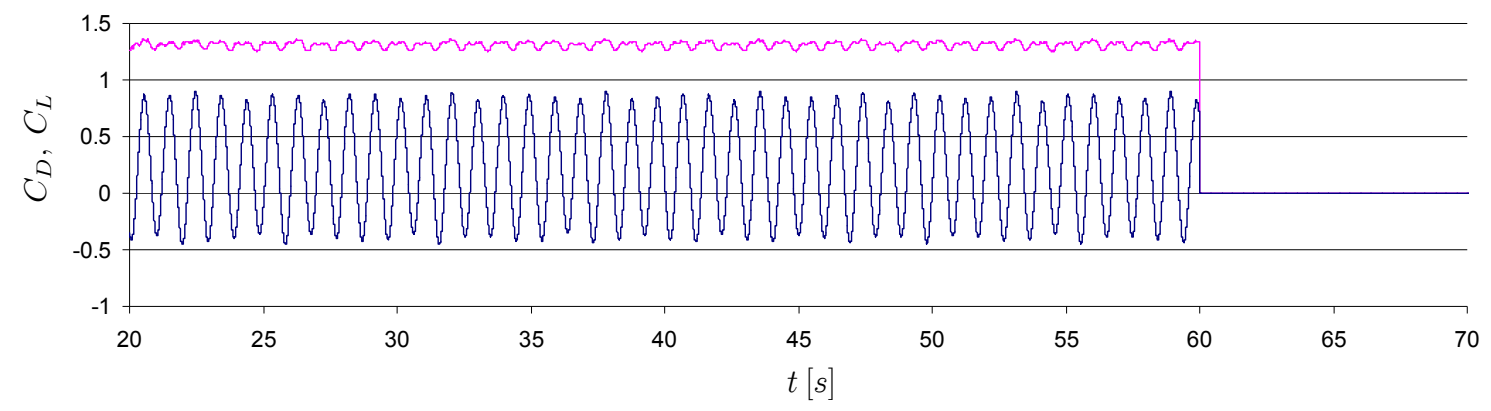

Figura A.38. Oscilación de los coeficientes de arrastre y de sustentación para la malla 3

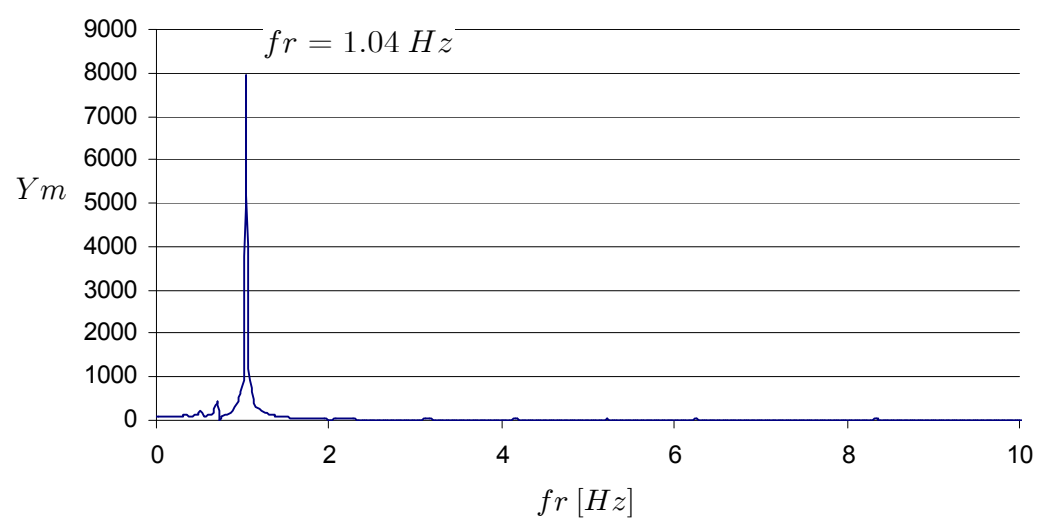

Figura A.39. Análisis FFT de la oscilación del coeficiente de sustentación para la malla 3 
Malla 4. 24064 elementos

- Sección de diámetro $2.2 \mathrm{~m}$

\begin{tabular}{|c|c|c|c|}
\hline \multicolumn{2}{|c|}{ Longitud $[m]$} & \multicolumn{2}{c|}{ Tamaño característico $[m]$} \\
\hline$\Gamma_{w}$ & 88 & $a$ & 2.20 \\
\hline$\Gamma_{d}$ & 44 & $b$ & 0.44 \\
\hline$l_{1}=l_{3}$ & 13.2 & $S$ & 0.20 \\
\hline
\end{tabular}

- Sección de diámetro $4.1 \mathrm{~m}$

\begin{tabular}{|c|c|c|c|}
\hline \multicolumn{2}{|c|}{ Longitud $[m]$} & \multicolumn{2}{c|}{ Tamaño característico $[m]$} \\
\hline$\Gamma_{w}$ & 164 & $a$ & 4.10 \\
\hline$\Gamma_{d}$ & 82 & $b$ & 0.82 \\
\hline$l_{1}=l_{3}$ & 24.6 & $S$ & 0.38 \\
\hline
\end{tabular}

Tabla A.5. Características geométricas y del mallado de los planos de fluido para la malla 4

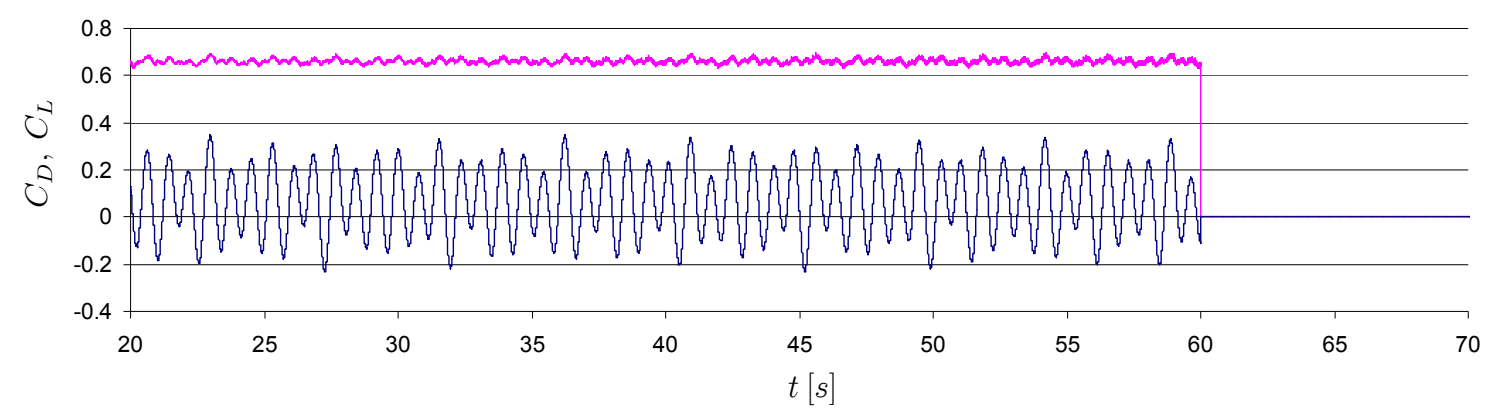

Figura A.40. Oscilación de los coeficientes de arrastre y de sustentación para la malla 4

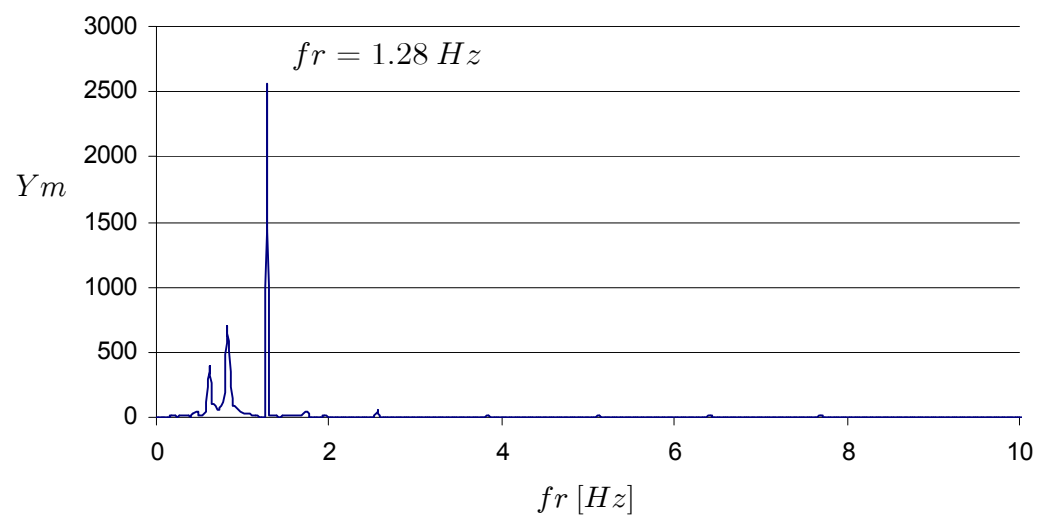

Figura A.41. Análisis FFT de la oscilación del coeficiente de sustentación para la malla 4
- Sección de diámetro $3.1 \mathrm{~m}$

\begin{tabular}{|c|c|c|c|}
\hline \multicolumn{2}{|c|}{ Longitud $[m]$} & \multicolumn{2}{c|}{ Tamaño característico $[m]$} \\
\hline$\Gamma_{w}$ & 128 & $a$ & 3.20 \\
\hline$\Gamma_{d}$ & 64 & $b$ & 0.64 \\
\hline$l_{1}=l_{3}$ & 19.2 & $S$ & 0.30 \\
\hline
\end{tabular}

- Sección de diámetro $5.2 \mathrm{~m}$

\begin{tabular}{|c|c|c|c|}
\hline \multicolumn{2}{|c|}{ Longitud $[m]$} & \multicolumn{2}{c|}{ Tamaño característico $[m]$} \\
\hline$\Gamma_{w}$ & 208 & $a$ & 5.20 \\
\hline$\Gamma_{d}$ & 104 & $b$ & 1.04 \\
\hline$l_{1}=l_{3}$ & 31.2 & $S$ & 0.48 \\
\hline
\end{tabular}


Malla 5. 26140 elementos

- Sección de diámetro $2.2 \mathrm{~m}$

\begin{tabular}{|c|c|c|c|}
\hline \multicolumn{2}{|c|}{ Longitud $[m]$} & \multicolumn{2}{c|}{ Tamaño característico $[m]$} \\
\hline$\Gamma_{w}$ & 88 & $a$ & 2.20 \\
\hline$\Gamma_{d}$ & 44 & $b$ & 0.44 \\
\hline$l_{1}=l_{3}$ & 13.2 & $S$ & 0.15 \\
\hline
\end{tabular}

- Sección de diámetro $4.1 \mathrm{~m}$

\begin{tabular}{|c|c|c|c|}
\hline \multicolumn{2}{|c|}{ Longitud $[m]$} & \multicolumn{2}{c|}{ Tamaño característico $[m]$} \\
\hline$\Gamma_{w}$ & 164 & $a$ & 4.10 \\
\hline$\Gamma_{d}$ & 82 & $b$ & 0.82 \\
\hline$l_{1}=l_{3}$ & 24.6 & $S$ & 0.28 \\
\hline
\end{tabular}

Tabla A.6. Características geométricas y del mallado de los planos de fluido para la malla 5

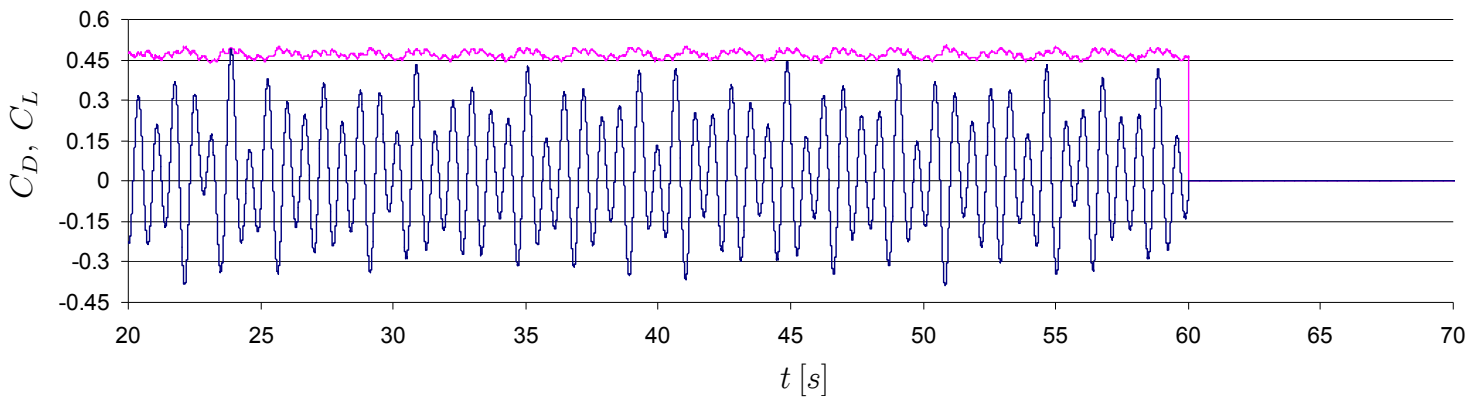

Figura A.42. Oscilación de los coeficientes de arrastre y de sustentación para la malla 5

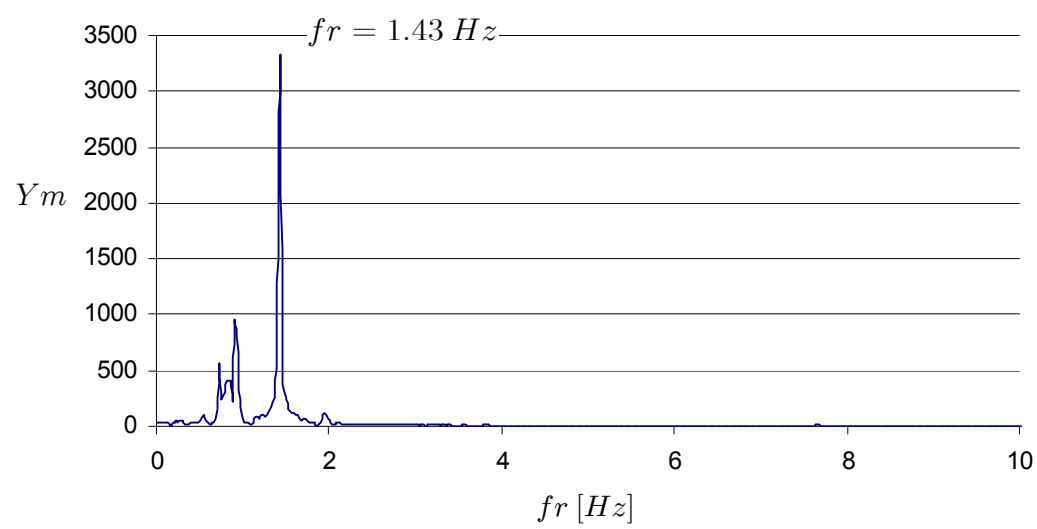

Figura A.43. Análisis FFT de la oscilación del coeficiente de sustentación para la malla 5 
Malla 6. 29694 elementos

- Sección de diámetro $2.2 \mathrm{~m}$

\begin{tabular}{|c|c|c|c|}
\hline \multicolumn{2}{|c|}{ Longitud $[m]$} & \multicolumn{2}{c|}{ Tamaño característico $[m]$} \\
\hline$\Gamma_{w}$ & 88 & $a$ & 2.20 \\
\hline$\Gamma_{d}$ & 44 & $b$ & 0.44 \\
\hline$l_{1}=l_{3}$ & 13.2 & $S$ & 0.10 \\
\hline
\end{tabular}

- Sección de diámetro $4.1 \mathrm{~m}$

\begin{tabular}{|c|c|c|c|}
\hline \multicolumn{2}{|c|}{ Longitud $[m]$} & \multicolumn{2}{c|}{ Tamaño característico $[m]$} \\
\hline$\Gamma_{w}$ & 164 & $a$ & 4.10 \\
\hline$\Gamma_{d}$ & 82 & $b$ & 0.82 \\
\hline$l_{1}=l_{3}$ & 24.6 & $S$ & 0.19 \\
\hline
\end{tabular}

Tabla A.7. Características geométricas y del mallado de los planos de fluido para la malla 6

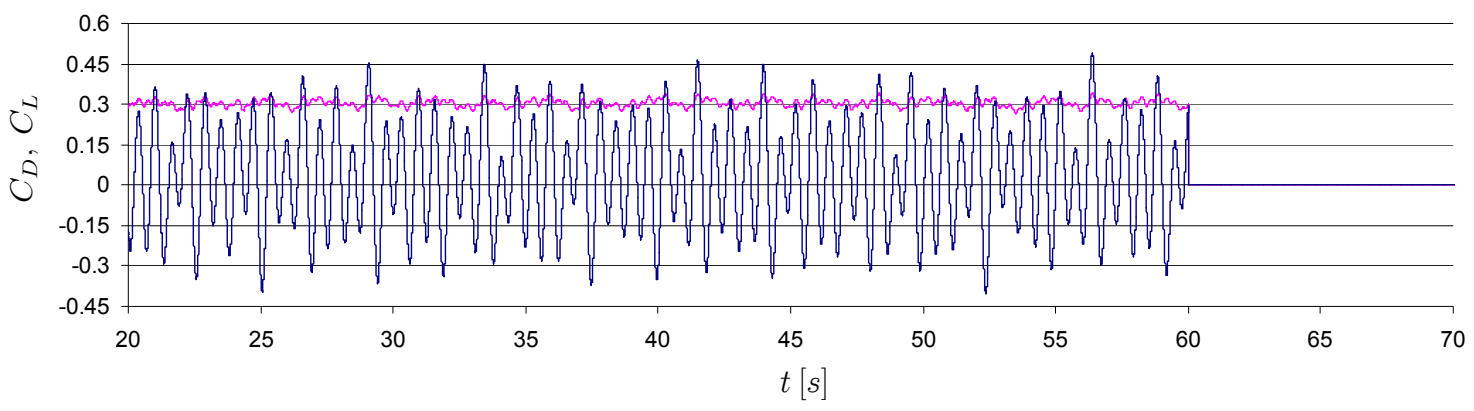

Figura A.44. Oscilación de los coeficientes de arrastre y de sustentación para la malla 7

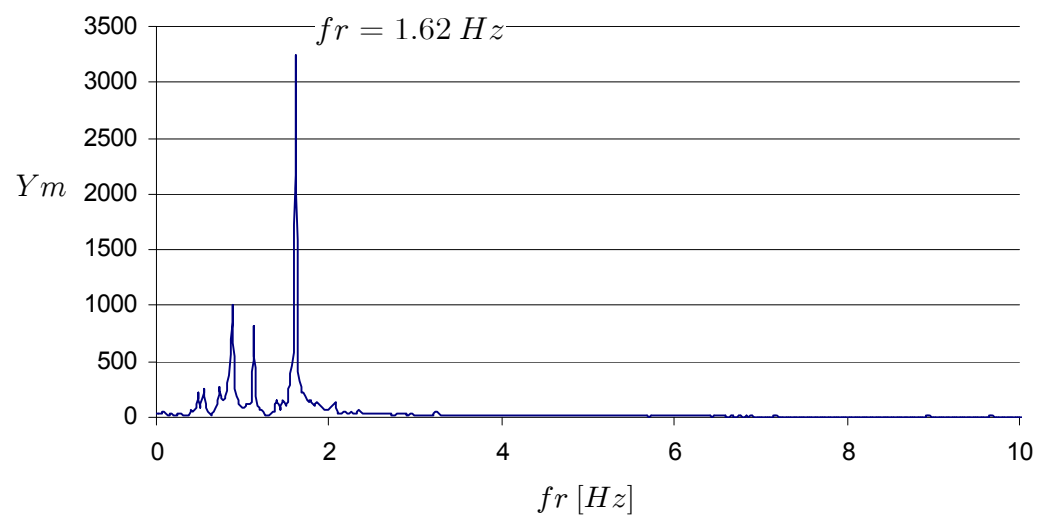

Figura A.45. Análisis FFT de la oscilación del coeficiente de sustentación para la malla 7
- Sección de diámetro $3.1 \mathrm{~m}$

\begin{tabular}{|c|c|c|c|}
\hline \multicolumn{2}{|c|}{ Longitud $[m]$} & \multicolumn{2}{c|}{ Tamaño característico $[m]$} \\
\hline$\Gamma_{w}$ & 128 & $a$ & 3.20 \\
\hline$\Gamma_{d}$ & 64 & $b$ & 0.64 \\
\hline$l_{1}=l_{3}$ & 19.2 & $S$ & 0.15 \\
\hline
\end{tabular}

- Sección de diámetro $5.2 \mathrm{~m}$

\begin{tabular}{|c|c|c|c|}
\hline \multicolumn{2}{|c|}{ Longitud $[m]$} & \multicolumn{2}{c|}{ Tamaño característico $[m]$} \\
\hline$\Gamma_{w}$ & 208 & $a$ & 5.20 \\
\hline$\Gamma_{d}$ & 104 & $b$ & 1.04 \\
\hline$l_{1}=l_{3}$ & 31.2 & $S$ & 0.24 \\
\hline
\end{tabular}


Malla 7. 52282 elementos

- Sección de diámetro $2.2 \mathrm{~m}$

\begin{tabular}{|c|c|c|c|}
\hline \multicolumn{2}{|c|}{ Longitud $[m]$} & \multicolumn{2}{c|}{ Tamaño característico $[m]$} \\
\hline$\Gamma_{w}$ & 88 & $a$ & 2.20 \\
\hline$\Gamma_{d}$ & 44 & $b$ & 0.22 \\
\hline$l_{1}=l_{3}$ & 13.2 & $S$ & 0.10 \\
\hline
\end{tabular}

- Sección de diámetro $4.1 \mathrm{~m}$

\begin{tabular}{|c|c|c|c|}
\hline \multicolumn{2}{|c|}{ Longitud $[m]$} & \multicolumn{2}{c|}{ Tamaño característico $[m]$} \\
\hline$\Gamma_{w}$ & 164 & $a$ & 4.10 \\
\hline$\Gamma_{d}$ & 82 & $b$ & 0.41 \\
\hline$l_{1}=l_{3}$ & 24.6 & $S$ & 0.19 \\
\hline
\end{tabular}

Tabla A.8. Características geométricas y del mallado de los planos de fluido para la malla 7

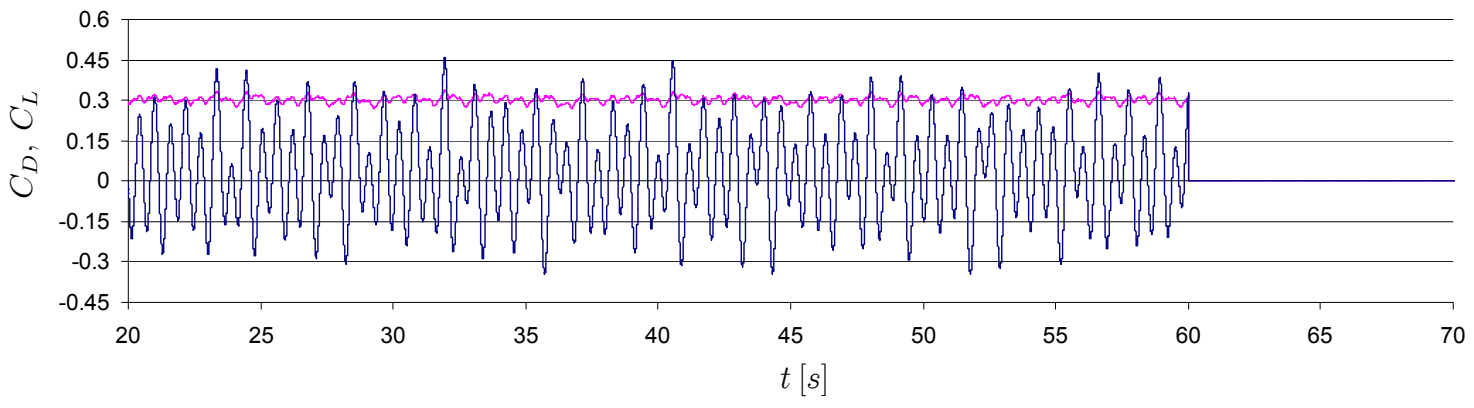

Figura A.46. Oscilación de los coeficientes de arrastre y de sustentación para la malla 7

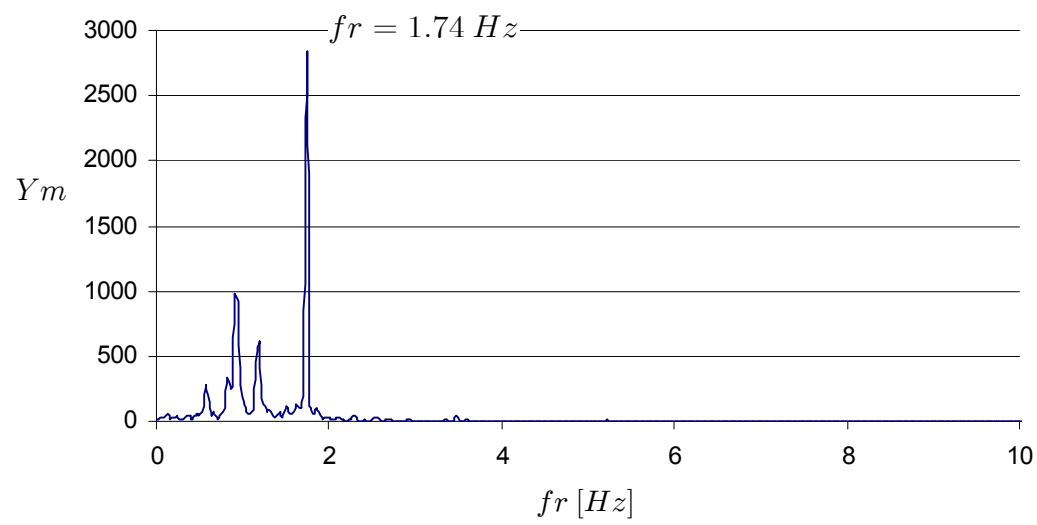

Figura A.47. Análisis FFT de la oscilación del coeficiente de sustentación para la malla 7 
En la Tabla A.9 se resumen los resultados obtenidos para cada una de las mallas utilizadas relativos al valor RMS del coeficiente de arrastre, la frecuencia de desprendimiento de los torbellinos y el número de Strouhal. Se observa que no se obtienen resultados coherentes con las gráficas del coeficiente de arrastre (Figura 2.4) y del número de Strouhal (Figura 2.8) mostradas en el Capítulo 2 hasta que el tamaño característico se hace lo suficientemente pequeño. Teniendo en cuenta esas gráficas se eligió la malla 6 para resolver el ejemplo de la chimenea, la cual tiene un menor número de elementos que la malla 7 y se obtienen resultados similares con un menor coste computacional.

\begin{tabular}{|c|c|c|c|c|c|}
\hline Malla & Tamaño característico $[m]$ & Número de elementos & $\boldsymbol{n}_{\boldsymbol{s}}$ & $\boldsymbol{S t}$ & $\tilde{\boldsymbol{C}}_{\boldsymbol{D}}$ \\
\hline 1 & 0.6 & 9348 & 0.76 & 0.13 & 1.45 \\
\hline 2 & 0.38 & 12792 & 0.94 & 0.17 & 1.36 \\
\hline 3 & 0.38 & 22236 & 1.04 & 0.18 & 1.31 \\
\hline 4 & 0.2 & 24064 & 1.28 & 0.23 & 0.66 \\
\hline 5 & 0.15 & 26140 & 1.43 & 0.25 & 0.47 \\
\hline 6 & 0.1 & 29694 & 1.62 & 0.28 & 0.30 \\
\hline 7 & 0.1 & 52282 & 1.74 & 0.31 & 0.30 \\
\hline
\end{tabular}

Tabla A.9. Comparación de los resultados obtenidos para las diferentes mallas para $R e=1.84 \cdot 10^{6}$

Finalmente, en la Figura A.48 y en la Figura A.49 se muestra gráficamente la variación del número de Strouhal y del coeficiente de arrastre respecto al número de elementos utilizados en la malla del fluido, respectivamente.

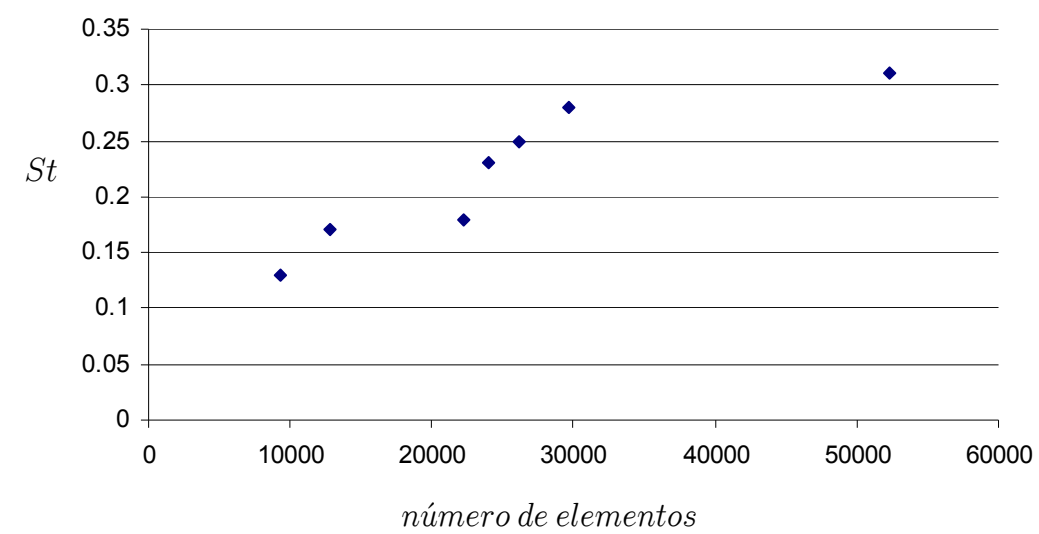

Figura A.48. Variación del número de Strouhal en función de la malla del fluido para $R e=1.84 \cdot 10^{6}$ 


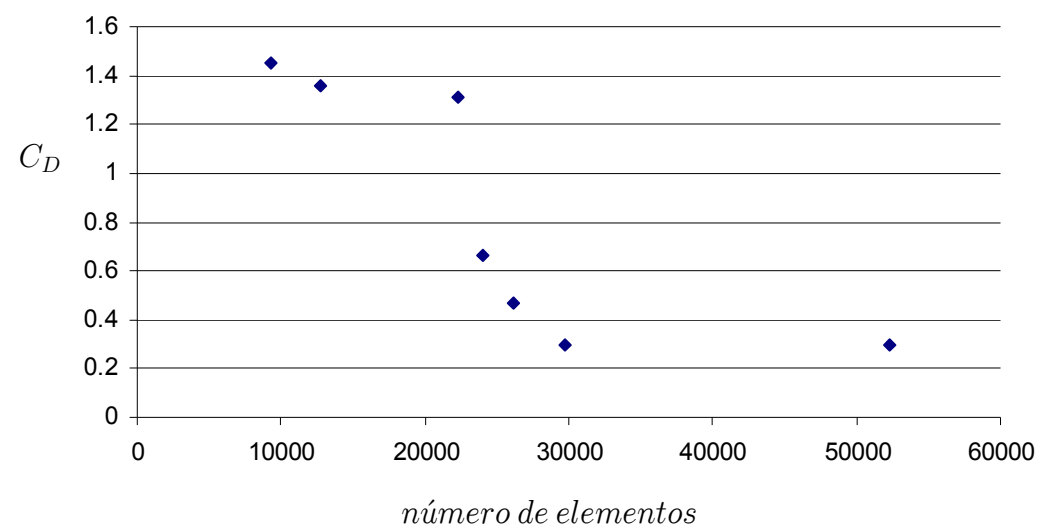

Figura A.49. Variación del coeficiente de arrastre en función de la malla del fluido para $R e=1.84 \cdot 10^{6}$ 


\section{Anexo J. Amplificación del coeficiente de arrastre}

\section{J.1. Amortiguamiento estructural 0.075}

- $U_{r}=3.5$

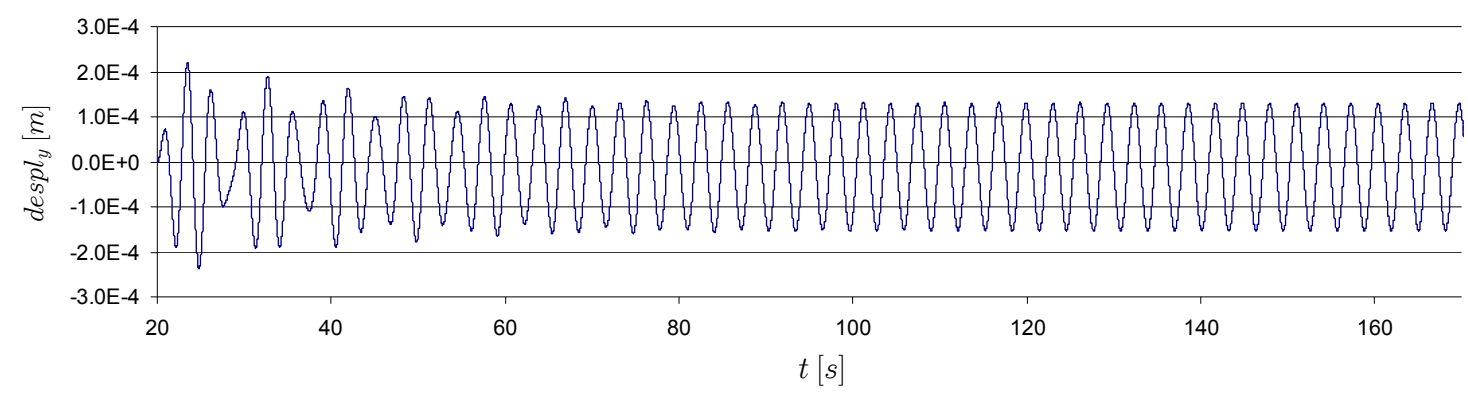

Figura A.50. Vibración transversal de la estructura

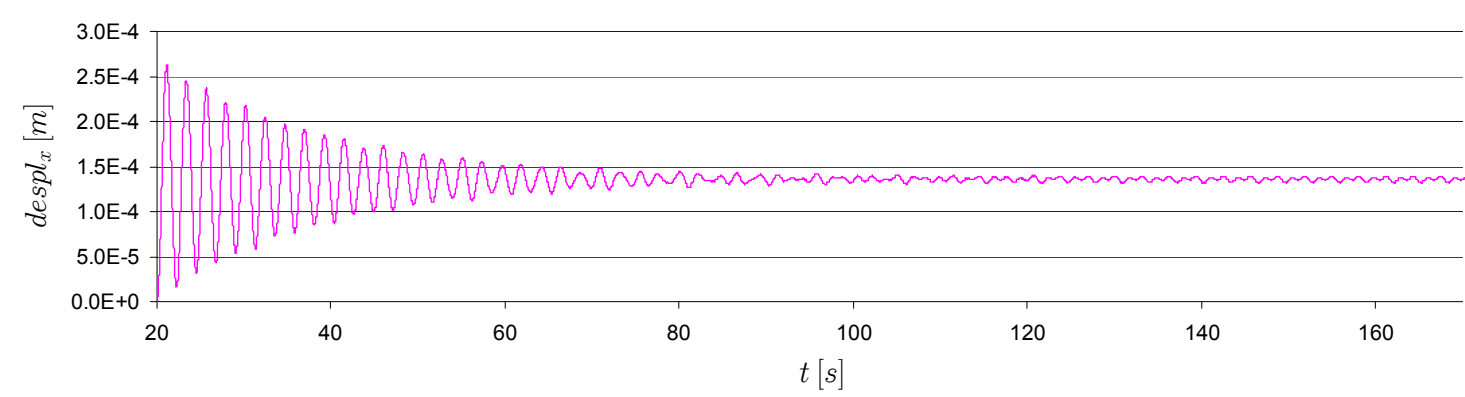

Figura A.51. Vibración en la dirección del flujo de la estructura

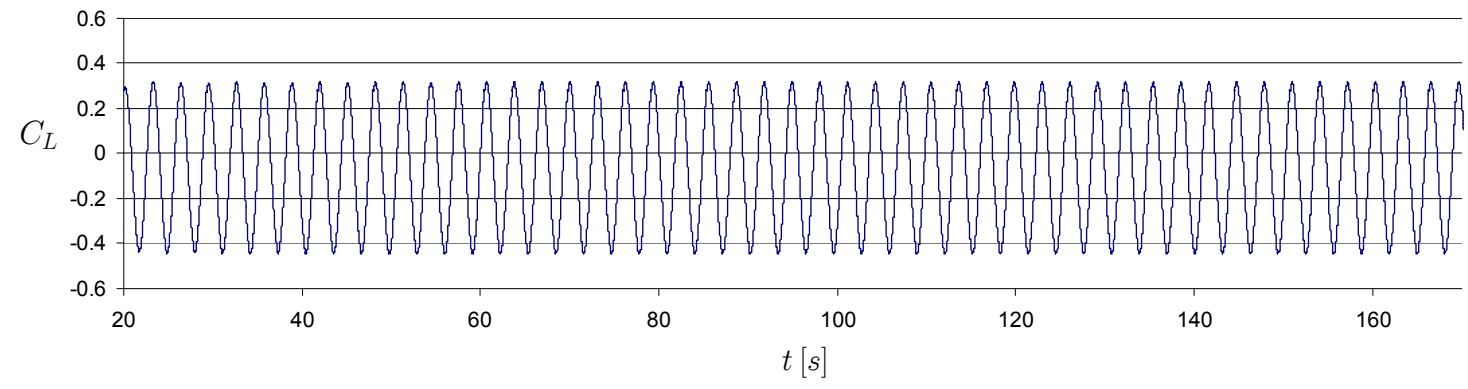

Figura A.52. Oscilación del coeficiente de sustentación

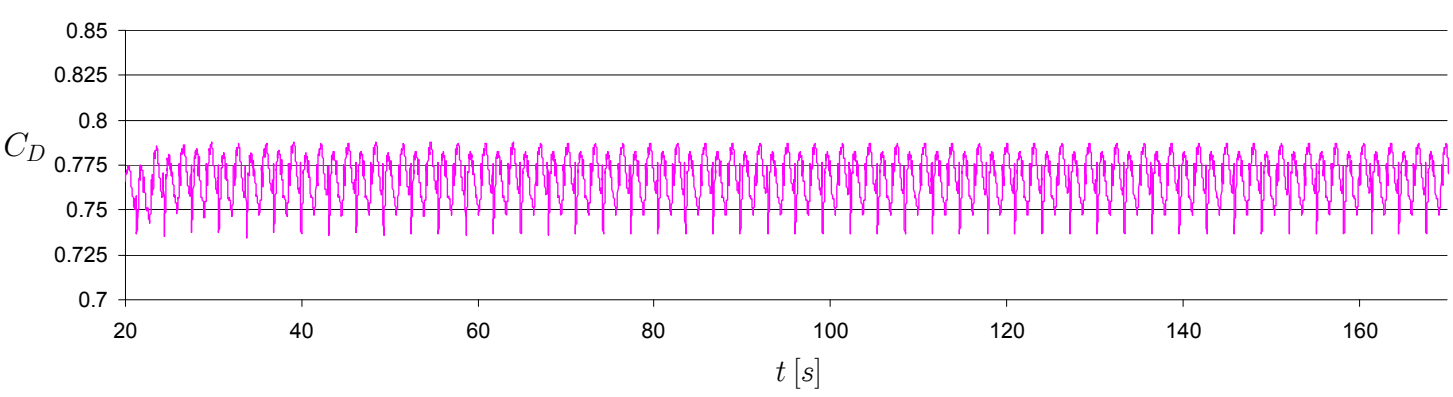

Figura A.53. Oscilación del coeficiente de arrastre 
- $U_{r}=4$

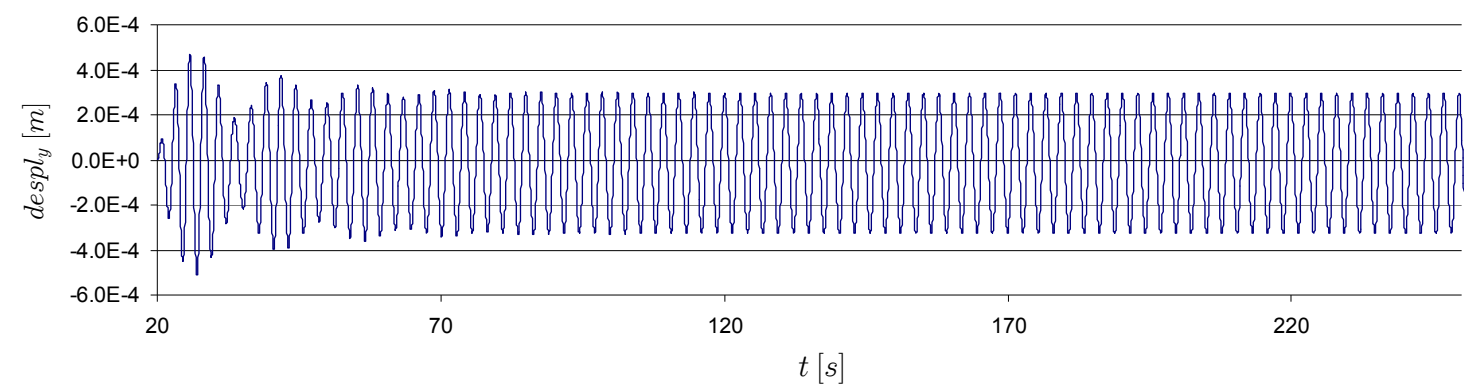

Figura A.54. Vibración transversal de la estructura

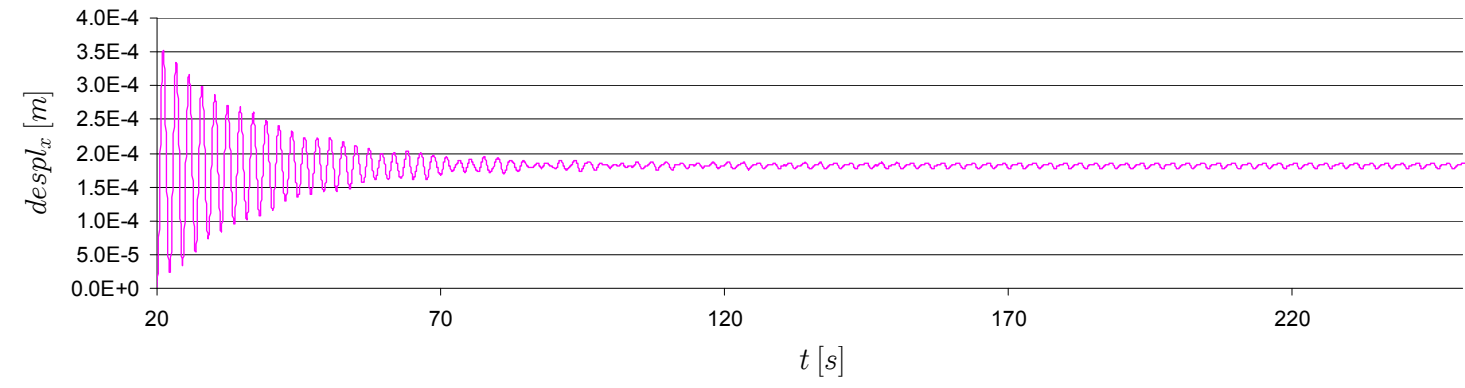

Figura A.55. Vibración en la dirección del flujo de la estructura

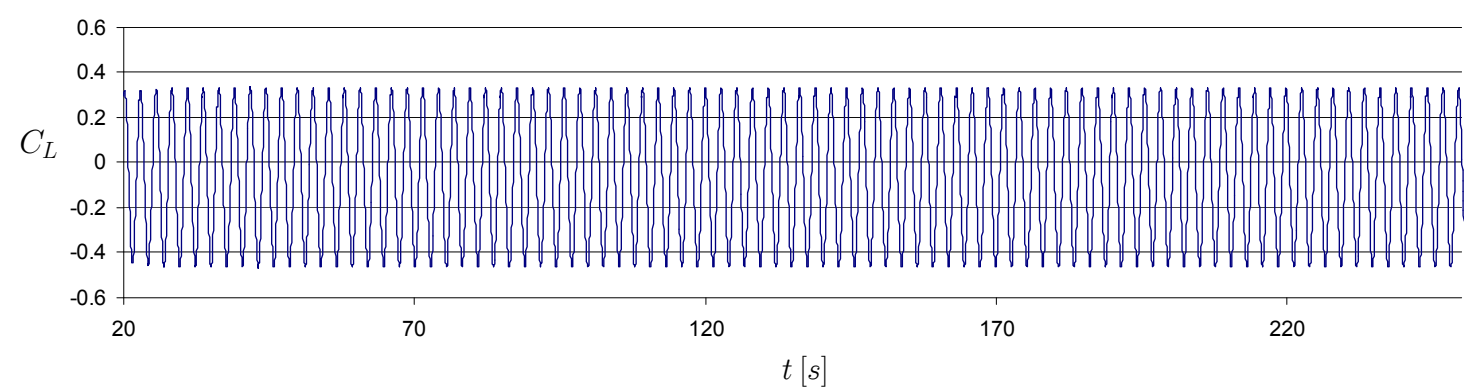

Figura A.56. Oscilación del coeficiente de sustentación

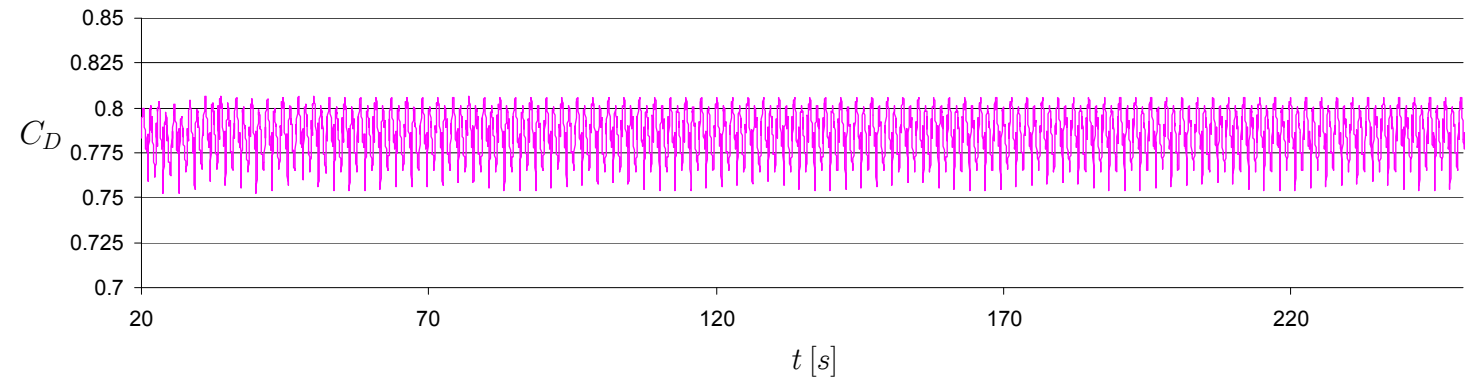

Figura A.57. Oscilación del coeficiente de arrastre 
- $U_{r}=4.5$

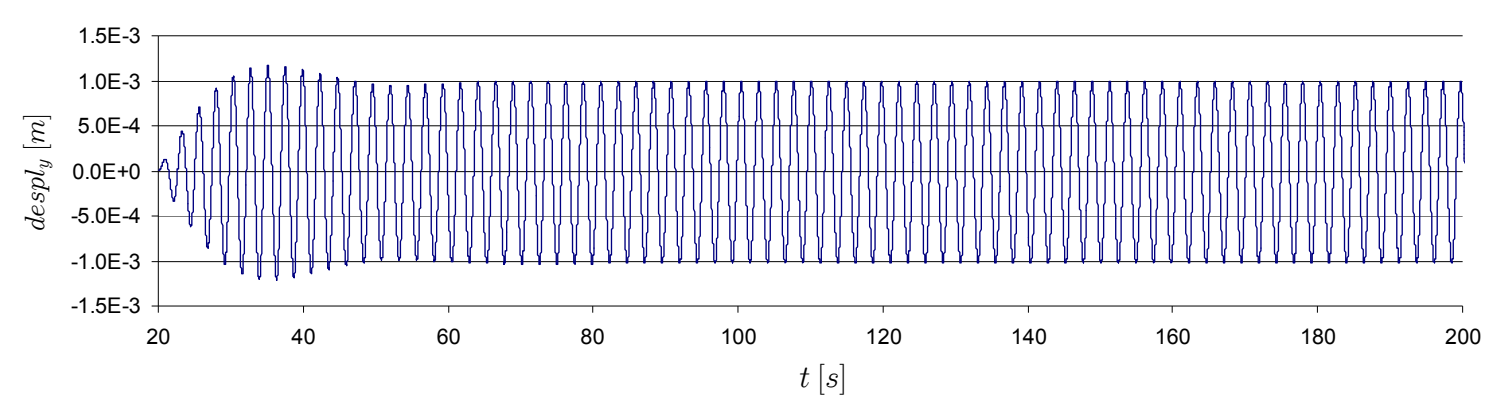

Figura A.58. Vibración transversal de la estructura

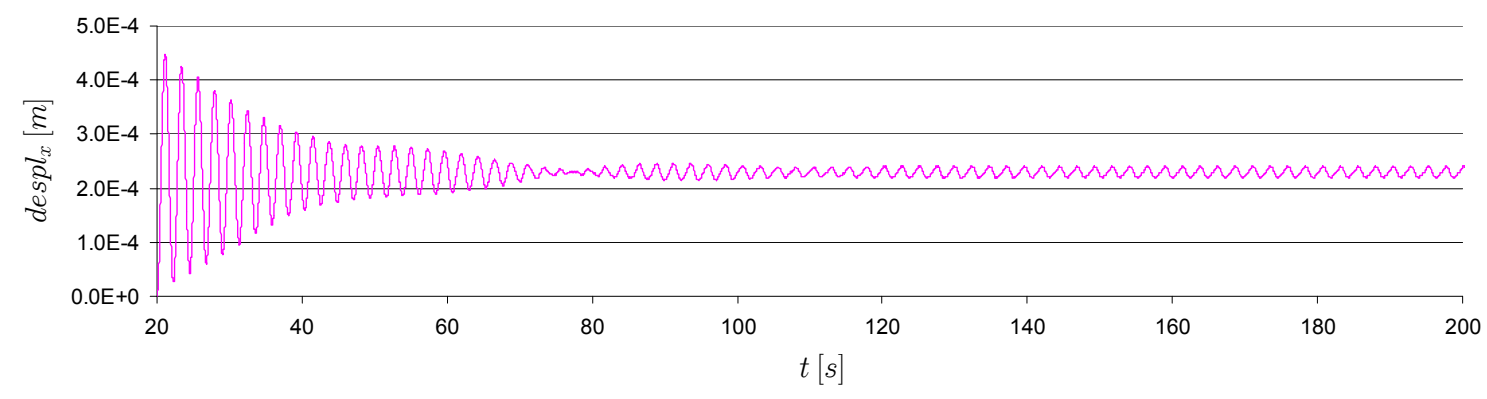

Figura A.59. Vibración en la dirección del flujo de la estructura

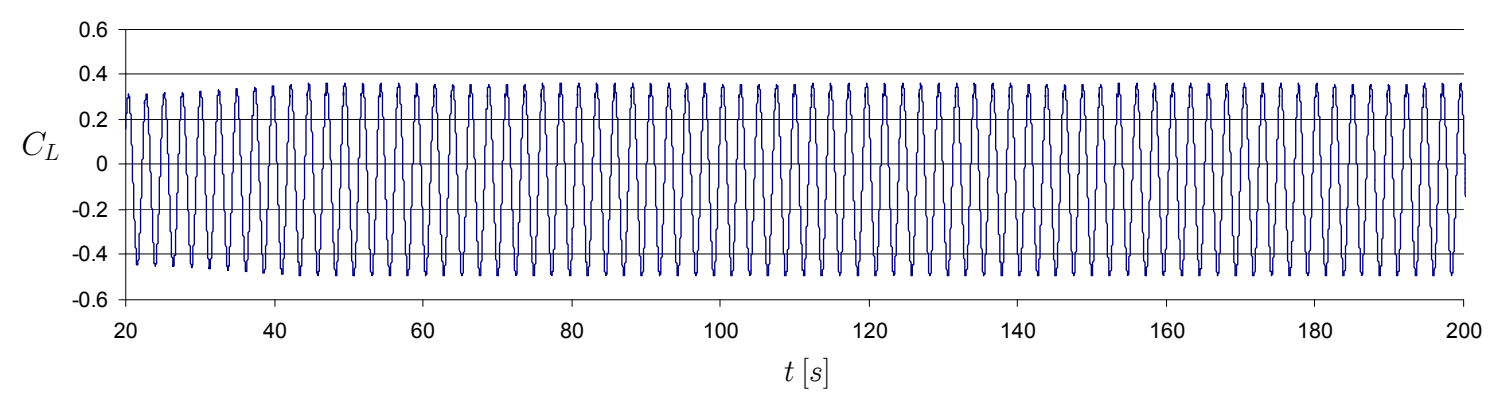

Figura A.60. Oscilación del coeficiente de sustentación

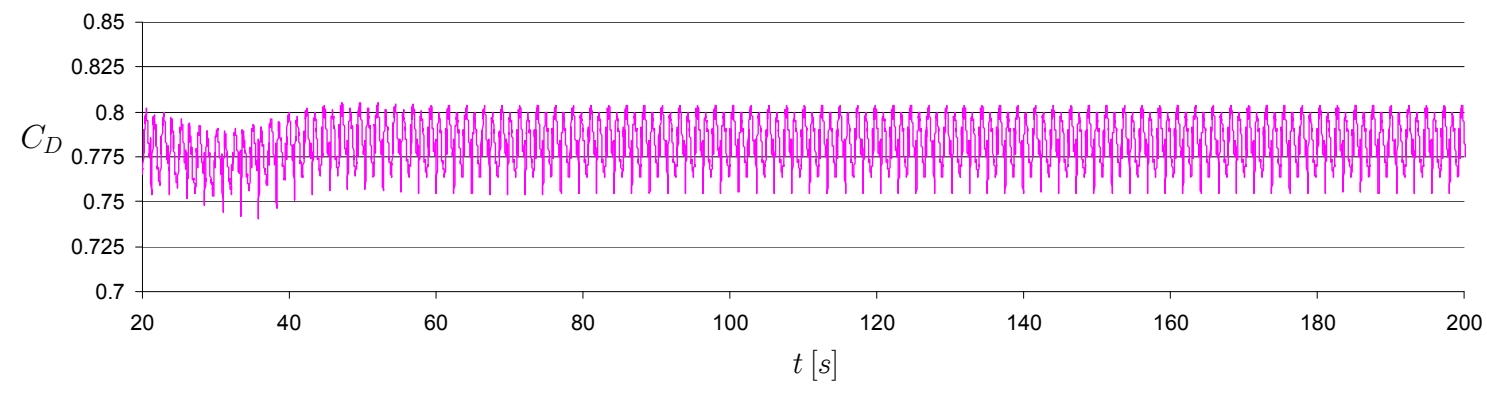

Figura A.61. Oscilación del coeficiente de arrastre 
- $U_{r}=5$

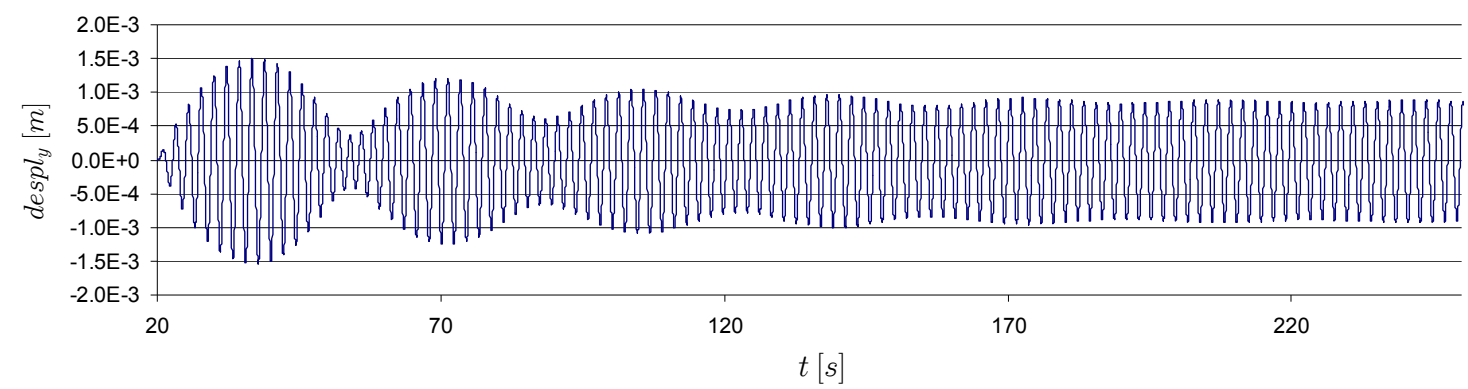

Figura A.62. Vibración transversal de la estructura

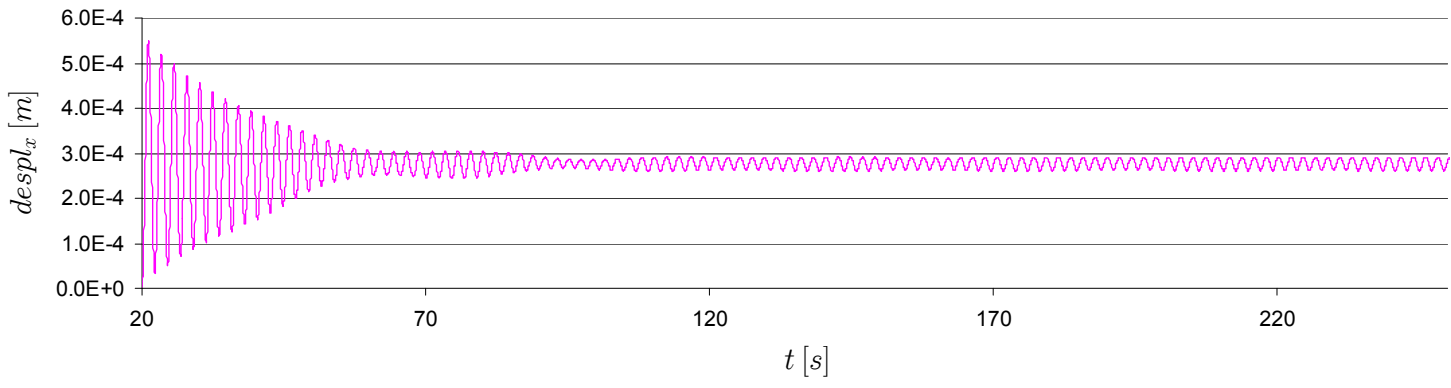

Figura A.63. Vibración en la dirección del flujo de la estructura

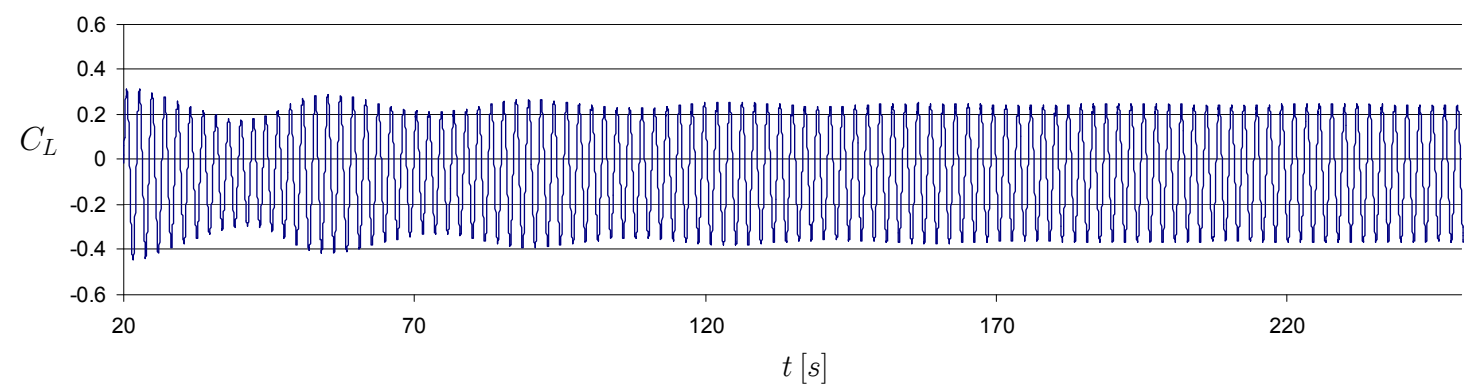

Figura A.64. Oscilación del coeficiente de sustentación

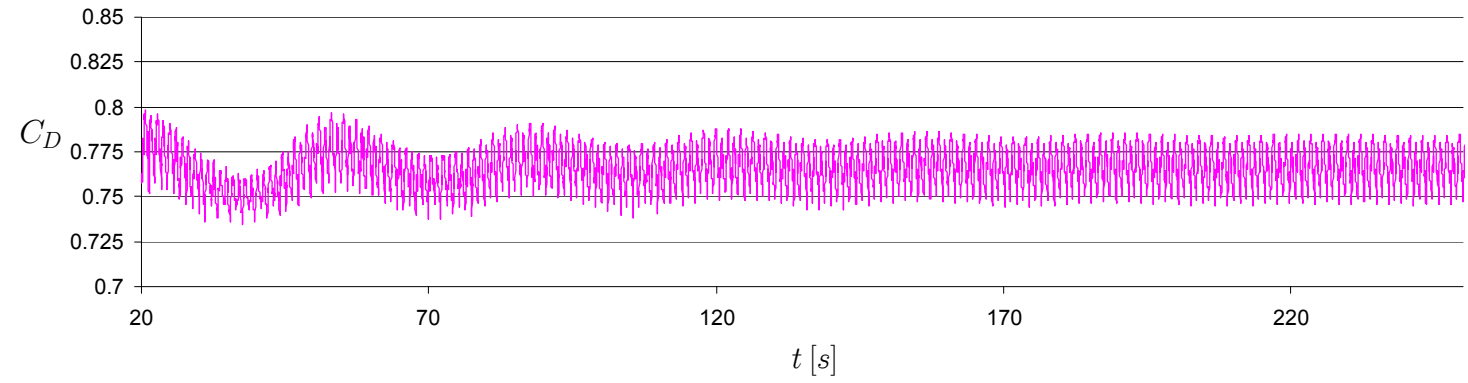

Figura A.65. Oscilación del coeficiente de arrastre 
- $U_{r}=5.5$

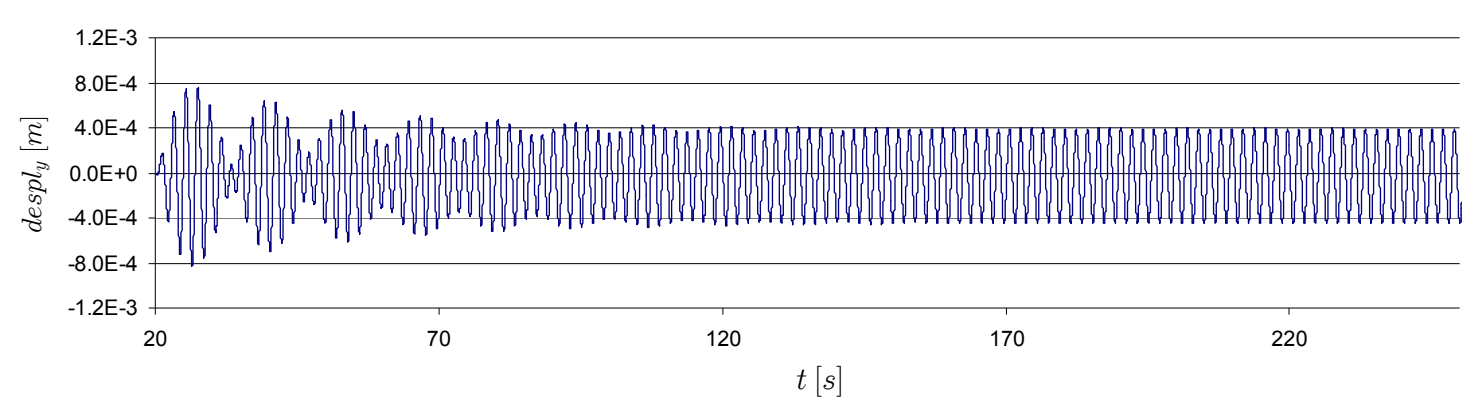

Figura A.66. Vibración transversal de la estructura

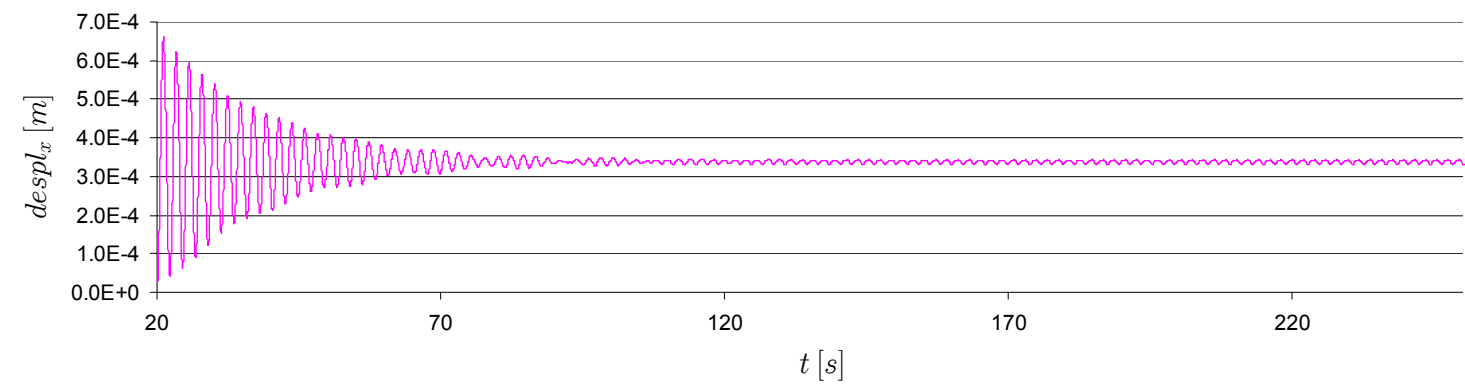

Figura A.67. Vibración en la dirección del flujo de la estructura

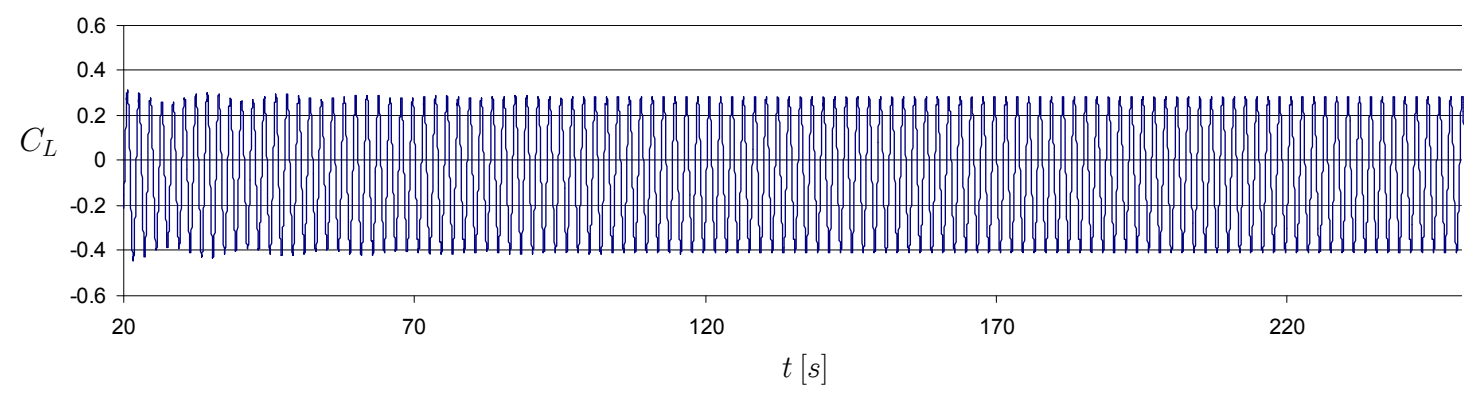

Figura A.68. Oscilación del coeficiente de sustentación

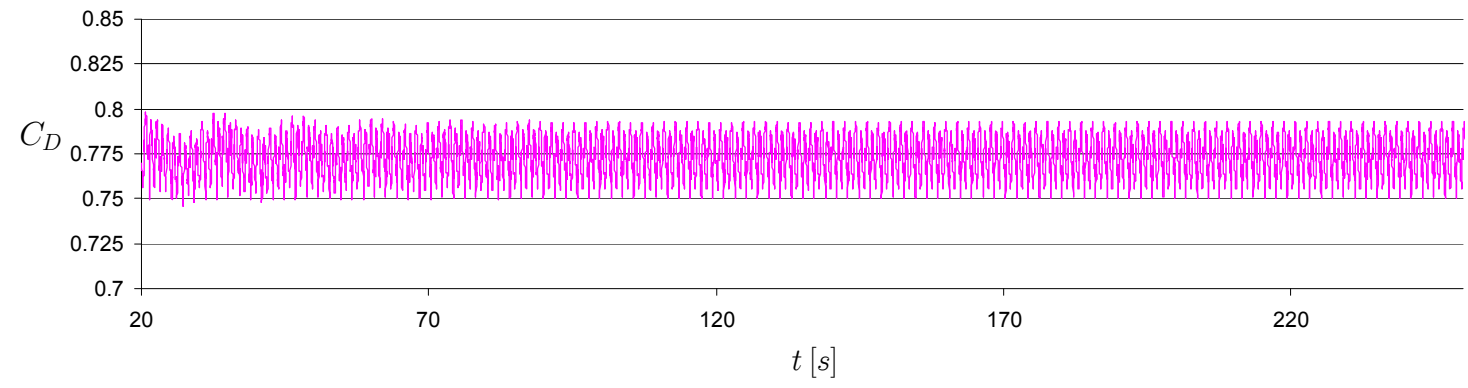

Figura A.69. Oscilación del coeficiente de arrastre 
- $U_{r}=6$

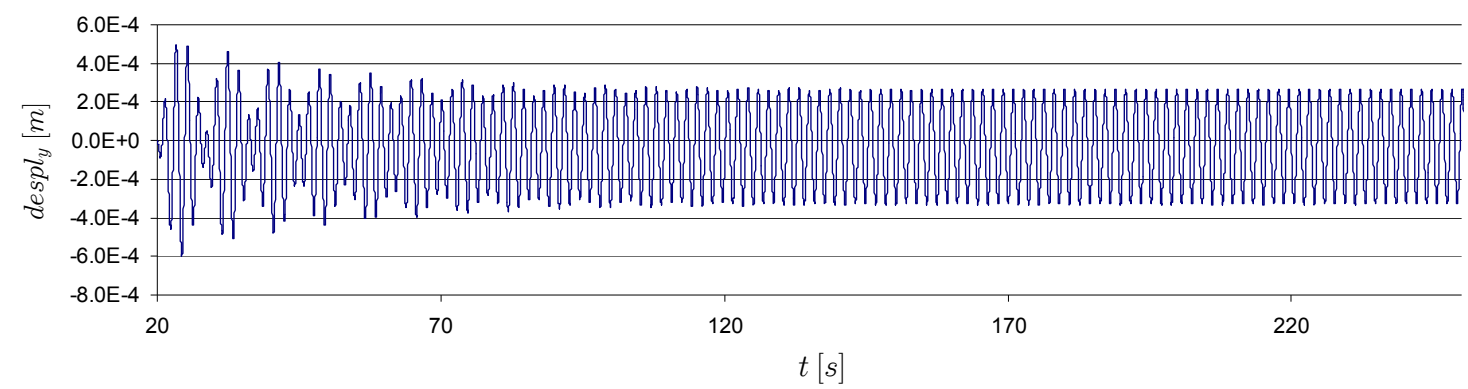

Figura A.70. Vibración transversal de la estructura

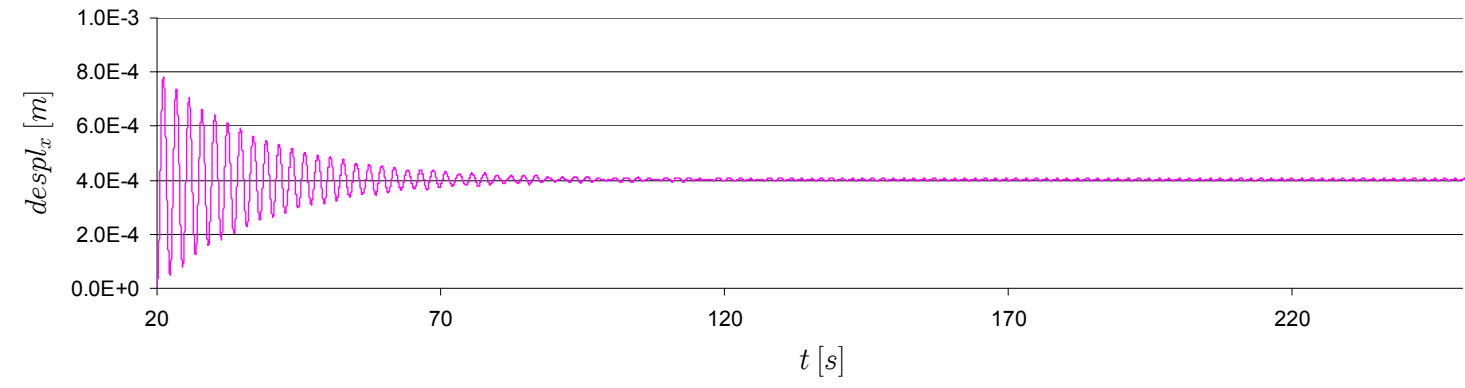

Figura A.71. Vibración en la dirección del flujo de la estructura

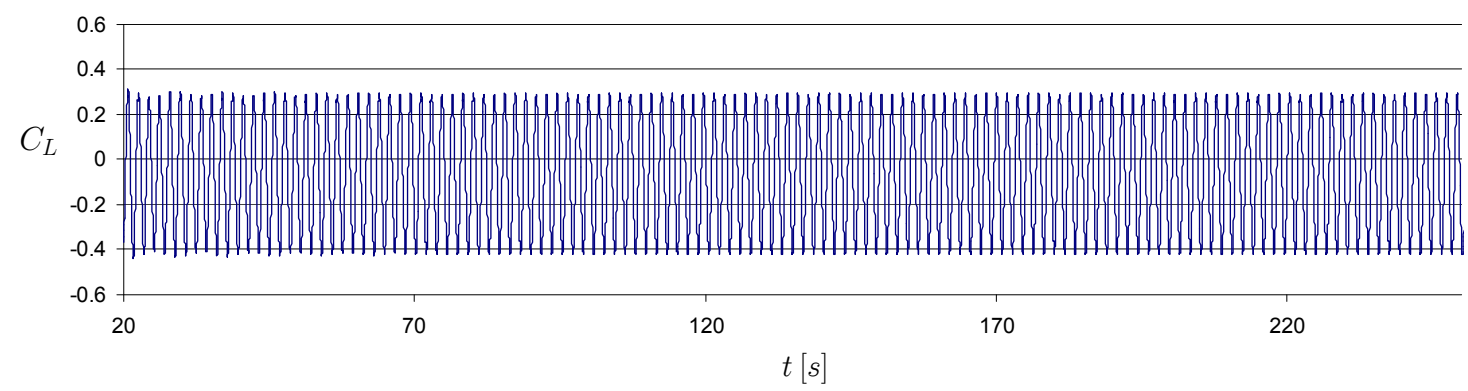

Figura A.72. Oscilación del coeficiente de sustentación

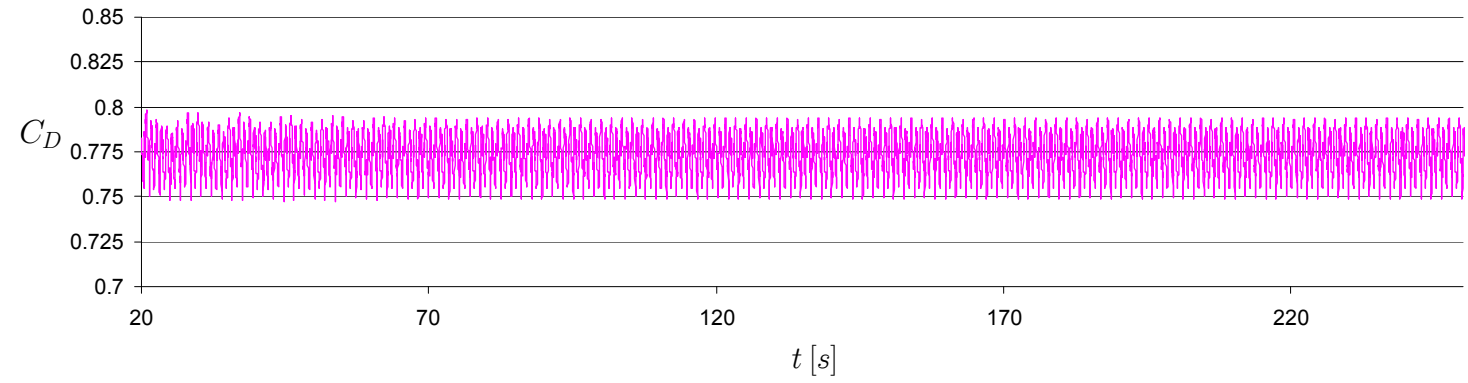

Figura A.73. Oscilación del coeficiente de arrastre 
- $U_{r}=7$

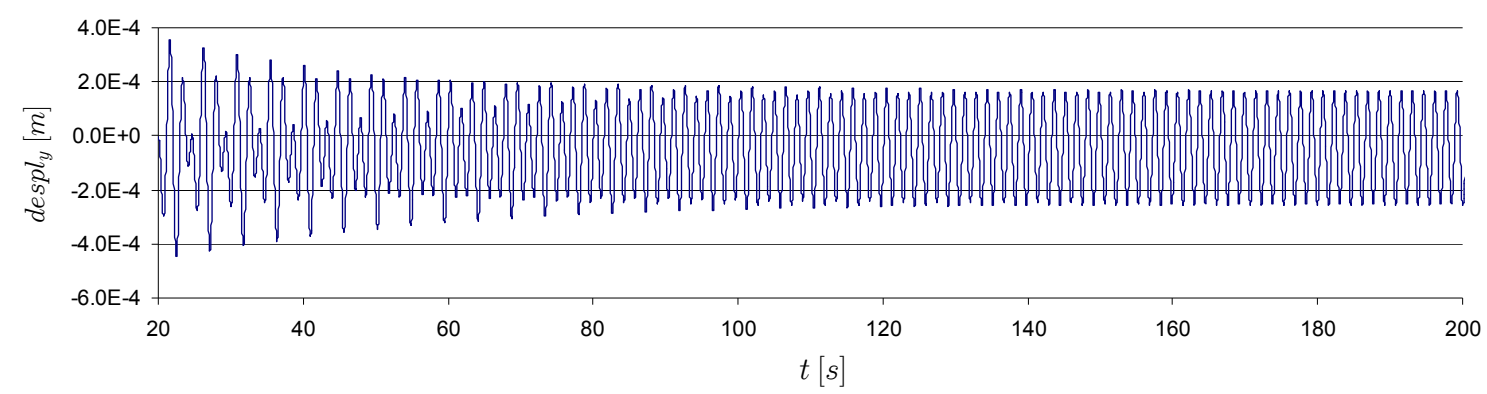

Figura A.74. Vibración transversal de la estructura

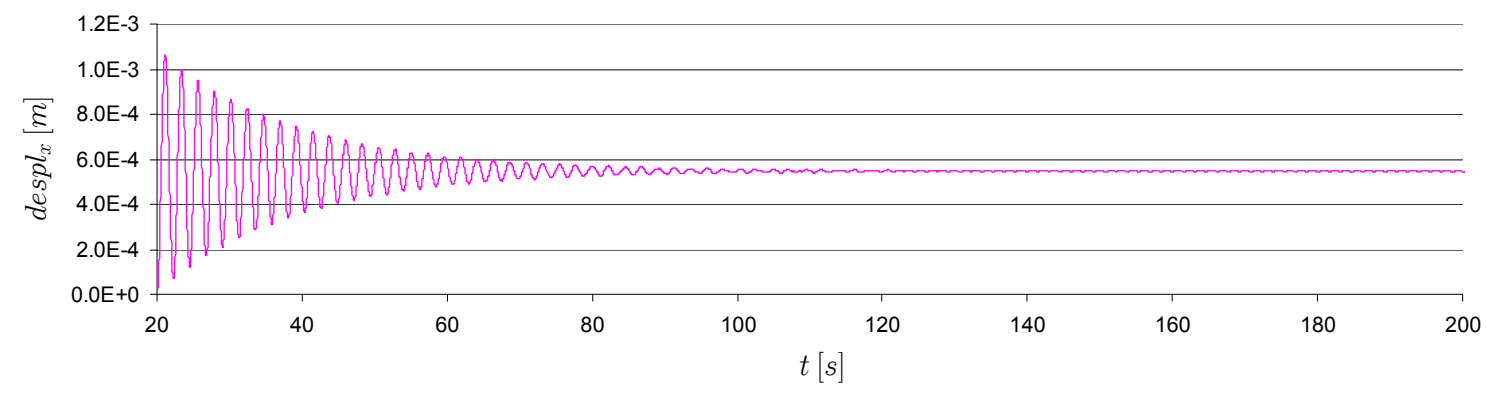

Figura A.75. Vibración en la dirección del flujo de la estructura

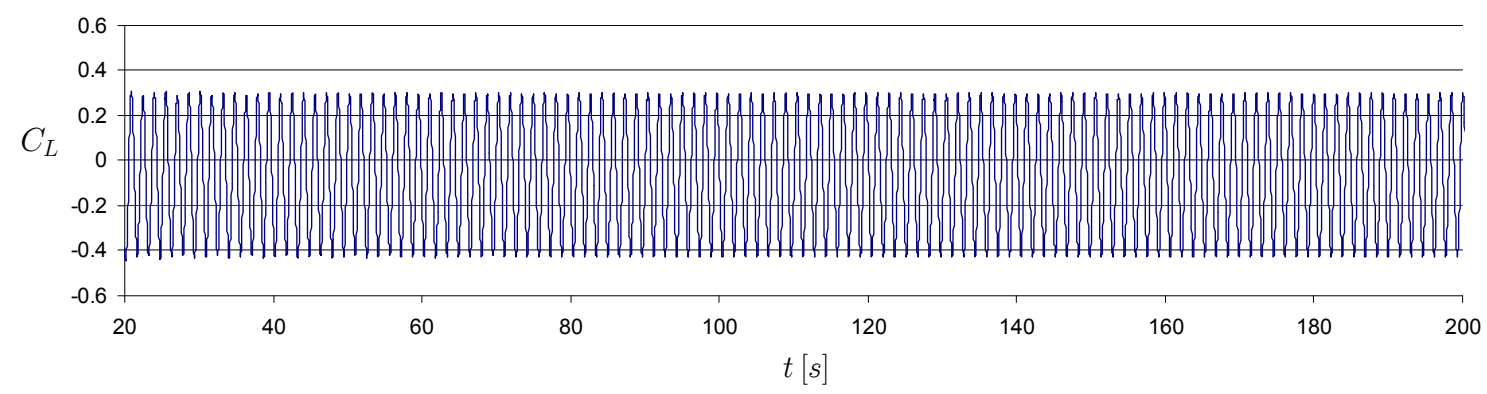

Figura A.76. Oscilación del coeficiente de sustentación

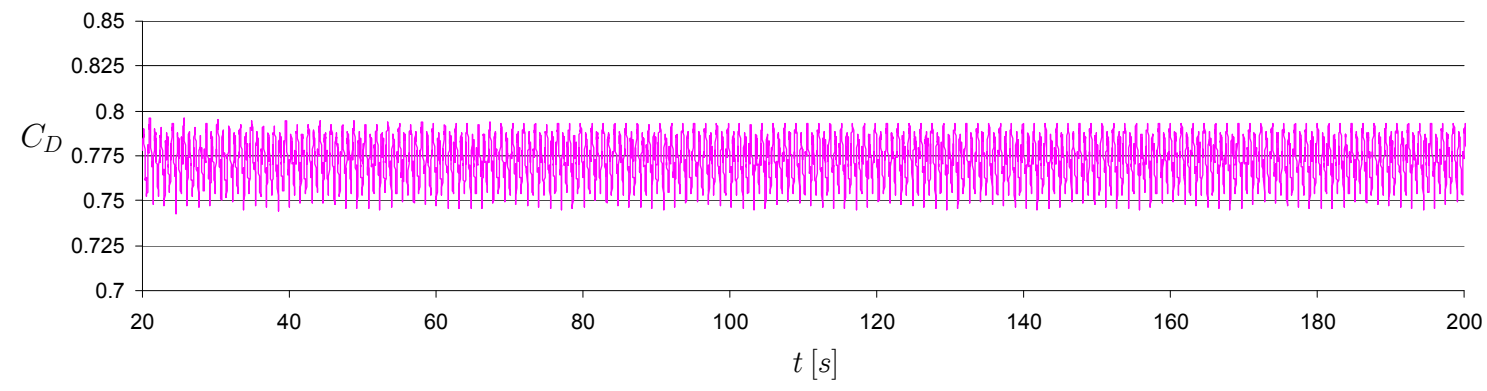

Figura A.77. Oscilación del coeficiente de arrastre 
- $U_{r}=8$

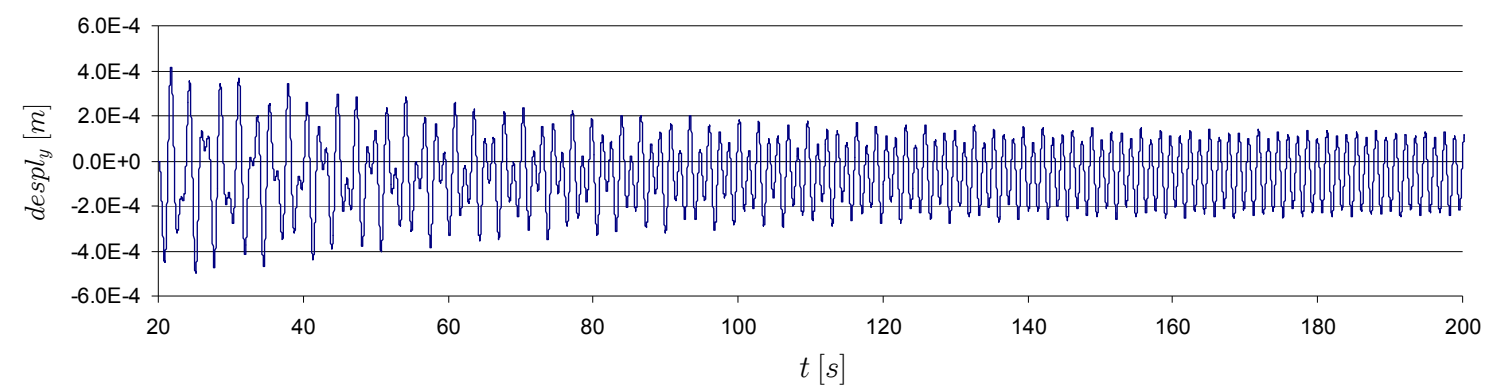

Figura A.78. Vibración transversal de la estructura

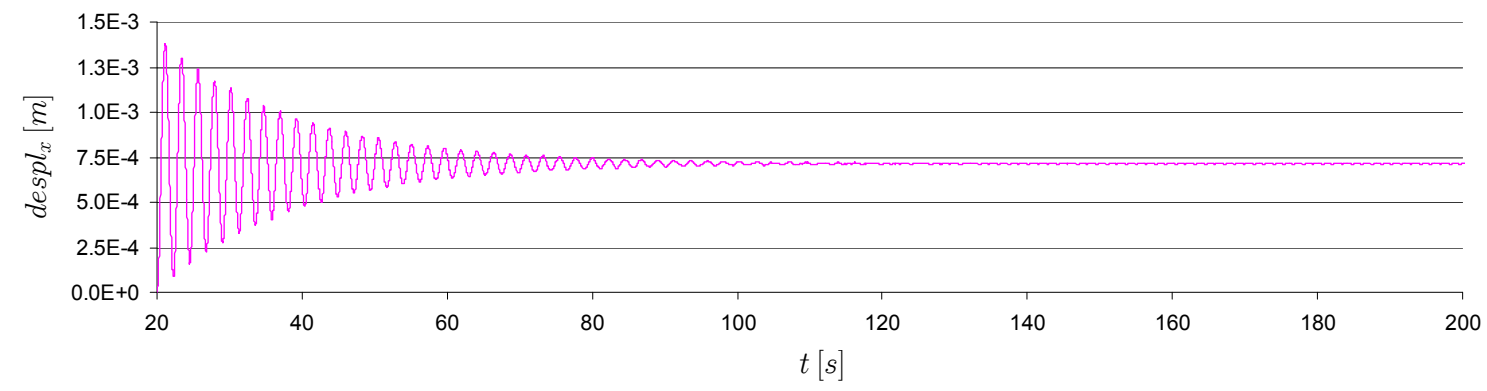

Figura A.79. Vibración en la dirección del flujo de la estructura

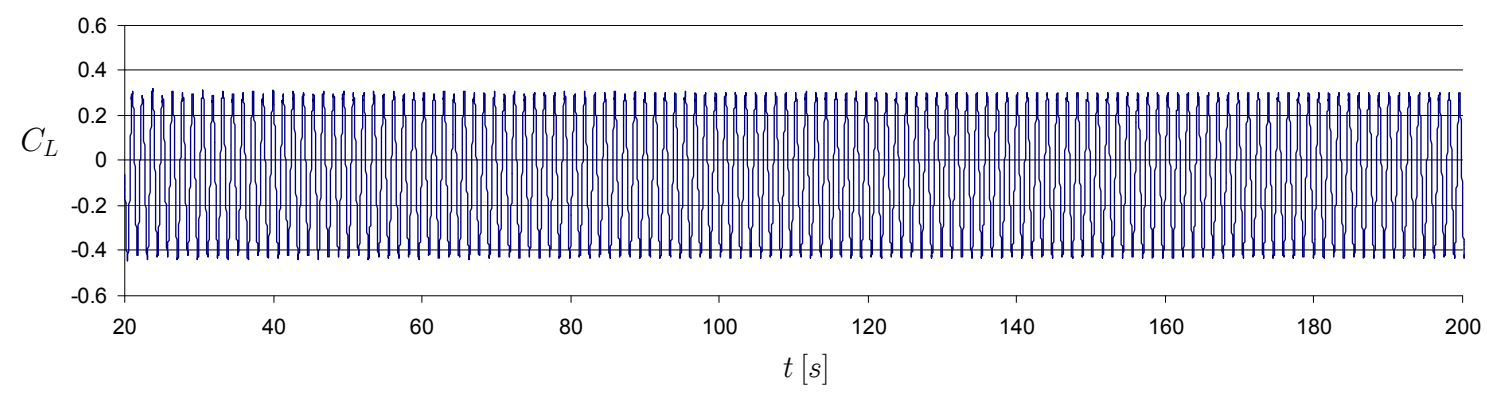

Figura A.80. Oscilación del coeficiente de sustentación

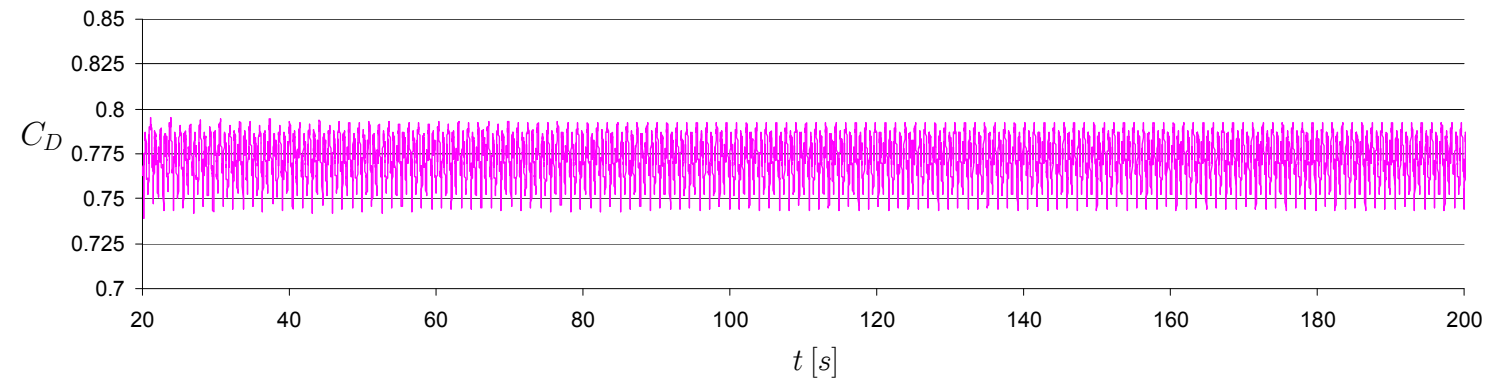

Figura A.81. Oscilación del coeficiente de arrastre 
- $U_{r}=9$

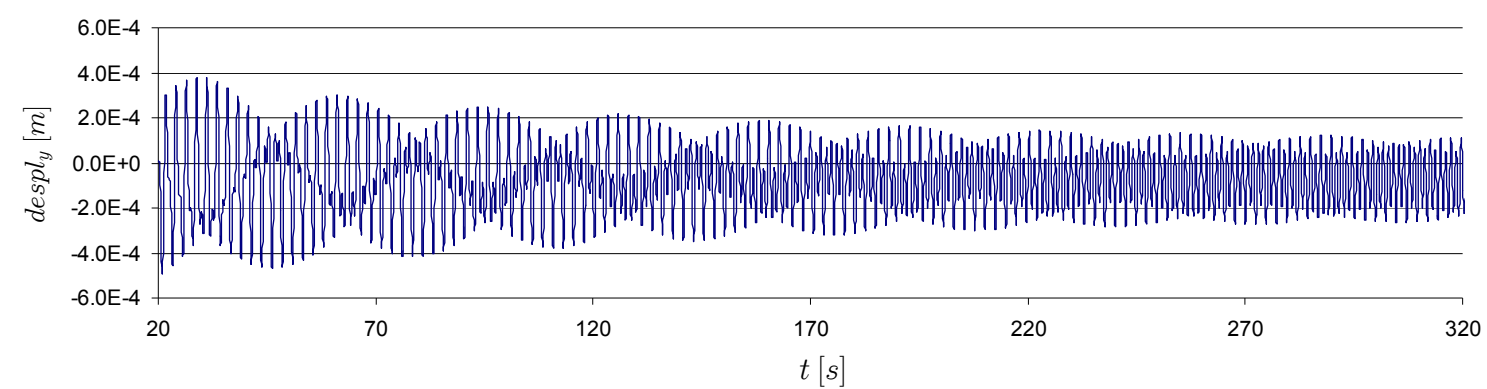

Figura A.82. Vibración transversal de la estructura

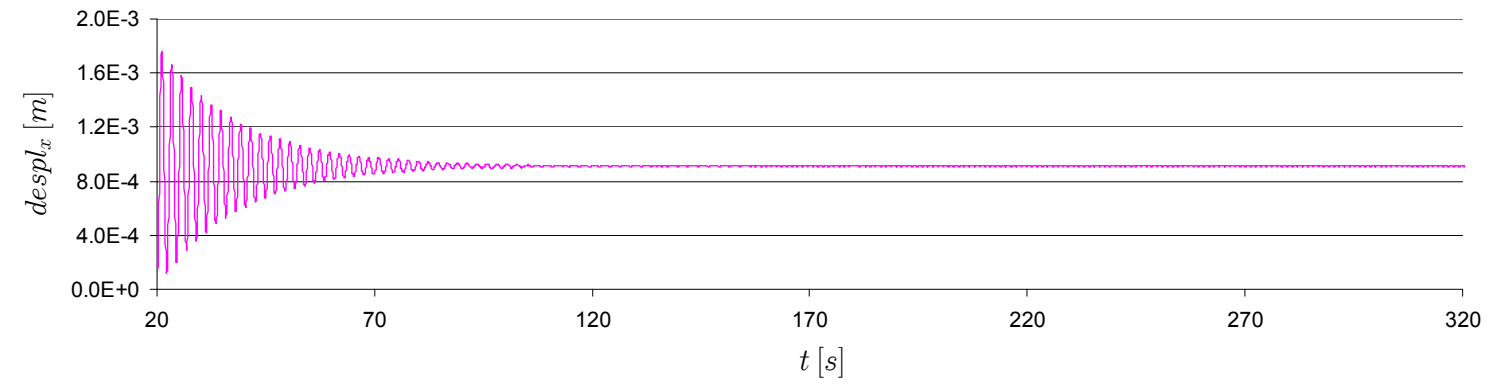

Figura A.83. Vibración en la dirección del flujo de la estructura

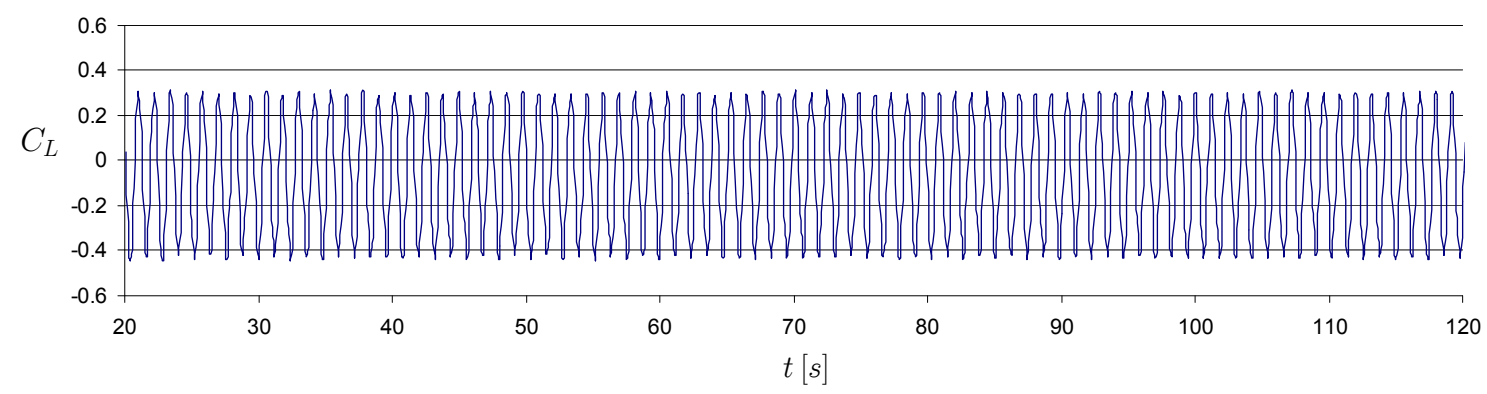

Figura A.84. Oscilación del coeficiente de sustentación

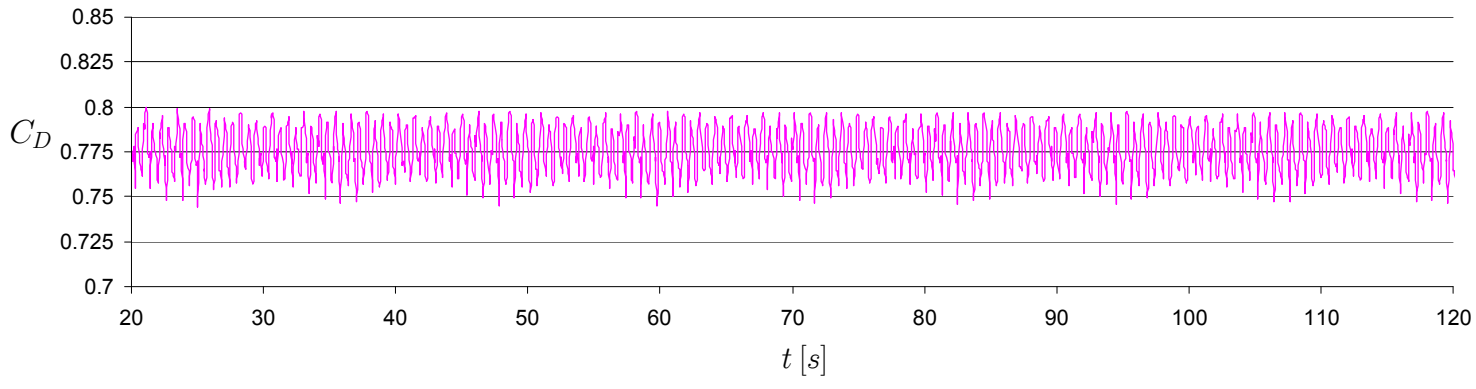

Figura A.85. Oscilación del coeficiente de arrastre 
- $U_{r}=10$

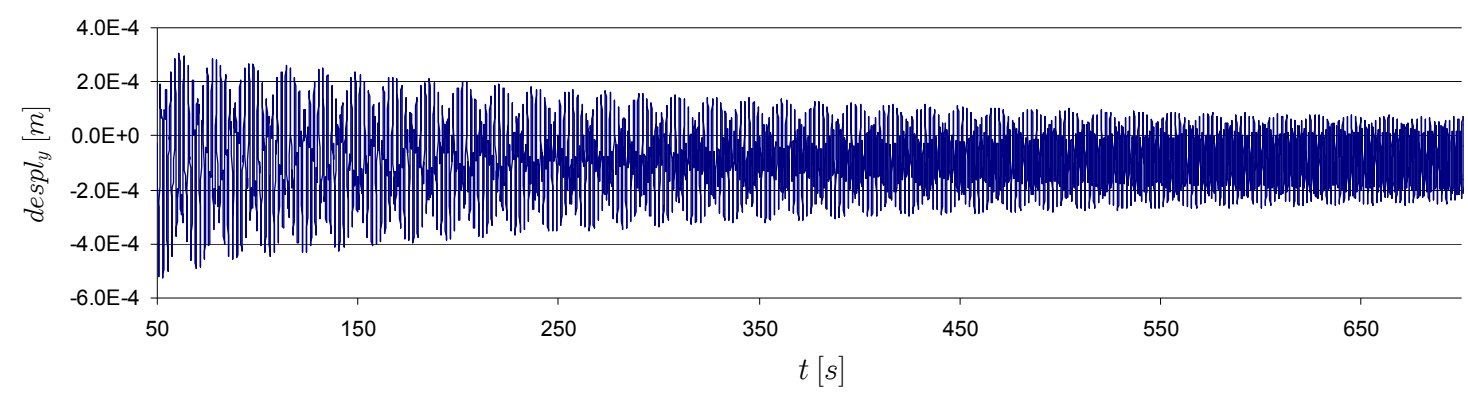

Figura A.86. Vibración transversal de la estructura

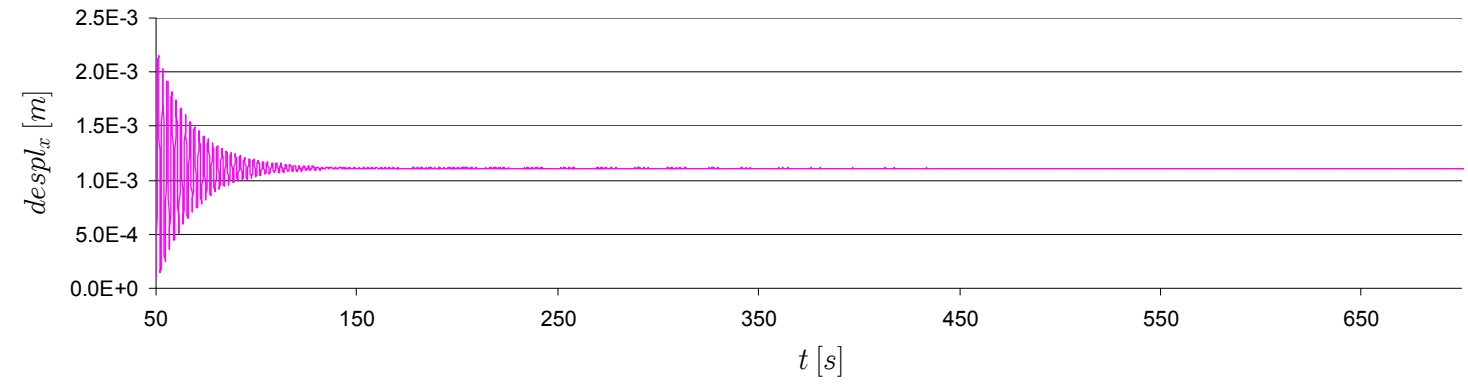

Figura A.87. Vibración en la dirección del flujo de la estructura

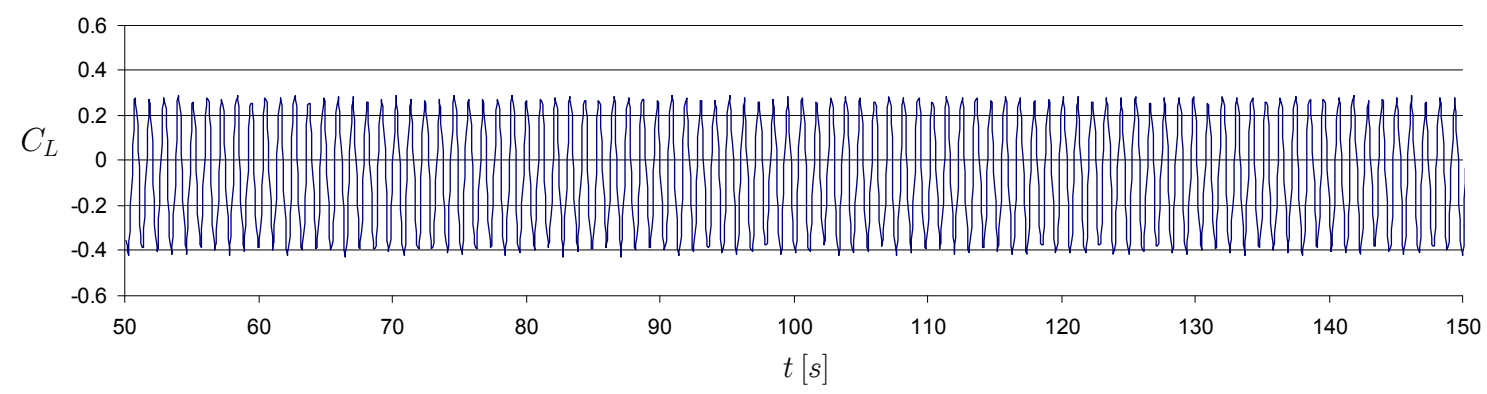

Figura A.88. Oscilación del coeficiente de sustentación

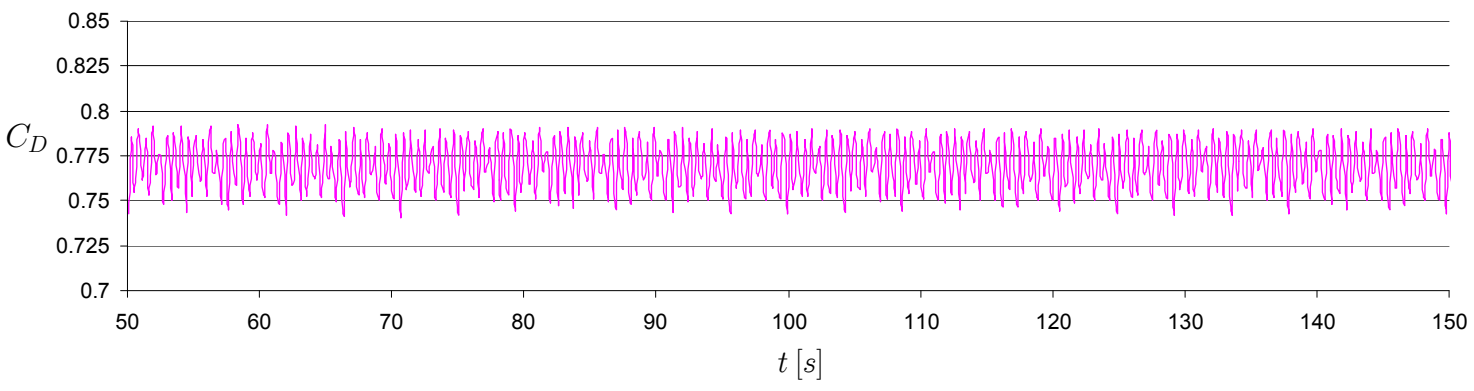

Figura A.89. Oscilación del coeficiente de arrastre 
- $U_{r}=11$

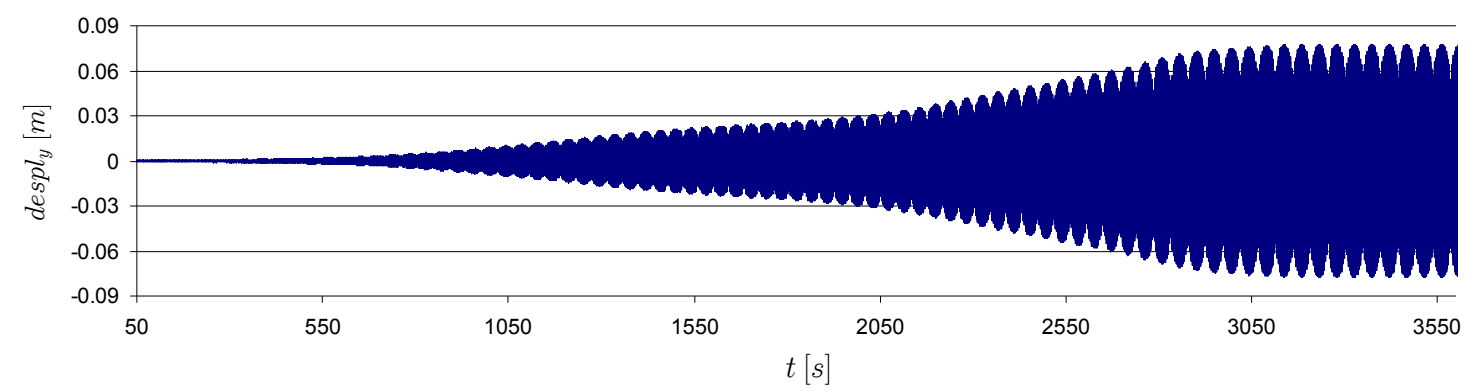

Figura A.90. Vibración transversal de la estructura

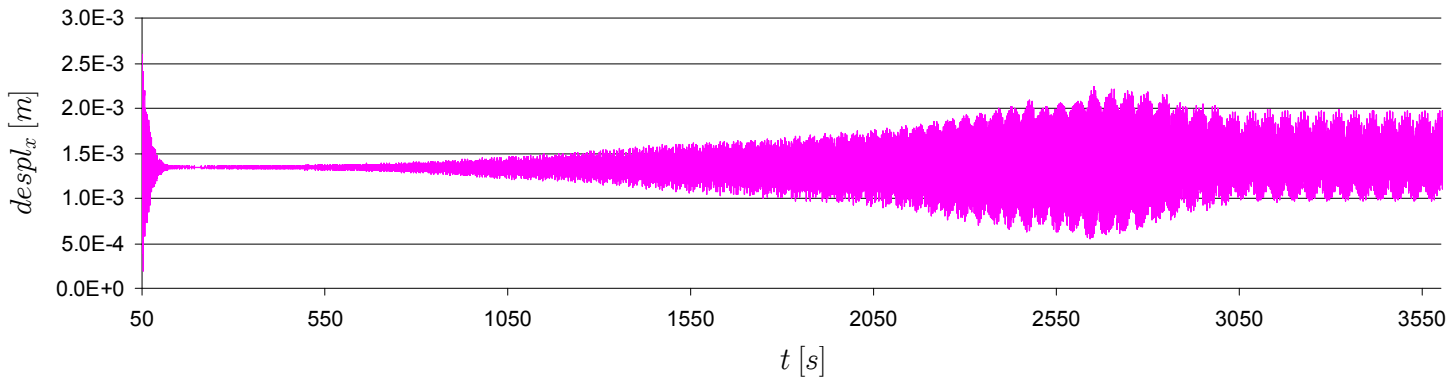

Figura A.91. Vibración en la dirección del flujo de la estructura

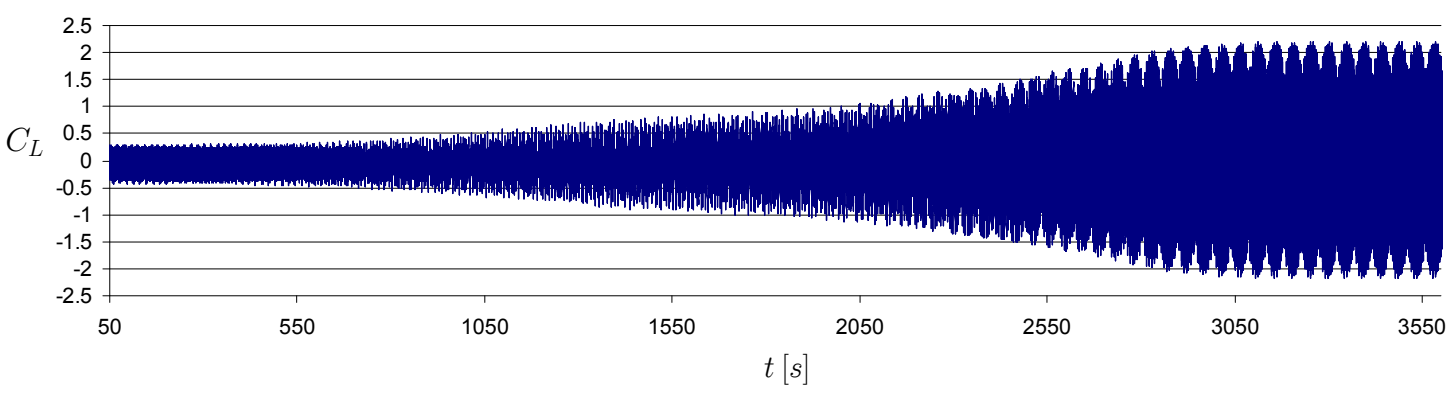

Figura A.92. Oscilación del coeficiente de sustentación

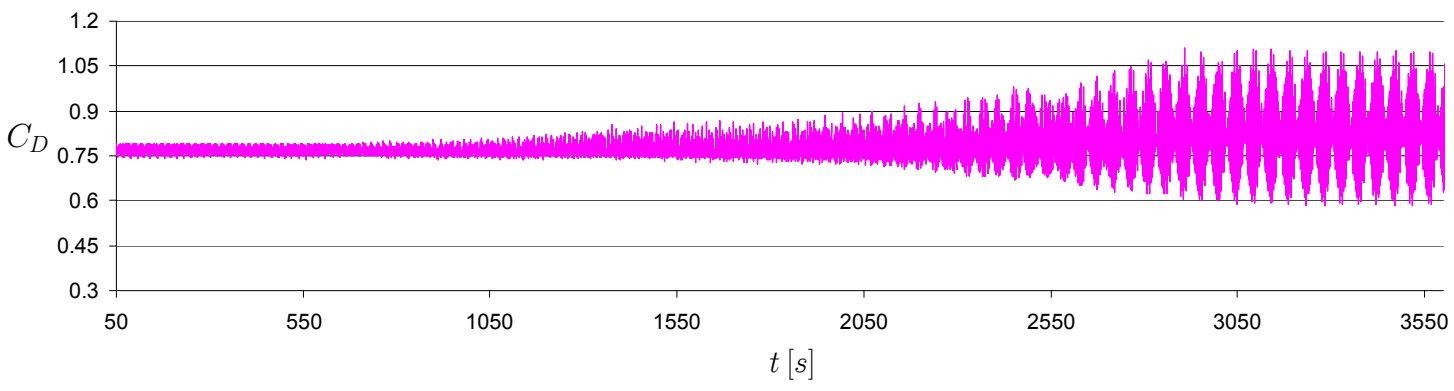

Figura A.93. Oscilación del coeficiente de arrastre 


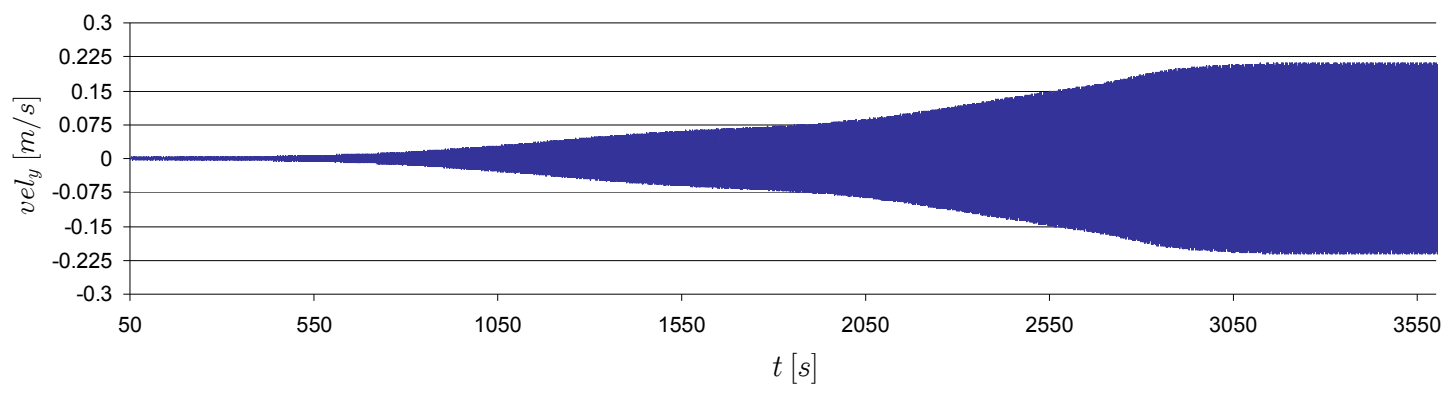

Figura A.94. Componente de la velocidad en la dirección transversal

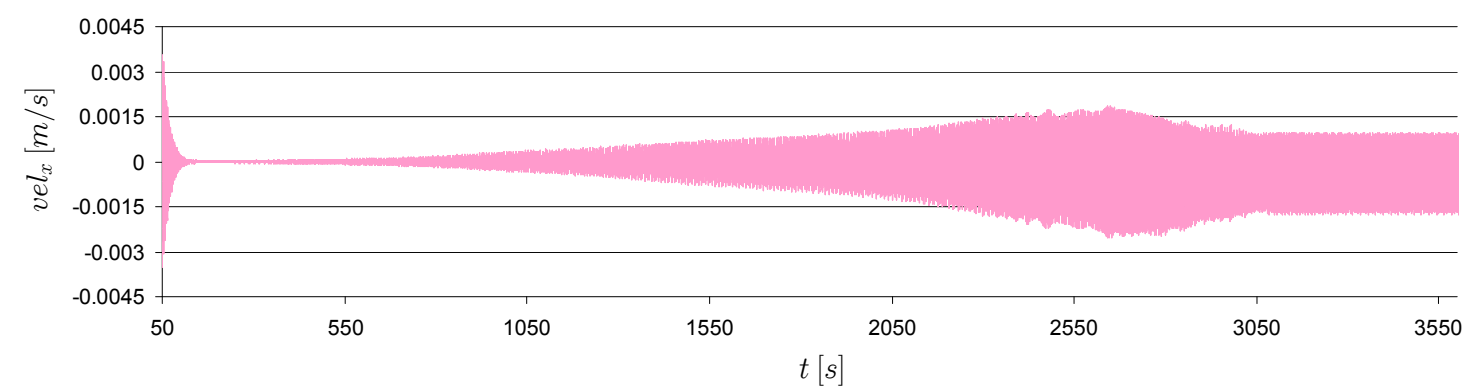

Figura A.95. Componente de la velocidad en la dirección del flujo

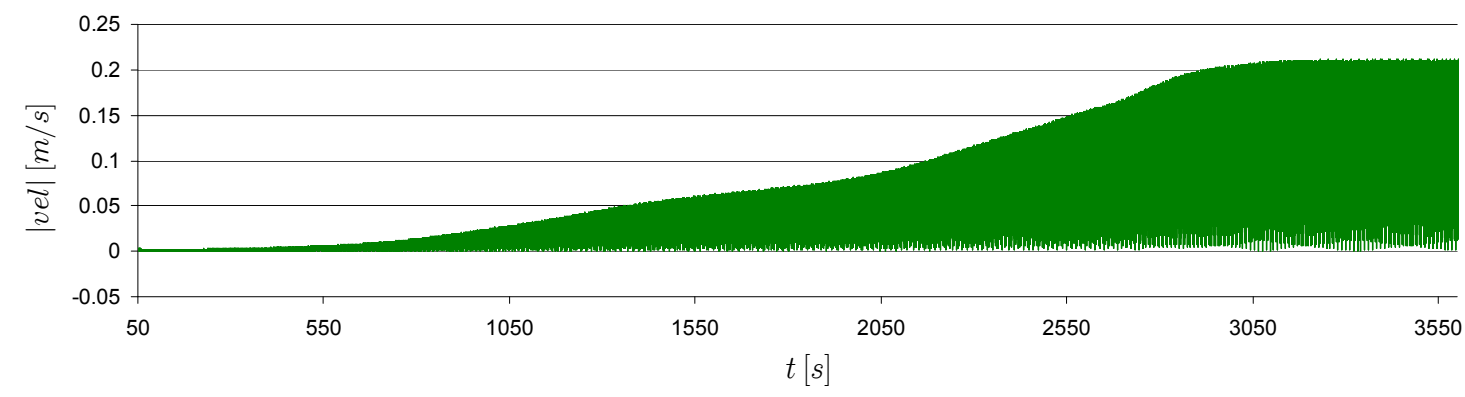

Figura A.96. Módulo de la velocidad 
- $U_{r}=13$

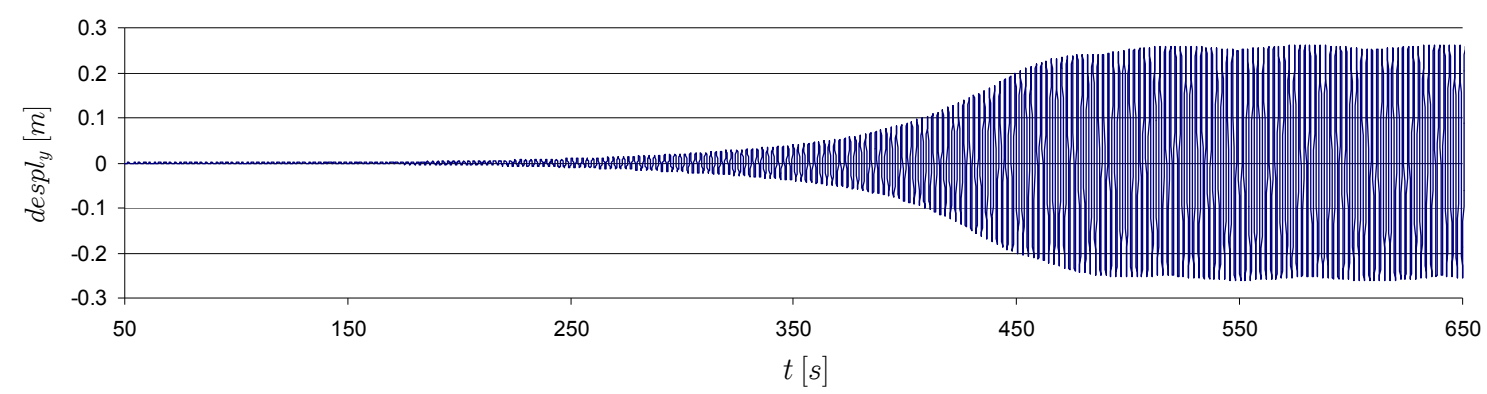

Figura A.97. Vibración transversal de la estructura

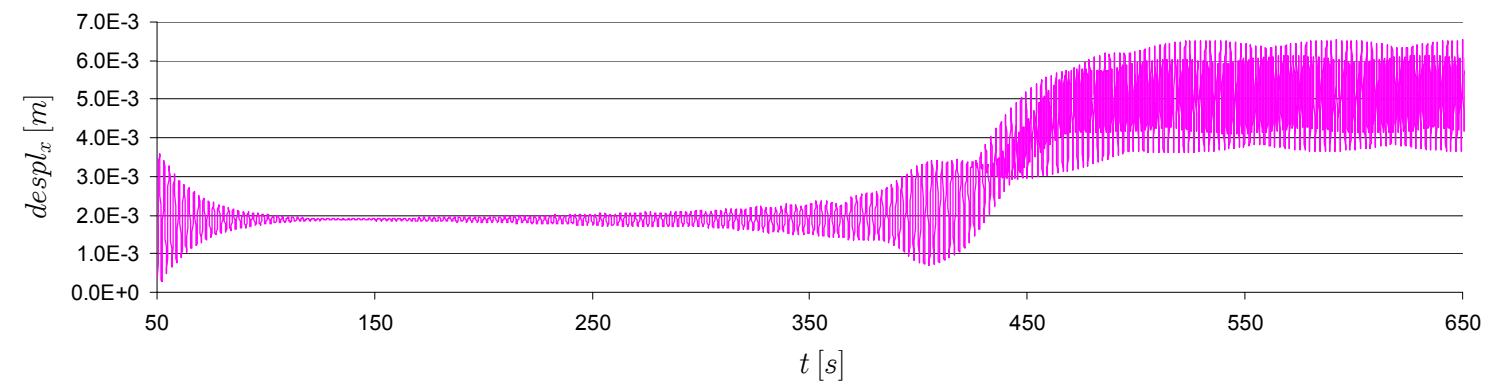

Figura A.98. Vibración en la dirección del flujo de la estructura

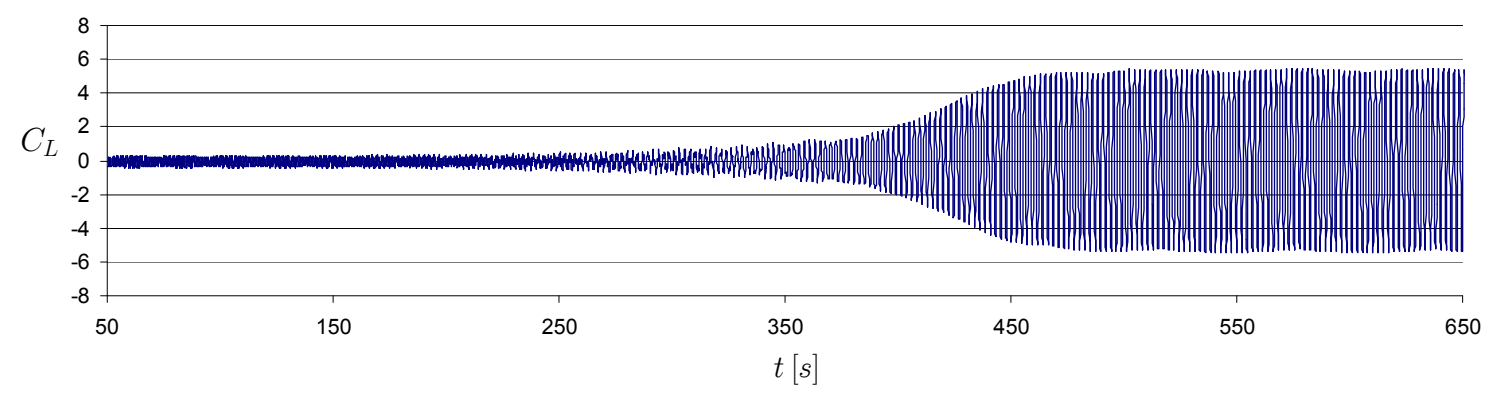

Figura A.99. Oscilación del coeficiente de sustentación

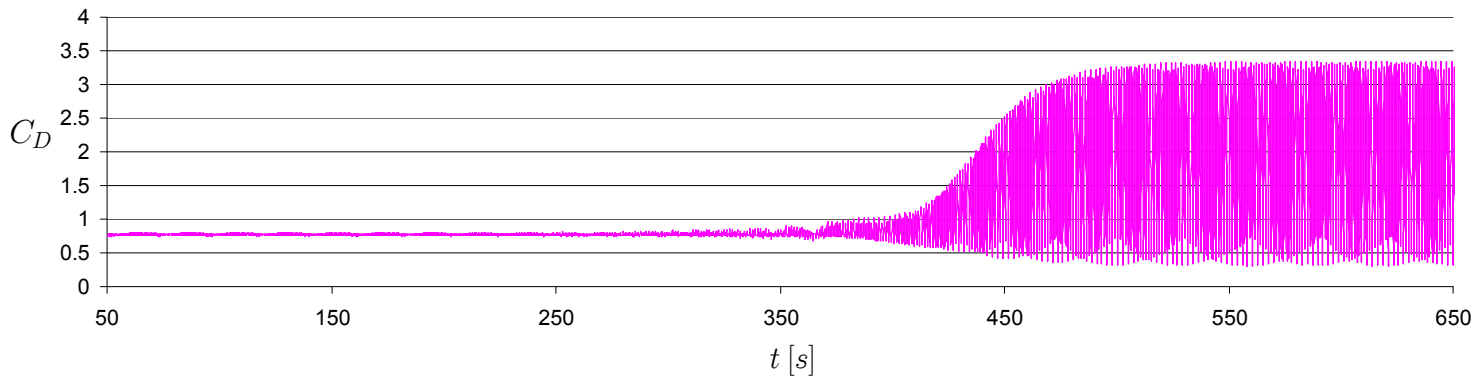

Figura A.100. Oscilación del coeficiente de arrastre 


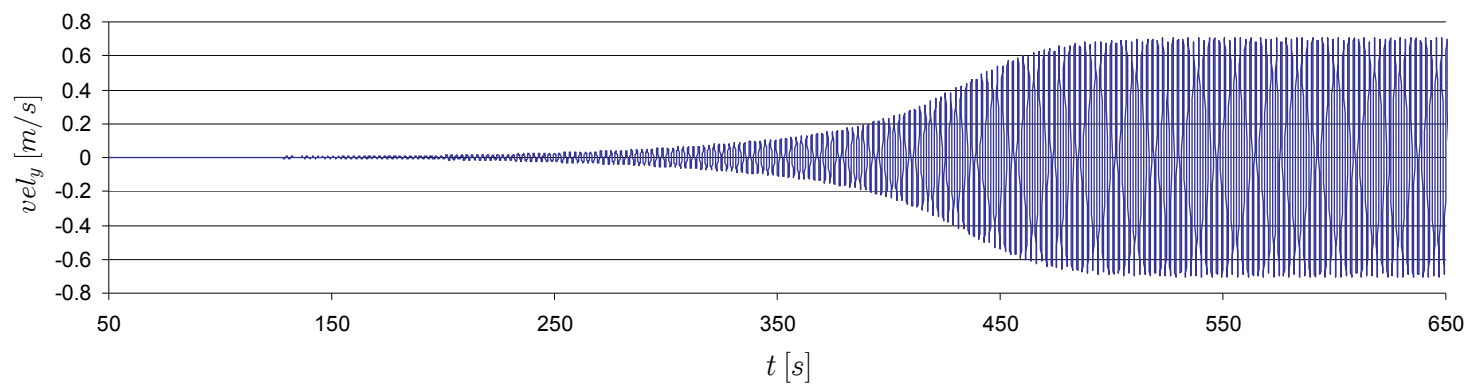

Figura A.101. Componente de la velocidad en la dirección transversal

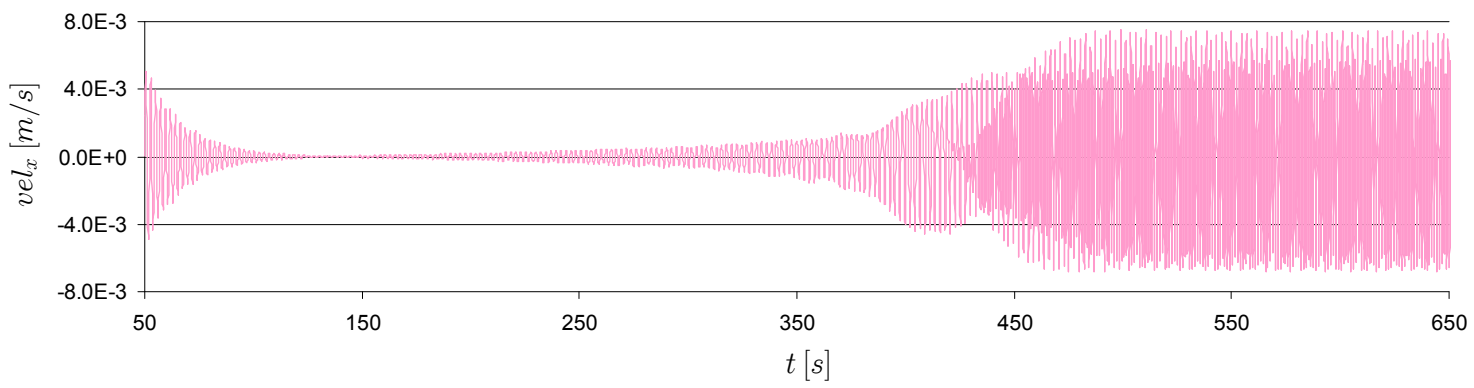

Figura A.102. Componente de la velocidad en la dirección del flujo

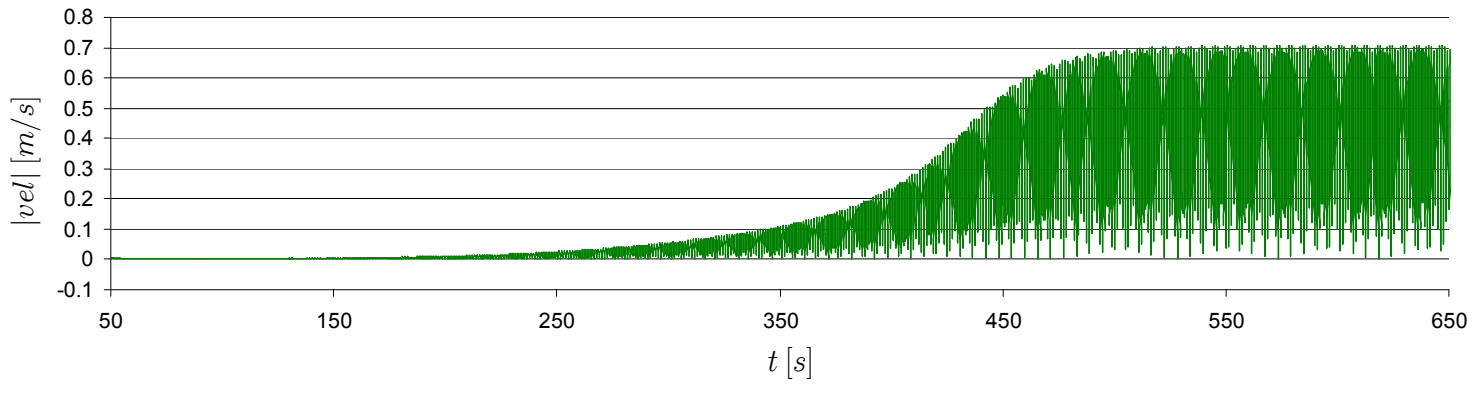

Figura A.103. Módulo de la velocidad 
- $U_{r}=15$

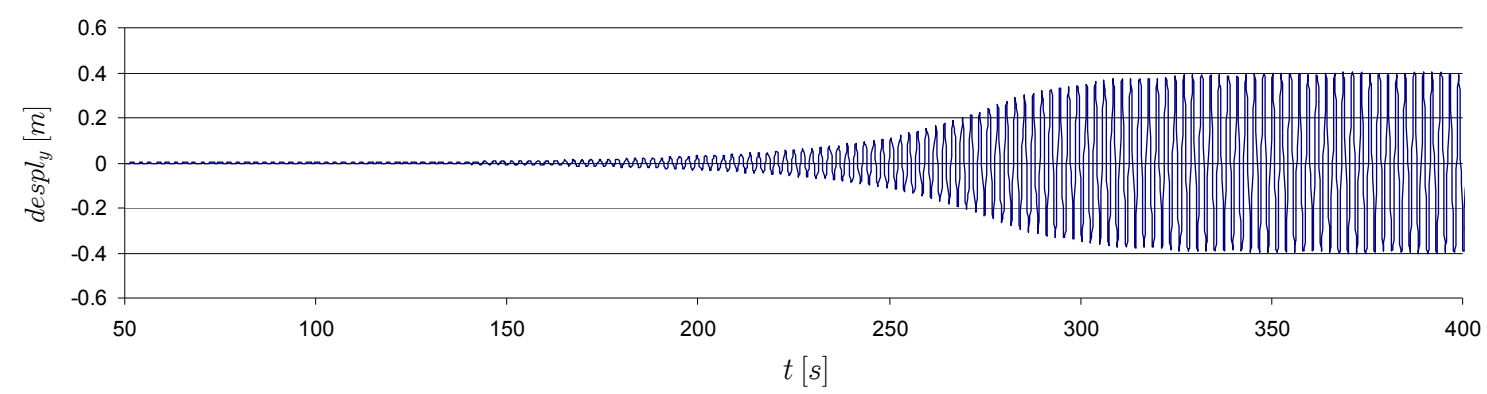

Figura A.104. Vibración transversal de la estructura

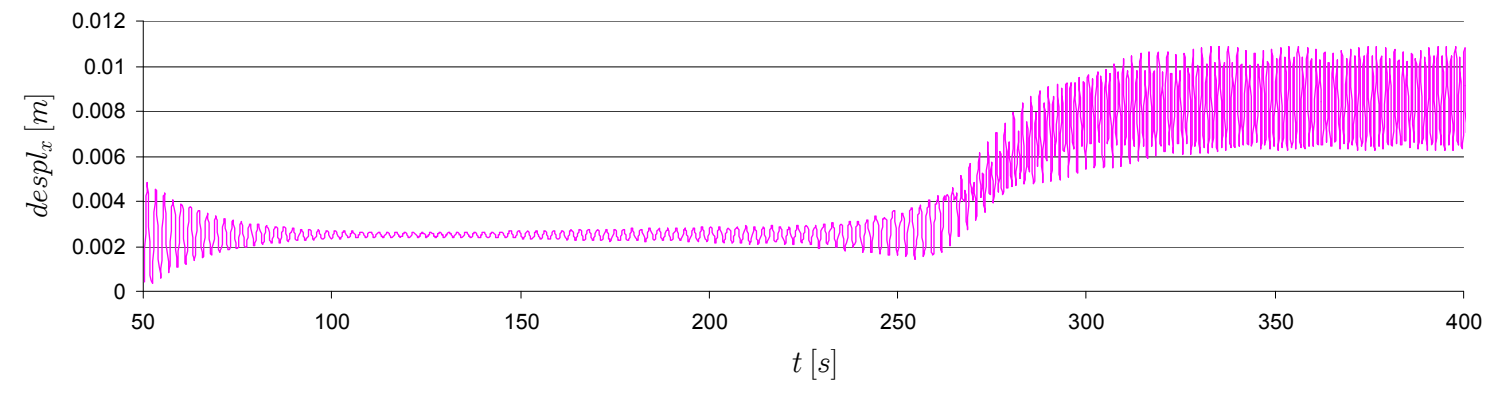

Figura A.105. Vibración en la dirección del flujo de la estructura

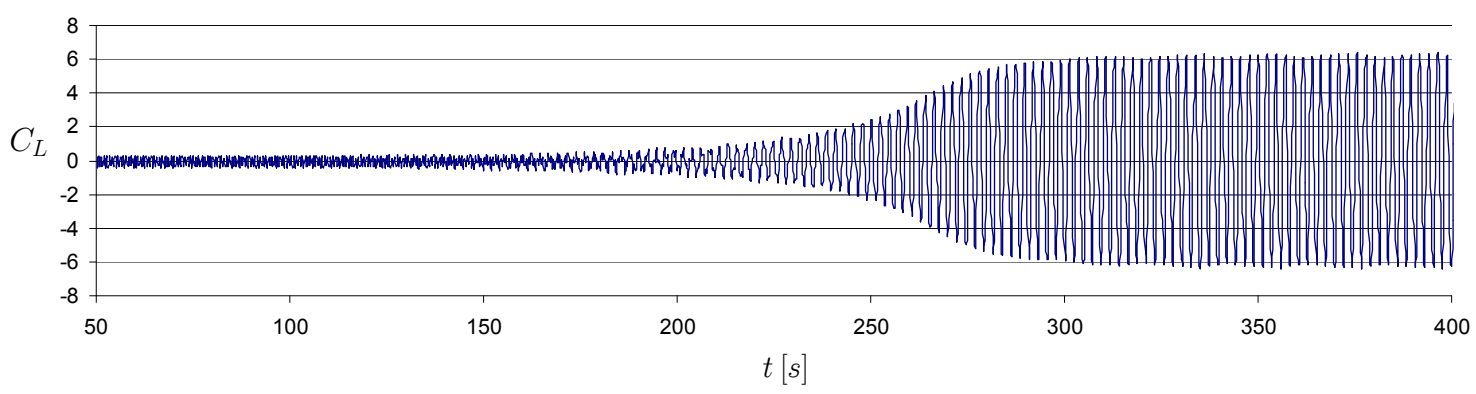

Figura A.106. Oscilación del coeficiente de sustentación

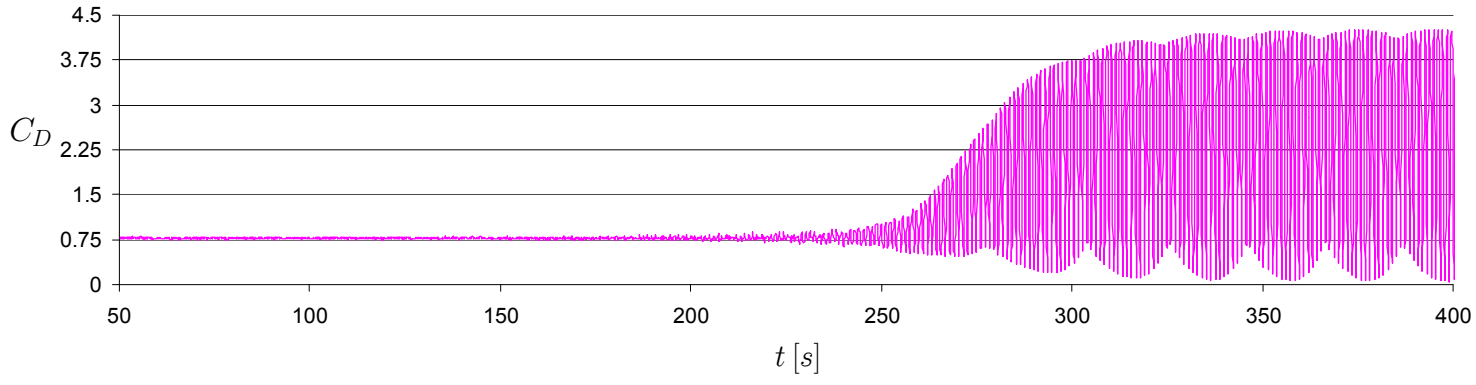

Figura A.107. Oscilación del coeficiente de arrastre 


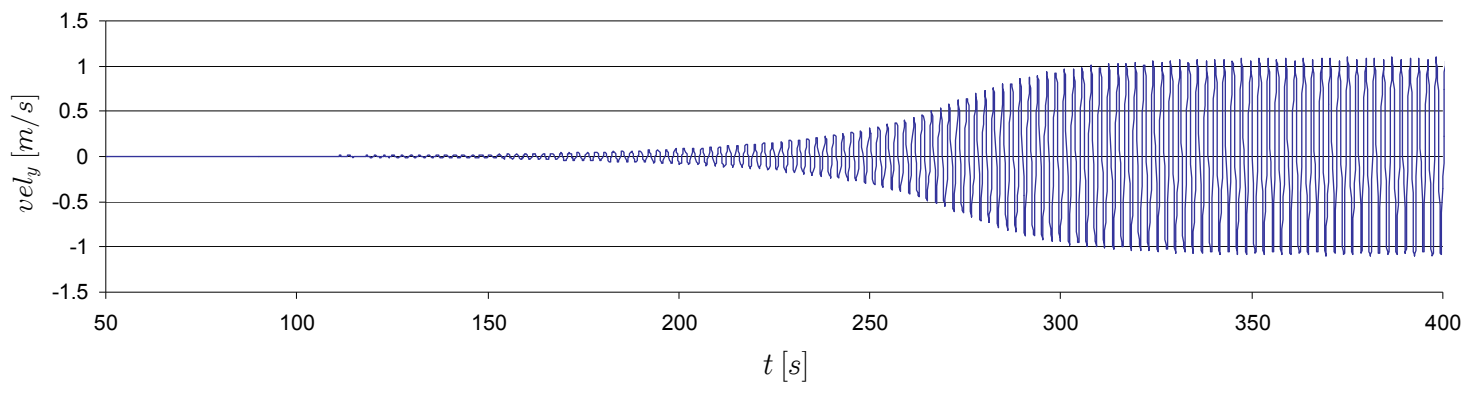

Figura A.108. Componente de la velocidad en la dirección transversal

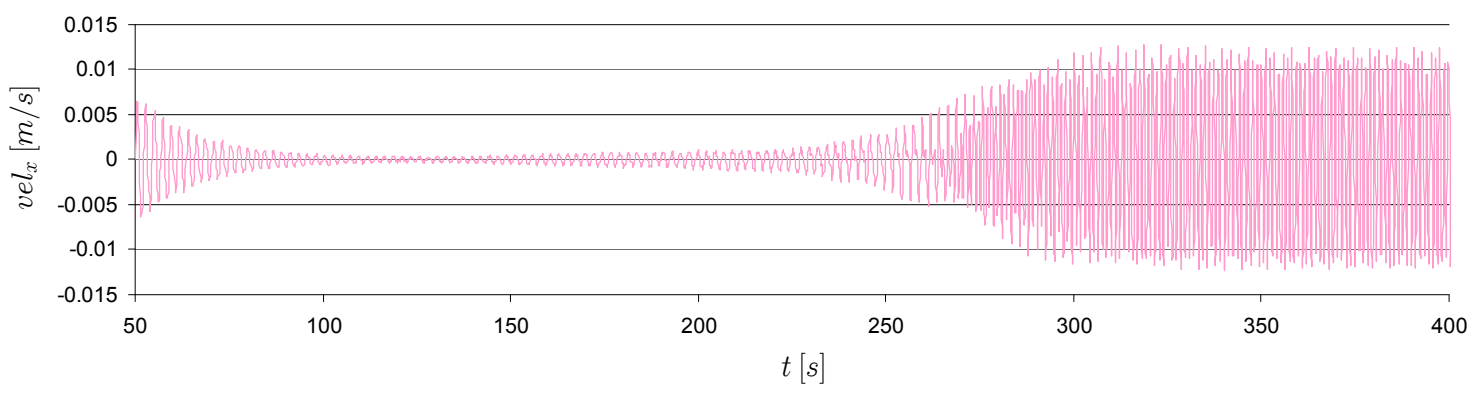

Figura A.109. Componente de la velocidad en la dirección del flujo

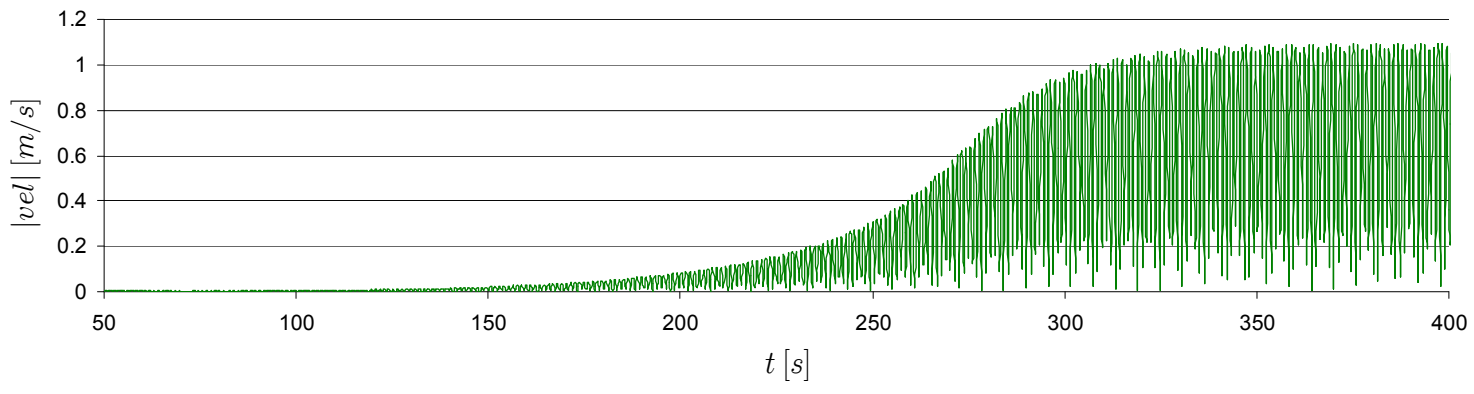

Figura A.110. Módulo de la velocidad 
- $U_{r}=17$

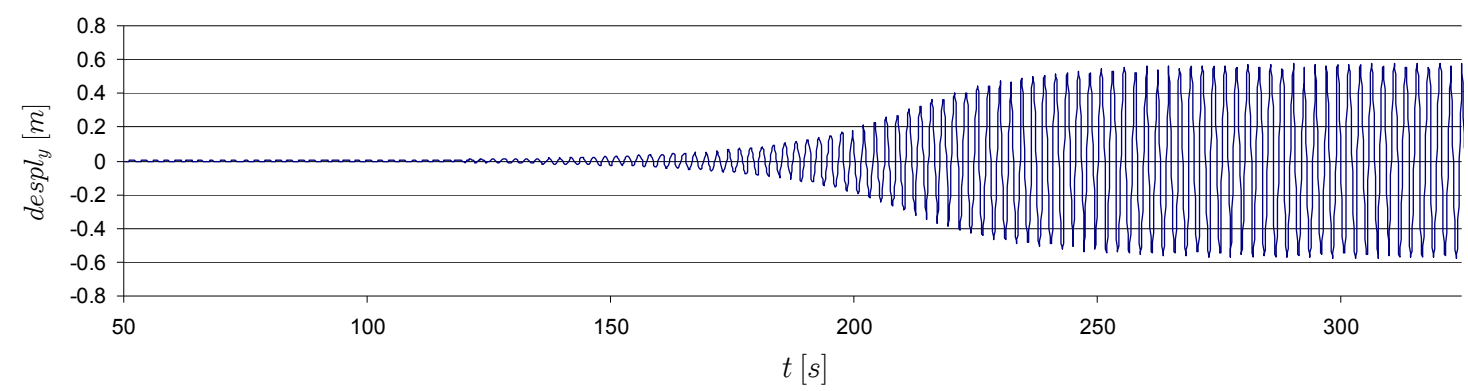

Figura A.111. Vibración transversal de la estructura

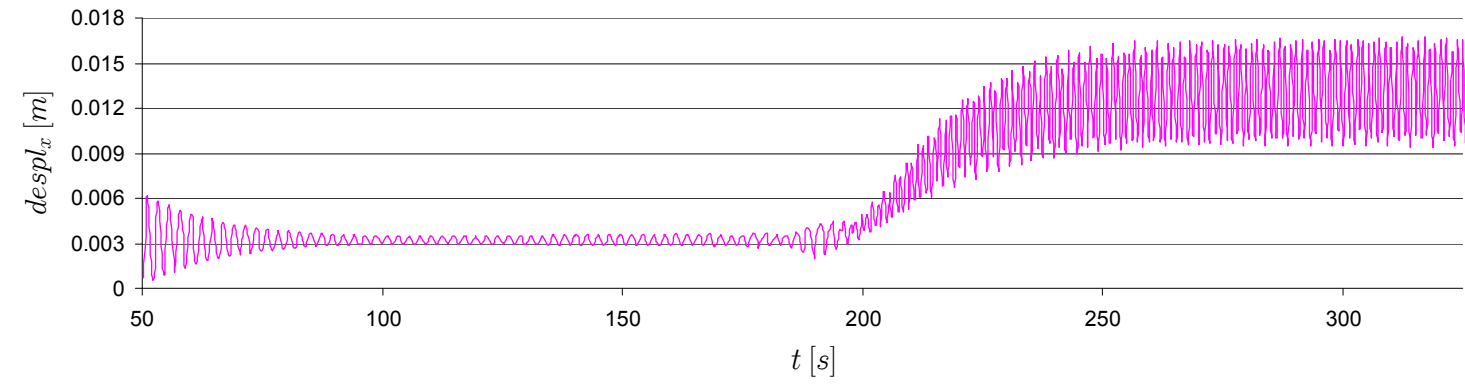

Figura A.112. Vibración en la dirección del flujo de la estructura

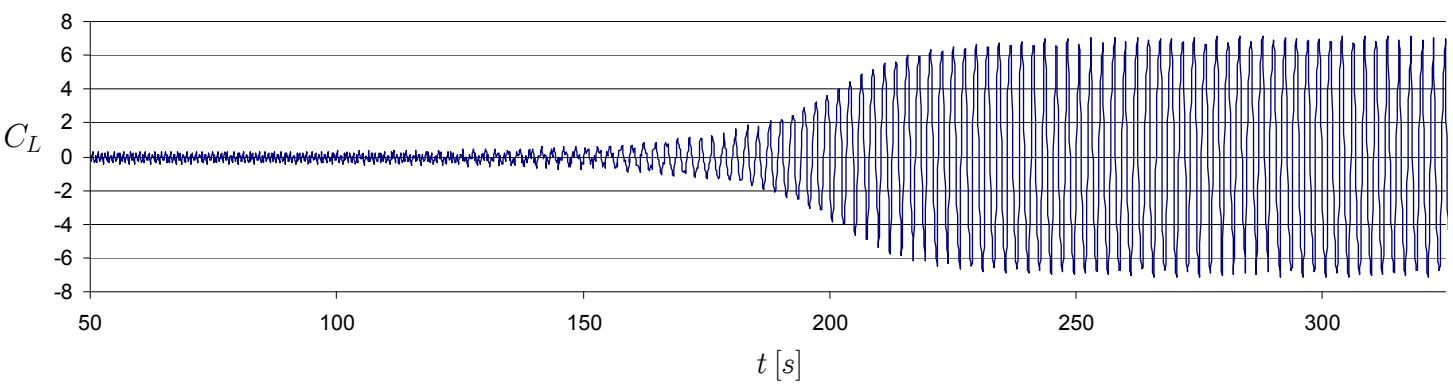

Figura A.113. Oscilación del coeficiente de sustentación

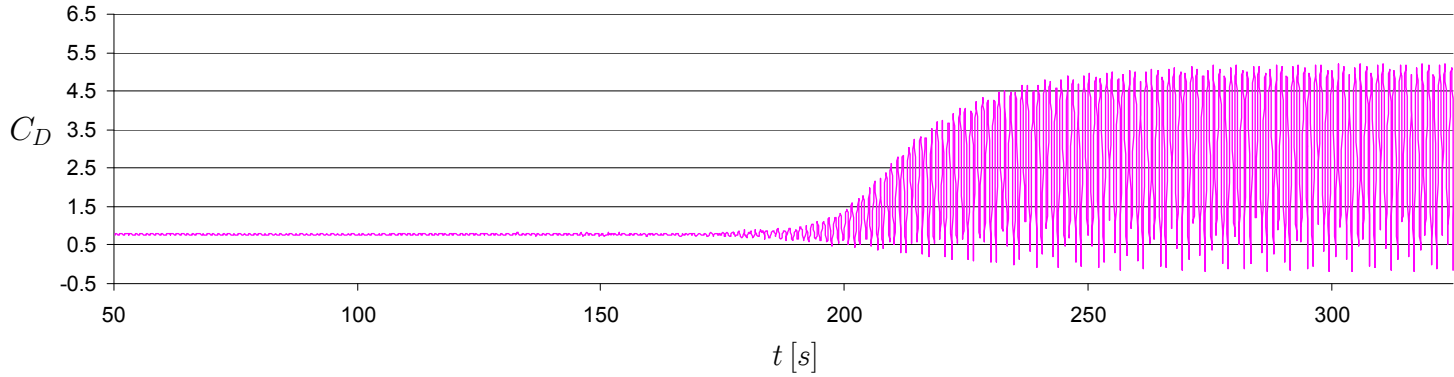

Figura A.114. Oscilación del coeficiente de arrastre 


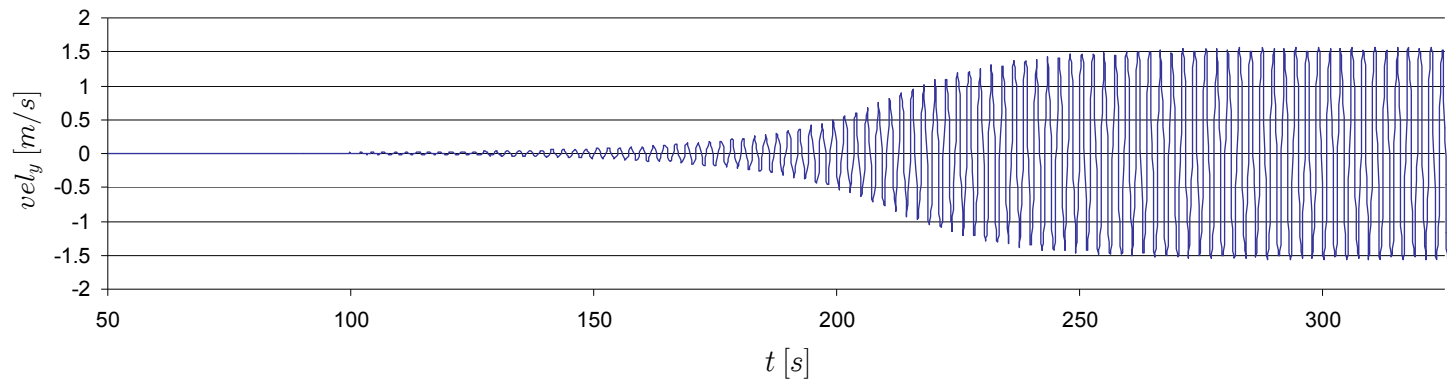

Figura A.115. Componente de la velocidad en la dirección transversal

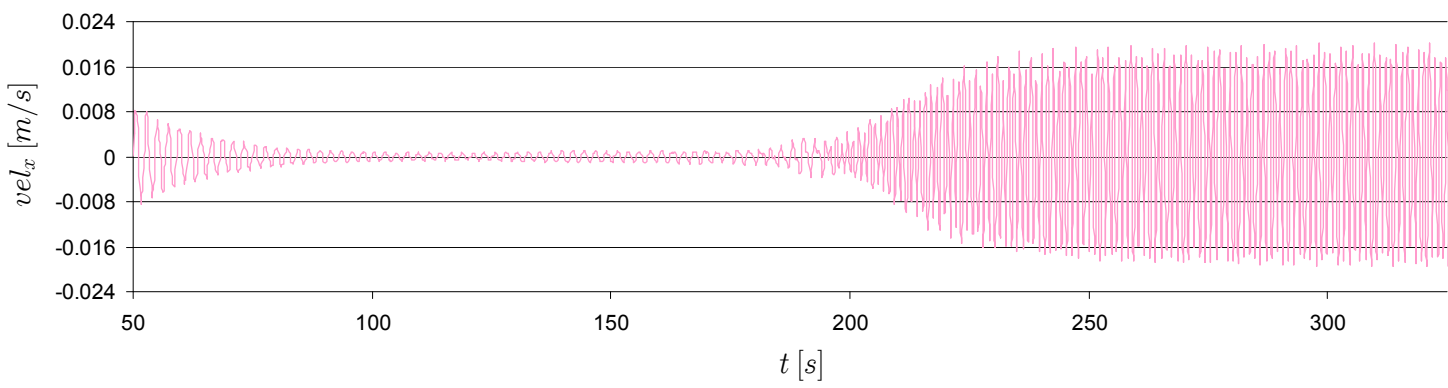

Figura A.116. Componente de la velocidad en la dirección del flujo

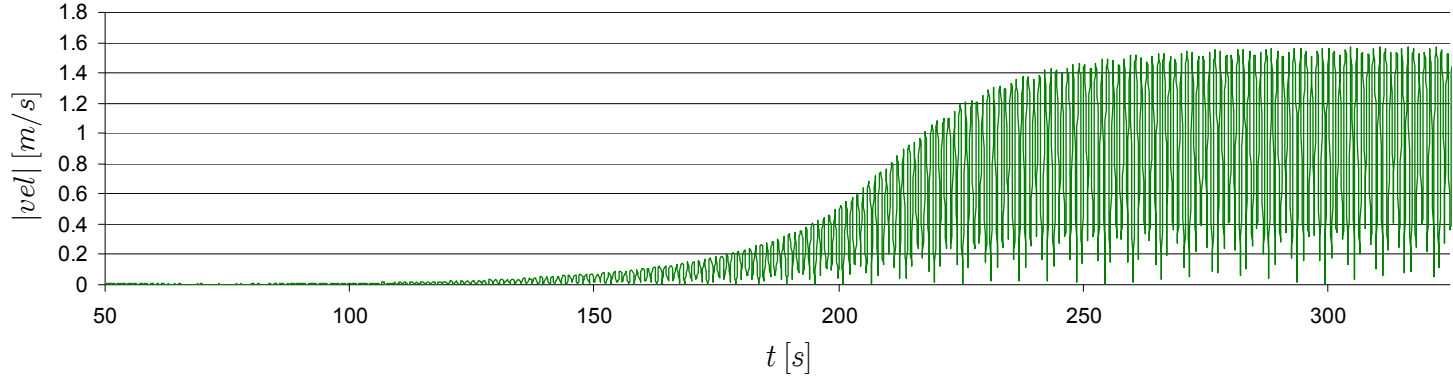

Figura A.117. Módulo de la velocidad 
- $U_{r}=19$

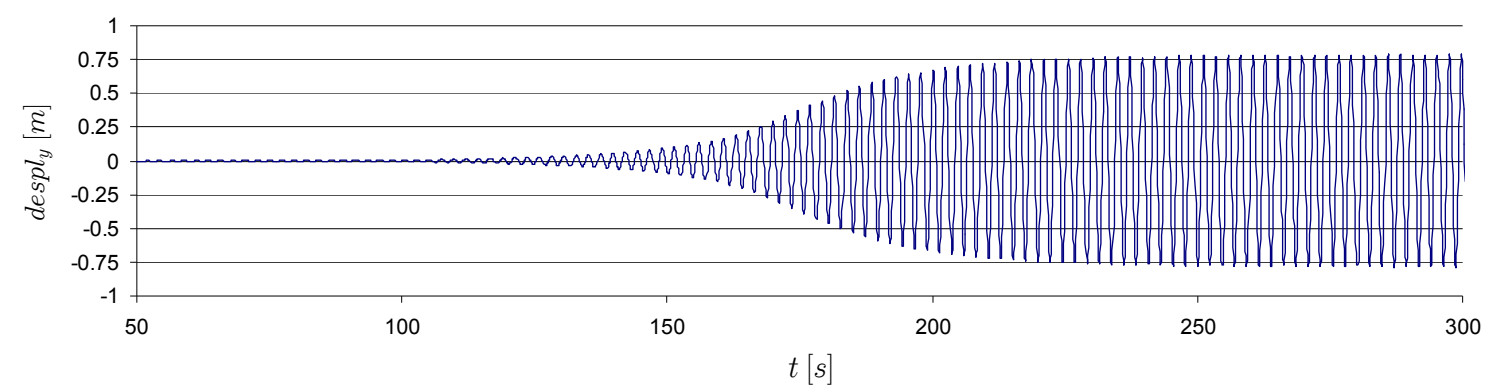

Figura A.118. Vibración transversal de la estructura

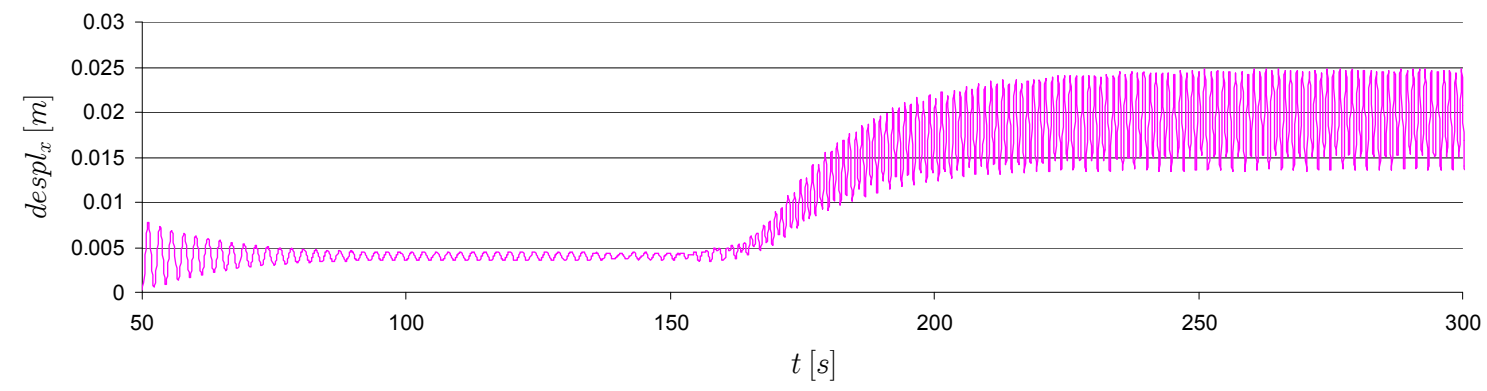

Figura A.119. Vibración en la dirección del flujo de la estructura

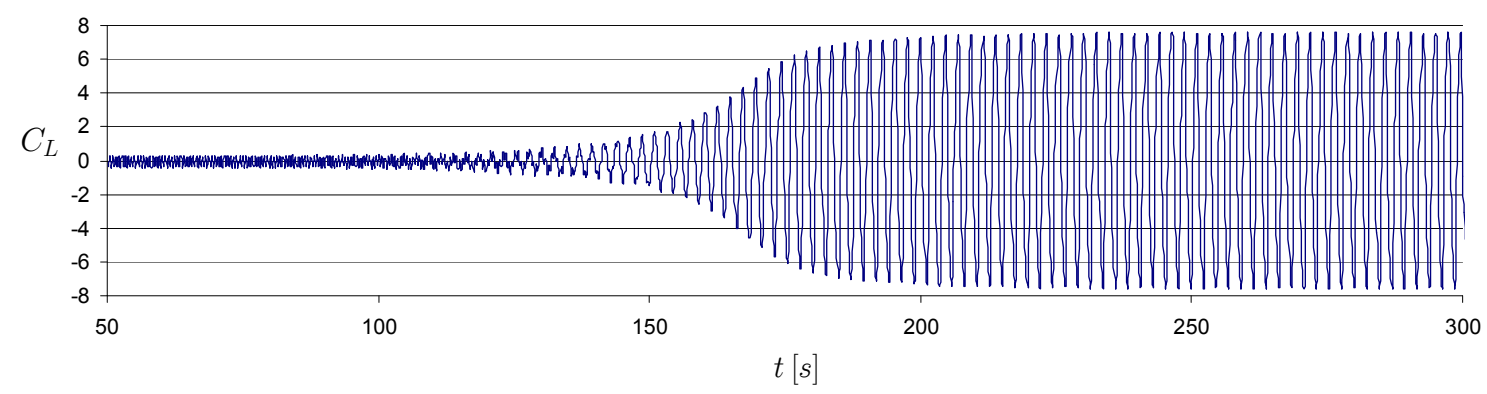

Figura A.120. Oscilación del coeficiente de sustentación

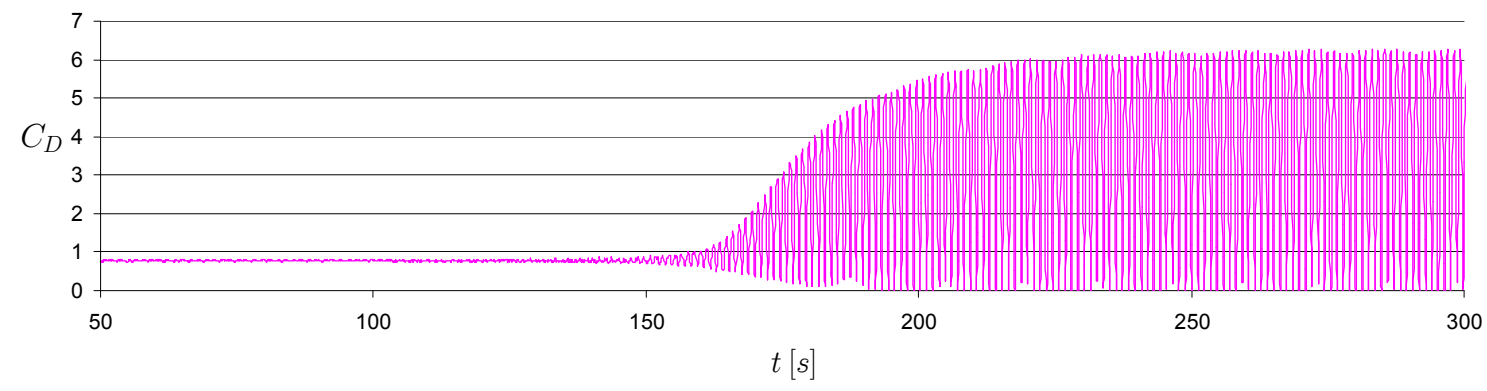

Figura A.121. Oscilación del coeficiente de arrastre 


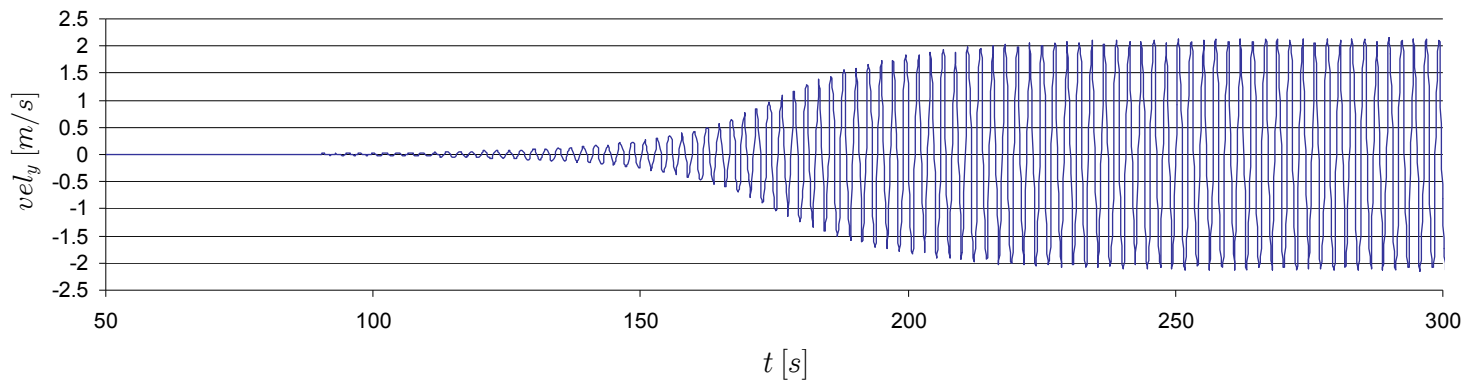

Figura A.122. Componente de la velocidad en la dirección transversal

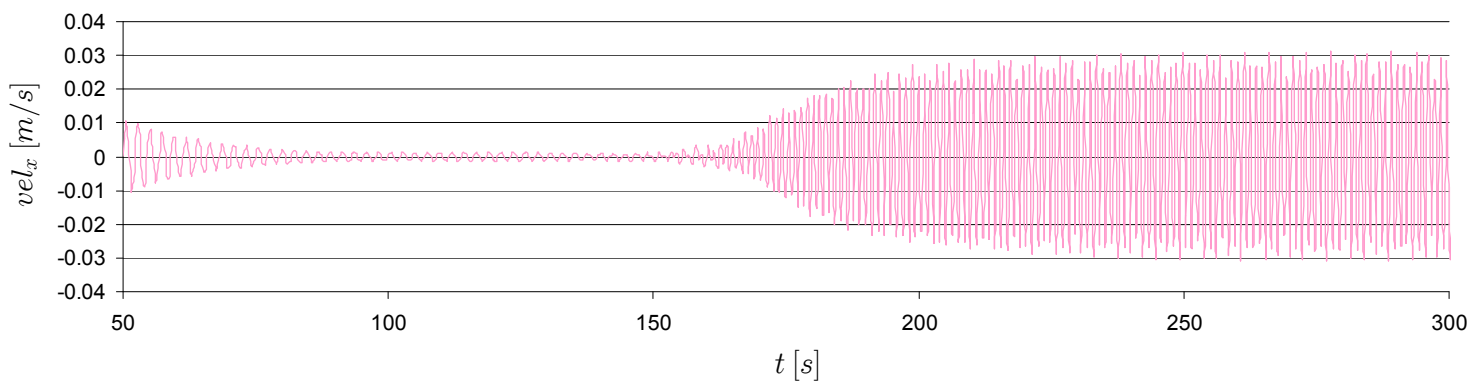

Figura A.123. Componente de la velocidad en la dirección del flujo

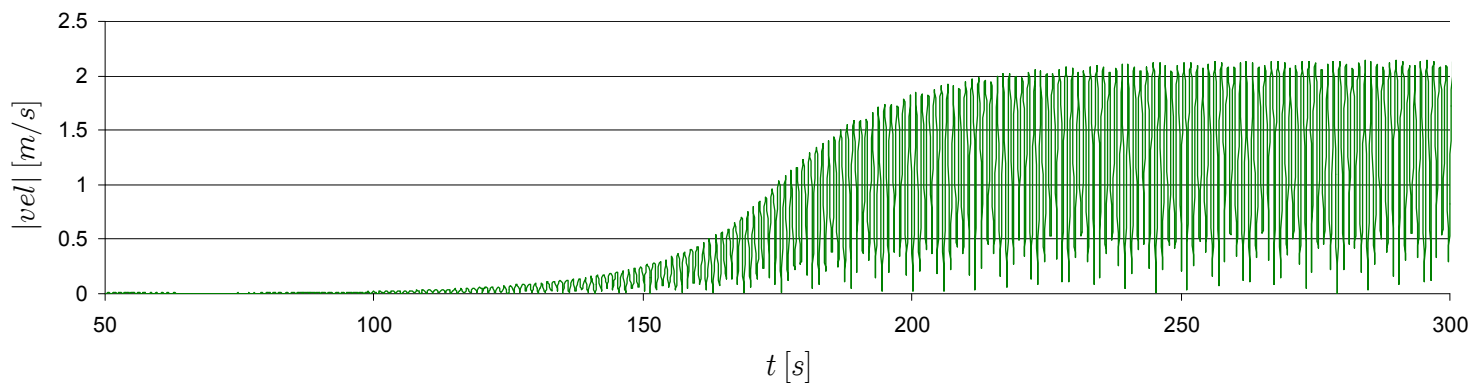

Figura A.124. Módulo de la velocidad 


\section{J.2. Amortiguamiento estructural 0.05}

- $U_{r}=3.5$

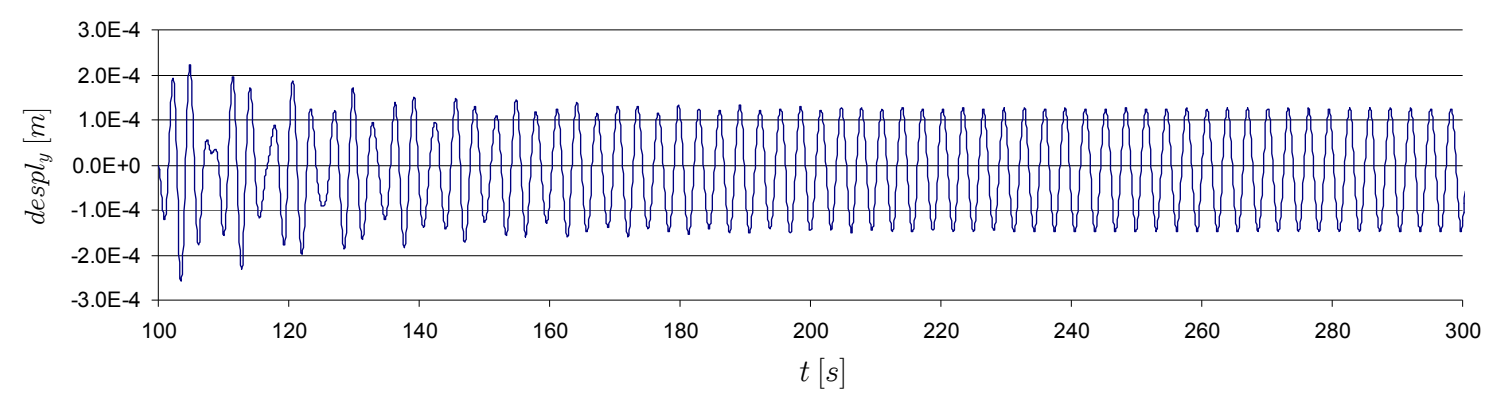

Figura A.125. Vibración transversal de la estructura

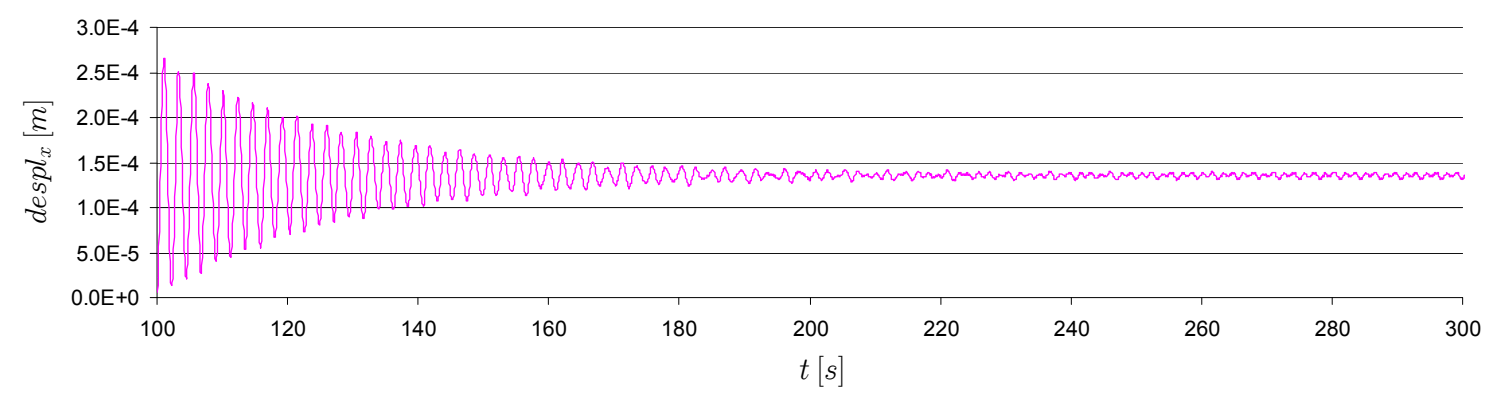

Figura A.126. Vibración en la dirección del flujo de la estructura

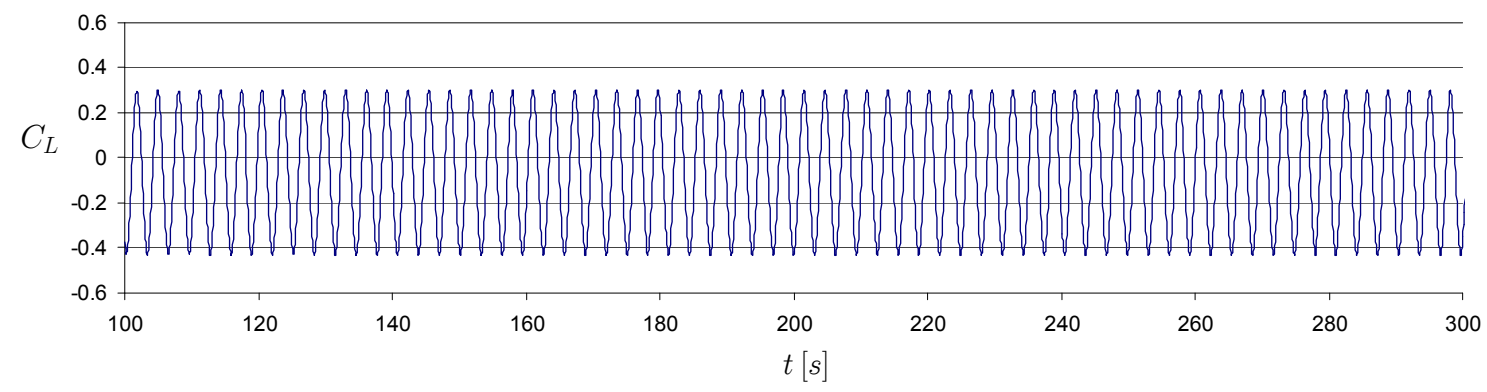

Figura A.127. Oscilación del coeficiente de sustentación

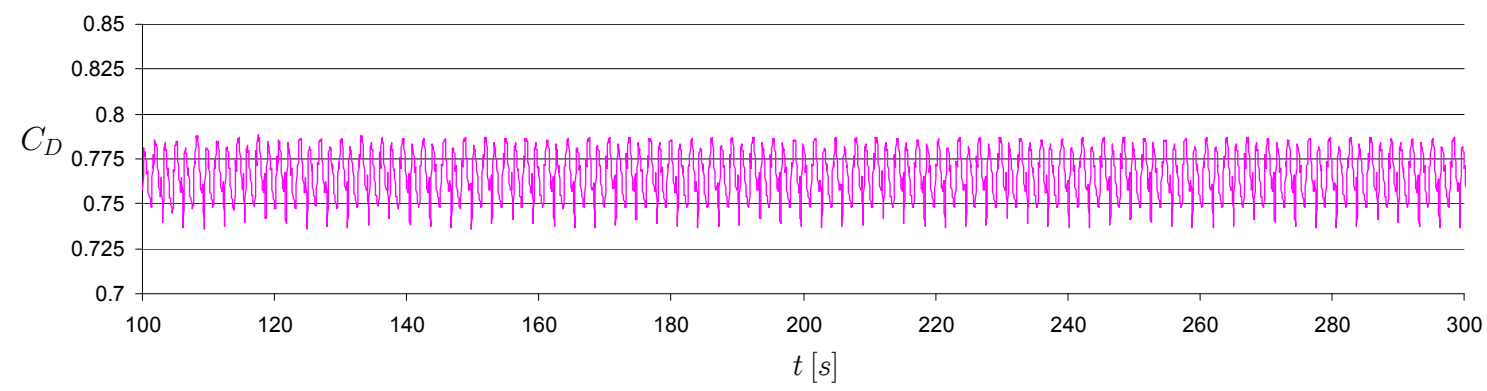

Figura A.128. Oscilación del coeficiente de arrastre 
- $U_{r}=4$

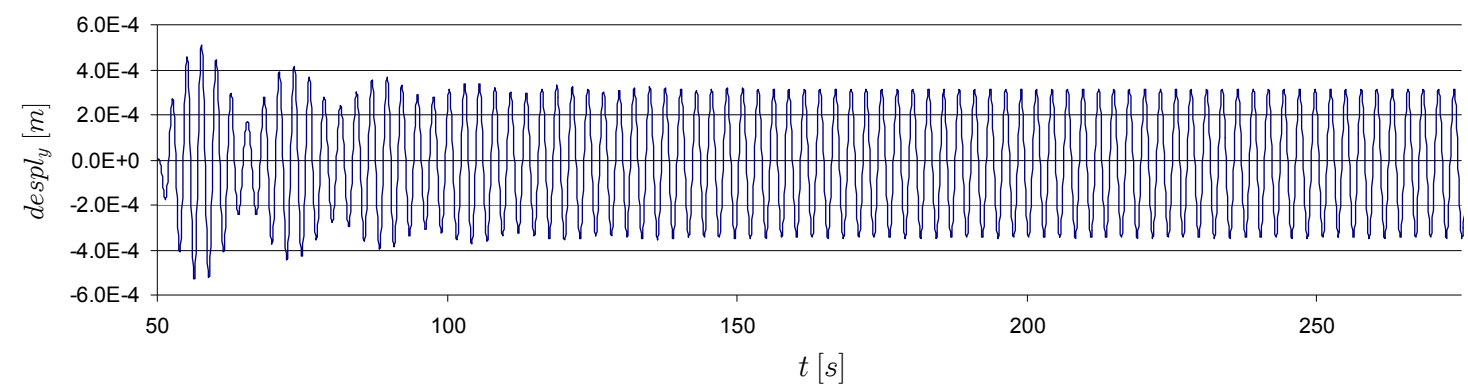

Figura A.129. Vibración transversal de la estructura

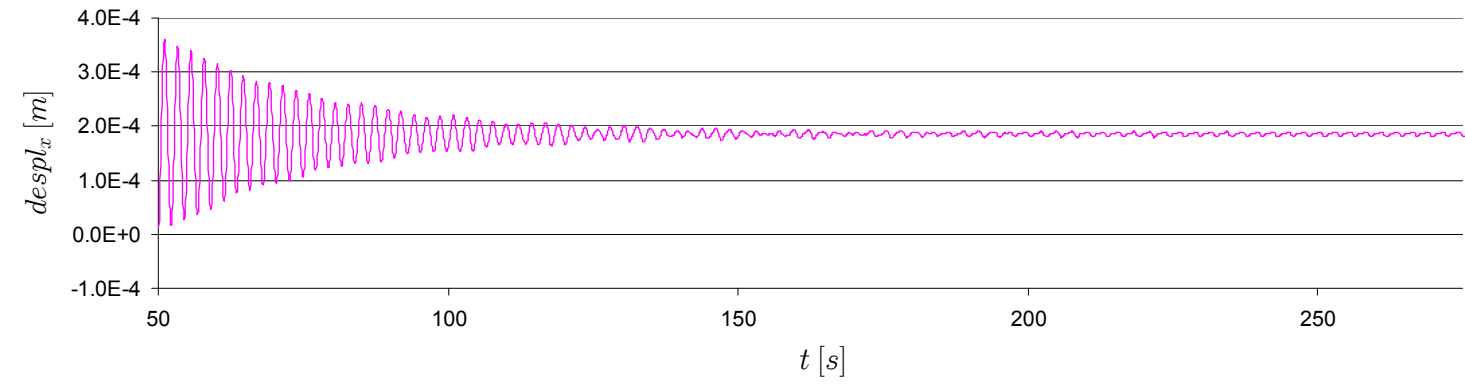

Figura A.130. Vibración en la dirección del flujo de la estructura

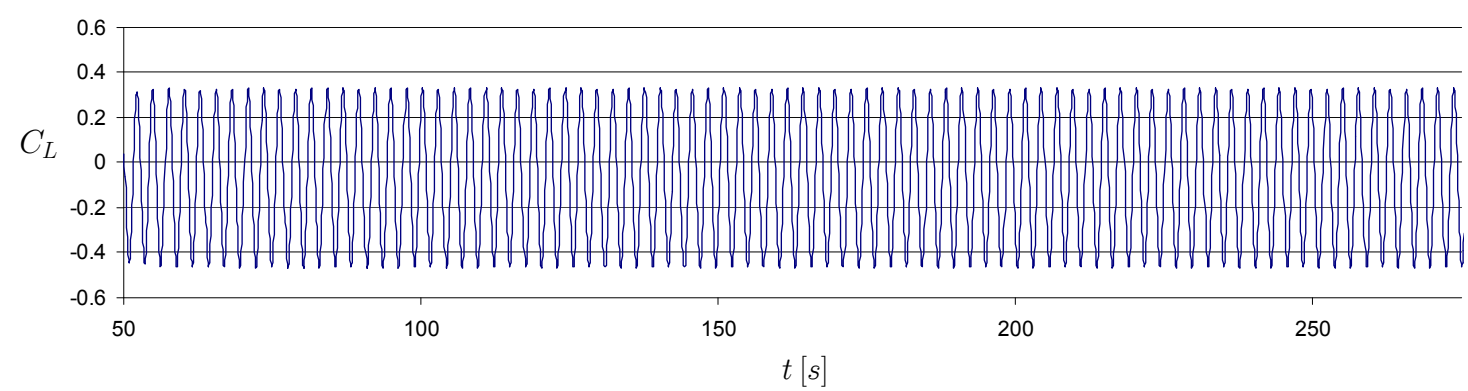

Figura A.131. Oscilación del coeficiente de sustentación

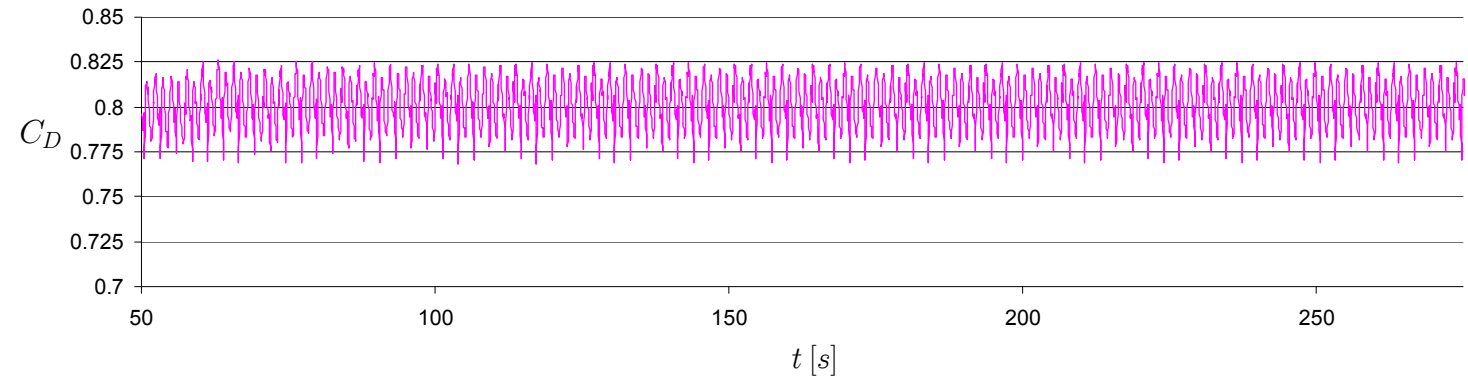

Figura A.132. Oscilación del coeficiente de arrastre 
- $U_{r}=4.5$

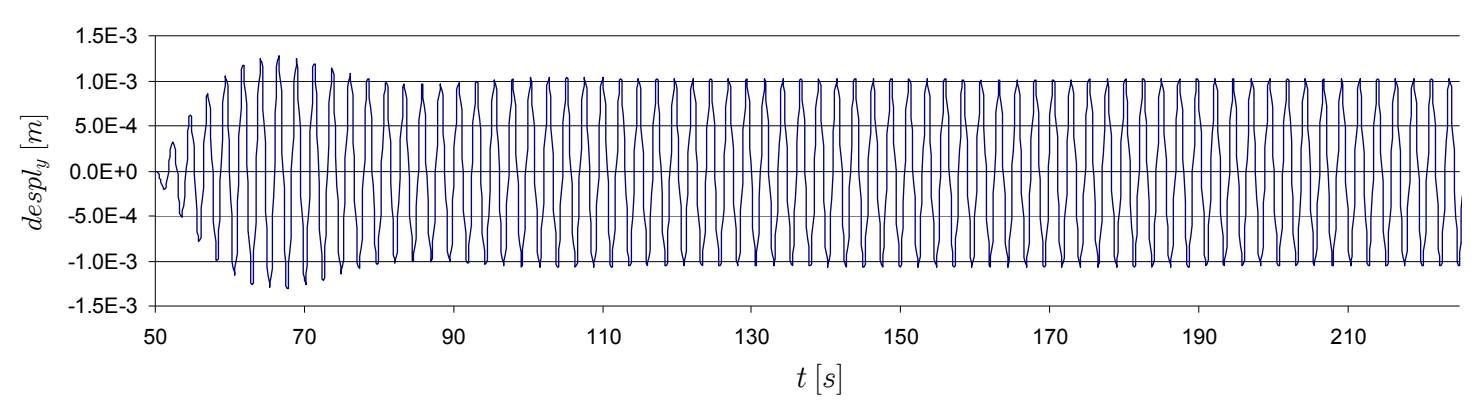

Figura A.133. Vibración transversal de la estructura

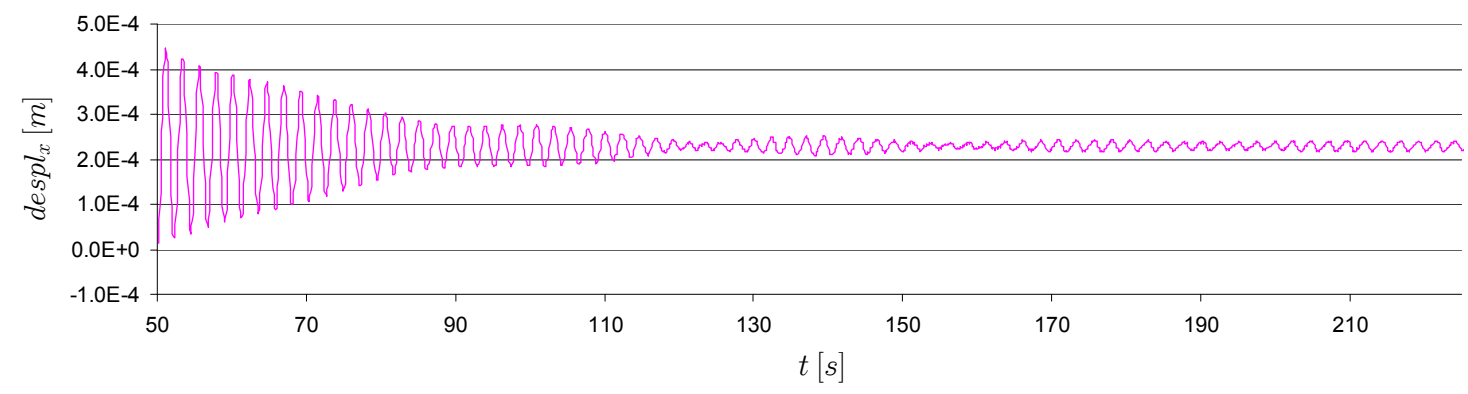

Figura A.134. Vibración en la dirección del flujo de la estructura

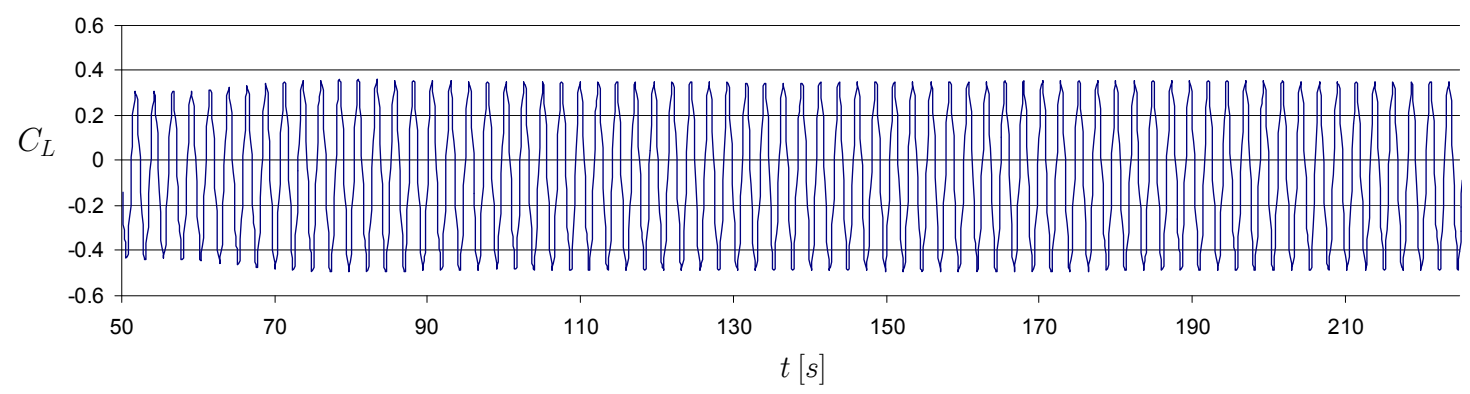

Figura A.135. Oscilación del coeficiente de sustentación

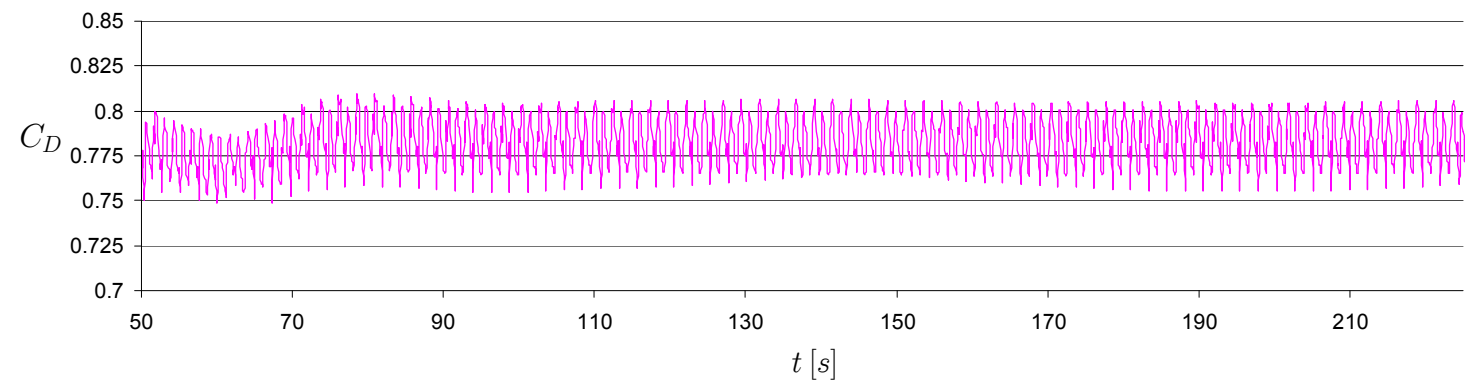

Figura A.136. Oscilación del coeficiente de arrastre 
- $U_{r}=5$

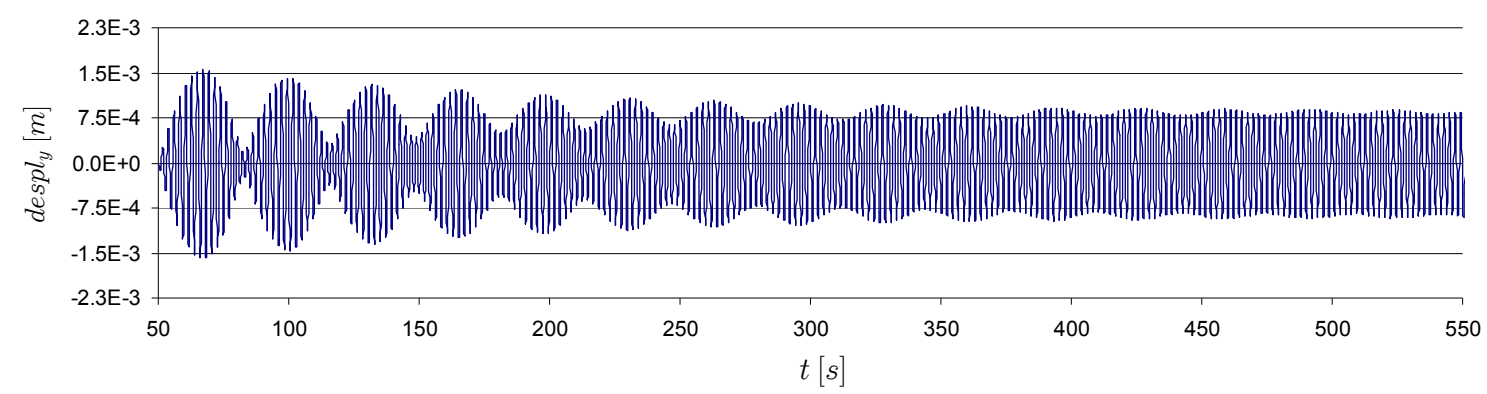

Figura A.137. Vibración transversal de la estructura

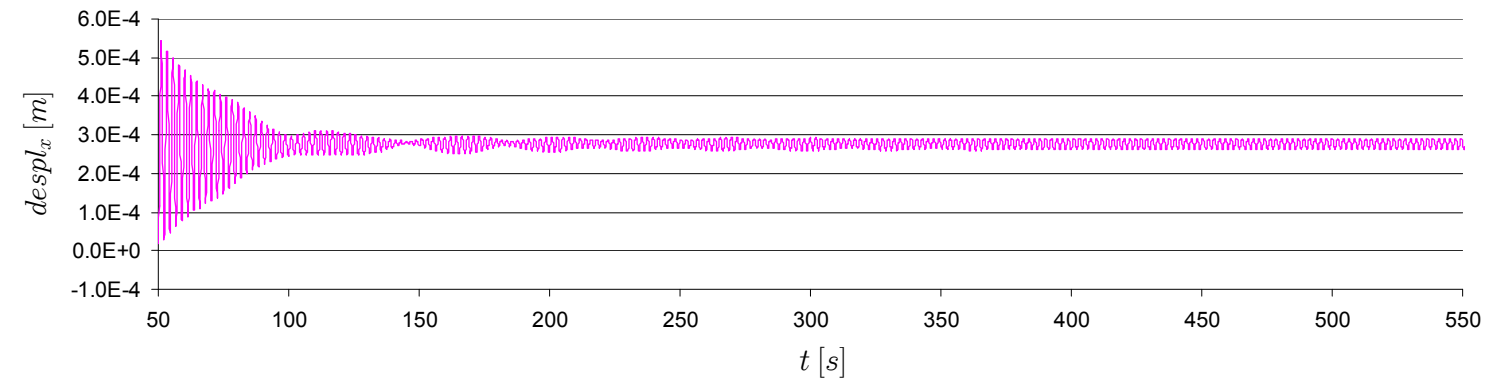

Figura A.138. Vibración en la dirección del flujo de la estructura

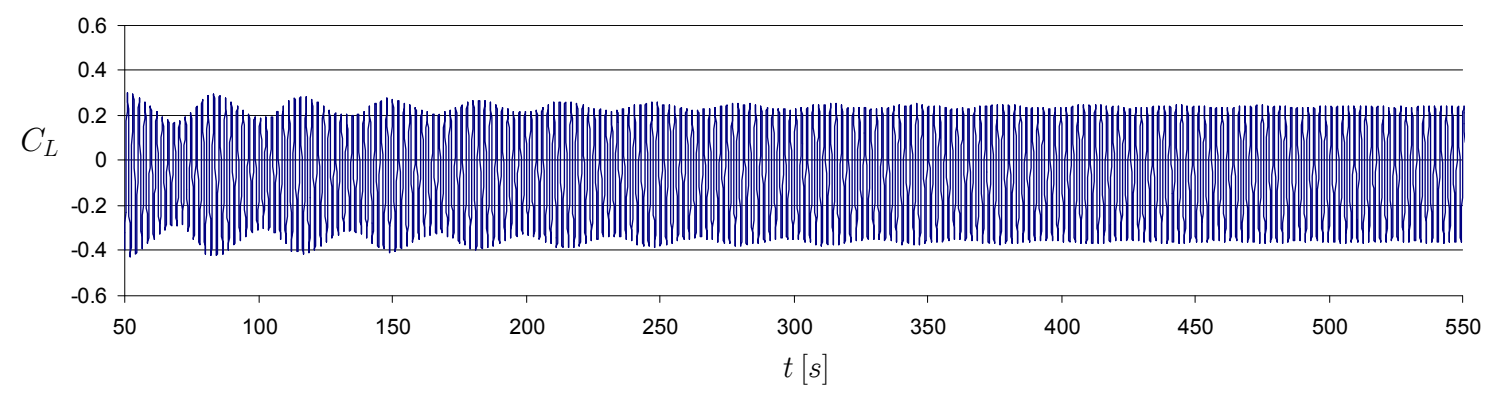

Figura A.139. Oscilación del coeficiente de sustentación

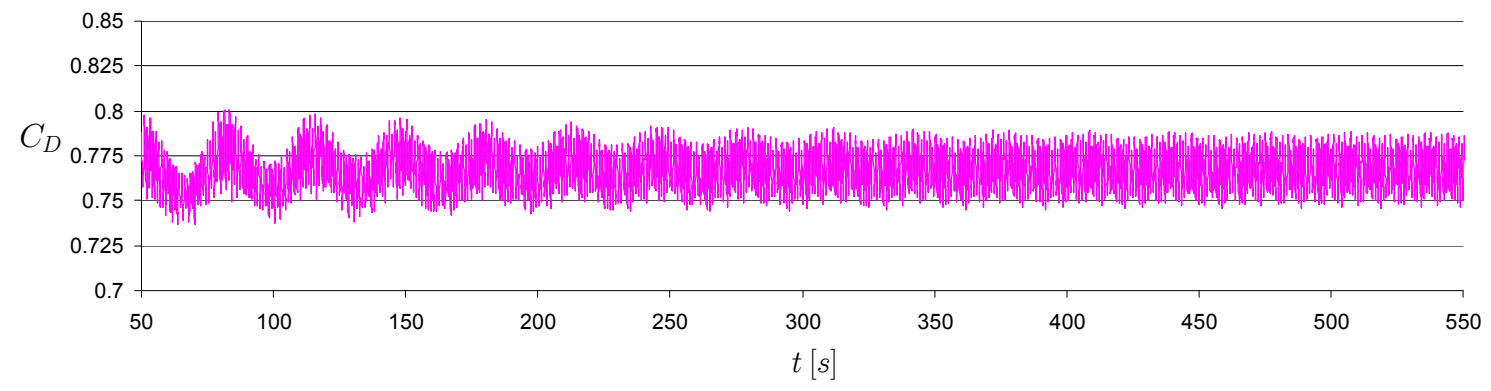

Figura A.140. Oscilación del coeficiente de arrastre 
- $U_{r}=5.5$

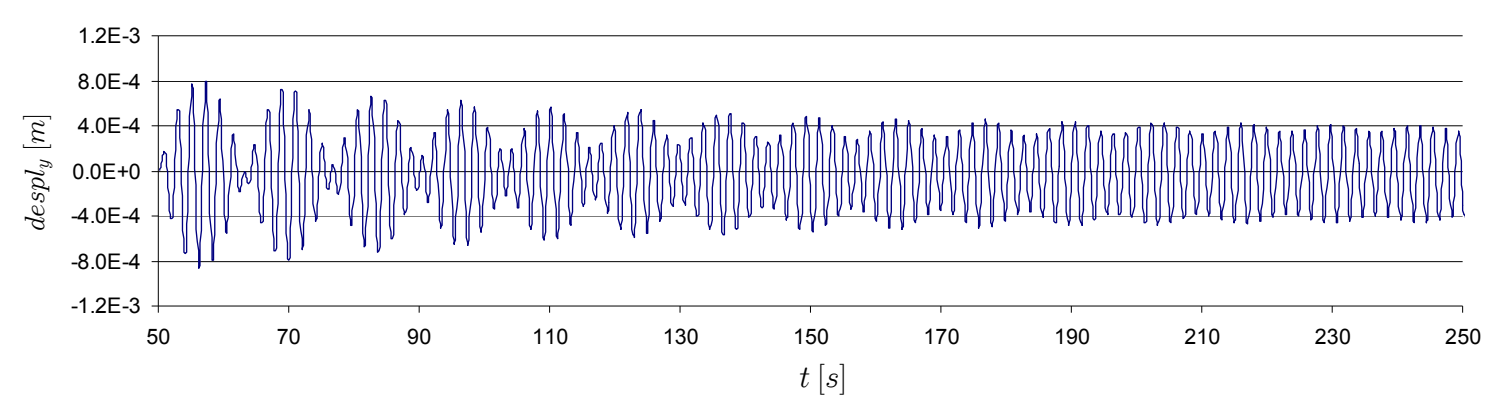

Figura A.141. Vibración transversal de la estructura

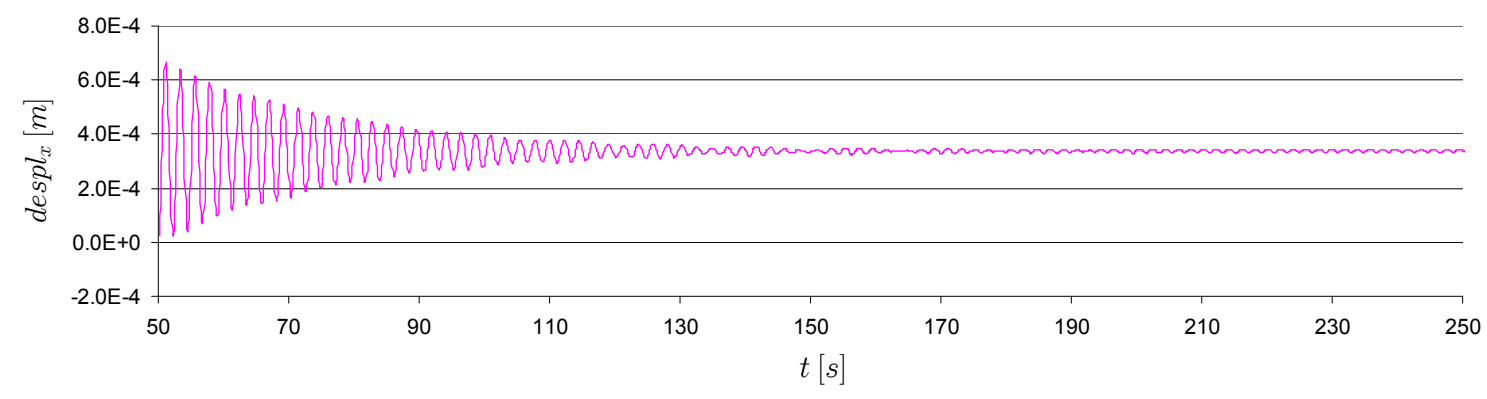

Figura A.142. Vibración en la dirección del flujo de la estructura

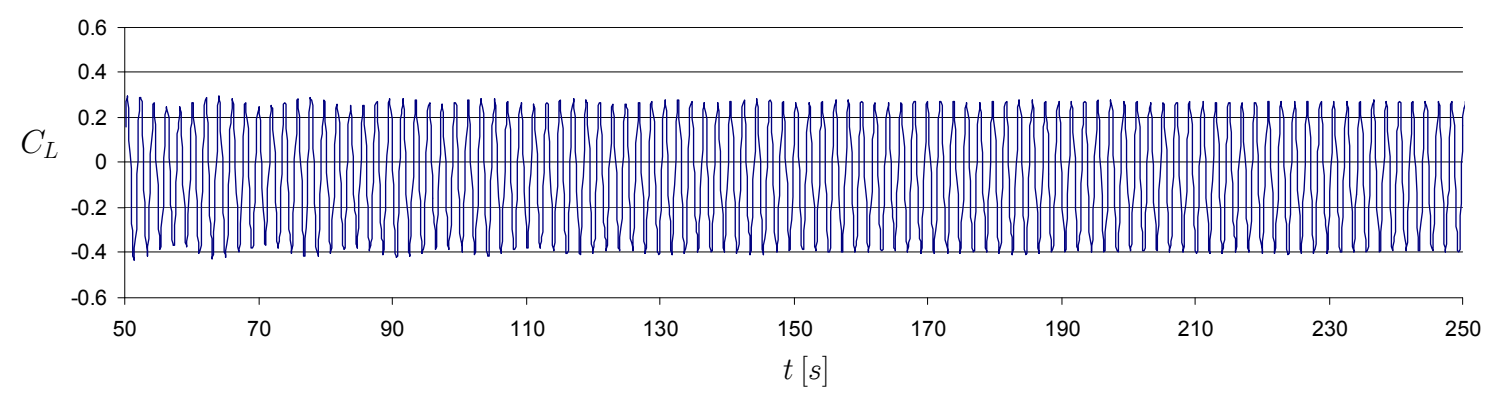

Figura A.143. Oscilación del coeficiente de sustentación

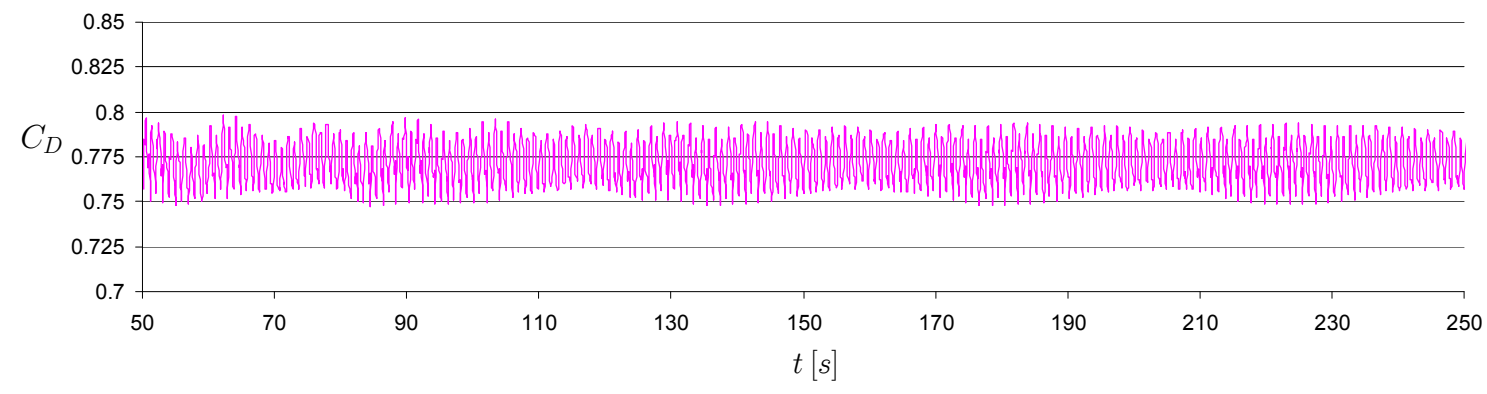

Figura A.144. Oscilación del coeficiente de arrastre 
- $U_{r}=6$

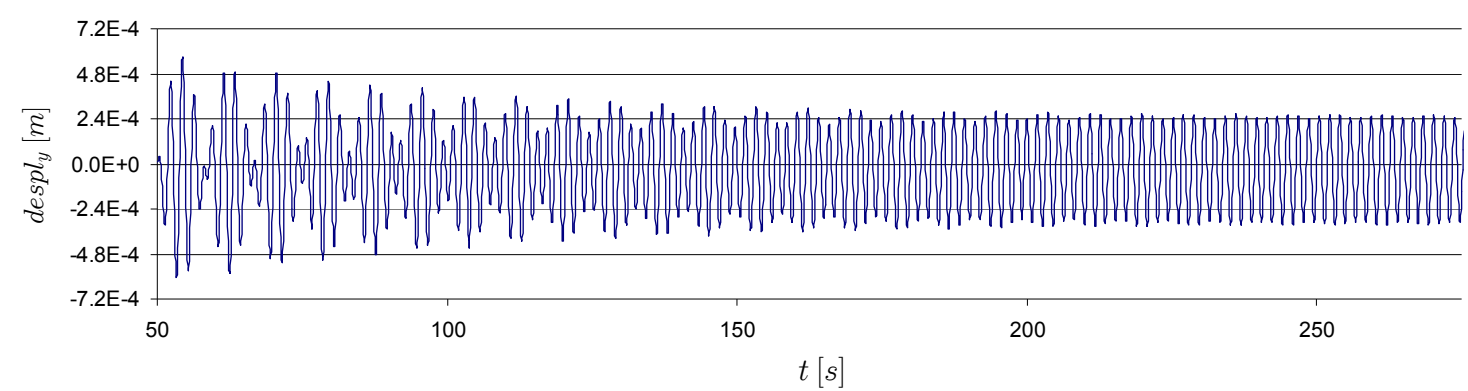

Figura A.145. Vibración transversal de la estructura

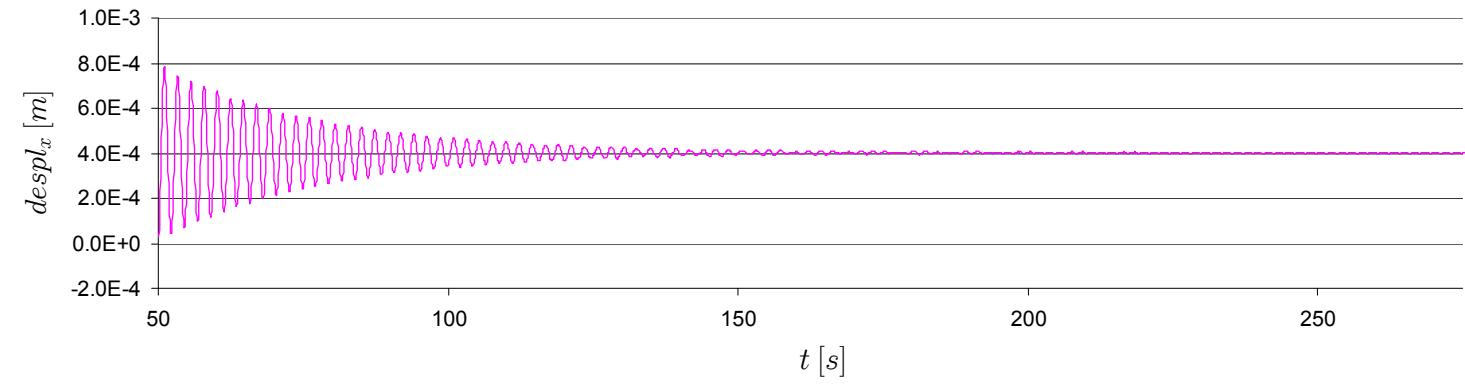

Figura A.146. Vibración en la dirección del flujo de la estructura

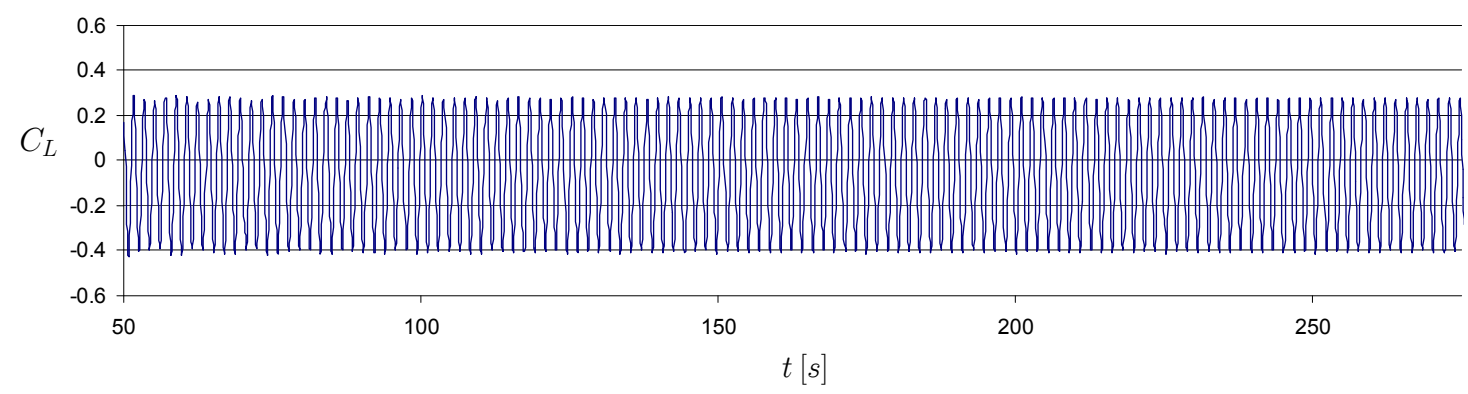

Figura A.147. Oscilación del coeficiente de sustentación

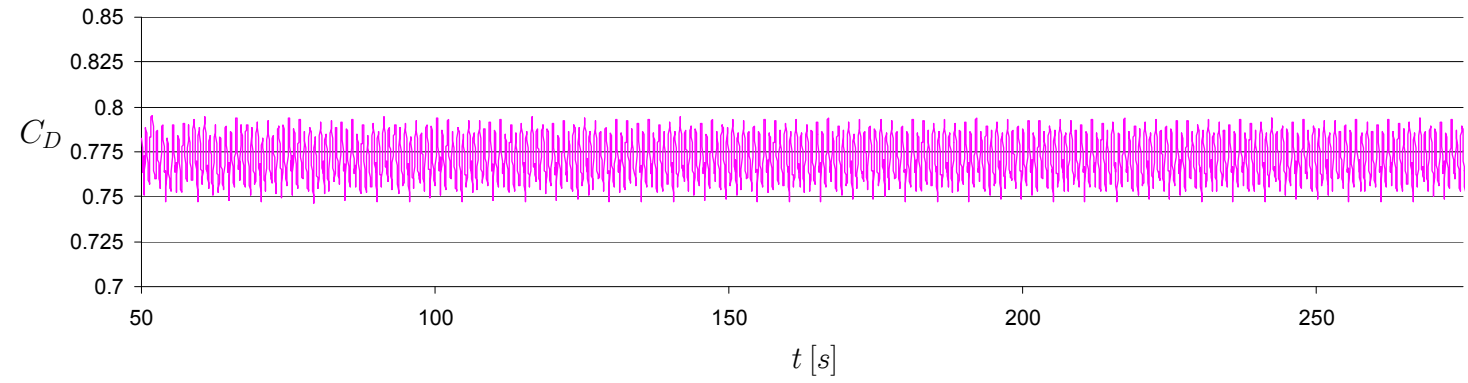

Figura A.148. Oscilación del coeficiente de arrastre 
- $U_{r}=7$

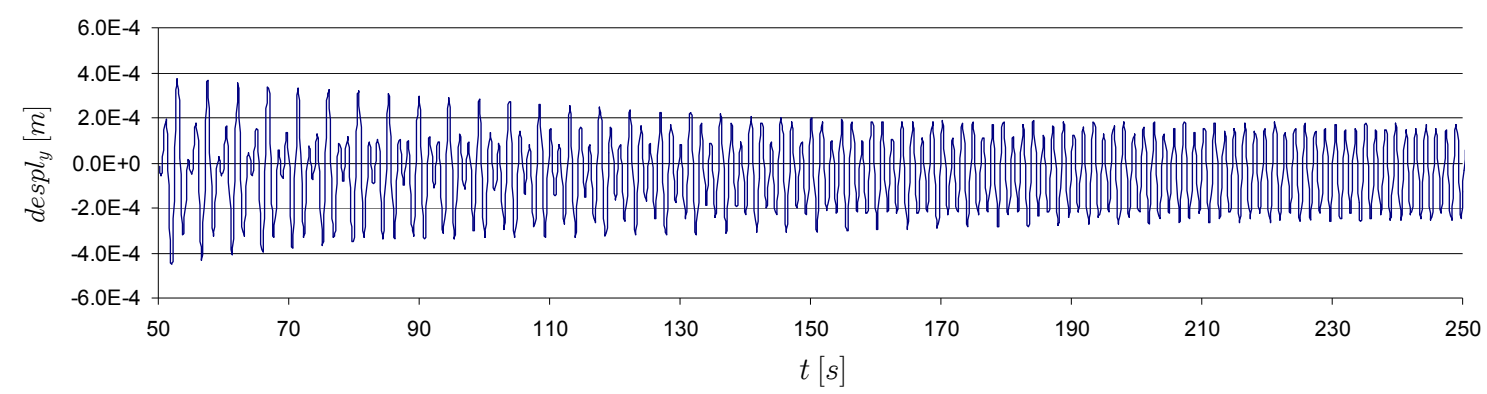

Figura A.149. Vibración transversal de la estructura

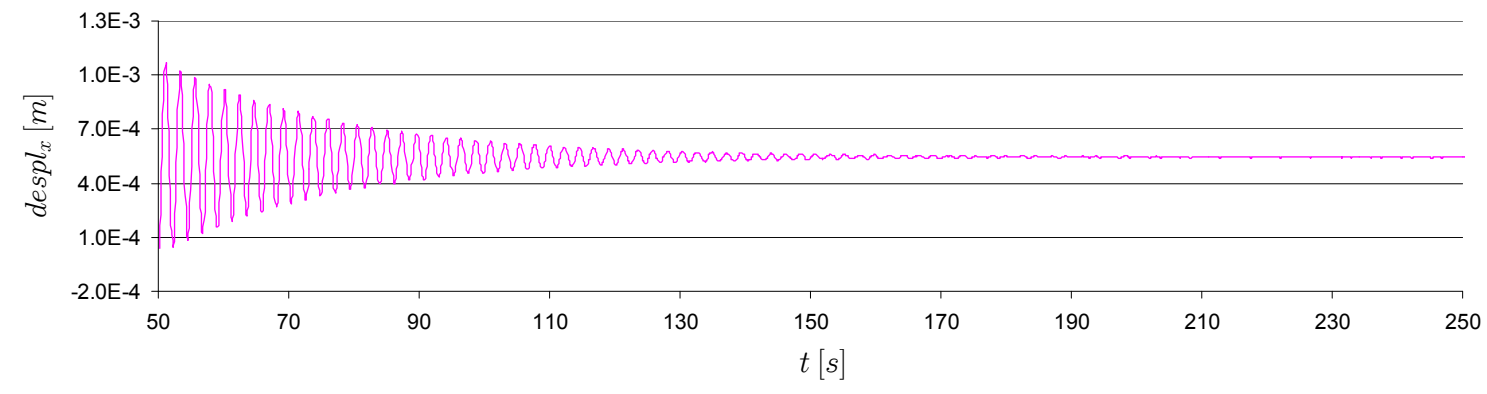

Figura A.150. Vibración en la dirección del flujo de la estructura

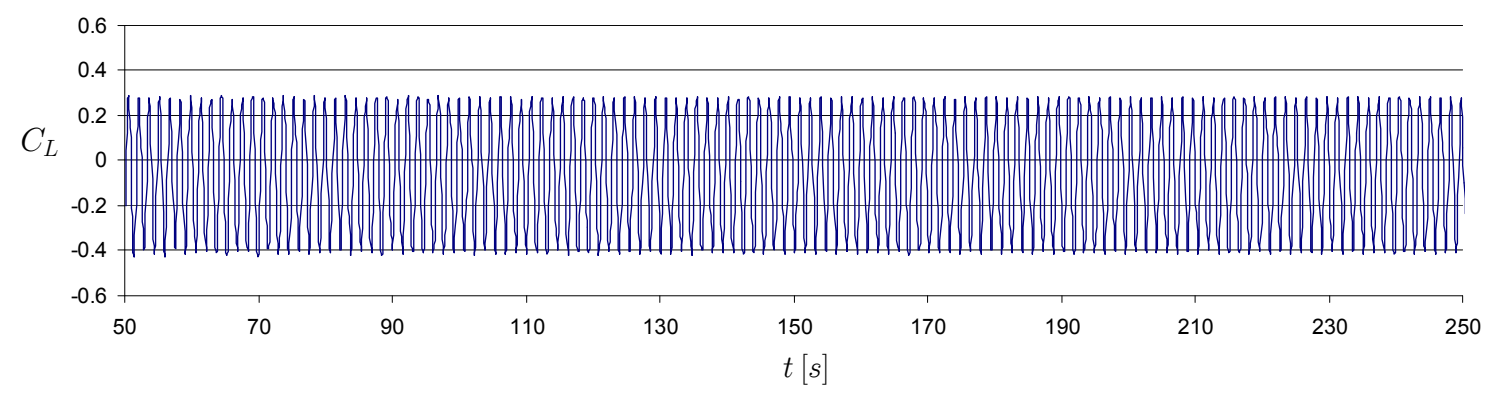

Figura A.151. Oscilación del coeficiente de sustentación

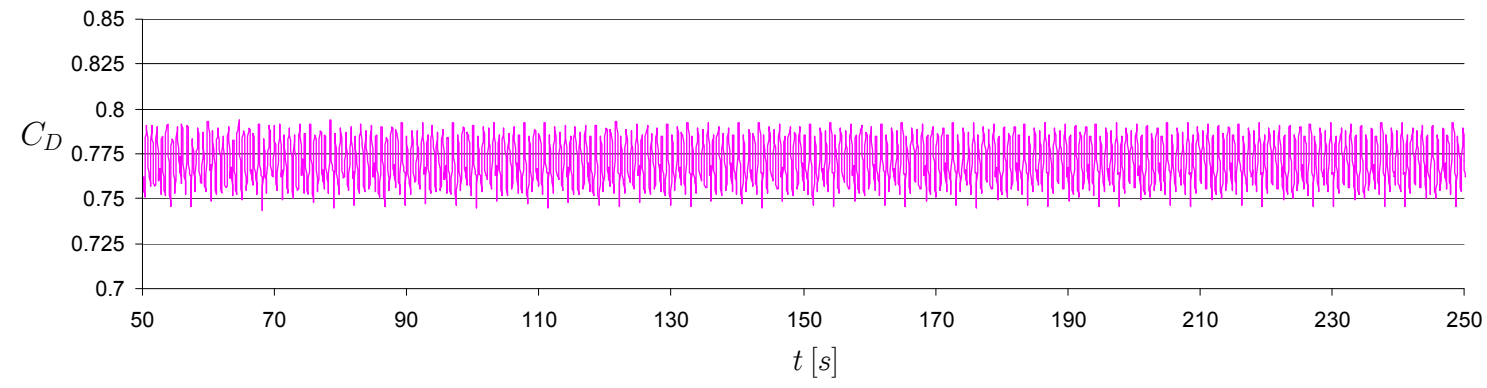

Figura A.152. Oscilación del coeficiente de arrastre 
- $U_{r}=8$

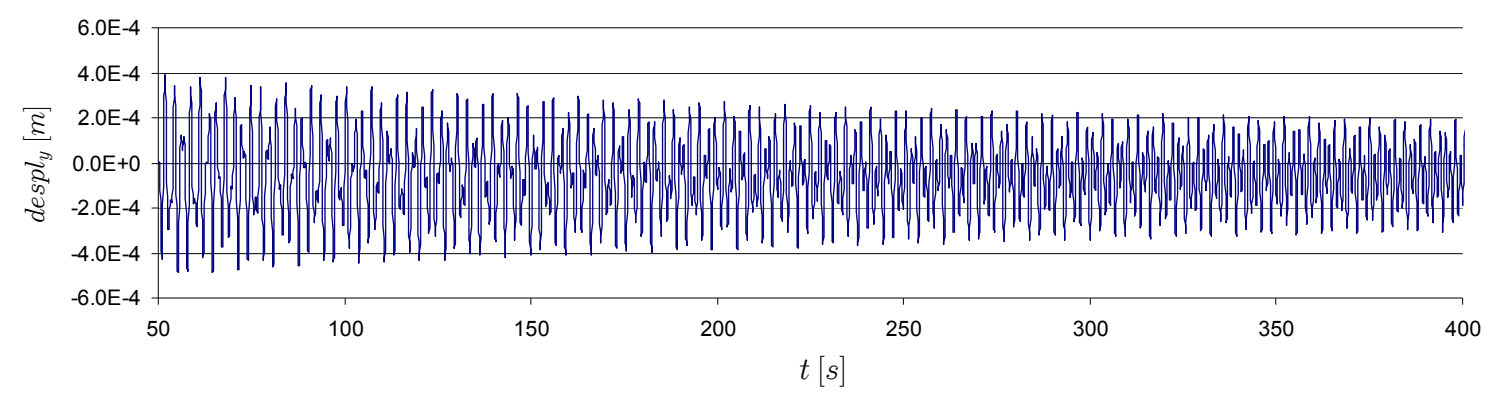

Figura A.153. Vibración transversal de la estructura

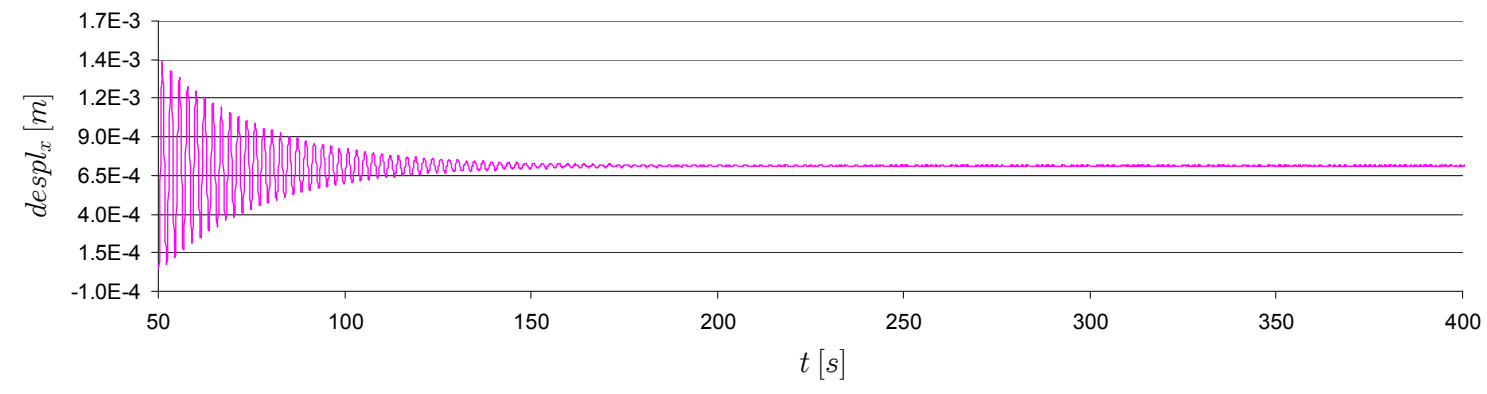

Figura A.154. Vibración en la dirección del flujo de la estructura

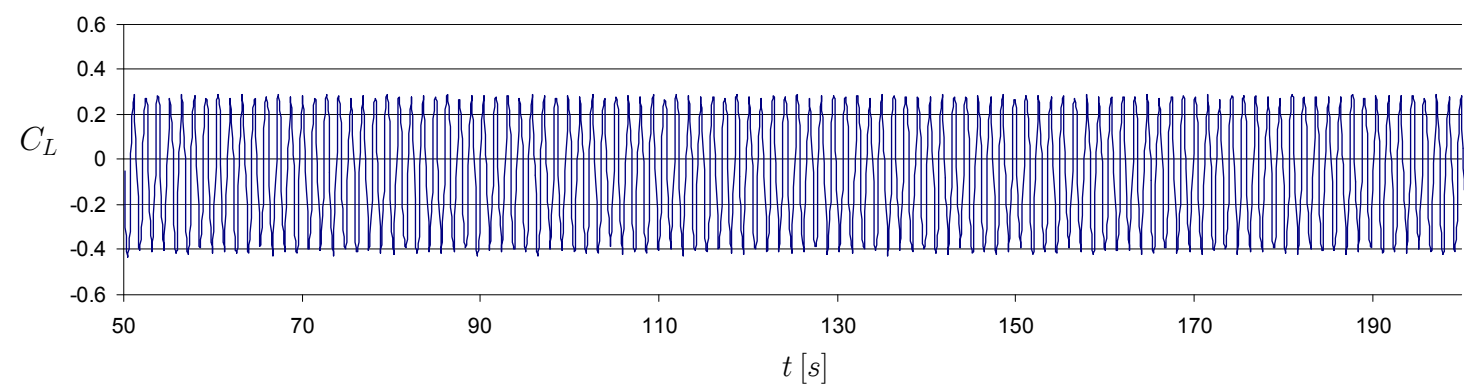

Figura A.155. Oscilación del coeficiente de sustentación

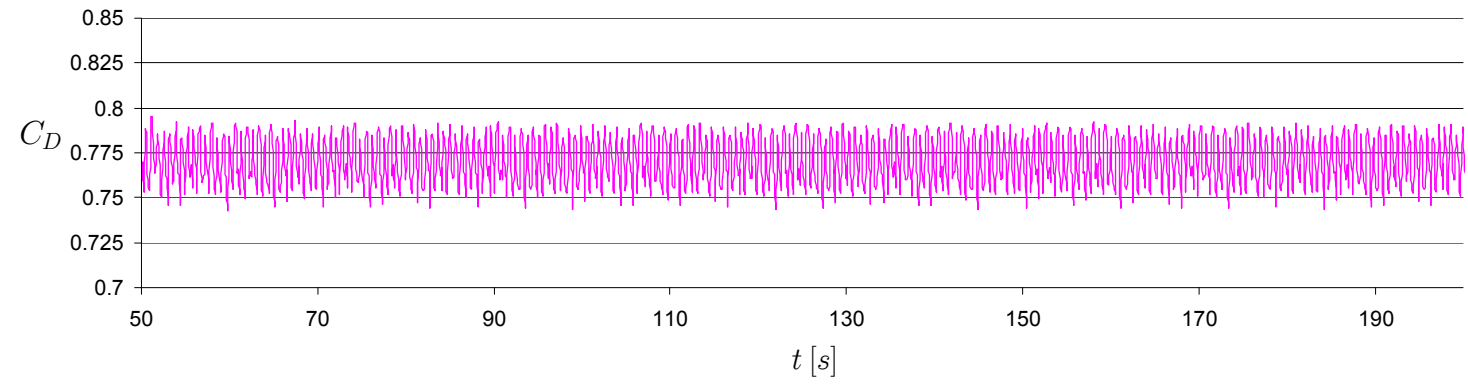

Figura A.156. Oscilación del coeficiente de arrastre 
- $U_{r}=9$

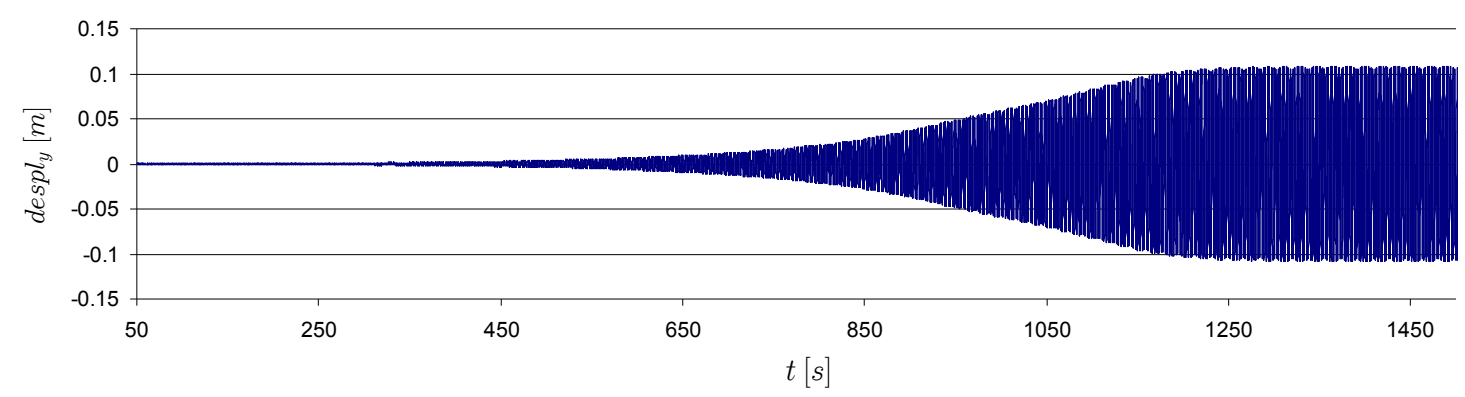

Figura A.157. Vibración transversal de la estructura

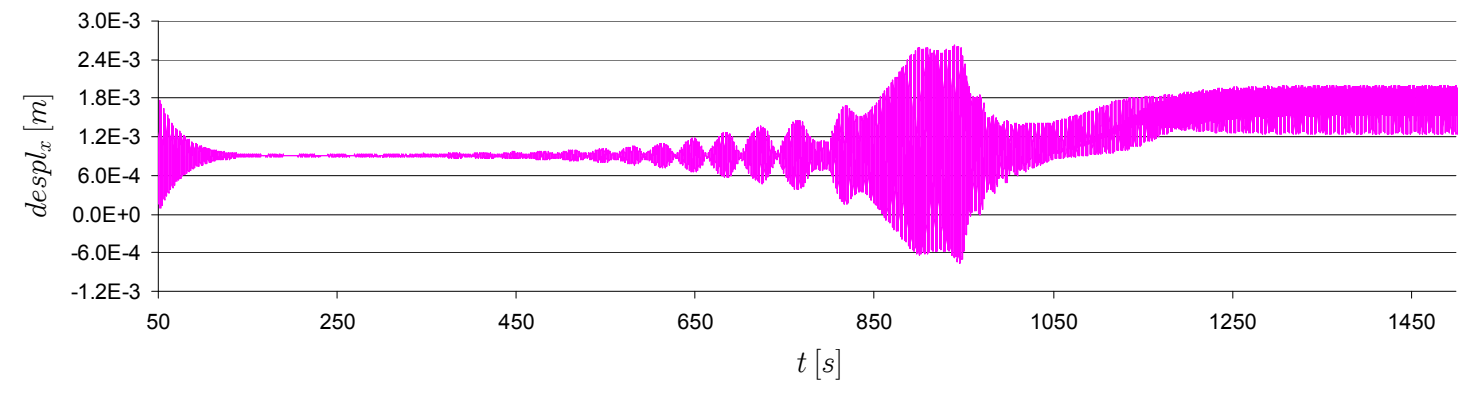

Figura A.158. Vibración en la dirección del flujo de la estructura

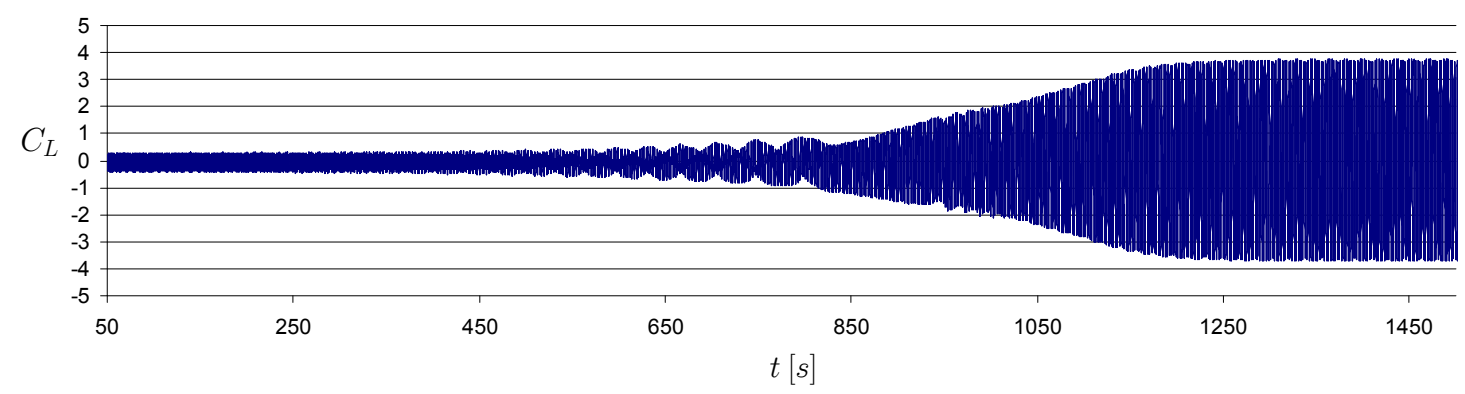

Figura A.159. Oscilación del coeficiente de sustentación

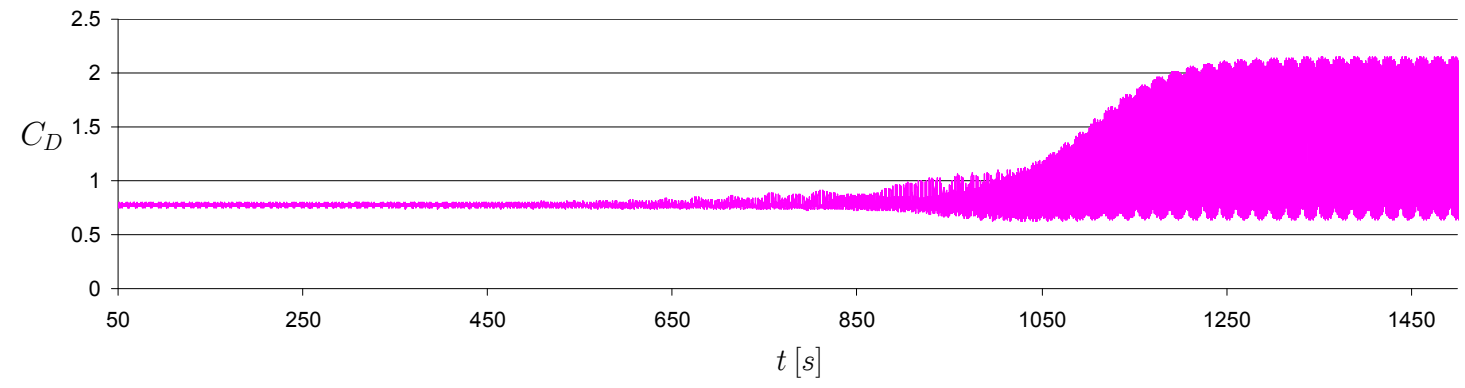

Figura A.160. Oscilación del coeficiente de arrastre 
- $U_{r}=11$

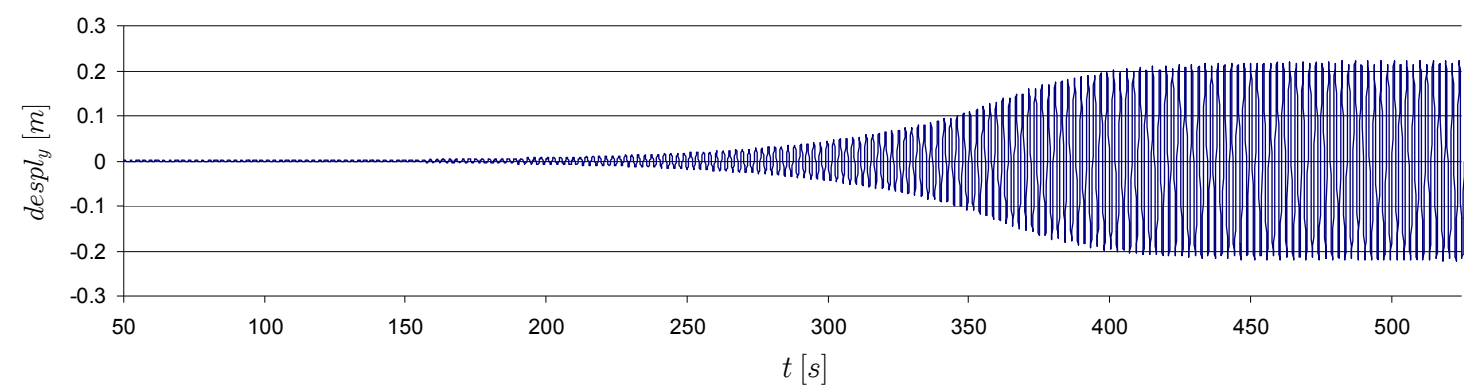

Figura A.161. Vibración transversal de la estructura

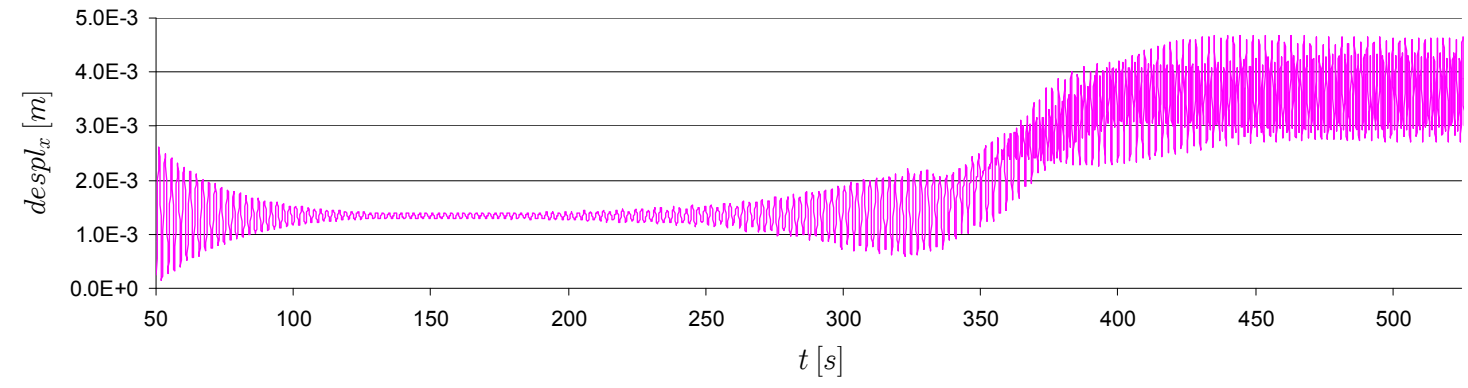

Figura A.162. Vibración en la dirección del flujo de la estructura

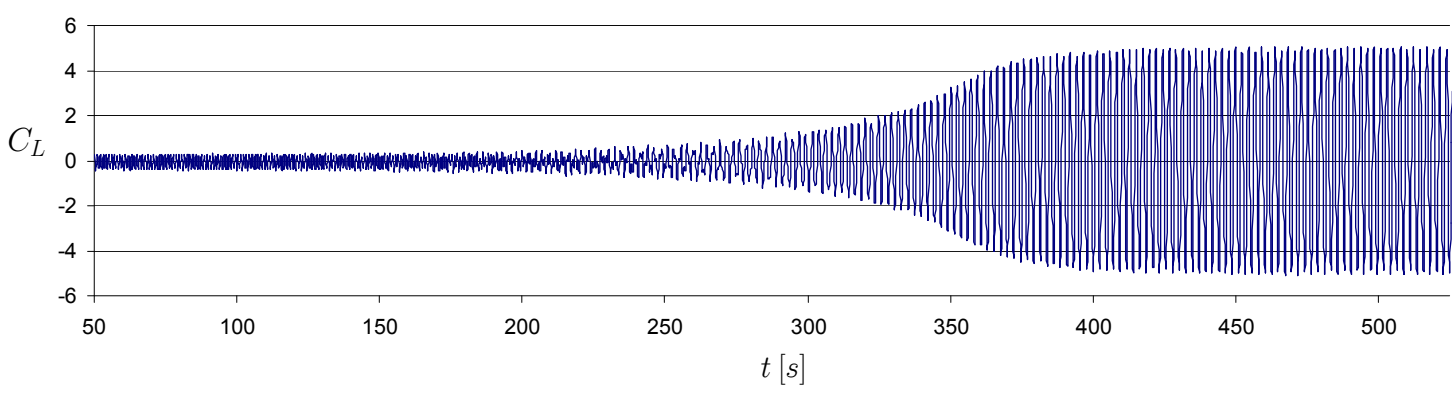

Figura A.163. Oscilación del coeficiente de sustentación

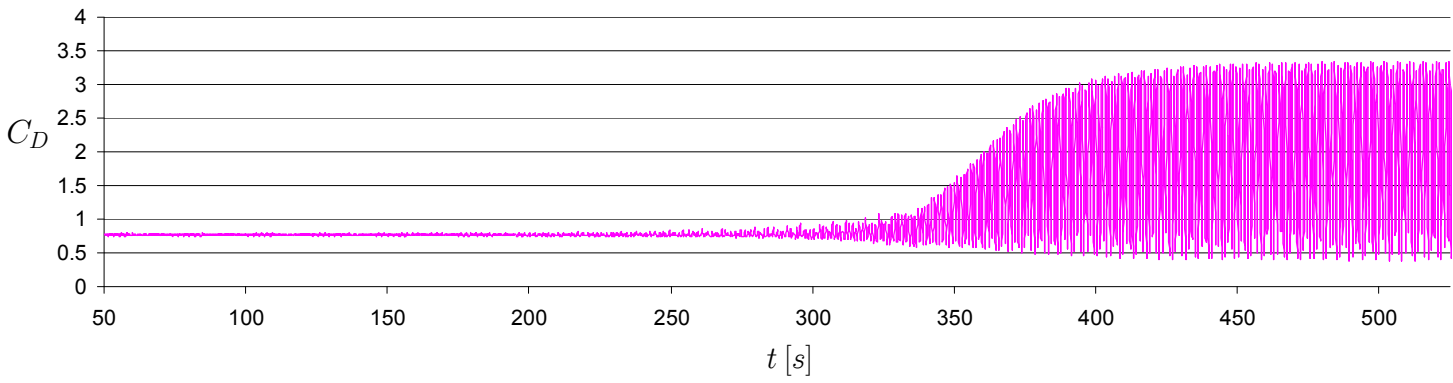

Figura A.164. Oscilación del coeficiente de arrastre 
- $U_{r}=13$

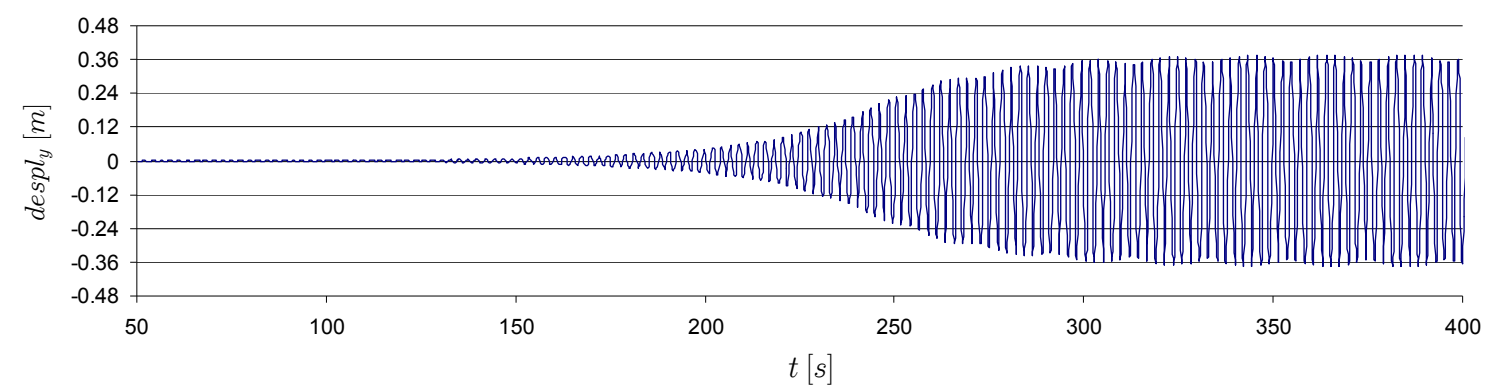

Figura A.165. Vibración transversal de la estructura

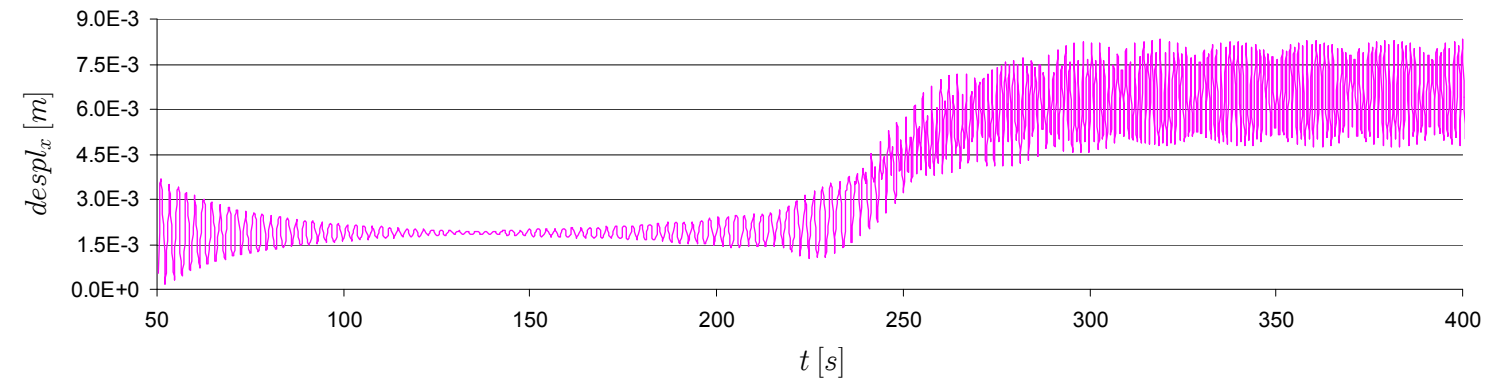

Figura A.166. Vibración en la dirección del flujo de la estructura

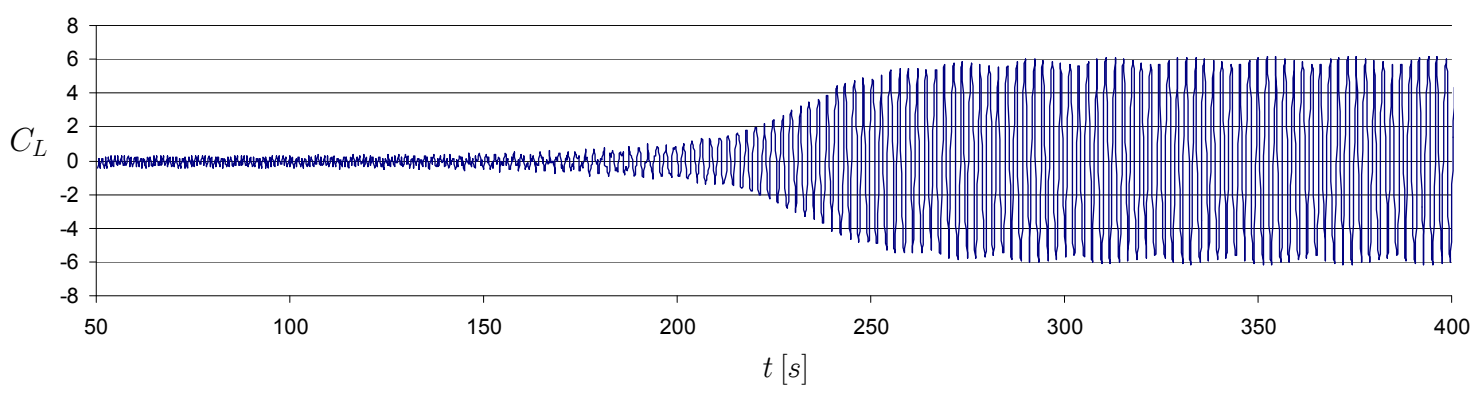

Figura A.167. Oscilación del coeficiente de sustentación

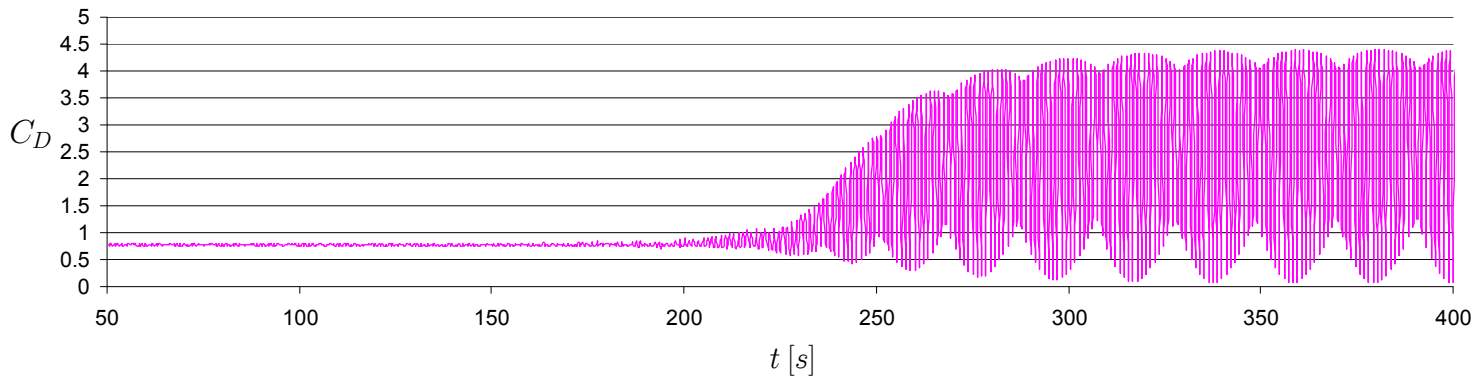

Figura A.168. Oscilación del coeficiente de arrastre 
- $U_{r}=15$

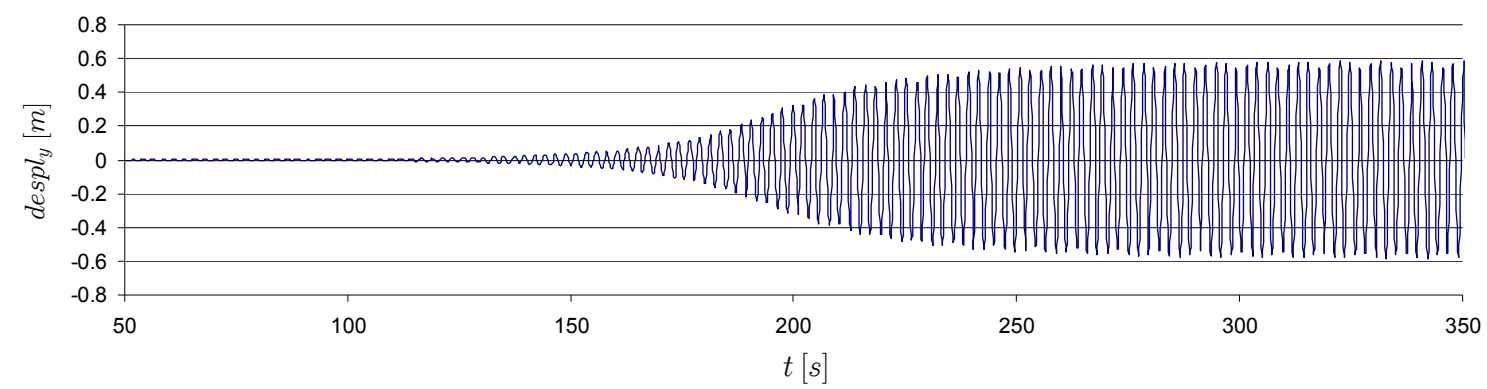

Figura A.169. Vibración transversal de la estructura

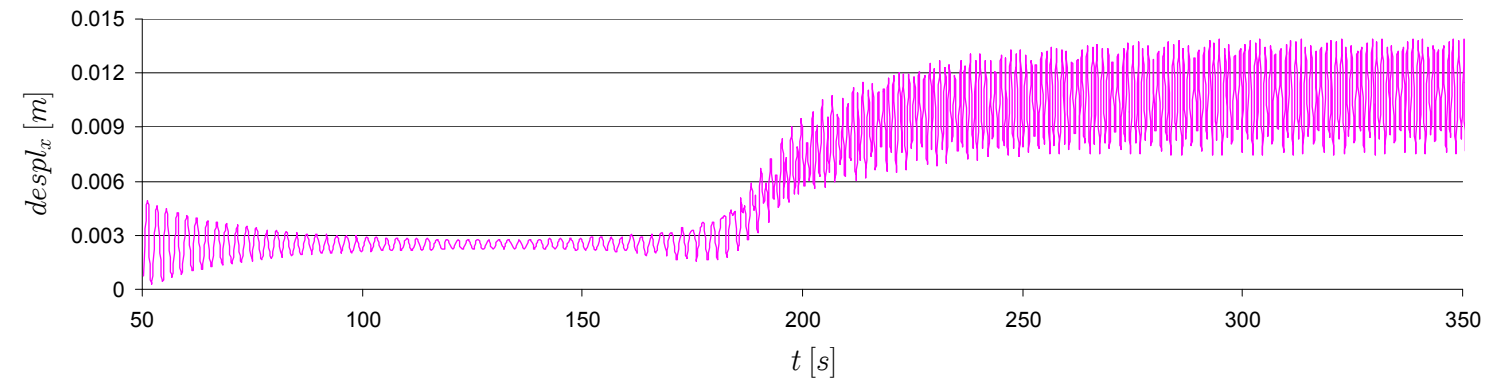

Figura A.170. Vibración en la dirección del flujo de la estructura

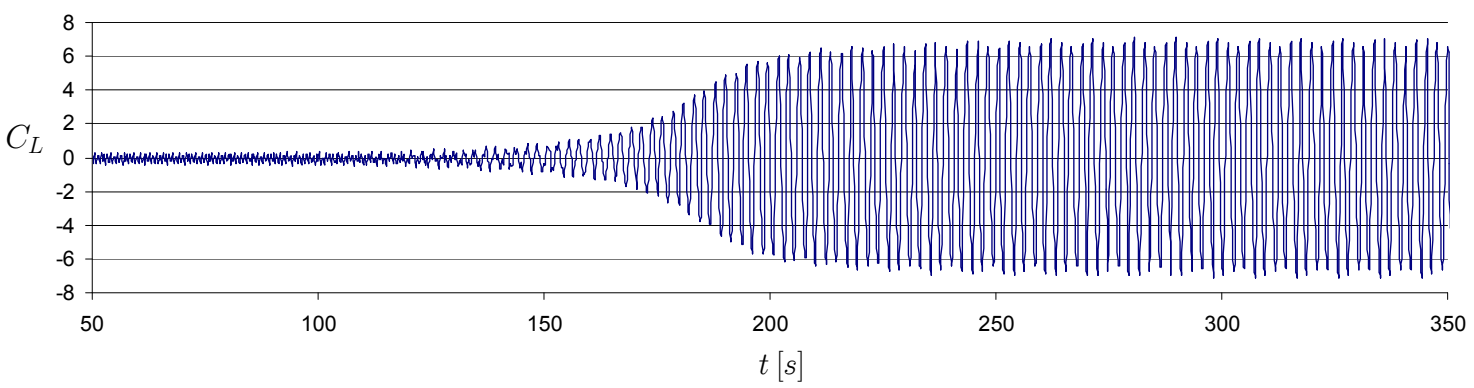

Figura A.171. Oscilación del coeficiente de sustentación

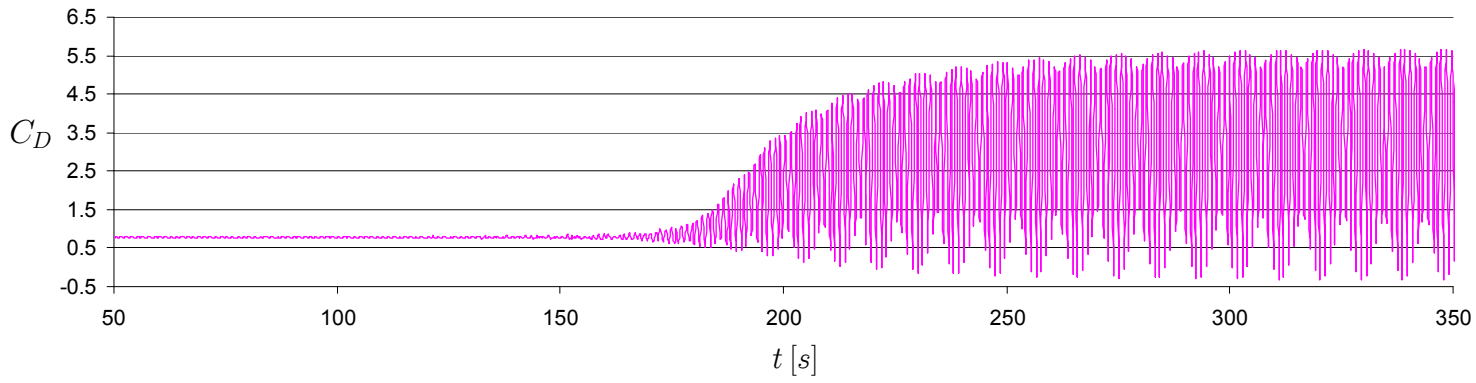

Figura A.172. Oscilación del coeficiente de arrastre 
- $U_{r}=17$

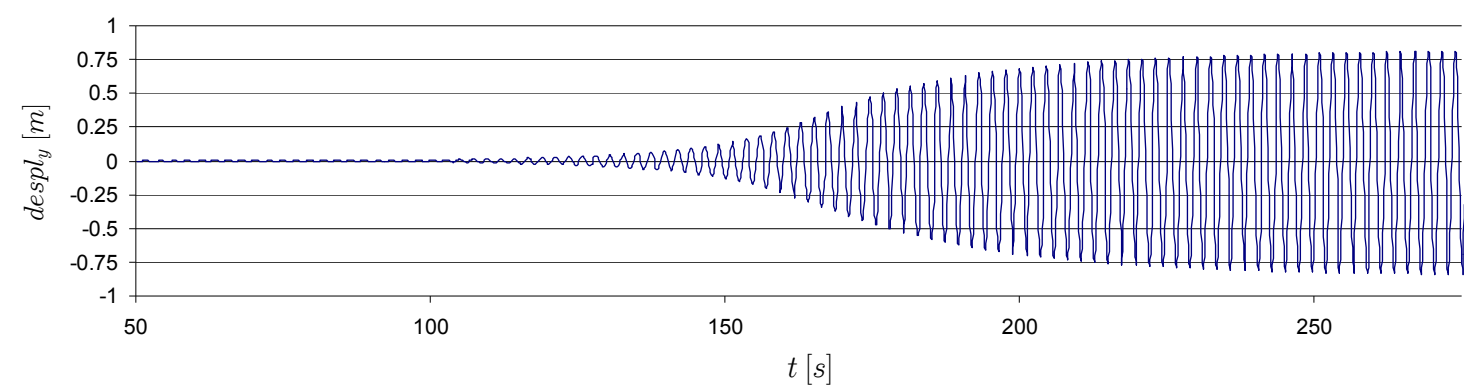

Figura A.173. Vibración transversal de la estructura

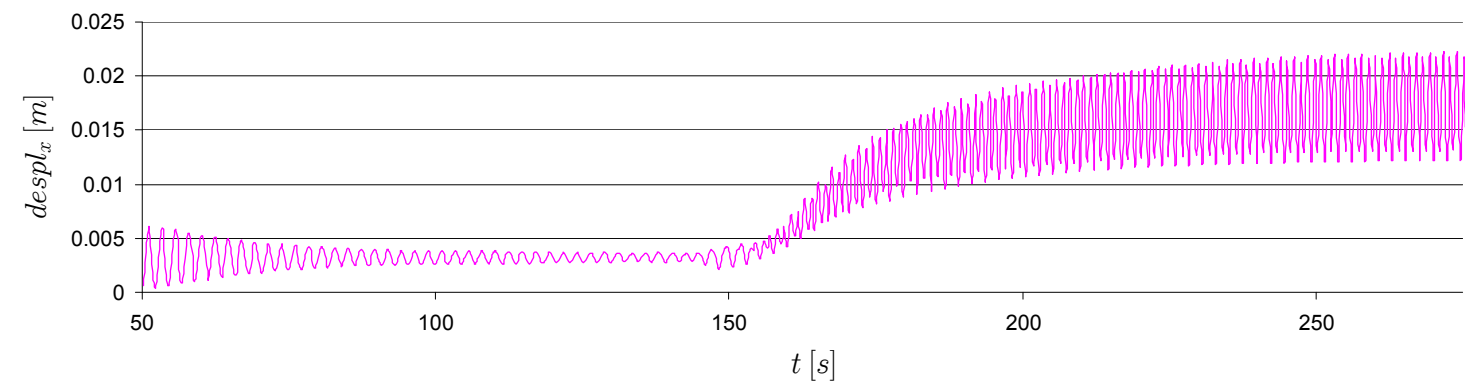

Figura A.174. Vibración en la dirección del flujo de la estructura

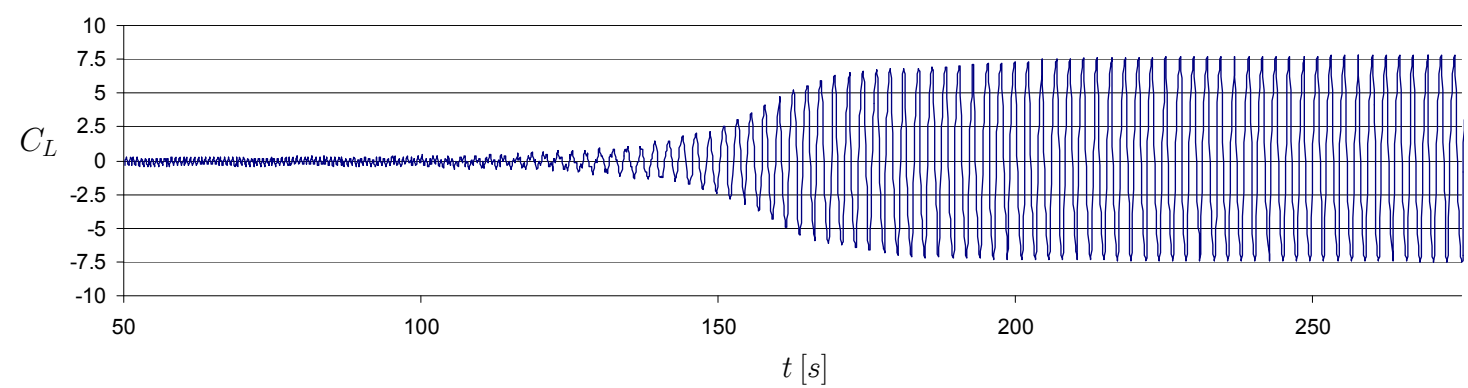

Figura A.175. Oscilación del coeficiente de sustentación

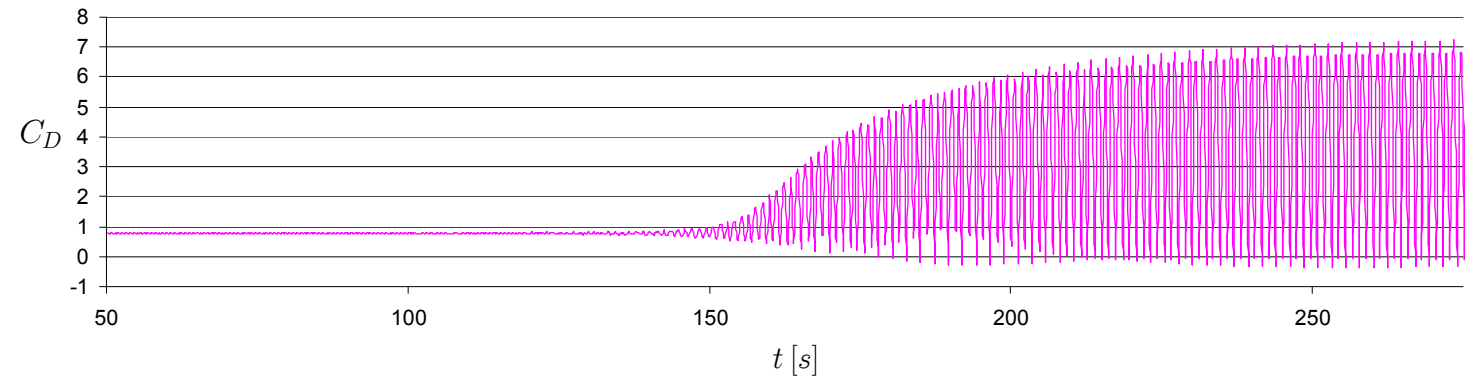

Figura A.176. Oscilación del coeficiente de arrastre 


\section{J.3. Amortiguamiento estructural 0.025}

- $U_{r}=3.5$

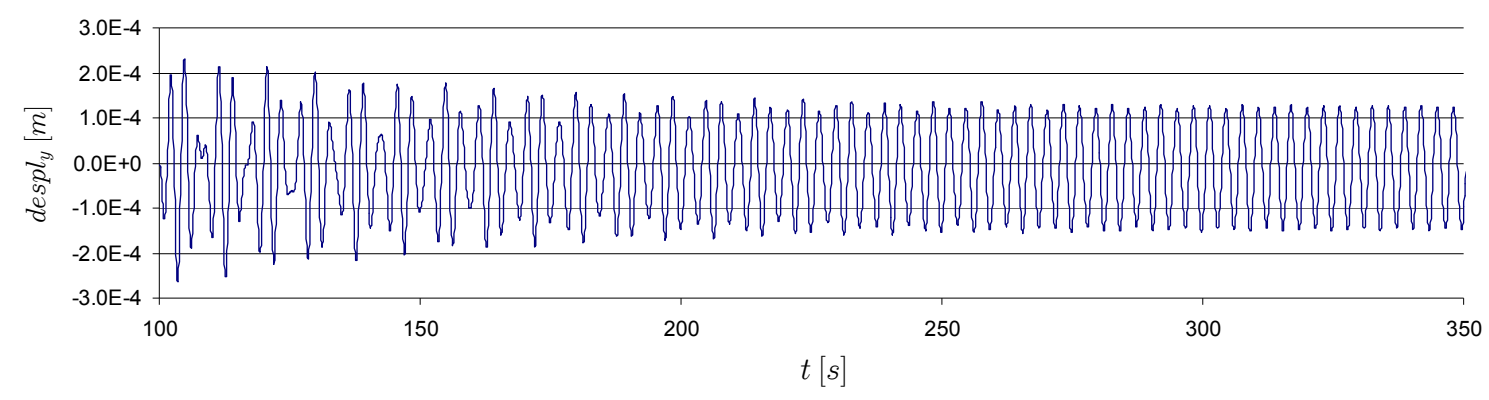

Figura A.177. Vibración transversal de la estructura

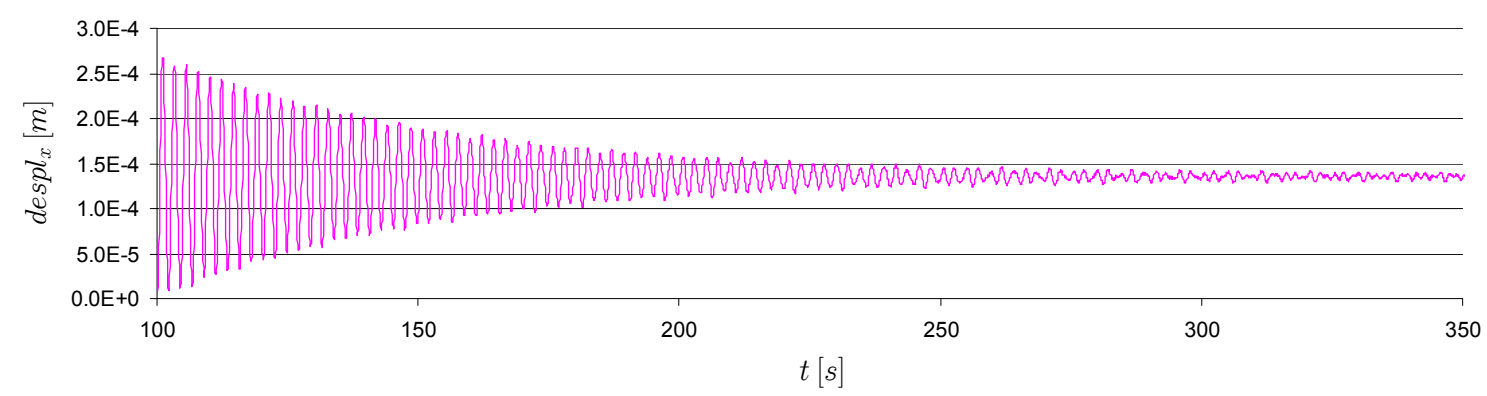

Figura A.178. Vibración en la dirección del flujo de la estructura

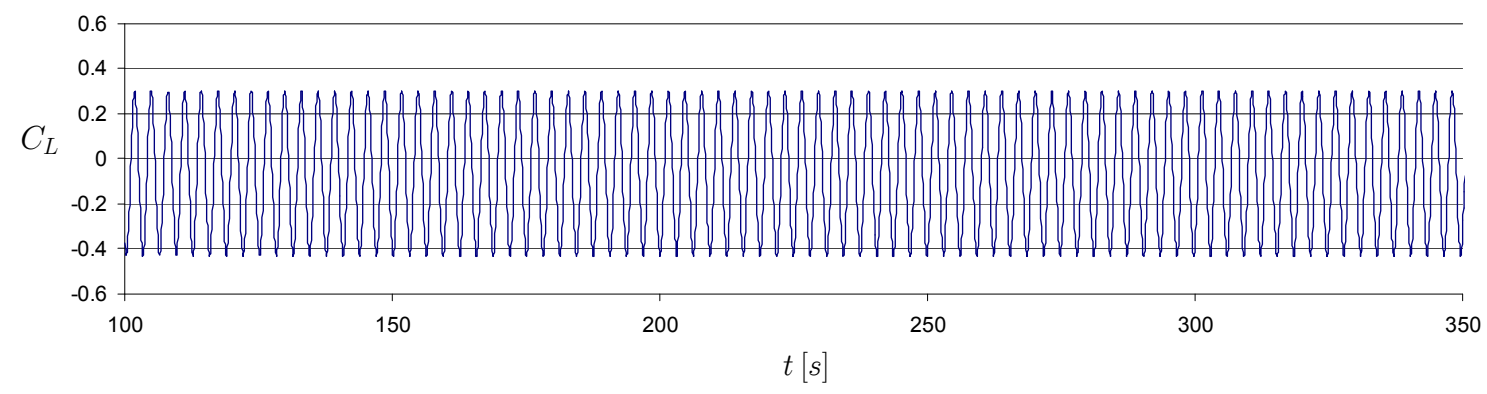

Figura A.179. Oscilación del coeficiente de sustentación

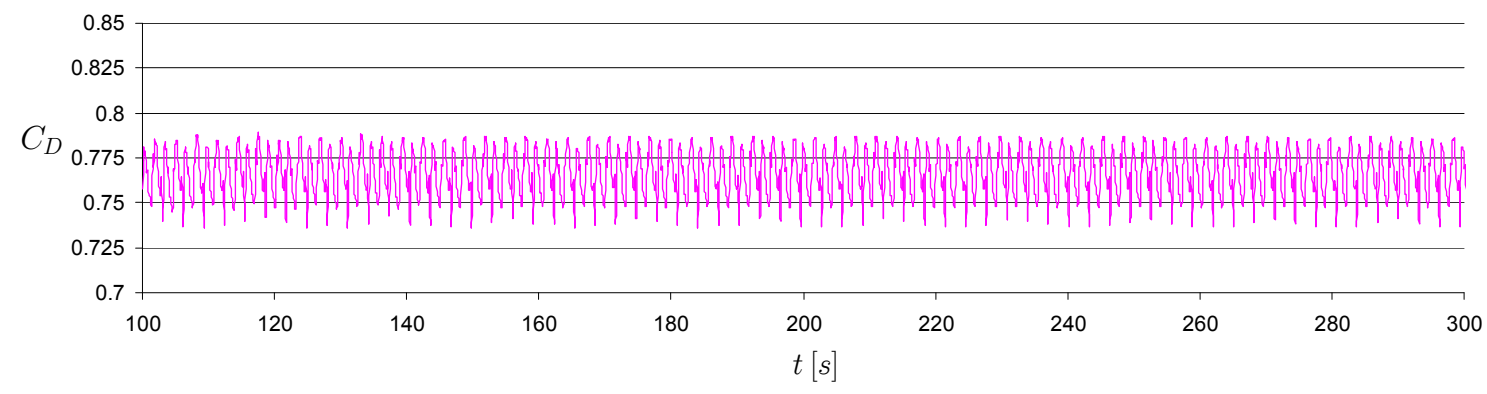

Figura A.180. Oscilación del coeficiente de arrastre 
- $U_{r}=4$

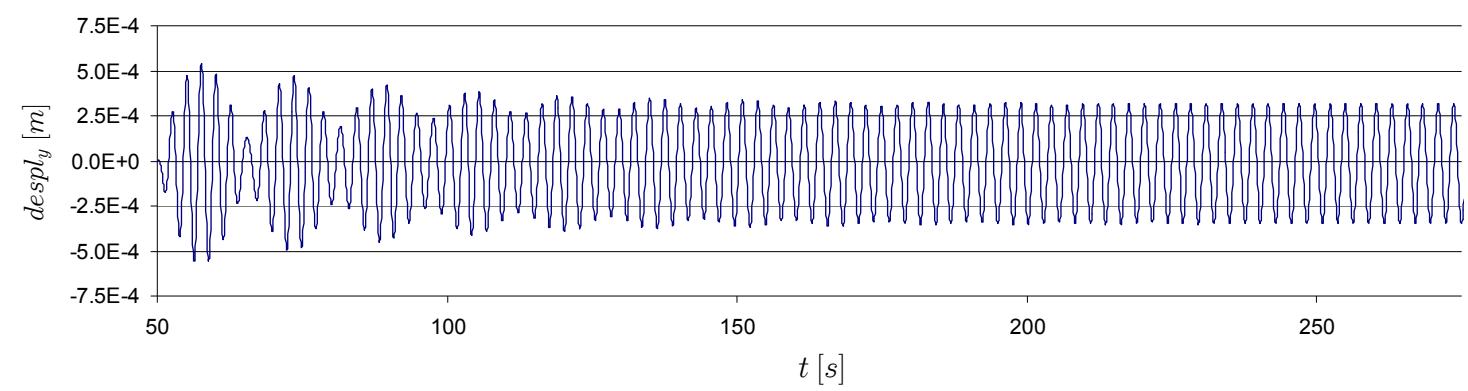

Figura A.181. Vibración transversal de la estructura

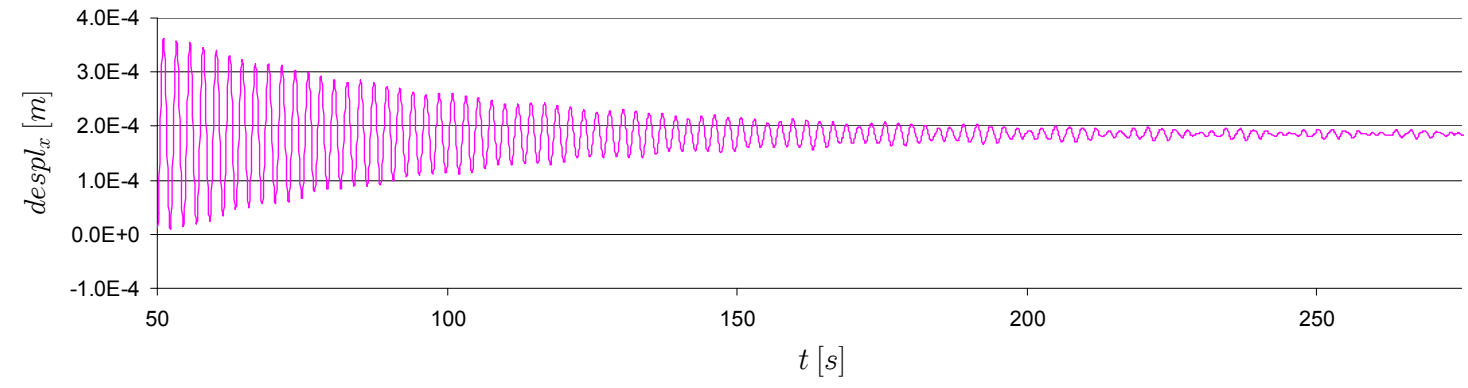

Figura A.182. Vibración en la dirección del flujo de la estructura

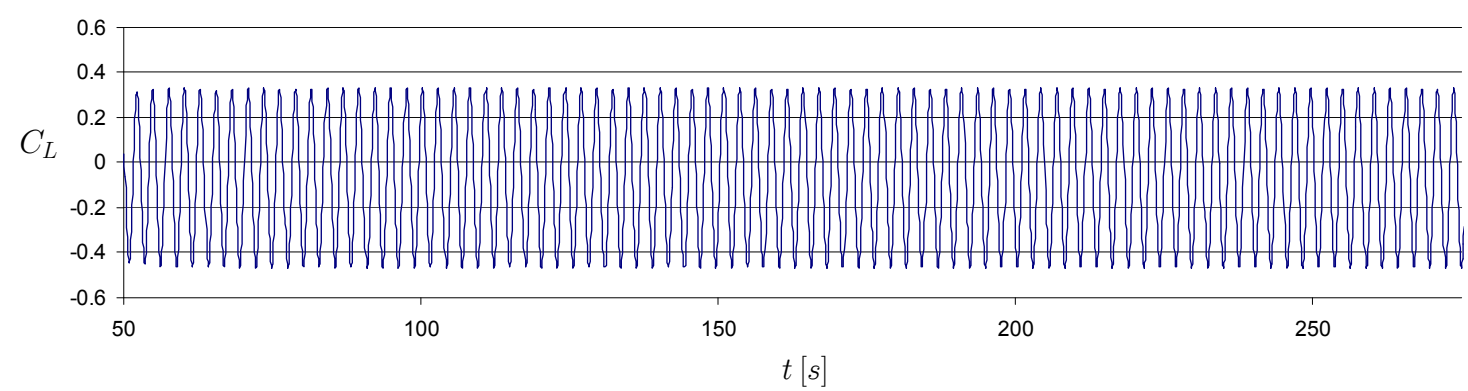

Figura A.183. Oscilación del coeficiente de sustentación

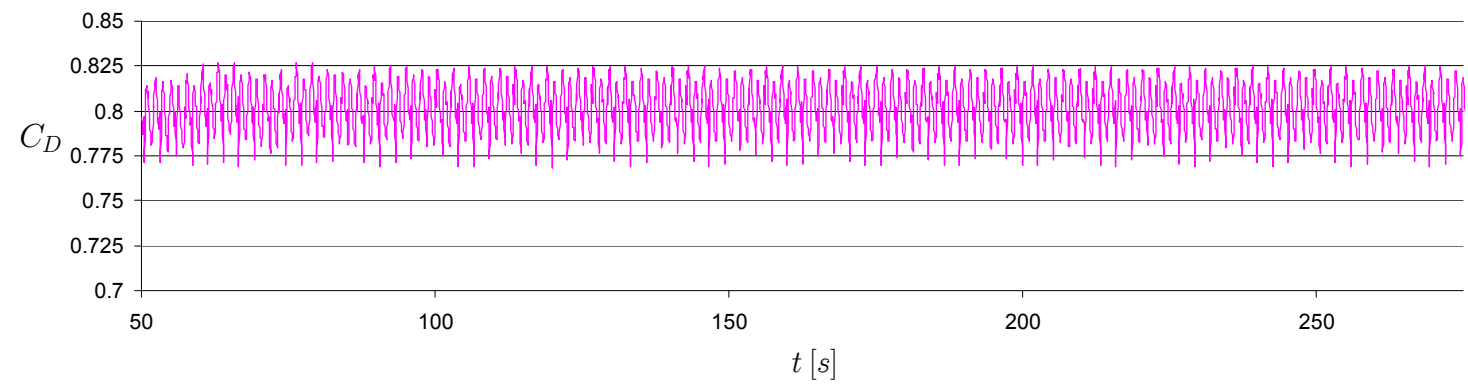

Figura A.184. Oscilación del coeficiente de arrastre 
- $U_{r}=4.5$

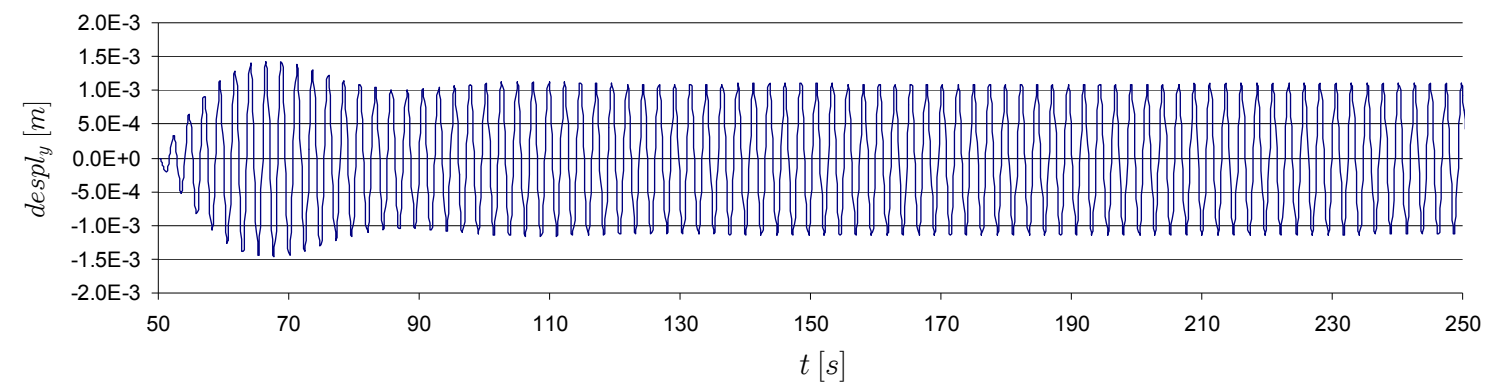

Figura A.185. Vibración transversal de la estructura

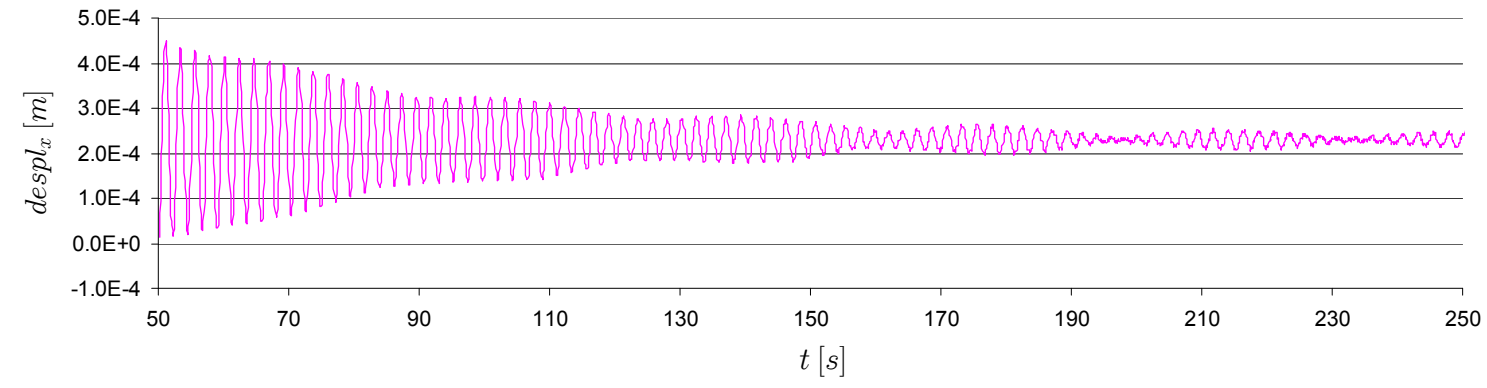

Figura A.186. Vibración en la dirección del flujo de la estructura

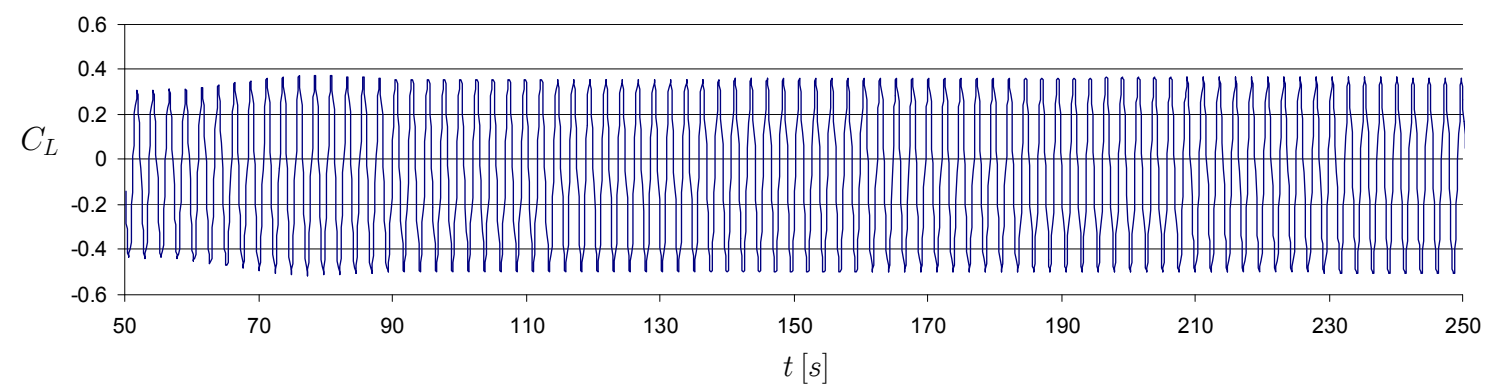

Figura A.187. Oscilación del coeficiente de sustentación

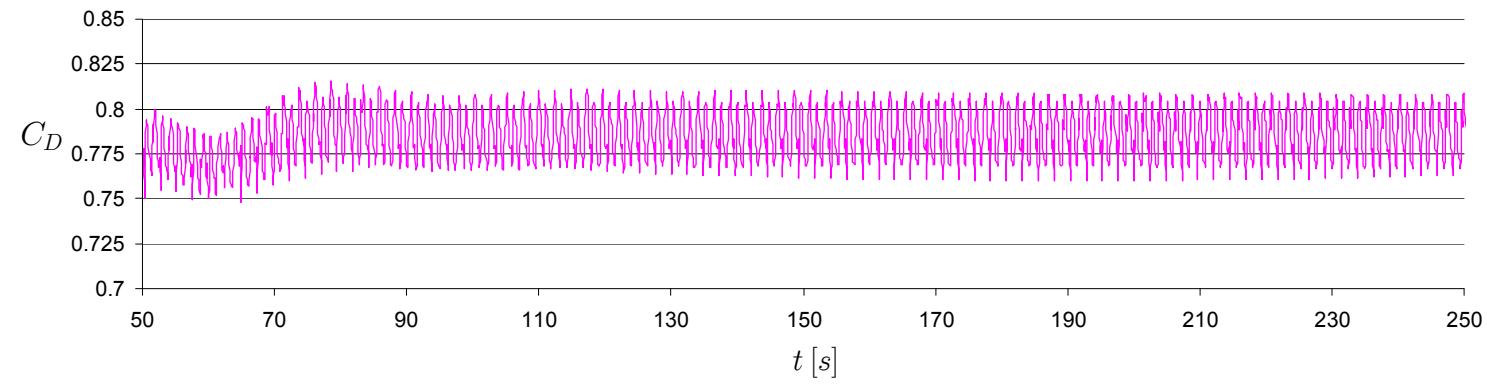

Figura A.188. Oscilación del coeficiente de arrastre 
- $U_{r}=5$

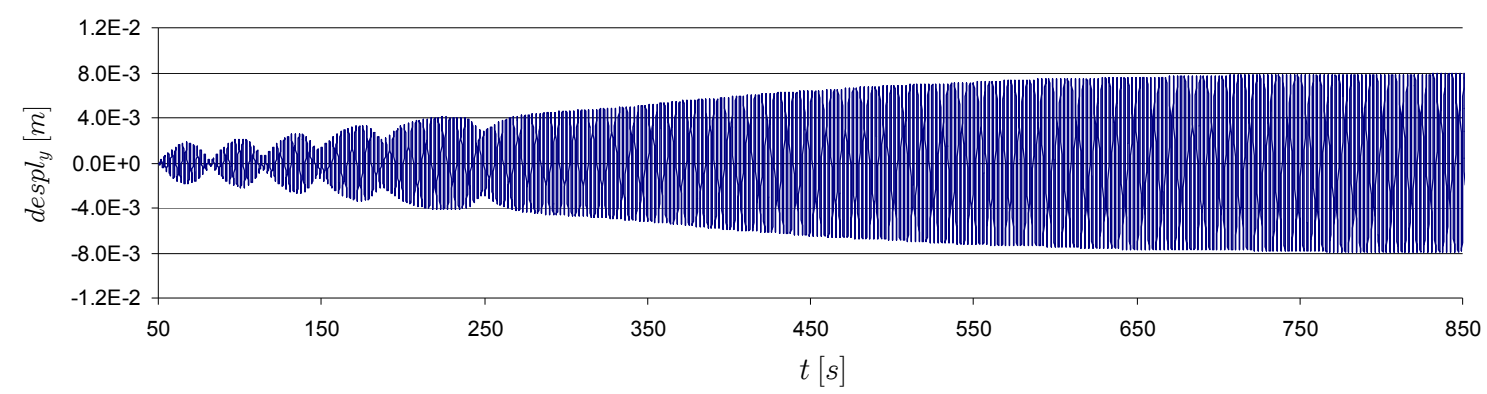

Figura A.189. Vibración transversal de la estructura

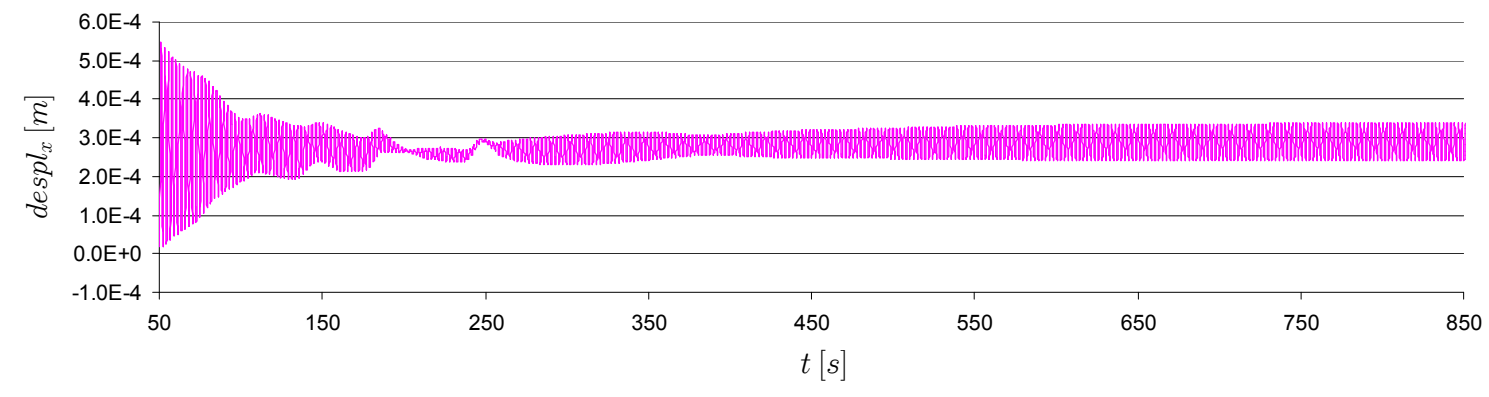

Figura A.190. Vibración en la dirección del flujo de la estructura

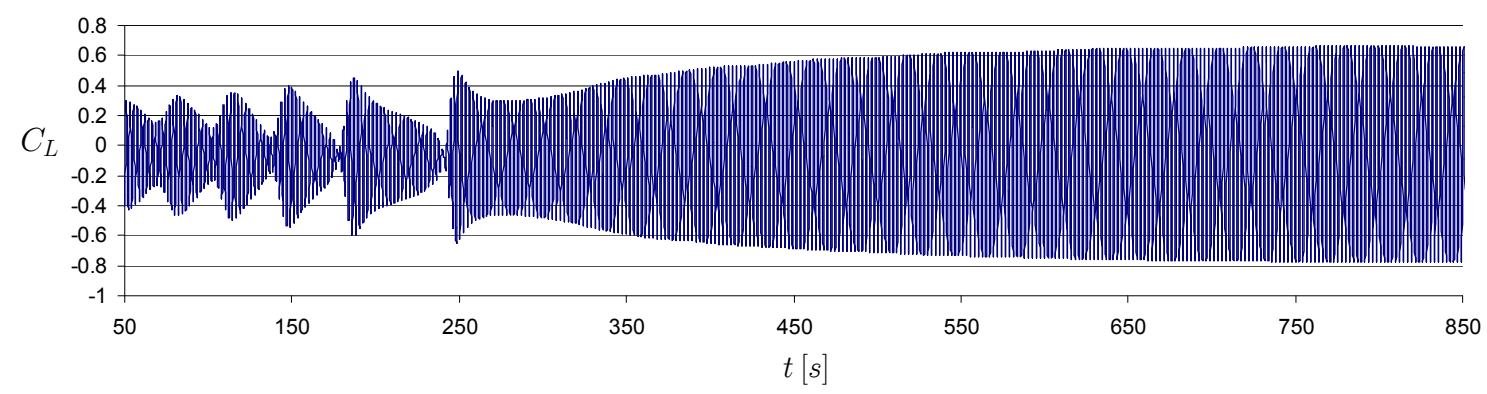

Figura A.191. Oscilación del coeficiente de sustentación

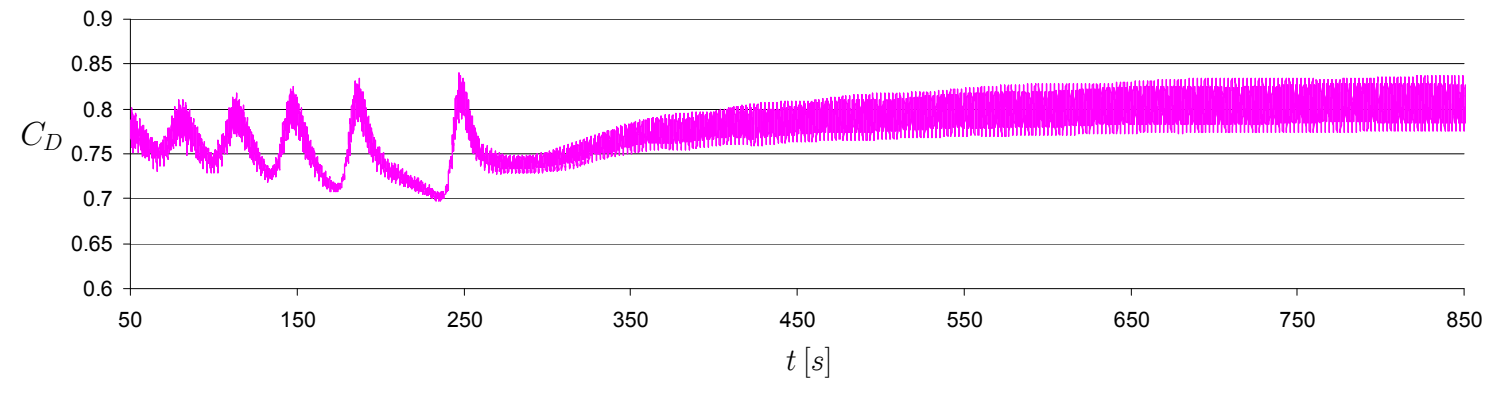

Figura A.192. Oscilación del coeficiente de arrastre 
- $U_{r}=5.5$

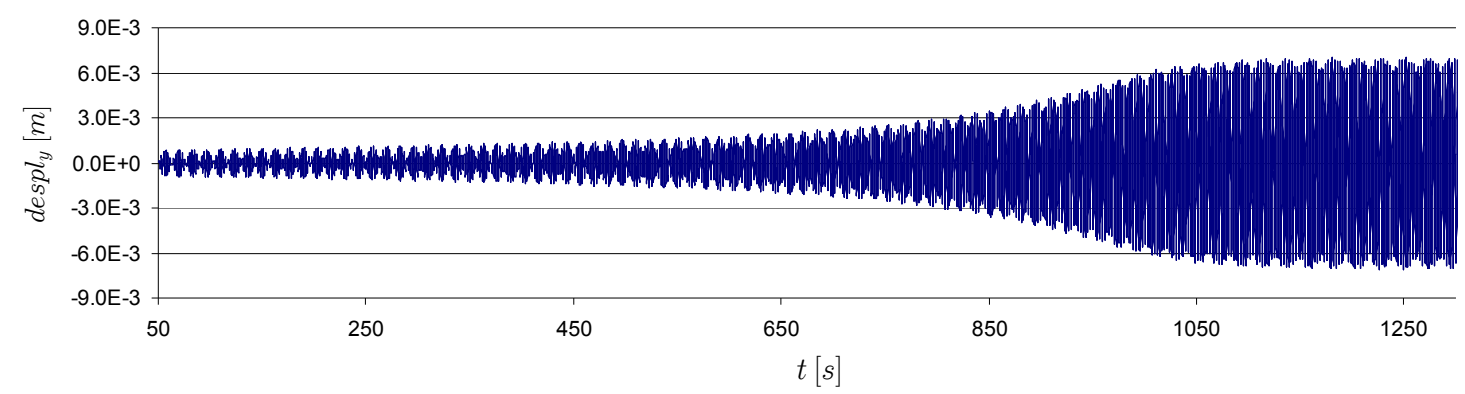

Figura A.193. Vibración transversal de la estructura

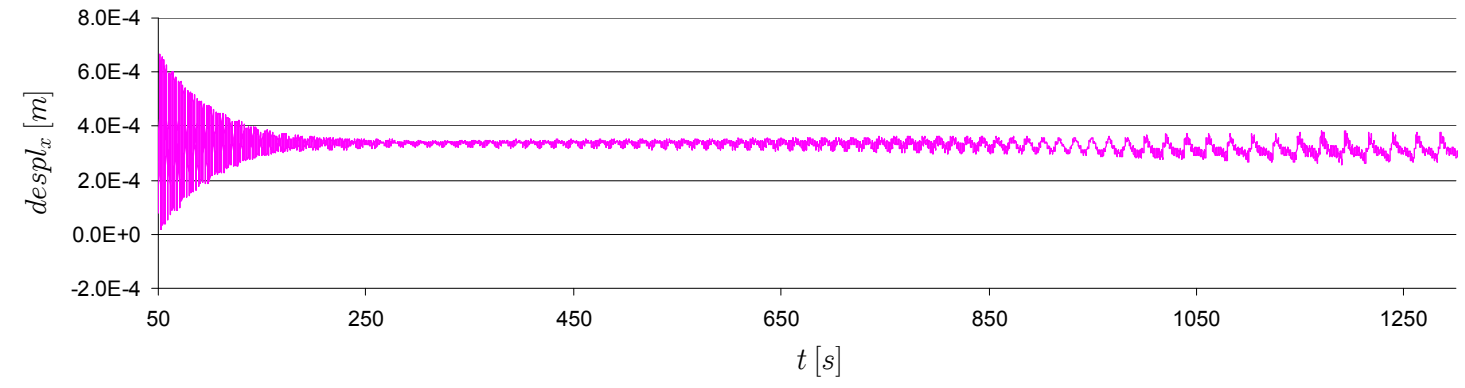

Figura A.194. Vibración en la dirección del flujo de la estructura

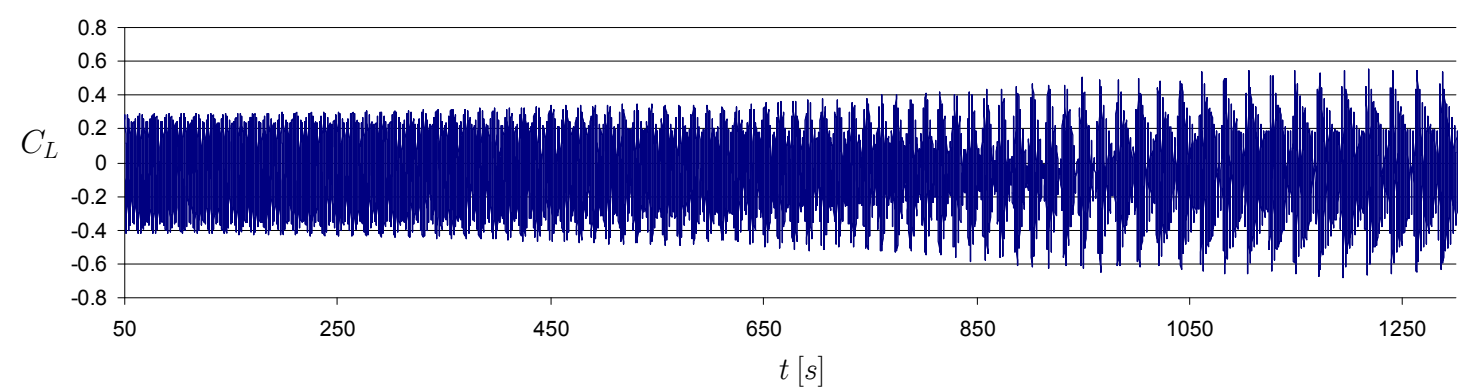

Figura A.195. Oscilación del coeficiente de sustentación

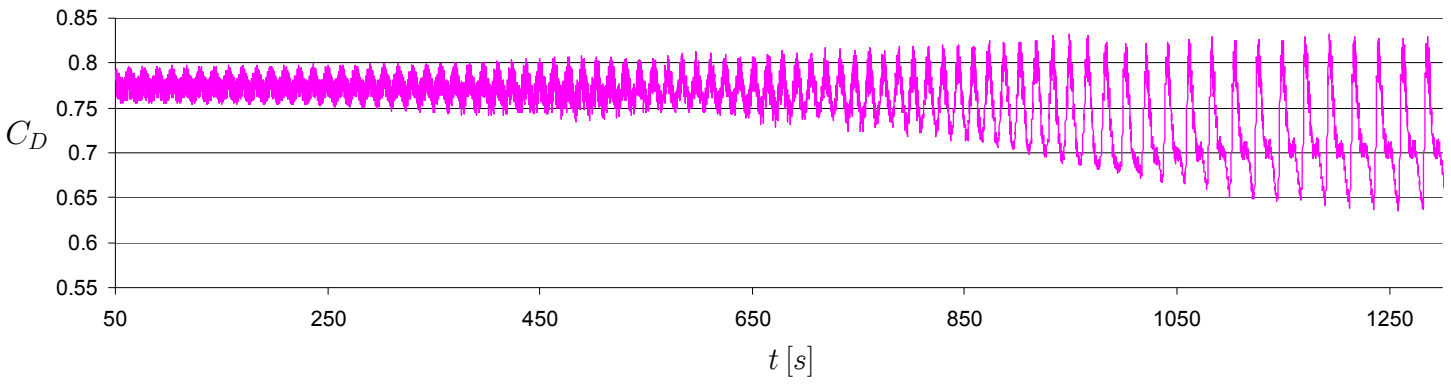

Figura A.196. Oscilación del coeficiente de arrastre 
- $U_{r}=6$

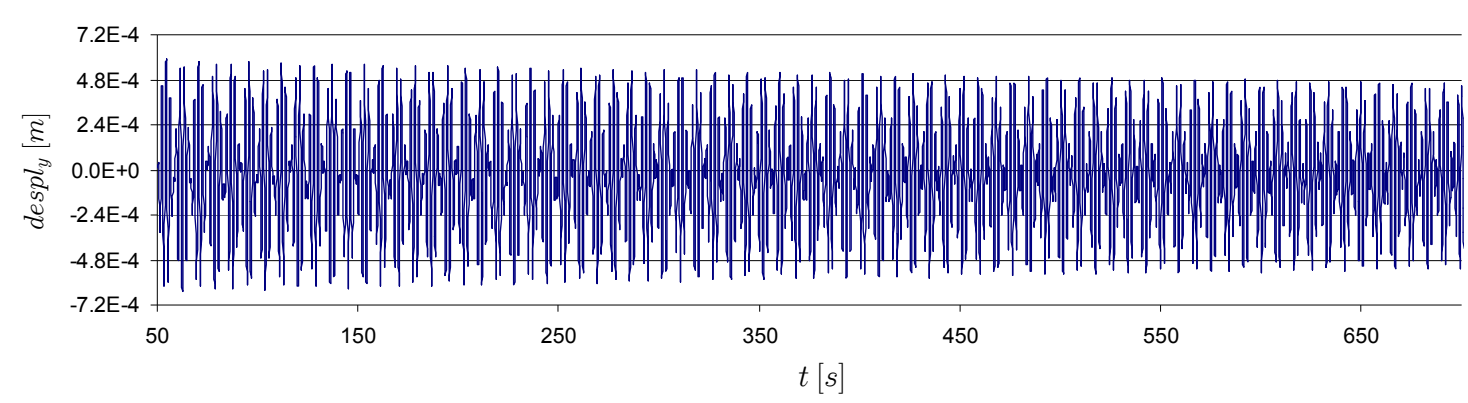

Figura A.197. Vibración transversal de la estructura

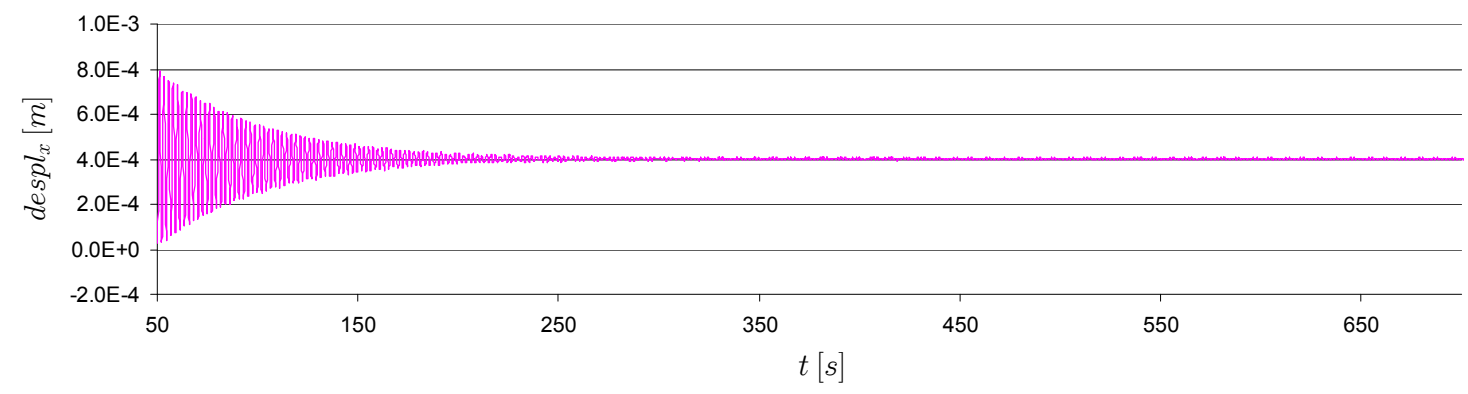

Figura A.198. Vibración en la dirección del flujo de la estructura

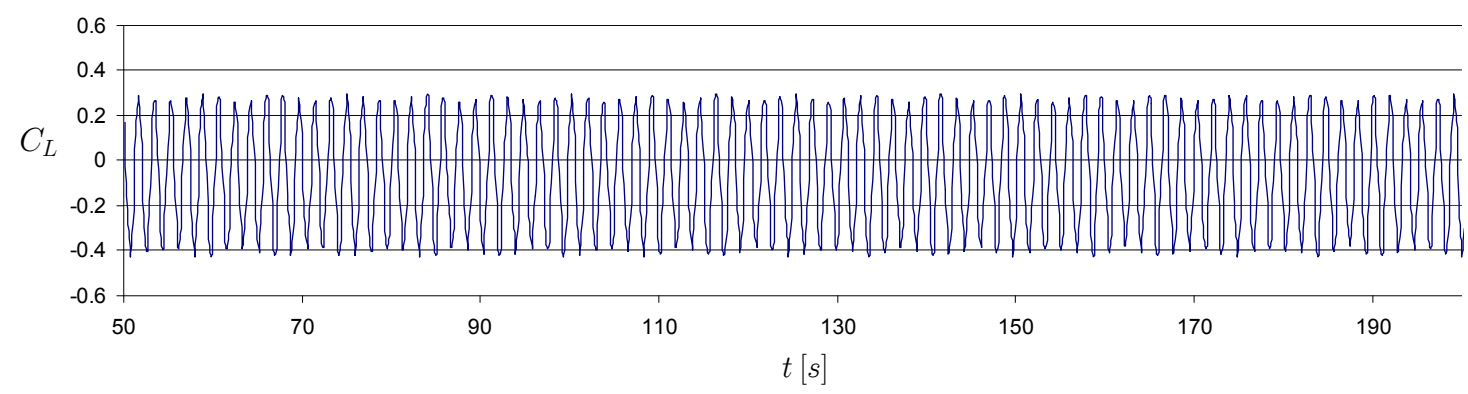

Figura A.199. Oscilación del coeficiente de sustentación

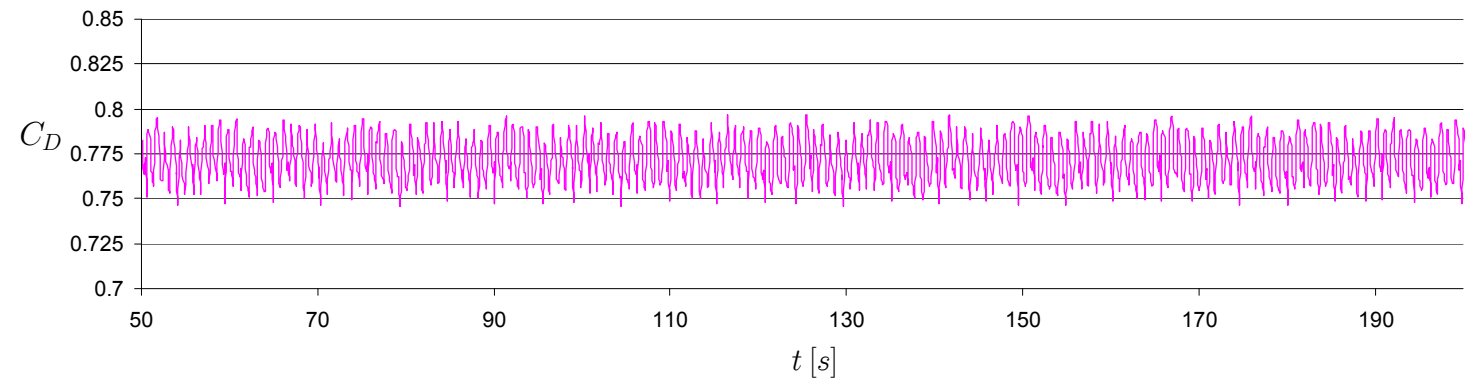

Figura A.200. Oscilación del coeficiente de arrastre 
- $U_{r}=6.5$

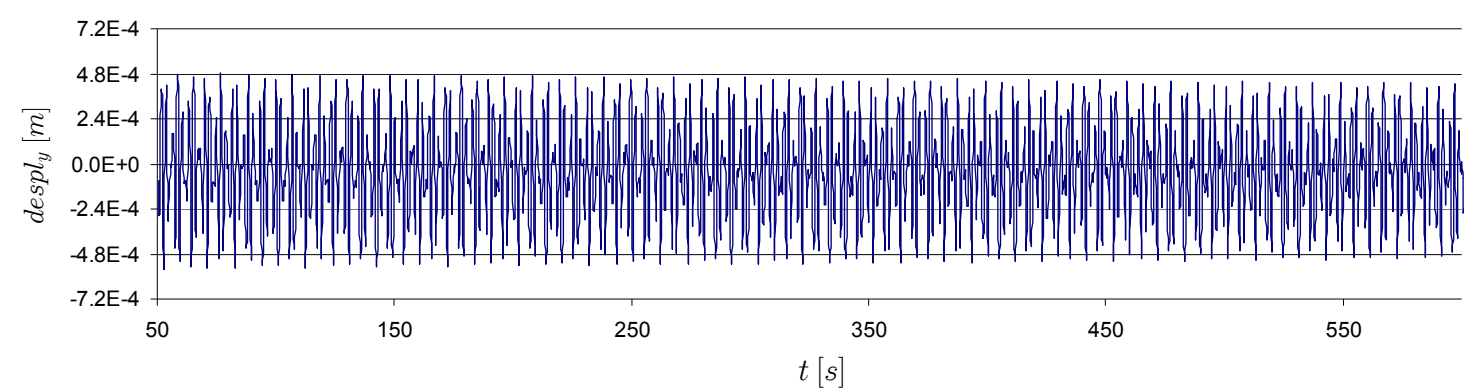

Figura A.201. Vibración transversal de la estructura

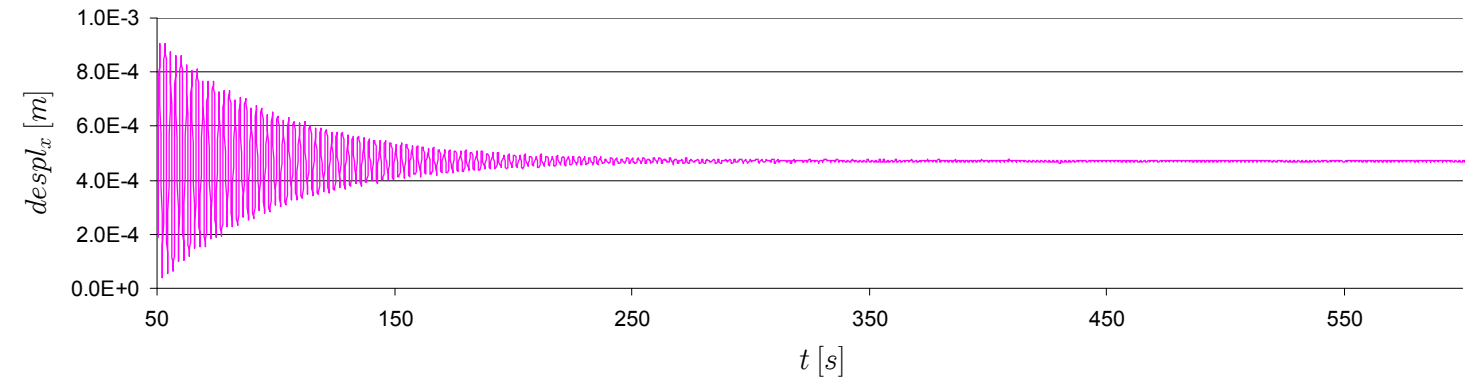

Figura A.202. Vibración en la dirección del flujo de la estructura

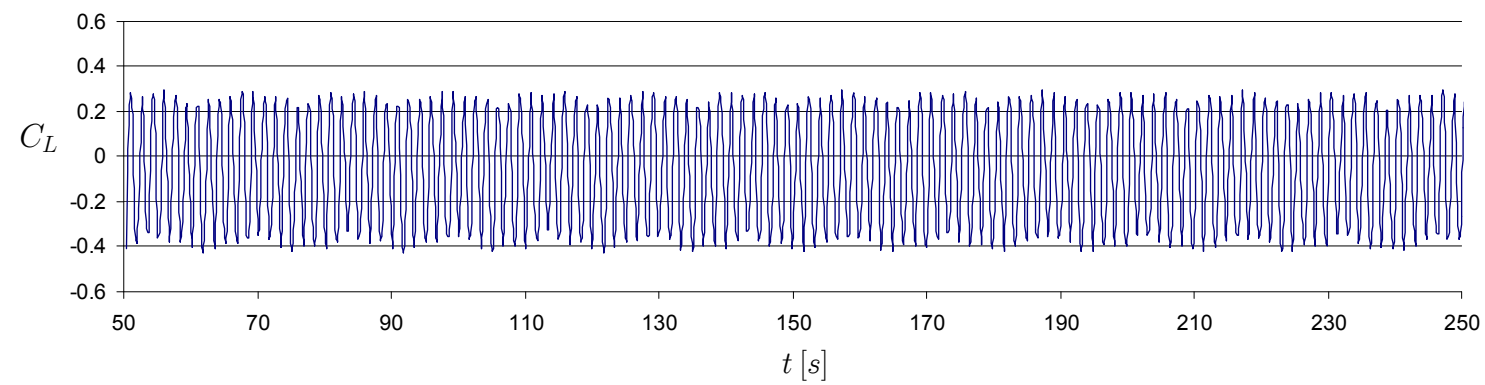

Figura A.203. Oscilación del coeficiente de sustentación

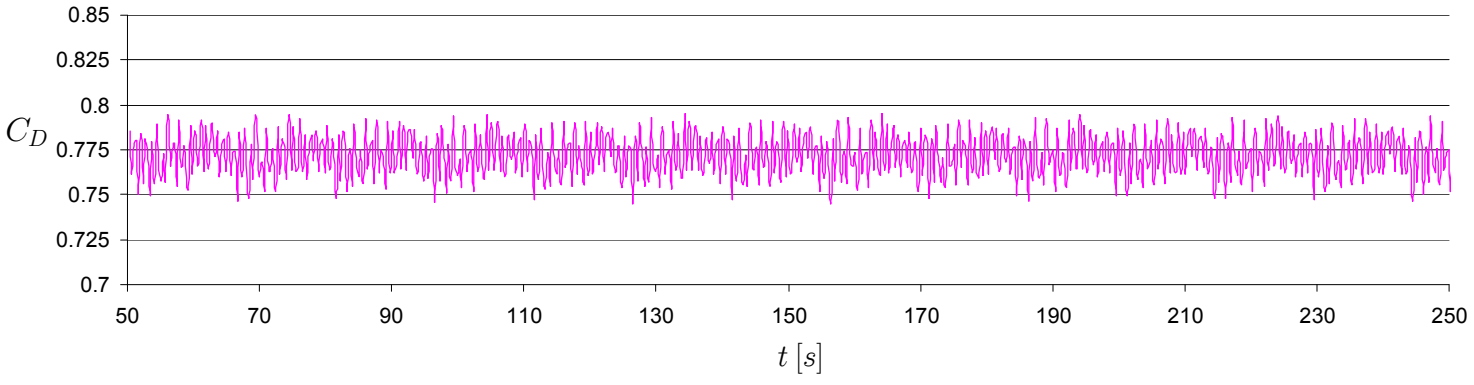

Figura A.204. Oscilación del coeficiente de arrastre 
- $U_{r}=7$

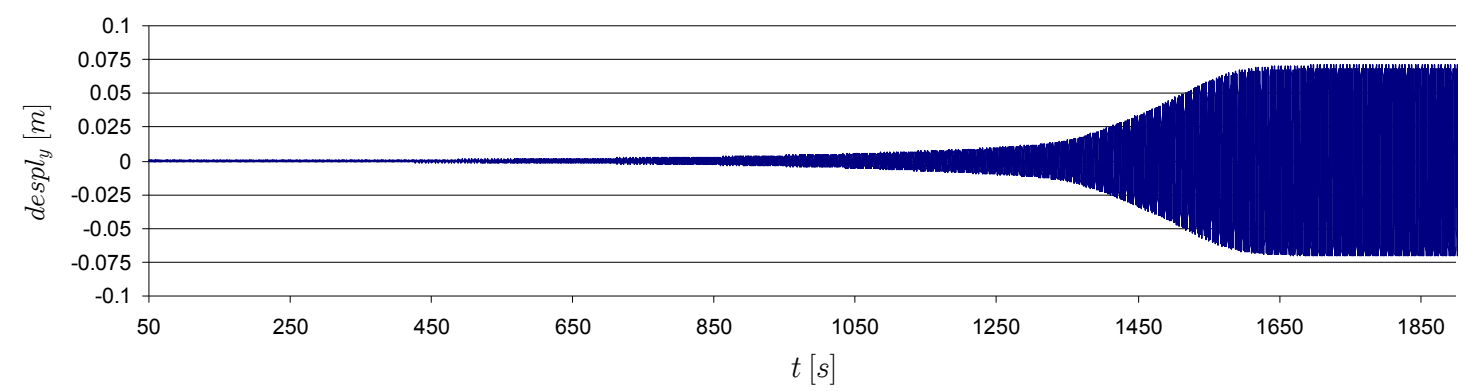

Figura A.205. Vibración transversal de la estructura

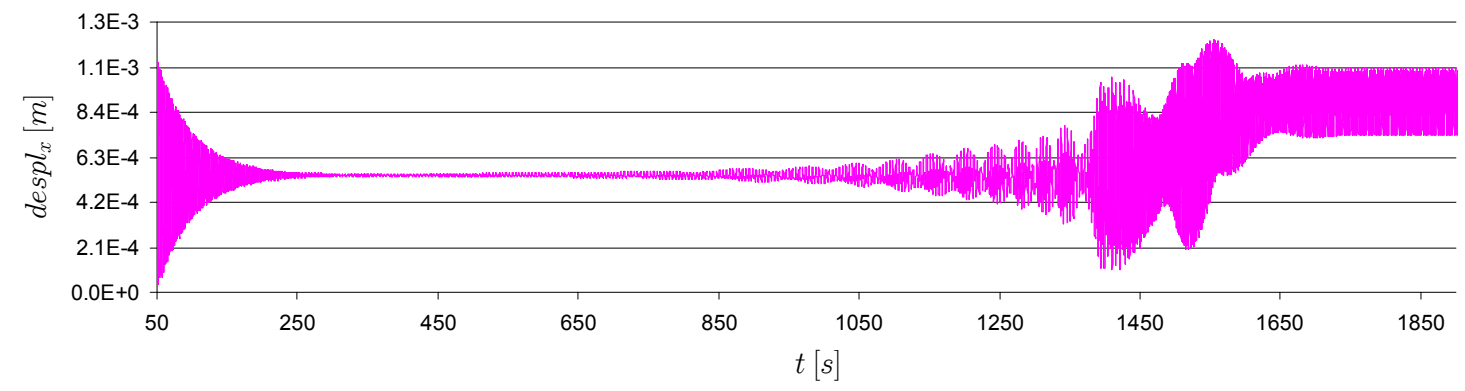

Figura A.206. Vibración en la dirección del flujo de la estructura

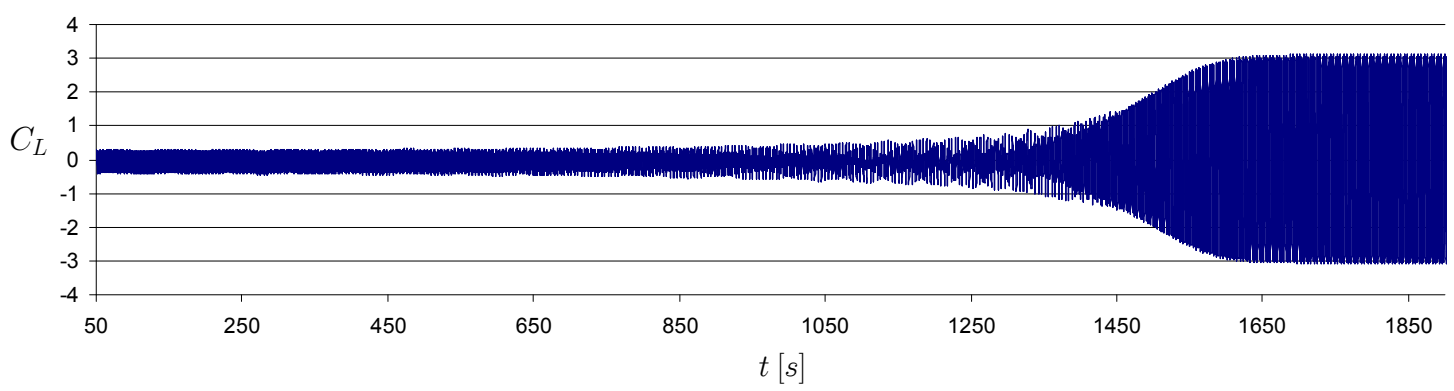

Figura A.207. Oscilación del coeficiente de sustentación

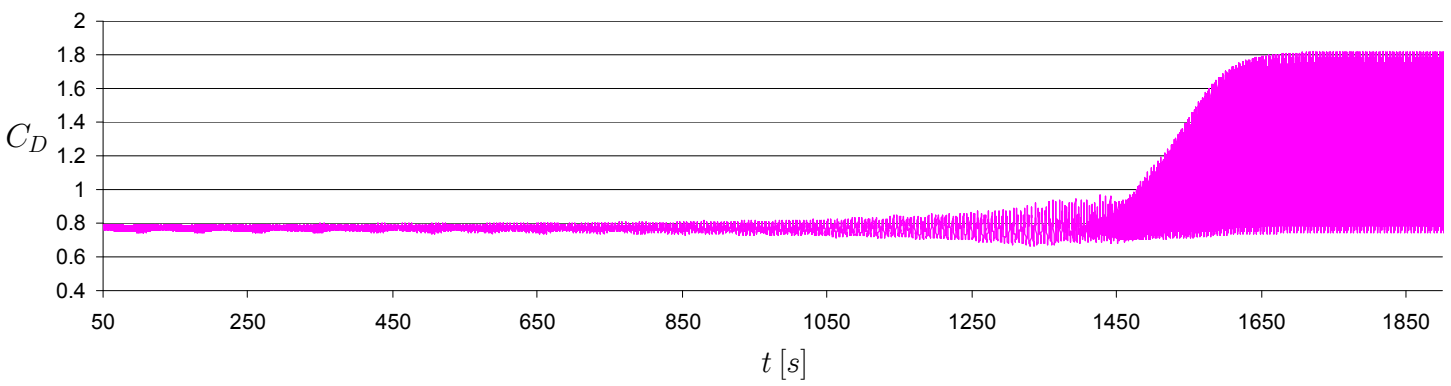

Figura A.208. Oscilación del coeficiente de arrastre 


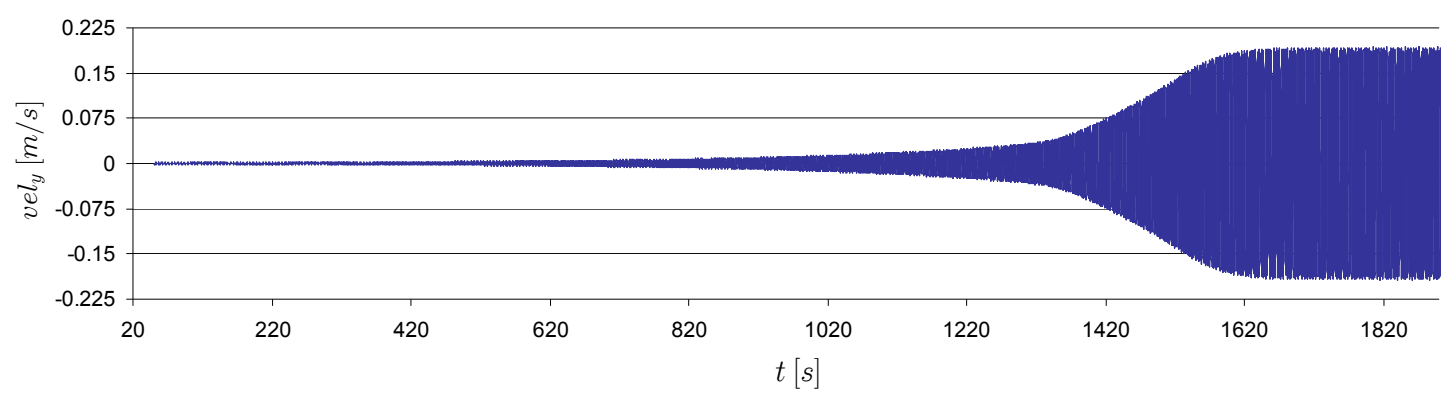

Figura A.209. Componente de la velocidad en la dirección transversal

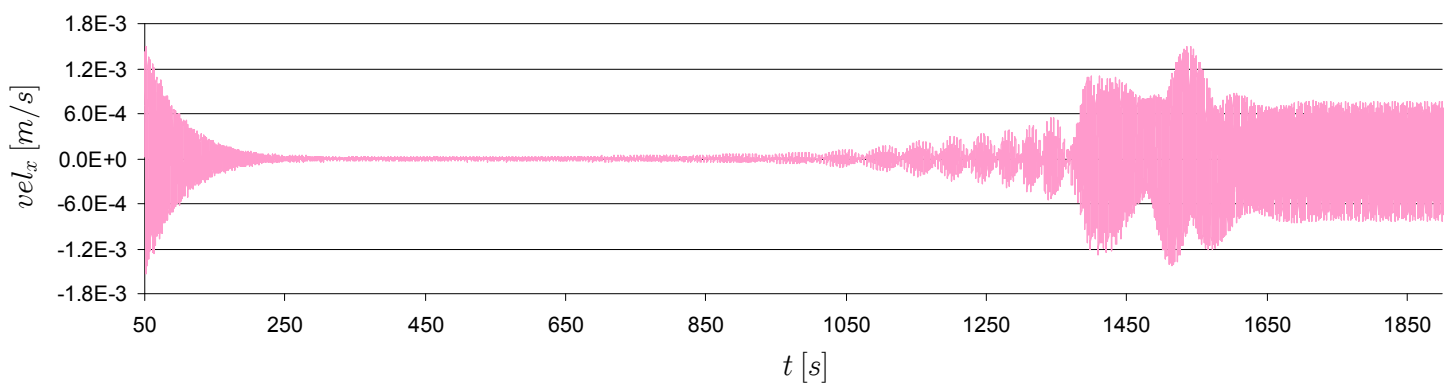

Figura A.210. Componente de la velocidad en la dirección del flujo

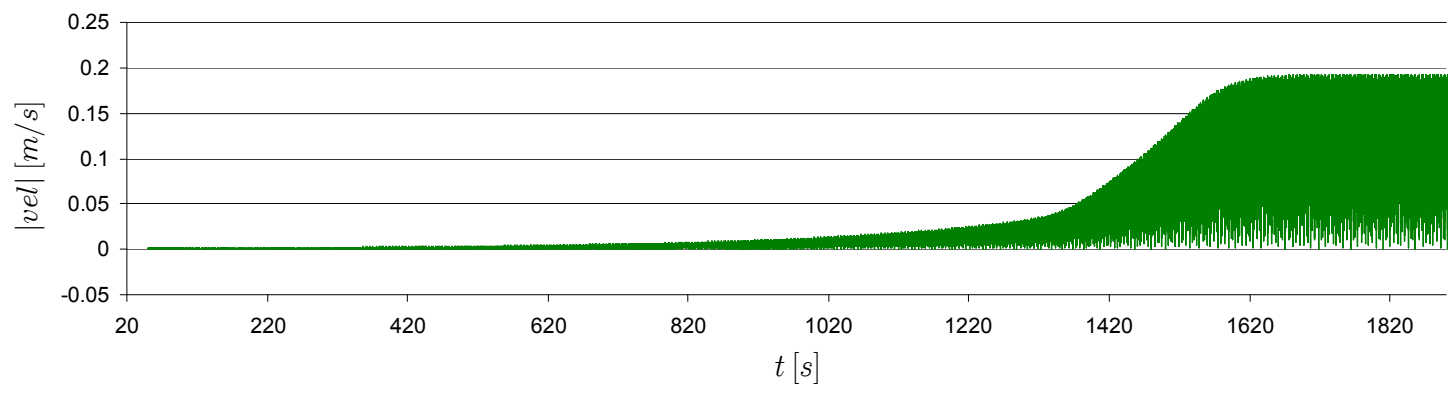

Figura A.211. Módulo de la velocidad 
- $U_{r}=8$

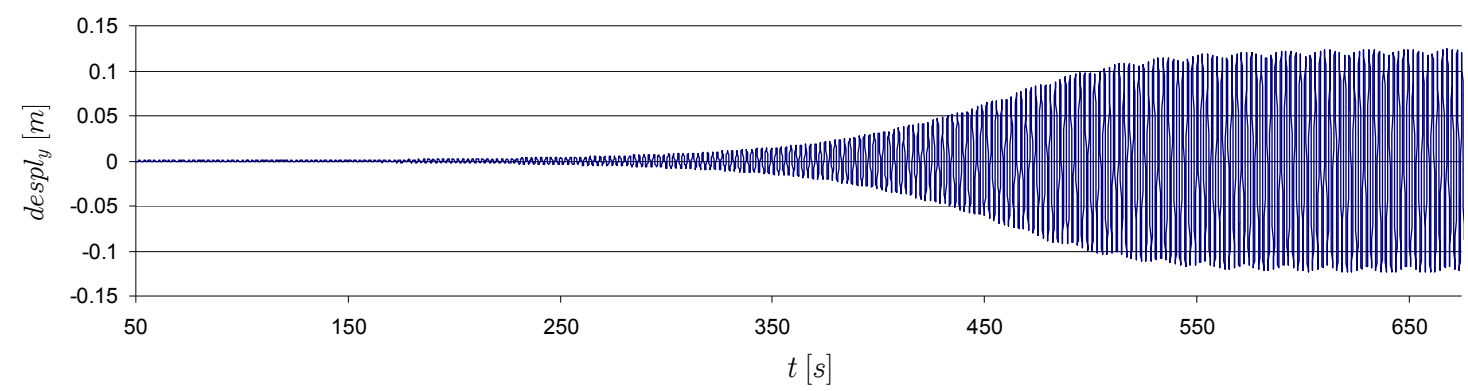

Figura A.212. Vibración transversal de la estructura

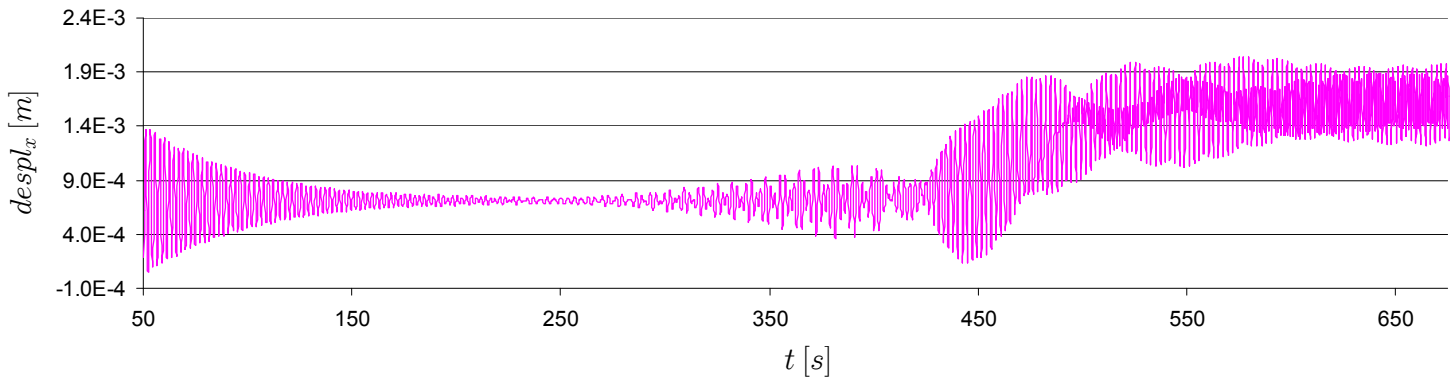

Figura A.213. Vibración en la dirección del flujo de la estructura

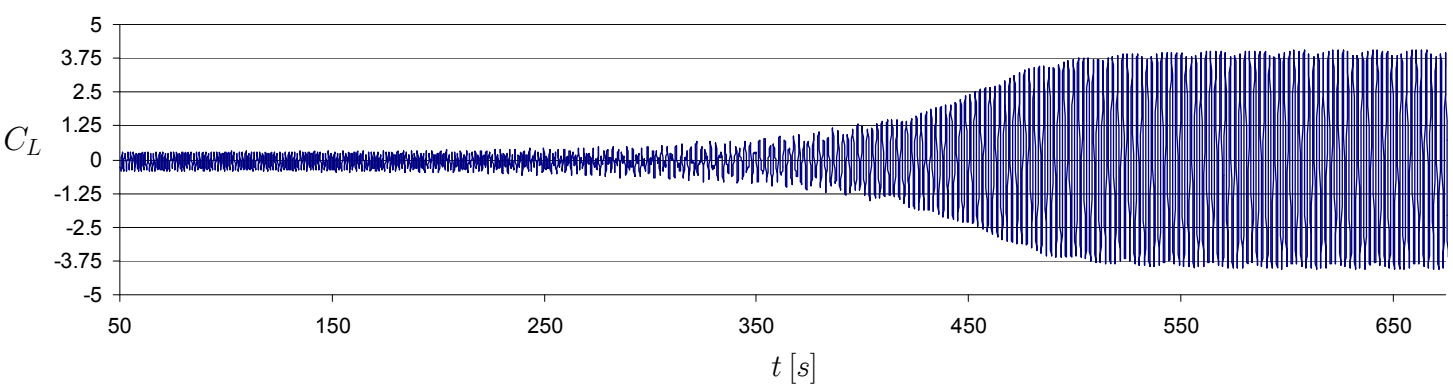

Figura A.214. Oscilación del coeficiente de sustentación

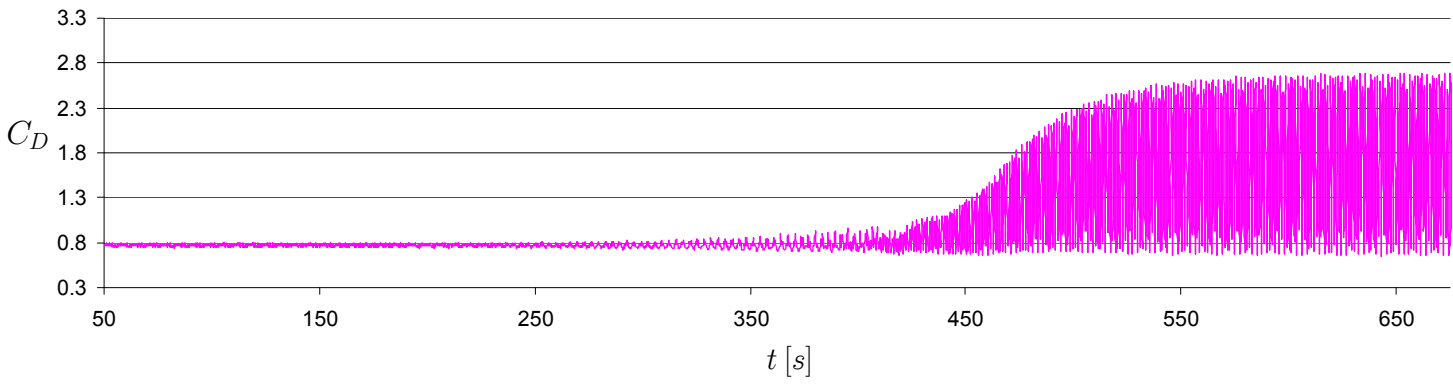

Figura A.215. Oscilación del coeficiente de arrastre 


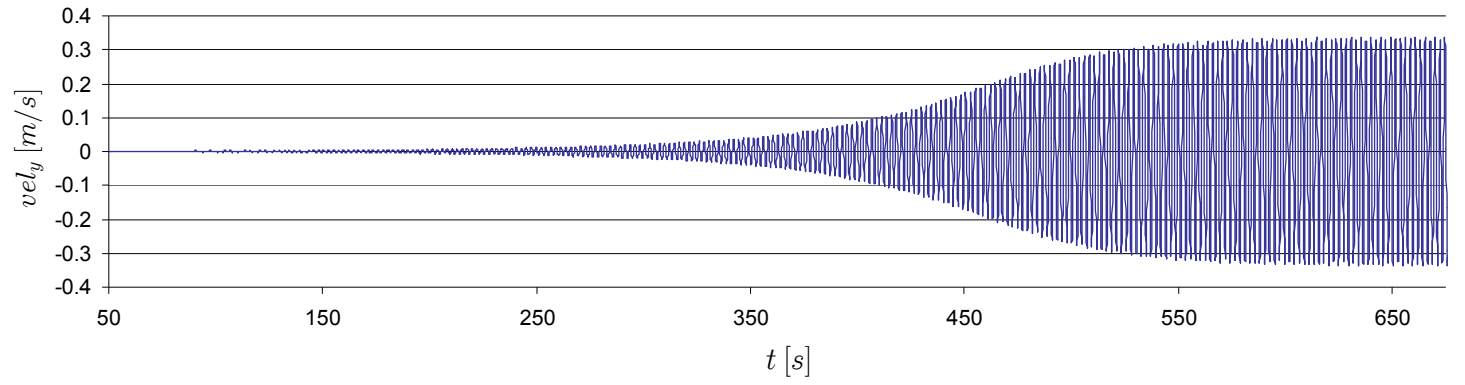

Figura A.216. Componente de la velocidad en la dirección transversal

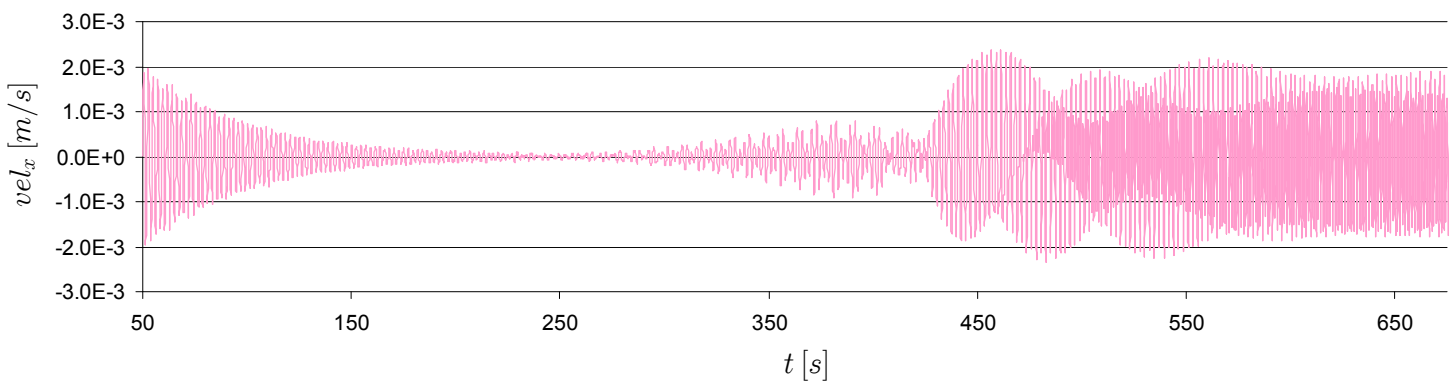

Figura A.217. Componente de la velocidad en la dirección del flujo

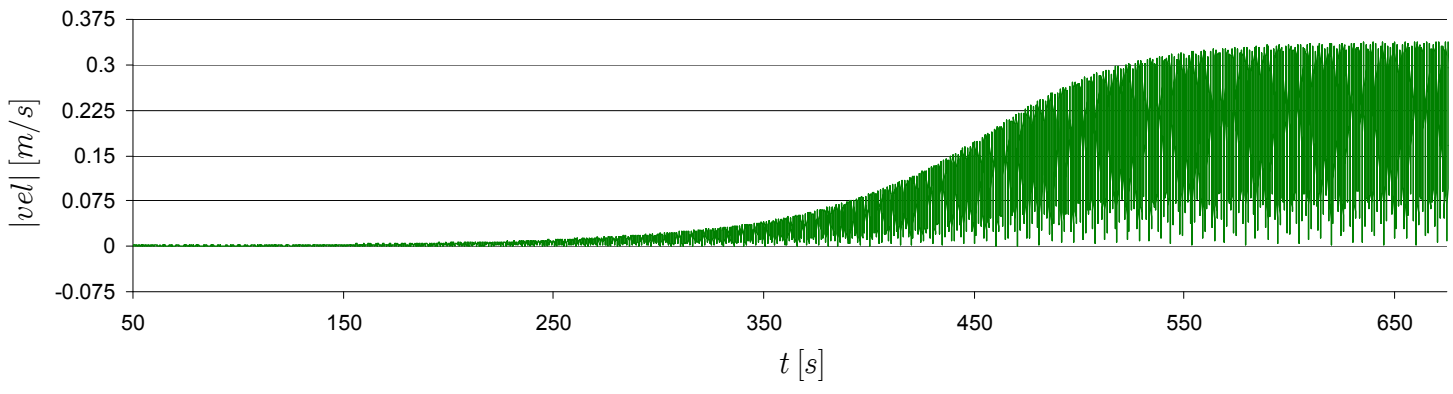

Figura A.218. Módulo de la velocidad 
- $U_{r}=9$

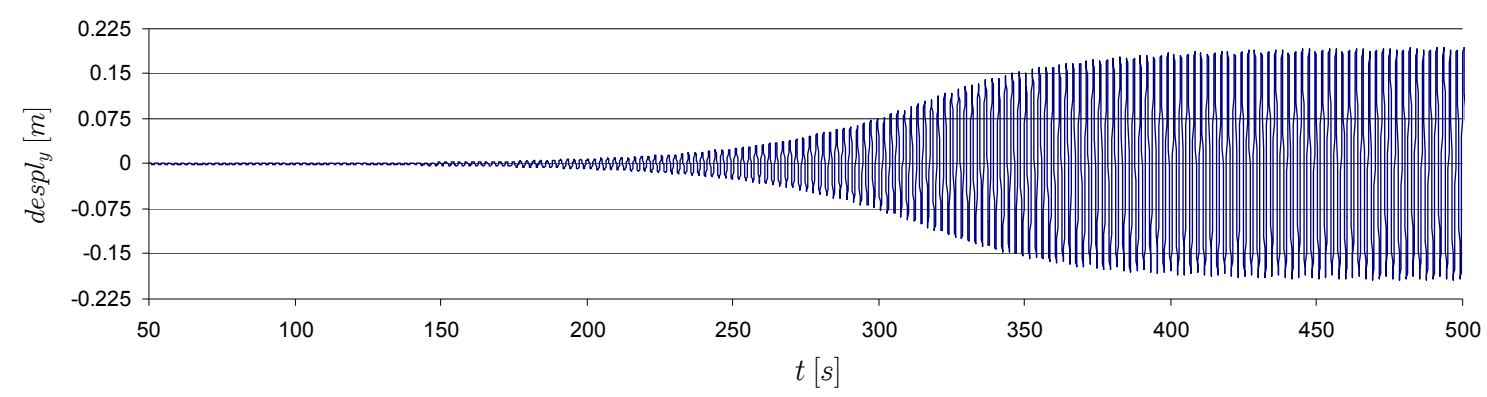

Figura A.219. Vibración transversal de la estructura

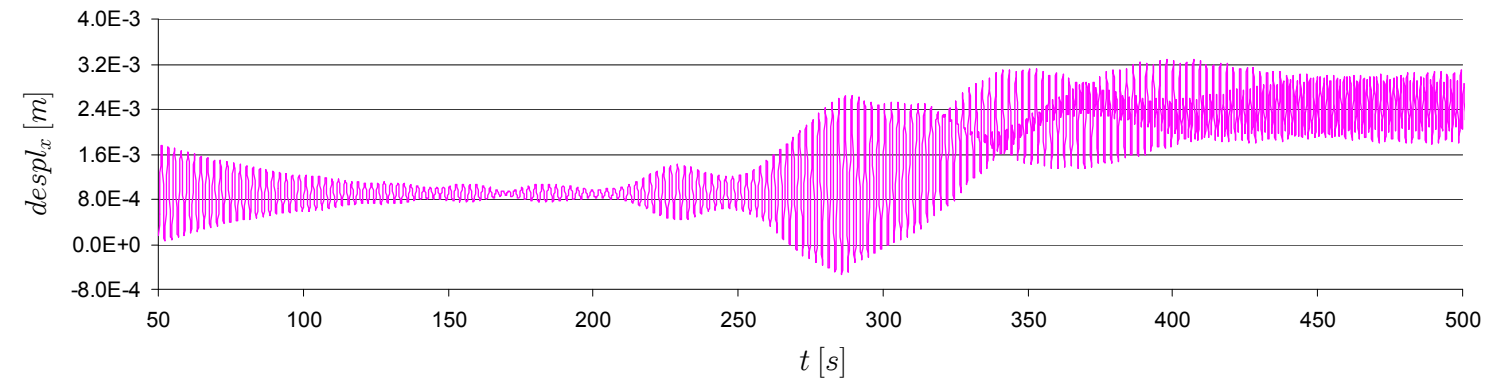

Figura A.220. Vibración en la dirección del flujo de la estructura

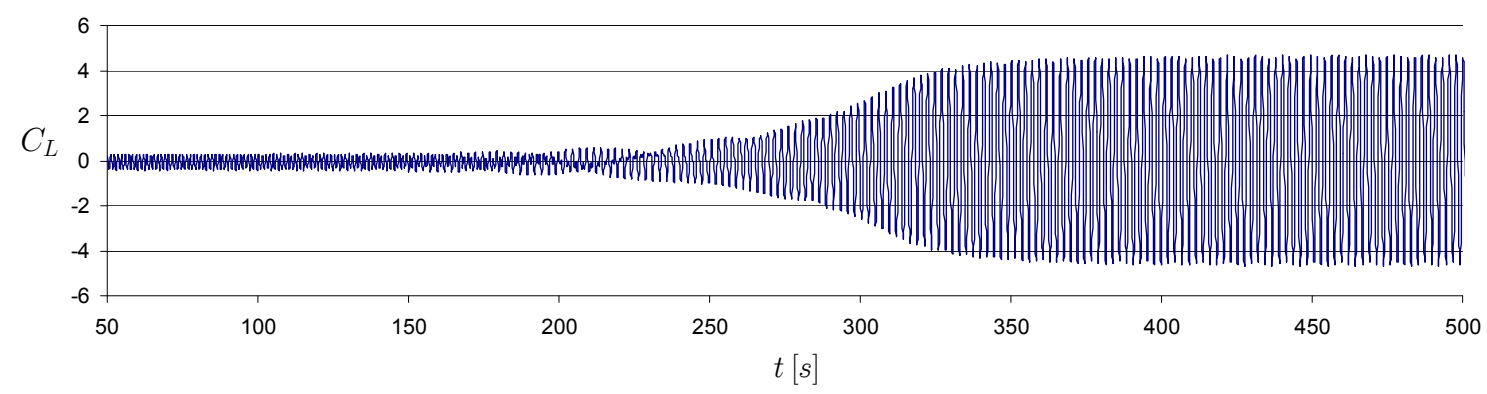

Figura A.221. Oscilación del coeficiente de sustentación

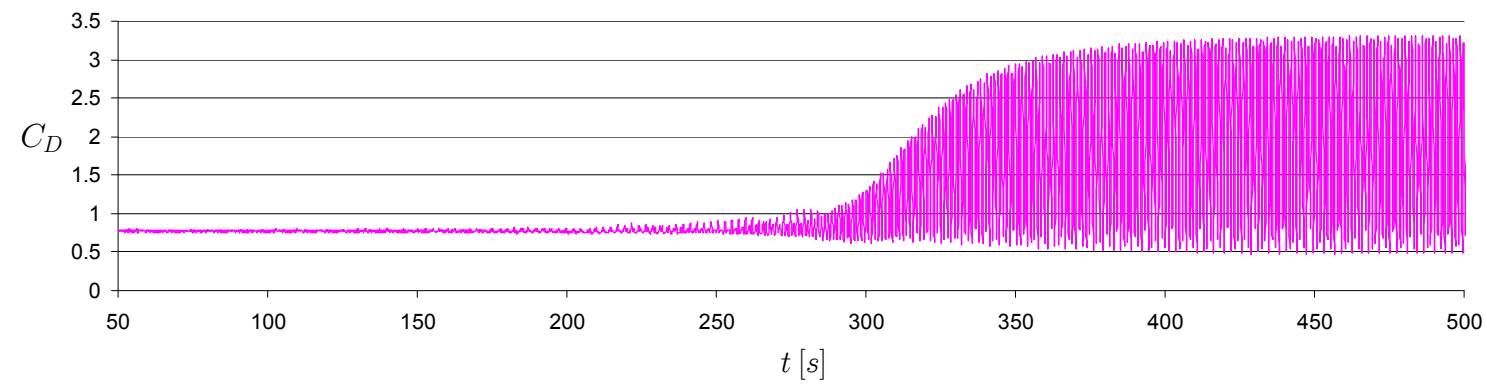

Figura A.222. Oscilación del coeficiente de arrastre 


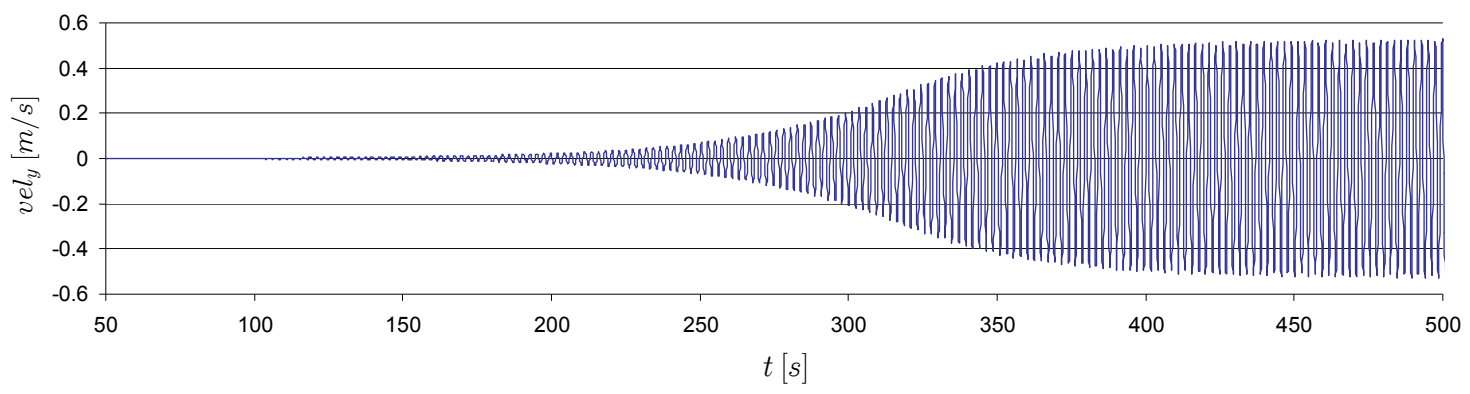

Figura A.223. Componente de la velocidad en la dirección transversal

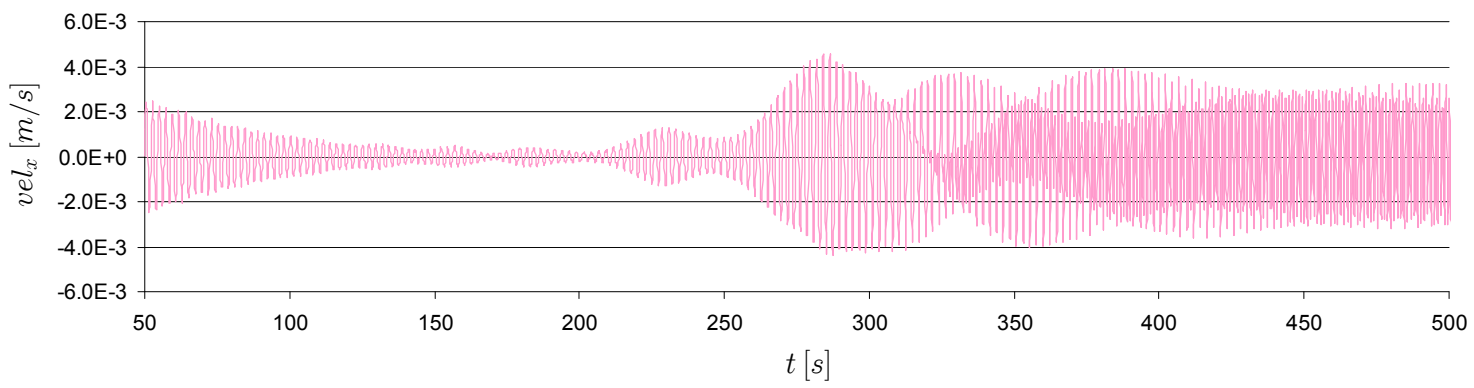

Figura A.224. Componente de la velocidad en la dirección del flujo

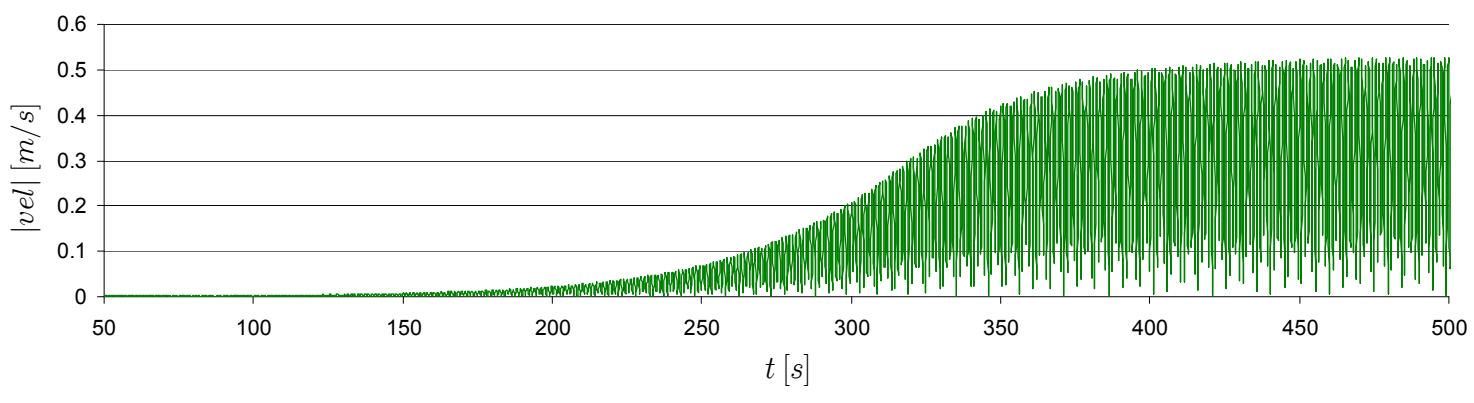

Figura A.225. Módulo de la velocidad 
- $U_{r}=11$

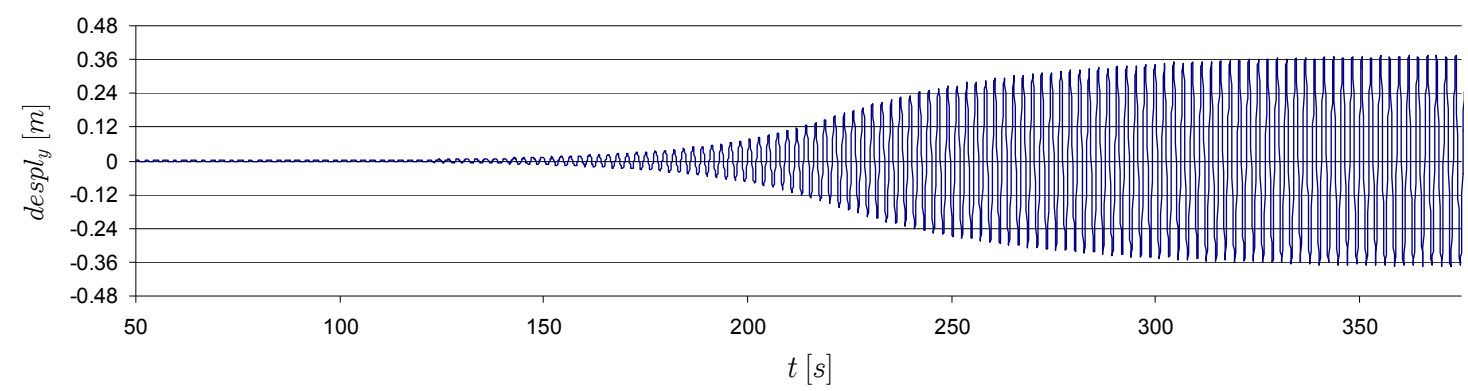

Figura A.226. Vibración transversal de la estructura

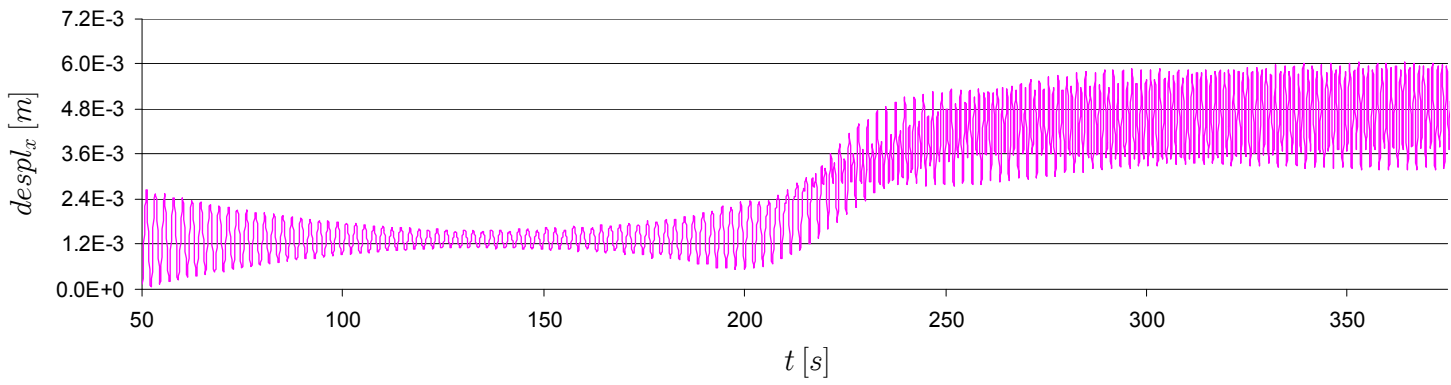

Figura A.227. Vibración en la dirección del flujo de la estructura

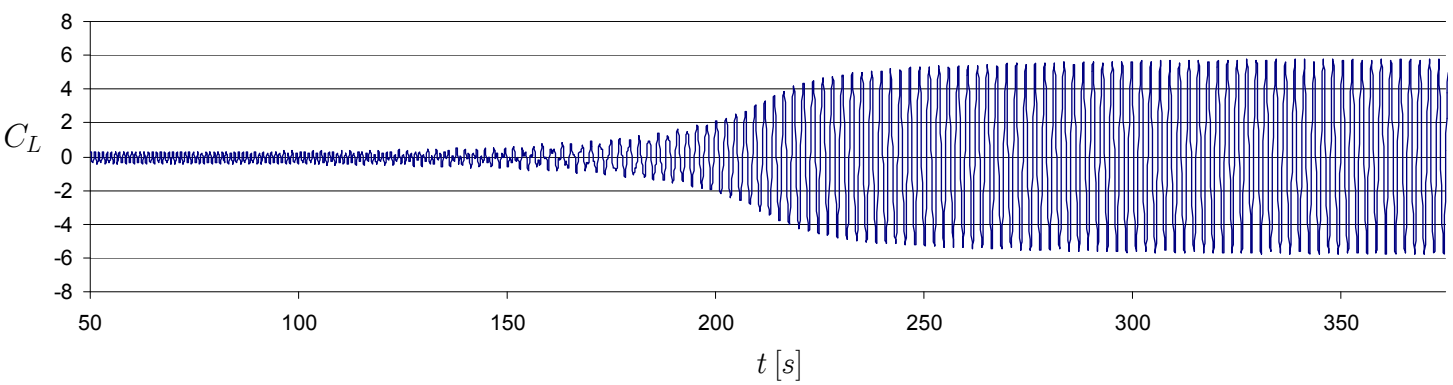

Figura A.228. Oscilación del coeficiente de sustentación

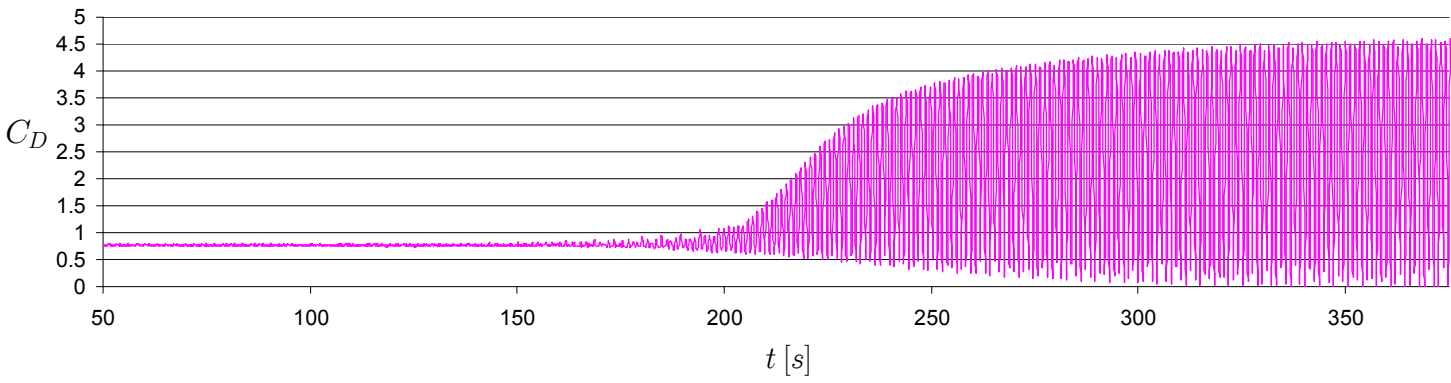

Figura A.229. Oscilación del coeficiente de arrastre 


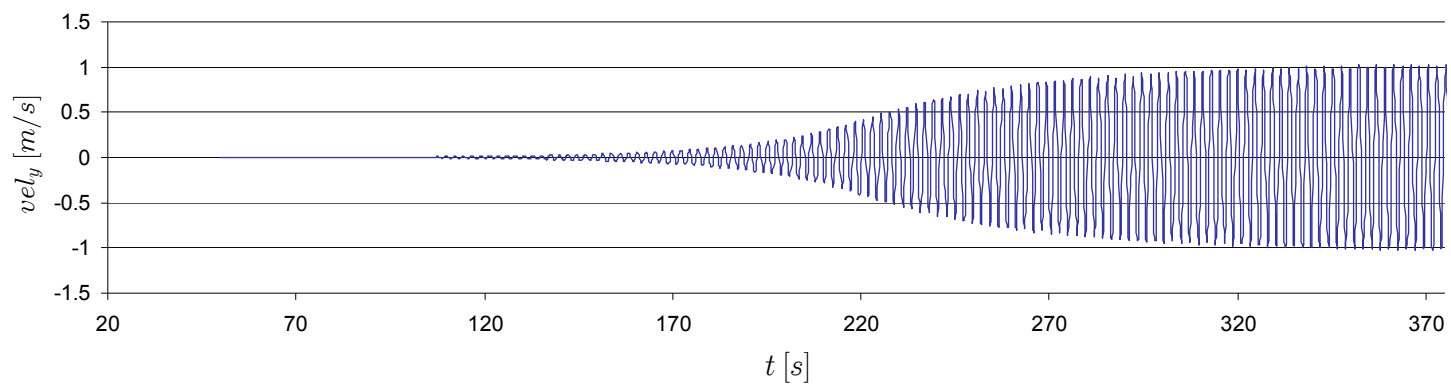

Figura A.230. Componente de la velocidad en la dirección transversal

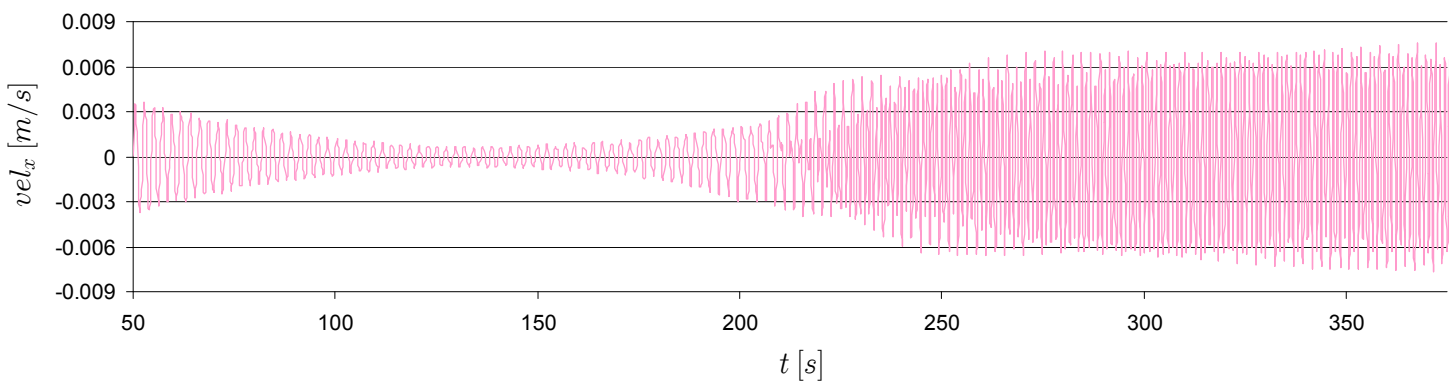

Figura A.231. Componente de la velocidad en la dirección del flujo

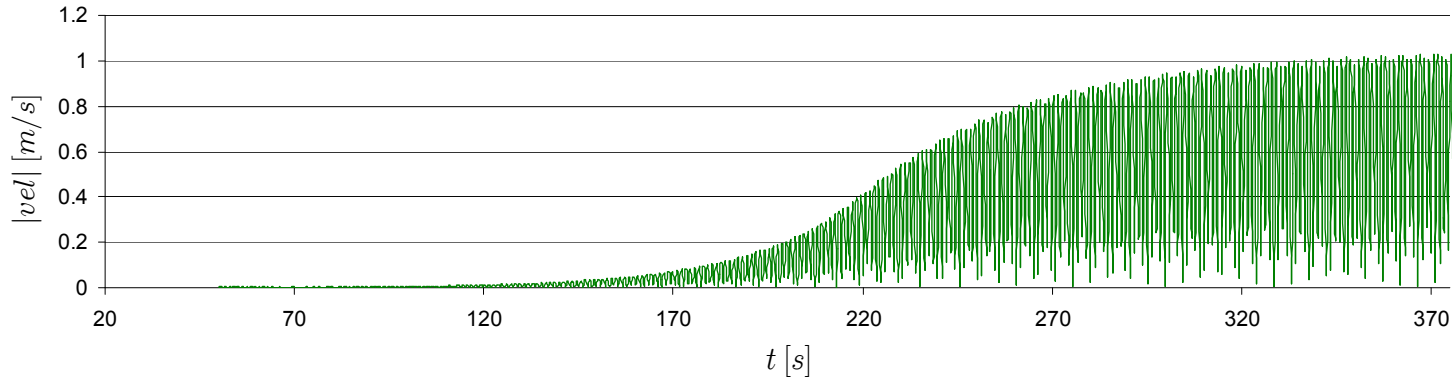

Figura A.232. Módulo de la velocidad 
- $U_{r}=13$

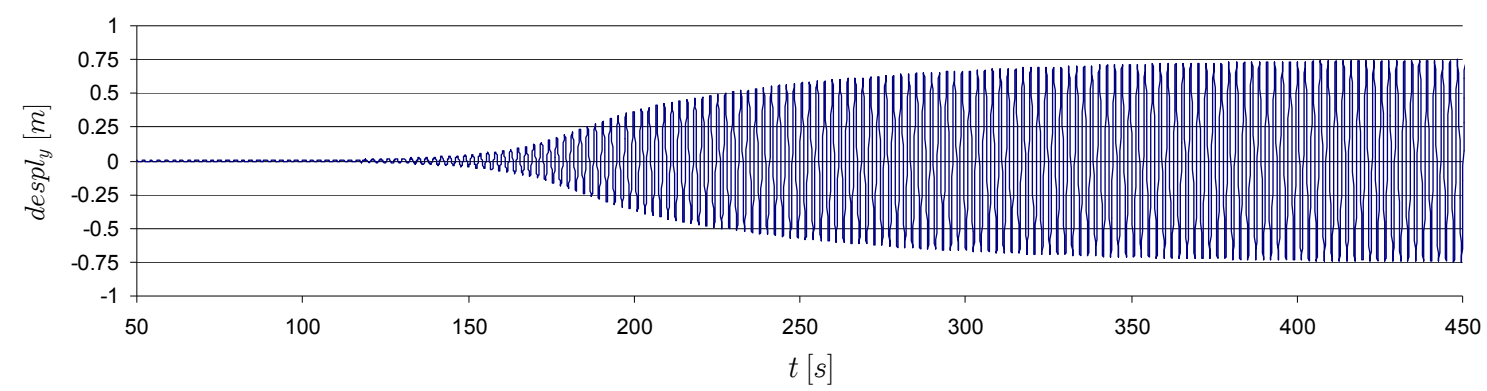

Figura A.233. Vibración transversal de la estructura

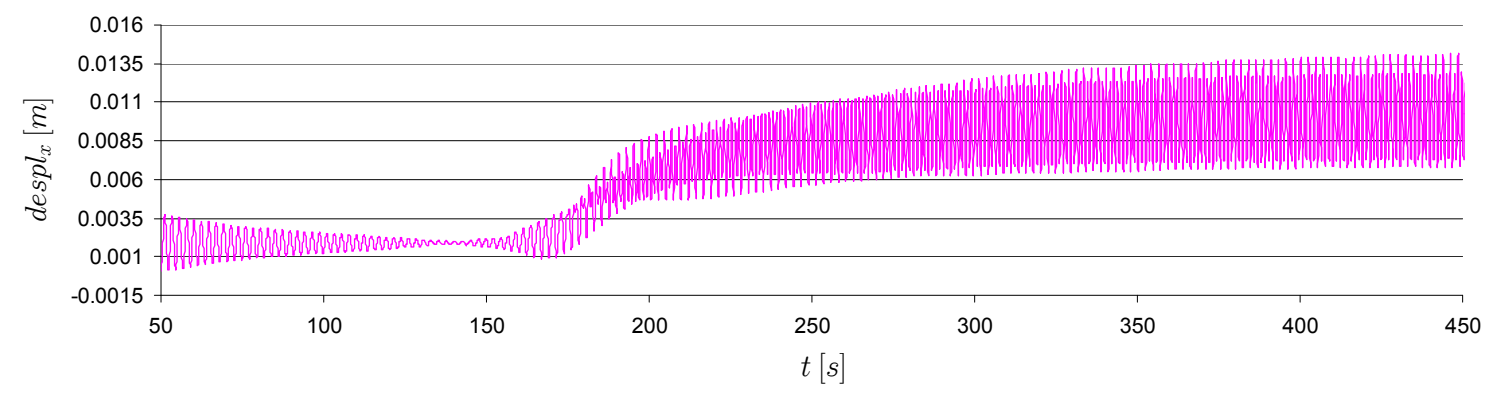

Figura A.234. Vibración en la dirección del flujo de la estructura

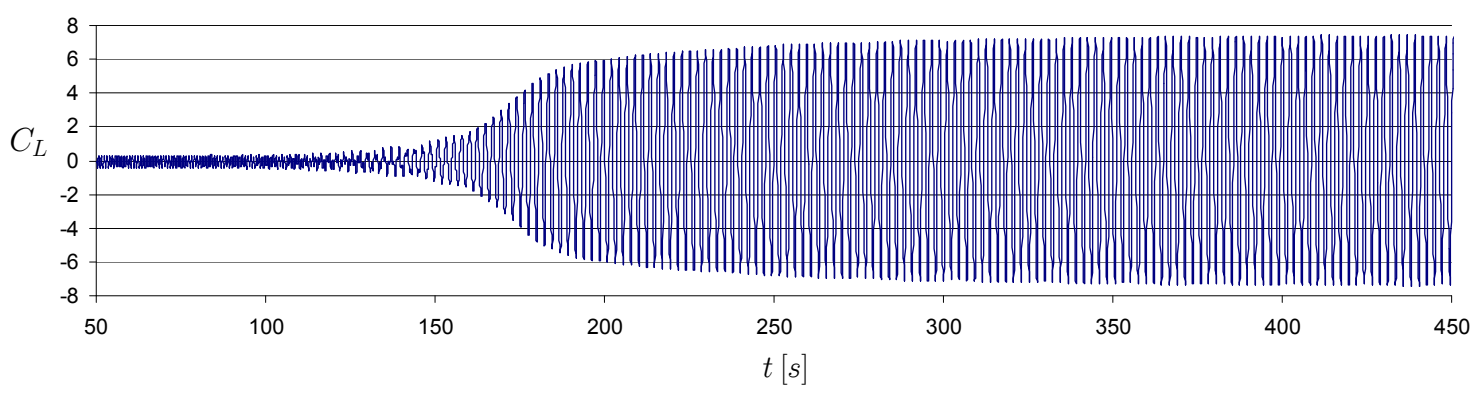

Figura A.235. Oscilación del coeficiente de sustentación

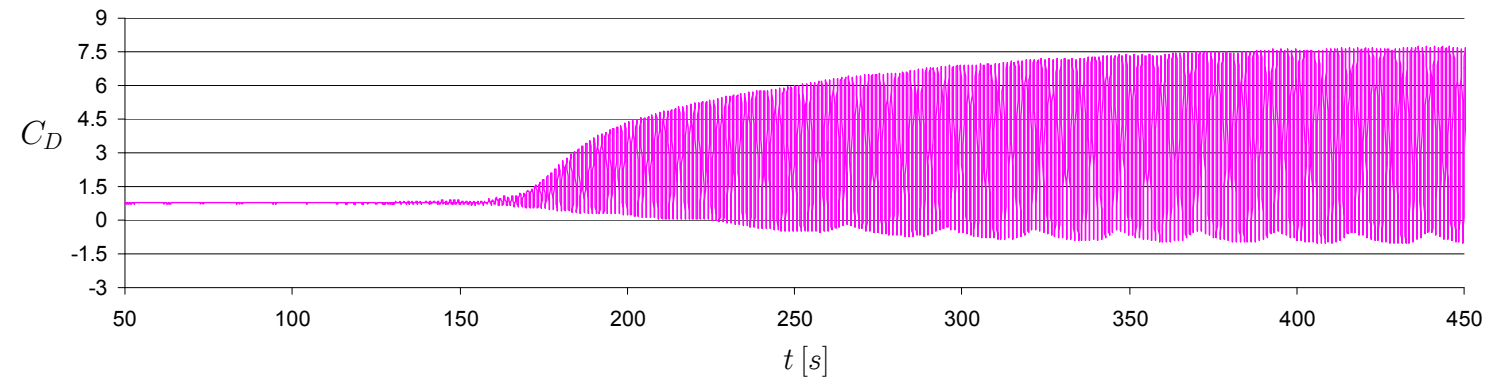

Figura A.236. Oscilación del coeficiente de arrastre 


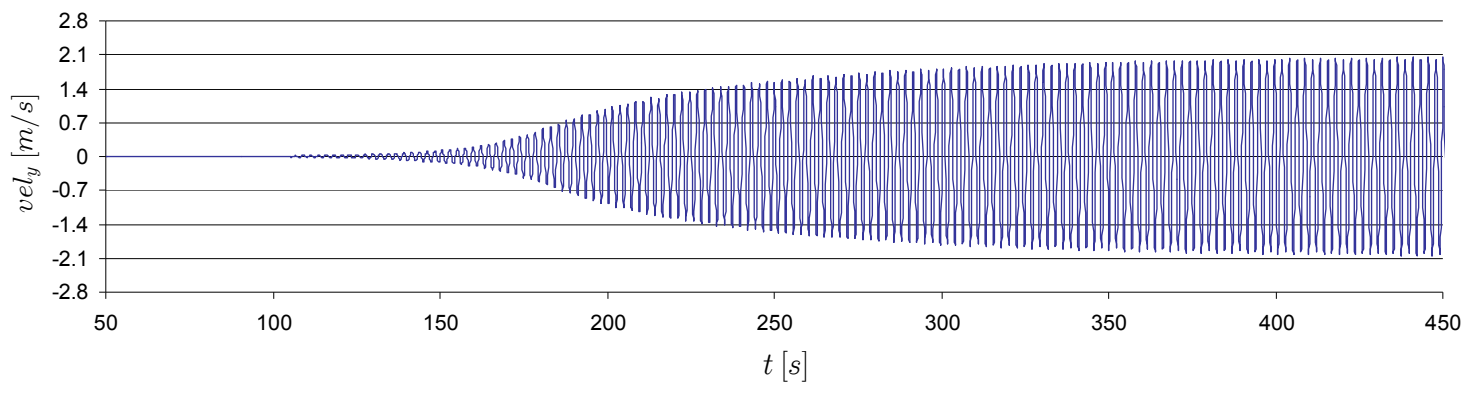

Figura A.237. Componente de la velocidad en la dirección transversal

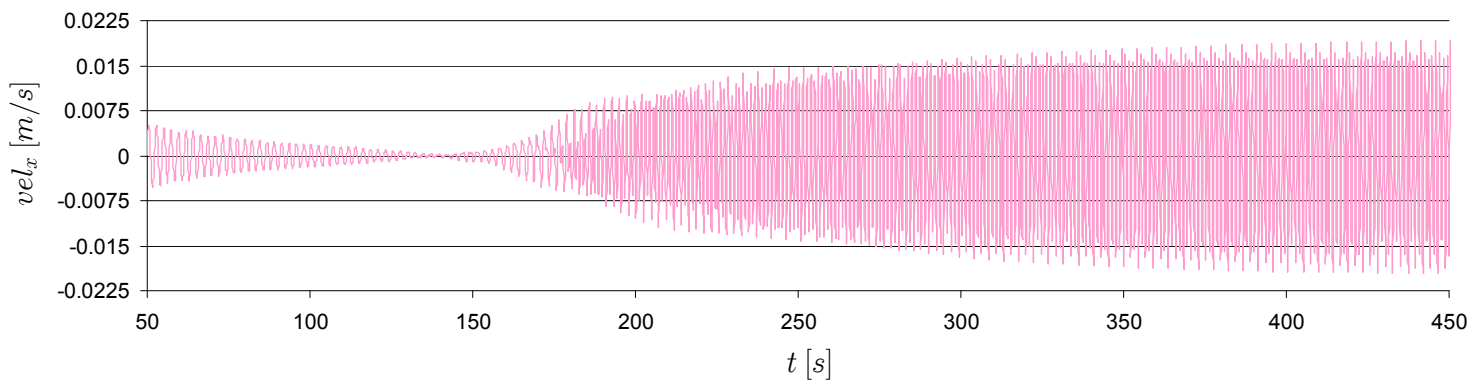

Figura A.238. Componente de la velocidad en la dirección del flujo

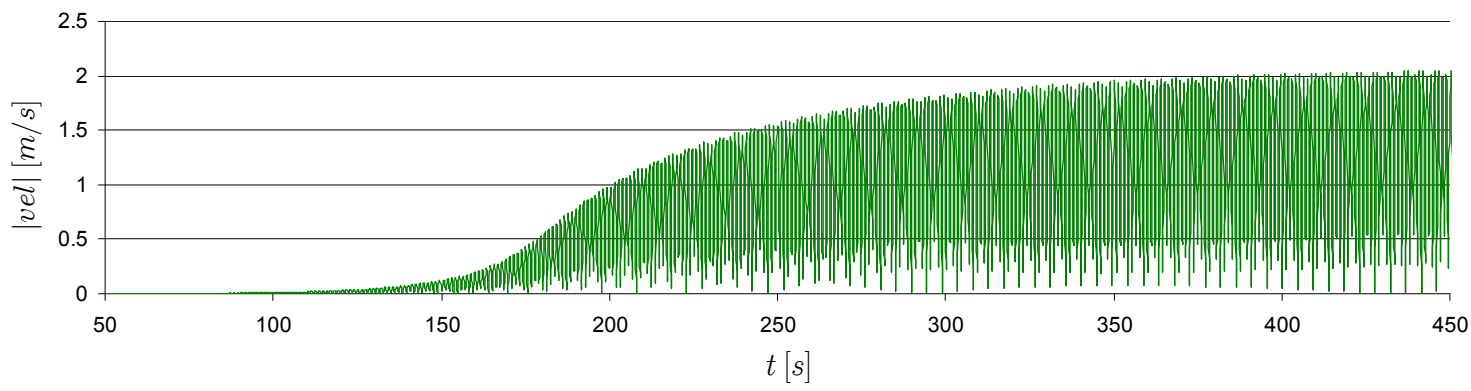

Figura A.239. Módulo de la velocidad 
- $U_{r}=15$

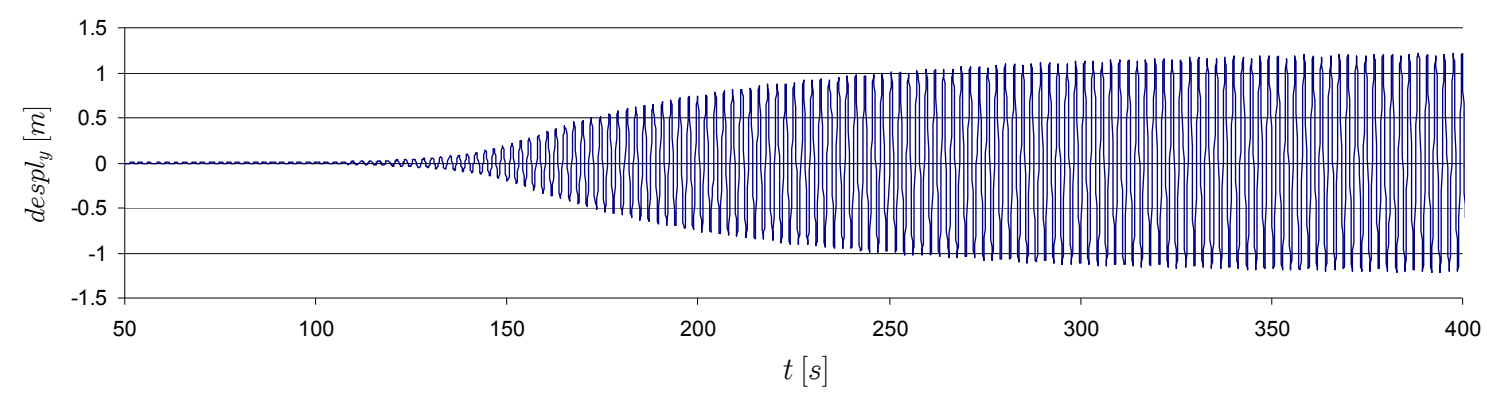

Figura A.240. Vibración transversal de la estructura

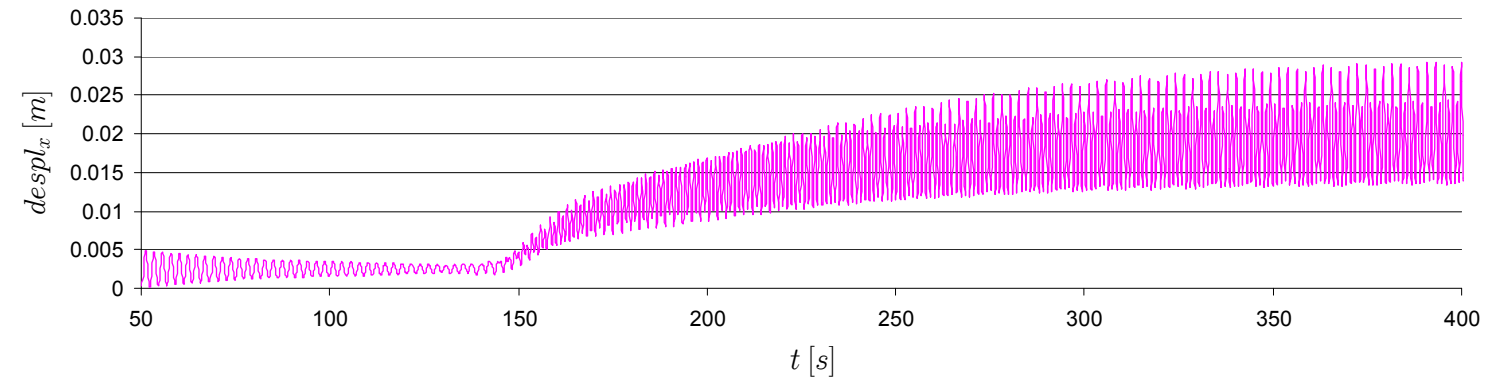

Figura A.241. Vibración en la dirección del flujo de la estructura

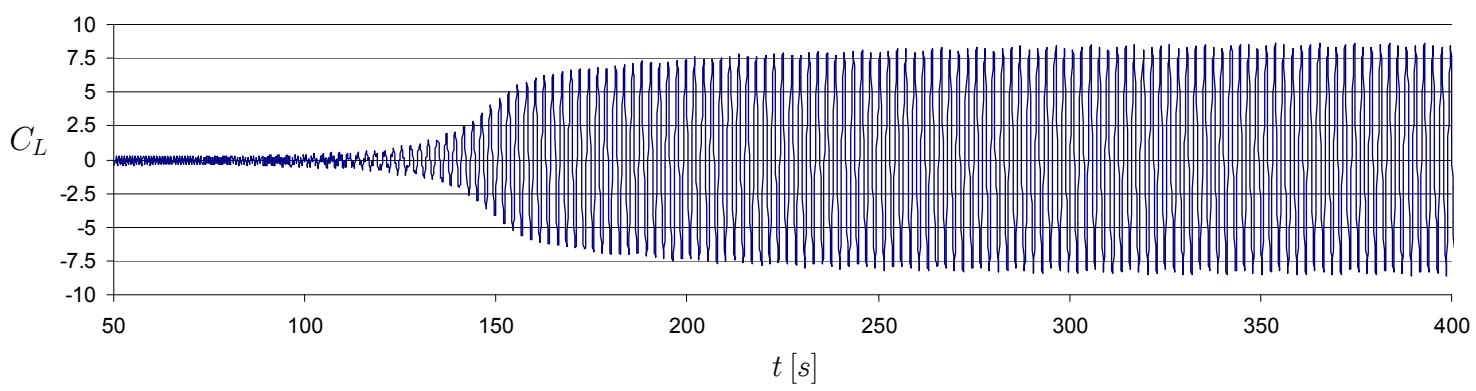

Figura A.242. Oscilación del coeficiente de sustentación

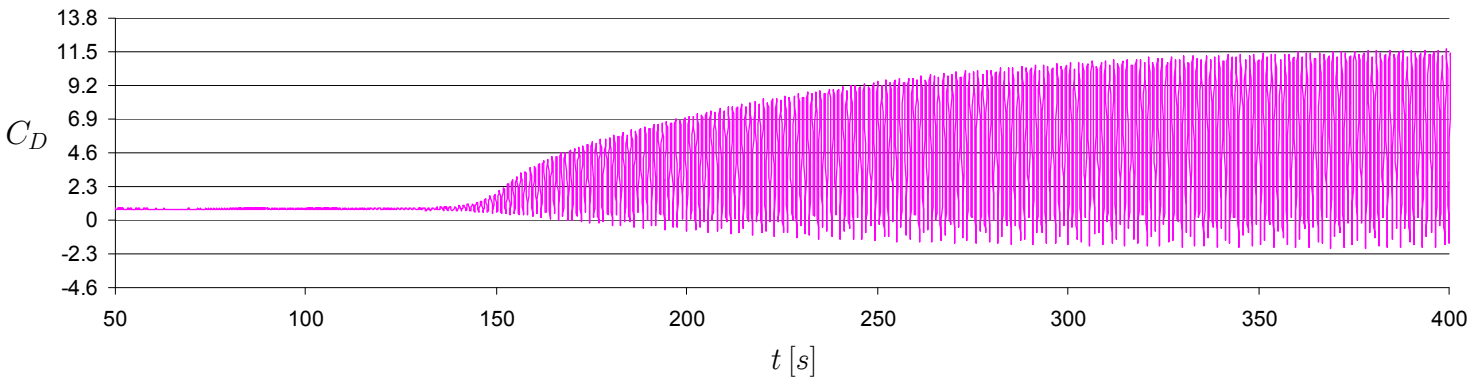

Figura A.243. Oscilación del coeficiente de arrastre 


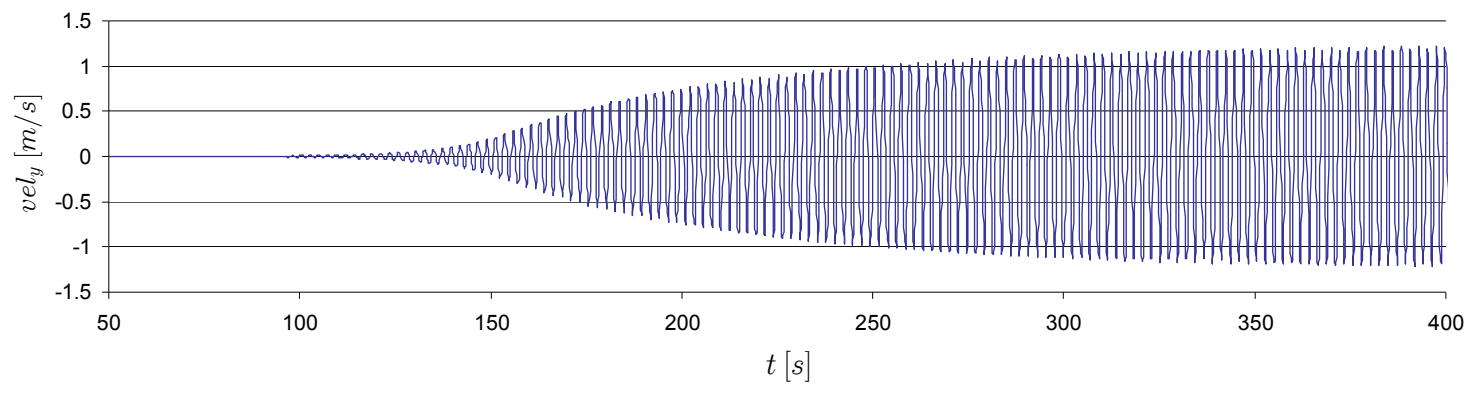

Figura A.244. Componente de la velocidad en la dirección transversal

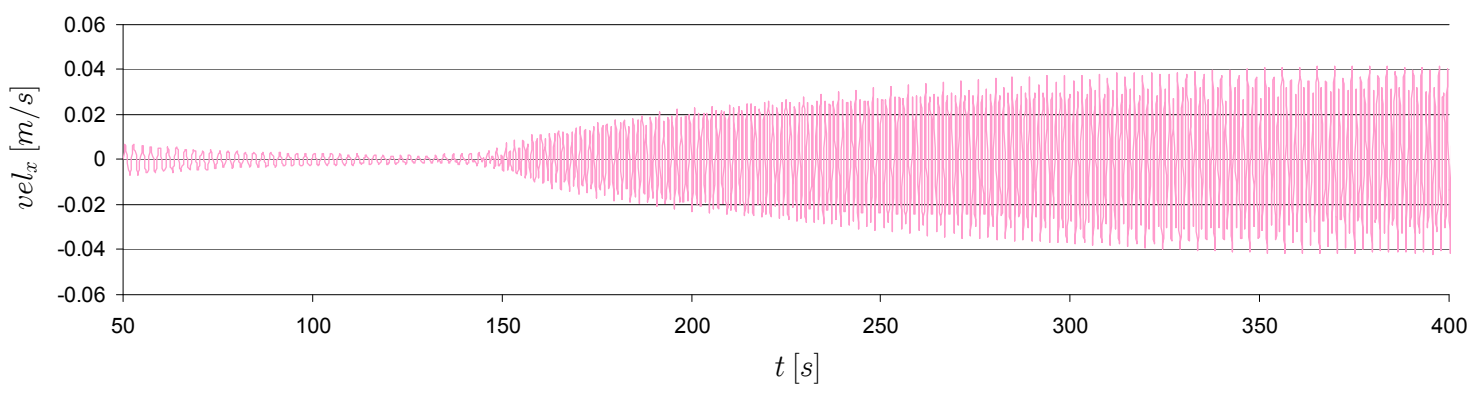

Figura A.245. Componente de la velocidad en la dirección del flujo

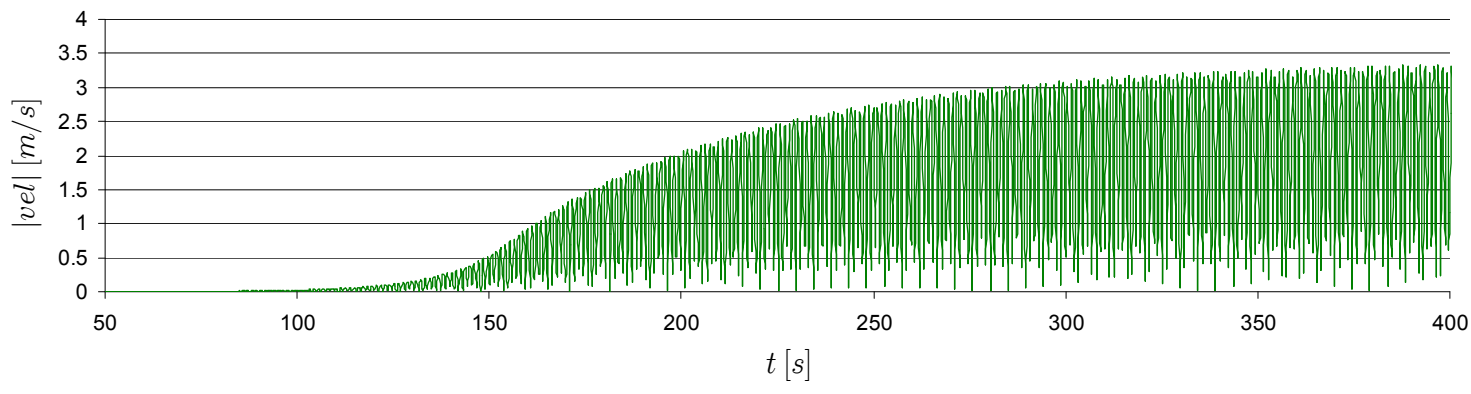

Figura A.246. Módulo de la velocidad 


\section{Anexo K. Curvas de Lissajous}

Para el ejemplo de la estructura empotrada-libre de $34 \mathrm{~m}$ de altura y sobre la cual se realizó el estudio del fenómeno de lock-in y de amplificación del coeficiente de arrastre se muestran a continuación las curvas de Lissajous de la trayectoria del extremo libre para diferentes valores de la velocidad reducida. Se ha supuesto amortiguamiento estructural $0.1 \mathrm{y}$ un sólo plano de fluido en el extremo libre.

La estructura se somete a la acción del viento durante $200 \mathrm{~s}$, entre $t=100 \mathrm{~s}$ y $t=300 \mathrm{~s}$. Transcurrido ese tiempo cesa la acción del viento y se estudia la respuesta libre de la estructura. Notar que durante los primeros $100 \mathrm{~s}$ la aplicación sólo resuelve el campo fluido con objeto de obtener una buena solución del fluido. Una vez que se ha obtenido esa solución, se puede resolver el problema acoplado de interacción fluido-estructura.

En la Figura A.247 se muestra el desplazamiento del extremo libre en la dirección en la que actúa el aire y en la Figura A.248 en la dirección transversal para $U_{r}=7$.

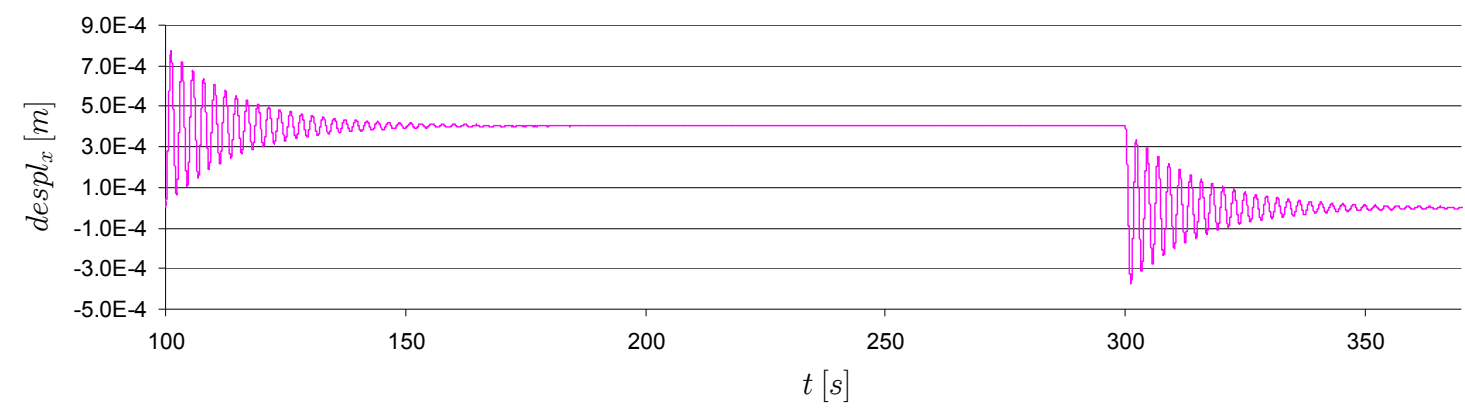

Figura A.247. Desplazamiento del extremo libre según la dirección del flujo

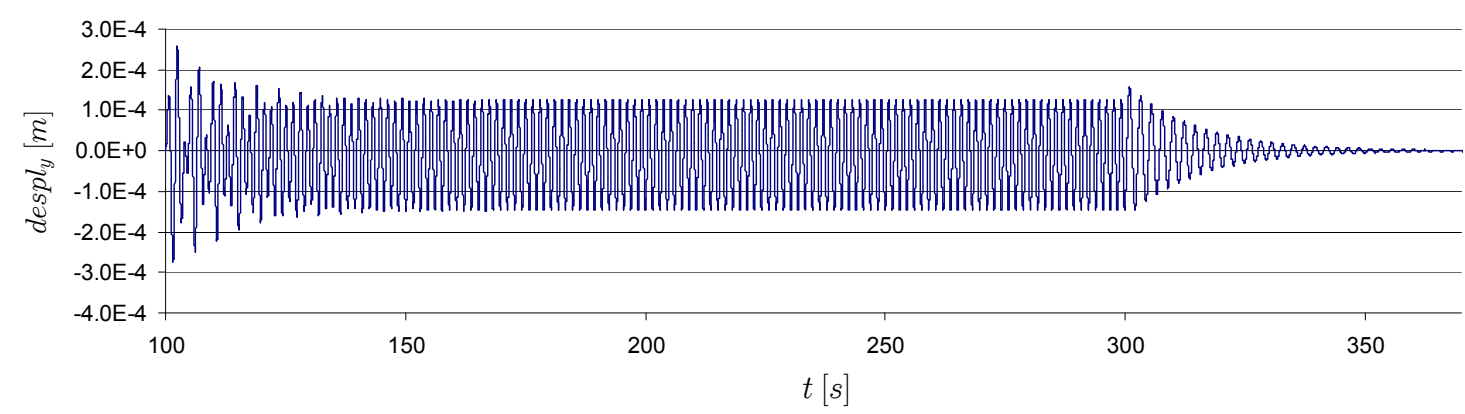

Figura A.248. Desplazamiento transversal del extremo libre 
En la Figura A.249 a la Figura A.251 se muestra la evolución en el tiempo de la trayectoria del extremo libre de la estructura mientras existe la acción del viento. Se observa que a partir de un determinado momento en el tiempo la estructura alcanza una nueva posición de equilibrio en el eje $O x$ respecto de la cual vibra.

Finalmente, en la Figura A.252 se muestra la trayectoria del extremo libre de la estructura una vez que ha cesado la acción del viento. Se observa cómo la estructura tiende a recuperar la posición de equilibrio inicial.

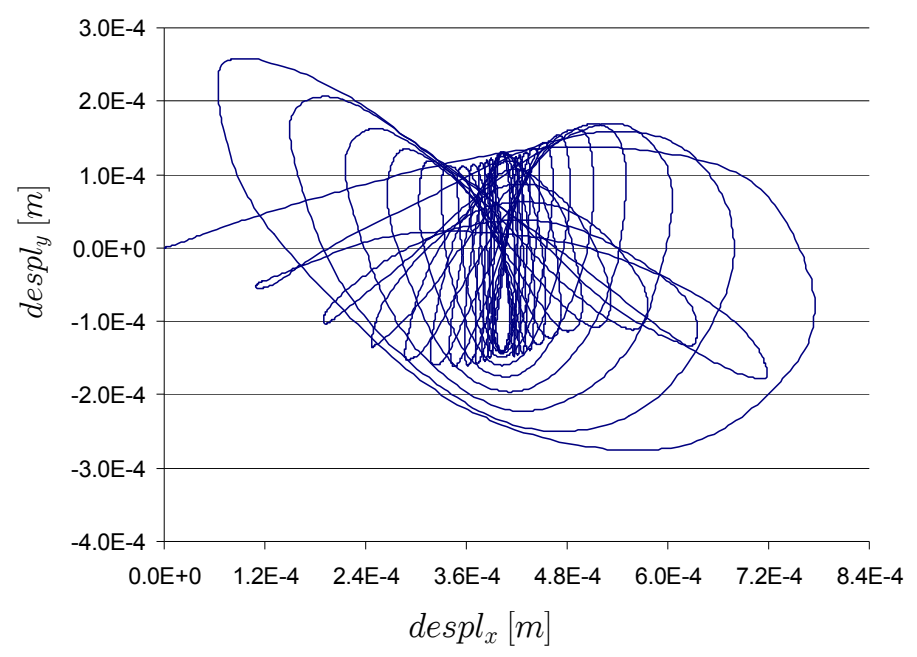

Figura A.249. Trayectoria del extremo libre en el intervalo de tiempo $100<t<150 \mathrm{~s}$

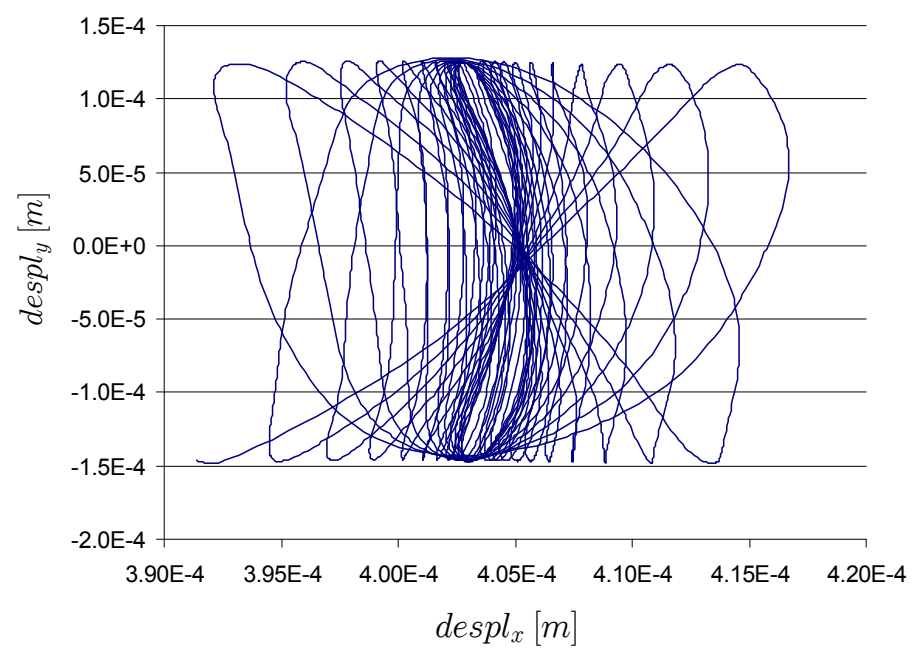

Figura A.250. Trayectoria del extremo libre en el intervalo de tiempo $150<t<250 \mathrm{~s}$ 


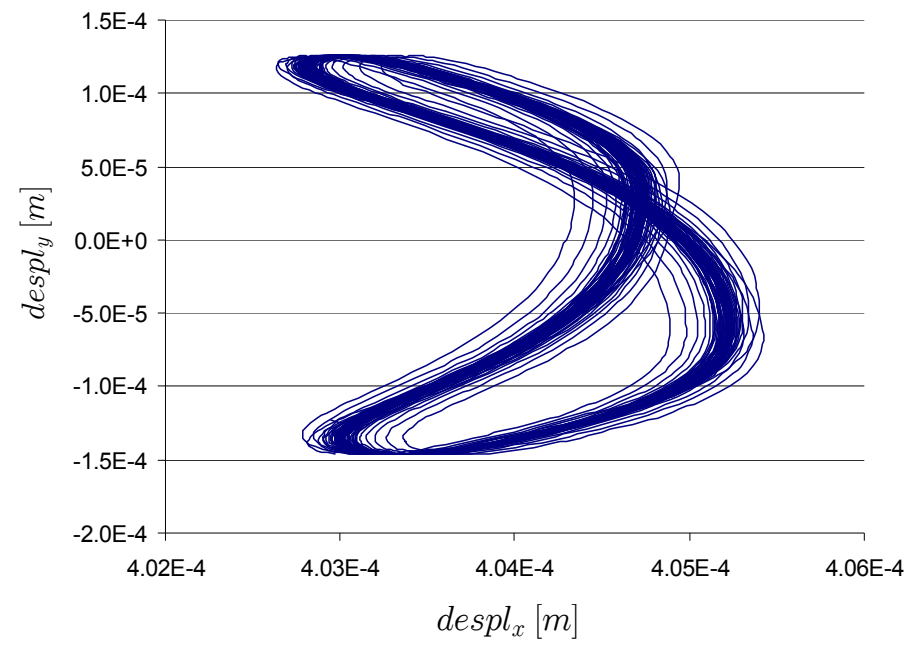

Figura A.251. Trayectoria del extremo libre en el intervalo de tiempo $200<t<300 \mathrm{~s}$

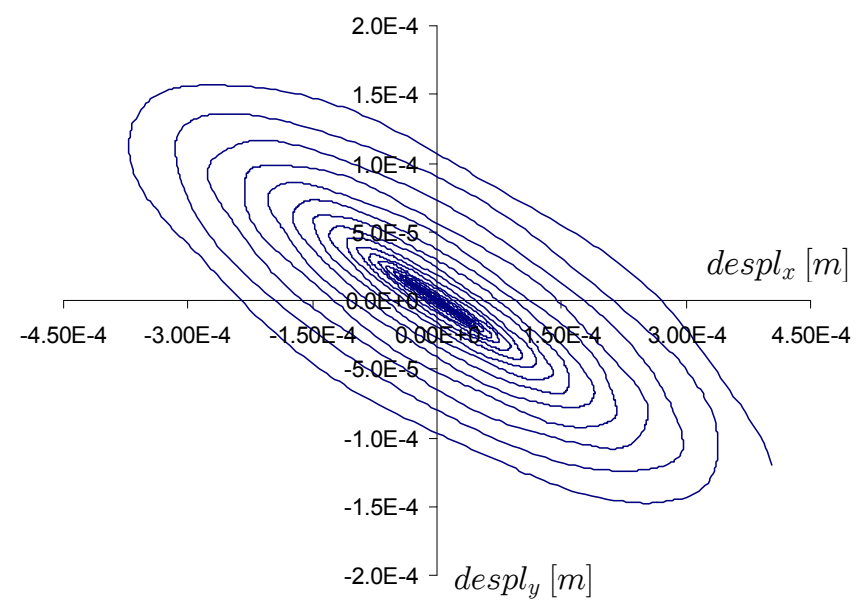

Figura A.252. Trayectoria del extremo libre para $t>300 \mathrm{~s}$

De forma análoga se muestran a continuación las curvas obtenidas para $U_{r}=5 \mathrm{y} U_{r}=3.5$.

- $U_{r}=5$

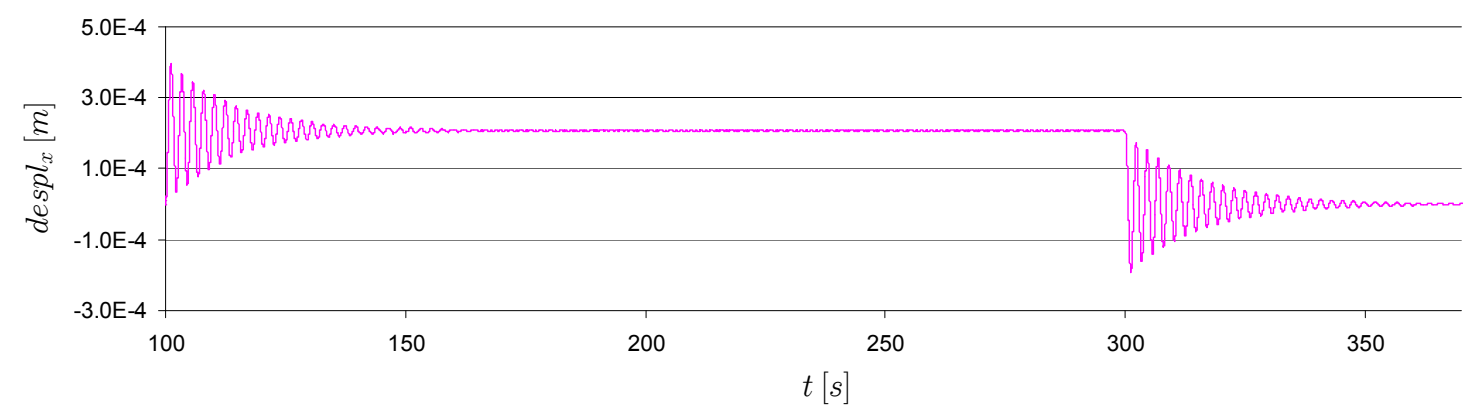

Figura A.253. Desplazamiento del extremo libre según la dirección del flujo 


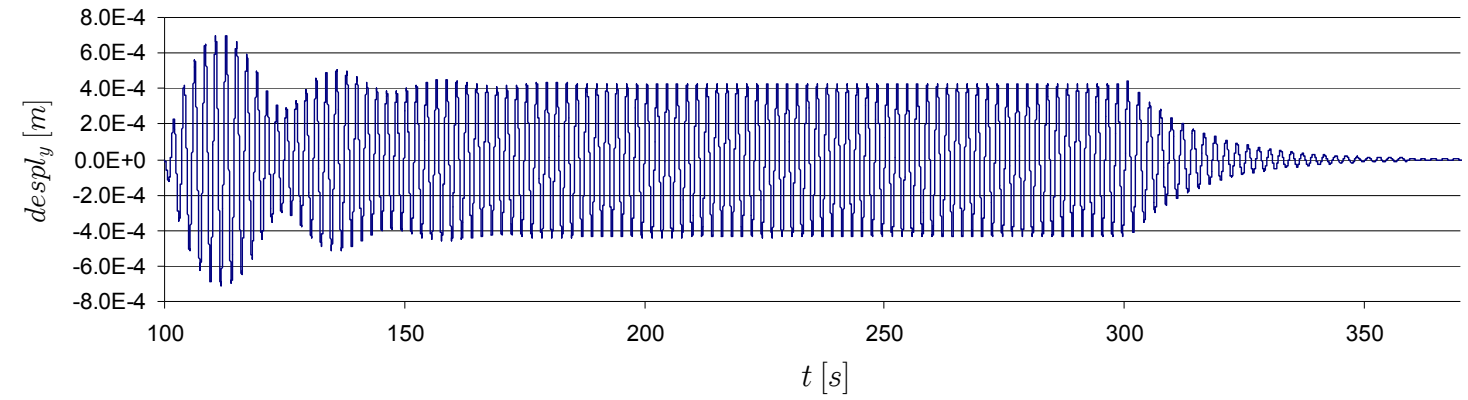

Figura A.254. Desplazamiento transversal del extremo libre

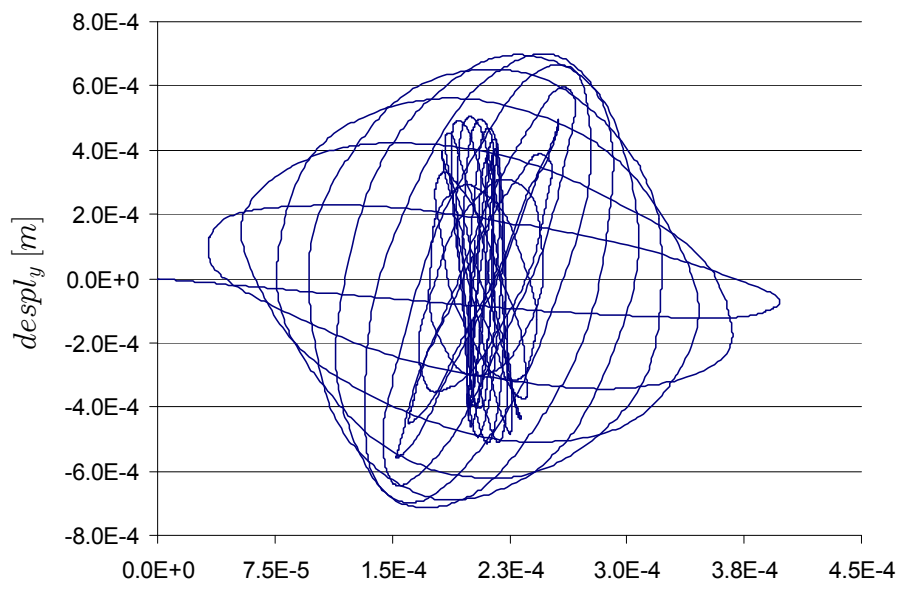

Figura A.255. Trayectoria del extremo libre en el intervalo de tiempo $100<t<150 \mathrm{~s}$

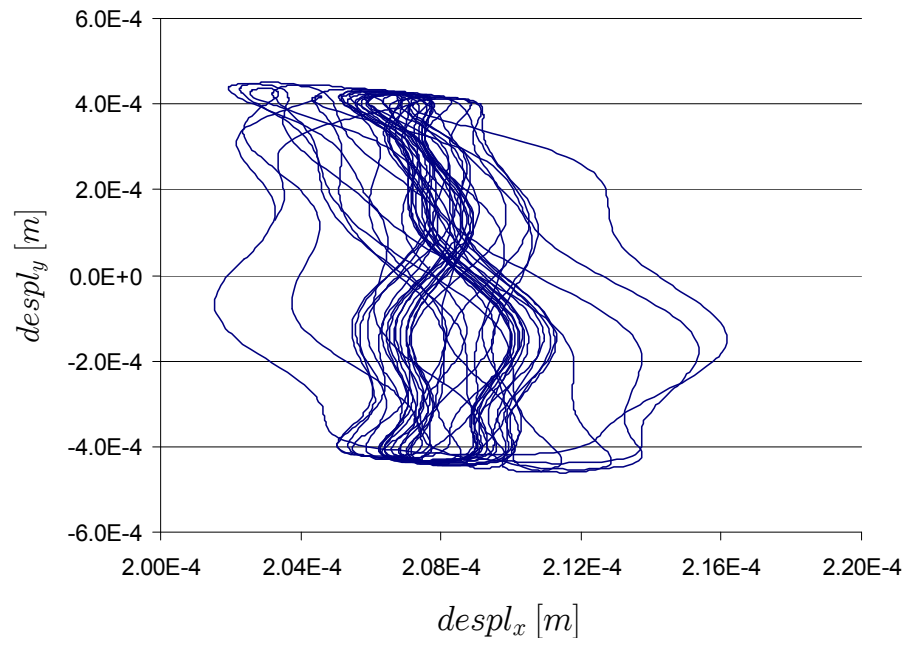

Figura A.256. Trayectoria del extremo libre en el intervalo de tiempo $150<t<250 \mathrm{~s}$ 


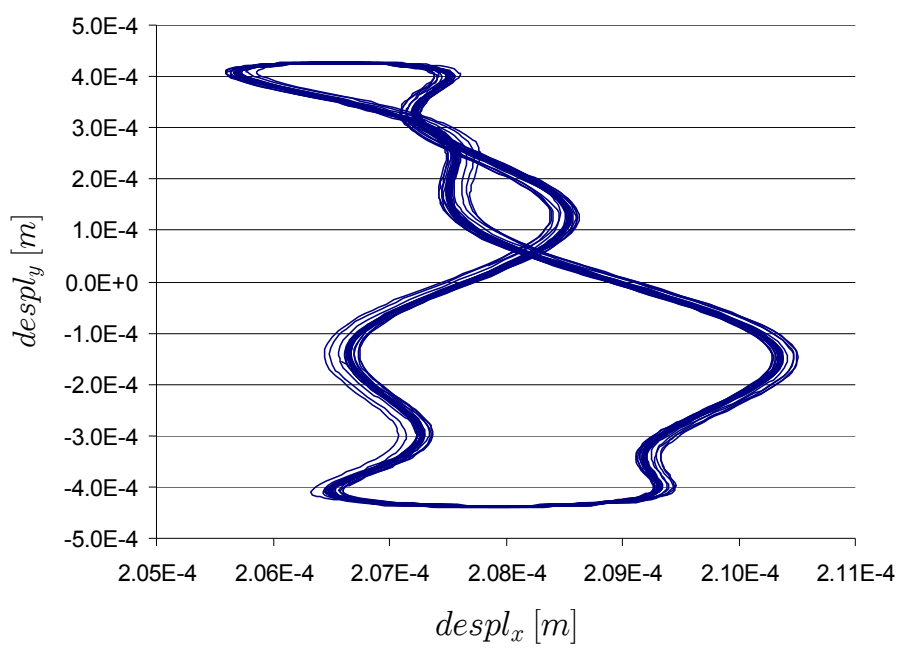

Figura A.257. Trayectoria del extremo libre en el intervalo de tiempo $200<t<300 \mathrm{~s}$

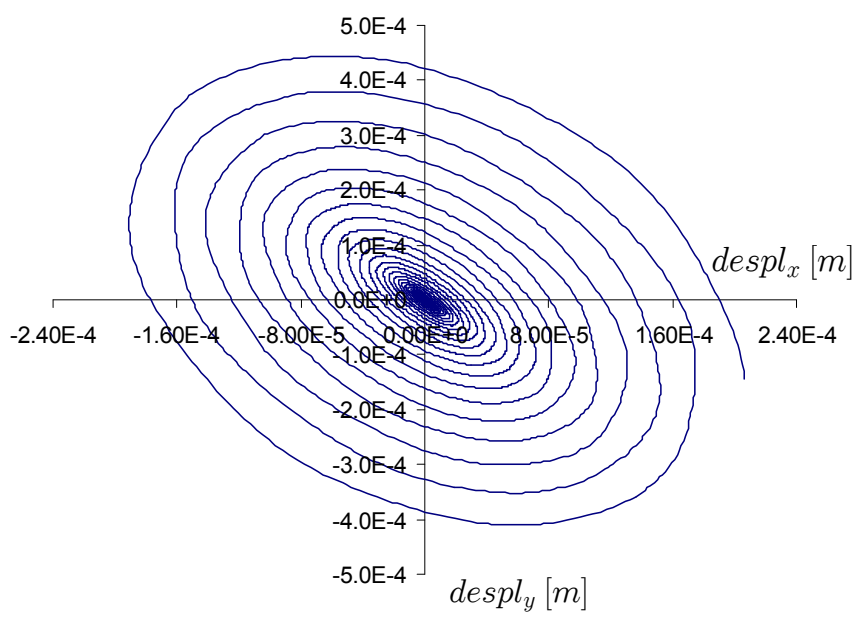

Figura A.258. Trayectoria del extremo libre para $t>300 \mathrm{~s}$

- $U_{r}=3.5$

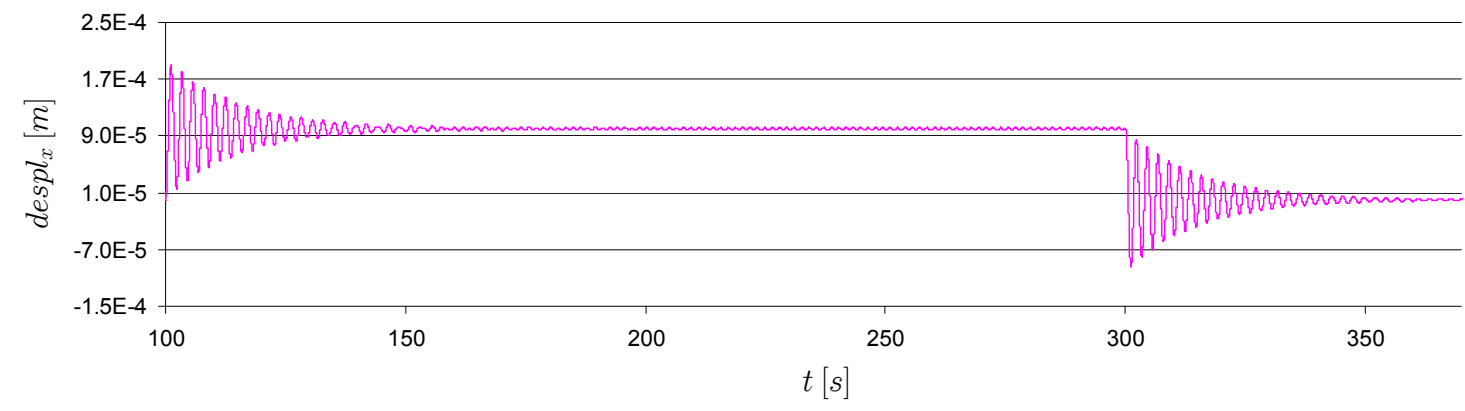

Figura A.259. Desplazamiento del extremo libre según la dirección del flujo 


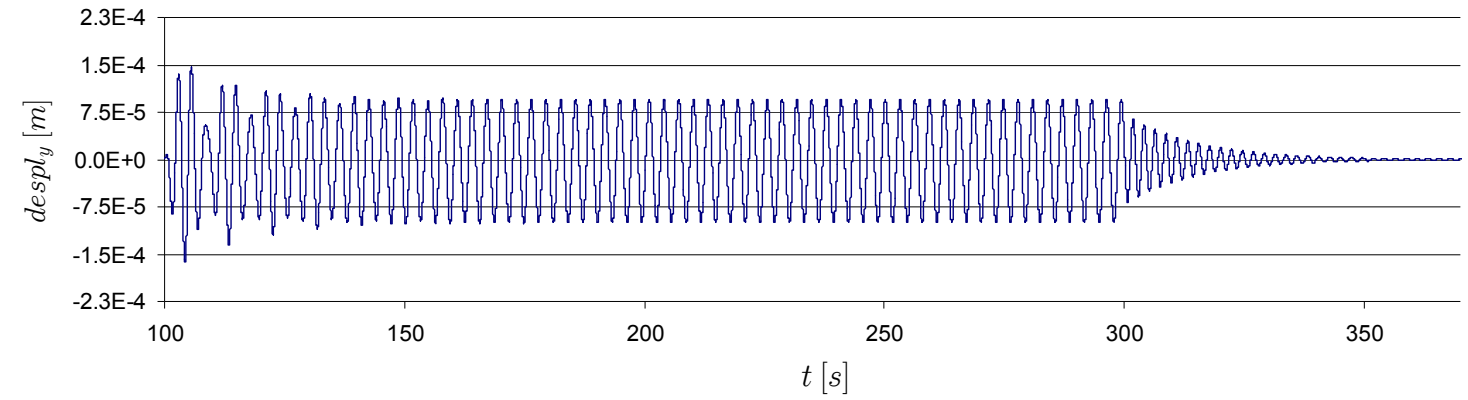

Figura A.260. Desplazamiento transversal del extremo libre

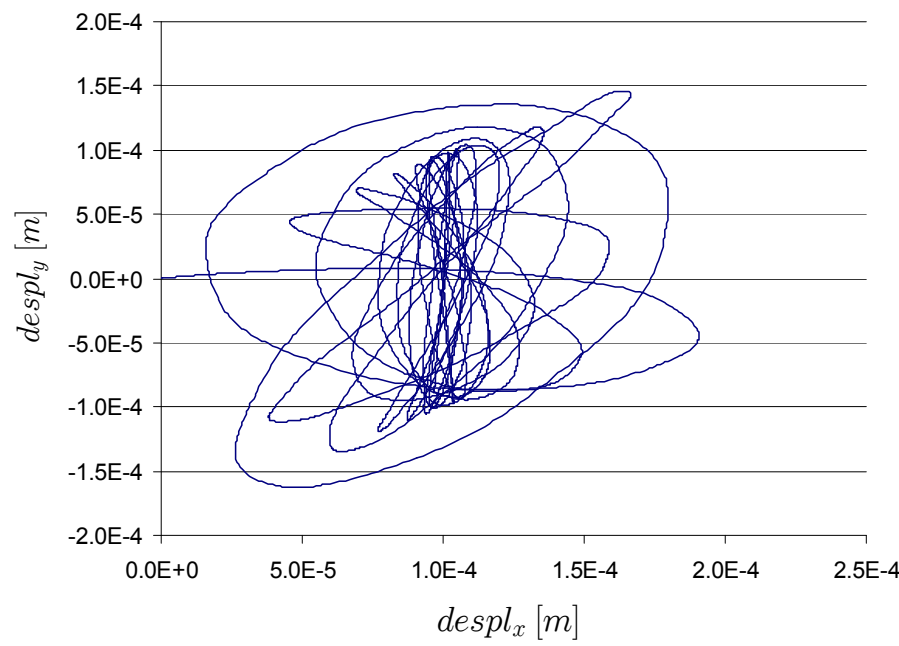

Figura A.261. Trayectoria del extremo libre en el intervalo de tiempo $100<t<150 \mathrm{~s}$

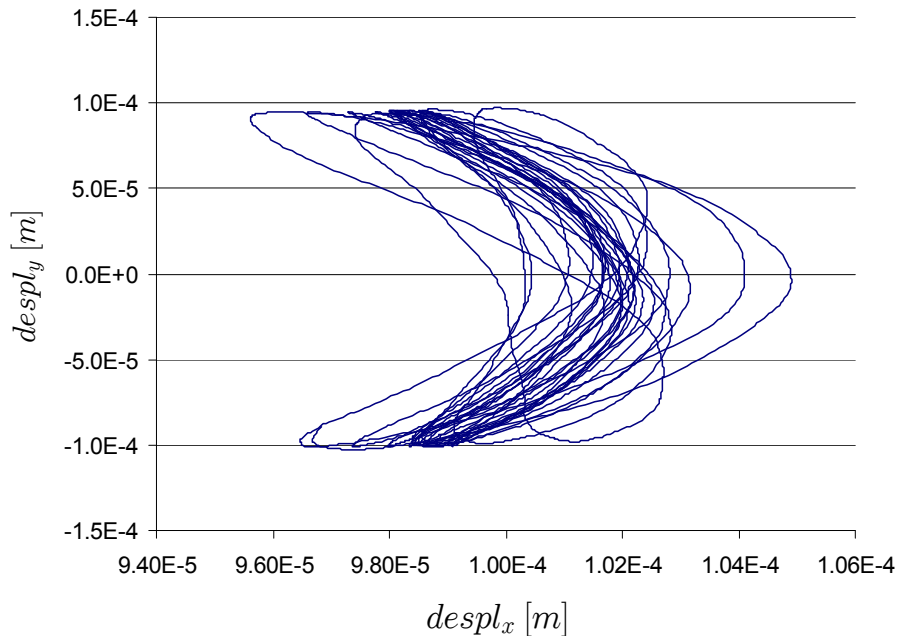

Figura A.262. Trayectoria del extremo libre en el intervalo de tiempo $150<t<250 s$ 


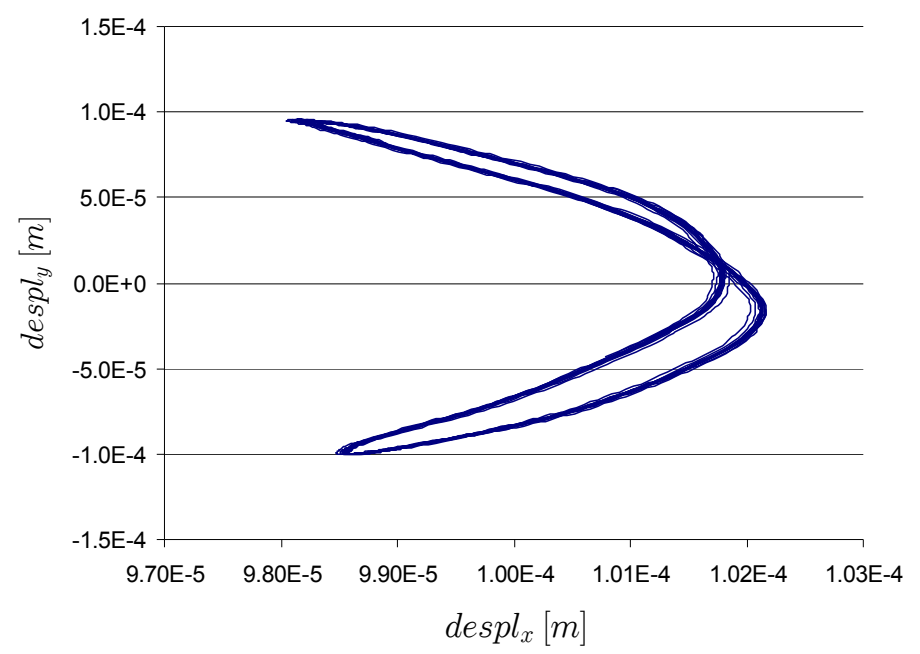

Figura A.263. Trayectoria del extremo libre en el intervalo de tiempo $200<t<300 \mathrm{~s}$

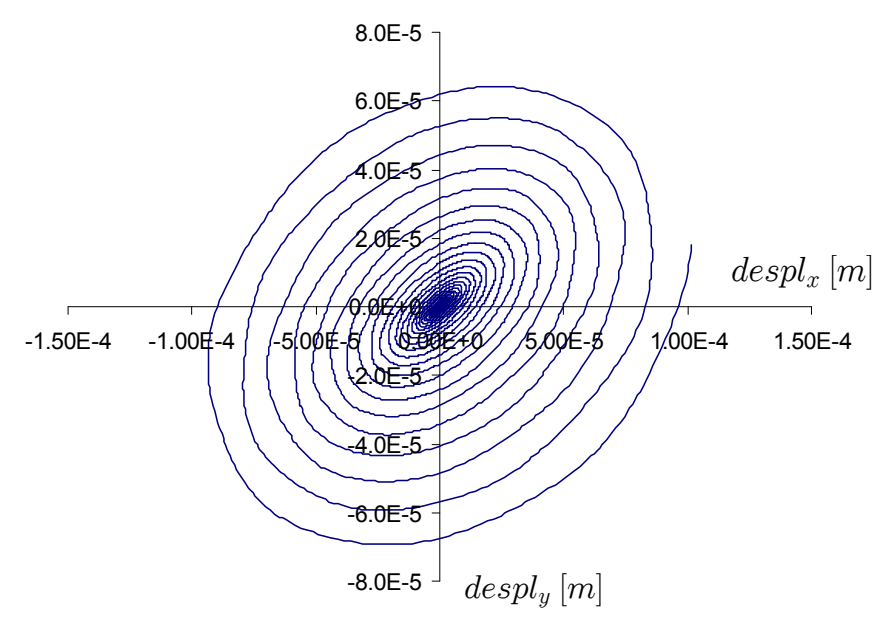

Figura A.264. Trayectoria del extremo libre para $t>300 \mathrm{~s}$

Finalmente, en la Figura A.265 se ha representado la respuesta en amplitud en función de la velocidad reducida y en la Figura A.266 se muestra una comparación de la trayectoria descrita por el extremo libre de la estructura para diferentes valores de la velocidad reducida y una vez que la estructura ha alcanzado la nueva posición de equilibrio respecto de la cual vibra.

Aunque para el valor del amortiguamiento estructural considerado en este ejemplo no se produce el fenómeno de lock-in, se comprueba que para $U_{r}=5$ se obtiene la mayor amplitud de la vibración de la estructura ya que para esa velocidad reducida la frecuencia de desprendimiento de los torbellinos está muy próxima a la frecuencia natural de la misma. También se observa que a 
medida que aumenta la velocidad del flujo se incrementa el desplazamiento de la estructura en la dirección éste, produciéndose la vibración transversal en cotas más alejadas del eje $O x$.

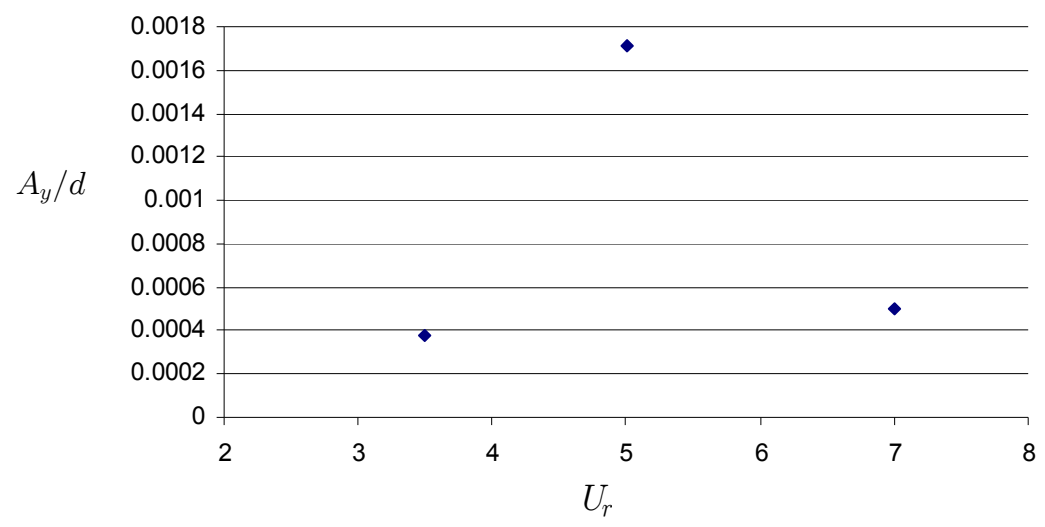

Figura A.265. Respuesta en amplitud de la estructura frente a la velocidad reducida

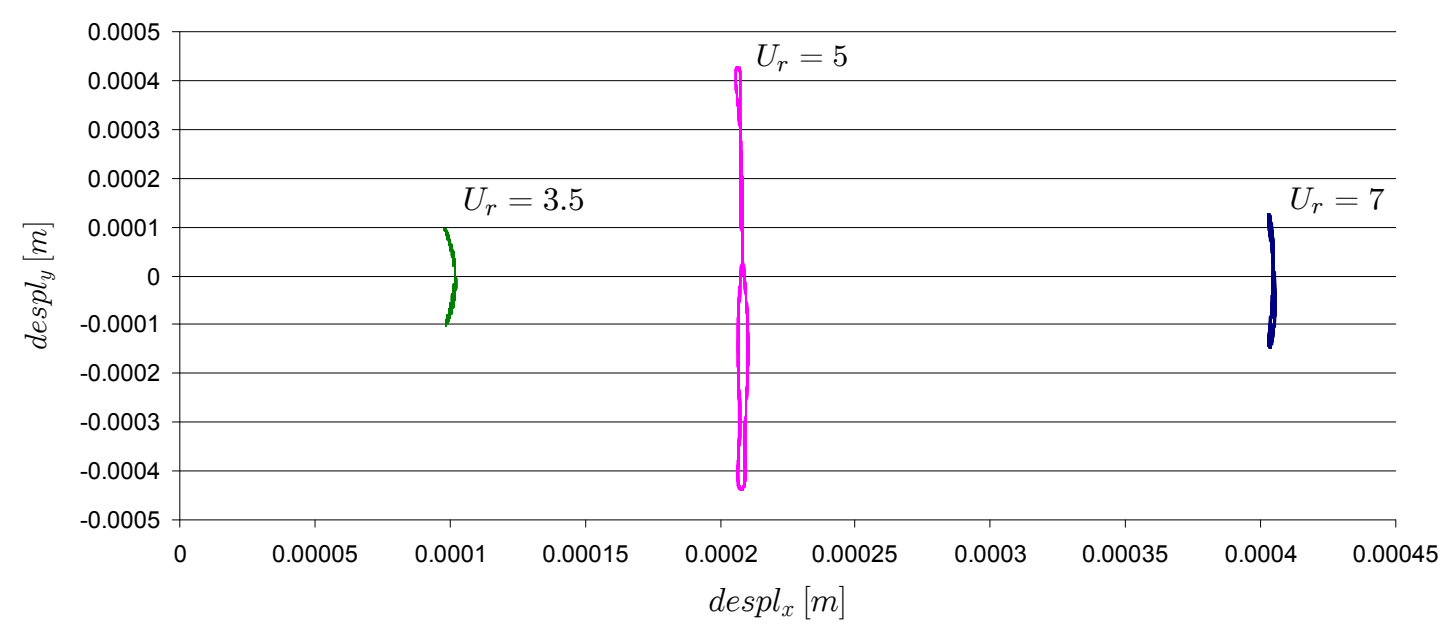

Figura A.266. Comparación de la trayectoria del extremo libre de la estructura para diferentes $U_{r}$ en el intervalo de tiempo $200<t<300 \mathrm{~s}$

A continuación se muestran las curvas de Lissajous en $2 \mathrm{D}$ y en 3D de la trayectoria que describen diferentes secciones de la estructura para $U_{r}=4, U_{r}=5.5$ y $U_{r}=13$. Se ha considerando el caso de amortiguamiento estructura 0.025 y un solo plano de fluido en el extremo libre.

Cabe destacar la notable diferencia que existe en la trayectoria que describe la estructura en función de la velocidad reducida. En la caso $U_{r}=4$ (Figura A.267 y Figura A.268) no se produce el fenómeno de lock-in mientras que para $U_{r}=5.5$ (Figura A.269 y Figura A.270) sí se produce. Para $U_{r}=13$ tiene lugar la amplificación del coeficiente de arrastre, lo cual se puede apreciar gráficamente en la Figura A.271 y en la Figura A.272. 
- $U_{r}=4$

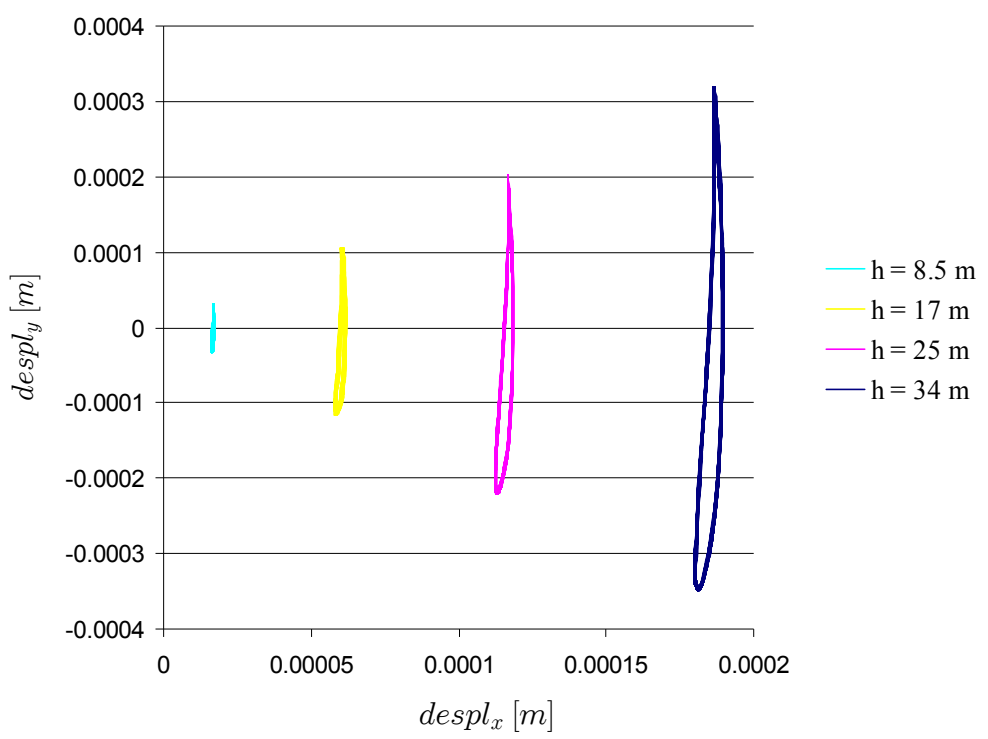

Figura A.267. Curvas de Lissajous 2D para $U_{r}=4$

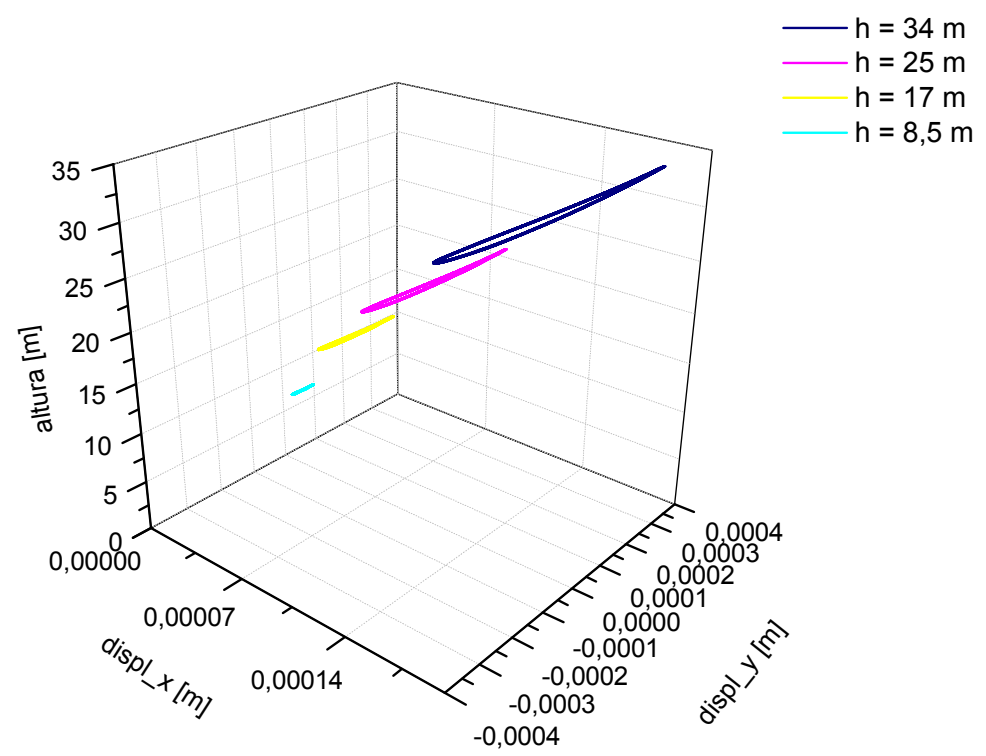

Figura A.268. Curvas de Lissajous 3D para $U_{r}=4$ 
- $U_{r}=5.5$

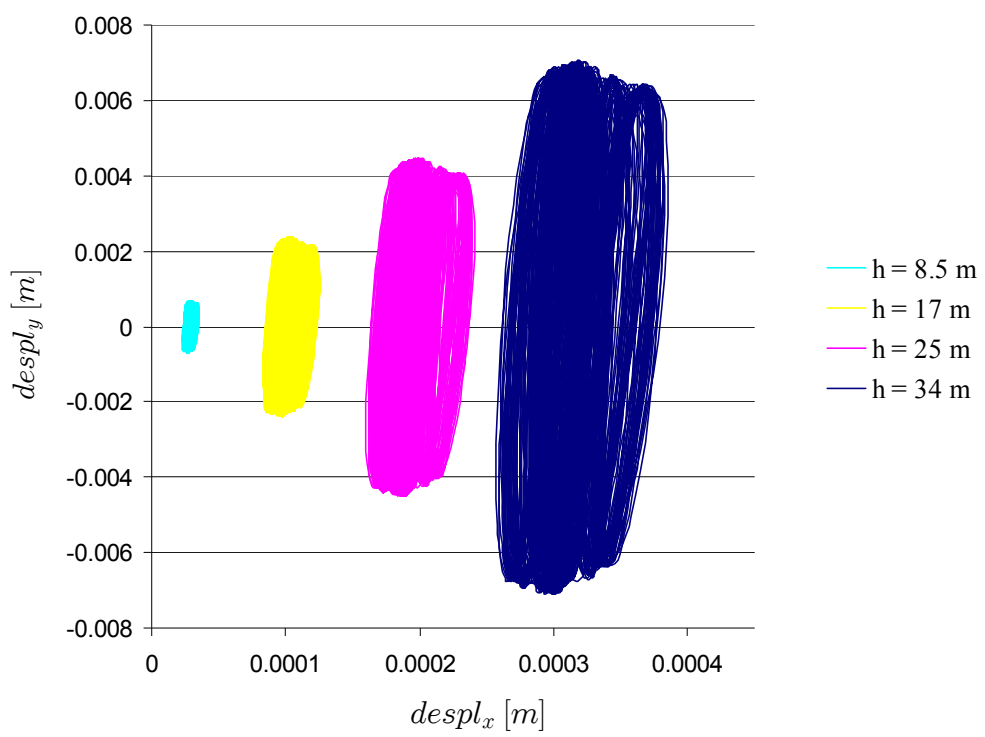

Figura A.269. Curvas de Lissajous 2D para $U_{r}=5.5$

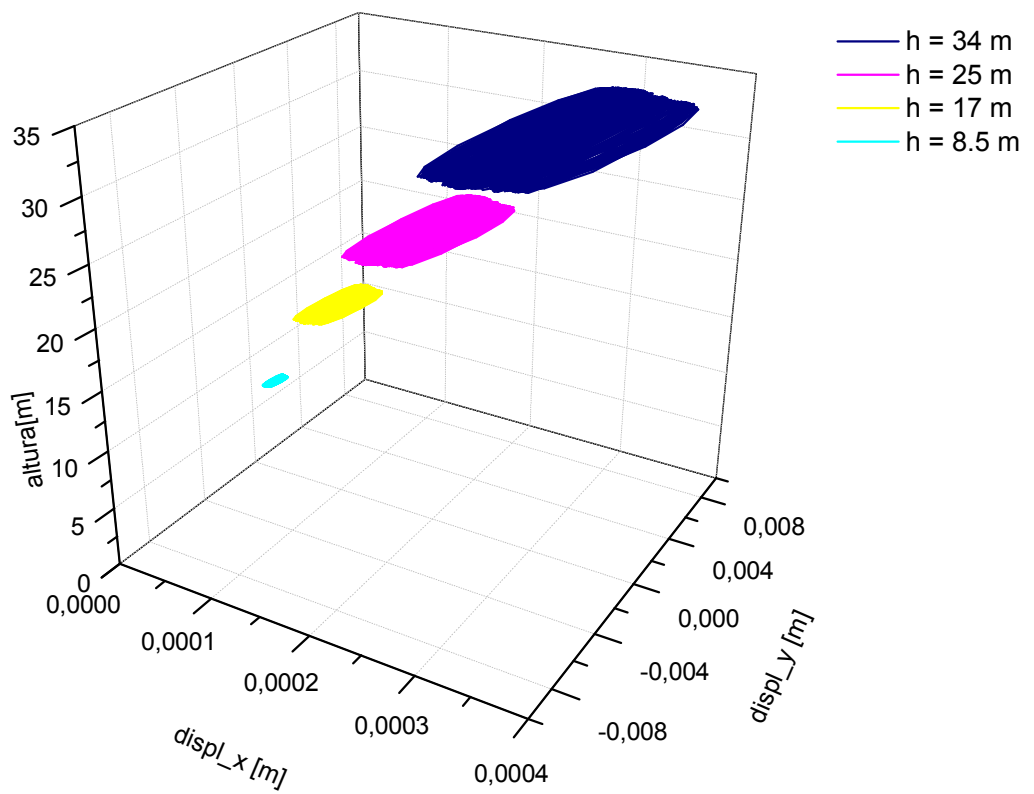

Figura A.270. Curvas de Lissajous 3D para $U_{r}=5.5$ 
- $U_{r}=13$

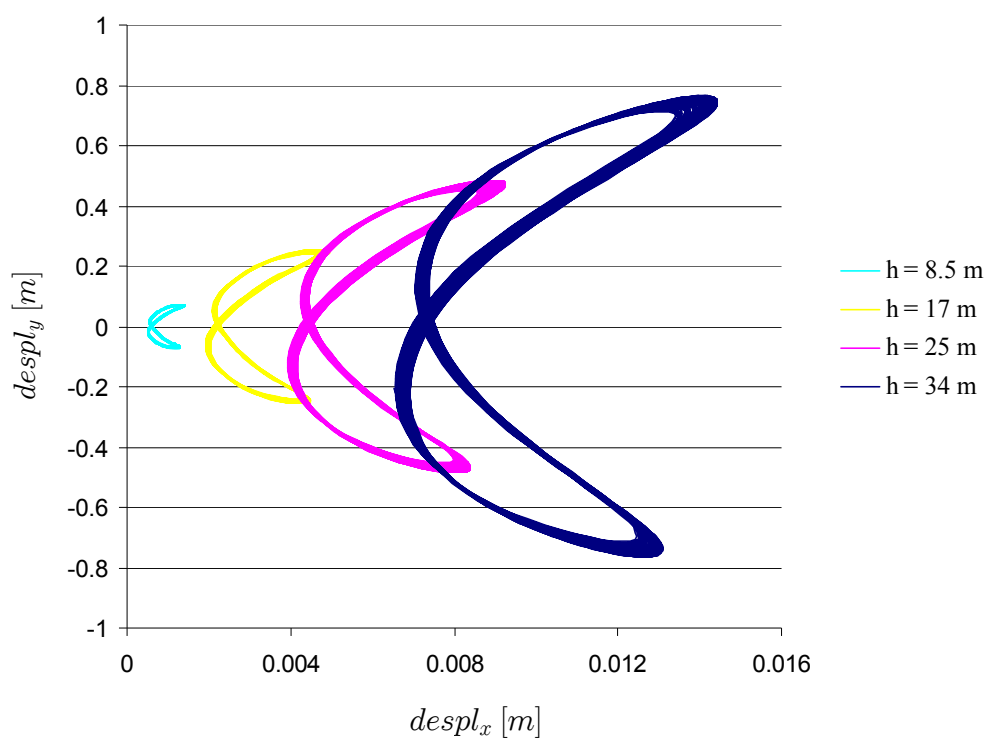

Figura A.271. Curvas de Lissajous 2D para $U_{r}=13$

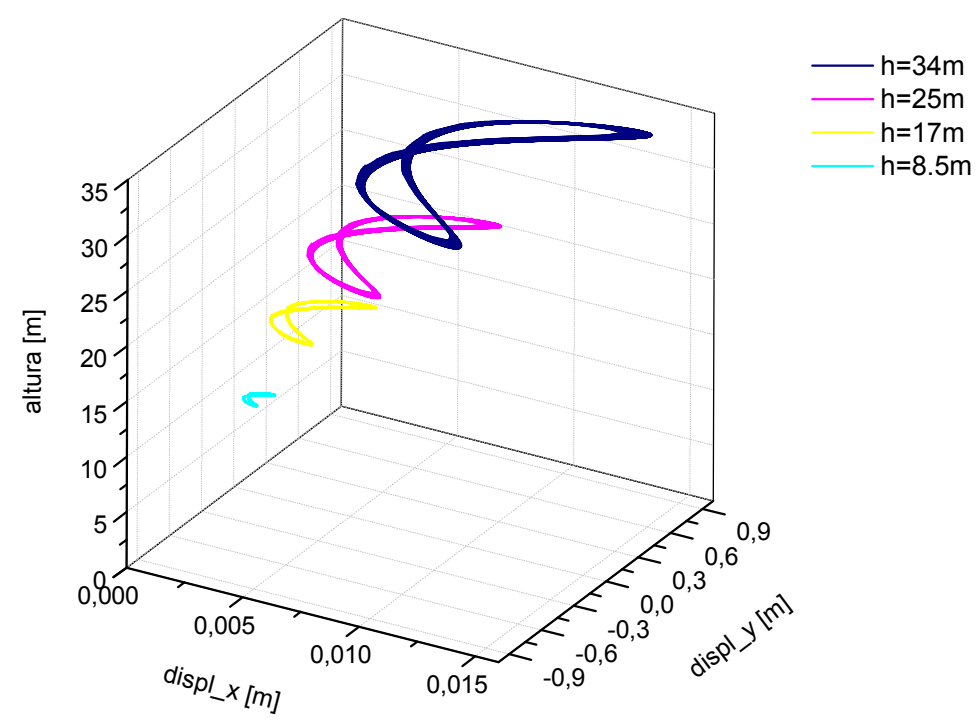

Figura A.272. Curvas de Lissajous 3D para $U_{r}=13$ 
Por último, en las figuras que se muestran a continuación se han representado las curvas de Lissajous para el caso de amortiguamiento estructural 0.1 y 8 planos de fluido.

- $U_{r}=4.5$

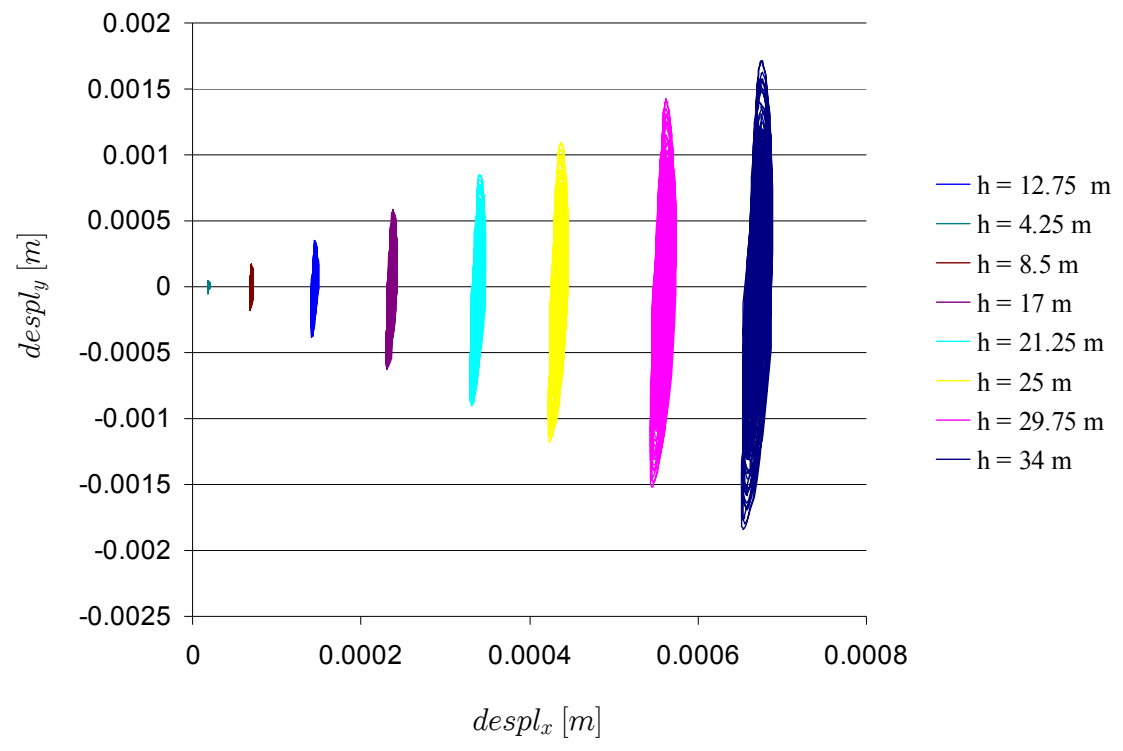

Figura A.273. Curvas de Lissajous 2D para $U_{r}=4.5$

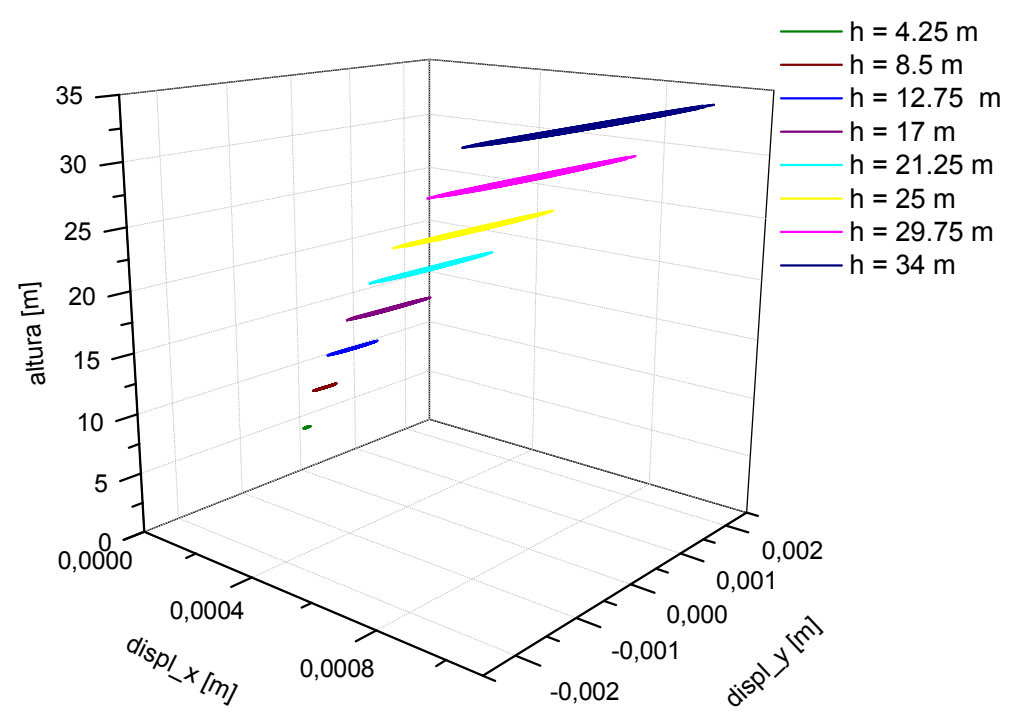

Figura A.274. Curvas de Lissajous 3D para $U_{r}=4.5$ 
- $U_{r}=5$

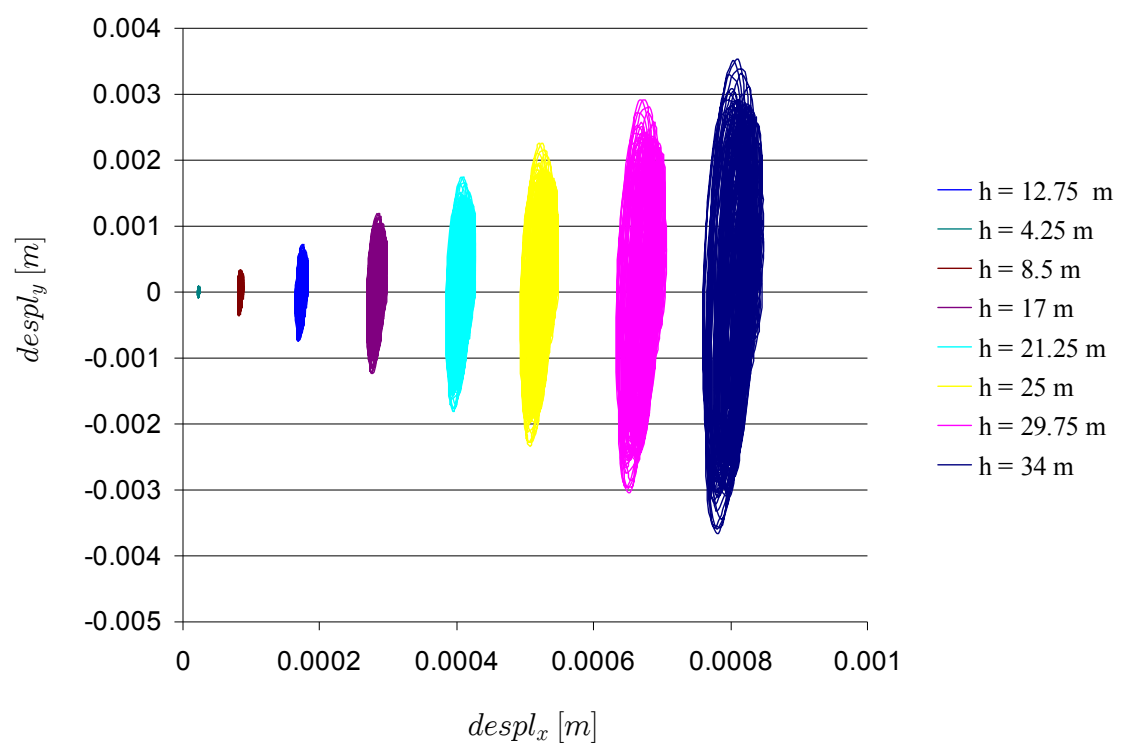

Figura A.275. Curvas de Lissajous 2D para $U_{r}=5$

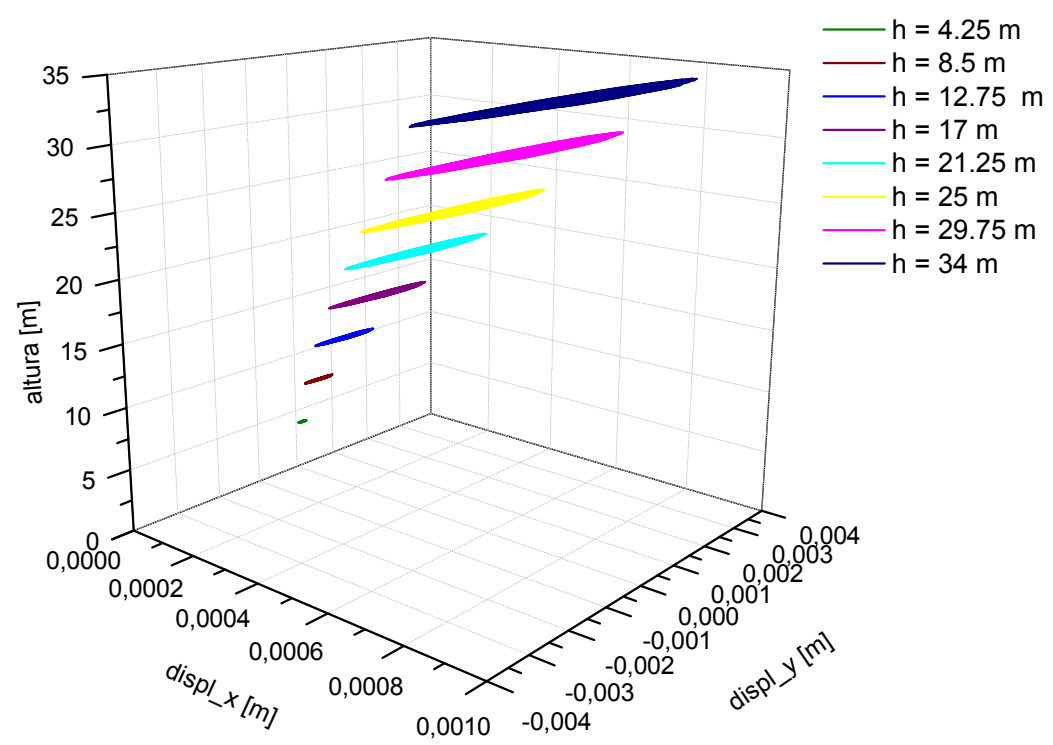

Figura A.276. Curvas de Lissajous 3D para $U_{r}=5$ 
- $U_{r}=7$

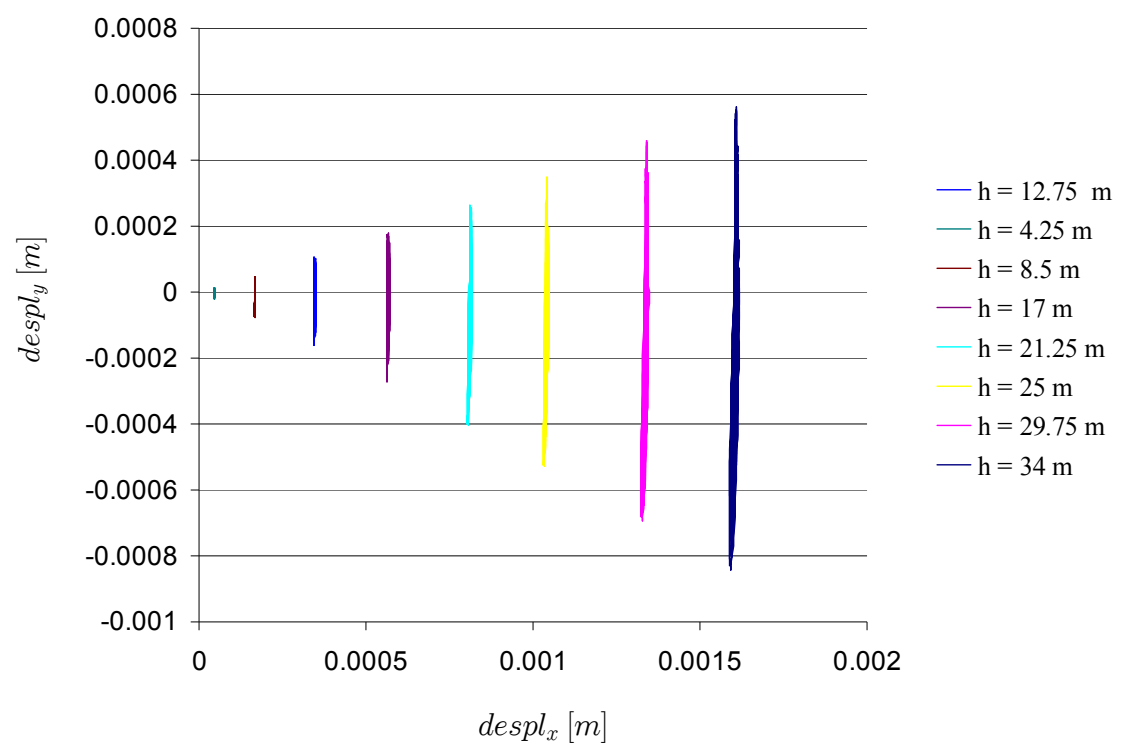

Figura A.277. Curvas de Lissajous 2D para $U_{r}=7$

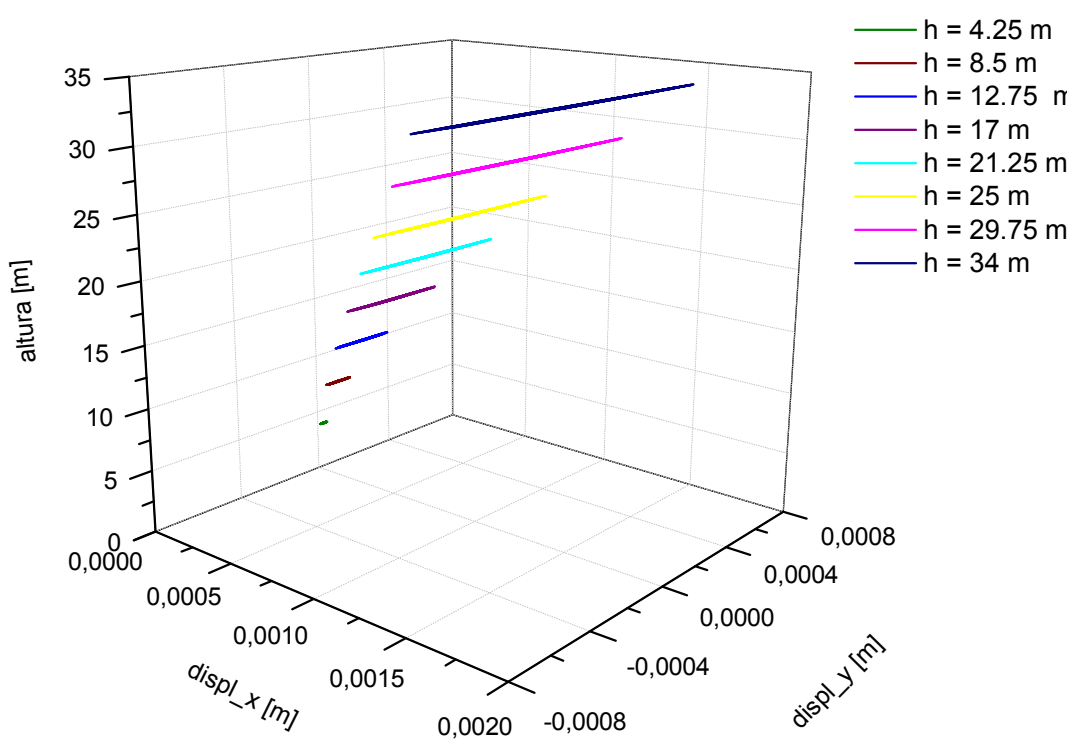

Figura A.278. Curvas de Lissajous 3D para $U_{r}=7$ 
- $U_{r}=13$

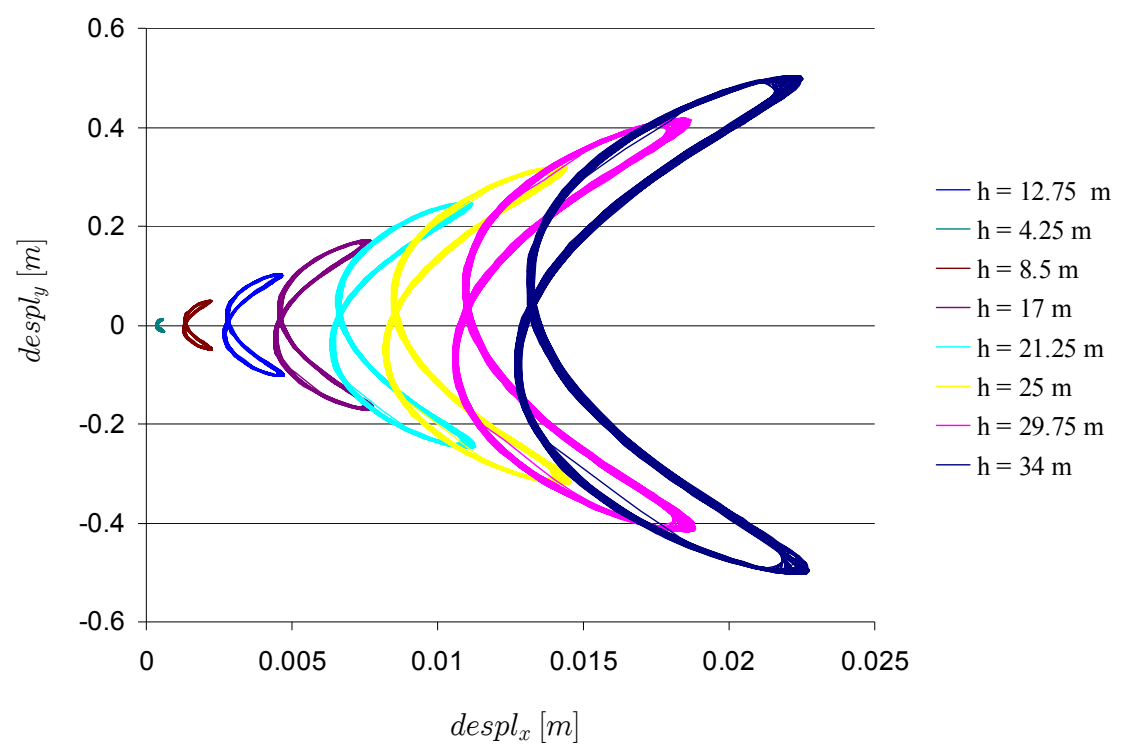

Figura A.279. Curvas de Lissajous 2D para $U_{r}=13$

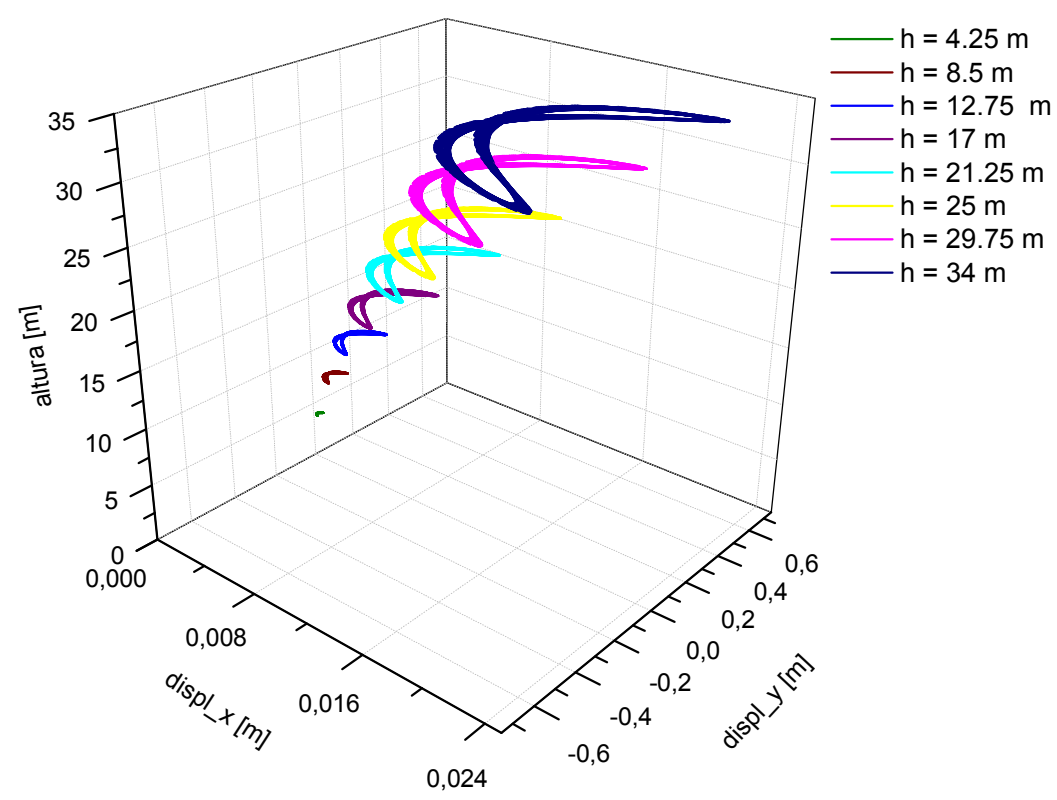

Figura A.280. Curvas de Lissajous 3D para $U_{r}=13$ 



\section{Referencias}

${ }^{1}$ Jung, S.N., Nagaraj, V.T., y Chopra, I., "Refined Structural Model for Thin- and Thick- Walled Composite Rotor Blades" American Institute of Aeronautics and Astronautics Journal, 40 (1), pp. 105-116, 2002.

${ }^{2}$ Chandra, R., y Chopra, I., "Experimental and Theoretical Analysis of Composite I Beams with Elastic Couplings", American Institute of Aeronautics and Astronautics Journal, 29 (12), pp. 2197 2206, 1991.

${ }^{3}$ Chandra, R., Stemple, D., y Chopra, I., "Thin-Walled Composite Beams Under Bending, Torsional, and Extensional Loads", Journal of Aircraft, 27 (7), pp. 619-626, 1990. 
